\title{
Eine kopfgesteuerte Phrasenstrukturgrammatik für spanische Nominalphrasen
}

Dissertation zur Erlangung des Doktorgrades der Philosophischen Fakultät der Georg-August-Universität zu Göttingen

\author{
vorgelegt von \\ Arne Reimar Kirchner \\ aus Concepción / Chile
}

Göttingen, im November 1999 
Inhaltsverzeichnis $\quad$ i

Abkürzungen iv

I. Einleitung 1

II. Einführung in die HPSG 6

1. Vorbemerkungen 6

$\begin{array}{lll}\text { 1.1. Ziele der Sprachbeschreibung } & 6\end{array}$

1.2. Syntaxtheorie 9

1.3. Kontextfreie Phrasenstrukturgrammatik (kf PSG) 13

1.4. Erweiterte PSG 16

$\begin{array}{ll}\text { 1.5. Die HPSG als Unifikationsgrammatik } & 18\end{array}$

2. Sprache als ein System von Zeichen: die HPSG 21

2.1. Merkmalstrukturen oder Attribut-Werte-Paare 21

2.2. Mathematische Eigenschaften von Merkmalstrukturen 24

2.2.1. Subsumtion und Extension 25

2.2.2. Unifikation 26

2.2.3. Disjunktion, Negation und Implikation 29

2.2.4. Sorten und Sortenhierarchie 30

2.3. Die syntaktische Struktur sprachlicher Zeichen 31

2.3.1. Köpfe, Kopfmerkmale und Kopftöchter 31

$\begin{array}{lll}\text { 2.3.2. } & \text { Subkategorisierung } & 34\end{array}$

2.3.3. Syntaktische Kategorien und Schemata 36

2.4. Konstituentenabfolge 45

$\begin{array}{lll}\text { 2.5. Subjekte } & 47\end{array}$

2.6. Semantik 52

2.6.1. Individuen, Sachverhalte und Situationen 52

2.6.2. Übersetzung in den Formalismus der Merkmalstrukturen 55

$\begin{array}{ll}\text { 2.6.3. Semantisches Prinzip } & 57\end{array}$

$\begin{array}{ll}\text { 2.6.4. Kongruenz } & 58\end{array}$

$\begin{array}{lll}\text { 2.7. Adjunkte } & 60\end{array}$

$\begin{array}{lll}\text { 2.8. Quantifizierer } & 64\end{array}$

$\begin{array}{lll}\text { 2.9. Possessiva } & 69\end{array}$

$\begin{array}{ll}\text { 3. Zusammenfassung } & \mathbf{7 1}\end{array}$ 
III. Die Nominalphrase 72

1. Das Nomen ohne Determinierer $\quad 72$

$\begin{array}{lll}\text { 1.1. Die Syntax } & 75\end{array}$

$\begin{array}{lll}\text { 1.2. Die Semantik } & 77\end{array}$

1.3. Integration in eine HPSG-basierte Deskription 84

1.4. Das Pronomen 85

$\begin{array}{llr}\text { 2. Determinierer } & 87\end{array}$

2.1. Klassifikation nach Alvarez Martínez (1986) 91

2.2. Die Klassifikationskriterien 98

$\begin{array}{ll}\text { 2.2.1. Genus und Numerus } & 98\end{array}$

2.2.2. Adverbialisierung 100

2.2.3. Komparation 101

2.2.4. Prädikation 102

2.2.5. Substantivierbarkeit 103

2.2.6. Quantifikation 109

2.2.7. Vor- und Nachstellung 111

$\begin{array}{ll}\text { 2.3. Kookkurrenz } & 114\end{array}$

2.3.1. Kookkurrenz nach Prado Ibán (1993) 114

2.3.2. Eine erste umfassendere Klassifikation 116

2.3.3. Klassifikation nach Allegranza (1998) und Quirk u.a. (1985) 120

2.3.4. Zentral- und Postdeterminierer 123

2.3.5. Abstufende Klassifikation 128

2.4. Semantische Aspekte 135

2.4.1. Demonstrativa 136

2.4.2. Possessiva 138

2.4.3. Die Artikel 139

2.4.4. Indefinitpronomina 143

$\begin{array}{ll}\text { 2.5. Semantisches und Kookkurrenz } & 145\end{array}$

2.6. Gesamtklassifikation 152

2.6.1. Klassifikationsmethode 156

2.6.2. Anwendung des Klassifikationsverfahrens 160

$\begin{array}{lll}\text { 2.7. } & 172\end{array}$

2.8. Exkurs: Vergleich mit dem deutschen Determinierersystem 175 
3. Spanische Determinierer und Nomina in der HPSG 183

$\begin{array}{lll}\text { 3.1. Valenz } & 184\end{array}$

$\begin{array}{ll}\text { 3.2. Kongruenz } & 194\end{array}$

3.3. Das Lexikon 199

3.3.1. Die Wortart Determinierer $\quad 199$

3.3.2. Die Wortart Nomen 203

$\begin{array}{lll}\text { 3.4. Wortstellung } & 204\end{array}$

3.5. Ein X-bar-Schema für die spanische NP 208

$\begin{array}{ll}\text { 3.6. Lexikoneinträge } & 210\end{array}$

3.7. Lexikalische Regeln 217

3.8. Die Struktur der NP 221

3.9. Die Semantik 222

4. Adjektive 22

4.1. Qualifizierende Adjektive in spanischen Grammatiken 231

4.2. Syntax von Adjektiven 233

4.3. Die Stellung nach Braselmann (1993) und Dietrich (1993) 235

4.4. Die Stellung nach Pelzing (1981) 240

4.5. Bipositionale Adjektive 242

4.6. Adjektive in der HPSG 245

$\begin{array}{ll}\text { 5. Präpositionen } & \mathbf{2 4 8}\end{array}$

IV. Zusammenfassung $\quad 253$

$\begin{array}{ll}\text { V. Bibliographie } & \mathbf{2 6 0}\end{array}$

$\begin{array}{lr}\text { VI. Das Korpus } & 274\end{array}$

1. Die Quellen 274

$\begin{array}{ll}\text { 2. Zuordnung der Beispiele } & 277\end{array}$

$\begin{array}{lr}\text { Namenindex } & 279\end{array}$

$\begin{array}{ll}\text { Sachindex } & 283\end{array}$ 


\section{Abkürzungen}

$1 / 2 / 3 \quad 1 . / 2 . / 3$. Person

A Adjektiv

Adj Adjektiv (Sorte)

adj_Det adjektivische Determinierer (Sorte)

ADJ_SUBST adjektivtypische Substantivierung

ADJ-DTR adjunkt-daughter

ADV

Adverbial

AGR

agreement

AKK

Akkusativ

AN

Adjektiv-Nomen-Abfolge

ARG

Argument

ARG-S

argument-structure

ARG-SLOT

argument-slot

AUX

Auxiliar

CASE

Kasus

CAT

Kategorie

C-INDICES

context-indices

COMP Komplement

COMP-DTR Komplementtochter

CONT content

DAT Dativ

DECL Deklination

Det Determinierer (Sorte)

DET / D Determinierer

det_Adj determinierende Adjektive (Sorte)

DET_SUBST determinierertypische Substantivierung

DISQUANT distinguished-quantifier

DP Determiniererphrase

DTR(S) daughters / Töchter

en

Eigennamen (Sorte) 


\begin{tabular}{|c|c|}
\hline fem & feminin \\
\hline fin & finit \\
\hline G & Grammatik \\
\hline GB & Government and Binding Theory \\
\hline GEN & Genitiv \\
\hline GPSG & Generalized Phrase Structure Grammar \\
\hline head-adj-str & head-adjunct-structure \\
\hline head-comp-str & head-complement-structure \\
\hline HEAD-DTR & Kopftochter \\
\hline headed-str & headed-structure \\
\hline HPSG & Head-driven Phrase Structure Grammar \\
\hline IND & Index \\
\hline $\inf$ & Infinitiv \\
\hline INST & instance \\
\hline $\mathrm{kf}$ & kontextfrei \\
\hline KOMB_1/2 & Kombinationssteuerer $_{1 / 2}$ \\
\hline KOMP & Komparation \\
\hline KONJ & Konjunktion \\
\hline LEX & lexikalisch \\
\hline LF & Logical-Form \\
\hline LFG & Lexical-Functional Grammar \\
\hline LOC & local \\
\hline mask & maskulin \\
\hline MOD & Modifizierer \\
\hline $\mathrm{N}$ & Nomen \\
\hline $\mathrm{n}$ & numeral \\
\hline $\mathrm{N}_{\mathrm{n}}$ & numerales Nomen \\
\hline NA & Nomen-Adjektiv-Abfolge \\
\hline $\mathrm{NACH}$ & Nachstellung \\
\hline NFORM & noun form \\
\hline NOM & Nominativ \\
\hline NONLOC & non-local \\
\hline
\end{tabular}




\begin{tabular}{|c|c|}
\hline NORM & normal \\
\hline NP & Nominalphrase \\
\hline NS & Nachstellung \\
\hline NTR & Neutrum \\
\hline NUM / num & Numerus \\
\hline PER & Person \\
\hline per_num & Person_Numerus \\
\hline PFORM & preposition form \\
\hline PHON & Phonologie \\
\hline $\mathrm{pl}$ & Plural \\
\hline pl-mass & plurale und Massennomina (Sorte) \\
\hline POL & Polarität \\
\hline $\operatorname{Pos}_{1}, \operatorname{Pos}_{2}$ & Possessiv $_{1}$ oder Possessiv 2 \\
\hline PP & Präpositionalphrase \\
\hline Präp / P & Präposition \\
\hline PRD & prädikativ \\
\hline pron & Pronomen (Sorte) \\
\hline PSG & Phrasenstrukturgrammatik \\
\hline psoa & parametrized state-of-affairs \\
\hline qfpsoa & quantifier-free-psoa \\
\hline QSTORE & quantifier store \\
\hline QUANTS & quantifiers \\
\hline $\mathrm{R}$ & Regel \\
\hline ref-obj & referentiell object \\
\hline Rel & Relativsatz \\
\hline RELN /reln & Relation \\
\hline RESTIND & restricted index \\
\hline RESTR & restriction \\
\hline RETRIEVED & retrieved quantifiers \\
\hline$S$ & Satz \\
\hline SEM & Semantik \\
\hline sg & Singular \\
\hline
\end{tabular}




\begin{tabular}{|c|c|}
\hline sg-count & singuläre zählbare Nomina (Sorte) \\
\hline sGEN & semantisches Genus \\
\hline sNUM & semantischer Numerus \\
\hline soa & state-of-affairs \\
\hline SPEC & specified \\
\hline sPER & semantische Person \\
\hline SPR & Spezifizierer \\
\hline SS & Syntax-Semantik \\
\hline ST & Status \\
\hline SUBCAT & Subkategorisierungsliste \\
\hline SUBJ & Subjekt \\
\hline SYN & Syntax \\
\hline SYNSEM & Syntax-Semantik \\
\hline TEMP & Tempus \\
\hline temp & temporal \\
\hline $\mathrm{TN} / \mathrm{tn}$ & transnumeral \\
\hline TRANS & Transposition \\
\hline V & Verb \\
\hline VAL & Valenz \\
\hline VAR & Variable \\
\hline VFORM & Verbform \\
\hline $\mathrm{V}_{\mathrm{n}}$ & nicht-terminales Vokabular \\
\hline VP & Verbalphrase \\
\hline VS & Voranstellung \\
\hline$V_{t}$ & terminales Vokabular \\
\hline $\mathrm{XP}$ & X-Phrase (unbestimmter Phrasentyp) \\
\hline Zahl & Zahlwort \\
\hline
\end{tabular}




\section{Einleitung}

Die Sprachwissenschaft will Probleme lösen, die mit der Struktur und dem Gebrauch von Sprache zusammenhängen. Die vorliegende Arbeit untersucht die Struktur und den Gebrauch der spanischen Nominalphrase, welche in mehrfacher Hinsicht ein spannendes Untersuchungsgebiet bildet. Ein erstes Problem ist bereits die Bezeichnung Nominalphrase. Darunter wird ein Gebilde verstanden, das aus einem Nomen und evtl. weiteren Wörtern besteht, wobei das Nomen eine herausragende Stellung einnimmt. Dies wird in der neueren Literatur seit Abney (1987) nicht immer so gesehen. Gerade im Bereich der Anglistik und Germanistik finden sich zahlreiche Untersuchungen (z.B. Abb 1994, Oepen 1994, Winhart 1997), die dem Nomen eine untergeordnete Stellung zuweisen und den Determinierer als dominant herausstellen. Somit muß für die spanische Nominalphrase überprüft werden, inwieweit es sich wirklich um eine Nominalphrase und keine Determiniererphrase handelt. Dabei muß die Struktur der gesamten Phrase berücksichtigt werden.

Als zweites findet sich in spanischen Grammatiken zumeist folgende Unterteilung der möglichen, in einer Nominalphrase außer Nomina auftretenden Elemente: Artikel und Adjektive. Da unter Artikel nur der definite Artikel verstanden wird (die Wortformen el, la, los und las), stellt sich die Frage, welche Wörter folglich unter die Wortart Adjektiv fallen. Spanische Grammatiken unterscheiden konsequenterweise sehr einheitlich zwischen determinierenden Adjektiven und qualifizierenden Adjektiven, so daß Wortarten wie Demonstrativa, Possessiva, Numeralia und Indefinitpronomina allesamt unter der Kategorie der determinierenden Adjektive zusammengefaßt werden. Dies steht im Kontrast zu Sprachen wie dem Deutschen oder Englischen (vgl. Engel 1982 und Quirk u.a. 1985), die eine Wortart Determinierer oder zumindest einzelne Wortarten wie Demonstrativa, Personalpronomina und eine klar abgegrenzte Wortart Adjektiv kennen. Gleiches gilt für das Französische (Grevisse 1993).

Auch diese Auffassung muß überprüft werden. Wir greifen dazu die Kriterien zur Differenzierung der Determinierer von Alvarez Martínez auf und modifizieren und ergänzen sie. Dabei werden morphologische, syntaktische und semantische Faktoren sowie die auch für qualifizierende Adjektive relevante Wortstellung berücksichtigt. Jeder Determinierer erhält bestimmte Eigenschaften zugesprochen, so daß eine Vergleichsgrundlage geschaffen wird, die weiteren Untersuchungen dienen kann. Wir können folglich alle Determinierer miteinander vergleichen und die Distanz mathe- 
matisch berechnen. In einem zweiten Schritt erfolgt eine Clusteranalyse, welche ähnliche Determinierer in einem Cluster, einer Klasse, zusammenfaßt. Auf diese Weise können wir nachweisen, daß die Annahme spanischer Grammatiker, Determinierer und Adjektive seien in einer Wortart zusammenzufassen, in vielen Punkten berechtigt und begründet ist. Eine klare Abgrenzung zwischen beiden Wortarten ist im Spanischen nicht möglich.

In einem Satz wie es un árbol können sowohl der indefinite Artikel un als auch der definite Artikel $e l$ sowie manch anderer Determinierer (u.a. algún, este, otro) vor árbol stehen. Dies verdeutlicht ein drittes Problem bei der Deskription der spanischen Nominalphrase: Neben der Abgrenzung Determinierer - Adjektiv steht die grundsätzliche Sonderstellung des definiten Artikels in spanischen Grammatiken. Obwohl zumindest die Distribution ähnlich ist, wird er z.B. bei Seco (1986) isoliert behandelt. Wir werden den definiten Artikel somit in die Klassifikation anhand der Kriterienliste einbeziehen, wobei sich zeigen wird, daß die Sonderbehandlung nicht gerechtfertigt ist.

Einen vierten interessanten Bereich der spanischen Nominalphrase bilden die Relationen zwischen den Elementen der Phrase. Wenn Determinierer auch als Adjektive, die in der Regel optionale Komponenten einer Nominalphrase darstellen, aufgefaßt werden können, dann könnte Optionalität auch auf Determinierer zutreffen. Folglich bliebe als einziges obligatorisches Element das Nomen übrig, welches dann auch als dominant gekennzeichnet wird. Nun kann aber leicht gezeigt werden, daß Determinierer nur unter bestimmten Umständen optional sind:

(a) Es un árbol.

(b) No hay árboles.

(c) *Es árbol.

Während (a) und (b) korrekte spanische Sätze darstellen, ist der Satz (c) ungrammatisch, gerade weil ein Determinierer fehlt. Somit stellt sich die Frage nach dem Status des Determinierers. Handelt es sich um Komplementation, wie es in der Regel für die Relation Determinierer - Nomen angenommen wird? Oder müssen wir von Adjungierung ausgehen, wie es in der Regel für die Relation Adjektiv - Nomen angenommen wird? Beide Möglichkeiten werden überprüft, wobei wir nach Zwicky (1993) eine Zwischenstellung in die Diskussion einbringen werden, die viele Vorteile bietet.

Im Zusammenhang mit der möglichen dominanten Stellung des Nomens innerhalb der Phrase war schon von der Phrasenstruktur die Rede. Dies ist der fünfte Be- 
reich, der besondere Beachtung verlangt. Wenn die Relation Determinierer - Nomen insofern nicht unproblematisch ist, als wir weder von Komplementation noch von Adjungierung sprechen können, und zudem Determinierer adjektivähnliche Eigenschaften haben können, stellt sich die Frage, wie die Struktur der Nominalphrase in einem Grammatikmodell zu beschreiben ist. Wir greifen dabei auf die kopfgesteuerte Phrasenstrukturgrammatik (Head-driven Phrase Structure Grammar, HPSG) ${ }^{1} \mathrm{zu}-$ rück, die uns besonders zur Deskription geeignet scheint. Dies liegt besonders in folgenden Eigenschaften begründet: Die HPSG ist eine Unifikationsgrammatik, welche syntaktisch-semantische Information in einer einzigen Attribut-Werte-Matrix darstellt. Die Koordination von Syntax und Semantik spielt dabei für die spanische Sprache eine wichtige Rolle, da semantische Information oft zur Erklärung sprachlicher Strukturen herangezogen werden muß, z.B. bei Infinitivkonstruktionen oder subjektlosen Sätzen. Gleiches gilt für die Eigenschaften von Determinierern und Adjektiven. Eine Berücksichtigung semantischer Informationen erleichtert die Deskription. Bei der adjektivischen Wortstellung sind zudem pragmatische Informationen relevant, die ebenfalls in der HPSG schon behandelt wurden (Engdahl / Vallduví 1996).

Da es sich bei dem Abgrenzungsversuch von Determinierer und Adjektiv um eine lexikalische Fragestellung handelt, spielt das Lexikon als grammatische Komponente eine herausragende Rolle. In der HPSG ist es üblich, linguistische Information über sogenannte Sortenhierarchien zu erfassen. Dabei stellt die oberste Sorte in einem Baumdiagramm die allgemeinste Information dar, nach unten nimmt der Informationsgehalt zu. Durch eine solche Hierarchie ist es möglich, feinere Abgrenzungen durch unterschiedliche Sorten sowie Überschneidungen durch Mehrfacheinträge zu erfassen.

Aus romanistischer Sicht stellt die Grammatiktheorie der HPSG zwar kein Neuland dar, die „Muttersprache“ der Theorie (Balari / Dini 1998: ii) ist aber eindeutig das Englische. Auch gibt es bereits zahlreiche Untersuchungen zum Deutschen (vgl. Nerbonne / Netter / Pollard 1994). Neben dem Sammelband „Romance in HPSG“ (Balari / Dini 1998) erschien bisher eine überschaubare Menge an Untersuchungen (u.a. Abeillé 1993 und Sag / Godard 1994 zum Französischen, Monachesi 1995 zu Klitika im Italienischen). Somit ist es als sechster Bereich interessant, das Modell anhand des Untersuchungsgegenstandes auf evtl. notwendige Modifikationen zu te-

\footnotetext{
${ }^{1}$ Wir werden im folgenden den weitaus geläufigeren englischen Terminus verwenden.
} 
sten. Dabei ist neben der Thematik Komplementation vs. Adjungierung vor allem die Kongruenz zu nennen.

Wenn wir als Untersuchungsgegenstand die Nominalphrase wählen, so impliziert dies, daß wir keine Satzstrukturen behandeln. Relativsätze, als mögliche Teilstrukturen von Nominalphrasen, werden aus diesem Grund nicht thematisiert. Präpositionalphrasen werden wir zwar kurz berücksichtigen, der Schwerpunkt der Arbeit liegt aber eindeutig auf den Wortarten Nomen, Determinierer und Adjektiv. Pronomen werden ebenfalls nur gestreift. Zudem werden diverse sprachliche Phänomene, die gewöhnlich in einer Einführung in die HPSG erläutert werden, nicht angeschnitten (Kontroll- und Bindungstheorie, gebundene und ungebundene Abhängigkeiten u.a.).

Ein besonderes Problem bei der Bearbeitung der spanischen Nominalphrase stellten die Beispielsätze dar. Zwar konnte eine Vielzahl an Grammatiken als Quelle dienen (bes. Matte Bon 1992, de Bruyne 1993, Alarcos Llorach 1994, RAE 1985 u.a.), besonders aber die Kookkurrenz von Determinierern erwies sich als problematisch, da befragte Muttersprachler selbst Beispielsätze aus der Untersuchung von Prado Ibán (1993) ablehnten. Also wurde ein Korpus erstellt, das aus sechs Teilen besteht. Die Referenz erfolgt über ein Kürzel. Die genaueren Angaben finden sich in Kapitel VI. Das Korpus besteht aus:

1. einer digitalen Ausgabe der Zeitung $A b c$ (vom 10.6.1999) (Kürzel A);

2. einer digitalen Ausgabe der Zeitung El Mundo (vom 9.6.1999) (M);

3. einer digitalen Ausgabe der Zeitung El País (vom 8.6.1999) (P);

4. dem Corpus argentino (vgl. Marcos-Marín 1994: 143ff., einführend 79ff.) (RA);

5. dem Corpus chileno (vgl. Marcos-Marín 1994: 148ff.) (Rch);

6. dem Proyecto Sherezade (Kurzgeschichten im Internet seit 1996) (S).

Sehr einfache Beispiele werden nicht belegt.

Die Untersuchung zur spanischen Nominalphrase ist wie folgt gegliedert:

Kapitel II. führt zunächst in die HPSG ein. Anhand einer kontextfreien Phrasenstrukturgrammatik werden die Besonderheiten der HPSG dargestellt (II.1.). Danach folgt eine Erläuterung der für den Untersuchungsgegenstand relevanten sprachlichen Phänomene und ihrer Darstellung im Formalismus der HPSG (II.2.).

Kapitel III. behandelt die spanische Nominalphrase. Zunächst steht das Nomen im Vordergrund (III.1.), seine Syntax und Semantik, die Bedingungen, unter denen ein einzelnes Nomen eigenständig eine Nominalphrase bilden kann, und ein Vergleich mit den Personalpronomen. Es folgt die Abgrenzung der Determinierer zu den Adjektiven (III.2.), wobei zunächst die Klassifikation von Alvarez Martínez (1986) be- 
sprochen wird, um dann Kriterien für eine Abgrenzung Determinierer - Adjektiv zu erstellen, die dem sprachlichen Material gerecht werden. Kookkurrenz und Semantik erfordern dabei besondere Aufmerksamkeit. Nach Analyse der Ergebnisse erfolgt zu Vergleichszwecken eine Klassifikation der deutschen Determinierer.

In Kapitel III.3. werden spanische Determinierer und Nomina in der HPSG erläutert. Dabei fallen der lexikalischen Komponente ebenso wie der Darstellung von Kongruenz und Wortstellung besondere Rollen zu. Kapitel III.4. geht auf qualifizierende Adjektive im Spanischen ein und referiert dabei besonders verschiedene Auffassungen über die Stellungsproblematik. Kapitel III.5. diskutiert Präpositionen, soweit sie unseren Untersuchungsgegenstand berühren. Kapitel IV. faßt die Ergebnisse zusammen. Nach der Bibliographie (Kap. V.) enthält Kap. VI. genauere Daten zum Korpus (VI.1.) und die Referenz der Beispielsätze auf das Korpus (VI.2.). Am Schluß folgen Namen- und Sachindex. 


\section{Einführung in die HPSG}

\section{Vorbemerkungen}

\subsection{Ziele der Sprachbeschreibung}

„La 'lingüística' es la ciencia del lenguaje. En este sentido, el término lenguaje comprende la facultad comunicativa propia de los humanos y las lenguas naturales en las que éste se manifiesta, junto con sus elementos constitutivos, las relaciones entre éstos, y las leyes o reglas de su funcionamiento, de describir y explicar sus formas y funciones como expresión del pensamiento humano y de la comunicación social“ (Alcaraz Varó / Martínez Linares 1997: s.v. lenguaje).

Das Ziel eines Erklärungssystems sprachwissenschaftlicher Prägung ist es, alle Ausdrücke zu erfassen, die ein Sprecher verwenden könnte. Dabei können wir von der sozialen Kommunikation und ihren verschiedenen Formen abstrahieren und uns auf die Kommunikationsmittel beschränken, d.h. wir müssen zunächst die konstituierenden Elemente eines Systems isolieren. Zwischen diesen Konstituenten bestehen syntaktische Relationen, die eruiert und untersucht werden müssen. Besonders interessant sind dabei alle Gesetzmäßigkeiten, die sich bei der Isolierung der Elemente wie auch bei den Relationen zwischen den Elementen entdecken lassen. Wenn dieser Prozeß abgeschlossen ist, gilt es, eine Beschreibung der Formen und Ausdrücke zu erstellen, die dem sprachlichen Material gerecht wird.

Wir nennen die sprachwissenschaftliche Disziplin für die Struktur von Sprache, wie sie oben beschrieben wurde, Syntax. Zwei Einschränkungen müssen nach den obigen Ausführungen für den Sprachgebrauch, wie er für den Kommunikationsalltag gebräuchlich ist, beachtet werden:

- Außersprachliche Faktoren können sprachliche Prozesse begrenzen, z.B. überfordern zu lange Sätze die menschliche Konzentrationsfähigkeit. Diese außersprachlichen Faktoren, seien sie nun eher der Psycholinguistik oder der Pragmatik zuzuordnen, können nicht berücksichtigt werden. Sie sind aber stets am Kommunikationsablauf beteiligt (vgl. Stein 1998: 10). 
- Wir müssen unterscheiden zwischen dem Sprachsystem, das ein Sprecher verinnerlicht hat, und seiner Anwendung (Kompetenz, vs. Performanz, vgl. Chomsky 1965: 14ff. und Grewendorf / Hamm / Sternefeld 1994: 31). Die Anwendung des Systems wird von den außersprachlichen Faktoren beeinflußt. Das System selbst wird nicht davon berührt. Es erscheint somit sinnvoller, sich auf die abstrakte Ebene des Systems zu begeben, um Sprache zu untersuchen. Aber auch eine höhere, abstraktere Untersuchungsebene bleibt natürlich weiterhin abhängig von deren Anwendung.

Die Definition von Sprache erweist sich als außerordentlich kompliziert (vgl. Crystal 1995: 396ff.). In Anlehnung an Borsley könnte Sprache vorsichtig folgendermaßen definiert werden (vgl. Borsley 1997: 7):

(1) Eine Sprache besteht aus einem Lexikon und einer endlichen Menge von Regeln und Prinzipien, welche der Menge von unendlich vielen, wohlgeformten Sätzen zugrunde liegen, die ein Sprecher gebrauchen könnte, sofern nicht außersprachliche Faktoren dies verhindern.

Diese Definition führt bereits zu dem hier präsentierten Untersuchungsgegenstand. Sie ist auf Sätze als nächstkleinere Einheit von Texten beschränkt. Allerdings kommunizieren Menschen grundsätzlich eher in Dialogen und Texten miteinander, so daß ein Satz intuitiv nicht als eine bestimmte Einheit verstanden wird. Mit Lexikon, Regel und Prinzip werden bereits noch zu klärende Termini verwandt, welche Konzepte beschreiben, die einem Sprecher nicht bewußt sind. Und auch die Annahme, daß eine Sprache eine unendliche Menge von Sätzen kennt, führt bereits zu der Hypothese, daß es ein System gibt, welches in der Lage ist, diese Menge an Sätzen zu erzeugen.

Die genannte Definition von Sprache ist als eine Arbeitshypothese zu betrachten, die keinen universellen Anspruch erhebt und je nach Forschungsinteresse variiert werden dürfte. Sie bleibt auf den Untersuchungsgegenstand dieser Arbeit begrenzt. Allerdings läßt sie noch zusätzlich zu bereits genannten Aspekten die „Bedeutung“ der Sätze außer acht. „Bedeutung“ wird insofern eine Rolle spielen, als die HPSG stets semantische Aspekte im Blick behält und im Gegensatz zu anderen Theorien die Semantik sogar in einem gemeinsamen Formalismus zusammen mit der Syntax darstellt. 
Jeder Sprecher einer Sprache wie z.B. der spanischen kennt eine endliche, sich im Laufe der Zeit verändernde Menge von Wörtern. Diese Menge wird ganz allgemein Lexikon genannt. Zudem kennt er eine Art Regelsystem, das er unbewußt grundsätzlich befolgt. Diese Regeln besagen z.B., daß im Spanischen Artikel grundsätzlich vor Nomina stehen. Sie werden den meisten Sprechern nicht bewußt sein, obwohl sie immer gelten und bei falscher Verwendung im Fremdsprachenunterricht korrigiert werden. Sie bewirken zudem, daß ein Sprecher problemlos Sätze als korrekt - d.h. wohlgeformt - erkennen kann, auch wenn er diese nie zuvor gehört oder geäußert hat. Es handelt sich um ein System, das augenscheinlich im Gehirn jedes Sprechers verankert ist. Dieses System gilt es zu erforschen.

Im Strukturalismus nach de Saussure werden neben der Unterscheidung zwischen dem Sprachsystem und seiner Realisierung (langue vs. parole) folgende Unterscheidungen hervorgehoben (vgl. Stein 1998: 8):

- Nicht-historische (synchrone) Sprachbetrachtung zu einem bestimmten Zeitpunkt hat Vorrang vor der historischen (diachronen) Sprachbetrachtung. Für diese Arbeit bleiben diachrone Details unberücksichtigt. Beispielsätze und -texte sind grundsätzlich neueren Datums.

- Einfache sprachliche Zeichen (z.B. Wörter im Gegensatz zu Verkehrsschildern u.a.) sind prinzipiell arbiträr und selten motiviert, d.h. sie sind nicht begründet, wie dies z.B. bei Verkehrsschildern, Piktogrammen und ähnlich gearteten Zeichen der Fall ist. Dies hat zur Folge, daß die Bedeutung von sprachlichen Zeichen zunächst einmal als gegeben und bekannt angesehen wird.

- Die Linguistik ist deskriptiv und nicht normativ (vgl. für einen teilweise normativen, lehrenden Ansatz Seco 1986), d.h. sie versucht, Sprache so zu beschreiben, wie sie ist und nicht wie sie sein sollte. Damit werden alle Sprachvarianten berücksichtigt, unabhängig von der Anzahl ihrer Sprecher, der politischen oder gesellschaftlichen Bedeutung einer Sprache oder sonstiger Faktoren, die eine Sprache als hochwertig oder unbedeutend qualifizieren könnten. In dieser Arbeit wird somit kein Unterschied gemacht, ob die Beispiele aus dem kastilischen Bereich, dem eher dialektal markierten Küstenbereich der Iberischen Halbinsel oder aus dem Hispanoamerikanischen mit seinen zahlreichen Facetten stammen. Ihr Ursprung muß als solcher gekennzeichnet werden, um den dialektalen Gebrauch zu erkennen und vom Standard abzugrenzen (vgl. einführend Metzeltin / Winkelmann 1992). 
Ein nicht unbedeutendes Ziel der Sprachwissenschaft in der Tradition Chomskys ist das Bemühen um universelle Regeln und Prinzipien (Chomsky 1957, 1965, 1981, für neuere Ansätze Chomsky / Lasnik 1995). Es wird eine Universalgrammatik postuliert, die eine angeborene Komponente des Menschen darstellt und mit einzelsprachlichen Parametern ergänzt wird. Konsequenterweise bedeutet diese Hypothese, daß es Regeln geben muß, die in allen oder fast allen Sprachen Verwendung finden, so wie auch spezielle Sonderausstattungen, die nur für eine Sprache oder Sprachfamilie zutreffen. Wie die universellen, anscheinend grundsätzlich für alle Sprachen geltenden Regeln im menschlichen Gehirn verankert sind oder sein könnten, ist umstritten.

\subsection{Syntaxtheorie}

Der Strukturalismus untersuchte Phoneme als kleinste Einheiten in einer Sprache, um sich dann über Morphologie und Syntax auf Semantik und Pragmatik zuzubewegen. Die Syntaxtheorie steht somit in einer Zwischenstellung: Sie setzt Phonologie als die Lehre der Klassifikation von Lauten einer Sprache und zum Teil auch die Morphologie als Lehre der Wortstrukturen und -bildung voraus ${ }^{2}$ und wird von semantischen und pragmatischen Faktoren beeinflußt. Sie arbeitet mit Sätzen als Teilen von Texten. Diese Sätze bestehen wiederum aus Wörtern. Sowohl die Definition als auch die Abgrenzung von Wörtern ist schwierig (vgl. die Übersicht verschiedener Ansätze in Crystal 1995: 91). Als vorläufige Arbeitsdefinition muß mangels besserer Alternative zunächst gelten:

(2) Ein Wort ist eine durch Leerstellen umrahmte Zeichenkette.

Diese Definition ist nicht befriedigend. Die schriftliche Darstellung kann grundsätzlich nicht der Ausgangspunkt einer sprachlichen Analyse sein. Eine Alternative könnte die phonetische Einheit des Wortes bilden. Dagegen spricht aber das französische mot phonétique (vgl. Stein 1998: 17): Wörter, die eine semantische oder syntaktische Einheit bilden, werden auch zusammengezogen und wie ein Wort ausge-

\footnotetext{
${ }^{2}$ Morphologie kann mitunter als Teilgebiet der Syntax verstanden werden. Der Terminus Morphosyntax kennzeichnet diesen Sachverhalt. Inwieweit die Wortbildung einen Teil der Morphosyntax bzw. auch der Syntax bildet, wird kontrovers diskutiert (vgl. Grewendorf / Hamm / Sternefeld 1994: 254ff.).
} 
sprochen. Folglich erhält der entsprechende Komplex nur einen Akzent: „Si on prend la langue parlée on n'y trouve pas les équivalents des blancs que l'on observe dans l'écrit. Dans une phrase, les sons s'assemblent, non en mots, mais en groupes unifiés par l'accent tonique ainsi que par la liaison, l'enchaînement et l'élision: J'ai rencontré mon voisin [3eRãkõtRe mõvwAz̃̃ ]“" (Grevisse 1993: 176).

Von daher scheint es sinnvoller, von Morphemen zu sprechen, die als kleinste bedeutungstragende Einheiten einer Sprache definiert werden. Problematisch ist in diesem Zusammenhang, daß in der Syntaxtheorie oft Wörter im Sinne der Definition (2) betrachtet werden. Eine morphologische Komponente ist in vielen Theorien nicht oder nur am Rande vorhanden (vgl. Riehemann 1993, Kathol 1995, Klenk 1996). Von daher muß zunächst mit der Arbeitshypothese gearbeitet werden, und komplexe Nomina wie el librepensador oder el gentilhombre sind genauso zu behandeln wie el coche, obwohl schon die Pluralbildung uneinheitlich ist (los librepensadores, los gentileshombres, los coches). Noch komplexere Nomen wie el correveidile ('der Petzer'), die einen ganzen Satz widerspiegeln $($ corre $+v e+y+d i+l e$ 'lauf, sieh und sag ihm' - alle Beispiele aus de Bryune 1993: 82f.), zeigen deutlich die Inadäquatheit der Arbeitshypothese. Idiomatische Redewendungen, die nur in ihrer speziellen Zusammensetzung anwendbar sind (no tener palabra 'wortbrüchig sein', pasar por alto 'übergehen'), verdeutlichen die Begrenzung der Arbeitshypothese auf semantischer Seite in der anderen Richtung: mehrere Wörter können als eine Einheit verstanden werden (zur dependenzgrammatischen Definition vgl. Weber 1992: 23ff.).

Die Syntaxtheorie versucht, Grammatiksysteme zu entwickeln, welche die Menge aller wohlgeformten Sätze einer Sprache generieren und dabei die nichtwohlgeformten ausklammern. Zudem sollen möglichst viele Prinzipien der Universalgrammatik erkannt und formuliert werden. Die dabei verwendeten Beschreibungsmethoden basieren auf Formalismen der Logik und der Mathematik (vgl. Allwood / Andersson / Dahl 1973). Sie müssen für den Leser überprüfbar, die Begründung muß nachvollziehbar und der Analyseprozeß durchsichtig sein. Diese Forderungen sind nur erfüllbar, wenn die Deskriptionsmethoden einsichtig sind. In der Folge wurden Formalismen entwickelt, deren einzelne Bestandteile und die Relationen der Bestandteile zueinander in einem wohldefinierten Verhältnis stehen. Einer natürlichsprachlichen Beschreibung sprachlicher Phänomene werden formale Beschreibungen vorgezogen. Ein Beispiel mag dies verdeutlichen: 
(3) „Como sabemos [...], el núcleo del sujeto es una palabra caracterizada por imponer su concordancia al núcleo del predicado. Si el núcleo del sujeto está en la forma singular, el del predicado tendrá que aparecer también en la forma singular. Si el primero está en plural, en plural irá también el segundo. La función de núcleo del sujeto está siempre desempeñada, según vimos, por una palabra que pertenece a la categoría de los sustantivos“ (Seco 1991: 87).

Die Vorgehensweise des Autors soll hier nicht kritisiert werden. Er wendet sich eher an einen über seine Sprache reflektierenden spanischen Muttersprachler denn an ein wissenschaftliches Publikum. In einer formalen Grammatik werden aber Definitionen klarer voneinander abgesetzt (palabra wird bei Seco ähnlich wie Morphem definiert, Seco 1991: 15, vgl. im Gegensatz dazu Grevisse 1993: 175).

Daraus folgt u.a.:

- Die Beziehung zwischen Subjekt und Prädikat (Subjektkongruenz) wird auch in formaler Darstellungsweise erläutert.

- Syntaktische (Kongruenz) und semantische (Rollen-)Phänomene werden getrennt betrachtet. Relationen und Funktionen werden in ein übergeordnetes System integriert (vgl. für gerade diesen Aspekt besonders hervorhebende Ansätze in Chomsky 1981 und Haegeman 1991: 139ff.).

- Es wird versucht, von Beginn an möglichst genau zu argumentieren. So kann der Kern einer Nominalphrase auch einer anderen, substantivierten Wortart entstammen (z.B. un buen hombre es un hombre bueno para los demás; aus de Bryune 1993: 235). Auf diese Möglichkeit muß zumindest hingewiesen werden.

Diese Forderungen unterscheiden formalisierte Grammatiken von der traditionellen Grammatikschreibung. Letztere ist nicht selten auf eine einzige Sprache bezogen und beschreibt diese, ohne auf formale Systeme zurückzugreifen. Die Ergebnisse dieser Beschreibungen sind meist weniger systematisch, aber deshalb nicht weniger brauchbar. In einer formalen Grammatik besteht hingegen die Gefahr, Formalia höher zu gewichten als die Sprachbeschreibung. Ein Leser kann den Eindruck gewinnen, natürlichsprachliche Fragestellungen, die nicht in das formale System passen, würden eher vernachlässigt. Die neueren Modelle seit Chomsky (1957) werden aber explizit als Ergebnisse einer erklärenden, empirischen Wissenschaft verstanden. 
Die Grundlage vieler Ideen und Konzepte der generativen Grammatik bilden die frühen Arbeiten von Chomsky: Syntactic Structures, 1957, und Aspects of the Theory of Syntax, 1965. Sie basieren auf Arbeiten zur Phonologie und erweitern die distributionellen Methoden zur Bestimmung von Phonemen sowie Wort- und Phrasenklassen. Für die syntaktische Komponente einer Grammatik wird für jeden Satz eine Tiefenstruktur angenommen, welche die semantische Interpretation des Satzes bestimmt, und eine Oberflächenstruktur, die seine phonetische Interpretation bestimmt. Tiefenstruktur und Oberflächenstruktur sind im allgemeinen distinkt, wobei die Oberflächenstruktur durch wiederholte Anwendung formaler Operationen, Transformationen genannt, determiniert wird. Ausgangspunkt der Einführung zweier unterschiedlicher syntaktischer Strukturen war es, die Disambiguierung zweideutiger Sätze zu ermöglichen (Chomsky 1965: 16ff. und Crystal 1995: 98, zur Geschichte der Phrasenstrukturgrammatik vgl. Egli / Egli-Gerber 1991: 7ff.).

Generell wird in den Arbeiten von Chomsky eine Konstituentenstruktur vorgeschlagen, die sich auf Ersetzungsregeln gründet. Wir werden hier zunächst eine solche kontextfreie Phrasenstrukturgrammatik (kf PSG) vorstellen, um dann die Unterschiede und Ähnlichkeiten mit einer kopfgesteuerten Phrasenstrukturgrammatik (HPSG) zu erläutern.

Für die Struktur von Phrasen wird in einer kf PSG wie auch in der HPSG auf die X-bar-Theorie verwiesen (Jackendoff 1977). Sie geht davon aus, daß alle Phrasenstrukturen unabhängig vom Typ (Nominal-, Verbal-, Präpositionalphrase u.a.) nach allgemeinen universellen Prinzipien aufgebaut sind, wobei mehrere Ebenen unterschieden werden. Die genannten Typen wie Nominal- oder Verbalphrase werden als maximale Projektion der zugrundeliegenden Kategorie Nomen (N) oder Verb (V) gesehen. $\mathrm{N}$ oder $\mathrm{V}$ sind Kategorien, die nur noch durch Lexeme ersetzt und als minimale Projektion oder von minimaler Komplexität bezeichnet werden. Die Notation ist $\mathrm{X}_{0}$ oder einfach $\mathrm{X}$ für die niedrigste Stufe, $\mathrm{X}_{1}, \mathrm{X}^{\prime}, \overline{\mathrm{X}}(\mathrm{X}$ Strich $=$ engl. $\mathrm{X}$ bar $)$ für die nächsthöhere Stufe und $\mathrm{X}_{2}, \mathrm{X}^{\prime \prime}$ oder $\overline{\overline{\mathrm{X}}}$ für die höchste Ebene, sofern man drei Stufen annimmt, wobei $\mathrm{X}$ als Platzhalter für eine beliebige Kategorie steht. Die mittlere Ebene $\mathrm{X}^{\prime}$ markiert Phrasen der Art coche nuevo, die in dieser Form unvollständig sind (und zu el / ese / un coche nuevo erweiterbar), aber keine lexikalische Ebene mehr darstellen. Ob drei die höchste Anzahl an Ebenen darstellen sollte, ist umstritten (vgl. Grewendorf / Hamm / Sternefeld 1994: 198ff.). In Strukturen, die komplexere Arten von Adjunktanbindung kennen (ein Adjektiv und ein Relativsatz 
an ein Nomen u.a.), scheinen mehr als drei Ebenen erforderlich. Gebräuchlich ist auch das rekursive Auftreten der Ebene X'.

\subsection{Kontextfreie Phrasenstrukturgrammatik (kf PSG)}

Wir erstellen an dieser Stelle eine kontextfreie Grammatik, die wir G abkürzen (zum Terminus kontextfrei vgl. Klenk 1980: 51ff. und 1995: 1584). Diese kf PSG namens $\mathrm{G}$ bildet eine Grammatik, die aus terminalen und nicht-terminalen Symbolen besteht. Die endliche Menge von Terminalsymbolen (Wörter oder Morpheme) wird mit $\mathrm{V}_{\mathrm{t}}$ für terminales Vokabular abgekürzt. Die ebenfalls endliche Menge von nicht-terminalen Symbolen enthält Symbole für syntaktische Kategorien wie Satz (S), Nominalphrase (NP), Nomen (N) oder Verb (V), d.h. sie symbolisieren Eigenschaften von Wörtern und auf höherer Ebene von Phrasen (abgek. $\mathrm{V}_{\mathrm{n}}$ für nicht-terminales Vokabular). Das terminale Vokabular wird im Vorfeld durch grammatische Kategorien wie Nomen $(\mathrm{N})$, Verb (V), Adjektiv (A), Präposition (P), Determinierer (Det) usw. klassifiziert. Über Elementen dieser Kategorien werden Konstituenten gebildet, die Teile einer mittels Ersetzungsregeln (R) erzeugten hierarchischen Struktur des abzubildenden Satzes sind. In (4) wird eine Nominalphrase aus Determinierer und Nomen gebildet, z.B. el coche (vgl. Chomsky 1965: 88ff.)

$$
\mathrm{NP} \rightarrow \operatorname{Det} \mathrm{N}
$$

Der Pfeil symbolisiert dabei den Prozeß der Ersetzung: Determinierer und Nomen werden aus der Nominalphrase (NP) abgeleitet, bzw. sie bilden zusammen eine Einheit, die eine NP darstellt.

Initiiert wird diese endliche Menge von Regeln durch ein Startsymbol, zumeist S. Dieses kennzeichnet den Beginn des Ableitungsprozesses. Wenn nur Nominalphrasen bearbeitet werden, kann das Startsymbol auch NP sein. Beginnend beim Startsymbol kann nun ein Ableitungsprozeß ablaufen, der Schritt für Schritt ein nichtterminales Symbol nach dem nächsten solange ersetzt, bis nur noch terminale Symbole, d.h. Wörter eines Satzes, übrigbleiben. Ist diese Ableitung möglich und wird der Prozeß nicht durch Irregularitäten derart unterbrochen, daß eine Ersetzung nicht 
möglich ist, dann ist der Satz nach der vorgegebenen Grammatik korrekt. Dieser Prozeß soll an dieser Stelle kurz beispielhaft erläutert werden.

So kann eine beliebige Grammatik $G$ als eine Struktur gesehen werden, die auf vier Elementen (Quadrupel) aufbaut: den terminalen und nicht-terminalen Symbolen, den Regeln und dem Startsymbol. G kann also definiert werden als:

$$
\mathrm{G}=\left(\mathrm{V}_{\mathrm{t}}, \mathrm{V}_{\mathrm{n}}, \mathrm{R}, \mathrm{S}\right)
$$

Ein Satz wird nun als eine Kette von Wörtern betrachtet. Ist jetzt diese Kette der von G erzeugten Sprache zugehörig, dann muß G die Kette aus dem Startsymbol erzeugen können. Durch die fortlaufende Ersetzung - zunächst des Startsymbols und in der Folge der Symbole auf der linken Seite einer Regel durch den Inhalt der rechten Seite derselben Regel - muß es möglich sein, am Schluß zu den Kategorien zu gelangen, die den Wörtern des Eingabesatzes zugeordnet sind. Wir illustrieren dies am Beispielsatz (6):

(6) El padre de Juan compró un coche nuevo.

Die kontextfreie Phrasenstrukturgrammatik, die diesen Satz erzeugen soll, sieht zunächst einmal so aus: 


$$
\begin{aligned}
& \mathrm{G}=\left(\mathrm{V}_{\mathrm{n}}, \mathrm{V}_{\mathrm{t}}, \mathrm{R}, \mathrm{S}\right) \text { mit } \\
& \mathrm{V}_{\mathrm{t}}=\{\text { coche, compró, de, el, Juan, nuevo, padre, un }\} \\
& \mathrm{V}_{\mathrm{n}}=\{\mathrm{A}, \mathrm{D}, \mathrm{N}, \mathrm{NOM}, \mathrm{NP}, \mathrm{P}, \mathrm{PP}, \mathrm{S}, \mathrm{V}, \mathrm{VP}\} \\
& \mathrm{R}=\{\mathrm{S} \rightarrow \mathrm{NP} \text { VP, } \quad \text { Nr. } 1 \\
& \mathrm{VP} \rightarrow \mathrm{V} \mathrm{NP}, 2 \\
& \mathrm{NP} \rightarrow \mathrm{D} \text { NOM, } \quad 3 \\
& \mathrm{NP} \rightarrow \mathrm{NOM}, \quad 4 \\
& \mathrm{NOM} \rightarrow \mathrm{NOM} \text { PP, } \quad 5 \\
& \mathrm{NOM} \rightarrow \mathrm{NOM} \mathrm{A,} \quad 6 \\
& \mathrm{NOM} \rightarrow \mathrm{N}, \quad 7 \\
& \mathrm{PP} \rightarrow \mathrm{P} \mathrm{NP}, \quad 8 \\
& \mathrm{~N} \rightarrow \text { coche } \mid \text { Juan } \mid \text { padre }{ }^{3}, \quad 9 \\
& \mathrm{D} \rightarrow \text { el } \mid \text { un, } \quad 10 \\
& \mathrm{~V} \rightarrow \text { comprar, } \quad 11 \\
& \mathrm{P} \rightarrow \text { de, } \quad 12 \\
& \text { A } \rightarrow \text { nuevo }\} \quad 13
\end{aligned}
$$

Die Grammatik (7) kann den Beispielsatz (6) erzeugen. Dabei wird nach dem Start mit der Regel 1, die als einzige das Startsymbol erhält, dieses durch die Kette NP VP ersetzt. Nun kann das Symbol NP durch Anwendung der Regel 3 oder 4 ersetzt werden, das Symbol NOM über die Regeln 5, 6 oder 7 usw. Nach welchem System Regeln verwendet werden und wie ein Automat an dieser Stelle vorgehen könnte, wird hier nicht näher betrachtet. (Wenn man die Darstellung in einem Baumdiagramm übernimmt, sind folgende Varianten möglich: vom Startsymbol als obersten Symbol nach unten, vom Satz als den untersten Symbolen aus nach oben oder beides abwechselnd; sowie von links nach rechts die Satzkette bearbeitend, von rechts nach links oder von einem bestimmten ausgewählten Satzteil in der Mitte, vgl. Naumann / Langer 1994). Anstelle eines einfachen Adjektivs (A) müßte nach der X-bar-Theorie richtigerweise eine Adjektivphrase angenommen werden, die auf lexikalischer Ebene ein Adjektiv beinhaltet. Dies kann aufgrund des einfachen Beispielsatzes hier vernachlässigt werden.

\footnotetext{
${ }^{3}$ Der senkrechte Strich | signalisiert verschiedene Optionen: coche oder Juan oder padre.
} 
$\mathrm{Daß}$ die Grammatik in (7) auch falsche Sätze erzeugen kann - z.B: *Padre de Juan compró coche nuevo -, liegt daran, daß z.B. die Determinierer grundsätzlich als fakultative Komponenten angenommen werden (statt Regel 3 kann auch immer Regel 4 in Kraft treten). Dies soll momentan nicht weiter stören. Zusätzlich gibt es viele spanische Sätze, die diese Grammatik nicht erzeugen kann (z.B. mit Hilfsverb, Adjektivphrasen, Adverbialen u.a.). Somit stellt Grammatik (7) zum einen eine Übergeneralisierung dar - es werden zuviele Strukturen erzeugt, die nicht alle wohlgeformt sind -, zum anderen ist sie zu schwach, um alle wohlgeformten Strukturen zu erzeugen. Das erste Problem könnte im genannten Beispiel durch die Tilgung der Regel 4 sehr einfach gelöst werden. Es muß dann immer ein Artikel vor dem Nomen stehen. Dies ist in Satz (6) der Fall, wenn auch nicht grundsätzlich in der spanischen Sprache (s. Kap. III.1.).

\subsection{Erweiterte PSG}

In Satz (6) können verschiedene Beobachtungen gemacht werden, die von unserer Grammatik (7) nicht beschrieben werden. So werden Kongruenz und syntaktische Funktionen nicht beschrieben. Kongruenz meint die Übereinstimmung der Merkmale Numerus und Person zwischen Subjekt und Verb. Innerhalb einer NP müssen die Merkmale von Numerus und Genus übereinstimmen. Dies wird von der Grammatik (7) bisher nur deshalb korrekt dargestellt, weil die femininen Formen der Determinierer und Nomina sowie unterschiedliche Verbformen noch nicht existieren. Diese müßten eingeführt werden, um z.B. Sätze der Art la madre de Juan compraba una máquina nueva behandeln zu können. Dafür bräuchte die Grammatik - abgesehen von den Wörtern und Regeln - auch Unterscheidungsmechanismen, die Konstruktionen wie *la padre verbieten. Wir könnten dies in den Ersetzungsregeln widerspiegeln und sie mit entsprechenden Merkmalen versehen:
(a) $\mathrm{S}$
$\rightarrow \quad \mathrm{NP}_{3, \mathrm{sg}}$
$\mathrm{VP}_{3, \mathrm{sg}}$
(b) $\mathrm{VP}_{3, \mathrm{sg}}$
$\rightarrow \quad \mathrm{V}_{3, \mathrm{sg}}$
NP
(c) $\mathrm{NP}_{3, \mathrm{sg}}$
$\rightarrow \quad \mathrm{D}_{\mathrm{sg}, \mathrm{mask}}$
$\mathrm{NOM}_{\text {sg, mask }}$
(d) $\mathrm{NOM}_{\mathrm{sg} \text {, mask }} \rightarrow \quad \mathrm{NOM}_{\mathrm{sg} \text {, mask }}$
$A_{\text {sg, mask }}$ 
Wenn wir die entsprechenden Regeln in (7) durch (8) ersetzen, erhalten wir eine Grammatik, die den Satz (6) mit den Merkmalen für Kongruenz beschreibt. Für plurale oder feminine Formen müßten korrespondierende Regeln eingeführt werden. Hier ist schon erkennbar, daß ein auf diese Weise arbeitendes System in ziemlich kurzer Zeit sehr komplex wird, wenn alle möglichen Kombinationen lediglich als neue Regeln addiert werden. Zudem wird viel redundante Information mitgeführt.

Dieses Problem wird in neueren Grammatikformalismen durch Variablen gelöst. Dabei werden die Werte durch beliebige Buchstaben oder Ziffern ersetzt, wobei gleiche Variablen auch gleiche Werte bedeuten. So könnten die entsprechenden Regeln in (7) auch durch die folgenden in (9) ersetzt werden. Die tiefgestellten Indizes stehen grundsätzlich in der Reihenfolge Person, Numerus, Genus. Wir verwenden an dieser Stelle bereits eine Attribut-Werte-Matrix zur Darstellung (vgl. Kap. III.2.1.): Die Abkürzungen stehen jeweils für die grammatische Kategorie, die Variable ist ihr Wert. Die Klammern verdeutlichen die Zugehörigkeit zur folgenden darüberstehenden Phrase.

(a) $\mathrm{S}$

$\rightarrow \mathrm{NP}$

VP

[ ]

$\left[\begin{array}{ll}\operatorname{PER} & a \\ \operatorname{NUM} & b\end{array}\right] \quad\left[\begin{array}{ll}\mathrm{PER} & a \\ \operatorname{NUM} & b\end{array}\right]$

(b) $\mathrm{VP} \rightarrow \mathrm{V} \rightarrow \mathrm{NP}$

$\left[\begin{array}{ll}\operatorname{PER} & a \\ \operatorname{NUM} & b\end{array}\right] \quad\left[\begin{array}{ll}\operatorname{PER} & a \\ \operatorname{NUM} & b\end{array}\right] \quad[]$

(c) $\mathrm{NP} \rightarrow \mathrm{D} \quad \mathrm{NOM}$

$\left[\begin{array}{ll}\text { NUM } & c \\ \text { GEN } & d\end{array}\right] \quad\left[\begin{array}{ll}\text { NUM } & c \\ \text { GEN } & d\end{array}\right] \quad\left[\begin{array}{ll}\text { NUM } & c \\ \text { GEN } & d\end{array}\right]$

(d) $\mathrm{NOM} \rightarrow \mathrm{NOM} \quad \mathrm{A}$

$\left[\begin{array}{ll}\text { NUM } & e \\ \text { GEN } & f\end{array}\right] \quad\left[\begin{array}{ll}\text { NUM } & e \\ \text { GEN } & f\end{array}\right] \quad\left[\begin{array}{ll}\text { NUM } & e \\ \text { GEN } & f\end{array}\right]$

Durch die Variablen wird gesichert, daß die entsprechenden Eigenschaften der Phrasen und ihrer Kategorien jeweils übereinstimmen müssen. Dabei bleiben die Werte der VP von (9a) zu (9b) identisch. So wird in (9a) die Subjektkongruenz zwischen NP und VP festgehalten. Wenn die Regeln (9c) und (9d) sich auf die NP in (9a) beziehen, stimmen die Werte überein, d.h. die Variable $b$ ist mit $c$ und $e$ identisch. Auf 
die NP in (9b) wird kein Einfluß ausgeübt. Die Werte $c$ und $d$ müssen mit $e$ und $f$ identisch sein, sofern es sich um dieselbe NP handelt.

Als zweites hatten wir bemerkt, daß die syntaktischen Funktionen wie Subjekt und Objekt in (7) weder berücksichtigt noch dargestellt werden. Diese Funktionen werden von der Verbform compró gefordert. Für dieses Thema wird auf Kapitel II.2.3.2. verwiesen.

Die Variablen zeigen, daß Lexeme und Phrasen mehr Eigenschaften besitzen, als ihnen die kf PSG in (7) zugestehen will. Diese Eigenschaften sind den Wortformen des Satzes inhärent. Deshalb wurde in neueren Grammatikmodellen (besonders GPSG, LFG und HPSG, s. Kap. II.1.5.) das Lexikon stärker eingebunden. Viele Merkmale sprachlicher Zeichen werden im Lexikon bereits zugeordnet, so daß diese Information nur auf syntaktisch höheren Ebenen ausgelesen und zusammengebunden werden muß. Damit bekommen auch Phrasen und Kategorien als nicht-terminale Symbole eine neue Funktion als „,verlängerter Arm“ (Projektion) der lexikalischen Einträge.

Ersetzungsregeln im beschriebenen Sinne wurden bei Pollard / Sag (1987: 147ff.) zunächst als Grammatikregeln in die HPSG eingeführt. Später wurden die Regeln als Schemata bezeichnet, welche die Informationsweitergabe steuern. Zunächst gehen wir auf die neue Form des Lexikons ein. Danach werden die Schemata und sich daraus ergebende Strukturen erläutert.

\subsection{Die HPSG als Unifikationsgrammatik}

Die HPSG wurde von Pollard und Sag (1987 und 1994) entwickelt. Sie basiert auf einer Reihe von anderen Grammatiktheorien, die in unterschiedlicher Art und Weise in die Entwicklung der HPSG eingeflossen sind. Dabei sind besonders folgende Grammatikmodelle zu nennen (vgl. Pollard / Sag 1994: 1): generative Transformationsgrammatik (Chomsky 1957; 1965); Government and Binding Theory (GB) (Chomsky 1981, vgl. auch Haegeman 1991 und Jones 1996); Generalisierte Phrasenstrukturgrammatik (GPSG) (Gazdar u.a. 1985); Kategorialgrammatik (vgl. Bach 1988, Steedman 1993 und im Kontrast zur Phrasenstrukturgrammatik Pollard 1988); Relationale Grammatik (vgl. Aissen / Perlmutter 1983); Lexikalisch-Funktionale Grammatik (LFG) (Bresnan 1982) und Parsing and Translation (PATR) (Shieber u.a. 
1983; für vergleichende Ansätze s. Egli / Egli-Gerber 1991, Sells 1985, Borsley 1993, Borsley 1996 und mit Bezug zu romanischen Sprachen Abeillé 1993; aktuell und umfassend Sag / Wasow 1999).

$\mathrm{Zu}$ den Unifikationsgrammatiken, auch deklarative Grammatiken genannt, werden insbesondere die LFG, die GPSG, merkmalsbasierte Ansätze innerhalb der Kategorialgrammatik und die HPSG gezählt. Neben einer Reihe von Unterschieden sind diesen Ansätzen folgende Aspekte gemeinsam:

- die Verwendung von Merkmalstrukturen zur Repräsentation linguistischer Inhalte. Dabei stellt die Vereinigung (Unifikation) von Merkmalstrukturen zu komplexeren Einheiten eine grundlegende Operation des Grammatiksystems dar;

- der weitgehende Verzicht auf Methoden der generativen Linguistik, in denen mit Oberflächen- und Tiefenstruktur zwei syntaktische Ebenen angenommen und durch Transformationen aufeinander bezogen werden.

Diese Aspekte sind auch durch den Einfluß der Computerlinguistik begründet, in der die HPSG als Basis für Parser ${ }^{4}$ herangezogen wird. Durch die besonders präzisen Erfordernisse, welche die Informatik an Grammatikmodelle stellt, wurde in der HPSG besonders auf mathematisch-logisch abgesicherte Formalismen geachtet.

Ein wichtiger Faktor in der Entwicklung dieses Grammatiktyps ist das Lexikon. Lexikalische Einheiten werden als komplexe Gebilde interpretiert, die linguistische Information beinhalten. Auf diese Information wirken syntaktische Regeln und Prinzipien ein. Dabei darf die Information in einer Struktur nur erweitert, aber nicht wie in der Transformationsgrammatik üblich - verändert oder gar getilgt werden. Es wird keine Reihenfolge für verschiedene Regelanwendungen postuliert, d.h. jede Regel kann jederzeit auf eine Struktur angewandt werden ohne Beschränkungen derart, daß bestimmte andere Regeln vorausgehen oder nachfolgen müßten.

In Opposition zu transformationsbasierten Formalismen wie der Government and Binding Theory von Chomsky (1981, vgl. auch die darauf basierende Weiterentwicklung der minimalistischen Grammatiktheorie von Chomsky / Lasnik 1995) verwenden Unifikationsgrammatiken keine unterschiedlichen syntaktischen Beschrei-

\footnotetext{
${ }^{4}$ Vgl. Stolzenberg (1992) oder das System in Copestake u.a. (1993). Linguistische Information wird allerdings seltener in HPSG-üblichen Matrizen dargestellt, da dies technisch bedingt relativ aufwendig ist. Für ein LFG-basiertes System vgl. Berman / Frank (1996: 2), für einen auf der HPSG basierenden Parser vgl. Cooper (1990: 160ff. und 207ff.). Zur katalanischen NP im Kontext der automatischen Übersetzung vgl. Badia (1994). Einführend in die Thematik Linguistik und EDV vgl. Figge, erscheint.
} 
bungsebenen. ${ }^{5}$ Alle Informationen, seien es phonologische, syntaktische oder semantische, werden grundsätzlich in der gleichen Art und Weise durch Merkmalstrukturen dargestellt. In Opposition zur LFG entsteht dabei nur eine einzige Matrix, die mit der funktionalen Struktur (f-structure) der LFG vergleichbar ist. Einen Strukturbaum, wie er im LFG-Modell als Konstituentenstruktur (c-structure) neben der $f$-structure üblich ist, gibt es in der HPSG nicht (vgl. neben Bresnan 1982 auch Berman / Frank 1996). Der gebräuchliche Beschreibungsmodus der HPSG sieht lediglich eine Attribut-Werte-Matrix vor, die über das Merkmal Töchter (Daughters, DTRS) die Konstituentenstruktur eines Zeichens darstellt. Die Bildung eines bekannten Strukturbaums ist aber relativ problemlos ableitbar, ebenso wie Phrasenstrukturregeln der Art S $\rightarrow$ NP VP. Sie sind dem System inhärent.

Pollard / Sag (1987) ist weiterhin als das grundlegende Werk für die HPSG anzusehen. Im zunächst als zweiten Band vorgesehenen Werk von 1994 wurden zahlreiche Modifikationen vorgenommen, sowie im neunten Kapitel (,Reflections and Revisions“) eine Vielzahl von weitreichenden Änderungen, die sich z.T. bewährt haben, diskutiert. Wir orientieren uns hier in der Regel am Werk von 1994, auch wenn das Buch von 1987 weiterhin die Grundlage stellt. Da sehr viele Verbesserungsvorschläge von 1994 und insbesondere des neunten Kapitels mittlerweile akzeptiert sind, werden wir diese einbeziehen. Vorschläge anderer Autoren werden diskutiert und z.T. in die Deskription spanischer Nominalphrasen integriert (vgl. z.B. als eine der ersten umfassenden Grammatikfragmente die Classification-based PSG von Cooper 1990).

\footnotetext{
${ }^{5}$ Für Einführungen in die HPSG mit der GB-Theorie als Ausgangspunkt vgl. Kiss (1995b) sowie für einen Vergleich der Theorien Borsley (1997).
} 


\section{Sprache als ein System von Zeichen: die HPSG}

\subsection{Merkmalstrukturen oder Attribut-Werte-Paare}

Unifikationsbasierte Formalismen verwenden Merkmalstrukturen zur Darstellung von Information: „feature structures are serving an informational function“ (Shieber 1986: 14). In der HPSG werden sprachliche Zeichen durch Merkmalstrukturen in Form von Matrizen repräsentiert. Pollard / Sag (1994: 31) unterscheiden zwischen phrasalen und lexikalischen Zeichen, wobei letztere einzelne Wörter kennzeichnen, während phrasale Zeichen Phrasen (NP, VP, PP usw.) und Sätze sind. Das sprachliche Zeichen und somit die Attribut-Werte-Paare stellen die grundlegende informationstragende Struktur in der HPSG dar (vgl. Pollard / Sag 1987: 8). Im Detail bedeutet dies, daß sprachliche Informationen der Art „dieses Nomen steht im Singular“ (z.B. coche) aufgegliedert werden. Die Information dieses Aussagesatzes, daß es sich um ein Nomen handelt, das den Wert Singular besitzt, welcher dem Attribut ${ }^{6}$ Numerus zugeordnet werden muß, wird folgendermaßen dargestellt:

$\left[\begin{array}{ll}\text { NUM } & s g\end{array}\right]^{7}$

Der Unterschied zwischen komplexen und atomaren Werten ist bedeutsam: Atomare Werte wie $s g$ für Singular können nicht weiter untergliedert werden, während komplexe Werte selbst weitere Merkmalstrukturen beinhalten. Wenn der Wert für den Numerus nicht bekannt ist, weil das alleinstehende Nomen ohne Determinierer ihn nicht festlegt, z.B. dt. Esel, wird dies durch eine leere Matrix gekennzeichnet:

[NUM [ ] ]

\footnotetext{
${ }^{6}$ Attribut und Merkmal werden in der HPSG-Literatur oft synonym verwendet.

${ }^{7}$ In der HPSG-Literatur sind verschiedene Notationen gebräuchlich. Wir setzen in dieser Arbeit die Merkmale recte und Werte kursiv. Sorten stehen innerhalb der Matrix oben links und werden verkleinert und kursiv gesetzt. Ein verweisender Index, ansonsten oft tiefergestellt, wird hier hinter den Wert mit Doppelpunkt gesetzt [Merkmal Wert : [Index] ] oder auch in dieser Form: [Merkmal [Index] [Wert] ], wenn es sich bei dem Wert um eine komplexere Matrix handelt, die mehrere untereinander plazierte Merkmale enthält.
} 
Merkmalstrukturen werden in der HPSG voll spezifiziert, indem neben der Angabe der Attribute und deren Werte auch die Sorte (auch Typ ${ }^{8}$ genannt) angegeben wird, d.h. daß jede Merkmalstruktur mit einem Sortensymbol etikettiert wird. Die Sorte sorgt dafür, daß komplexere Informationen kürzer dargestellt werden können, indem per Definition bei gleicher Sorte nur ein bestimmter Aufbau eines Zeichens zugelassen wird. Die allgemeinste Sorte eines sprachlichen Zeichens ist zeichen (engl. sign).

Ein allgemeines sprachliches Zeichen beinhaltet in erster Linie zwei Informationen: phonologische (PHON) und syntaktisch-semantische (SYNSEM), welche fast immer in Attribut-Werte-Paaren dargestellt werden. (In Pollard / Sag 1987 werden Syntax und Semantik noch getrennt, so daß es drei Ebenen gibt: PHON, SYN und SEM.) Da es sich hier nur um geschriebene Wörter und Sätze handelt und über die phonologische Seite nichts gesagt werden wird, könnte auch eine entsprechende Abkürzung für Orthografie gesetzt werden (vgl. Copestake u.a. 1993). Wir bleiben hier beim HPSG-Standard. Auch die englischen Abkürzungen für Merkmale und Werte werden beibehalten. Werte sind atomar (12) oder komplex (13):

$$
\left[\begin{array}{l}
\text { zeichen } \\
\text { PHON coche } \\
\text { SYNSEM [] }
\end{array}\right]
$$

$$
\left[\begin{array}{l}
\text { zeichen } \\
\text { PHON coche } \\
\text { SYNSEM } \mid \text { LOCAL } \mid \text { CAT } \mid \text { HEAD }\left[\begin{array}{l}
\text { nomen } \\
\text { NUM sg }
\end{array}\right]
\end{array}\right]
$$

Genau genommen müßten in (13) eingebettete Matrizen mit jeweils neuem Attribut stehen: nach SYNSEM eine Matrix mit dem Attribut LOCAL (lokal), nach LOCAL eine Matrix mit dem Attribut CATEGORY (Kategorie, CAT) bis zu HEAD mit der Sortenangabe nomen. Diese Folge von Attributen wie in der Kette SYNSEM| LOCAL | CAT | HEAD wird Pfad genannt. Der senkrechte Strich zeigt dabei nur den Weg, der beschritten wird. So könnte neben LOCAL noch NON-LOCAL als Attribut für SYNSEM erscheinen, wie auch neben CAT noch CONTENT oder

\footnotetext{
${ }^{8}$ Auch die Termini Sorte und Typ werden in der HPSG-Literatur synonym verwendet.
} 
CONTEXT als Attribut für LOCAL. Diese werden aber ausgespart, wenn für den jeweiligen Darstellungszusammenhang nur der aufgezeichnete Pfad nötig ist.

Die Sorte einer Merkmalstruktur wird in der HPSG in eine Sortenhierarchie eingebettet. Diese Hierarchie kann durch eine Disjunktion dargestellt werden. Dabei wird das bei Pollard / Sag (1987) regelmäßig verwendete und später eliminierte Attribut [LEX +] für lexikalische Zeichen gern als einfaches Unterscheidungsmerkmal gebraucht (vgl. Müller 1999: 2). Die Relation von z.B. zeichen zu lexikalisches_zeichen kann als eine Relation von Supertyp zu Subtyp bezeichnet werden.

$$
\begin{array}{ll}
\text { zeichen } & =\text { lexikalisches_zeichen } \vee \text { phrasales_zeichen } \\
\text { lex_zeichen } & =\text { nomen } \vee \text { verb } \vee \text { präposition } \vee \ldots \\
\text { verb } & =\text { vollverb } \vee \text { hilfsverb }
\end{array}
$$

Ein Baumdiagramm kann die gleiche Information auch darstellen:

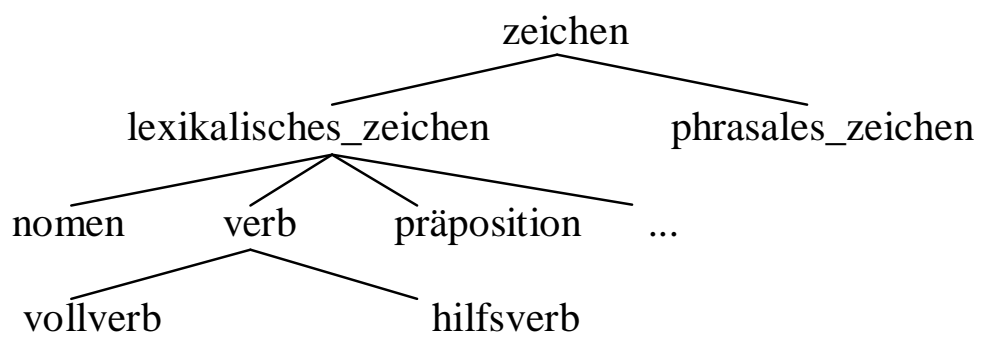

Der Aufbau einer Matrix zur Beschreibung eines sprachlichen Zeichens wird in der HPSG folgendermaßen normiert:

$$
\left.\left[\begin{array}{l}
\text { zeichen } \\
\text { PHON ... }
\end{array}\left[\begin{array}{l}
\text { LOC }\left[\begin{array}{l}
\text { HEAD ... } \\
\text { SUBCAT ... }
\end{array}\right] \\
\text { CONTENT ... } \\
\text { CONTEXT ... }
\end{array}\right]\right]\right]
$$

Wir bekommen neben der phonologischen eine detailliert untergliederte Matrix für die syntaktisch-semantische Information. Diese Information (SYNSEM) wird zunächst in lokale (LOCAL) und nicht-lokale Merkmale (NON-LOCAL) unterteilt. Die 
lokale Information untergliedert sich wiederum in kategorielle (CAT), semantische (CONTENT) und kontextuelle Information (CONTEXT). Anstelle von kontextueller können wir auch von pragmatischer Information sprechen. Die Kategorie wird genauer spezifiziert durch die Angaben zum Kopf der Phrase (HEAD) und ihrer Subkategorisierungsliste (SUBCAT, in neueren Ansätzen Valenz genannt).

Bevor wir auf weitere linguistische Fragen in den Kapiteln II.2.3. bis II.2.9. eingehen, werden die mathematischen Eigenschaften und Möglichkeiten von Merkmalstrukturen erläutert.

\subsection{Mathematische Eigenschaften von Merkmalstrukturen}

Merkmalstrukturen haben mathematisch definierte Eigenschaften. Insbesondere sind Subsumtion und Unifikation zu nennen, die einen weitreichenden Einfluß auf das Arbeiten mit Merkmalstrukturen besitzen (für eine formale Einführung vgl. King 1989 und Carpenter 1992).

Im Mittelpunkt steht die Wohlgeformtheit eines sprachlichen Zeichens. Auch Merkmalstrukturen müssen wohlgeformt sein und eine linguistisch korrekte Entität widerspiegeln. Zudem dürfen sie sich nicht selbst widersprechen. Strukturen wie in (17a) mit dem Attribut $f$ oder in (17b) sind nicht korrekt, gekennzeichnet durch einen Asterisk *, da innerhalb derselben Merkmalstruktur einem Attribut zwei verschiedene Werte zugeschrieben werden:

$$
*\left[\begin{array}{ll}
\mathrm{f} & a \\
\mathrm{f} & b
\end{array}\right]
$$

$$
*\left[\begin{array}{l}
\text { NUM } s g \\
\text { NUM } p l
\end{array}\right]
$$

Sehr wohl möglich ist natürlich wie in allen mathematischen Operationen das Verwenden von Variablen. 


\subsubsection{Subsumtion und Extension}

Wir betrachten zunächst die folgenden zwei Matrizen:

$\left[\begin{array}{ll}\text { PHON } & \text { coche } \\ \text { SYNSEM }[]\end{array}\right]$

$$
\left[\begin{array}{l}
\text { PHON } \\
\text { SYNSEM } \mid \text { LOCAL } \\
\text { LAT } \mid \text { HEAD nomen }
\end{array}\right]
$$

Die Merkmalstruktur (18b) ist reicher an Information als (18a). Man sagt: (18b) ist eine Extension von (18a) (ist spezieller als (18a)). Im Gegenzug subsumiert die Matrix (18a) die Matrix (18b), formal dargestellt: (18a) $\preceq(18 b)$. Die Subsumtionsrelation ist wichtig, da, wie oben gezeigt, im HPSG-Formalismus Sortenhierarchien aufgestellt werden. Folglich subsumiert die oberste Sorte in der Hierarchie zeichen alle anderen Sorten für sprachliche Ausdrücke, da sie speziellere Informationen beinhalten als zeichen. Alle anderen Sorten beinhalten aber auch die Gesamtheit der Informationen, die zeichen enthält. Die Sorte verb hingegen ist eine Extension von zeichen, da sie mehr Informationen enthält. Verb subsumiert hingegen die Sorte vollverb, welche tiefer in der Hierarchie steht. Wir bilden somit Klassen, die zueinander in einer Teilmengenrelation stehen.

Aus den obigen Ausführungen folgt, daß es Bedingungen für eine Subsumtionsrelation gibt. So müssen beide Merkmalstrukturen konsistent sein.

(19) A subsumiert B, d.h. $\mathrm{A} \preceq \mathrm{B}$ genau dann, wenn

B alle Informationen enthält, die A enthält.

A werde instantiiert durch (18a), B durch (18b).

Aus (19) folgt, daß

1. alle Pfade von (18a) auch Pfade in (18b) sind;

2. beide Merkmalstrukturen Sortenidentität aufweisen;

3. die Strukturen identisch sein müssen (der Unterstrich symbolisiert eine mögliche Gleichheit), wenn es sich um atomare Merkmalstrukturen handelt:

Wenn $\mathrm{A} \preceq \mathrm{B}$, wobei $\mathrm{A}$ und $\mathrm{B}$ atomar, dann $\mathrm{A}=\mathrm{B}$

(vgl. auch Naumann / Langer 1994: 230, und die Definition von Kiss 1995a: 43). 
Die Bedingung besagt lediglich, daß B alle Informationen enthalten muß, die auch A enthält. So darf keine widersprüchliche Information vorkommen, die Pfadanfänge müssen gleich sein, und es muß einen (beliebigen) Supertyp beider Strukturen in einer Hierarchie geben. Die letzte Folgerung verdeutlicht, daß zwei Strukturen identisch sein müssen, wenn sie jeweils nur einen atomaren Wert besitzen. Sie werden sich dann gegenseitig subsumieren.

Wir können für die Matrix in (18) folgende Subsumtionsrelation aufstellen:

$$
[] \preceq\left[\begin{array}{l}
\text { PHON coche } \\
\text { SYNSEM }[]
\end{array}\right] \preceq\left[\begin{array}{l}
\text { PHON coche } \\
\text { SYNSEM } \mid \text { LOCAL } \mid \text { CAT } \mid \text { HEAD nomen }
\end{array}\right]
$$

Die leere Menge bleibt stets allgemeinstes Element einer solchen Relation. Sie wird als T (sprich: top) bezeichnet, da sie alle anderen Elemente subsumiert. Elemente, die inkonsistente Informationen tragen oder bei gleichem Pfad verschiedene Werte aufweisen, können nicht zueinander in Beziehung gesetzt werden. Dafür steht das Symbol $\perp$ (sprich: bottom).

Die Beziehung zwischen zeichen, phrasales_zeichen und lexikalisches_zeichen läßt sich ebenfalls in einer Subsumtionsrelation darstellen, wobei zeichen auch als Supertyp der Subtypen phrasales_zeichen und lexikalisches_zeichen bezeichnet wird:

$$
\text { zeichen } \preceq \text { lexikalisches_zeichen, zeichen } \preceq \text { phrasales_zeichen }
$$

\subsubsection{Unifikation}

Die Unifikation ist die zentrale Operation der HPSG wie aller anderen Unifikationsgrammatiken. Sie steht in engem Zusammenhang mit der Subsumtion, denn auch hier müssen die Werte und die Pfade konsistent sein. Wir nehmen folgendes Beispiel: In einer Matrix wird Kongruenz (AGREEMENT, AGR) mit Numerus und Person spezifiziert, in einer anderen mit Numerus und Genus. Die Werte müssen widerspruchsfrei sein, d.h. in diesem Fall müssen beide Numeruswerte identisch sind. Da Numerus jeweils auf AGR folgt, ist die Pfadangabe ebenfalls konsistent. Die beiden 
Matrizen können nun in einer vereinigt werden $(\cup$ bezeichnet hier die Operation der Unifikation):

$$
\begin{aligned}
{\left[\text { AGR }\left[\begin{array}{ll}
\text { NUM } & s g \\
\text { PER } & 3
\end{array}\right]\right] \cup\left[\text { AGR }\left[\begin{array}{ll}
\text { NUM } s g \\
\text { GEN } & f e m
\end{array}\right]\right] } & \Rightarrow\left[\text { AGR }\left[\begin{array}{ll}
\text { NUM } s g \\
\text { PER } & 3 \\
\text { GEN } & f e m
\end{array}\right]\right] \\
\mathrm{A} & \cup \quad \mathrm{B} \quad \mathrm{C}
\end{aligned}
$$

Nach den im vorigen Unterkapitel aufgestellten Prinzipien von Subsumtionsrelationen muß nun gelten, daß jede der beiden Matrizen A und B jeweils $C$ subsumiert, da $\mathrm{C}$ durch die Operation der Unifikation nun mehr Information spezifiziert als A oder B (vgl. Shieber 1986, 17f.). Umgekehrt ist C eine Extension sowohl von A wie auch von B. Sind die Werte der zu unifizierenden Matrizen nicht konsistent, scheitert die Unifikation. Dies wird mit dem Symbol $\perp$ (fail) dargestellt.

$$
\begin{aligned}
& \text { AGR } \left.\left[\begin{array}{ll}
\text { NUM } s g \\
\text { PER } & 3
\end{array}\right]\right] \cup\left[\text { AGR }\left[\begin{array}{l}
\text { NUM } p l \\
\text { GEN fem }
\end{array}\right]\right] \Rightarrow \perp \\
& \text { A } \cup \quad \text { B } \quad \Rightarrow \text { C }
\end{aligned}
$$

Wenn nun zwei Strukturen, die in einem größeren Zusammenhang stehen, identische Werte aufweisen, besteht die Möglichkeit, sie über eine Variable zu indizieren. Der Wert wird dann von beiden Strukturen geteilt. Pollard / Sag (1994: 19) nennen dies structure sharing (Darstellung nach Kiss 1995a: 41):

$$
\left[\begin{array}{l}
\text { SUBJ } \mid \text { AGR }\left[\begin{array}{lr}
\text { NUM } & s g \\
\text { PER } & 3
\end{array}\right] \\
\text { AGR }\left[\begin{array}{lc}
\text { NUM } & s g \\
\text { PER } & 3
\end{array}\right]
\end{array}\right]
$$

$$
\left[\begin{array}{l}
\text { SUBJ } \mid \text { AGR [1] }\left[\begin{array}{lr}
\text { NUM } & s g \\
\text { PER } & 3
\end{array}\right] \\
\text { AGR [1] }
\end{array}\right]
$$

Im Fall von (24a) sind zwar beide Agreement-Werte identisch, aber nicht voneinander abhängig. Hier können wir von Typ-Identität (type-identity) sprechen. Im zweiten Fall (24b) besteht durch Indices eine gegenseitige Abhängigkeit, da sich beide Zeichen dieselben Merkmale teilen. Dieser Fall wird strikte Identität (token-identity) genannt (vgl. Kiss 1995a: 40f.). Er tritt z.B. bei der Subjekt-Verb-Kongruenz auf, für 
deren Beschreibung (24b) ein Beispiel ist. Eine logische Folge der strikten Identität besteht darin, daß nach Modifikation eines Wertes die Aktualisierung für beide Pfade gilt. Somit darf strikte Identität nur bei Pfaden angenommen werden, die auch in Abhängigkeit zueinander stehen. Wenn die Pfade in (24a) sich auf zwei verschiedene Konstruktionen beziehen und nicht voneinander abhängig sind, so kann zwar von Typ-Identität gesprochen werden, der strukturteilende Index wäre aber falsch.

In der HPSG wird sehr viel mit Indizes gearbeitet, da sprachliche Information auf diese Weise nur einmal dargestellt werden muß. An anderen Stellen wird per Index auf diese Information referiert, ohne sie nochmals explizit formulieren zu müssen.

Theoretisch ist es möglich, daß eine Merkmalstruktur zyklisch ist, indem sich ein Index auf eine Struktur innerhalb des eigenen Geltungsbereichs bezieht. Diese Strukturen beschreiben eine unendliche Rekursivität. Seien $f, g$ und h beliebige Variablen:

$$
\text { f [1] } \left.\left[\begin{array}{cc}
\mathrm{g} & a \\
\mathrm{~h} & {[1]}
\end{array}\right]\right]
$$

In der obigen Matrix referiert der Index der Wertematrix von f auf den Wert von h. Da h zugleich auch einen Teilwert von $\mathrm{f}$ darstellt, ergibt sich eine zyklische Struktur.

In Merkmalsstukturen werden auch Listen und Mengen von Merkmalstrukturen als Werte erlaubt. Das Attribut Subkategorisierung (SUBCAT) wird grundsätzlich durch eine Liste (d.h. eine geordnete Menge) an Werten spezifiziert (Notation durch spitze Klammern $<>$ ). Ebenfalls möglich ist eine Menge an Werten (Notation durch geschweifte Klammern \{ \} ). Subsumtions- und Unifikationsregeln sind mit der Relation append ('anhängen') entsprechend anzuwenden (vgl. Kiss 1995a: 45):

(a) Listenstrukturen: <XP, YP >

Mengenstrukturen: $\{\mathrm{XP}, \mathrm{YP}\}$

(b) Die Unifikation zweier Listenstrukturen gelingt nur bei gleicher Anzahl der Elemente: Seien $A=<A_{1}, \ldots, A_{n}>, B=\left\langle B_{1}, \ldots, B_{n}>\right.$ und $C=<C_{1}$, $\ldots, \mathrm{C}_{\mathrm{n}}>$ Listen von Merkmalstrukturen. $\mathrm{C}$ ist die Unifikation von $\mathrm{A}$ und $B(C=A \cup B)$ gdw. für jedes $C_{i}$ gilt: $C_{i}=A_{i} \cup B_{i}$; die Unifikation von Listen wird unter Beachtung der Reihenfolge elementweise durchgeführt (Pollard / Sag 1987: 45f.). 
(c) Die Unifikation zweier Mengen $\mathrm{M}_{1}$ und $\mathrm{M}_{2}$ von Merkmalstrukturen ist jede konsistente Menge $\mathrm{M}$, die sowohl durch $\mathrm{M}_{1}$ als auch durch $\mathrm{M}_{2}$ beschrieben werden könnte. Pollard / Sag (1987: 47) verwenden folgendes Beispiel, um die Unifikation zweier Mengen zu erläutern. Das Ergebnis $\mathrm{C}$ muß eine Disjunktion sein, da die Zuordnung der Merkmale aus A und $\mathrm{B}$ nicht festgelegt ist:
A $\{[$ MAKE TOYOTA], [MAKE DATSUN] $\}$
B $\{[$ COLOR WHITE $],[$ COLOR RED $]\}$
C $\left\{\left[\begin{array}{l}\text { MAKE } \\ \text { COLOR WOYOTA }\end{array}\right],\left[\begin{array}{l}\text { MAKE } \\ \text { COLOR RATSUN }\end{array}\right]\right\} \vee$
$\left\{\left[\begin{array}{l}\text { MAKE TOYOTA } \\ \text { COLOR RED }\end{array}\right],\left[\begin{array}{ll}\text { MAKE } & \text { DATSUN } \\ \text { COLOR WHITE }\end{array}\right]\right\}$

\subsubsection{Disjunktion, Negation und Implikation}

In einigen Fällen ist es nötig, den Wert eines Attributs durch eine Disjunktion zu beschreiben. Das deutsche Wort Mann stellt dafür ein gutes Beispiel dar (nach Kiss 1995a: 47):

$$
\begin{aligned}
& \text { Mann: } \\
& {\left[\text { CAT }\left[\begin{array}{l}
\text { NFORM NORM } \\
\text { CASE NOM } \vee D A T \vee A K K
\end{array}\right]\right.}
\end{aligned}
$$

Die Negation kann u.U. eine Beschreibung abkürzen. Die Darstellung (27) läßt sich auch durch eine Negation beschreiben (Kiss 1995a: 47):

$$
\left[\mathrm{CAT}\left[\begin{array}{ll}
\text { NFORM } & \text { NORM } \\
\text { CASE } & \neg G E N
\end{array}\right]\right]
$$

Hier wird ein Verfahren der Unterspezifizierung angewandt. Ein Merkmal kann nicht genau spezifiziert werden, da es ambig ist und erst der Kontext eine genauere Beschreibung ermöglicht. So erlaubt das deutsche Nomen Mann drei Kasus, was erst durch einen der drei möglichen Artikel (der für NOM, dem für DAT, den für AKK) 
festgelegt wird. Um mit positiven Kategorien zu arbeiten, wird in der Computerlinguistik zuweilen eine künstliche Zwischenklasse angenommen, die aus den drei möglichen Kasus besteht und erst später durch den Kontext aufgelöst wird.

Die Implikation besitzt die Form $\mathrm{A}=>\mathrm{B}$, d.h. wenn ein Objekt A entspricht, entspricht es auch B. Die Implikation wird bei den Prinzipien und Regeln der HPSG verwendet.

Diese logischen Operationen werden bei der Deskription eines sprachlichen Zeichens in der HPSG ständig angewandt. So werden öfter lexikalische Regeln (als Operation der Implikation) verwendet, um Lexeme in andere Kategorien zu überführen, die sich in der Folge mit anderen Lexemen zu Phrasen verbinden. Kiss (1995a: 44) definiert diese Beziehung nach Pollard / Sag (1987: 43) wie folgt (für eine formale Einführung vgl. Carpenter 1991):

\section{Implikation:}

Die Unifikation einer Implikation $A=>B$ mit einer beliebigen Struktur C entspricht der Unifikation von B mit C, wenn C eine Subsorte von A ist; d.h. $(\mathrm{A}=>\mathrm{B}) \cup \mathrm{C}=\mathrm{B} \cup \mathrm{C}$, wenn $\mathrm{A} \preceq \mathrm{C}$.

Wenn A durch eine Regel in B umgewandelt und danach mit C unifiziert wird, dann ist das Resultat identisch mit einer sofortigen Unifikation von B mit C.

\subsubsection{Sorten und Sortenhierarchie}

Wie bereits angesprochen, werden die Merkmalstrukturen in der HPSG etikettiert. Damit wird festgelegt, daß nur bestimmte Merkmale zu der Merkmalstruktur einer Sorte gehören, nämlich diejenigen, die als angemessen deklariert werden. Andere sind nicht akzeptabel. Zudem werden die Sorten in eine Hierarchie überführt, so daß zwischen Sorten immer eine Relation besteht. In (15) wurde z.B. die Relation zwischen zeichen und lexikalisches_zeichen als eine von Supertyp und Subtyp bezeichnet. Pollard / Sag (1994: 396ff.) stellen eine universelle Sortenhierarchie auf. Das Ziel ist es, möglichst wenig redundante Information darstellen zu müssen. Sag / Wasow (1999: 139) definieren (T steht für type): 
„If $\mathrm{T}_{2}$ is a subtype of $\mathrm{T}_{1}$, then

a. every feature specified as appropriate for $T_{1}$ is also appropriate for $T_{2}$, and

b. every constraint associated with $\mathrm{T}_{1}$ affects all instances of $\mathrm{T}_{2}$.“

Auf diese Weise wird das Lexikon in einer effektiven Weise organisiert. Wir skizzieren hier einen Ausschnitt aus dieser Hierarchie, der mögliche Kopfstrukturen zeigt (vgl. Flickinger 1987: 5-54, bes. 20):

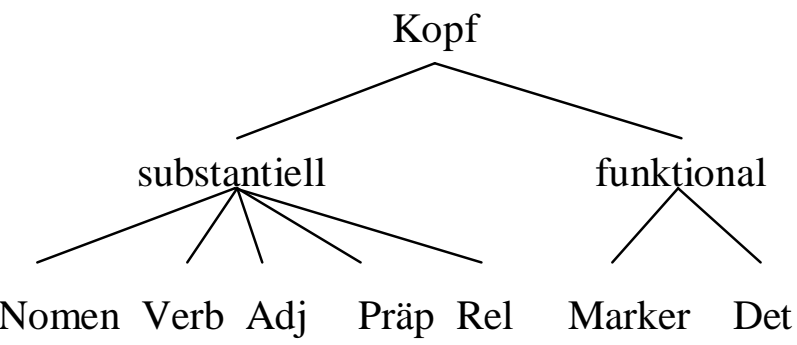

In der HPSG wird der Begriff „Kopf“ besonders hervorgehoben. Jedes sprachliche Zeichen besitzt einen Kopf, der seine Merkmale auf das gesamte Zeichen vererbt. In der obigen Hierarchie wird eine Aufteilung vorgestellt, die Köpfe in substantiell und funktional gliedert. Die substantiellen Köpfe sind lexikalische Einheiten. Funktionale Köpfe übernehmen vorwiegend syntaktische Funktionen.

\subsection{Die syntaktische Struktur sprachlicher Zeichen}

\subsubsection{Köpfe, Kopfmerkmale und Kopftöchter}

In der kontextfreien PSG hatten wir terminale und nicht-terminale Symbole, um Wörter einer Sprache zu erfassen. Wir nennen die terminalen Symbole in ihrer Gesamtheit nun Lexikon. Unter einem Lexikon verstehen wir eine Liste aller Wörter und Affixe, die in dieser Sprache vorkommen und die mit bestimmten Eigenschaften versehen werden können. Somit werden sowohl lexikalische Einheiten mit semantischer Bedeutung erfaßt wie auch morphologische Einheiten, die lediglich grammatische Kategorien ohne spezielle Bedeutung darstellen. Diese morphologischen Einheiten werden mit lexikalischen verknüpft, wobei sie deren ursprüngliche Eigenschaften verändern und eigene hinzufügen können. 
Eine Merkmalstruktur nach Pollard / Sag (1994: 29) für spanisch coche kann folgendermaßen aussehen:

$$
\left[\text { SYNSEM } \mid \text { LOC } \mid \text { CAT }\left[\begin{array}{l}
\text { HEAD }\left[\begin{array}{l}
\text { nomen } \\
\text { NFORM normal }
\end{array}\right] \\
\text { SUBCAT }\langle\text { DET }\rangle
\end{array}\right]\right]
$$

Die lokalen Merkmale eines lexikalischen Zeichens werden im Attribut HEAD und in der Subkategorisierung festgehalten. HEAD bestimmt, wie das Zeichen spezifiziert ist, d.h. welche Eigenschaften es trägt. Diese Eigenschaften können vom Kontext abhängig und zunächst unterspezifiziert sein, d.h. es werden nicht alle Merkmale in gewohnter Weise determiniert, sondern offen gelassen.

Das HEAD-Merkmal kann Werte der Sorten lexikalisch (substantive) oder funktional (functional) beinhalten. Lexikalisch umfaßt Nomina, Verben, Adjektive und Präpositionen - Wörter, von denen eine lexikalische Bedeutung erwartet wird -, funktional sind Determinierer und Marker (d.h. Komplementierer). Die lexikalischen Attribute werden durch ihre jeweilige Form - sprich: KASUS für Nomina und Adjektive, VFORM für Verben, PFORM für Präpositionen usw. - genauer bestimmt. Oft wird aber wie in (32) nur die Sorte des Kopfes angegeben. Sie entspricht der Wortart.

Das Attribut NOUNFORM (NFORM) eines spanischen Nomens kann nach Pollard / Sag (1987: 62) nur den Wert NORMAL anzeigen, da es die explitiven Pronomen (there, it, es) des Englischen und Deutschen, die bei Pollard / Sag alternativ stehen, im Spanischen nicht gibt. Allerdings kann ein Infinitiv in einer Weise nominal verwendet werden, daß nur eine Beschreibung als [NFORM inf] adäquat ist, z.B. in: Verás un siempre temer, / Un eterno idolatrar, / Un diestro lisonjar / Y un incierto pretender (Cervantes, zit. n. RAE 1985: 484). Da die alternative Darstellung über KASUS aus Pollard / Sag (1994) im Spanischen nur bei Personalpronomen möglich ist, erhält das Attribut NFORM weiterhin eine gewisse Berechtigung.

Analog gibt es für Verben ein Attribut VERBFORM (VFORM), das sich in die Werte finite (fin), infinitive (inf), past participle und gerund untergliedert. Pollard / Sag (1987: 60) unterscheiden noch die base-, present participle- und passive parti- 
ciple-Form ${ }^{9}$ eines Verbs, die im Spanischen nicht existieren. Zuletzt sind noch die booleschen ${ }^{10}$ Attribute AUX für die Beschreibung von Hilfsverben (+ oder -) und PRD für prädikative Konstruktionen, ebenfalls (+ oder -), zu nennen.

Die Merkmale des Kopfes einer Phrase werden an die gesamte Phrase weitervererbt. Damit muß eine Phrase alle Eigenschaften aufweisen, die auch ihr Kopf besitzt. Dies wird im Kopf-Merkmal-Prinzip festgehalten (Head Feature Principle; vgl. auch Sag / Pollard 1989: 139ff.). Zuvor müssen aber die Termini head, HEAD und HeadDaughter noch klarer differenziert werden. HEAD ist ein Attribut von CATEGORY und hat als Wert die Merkmale des entsprechenden Kopfes. Dieser Kopf wird in der HPSG-Literatur meist allgemein als head bezeichnet und hier zur besseren Unterscheidung einfach dt. Kopf genannt. In Anlehnung an die X-bar-Theorie war in der ersten Fassung der HPSG (Pollard / Sag 1987) der Kopf eines Ausdrucks in der Regel ein Wort mit dem Merkmal [LEX +$]^{11}$. Eine Kopftochter (Head-Daughter) enthält die Konstituente, die als Kopf der beschriebenen Struktur bezeichnet werden muß. Als Standards gelten folgende Festlegungen für Köpfe:

Kopf

- eines Satzes /

einer Verbalphrase: das am wenigsten untergeordnete Verb

- einer Nominalphrase: das am wenigsten untergeordnete Nomen

- einer Präpositionalphrase: die am wenigsten untergeordnete Präposition

- einer Adjektivphrase: das am wenigsten untergeordnete Adjektiv

\footnotetext{
${ }^{9}$ Die base-Form ist zudem meist mit dem Infinitiv, die past participle-Form mit der passive participle-Form identisch.

${ }^{10}$ Binär im Sinne von boolesch: entweder positiv (+) oder negativ (-), vgl. auch Hadlich (1975: 118).

${ }^{11}$ Das Attribut LEXICAL (LEX) (Pollard / Sag 1987: 72) wurde nur für die Wortstellung und die Differenzierung von phrasalen und lexikalischen Zeichen eingeführt, die in neueren Versionen über die Sorten stattfindet. Es wird meist weggelassen (vgl. Pollard / Sag 1994: 22). Wichtige Konstellationen der Wortstellung können mitunter auch über die Bindungsmerkmale erklärt werden und gelten als einzelsprachliche Phänomene.
} 
Das Kopf-Merkmal-Prinzip besagt nun folgendes:

(34) Der Wert des HEAD-Attributs der Kopftochter stimmt mit dem Wert des HEAD-Attributs der gesamten Konstituente überein.

Head-Feature-Principle:

$$
[\text { DTRS headed - structure }] \rightarrow\left[\begin{array}{l}
\text { SYNSEM } \mid \text { LOC } \mid \text { CAT } \mid \text { HEAD [1] } \\
\text { HEAD - DTR } \mid \text { SYNSEM } \mid \text { LOC } \mid \text { CAT } \mid \text { HEAD [1] }
\end{array}\right]
$$

\subsubsection{Subkategorisierung}

Die Subkategorisierungsliste enthält Verweise auf die Zeichen, die noch zur Sättigung der Konstituente nötig sind (vgl. Sag / Pollard 1989). Im speziellen Fall des Beispielsatzes el padre de Juan compró un coche nuevo in (6) bedeutet dies, daß ein Determinierer gebraucht wird, damit aus dem Nomen coche eine Nominalphrase wird. Für Verben benennt die Liste die noch gesuchten Komplemente und das Subjekt. Sie werden in gewohnter Abfolge - und im Gegensatz zu Pollard / Sag (1987) von links nach rechts in aufsteigender Obliquitätshierarchie aufgeführt, d.h. das Subjekt steht an erster Stelle, dann folgen die möglichen Objekte (zur Obliquitätshierarchie vgl. auch Langer / Thümmel 1994: 103). Für ein Verb wie comprar sieht dieser, zunächst nur die Syntax betreffende Eintrag folgendermaßen aus:

$$
\left[\text { SYNSEM } \left[\text { LOC }\left[\text { CAT }\left[\begin{array}{l}
\text { HEAD verb } \\
\operatorname{SUBCAT}\langle(\mathrm{NP}[N O M]), \mathrm{NP}[A K K]\rangle]
\end{array}\right]\right]\right.\right.
$$

Die Subkategorisierungsliste besagt, daß eine NP als Subjekt und eine NP als Objekt erwartet wird. Da im Spanischen das Subjektpronomen dem Verb inhärent ist und ein explizites Subjekt entsprechend fehlen kann, kann diese Optionalität durch eine runde Klammer um die Subjekt-NP dargestellt werden (vgl. zur Optionalität Kiss 1991).

Auch die Subkategorisierung kann in einem Prinzip festgehalten werden: 
(36) Der SUBCAT-Wert der Kopfkonstituente ist die Vereinigung des SUBCAT-Wertes der gesamten Konstituente mit den Werten der realisierten Komplementtöchter.

Subkategorisierungsprinzip:

[DTRS headed - structure $] \rightarrow$

$$
\left[\begin{array}{l}
\text { DTRS }\left[\begin{array}{l}
\text { HEAD - DTR } \mid \text { LOC } \mid \text { CAT }[\text { SUBCAT append }([1],[2])] \\
\text { COMP - DTRS [2] }
\end{array}\right]
\end{array}\right]
$$

Das Attribut Töchter (DAUGHTERS, DTRS) mit seinen Subtypen Kopftochter und Komplementtöchter (Adjunkttöchter, Markertöchter usw.) bezeichnet die Verzweigungen, die in einem Strukturbaum üblich sind. Dabei wird jede Konstituente nach ihrer Relation zum Kopf der übergeordneten Konstituente benannt.

Es folgen die Subkategorisierungslisten einiger spanischer Wörter:

$$
\begin{aligned}
\text { (a) llueve }< & > \\
\text { (b) fácil }< & (\mathrm{PP}[a \vee \text { de } \vee \text { para }+\mathrm{NP} \vee \mathrm{INF}] \vee \\
& \mathrm{KONJ}[q u e+\mathrm{SUBJUNTIVO}])> \\
\text { (c) hombre }< & \mathrm{DET}> \\
\text { (d) come }< & (\mathrm{NP}[N O M, N O R M]),(\mathrm{NP}[\text { AKK,NORM }])> \\
\text { (e) compra }< & (\mathrm{NP}[N O M, N O R M]), \mathrm{NP}[\text { AKK, NORM }]> \\
\text { (f) obligó }< & (\mathrm{NP}[N O M, N O R M]), \mathrm{XP}[\text { AKK, NORM }], \mathrm{PP}[a+\mathrm{INF}]>\vee \\
< & (\mathrm{NP}[N O M, N O R M]), \mathrm{XP}[\text { AKK, NORM }], \\
& \operatorname{PP}[a+\mathrm{KONJ}+\mathrm{SUBJUNTIVO}]>
\end{aligned}
$$

Das erste Beispiel zeigt das nullwertige Verb llover, welches kein Komplement zu sich nimmt. Fácil kann als Adjektiv eine Präpositionalphrase (Para España será muy fácil de cumplir, M-13) oder einen Subjektsatz (Es fácil que venga hoy, RAE 1992: 943) nach sich ziehen. Da Subkategorisierung bei Adjektiven nur fakultativ auftritt, stehen beide Optionen in runden Klammern. Gleiches gilt für mögliche Subjekte bei den Verben in (d) bis (f). Das Nomen hombre subkategorisiert einen Artikel (vgl. 
Kapitel III.1.). Bei come ist das Akkusativobjekt fakultativ. Compra hingegen verlangt ein Objekt. Beide Objekte werden als NP $[A K K]$ bezeichnet.

Obligar kann sich auch auf Personen beziehen, wobei das Akkusativobjekt dann mit der Präposition $a$ konstruiert und als PP dargestellt wird. So ist eine Beschreibung mit XP nötig, als Abkürzung für NP $\vee$ PP. Möglich wäre auch eine künstliche Klasse Akkusativphrase, die als Supertyp von NP und PP fungiert und beide Phrasentypen subsumiert. Diese Konstituente wäre in bezug auf ihre linguistischen Sorte unterspezifiziert.

Für Verben, die Konstituenten binden, die als Verbalphrasen zu bezeichnen sind (z.B. Infinitiv- bzw. Gerundialkonstruktionen), wie z.B. obligar, muß eine Disjunktion eingeführt werden: Je nach Konstruktion mit gleichem Subjekt wie im Matrixsatz oder eigenem Subjekt folgt eine Infinitivphrase oder ein Nebensatz im Konjunktiv. Das fakultative Subjekt des Spanischen wird hier nicht weiter thematisiert.

\subsubsection{Syntaktische Kategorien und Schemata}

Die Subkategorisierungsliste und der Kopf eines Zeichens besitzen besondere Bedeutung als mögliche Klassifikationskriterien für Wortarten. Das Merkmal HEAD charakterisiert und klassifiziert die Kopfmerkmale einer Phrase. Die Subkategorisierung legt nicht nur die Anzahl, Art und Obliquitätshierarchie der Komplemente fest, sondern identifiziert auch die syntaktische Kategorie. So können nur Determinierer, Nominalphrasen und Sätze gesättigt sein, während Nomina und Verbalphrasen sowie Verben zumindest ein Komplement erfordern. Die Obliquitätshierarchie ordnet dabei die Komplemente eines Kopfes nach ihrer syntaktischen Nähe zum Kopf. So sind Dativ- und Akkusativobjekt weitaus enger an das Verb angelehnt (ihr ein Buch geben) als das Subjekt (vgl. die Beispiele und Ausführungen bei Langer / Thümmel 1994: 102ff. und Klenk 1997: 314ff.).

Aus diesen HEAD-Attributen lassen sich zusammen mit der Subkategorisierungsliste die traditionellen grammatischen Symbole definieren, die in der kf PSG als nicht-terminale Symbole beschrieben wurden (vgl. auch Pollard 1988: 398): 
(a) DET $\left[\operatorname{SYNSEM} \mid\right.$ LOC $\left.\mid \mathrm{CAT}\left[\begin{array}{l}\mathrm{HEAD} \text { det } \\ \operatorname{SUBCAT}\langle\rangle\end{array}\right]\right] \quad$ uno, el

(b) $\quad \mathrm{N} \quad\left[\operatorname{SYNSEM} \mid\right.$ LOC $\left.\mid \mathrm{CAT}\left[\begin{array}{l}\mathrm{HEAD} \text { noun } \\ \operatorname{SUBCAT}\langle\mathrm{DET}\rangle\end{array}\right]\right]$ crisis, chico malo

(c) NP $\quad\left[\right.$ SYNSEM $\mid$ LOC $\mid$ CAT $\left.\left[\begin{array}{l}\text { HEAD noun } \\ \text { SUBCAT }\langle\rangle\end{array}\right]\right] \quad$ la crisis, el chico malo

(d) VP $\quad\left[\right.$ SYNSEM $\mid$ LOC $\mid$ CAT $\left.\left[\begin{array}{l}\text { HEAD verb } \\ \text { SUBCAT }(\mathrm{NP})\rangle\end{array}\right]\right]$ anda, compra un coche ${ }^{12}$

(e) $\quad\left[\right.$ SYNSEM $\mid$ LOC $\mid$ CAT $\left.\left[\begin{array}{l}\text { HEAD verb } \\ \text { SUBCAT }\rangle\rangle\end{array}\right]\right] \quad \begin{aligned} & \text { Juan se va, } \\ & \text { Maribel come }\end{aligned}$

Aus Tabelle (38) wird ersichtlich, daß die HPSG keine neuen lexikalischen Einheiten einführt. Alle aus der kf PSG bekannten, syntaktisch relevanten nicht-terminalen Symbole werden bearbeitet und in Attribut-Werte-Paaren dargestellt. Allerdings findet eine Verschiebung der Betrachtungsweise statt. Während vorher eher das sprachliche Zeichen als ganzes gesehen wurde, wird es nun von vornherein untergliedert und in seine Merkmale zerlegt. Die Funktionen von Kopf und Subkategorisierung spielen eine entscheidende Rolle für die Zugehörigkeit zu einer bestimmten Wortart, so daß in der Folge die strikte Trennung zwischen terminalen und nicht-terminalen Symbolen in dieser Form nicht mehr Bestand hat. Das Ergebnis ist oft ähnlich, bzw. nur methodisch in der Generierung der linguistischen Information unterschiedlich. So verwundert es nicht, daß geläufige Symbole der kf PSG als Abkürzung für komplexere Einheiten verwendet werden, nachdem sie zuvor im HPSG-Modus definiert wurden. Folgende grammatische Symbole werden in einer verkürzenden Form genutzt (vgl. Pollard / Sag 1994: 28):

\footnotetext{
${ }^{12}$ Da sich die Subkategorisierungsliste auf das Subjekt bezieht, muß dieses als fakultativ markiert werden.
} 


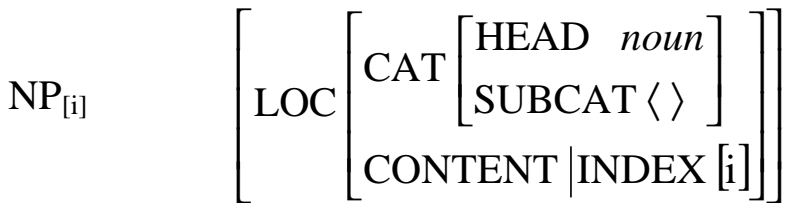

$$
\begin{aligned}
& \mathrm{S}_{[\mathrm{i}]} \quad\left[\operatorname{LOC}\left[\begin{array}{l}
\text { CAT }\left[\begin{array}{l}
\text { HEAD verb } \\
\text { SUBCAT }\langle\rangle
\end{array}\right] \\
\text { CONTENT [i] }
\end{array}\right]\right] \\
& \operatorname{VP}_{[\mathrm{i}]} \quad\left[\mathrm{LOC}\left[\begin{array}{l}
\text { CAT }\left[\begin{array}{l}
\text { HEAD verb } \\
\text { SUBCAT }\langle\text { synsem }\rangle
\end{array}\right] \\
\text { CONTENT [i] }
\end{array}\right]\right]
\end{aligned}
$$

Alle drei Phrasentypen werden mit syntaktischer und semantischer Information dargestellt, so daß die Matrizen in ihrer Gesamtheit von der Sorte synsem sind. Die gleiche Sorte wird als Wert der Subkategorisierungsliste bei Verbalphrasen verlangt [SUBCAT < synsem >]. Synsem zeigt dabei lediglich an, daß das in der Subkategorisierungsliste geforderte Element mit syntaktischer und semantischer Information behaftet sein muß. Über mögliche Inhalte und Anzahl der Elemente wird nichts ausgesagt.

Im folgenden werden Attribute, die die leere Menge beinhalten, nicht realisiert. Zudem wird auf das PHON-Attribut verzichtet, soweit es nicht zum Verständnis erforderlich ist. Pollard und Sag sagen kaum etwas über phonologische Komponenten in ihrer Grammatiktheorie aus, verweisen aber bisweilen auf mögliche Anwendungsmöglichkeiten der Phonologie, besonders für die Satzstellung (Pollard / Sag 1994: 15 und 376, vgl. für einen erweiterten Ansatz der phonologischen Komponente in der HPSG Bird / Klein 1993).

Die syntaktischen Darstellungsmöglichkeiten sind nun weit genug differenziert, um den Satz el padre de Juan compró un coche nuevo aus (6) darzustellen. Die Struktur erscheint folgendermaßen (vgl. auch Kiss 1995a: 58f.): 


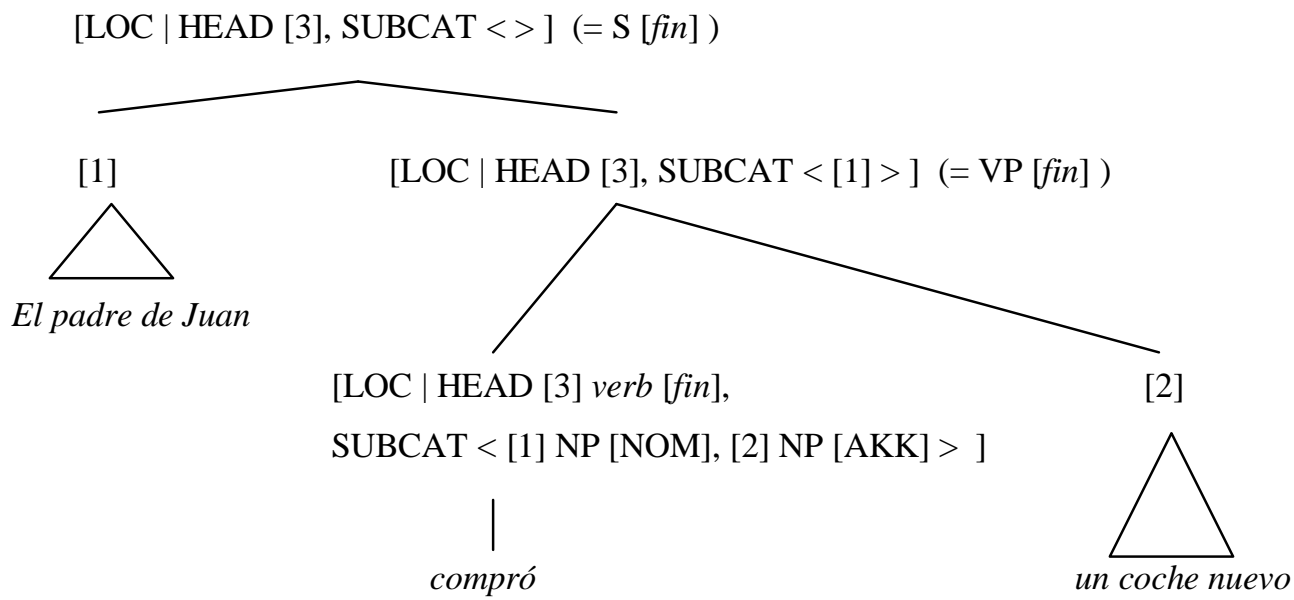

Das Verb ist der Kopf des gesamten Satzes. Auf unterster Stufe wird der Kopf als finites Verb definiert, und die Subkategorisierungsliste verlangt eine Nominalphrase im Nominativ und eine im Akkusativ. Auf der mittleren Stufe wurde das Akkusativobjekt angebunden. Die Subkategorisierungsliste verringert sich um die NP mit dem Index [2], der das Akkusativobjekt kennzeichnet. Es wandern nur noch der Index [1] für die Subjektphrase sowie die Merkmale des Kopfes mit zum nächsthöheren Knoten. Beim Knoten $\mathrm{S}$ ist die Subkategorisierungsliste leer. Der Kopf wird weiterhin durch den Index [3] vermerkt.

Das Subkategorisierungsprinzip besagt, daß der SUBCAT-Wert eines Kopfes (hier das Verb compró) die Summe aus dem SUBCAT-Wert des darüberliegenden Knotens (hier der VP-Knoten) und dem Wert der realisierten Konstituente (hier die NP un coche nuevo) darstellt. Wir können auch sagen, daß das Verb comprar in einer bestimmten Lesart zwei Komplemente benötigt und seine SUBCAT-Liste auf dem darüberstehenden Knoten um die bereits realisierten Tochterkonstituenten (hier das Akkusativobjekt) verringert wird.

Die HPSG kennt keine explizite Baumstruktur. Ein Baumdiagramm kann aber stets konstruiert und mit den entsprechenden, je nach Darstellungsintention wichtigen Merkmalen etikettiert werden. Auf diese Weise werden auch PSG-Regeln abgeleitet, auch wenn die HPSG sie nicht direkt zur Deskription verwendet. Beide Vorgehensweisen sind in der HPSG-Literatur gebräuchlich (vgl. z.B. Kiss 1995a: 58ff.). Das Baumdiagramm in (40) wird in der HPSG wie folgt dargestellt. Dabei verzichten wir aus Gründen der Übersichtlichkeit auf das Tochterattribut DTRS, welches sich ohnehin nur in speziellere Töchter wie HEAD-DTR und COMP-DTR aufteilt. SYNSEM wird mit SS abgekürzt: 


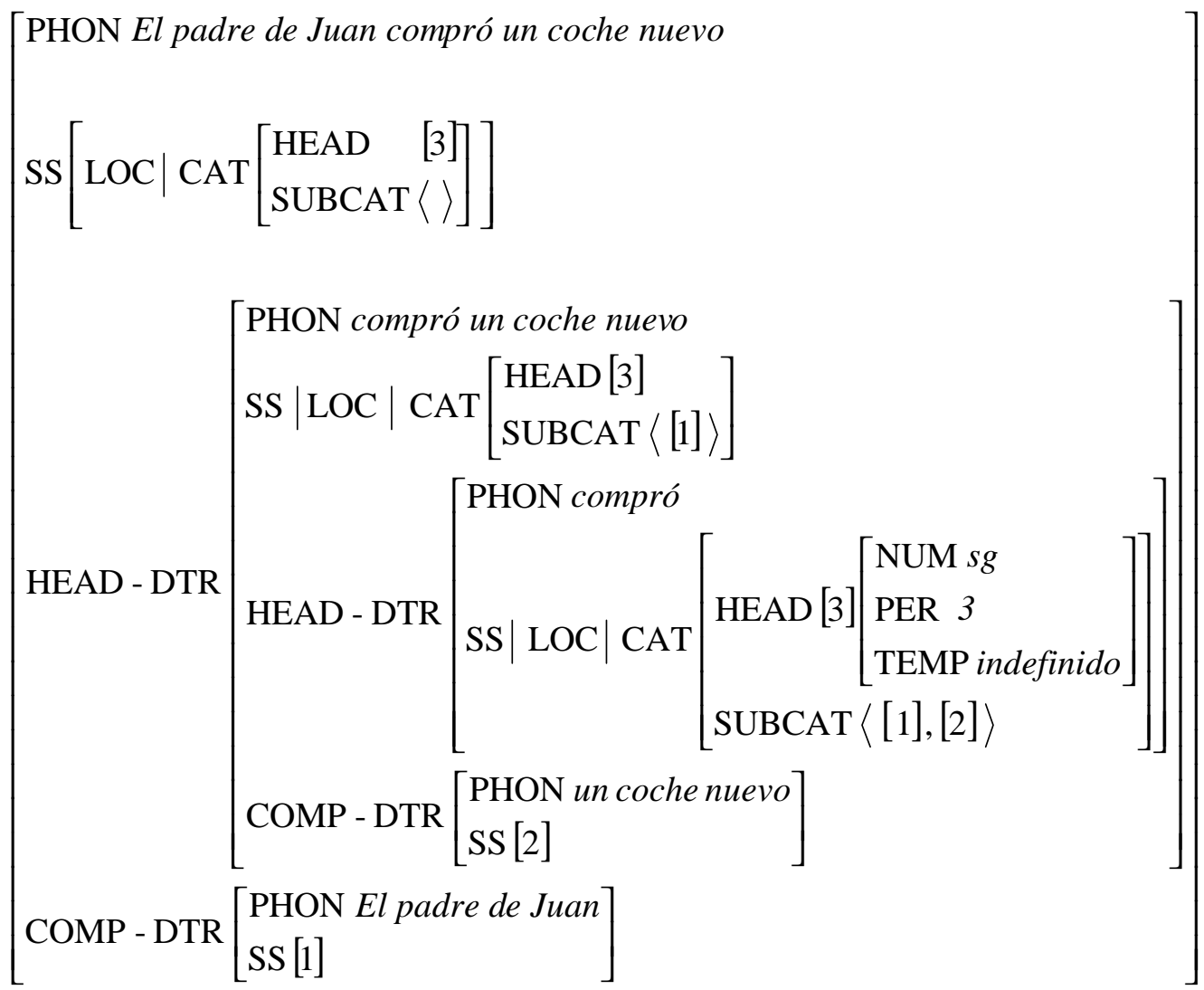

Die Indizes der Baumstruktur (40) und der Matrix (41) beziehen sich auf dieselben Konstituenten. Der Index [3] markiert den Kopf des Satzes (das Verb compró), der Index [1] das Subjekt (el padre de Juan) und der Index [2] das Akkusativobjekt (un coche nuevo). Während ein Baumdiagramm von oben nach unten zu lesen ist, wird in einer Merkmalstruktur der HPSG die gleiche Information von links nach rechts dargestellt. Die lexikalische Information steht am weitesten rechts in der Matrix. Eine Verzweigung im Baumdiagramm wird über die Merkmale HEAD-DTR und COMPDTR (alg. DTRS) ausgedrückt.

Wir hatten Sorten für lexikalische Kategorien verwendet. Wir können sie nun auch für syntaktische Strukturen verwenden. So kann die Struktur für den Satz (6) als Kopf-Komplement-Struktur (head-comp-str) bezeichnet werden. Sie steht damit im Kontrast zu Kopfstrukturen (headed-str), über die nicht mehr ausgesagt werden kann, als daß sie einen Kopf besitzen, z.B. bei einem Satz ohne Subjektrealisierung, der nur aus einem Verb besteht: vienen. Diese Struktur subsumiert zudem die KopfKomplement-Struktur, da sie weniger spezifiziert ist. Wir können somit eine Hierarchie von Strukturen erzeugen (nach Sag 1997: 443) ${ }^{13}$ :

\footnotetext{
${ }^{13}$ Bei Sag steht phrase anstelle von structure.
} 


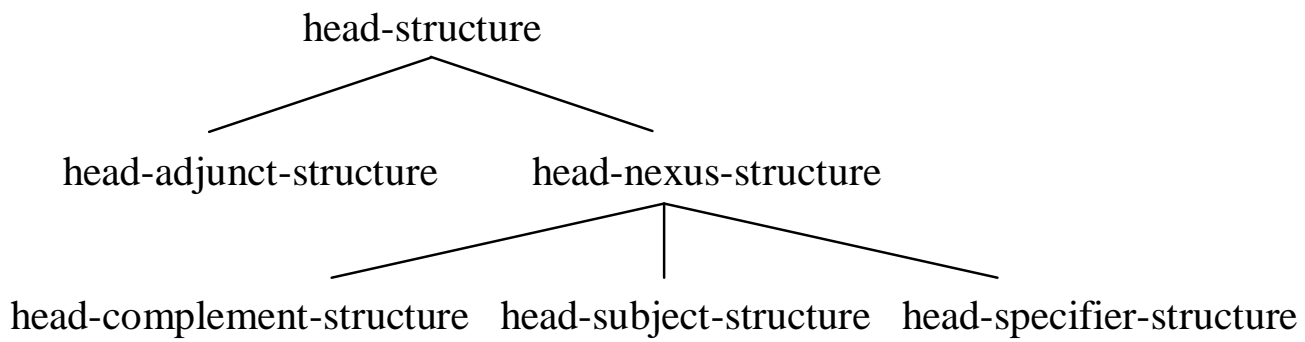

Wie oben bereits angedeutet, sind Syntaxregeln der HPSG nicht völlig fremd. Sie werden in Schemata umgewandelt, die auf die Konstituentenstruktur einwirken, und liefern die Bedingungen, unter denen eine Verbindung zweier Teilstrukturen zu einer neuen Struktur möglich ist. In Pollard / Sag (1987: 149ff.) wurden die Schemata noch Grammatikregeln genannt, weil sie den kontextfreien Regeln sehr ähneln und analog zu den Schemata der Government and Binding Theory von Chomsky (1981: 153ff.) operieren. Das Prinzip der Unmittelbaren Dominanz (Immediate-DominancePrinciple, IDP) legt fest, daß immer eines dieser Schemata zutreffen muß (Pollard / Sag 1994: 399, vgl. auch Müller 1999: 15ff.):

\section{Prinzip der Unmittelbaren Dominanz}

(Immediate-Dominance-Principle, IDP):

Jede Phrase mit einem Kopf muß genau eines der Immediate-DominanceSchemata erfüllen.

(44) Schema 1

kennzeichnet eine gesättigte Phrase ([SUBCAT <>]), deren Wert des Attributs DTRS vom Typ Kopf-Komplement-Struktur (head-comp-struc) ist. Die Kopftochter (HEAD-DTR) ist ein phrasales Zeichen ([LEX - ]) und die Komplementtöchter (COMP-DTRS) eine Liste der Länge eins ([SUBCAT < synsem >]).

Dieses Schema läßt sich mit kf PSG-Regeln erläutern (vgl. Pollard / Sag 1994: 38f.):

$$
\begin{aligned}
& \mathrm{S} \rightarrow \mathrm{NP} \mathrm{VP} \\
& \mathrm{NP} \rightarrow \mathrm{D} \mathrm{NOM}
\end{aligned}
$$


Wir haben eine gesättigte Phrase, die im HPSG-Formalismus durch eine leere Subkategorisierungsliste gekennzeichnet ist. Diese Phrase besitzt eine Kopftochter, die ebenfalls aus einer Phrase besteht ( [LEX -] ), diesmal gekennzeichnet durch eine nichtleere Subkategorisierungsliste. Die besagte Phrase besitzt nicht nur eine Kopftochter, sondern auch ein Komplement - deshalb die Sorte Kopf-KomplementStruktur -, über das lediglich gesagt wird, daß es sich nur um ein einzelnes handelt. Dieses Komplement kann sowohl ein lexikalisches Zeichen als auch eine Phrase sein.

Die in (45) genannten kontextfreien Regeln spezifizieren diese Information. Auf der linken Seite der Regel steht ein phrasales Zeichen (S und NP), dessen Kopftochter auf der rechten Seite ebenfalls ein phrasales Zeichen (VP und NOM) ist. Die jeweiligen Komplemente (NP und D) bestehen aus nur einem Element und können sowohl phrasal wie auch lexikalisch sein. Der oberste Knoten in (40) S [fin] erfüllt die Anforderungen von Schema 1.

Im Formalismus der HPSG sieht eine entsprechende Matrix folgendermaßen aus:

$$
\left[\begin{array}{l}
\operatorname{SYNSEM} \mid \text { LOC } \mid \text { CAT }[\operatorname{SUBCAT}\langle\rangle] \\
\text { DTRS }\left[\begin{array}{l}
\text { HEAD - DTR }|\operatorname{SYNSEM}| \text { LOC } \mid \text { CAT }\left[\begin{array}{l}
\text { LEX - } \\
\operatorname{SUBCAT}\langle[]\rangle
\end{array}\right] \\
\text { COMP - DTRS }\{[]\}
\end{array}\right]
\end{array}\right.
$$

Die Notation < [ ] > besagt dabei, daß ein Element in einer Liste zwar auftritt, aber nicht näher spezifiziert wird. Die leer notierte Klammer steht als Variable für die Liste, nicht als standardisiertes leeres Element.

Die Subkategorisierungsliste der gesamten Phrase ist leer, ihr Komplement spaltet sich in Kopf- und Komplementtöchter. Das LEX-Attribut ([LEX -]) besagt, daß es sich bei der Kopftochter nicht um ein lexikalisches Element handelt. Die mit einer noch näher zu spezifizierenden eckigen Klammer gefüllte Komplementtochter kennzeichnet die Menge als einelementig.

Das zweite Schema beschreibt die nächste Verzweigung der Verbalphrase in (40). Es handelt sich um einen lexikalischen Kopf - in diesem Fall zumeist ein Verb -, über den ausgesagt wird, daß auf jeden Fall noch ein Komplement realisiert werden muß (im Fall des Beispielsatzes (6) das Subjekt), unabhängig von den bereits realisierten Komplementen. 
kennzeichnet eine fast gesättigte Phrase (Subkategorisierungsliste der Länge eins, SUBCAT < [ synsem ] >), deren Wert des Attributs DTRS ebenfalls vom Typ Kopf-Komplement-Struktur ist. Die Kopftochter (HEAD-DTR) ist ein lexikalisches Zeichen ( [LEX +] ).

Das zweite Schema (Pollard / Sag 1994: 38) steht für die kontextfreien Regeln

$$
\begin{aligned}
& \mathrm{VP} \rightarrow \mathrm{V} \mathrm{NP} \\
& \mathrm{P} \rightarrow \mathrm{P} \mathrm{NP}
\end{aligned}
$$

Im HPSG-Formalismus kann dies so dargestellt werden:

$$
\left[\begin{array}{l}
\text { SYNSEM } \mid \text { LOC } \mid \text { CAT } \mid \text { SUBCAT }\langle[]\rangle \\
\text { DTRS }[\text { HEAD - DTR } \mid \text { SYNSEM } \mid \text { LOC } \mid \text { CAT }[\text { LEX + }]]
\end{array}\right]
$$

Die Subkategorisierungsliste der Phrase ist nicht leer, sondern von der Länge eins, was das Merkmal [ SUBCAT < [ ] > ] anzeigt. Der Kopf ist bereits ein lexikalisches Zeichen, welches in einem letzten Schritt durch ein Lexem ersetzt wird. In (48) trifft dies auf die Kategorien $\mathrm{P}$ und V zu. Über die Art der Komplemente wird keine Aussage gemacht.

Als drittes Schema nennen Pollard / Sag (1994: 40) noch die Möglichkeit, daß alle Komplemente gleichrangig realisiert werden:

\section{Schema 3}

kennzeichnet eine gesättigte Phrase ( [SUBCAT <>] ) mit dem Attribut DTRS vom Typ Kopf-Komplement-Struktur. Die Kopftochter ist ein lexikalisches Zeichen $([$ LEX +] ).

Dieses Schema soll vor allem für invertierte Strukturen des englischen Fragesatzes eine Lösungsmöglichkeit bieten, da dort ein Hilfsverb [AUX +, INV +] auf die gleiche Ebene mit seinen Komplementen in einem Baumdiagramm gestellt werden muß (Can Kim go?, Pollard / Sag 1994: 42). Alle Komplemente werden somit gleichrangig neben dem Kopf in einer flachen, nicht-binären Struktur realisiert. Es gibt auch keine Beschränkung der Subkategorisierungsliste auf ein Element. Für spanische 
Fragesätze ist dieses Schema ebenfalls dann brauchbar, wenn sich Änderungen an der Konstituentenreihenfolge ergeben: Usted quiere comer - ¿Quiere usted comer? Aber auch für Auxiliarverben mit Infinitivkonstruktionen, die wiederum Komplemente subkategorisieren, kann Schema 3 von Bedeutung sein, da innerhalb eines Satzes eine zweite Verbalphrase auftaucht (Pollard / Sag 1994: 41). 


\subsection{Konstituentenabfolge}

Die erste HPSG-Version beschreibt lineare Abfolgen über separate Regeln (Pollard / Sag 1987: 169ff.). Köpfe werden in bezug auf ihre Komplemente vor- oder nachgestellt. Dies kann sehr einfach dargestellt werden (im Gegensatz zu Pollard / Sag, die nur hier LEX als Attribut von HEAD interpretieren):

$$
[\mathrm{LEX}+]<[]
$$

Mit Regel (51) stehen alle lexikalischen Köpfe vor ihren Komplementen. Freilich ist dies nicht immer der Fall. Zum einen gibt es Köpfe, die nach ihren Komplementen stehen (z.B. folgen Nomina den von ihnen subkategorisierten Determinierern), zum anderen wird nichts über gleichrangige Komplemente ausgesagt, die Frage etwa, ob ein Dativobjekt vor oder nach einem Akkusativobjekt steht.

Das erste Problem kann mit noch genaueren Angaben zum Kopf gelöst werden, so daß z.B. ein Nomen nach seinem Komplement steht:

$$
\text { [ ] }<\left[\begin{array}{l}
\text { LEX }+ \\
\text { HEAD nomen }
\end{array}\right]
$$

Das zweite Problem erfordert genauere Angaben zum Komplement. Das Zeichen « steht für eine lineare Abfolgeregel, die sich auf die Obliquitätshierarchie bezieht. Dabei wäre das linke Komplement weniger oblique als das rechte:

\section{Komplement « Komplement}

Mit diesem Regelapparat können genügend Abfolgeregeln so konstruiert werden, daß eine lineare Beschreibung der spanischen Nominalphrase möglich ist. Voraussetzung ist ein genaues Studium der spanischen NP, so daß die Regeln die sprachlichen Gegebenheiten widerspiegeln. Für die spanische Nominalphrase könnten in einem ersten Ansatz zwei Regeln aufgestellt werden: 
Linearitätsregel 1

SPR $[$ HEAD det $]<\left[\begin{array}{l}\text { LEX }+ \\ \text { HEAD nomen }\end{array}\right]$

\section{Linearitätsregel 2}

$\left[\begin{array}{l}\text { LEX }+ \\ \text { HEAD nomen }\end{array}\right]<$ ADJUNCT [HEAD prep $\vee$ rel $]$

Die erste Regel besagt, daß Spezifizierer (SPR) mit einem Determinierer als Kopf vor dem Nomen als Kopf der gesamten NP stehen müssen. Die zweite Regel besagt, daß das Nomen als Kopf stets vor Adjunkten steht, die entweder Präpositionalphrasen oder Relativsätze darstellen.

Bei dieser Darstellung werden die Abfolgeregeln in einem separaten Formalismus erfaßt. Diese Abtrennung ist unbefriedigend. In Reape (1994) und einigen folgenden Aufsätzen (Kathol 1995, Richter / Sailer 1995, Reape 1996, Richter 1997, Müller 1999) wird das Konzept der Domäne vorgeschlagen, welches in den HPSGFormalismus integriert werden kann. Danach bildet ein lexikalisches Zeichen eine Domäne. Wenn sich zwei Lexeme zu einer Phrase zusammenschließen, bilden sie eine neue Domäne (nach Kathol 1995; Reape 1994 erstellt erst aus zwei Lexemen eine Domäne). Prinzipien der Zusammenführung regeln die Verkettung in einer sehr ähnlichen Weise, wie dies in den Abfolgeregeln (54) geschieht. Dieser Ansatz wird hier nicht weiter verfolgt, da er lediglich ein zusätzliches Merkmal für die Konstituentenreihenfolge einführt, was allerdings für komplexe Sätze seine Berechtigung hat. Es sollte aber möglich sein, aufgrund der beteiligten Lexeme die Konstituentenreihenfolge innerhalb einer Phrase festzulegen, wie es die obigen Regeln vorsehen. Zudem ist gerade für romanische Sprachen eine Integration der phonologischen Komponente an dieser Stelle wünschenswert, da die Intonation und die Akzentuierung morphophonologische Interferenzen mit sich bringen (vgl. obige Angaben zum mot phonétique). Dies gilt für das Französische noch stärker als für das Spanische (vgl. Grevisse 1993: 175ff.). Für komplexere spanische Nominalphrasen muß dabei zusätzlich ein besonderes Gewicht auf lineare Strukturen bei Nominalphrasen mit Adjektiven gelegt werden, da Adjektive in der Stellungsfrage von pragmatischer Information abhängen (z.B. subjektiven vs. objektiven Gebrauch, vgl. Kap. III.4.). 


\subsection{Subjekte}

Im Spanischen sind folgende Konstruktionen üblich:

(a) Señora, no entiendo lo que me quiere decir. (S-9)

(b) Qué le vamos a hacer. (M-2)

(c) Todos los hombres buscamos esta experiencia básica y fundamental. (Rch-8)

(d) Me olvidé de comprar flores. (S.4)

(e) (Pero yo no te creo nada.) Me niego a aceptar que has olvidado. (S-11)

Allen diesen Sätzen ist gemeinsam, daß das Subjekt syntaktisch nicht realisiert wird. Die Subjektfunktion ist der Morphologie des Verbs inhärent, so daß eine anaphorische Referenz wie in den Sätzen (a) und (e) möglich ist. Sprachen, die wie das Spanische diese Eigenschaft aufweisen, werden als subject-drop-languages bezeichnet (vgl. Mallén 1995: 229). Konsequenterweise bedeutet dies für die bisherige Subkategorisierungsliste, daß ein in der Standard-HPSG als obligatorisch betrachteter Eintrag nicht realisiert sein muß.

Die in der Endung des Verbs morphologisch realisierten Informationen müssen für eine Darstellung ausreichen. Die SUBCAT-Liste muß so modifiziert werden, daß ein Subjekt nicht notwendigerweise subkategorisiert wird, aber die Endung des Verbs mit Person und Numerus auf dieses Subjekt verweist. Somit kann das Subjekt nicht als fakultative Konstituente verstanden werden. Allerdings lassen sich die Merkmale Person und Numerus im Verb morphologisch nicht isolieren, sondern sind mit Tempus und Modus vermischt. Es muß also von einem syntaktisch unter bestimmten Umständen nicht realisierten Subjekt ausgegangen werden.

Klenk (1996) nimmt an dieser Stelle Regeln an, die das entsprechende Verb vom Infinitiv in eine Flexionsform überführen und die Merkmale für Person und Numerus in der Subkategorisierungsliste an die Stelle des Nominativobjekts stellen, eine Funktion per_num (per für Person und num für Numerus), die als Alternative für NP $[N O M]$ steht und einen zweiten lexikalischen Eintrag erzeugt. Diese Art einer grammatischen Regel wird allgemein als lexikalische Regel bezeichnet. Dabei wird die SUBCAT-Liste in ARGUMENTE umbenannt, da es sich nicht mehr um Komplementphrasen handelt. Im Lexikon stehen dann zwei Einträge für die finite Form z.B. bei comprar: 
(56) Infinitiv:

a. [SYNSEM | LOC | CAT | HEAD [VFORM inf] ]

Verbformen:

[SYNSEM | LOC | CAT | HEAD [VFORM fin] ] $\cup$

b. [SYNSEM | LOC | CAT | ARGUMENTE < NP [NOM], NP [AKK] >] $\vee$

c. [SYNSEM | LOC | CAT | ARGUMENTE < per_num, NP $[A K K]>]$

Eine lexikalische Regel gilt als eine Operation der Umwandlung von Lexemen (vgl. Pollard / Sag 1987: 191ff., Frank 1991: 44 und Flickinger 1987: 103ff.). Das Lexikon in der HPSG ist grundsätzlich hierarchisch angelegt. Eigenschaften wie [SYNSEM | LOC [ LEX +] ] werden von allen Lexemen geteilt, da dieser Pfad nur die Eigenschaft darstellt, ein Lexem zu sein. Andere Eigenschaften wie [HEAD nomen] werden nur von Nomina geteilt. Lexikalische Regeln operieren nun auf dieser Grundlage und überführen Lexeme mit bestimmten Eigenschaften in ähnliche Lexeme mit anderen, divergierenden Eigenschaften. In (56) muß eine lexikalische Regel dafür sorgen, daß der Infinitiv in eine finite Verbform überführt wird. Der Ansatz von Klenk (1996) fordert an dieser Stelle zwei Regeln: einmal für ein realisiertes Subjekt, das andere Mal für ein nicht-realisiertes Subjekt. Eine ähnliche Regel wäre auf syntaktischer Ebene für Akkusativobjekte nötig, die je nach ihrer Eigenschaft des Bezugs auf eine Person (oder als eine Person betrachtetes Lebewesen) mit der Präposition $a$ stehen müssen und so eine Präpositionalphrase statt einer Nominalphrase bilden.

Klenk (1996) führt zusätzlich das Attribut MORPH ein, um morphologische Komponenten, die in anderen Sprachen als dem Englischen eine große Rolle spielen, ebenfalls erfassen zu können (vgl. auch Krieger / Nerbonne 1993: 105ff. zur deutschen Morphologie sowie Riehemann 1993: 45ff.). Dieses Attribut könnte in einer detaillierten Weiterentwicklung zu einer zufriedenstellenderen Definition des Wortes führen, als sie in (2) gegeben ist.

Die Standard-HPSG ist bei der Deskription von Subjekten einen ähnlichen Weg wie Klenk (1996) gegangen. Bereits 1987 formuliert Borsley (vgl. auch Pollard 1989: 186) die Forderung nach einer Trennung von SUBCAT und SUBJECT (SUBJ), wobei SUBJ dasjenige Attribut darstellt, welches die Subjektfunktion einer NP in der Rektionsliste eines Verbs abbildet. Compra bekäme dann anstelle von (57a) den Eintrag von (57b) (nach Borsley 1987: 6): 
(a) $\mathrm{SUBCAT}<\mathrm{NP}[3$, sing $], \mathrm{NP}>$

(b) SUBJ $\quad<\mathrm{NP}[3$, sing $]>$; SUBCAT $<\mathrm{NP}>$

Borsley will mit dieser Analyse einige Probleme der Standard-HPSG-Theorie aufzeigen. Pollard / Sag (1994: 344ff.) nehmen seine Anregungen auf und skizzieren einen revidierten Ansatz, der die folgenden Schwierigkeiten erklären soll:

- die Charakterisierung von Nicht-Kopf-Kategorien,

- die Behandlung von Verbalphrasen, die als gesättigt angesehen werden müssen,

- von Präpositionen, die vor der subkategorisierten NP stehen, und

- von Komplementiererphrasen, die in Subjektposition stehen.

Besonders der zweite Punkt ist an dieser Stelle wichtig. Das Attribut SUBJ erlaubt eine unterschiedliche Klassifikation von Subjekt und Objekten, die dem sprachlichen Material gerecht wird (vgl. auch Warner 1993: 70). Viele infinitive Konstruktionen scheinen kein syntaktisches Subjekt zu benötigen, wenn es auch prinzipiell stehen kann.

Kiss (1995a: 79) greift den Ansatz von Borsley auf und modifiziert ihn in der Weise, daß nun das SUBJ-Attribut einen Wert von HEAD darstellt. Ein Eintrag für das Verb comprar stellt sich nach Kiss wie folgt dar:

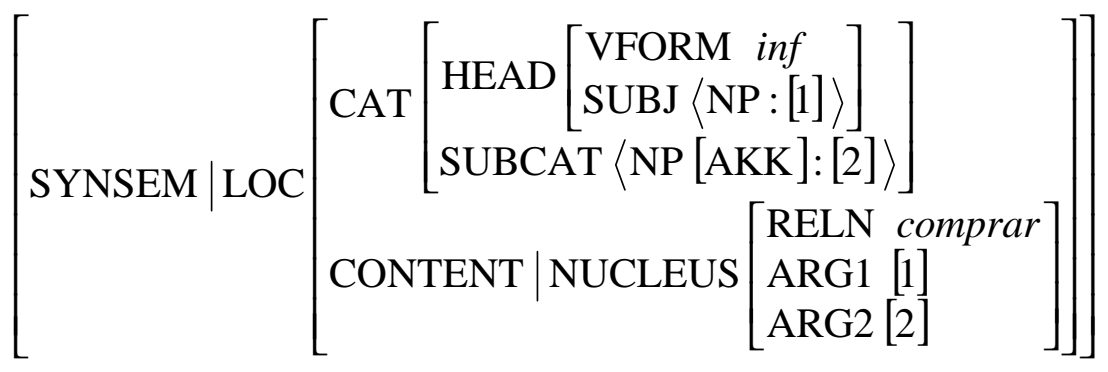

Zudem geht er von einer lexikalischen Regel für Finitheit (Kiss 1995a: 79) aus, die den Wert von SUBJ in die Subkategorisierungsliste des finiten Verbs überführt. Der Infinitiv braucht somit kein Subjekt. 


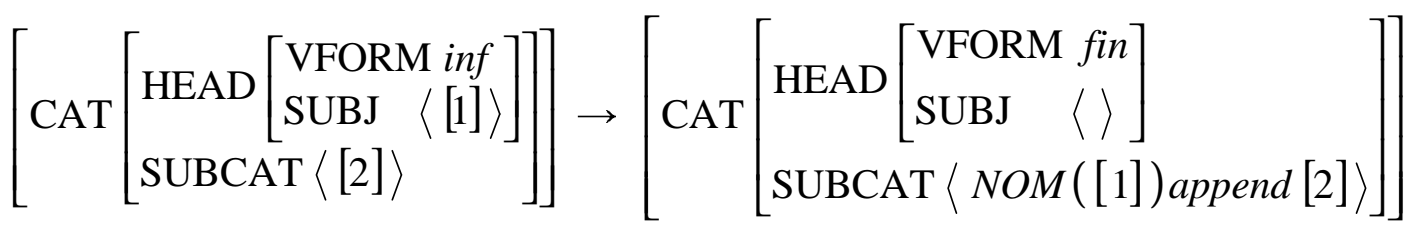

Der Wert des SUBJ-Attributs kann auch realisiert werden, wenn er in die SUBCATListe übernommen wird. Das läßt den Schluß zu, daß es sich letztlich um eine Beschreibung handelt, die positiv oder negativ die syntaktische Existenz des Subjekts angibt. Für spanische Verbalphrasen könnte so ein SUBJ-Attribut angenommen werden, das bei negativem Wert eine saturierte Verbalprojektion angibt, bei positivem jedoch die Angabe in die Subkategorisierungsliste überführt und nun anzeigt, daß das Verb mit dem Wert des SUBJ-Attributs kongruiert.

In Kapitel 9 von Pollard / Sag (1994) wird aufgrund des Vorschlags von Borsley (1987) zwischen SUBJ, COMPS (Komplemente) und SPR differenziert. Sie ersetzen die SUBCAT-Liste der ersten Kapitel durch das Sammelattribut VALENZ (VAL). Spezifizierer kennzeichnen Nominalphrasen, Subjekte und Komplemente eher Verben. Das zusätzliche Attribut für die Kongruenzstruktur (AGREEMENTSTRUCTURE, AGR-S) ersetzt die Subkategorisierungsliste. Es ist weiterhin nötig, um die Obliquitätshierarchie der Komplemente zu erfassen. Dies vereinfacht die Beschreibung von präpositionalen Akkusativobjekten.

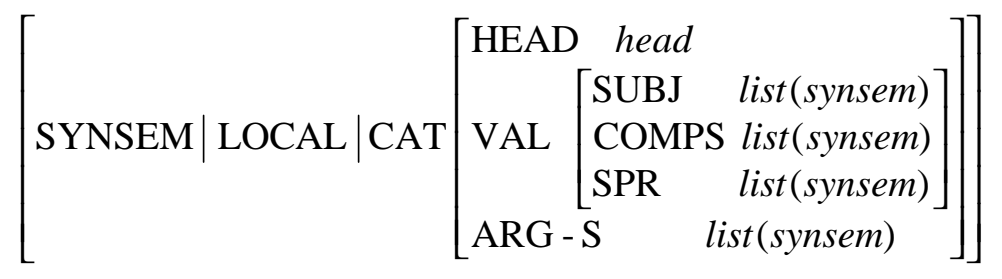

Ziel dieser Änderung ist es erstens, subjektlose Sätze zu erfassen, die vorher als ungesättigte Verbalphrasen eingeordnet werden mußten, und zweitens die Abhängigkeiten zwischen finiten Verben und ihren Komplementen, Infinitivkonstruktionen und deren Subjekten sowie Nomina und ihren Spezifizierern besser zu beschreiben. Durch Einbeziehen eines Attributs für Spezifizierer können jegliche Arten von Subkategorisierung, auch nominale, differenziert werden. Dies hat sich als praktikabel erwiesen. 
Offen blieb bisher, inwieweit Prinzipien und Ansätze der HPSG modifiziert werden müssen. So wird die Strukturhierarchie modifiziert: die Kopf-KomplementStruktur bleibt zwar erhalten, bezieht sich aber nur noch auf Objekte ohne das Subjekt. Der neue Typ Kopf-Subjekt-Struktur wird in die Strukturhierarchie integriert. Schema 1 und 2 werden nach Pollard / Sag (1994: 347f.) folgendermaßen modifiziert:

(61) Schema 1

kennzeichnet eine Phrase, deren Attribut DTRS vom Typ Kopf-SubjektStruktur ist. Die Kopftochter ist ein phrasales Zeichen.

\section{Schema 2}

kennzeichnet eine Phrase, deren Attribut DTRS vom Typ Kopf-KomplementStruktur ist. Die Kopftochter ist ein lexikalisches Zeichen.

Die geringe Komplexität dieser Schemata spricht für sich. Sie beweist, daß die hierarchische Gliederung von Strukturtypen sinnvoll ist, vorausgesetzt, daß sie den sprachlichen Gegebenheiten entspricht.

Das Subkategorisierungsprinzip wird in Valenzprinzip umbenannt und operiert mit der Variablen $F$, die für die Valenzmerkmale SUBJ, COMPS und SPR steht (vgl. Pollard / Sag 1994: 348, 358ff.).

\section{Valenzprinzip}

Für jedes Valenzmerkmal $F$ einer Phrase ist der $F$-Wert der Kopftochter die Verkettung des F-Werts der Phrase mit der Liste der SYNSEM-Werte ihrer F-Töchter (DTRS).

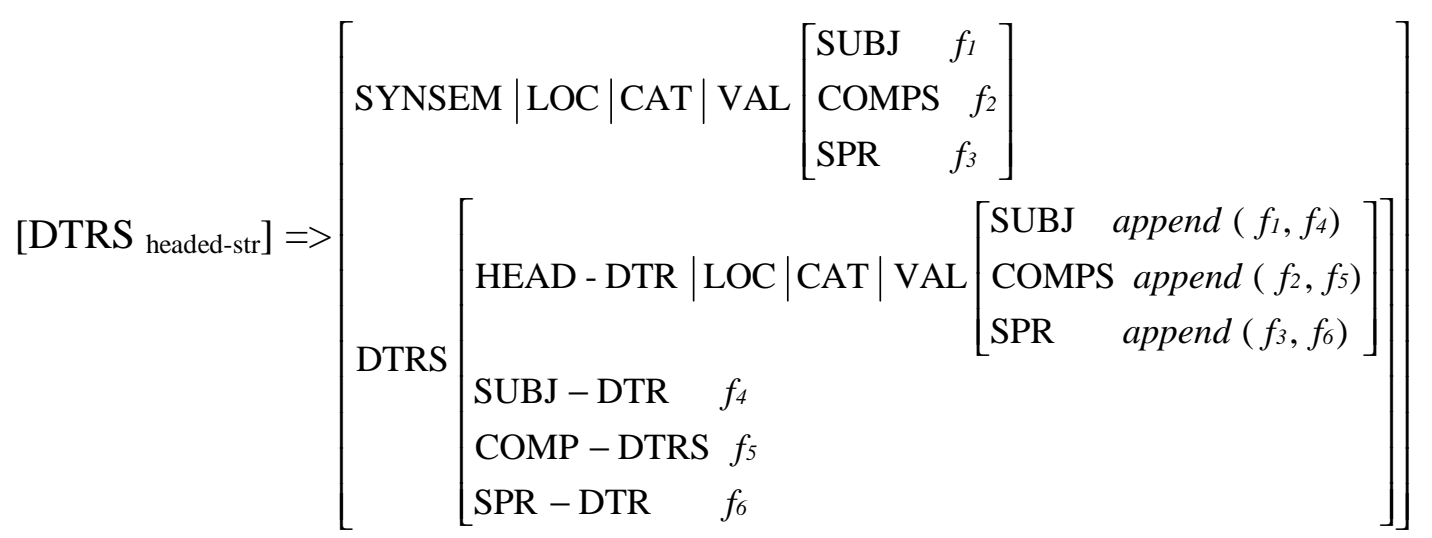


Das Prinzip besagt lediglich, daß die Werte des Pfades DTRS | HEAD-DTR | LOC | CAT | VAL durch eine append-Opperation aus den Werten der übergeordneten Phrase und denen der Töchter gebildet werden. Es findet eine Verknüpfung der Informationen statt.

Mit diesen Änderungen ist es möglich, einige sprachliche Details genauer zu erfassen. Nach dem folgenden Kapitel über Semantik werden wir am Beispielsatz $E l$ padre de Juan compró el coche nuevo Details erläutern. Wir werden weiterhin die Prinzipien der Standard-HPSG als Arbeitsgrundlage verwenden.

\subsection{Semantik}

Ziel der semantischen Komponente innerhalb einer Grammatik ist es, die Bedeutung eines Satzes zu ermitteln. Es gibt viele sprachwissenschaftliche Ansätze, um dies zu tun. Pollard / Sag (1987) stützen sich im wesentlichen auf die Situationssemantik von Barwise / Perry (1987) und übertragen die Ergebnisse in den Formalismus der HPSG.

\subsubsection{Individuen, Sachverhalte und Situationen}

Individuen sind Dinge, die existieren, zur Ordnung der bekannten Welt gehören, als solche wahrgenommen werden, in irgendeiner Form agieren und dadurch Reaktionen hervorrufen (vgl. hierzu und zum folgenden Müller 1999: 24ff. sowie Cooper 1990: 99ff.). $\mathrm{Zu}$ den Individuen werden neben Personen (Carlos, Maria) und Konkreta (der Tisch, das Auto) auch abstrakte Konzepte gezählt (die Angst, der Wunsch). Individuen besitzen Eigenschaften (schön, elegant, unglücklich). Man könnte Eigenschaften auch als einstellige Relationen verstehen, mit denen Fakten über Individuen ausgesagt oder Bezüge auf Individuen erstellt werden. Relationen werden durch verbale Bezüge dargestellt und können nullstellig (regnen, llover), einstellig (schlafen, dormir, morir), zweistellig (lieben, querer), dreistellig (geben, dar) oder vierstellig (kaufen, comprar) sein. Diese semantischen Relationen dürfen nicht mit der Subkategorisierungsliste der entsprechenden Verben verwechselt werden, die syntaktische Informationen beinhaltet. So ist die Subkategorisierungsliste von comprar in Voy a 
comprar leer, da hier comprar das Einkaufen in einem sehr allgemeingültigen Sinne meint und das Subjekt im Auxiliar ir enthalten ist. Ansonsten kann comprar aus semantischer Perspektive vierstellig sein: Es gibt einen Käufer, einen Verkäufer, eine Ware und einen Preis (El padre de Juan compró un coche nuevo por 300000 pts del taller Rodríguez ${ }^{14}$ ). Individuen, Eigenschaften und Relationen können auch als Charakterisierungsschema für eine bestimmte, gegebene Welt verstanden werden.

Für die Situationssemantik nennt Bußmann (1990, s.v. ebd.) folgende Grundannahmen (vgl. auch Schwarz / Chur 1996: 123ff.):

1. „Eigenschaften und Relationen werden als Grundbegriffe angenommen und nicht mengentheoretisch aus anderen Einheiten konstruiert;

2. es gibt eine einzige Welt, nämlich die wirkliche, und nicht eine Vielzahl von möglichen Welten;

3. gültige Aussagen beziehen sich entweder auf diese Welt oder auf ihre Teile, die „Situationen“ genannt werden;

4. die Bedeutung eines deklarativen Satzes $S$ ist eine Relation zwischen dem Typ von Situationen, in denen $S$ behauptend geäußert wird, und dem Typ, dem diejenigen Situationen zugehören, die dabei beschrieben werden $[. . .]^{\text {“. }}$

Die Welt besteht aus Situationen, in denen Individuen Eigenschaften besitzen und in Relationen zueinander stehen. Diese Welt kann sich in verschiedenen Zuständen befinden, die auch als Sachverhalte bezeichnet werden. Ein solcher möglicher Sachverhalt wird in der Situationssemantik state of affairs genannt und im folgenden mit soa abgekürzt.

Ein einfacher Sachverhalt wird bestimmt durch eine Relation seiner Argumente und weitere, äußere Komponenten wie Ort, Zeit u.a. Hinzu tritt das Merkmal Polarität für die Existenz oder Nicht-Existenz des Sachverhalts, dargestellt durch 0 für die Nicht-Existenz und 1 für die Existenz. Eine formale Repräsentation umfaßt demnach die Angabe der Relation, der weiteren Komponenten und ihrer Rollen sowie der Polarität. Pollard / Sag verwenden Namen, die dem Verb und seinen möglichen Relationen entstammen, so daß bei comprar die thematischen Rollen des Kaufenden $\mathrm{El}$ Que-Compra und des Gekauften Lo-Comprado genannt werden. In Pollard / Sag (1994: 342f.) wird zwar eine thematische Einteilung in SOURCE, GOAL und AGENS u.a. diskutiert, dies verlangt aber eine weitergehende Bearbeitung wie in

\footnotetext{
${ }^{14}$ Dieser Satz ist zwar konstruiert, vgl. aber aus dem Korpus: NH Hoteles compra el 19 por ciento de la italiana Jolly Hotels por 5.100 millones (A-2).
} 
Kunze (1991), die hier nicht geleistet werden kann (vgl. auch Müller 1999: 25 und neuere Ansätze von Sag 1997).

Sachverhalte können in einer an die Prädikatenlogik der ersten Stufe angelehnten Darstellungsweise formuliert werden (vgl. Allwood / Andersson / Dahl 1973: 60ff.). Der Begriff Instanz (Instance) beschreibt dabei die Inhaltskomponente von Nomina (nach Pollard / Sag 1994: 87ff.).

(a) «llamar, La que llama: Ana, La que es llamada: María; 1» 'der Sachverhalt, daß Ana María ruft'

(b) «perro, Instance: Elvis; 1» 'der Sachverhalt, daß Elvis ein Hund ist'

(c) «gato, Instance: Elvis; 0»

'der Sachverhalt, daß Elvis keine Katze ist'

(d) «desear, El que desea: Pedro, Lo deseado: «comer, El que come: Francisco, Lo comido: pastel; 1»; 0» 'der Sachverhalt, daß Pedro nicht wünscht, daß Francisco Kuchen ißt'

Bei der an den prädikatenlogischen Formalismus angelehnten Deskription handelt es sich um eine linguistische Beschreibung, für welche die Tatsache, ob einem Sachverhalt ein Wahrheitswert zugeordnet werden kann oder nicht, weniger entscheidend ist. Im folgenden Sachverhalt

(a) «perro, Instance: Elvis; 1»

(b) perro (Elvis)

bekommt (64a) nicht im prädikatenlogischen Sinne einen Wahrheitswert zugesprochen, da es lediglich den Sachverhalt anzeigt, daß Elvis ein Hund ist, unabhängig davon, ob dies wahr ist. In anderen Worten: Durch die Polarität ist es möglich, negative Aussagen $\mathrm{zu}$ treffen. Demgegenüber kennzeichnet die prädikatenlogische Struktur (64b) etwas, das entweder wahr oder falsch ist, je nachdem ob Elvis wirklich ein Hund ist oder nicht. In (63d) als eine im Vergleich zu (64a) komplexere Form ist die Zuordnung eines Wahrheitswertes für Lo deseado auch gar nicht möglich, da jemand nicht wahr oder falsch etwas wünschen kann. 


\subsection{2. Übersetzung in den Formalismus der Merkmalstrukturen}

Semantische Sachverhalte werden in den HPSG-Formalismus übersetzt, indem neben das Attribut CATEGORY das Attribut CONTENT für die Semantik tritt. Eine Nominalphrase erhält einen INDEX-Wert, um anzuzeigen, daß ihr eine thematische Rolle zugesprochen wird. Dieses Attribut wird ergänzt durch den Wert der RESTRICTION des Nomens, welcher eine Menge von parametrized states-of-affairs (psoa, Umstände) kennzeichnet. Der Unterschied zu soa besteht in der Möglichkeit, eine Variable an die Stelle der Instanz zu plazieren, z.B.:

«borracho, Instance: X; 1»

'der Sachverhalt, daß X betrunken ist'

Dies ist linguistisch notwendig, um auf Sachverhalte zu referieren, die nicht sofort instantiiert werden. Die Variable X ist zunächst nicht an bestimmte Referenten gebunden. Ihre Namenswahl ist willkürlich.

Die Sorte psoas markiert referentielle Nomina, aber nicht unbedingt Pronomen, da diese sich anaphorisch auf ein Voriges beziehen. Pronomen werden als Subtypen der referentiellen Objekte behandelt. Im folgenden Beispiel verweist die Sorte referentiell-object (ref-obj) auf ein Lexem, das auf eine Entität referiert. Damit werden Expletiva ausgeschlossen (vgl. Pollard / Sag 1994: 25).

Im Formalismus der HPSG wird ein Pfad angenommen, der als Wert des Merkmals SYNSEM | LOCAL | CONTENT die Merkmale INDEX und RESTRICTION (RESTR) erhält. INDEX enthält die Kongruenzattribute, RESTRICTION übernimmt die Darstellung der Restriktionen, wie sie z.B. durch Adjektive eingeführt werden (vgl. Pollard / Sag 1994: 47ff.). 
(66)

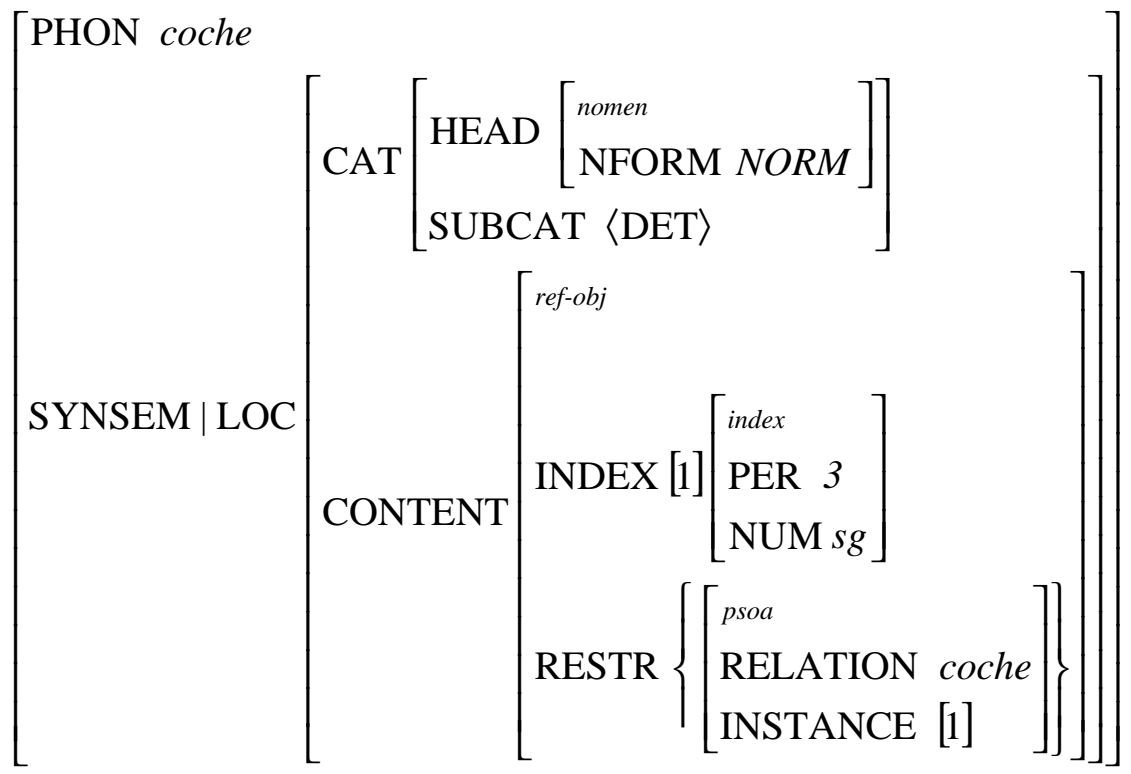

Polarität wird nur angegeben, wenn ihr Wert 0 ist. 1 wird als Vorgabe (default) angenommen.

Die Referenz auf eine Entität kann verschieden aussehen. Verschiedene Typen von Referentialität werden in einer Hierarchie erfaßt. Dabei kann die oberste Einteilung, die Differenzierung zwischen referentiell und expletiv (vgl. Müller 1999: 28), im Spanischen vernachlässigt werden, da es keine Entsprechung zu den deutschen oder englischen Expletiva es, there, it gibt (vgl. Pollard / Sag 1994: 249).

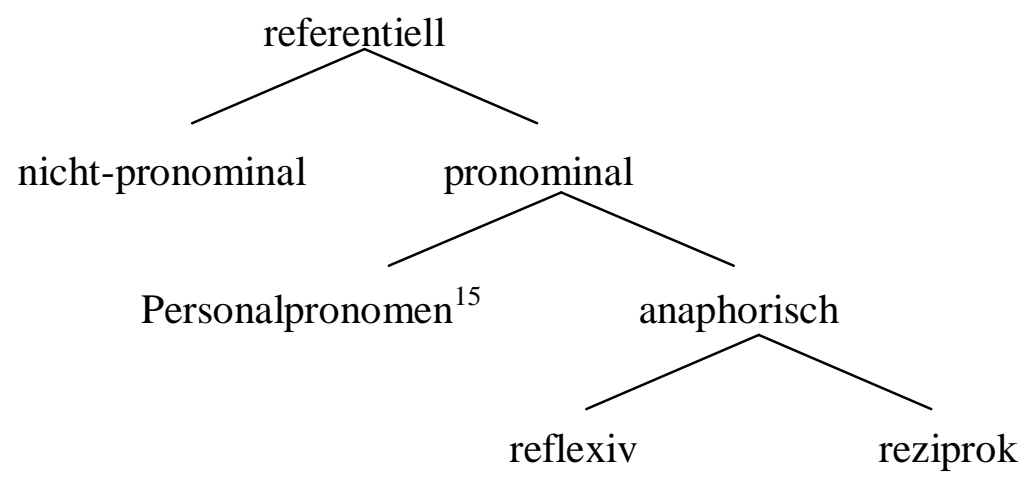

Ein gewöhnliches Nomen wie coche wird als referentiell und nicht-pronominal eingeordnet. Falls dieses Nomen noch mit einem Adjunkt versehen wird, bekommt das Nomen eine zweite Restriktion, und das entsprechende Attribut erhält einen zweiten Wert in der Menge der psoa-Argumente (z.B: coche nuevo, dabei führt nuevo eine zweite Matrix mit dem Instance-Attribut [1], das sich auf den Index bezieht, mit der Relation nuevo ein).

\footnotetext{
${ }^{15}$ Personalpronomen bezeichnen hier Pronomen, die nicht anaphorisch sind, z.B. yo, tú u.a.
} 
Die Darstellung von Eigennamen wird ebenfalls über eine Relation ausgedrückt:

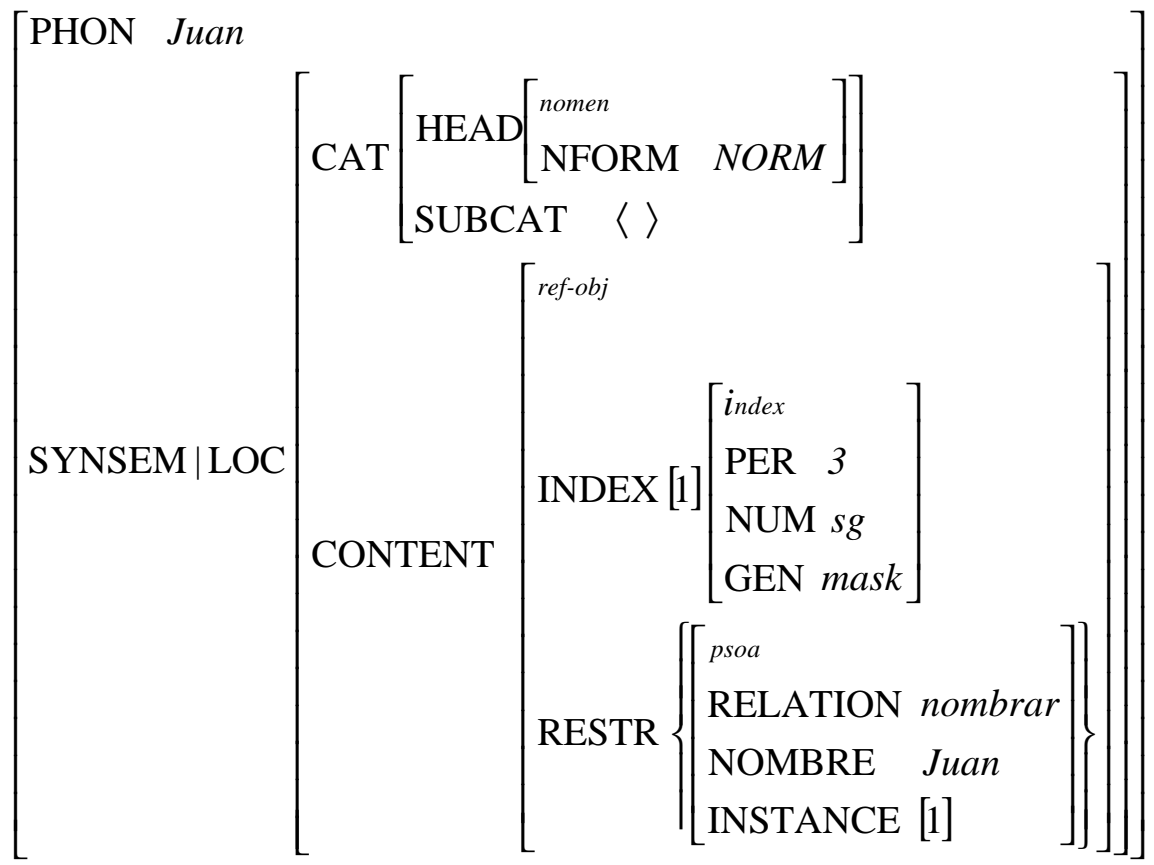

Die Darstellung des Verbs compra, ergänzt durch die semantische Komponente, wird wie folgt behandelt. Die Verbindung von Syntax und Semantik wird durch die Indizierung dargestellt. Da es sich allerdings um zwei verschiedene Aspekte der gleichen Komponente handelt, verweisen die Indizes der Subkategorisierungsliste auf die semantische Ebene.

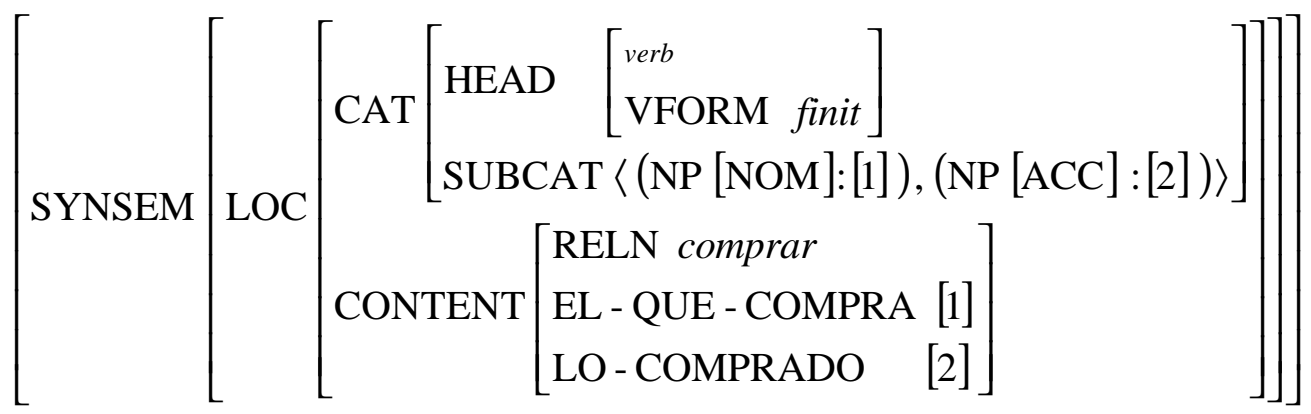

\subsubsection{Semantisches Prinzip}

Nach den Grundsätzen der HPSG muß es auch ein Prinzip für die Semantik geben unabhängig davon, ob es sich um eine Merkmalstruktur mit Komplement oder Adjunkt handelt (Pollard / Sag 1994: 319): 
(a) [DTRS head-comp-structure $] \rightarrow$ $\left[\begin{array}{l}\text { SYNSEM } \mid \text { LOC } \mid \text { CONTENT [1] } \\ \text { HEAD-DTR } \mid \text { SYNSEM } \mid \text { LOC } \mid \text { CONTENT [1] }\end{array}\right]$

(b) [DTRS head-adj-structure $] \rightarrow$ $\left[\begin{array}{l}\text { SYNSEM } \mid \text { LOC } \mid \text { CONTENT [1] } \\ \text { ADJ - DTR } \mid \text { SYNSEM } \mid \text { LOC } \mid \text { CONTENT [1] }\end{array}\right]$

Das Prinzip besagt lediglich, daß die semantischen Werte einer Struktur mit den Werten ihrer semantischen Köpfe oder Adjunkte übereinstimmen müssen. Müller (1999: 33ff.) erweitert diese Strukturen bereits an dieser Stelle um die kontextuelle Komponente und kritisiert den HPSG-Ansatz für Quantifikation. Der Bereich Semantik nimmt in der HPSG zwar einen breiten Raum ein, ist aber in vielen Details auch nicht unproblematisch. So stellt sich die Frage, inwieweit die Semantik wirklich in die Theorie eingebettet ist und nicht nur syntaktische Lücken füllt.

\subsubsection{Kongruenz}

Die Kongruenz zwischen Subjekt und Prädikat stellen Pollard / Sag (1994: 60ff.) in einem unifikationsbasierten Ansatz (constraint-based approach to agreement) dar. Dabei soll die Information aus zwei verschiedenen Quellen kompatibel sein und durch Index verbunden werden. Die Verlagerung dieser Information in die Semantik ist allerdings für Sprachen, die ein grammatisches Genus kennen, problematisch.

Syntaktische Kongruenz kann nicht in der Semantik beschrieben werden, wie es Pollard / Sag (1994) vorsehen. Es lassen sich problemlos Beispiele finden, in denen sich Kongruenz (engl. agreement) syntaktisch und semantisch unterscheidet. Nach Kathol (1999: 12ff., vgl. auch Kiss 1995a: 49) wird deshalb ein Agreement-Merkmal (AGREEMENT, AGR) im Kopfattribut HEAD angenommen, welches die Kongruenzmerkmale Person, Numerus, Genus und Kasus enthält.

Die folgenden Beispiele zeigen, wie besonders Genus oder Numerus syntaktisch und semantisch differieren können. Im Spanischen bezeichnet gente 'Leute' eine Menge an Personen, auch wenn das Lexem syntaktisch im Singular steht. Die französische Anrede vous wird syntaktisch im Plural verwendet, gilt aber auch als höfli- 
che Anredeform für eine Einzelperson. Im Deutschen muß oft zwischen syntaktischem und semantischem Genus unterschieden werden. Mädchen ist syntaktisch neutrum und semantisch feminin. Zur Differenzierung der Bezeichnungen werden die Kongruenzmerkmale im INDEX als semantisch (sNUM, sPER und sGEN) etikettiert.

$$
\begin{aligned}
& \text { (a) sp. gente: } \quad\left[\begin{array}{l}
\text { CAT } \mid \text { HEAD } \mid \text { AGR }[\text { NUM } s g] \\
\text { CONTENT } \mid \text { INDEX }[\text { SNUM } p l]
\end{array}\right] \\
& \text { (b) frz. vous: } \quad\left[\begin{array}{l}
\text { CAT } \mid \text { HEAD } \mid \text { AGR }\left[\begin{array}{ll}
\text { PER } & 2 \\
\text { NUM } & p l
\end{array}\right] \\
\text { CONTENT } \mid \text { INDEX [SPER 2 }]
\end{array}\right] \\
& \text { (c) dt. Mädchen: }\left[\begin{array}{l}
\text { CAT } \mid \text { HEAD } \mid \text { AGR }[\text { GEN } n t r] \\
\text { CONTENT } \mid \text { INDEX }[\text { SGEN fem }]
\end{array}\right]
\end{aligned}
$$

Kathol (1999) zeigt, daß Kongruenz ein komplexes Phänomen ist. Wenn prädikative Strukturen berücksichtigt werden, dann läßt sich zeigen, daß Kongruenz immer sowohl semantisch wie auch syntaktisch erklärt werden muß:

(a) Su majestad suprema está contento.

(b) Vous êtes belle.

Im spanischen Beispielsatz (72a) zeigt sich, daß syntaktische und semantische Information in Opposition stehen. Das Adjektiv contento bezieht sich auf die männliche Person, die sich hinter dem femininen Nomen majestad verbirgt. Im Beispiel (72b) ist vous als semantisch unterspezifiert anzusehen. Es steht grundsätzlich in zweiter Person Plural und kongruiert bei diesem Merkmal mit dem Verb. Das Adjektiv belle ist als feminin im Singular markiert und kongruiert semantisch mit dem Subjekt. Es weist somit dem Index neue Aspekte zu, die zuvor nicht enthalten waren. Damit entspringt die Information des Satzes verschiedenen Quellen.

Die allgemeine Struktur von Nomina wird in Anlehnung an Kathol (1999) nun folgendermaßen modifiziert. Für die Einführung in weitere Beschreibungsmechanismen bleiben wir aber zunächst bei den Konventionen der Standard-HPSG von Pollard / Sag (1994). 


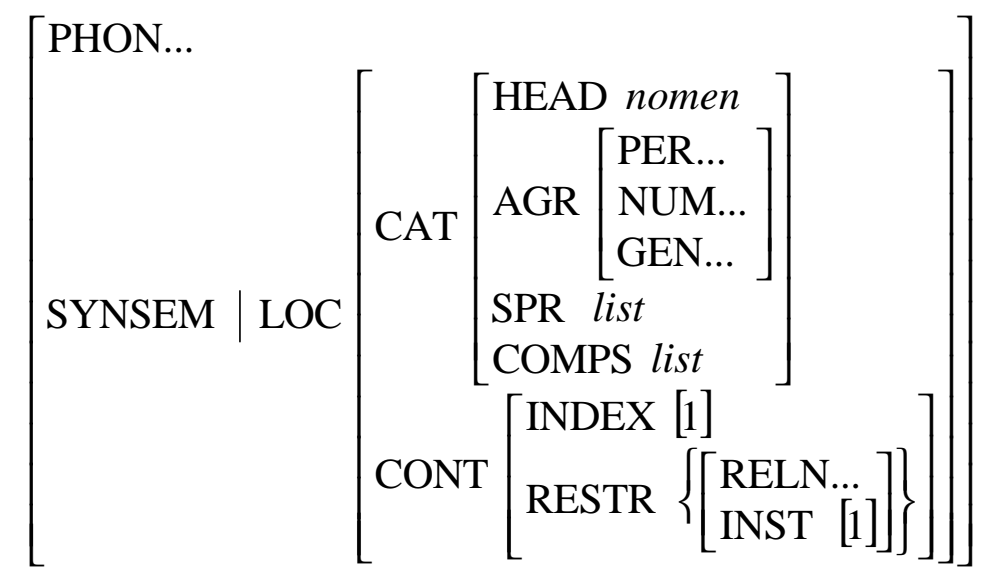

\subsection{Adjunkte}

Adjunkte sind freie Angaben, die in der Regel fast überall auftreten können. Das gilt insbesondere für Zeit- und Ortsangaben. Manche Verben verlangen allerdings eine entsprechende Angabe, wie z.B. dt. wohnen, das grundsätzlich den Ort des Wohnens verlangt. In diesen Fällen handelt es sich nicht um Angaben, sondern um notwendige Komplemente, die in der Subkategorisierungsliste des Verbs erscheinen. Zusätzlich müssen Adjunkte von fakultativen Komplementen unterschieden werden. Nach Flickinger (1987: 69ff.) ist dies dadurch möglich, daß z.B. bei der Passivbildung einer Ergänzung (als fakultativem Komplement) die thematische Rolle des Agens zugeschrieben wird. Dies ist bei Adjunkten nicht möglich. Adjunkte stehen also als freie Angabe neben obligatorischen und fakultativen Komplementen.

Pollard / Sag waren zunächst davon ausgegangen, daß ein Kopf sein Adjunkt wählt (Pollard / Sag 1987: 157ff., vgl. auch Abb / Maienborn 1994). Es mag zwar semantisch so sein, daß ein Kopf gewisse Restriktionen ausübt, grundsätzlich ist es aber unmißverständlicher, davon auszugehen, daß ein Adjunkt seinen Kopf wählt. Somit würde das Adjunkt die semantischen Restriktionen des Kopfes akzeptieren.

Adjunkte werden in der HPSG ähnlich behandelt wie Komplementierer. Sie verweisen auf den Kopf, den sie beschreiben, und führen ein Merkmal mit sich, daß sie als Adjunkt kennzeichnet. Damit dies geschehen kann, führen Pollard / Sag (1994: 55ff.) das Attribut MODIFIER (MOD) ein, ähnlich dem Attribut SPEC für Marker. Wir behandeln hier zunächst einmal hauptsächlich Adjektive, die in einer Nominalphrase Adjunktfunktion übernehmen, z.B. in el coche nuevo. 
Das Attribut PREDICATIVE (PRD) zeigt an, ob es sich im Falle von Adjektiven um ein prädikatives Adjektiv handelt oder nicht. Drei Klassen von Adjektiven werden bei Pollard / Sag (Pollard / Sag 1994: 55) unterschieden: Adjektive, die

1. nur prädikativ zu Kopulaverben erscheinen;

2. nur attributiv auftreten;

3. sowohl prädikativ als auch attributiv erscheinen.

Für das Spanische ist die Distinktion zwischen Adjektiven, die vor oder nach dem Nomen auftreten können, relevant. Die Semantik muß eine Disjunktion zwischen subjektiver oder objektiver Sichtweise des Sprechers ermöglichen. Für genauere Untersuchungen wird auf Kapitel III.4. verwiesen. Wir können zunächst grundsätzlich unterscheiden zwischen Adjektiven, die:

1. nur vor dem Nomen stehen;

2. nur nach dem Nomen stehen;

3. sowohl vor als auch nach dem Nomen stehen.

Renz (1993: 76) fordert für die Klassifikation von Adverbialen, daß zwischen Ergänzung und Angabe unterschieden wird. Dies war in der ersten HPSG-Version (Pollard / Sag 1987) noch nicht möglich, wird aber in der neuen Version eher deutlich: Angaben selektieren ihren HEAD und werden mit dem MOD-Attribut wie in der folgenden Matrix (74) dargestellt. Ergänzungen stehen demgegenüber in der SUBCAT-Liste und müssen obligatorisch realisiert werden. Wenn Ergänzungen fakultativ sind, wird dies durch runde Klammern angezeigt.

In Opposition zu einer Analyse für das Englische müssen spanische Adjektive auch Genus und Numerus markieren, um die Kongruenz mit dem Nomen darzustellen. Der semantische Inhalt von nuevo ist dann lediglich eine Vereinigung der Merkmale des Nomens mit der Restriktion des Adjektivs. Das Adjektiv nuevo erhält nach Pollard / Sag (1994: 55) folgendes SYNSEM-Attribut: 
(74)

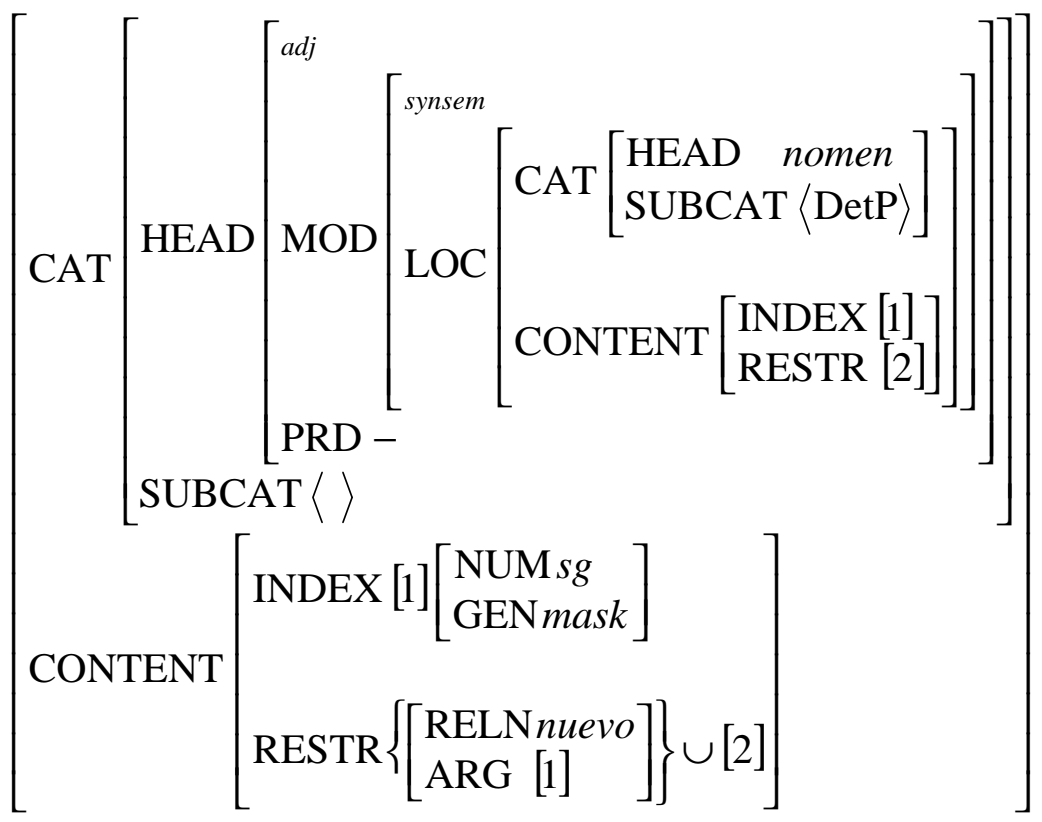

Im Kopfattribut des Adjektivs erscheint ein Attribut Modifizierer (MOD), das auf den $\mathrm{zu}$ modifizierenden Kopf und seine Eigenschaften verweist. Das Attribut PRÄDIKATIV (PRD) zeigt an, ob ein Adjektiv prädikativ verwendet wird. Semantisch wird lediglich festgehalten, daß der Index des zu beschreibenden Objekts von zwei Attributen Werte erhält: dem Adjektiv und dem zugehörigen Nomen, auf das hier nur verwiesen wird.

Daraus folgt das Schema 5 für Adjunkte (Pollard / Sag 1994: 56) ${ }^{16}$ :

\section{Schema 5}

kennzeichnet eine Phrase vom Typ Kopf-Adjunkt-Struktur (head-adjunctstructure, head-adj-struc). Der Wert des Attributs MOD der Adjunkttochter ist identisch mit dem SYNSEM-Wert der Kopftochter.

Die semantische Analyse der Phrase coche nuevo folgt in (76).

\footnotetext{
${ }^{16}$ Wir bleiben bei der Zählung von Pollard / Sag (1994), die in Schema 4 Komplementierer als Marker erläutern, welches hier unberücksichtigt bleiben kann.
} 
(76)

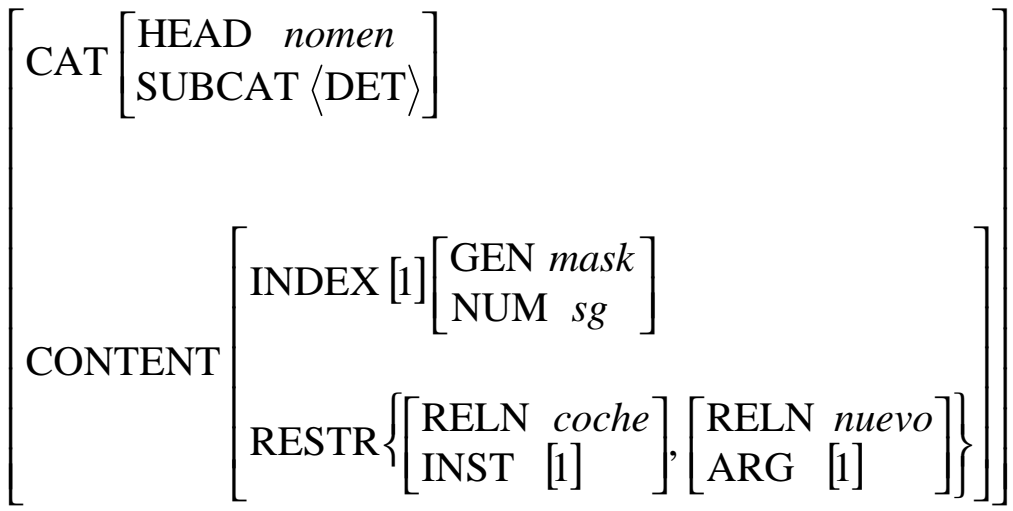

Grundsätzlich ist in diesem Zusammenhang die Frage des präpositionalen Anschlusses wichtig. Nominalphrasen können durch Präpositionalphrasen genauer bestimmt werden. Sogar nominale Infinitivkonstruktionen können präpositionale Attribute mit der Präposition de nach sich ziehen, wie Beispiel (77a) zeigt:

(a) el dulce lamentar de los pastores (Seco 1989: 231)

(b) la moda del arte joven (A-1)

(c) Respiró alegre el purísimo aire del lugar. (S-9)

(d) café con leche

In den ersten drei Fällen bezieht sich de attributiv auf das vorhergehende Nomen und spezifiziert dieses mit einer NP, die von der Präposition subkategorisiert wird. In (77d) modifiziert die Phrase con leche das vorangehende Nomen café. Nach Bierwisch (1988: 8) und Kaufmann (1995: 18-34) kann eine semantische Relation angenommen werden, die als Argumente die Präpositionalphrase und die modifizierte NP besitzt. Zwar untersuchen beide Autoren nur lokale Präpositionen, zeigen aber den Beziehungscharakter von Präpositionalphrasen, der je nach Präposition und Zusammenhang genauer determiniert werden müßte. Im Beispiel (77d) erstellt folglich die Präposition con eine semantische Relation, welche zwei Argumente kennt: die von der Präposition subkategorisierte NP (leche) und die NP, auf welche sich die PP bezieht (café). Da es sich aber um eine Relation handelt, die nur zwischen Nominalphrasen in dieser Form besteht, gehen wir davon aus, daß dieses Gebiet genauere Untersuchungen erfordert. Für verbale Elemente wären ohnehin noch andere Aspekte zu beachten (vgl. auch Verspoor 1996 speziell für PPs in der Funktion eines Dativobjekts). Die Syntax einer attributiven Präpositionalphrase kann wie folgt dargestellt werden (nach Pollard / Sag 1994: 342, 347): 


$$
\left[\operatorname{CAT}\left[\begin{array}{c}
\operatorname{HEAD}\left[\begin{array}{l}
\text { prep } \\
\text { MOD N': [1] } \\
\text { PRD - }
\end{array}\right] \\
\text { SUBCAT }\langle\mathrm{NP}:[2]\rangle
\end{array}\right]\right]
$$

(78) stellt eine Ausgangshypothese zur Beschreibung von Präpositionalphrasen dar. Die Komplexität von präpositionalen Konstruktionen kann nicht abgedeckt werden. Die Matrix bleibt ein Hilfskonstrukt, zumal Pollard / Sag (1994: 396n) ausdrücklich darauf hinweisen, noch keine vollständige Liste für nichtprädikative Präpositionen zu kennen. Badia (1998) unterstreicht bei katalanischen Präpositionalphrasen die notwendige Differenzierung zwischen semantisch vollwertigen und semantisch leeren Präpositionen. Eine detaillierte Untersuchung der sprachlichen Gegebenheiten, wie sie Badia unternimmt und welche auch andere, nicht nur nominale Kontexte berücksichtigt, ist Voraussetzung für eine angemessene syntaktisch-semantische Deskription von Präpositionen. Auch wenn wir in Kapitel III.5. nochmals auf Präpositionen eingehen, bleibt der Schwerpunkt dieser Arbeit auf der Nominalphrase aus Nomen, Determinierer und Adjektiv.

\subsection{Quantifizierer}

Der Begriff der Quantifizierung stammt aus der formalen Logik (vgl. Allwood / Andersson / Dahl 1973: 1ff. und Cooper 1990: 143ff.). Es wird insbesondere zwischen dem Existenzquantor ( $\exists$, 'un, ein') und dem Allquantor ( $\forall$, 'todos, alle’) unterschieden. Der Existenzquantor besagt, daß mindestens einem Element des jeweiligen Bezugsbereichs eine Eigenschaft zugeordnet wird. Der Allquantor spricht diese Eigenschaft allen Elementen des Bezugsbereichs zu. Das Spanische kennt nun viele Wörter, die als Quantifizierer bezeichnet und zwischen den zwei Extremen Existenzquantor und Allquantor eingeordnet werden müssen: cualquiera, alguno, varios, mисho usw. Sie werden oftmals ebenfalls durch den Existenzquantor erfaßt. Während die semantische Differenzierung dieser Quantifizierer etwas ungenau bleibt, erlauben die logischen Quantoren die genaue Beschreibung von Gültigkeitsbereichen bei Ambiguitäten, wie z.B. im Satz cada persona sabe una canción. 
Dies bedeutet, daß ein Quantor auf etwas einwirkt, das als Variable innerhalb seines Bezugsbereichs gesehen werden kann. Damit verändert sich auch die Semantik dieses Wortes, in dem hier betrachteten Fall des Nomens. Es gerät sozusagen in den Gültigkeitsbereich des Quantors. In der Standard-HPSG wurde der semantische Gehalt (CONTENT) des Nomens coche wie folgt dargestellt (in der weiterhin verwendeten Notation der Standard-HPSG). Die Sorte des Attributs RESTRICTION (RESTR) ist grundsätzlich ein parametrized state of affairs (psoa). Diese Angabe stellt entsprechend Umstände dar, die in irgendeiner Form eingeschränkt werden (Pollard / Sag 1994: 47):

$$
\left.\left[\begin{array}{l}
\text { INDEX [1] }\left[\begin{array}{l}
\text { index } \\
\text { PER 3 } 3 \\
\text { NUM sing } \\
\text { GEN mask }
\end{array}\right] \\
\text { RESTR }\left\{\left[\begin{array}{l}
\text { psoa } \\
\text { RELATION coche } \\
\text { INSTANCE }[1]
\end{array}\right]\right.
\end{array}\right]\right\}
$$

Quantoren zeichnen sich nun darin aus, daß sie die INDEX-RESTRICTION-Relation genauer bestimmen. Dafür wird erstens das Attribut Restringierter-Index (RESTRICTED-INDEX, RESTIND) und zweitens ein „Speicher für Quantifizierer“ eingerichtet (QUANTIFIERSTORE, QSTORE, vgl. Cooper 1983: 52-78). Beide Attribute stehen in dem Zusammenhang, daß ein an einer bestimmten Stelle noch nicht realisierter Quantifizierer im „Speicher“ registriert wird, bis die Realisierung und damit die Freigabe stattfindet. Dieser im Speicher befindliche Wert entspricht dem Restringierten-Index und ist mit ihm koindiziert.

Ziel ist es, alle quantifizierenden Elemente an entsprechenden Stellen in einem Strukturbaum so aus dem Speicher zu geben, daß sie ihren Bezugsbereich determinieren. Nomina subkategorisieren Artikel, aber Artikel spezifizieren zugleich auch die Nomina (vgl. auch Kiss 1995a: 59ff.). Da nun durch den Gültigkeitsbereich des Quantors eine Aufteilung in den Kernbereich einer Phrase oder eines Lexems und den weiterführenden Bezugsbereich nötig ist, wird das Attribut NUCLEUS ('Kern') als Teilwert zu CONTENT eingeführt. Es trennt die nicht-quantifizierte Information von den Quantifizierern, welche im neuen Attribut Quantifizierer (QUANTIFIERS, 
QUANTS) enthalten sind. Letzteres beinhaltet eine Liste von Quantifizierern in der Reihenfolge ihrer Bezugsbereiche. Für den Kern (NUCLEUS) wird eine Subsorte der Sorte psoa eingeführt, die den beschriebenen Situationstyp einer Äußerung determiniert, die Subsorte quantifiziererfreie psoa (quantifier-free-psoa, qfpsoa).

Wir bekommen somit neue Merkmale für das Attribut CONTENT:

$$
\left[\begin{array}{ll}
\text { QUANTS } & \langle\rangle \\
\text { NUCLEUS } & {[]} \\
\text { QSTORE } & \{\}
\end{array}\right]
$$

Die Quantifizierer (QUANTS) kennzeichnen eine Liste, der Kern (NUCLEUS) ist ein einzelner Wert. QSTORE kennzeichnet eine Menge. Der semantische Gehalt des Satzes yo sé una canción kann wie folgt dargestellt werden (nach Pollard / Sag 1994: 320). Der Index [2] verweist auf den Sprecher:

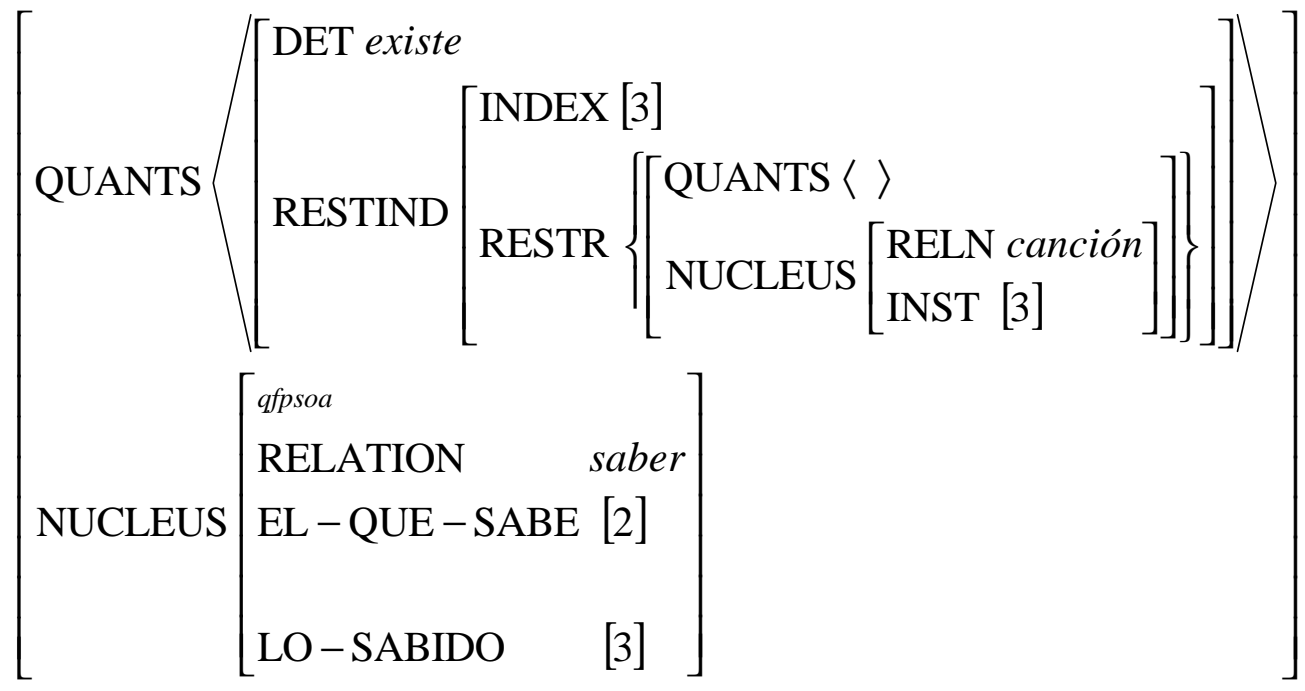

Entscheidend für eine Aufschlüsselung im Baumdiagramm ist die Beziehung zwischen QSTORE und QUANTS in (80), um aufzeigen zu können, an welchem Knoten eines Baumdiagramms welcher Bezugsbereich eines Quantifizierers erkennbar ist. Diese Relation des Ein- und Auslagerns von Informationen über den Bezugsbereich des Quantifizierers leistet das Attribut RETRIEVED-QUANTIFIERS (RETRIEVED, 'Wiederhergestellte Quantifizierer'), welches eine Liste von Quantifizierern beinhaltet. Alle diese Erweiterungen sind notwendig, um die Ambiguität des 
Beispielsatzes Cada persona sabe una canción beschreiben zu können:

(82) Cada persona sabe una canción.

(a) $(\forall \mathrm{x} \mid\{$ persona $(\mathrm{x})\})(\exists \mathrm{y} \mid\{$ canción $(\mathrm{y})\})$ saber $(\mathrm{x}, \mathrm{y})$

(b) $(\exists \mathrm{y} \mid\{$ canción $(\mathrm{y})\})(\forall \mathrm{x} \mid\{$ persona $(\mathrm{x})\}) \operatorname{saber}(\mathrm{x}, \mathrm{y})$

\section{Interpretation:}

von (82a): Für jedes $x$ ( $x$ ist eine Person) gibt es ein y (y ist ein Lied), wobei gilt: x kennt y. (d.h., jeder kennt irgendein Lied)

von (82b): Es gibt ein y (y ist ein Lied), so daß für alle $\mathrm{x}$ ( $\mathrm{x}$ ist eine Person) gilt: $x$ kennt $y$. (d.h., ein bestimmtes Lied kennen alle)

Die Interpretationen fußen auf unterschiedlichem Skopus des All- bzw. Existenzquantors. Das Baumdiagramm (83) zeigt den Satz Cada persona sabe una canción, wobei nach der Konstituente V in (84a) die Konstituenten VP in (84b) und S in (84c) unterschiedlich interpretiert werden (in Anlehnung an Pollard / Sag 1994: 324f. und Kiss 1995a: 65ff.).

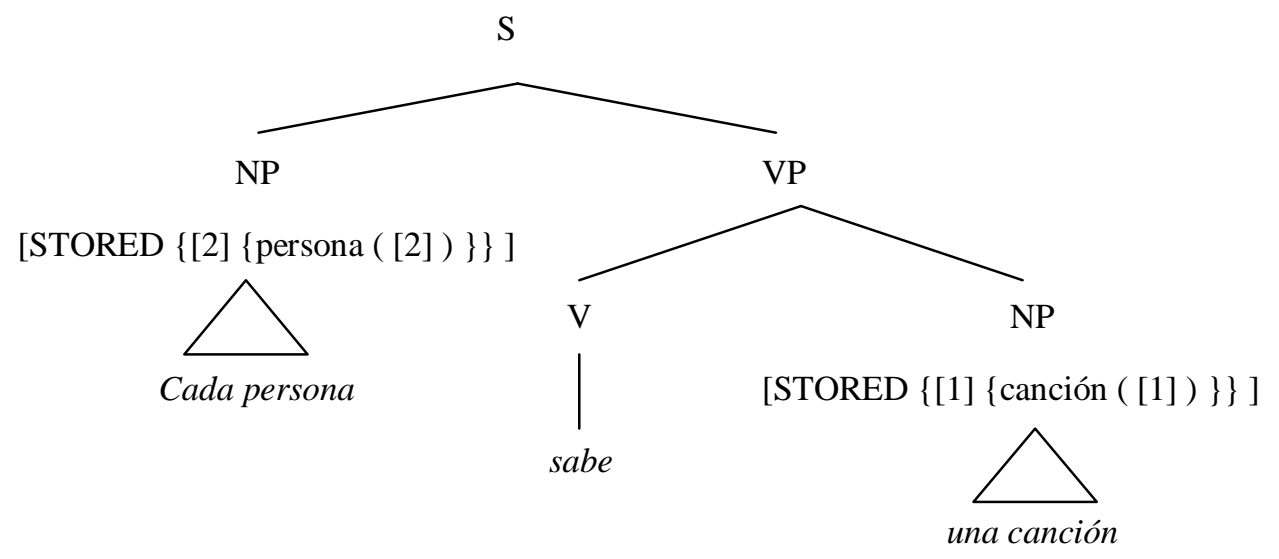

Bei der Analyse von (82a) werden alle Quantoren sofort abgebunden, so daß das Attribut QSTORE | STORED der Verbalprojektion nie gefüllt wird. Bei der zweiten Interpretation muß der Existenzquantor in dieses Attribut überführt werden, um den Skopus zu verdeutlichen. Wir zeigen von daher an dieser Stelle die zweite Interpretation (82b). Beim Verb sind die Merkmale STORED und RETRIEVED noch leer. Die Matrizen stellen jeweils die CONTENT-Werte dar. 
(84a) V

$$
\left[\begin{array}{l}
\text { QUANTS }\langle\rangle \\
\text { NUCLEUS [3] }\left[\begin{array}{lc}
\text { RELN } & \text { saber } \\
\text { EL-QUE - SABE [2] } \\
\text { LO-SABIDO } & {[1]}
\end{array}\right] \\
\text { QSTORE }\left[\begin{array}{l}
\text { STORED }\{\} \\
\operatorname{RETRIEVED}\langle\rangle
\end{array}\right]
\end{array}\right]
$$

Auf der Ebene VP befindet sich der Existenzquantor im Attribut STORED. Um die Interpretation 'ein bestimmtes Lied kennen alle' zu erreichen, darf der Existenzquantor erst auf der obersten Ebene, der Satzebene, abgebunden werden.

(84b) VP

$$
\left[\begin{array}{l}
\text { QUANTS }\langle\rangle \\
\text { NUCLEUS [3] } \\
\text { QSTORE }\left[\begin{array}{l}
\text { STORED }\{\exists[1]\{\operatorname{cancion}([1])\} \\
\operatorname{RETRIEVED}\langle\rangle
\end{array}\right.
\end{array}\right]
$$

Auf der Ebene des Satzes werden alle Quantoren abgebunden, auch der vorher gespeicherte Existenzquantor, und dem Quantifiziererattribut zugeführt:

$(84 c) \quad S$

$$
\left[\begin{array}{l}
\text { QUANTS }\langle\exists[1]\{\text { cancion }([1])\}, \forall[2]\{\text { persona }([2])\}\rangle \\
\text { NUCLEUS [3] } \\
\text { QSTORE }\left[\begin{array}{l}
\operatorname{STORED}\{\} \\
\operatorname{RETRIEVED}\langle\exists[1]\{\text { cancion }([1])\}, \forall[2]\{\text { persona }([2])\}\rangle
\end{array}\right]
\end{array}\right]
$$

Pollard / Sag müssen so ein neues semantisches Prinzip formulieren (vgl. Pollard / Sag 1994: 323). In Pollard / Yoo (1997) wird der hier kurz skizzierte Ansatz kriti- 
siert, weil er nicht in der Lage ist, Ambiguitäten in Anhebungskonstruktionen ${ }^{17} \mathrm{zu}$ erläutern. Da wir in dieser Arbeit nicht auf ganze Sätze eingehen werden, sondern nur auf nominale Teilstrukturen, bleibt dieses Teilgebiet unbearbeitet. Wir werden im Zusammenhang mit dem Skopus von mehreren an ein einzelnes Nomen adjungierten Adjektiven in einer Nominalphrase auf dieses Thema zurückkommen (vgl. Kap. III.2.).

\subsection{Possessiva}

Während bei den Quantifizierern mit uno und cada zwei Determinierer betrachtet wurden, deren prädikatenlogische Einordnung problemlos möglich ist, stellen die Possessiva höhere Anforderungen. $\mathrm{Mi}$, tu, su, nuestro und vuestro beinhalten den definiten Artikel und damit einen nur sehr schwer prädikatenlogisch zu klassifizierenden Determinierer.

Pollard / Sag (1994: 51ff.) untersuchen bei den Possessiva engl. my und Konstruktionen wie engl. Mary's. Die Analyse von spanisch $m i$ als vorangestelltem, unflektiertem Possessivpronomen gleicht der von Pollard / Sag für das englische Äquivalent my (nach Pollard / Sag 1994: 51). Die Matrix enthält den Wert für den Pfad SYNSEM | LOC. Wir bleiben dabei und im folgenden bei der Darstellung von Pollard / Sag (1994). Die Modifikationsvorschläge des vorigen Kapitels über Quantifizierer bleiben unberücksichtigt:

\footnotetext{
${ }^{17}$ Unter Anhebung wird die nicht erfolgende Zuweisung einer thematischen Rolle des Modalverbs an das Subjekt verstanden, vgl. dazu ausführlich Kiss (1995a: 6ff.). Der entsprechende Beispielsatz bei Pollard / Yoo (1997: 420) lautet A unicorn appears to be approaching.
} 


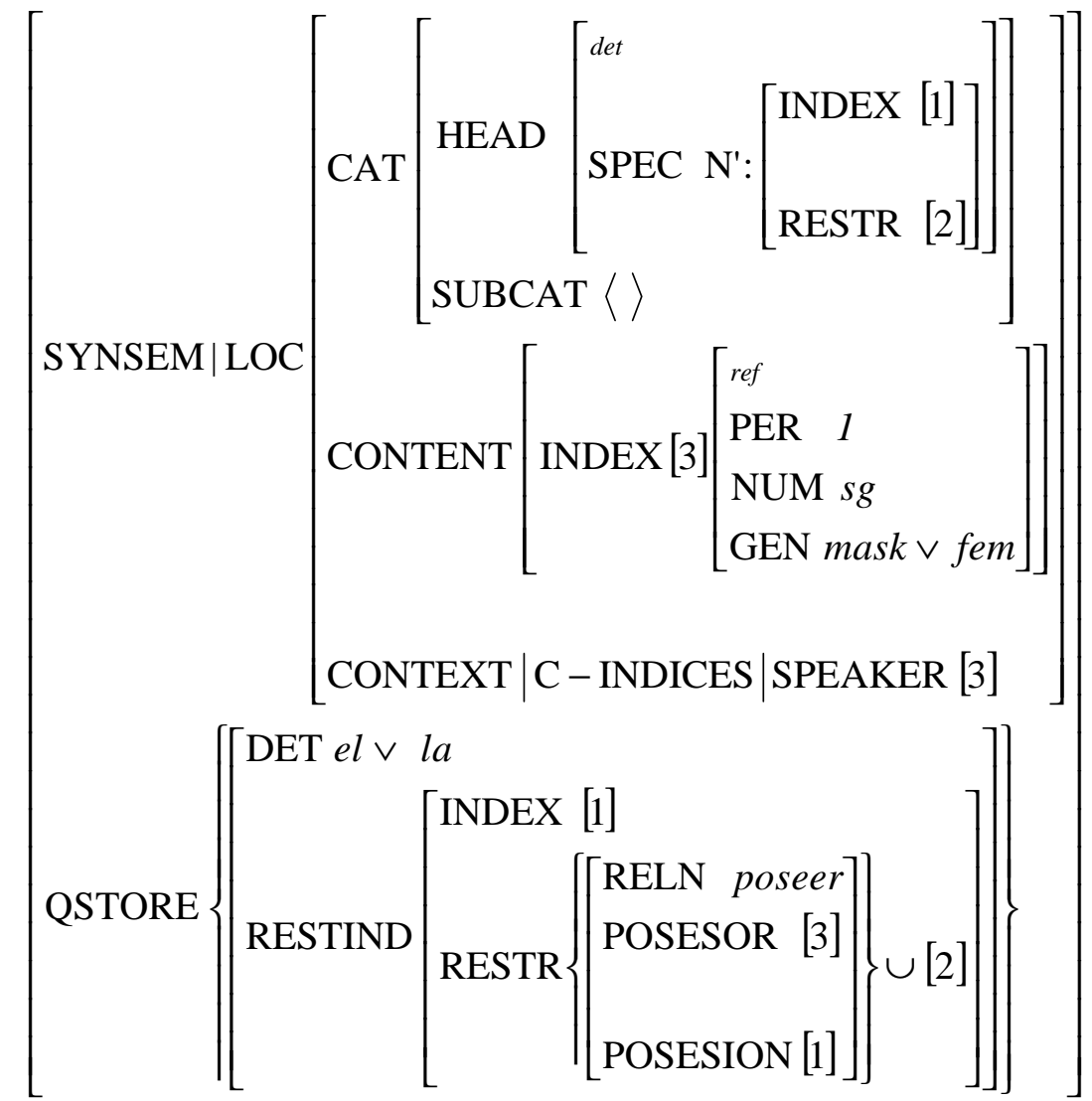

Das Possessivpronomen $m i$ gibt es sowohl als Determinierer, der als Quantifizierer dargestellt wird, als auch in der Funktion eines Personalpronomens (mí mit Akzent). Das an dieser Stelle neue Attribut Kontext (CONTEXT) verweist auf pragmatische Informationen, die mit dem beschriebenen Lexem verknüpft sind. So kann behauptet werden, daß das Personalpronomen auf den Sprecher verweist und damit den Eintrag CONTEXT | CONTEXT-INDEX (C-INDEX) | SPEAKER (Kontext | Kontextin$\operatorname{dex} \mid$ Sprecher) wie in (85) erhält. Unter QSTORE findet die Restriktion des Determinierers statt, der mit den Indizes [1] und [2] auf das folgende Nomen verweist und mit dem Index [3] auf den Sprecher, der sich durch die Verwendung des Pronomens zum Eigentümer eines Objekts oder einer Eigenschaft erklärt.

Da die Form mío zwar die possessiven, aber nicht die determinierenden Eigenschaften von $m i$ besitzt, wird seine Beschreibung anders aussehen. Hinzu tritt die Tatsache, daß mío als grundsätzlich dem Nomen nachgestellte und immer mit vorangestelltem Determinierer auftretende Wortform adjektivische Merkmale aufweist, die seine Klassifikation als Determinierer zweifelhaft machen. Wir werden dieses Problem in Kapitel III.2. angehen. 


\section{Zusammenfassung}

Wir haben in diesem Kapitel eine Einführung in die HPSG gegeben. Zunächst wurde eine einfache kontextfreie Phrasenstrukturgrammatik konstruiert, deren Elemente dann anhand eines Beispielsatzes schrittweise in einer kopfgesteuerten Phrasenstrukturgrammatik erläutert wurden. Dabei zeigt sich, daß lexikalische Elemente in wesentlich größerem Umfang an der HPSG beteiligt sind, als dies in der kontextfreien PSG der Fall ist. Grundsätzlich wird in der HPSG versucht, lexikalische Entitäten so weit und so genau wie möglich mit ihren syntaktischen und semantischen Möglichkeiten einzugrenzen. Auf diesen Informationen beruhen lexikalische Regeln, welche diese Information kontextgebunden verändern können.

Die Prinzipien, hauptsächlich das Kopfmerkmalsprinzip und das Subkategorisierungs- oder Valenzprinzip, versuchen elementare Verknüpfungsmuster in Sätzen zu erfassen. Die Schemata als einzelsprachliche Komponenten steuern die möglichen Kombinationen von Phrasen. Damit werden Ersetzungsregeln unnötig, sind aber aus den Strukturen ableitbar.

Ungebundene Abhängigkeiten werden wie die Kontroll- und Bindungstheorie nicht erörtert. Sie spielen im Zusammenhang mit Pronomen eine bedeutende Rolle. Da wir den Kontext über die engere NP hinaus ausblenden, Satzstrukturen nicht bearbeiten und diese Arbeit eher syntaktisch ausrichten, bleiben diese Phänomene auBen vor.

Als in dieser Form noch unbefriedigend erscheinen die Beschreibungsstrukturen für Adjunkte, insbesondere für Präpositionalphrasen. Adjektivische Adjunkte müssen noch unter pragmatischen Gesichtspunkten näher betrachtet werden. Für die Subkategorisierungsliste wurde ein neuer Vorschlag unterbreitet, der die Teilgruppen (Subjekt, Komplement, Spezifizierer) erläutert. 


\section{Die Nominalphrase}

Die Bearbeitung der spanischen Nominalphrase fokussiert vor allem die Wortarten Nomen, Determinierer und Adjektiv. Der Terminus Adjektiv wird in traditionellen spanischen Grammatiken als Oberbegriff sowohl für qualifizierende Adjektive als auch für quantifizierende Determinierer verwandt. Wir werden diese beiden Klassen voneinander abgrenzen. Präpositionen werden wir nur kurz behandeln, Relativsätze ganz außer acht lassen. Pronomina als eigenständige Vertreter für die NP werden nur gestreift.

Wir beginnen die Untersuchung mit dem alleinstehenden Nomen (III.1.), um dann die Abgrenzung Determinierer vs. Adjektiv anzuschließen (III.2.). Fragestellungen des grundsätzlichen syntaktischen Aufbaus der spanischen Nominalphrase bleiben stets im Blickfeld und werden in III.3. thematisiert. Abschließend folgen Kapitel über qualifizierende Adjektive (III.4.) und Präpositionen (III.5.).

\section{Das Nomen ohne Determinierer}

Mit Nomen ist in erster Linie die Wortart Nomen oder Substantiv gemeint. Ebenfalls als nominal einzustufende Wortklassen wie Determinierer, Pronomina und Adjektive werden später behandelt. Mit Nomen bezeichnen wir zunächst Gattungsnomina (Appellativa) und grenzen diese von Eigennamen und Pronomina ab (für die Besonderheiten von Eigennamen vgl. Longobardi 1994).

Nomina machen in der Regel einen Großteil des Wortschatzes einer Sprache aus. Berschin / Fernández-Sevilla / Felixberger (1987: 288) beziffern ihn auf 50,5 \% des Gesamtwortschatzes. Nach Seco (1991: 87) ,podemos, pues, definir el sustantivo como una palabra capaz de funcionar como núcleo del sujeto en una oración. Hay que distinguir, no obstante, entre aquellas palabras que son sustantivos por naturaleza - nombres y pronombres - y aquellas que lo son funcionalmente, es decir, que están habilitadas (por el procedimiento de traslación [...]) para desempeñar funciones propias de los verdaderos sustantivos“. Nach Helbig / Buscha (1994: 229) kann ein Sub- 
stitutionsrahmen für die Zuordnung zur Wortklasse „Substantivwörter“ angenommen werden:

Das ... war gut. (Essen)

Ich sehe den ... (Freund).

Sie sprachen von der .... (Reise).

Ähnlich verfährt Bosque (1993: 41f.), um Nomina als solche kenntlich zu machen:

(2) _ es bueno.

Juan recordó_.

Sus_grandes.

Sowohl Seco als auch Helbig / Buscha und Bosque definieren das Nomen über den Kontext, in Abhängigkeit seiner vom Verb zugewiesenen Funktion (bei Helbig / Buscha: Subjekt, Akkusativ- und Präpositionalobjekt). Zudem müssen zwei Differenzierungen betrachtet werden:

1. zwischen Nomen und Pronomen: Pronomina können anstelle einer Nominalphrase auftreten, nicht anstelle eines Nomens. Sie sind hinsichtlich Numerus, Person und Kasus spezifiziert. Die Kombination Determinierer / Adjektiv und Pronomen ist in der Regel nicht erlaubt. Bei Martínez (1989) werden „Determinierer“ und „Pronomen“ manchmal synonym verwandt (vgl. auch Luján 1980: 81, Hudson 2000: 22f. fordert dies explizit).

2. zwischen ,echten“ Nomina und substantivierten, sonst anderen Wortklassen zugeordneten Wörtern (z.B. Infinitive ${ }^{18}$ ).

In den folgenden Abschnitten soll die Frage beantwortet werden, unter welchen Bedingungen ein einzelnes Nomen eine Nominalphrase bildet. Es kann evtl. noch durch ein oder mehrere Adjektive modifiziert werden, steht aber ohne Determinierer (vgl. z.B. die Abwandlung des Beispielsatzes von Helbig: Ich sehe Freunde; ich sehe gute Freunde). Dabei sind nach Iturrioz (1996: 383) folgende Erscheinungen zu berïcksichtigen:

(3) „El uso de los artículos y en especial la ausencia de los mismos no puede explicarse mediante un rasgo semántico simple, sino que más bien está

\footnotetext{
${ }^{18}$ Zur Diskussion über den Status von Infinitiven vgl. Plann 1981, Yoon / Bonnet-Farran 1991, Klenk 1998 sowie zum englischen Gerundium Malouf 1998.
} 
regulado por varios factores de orden

1. semántico-lexical: significado del nombre núcleo del SN [hier: NP], significado del verbo o predicado;

2. morfo-sintáctico: subcategorías nominales, interacción con otros determinantes, jerarquía de los roles, orden de los constituyentes de la oración;

3. pragmático-discursivo: grado de topicidad, concentración de la información, formación de macroestructuras;

4. grado de gramaticalización y lexicalización.

5. En todo caso es necesario integrar el uso de los artículos en el marco más general de la operación de DETERMINACIÓN y de la interacción de ésta con otras funciones como INDIVIDUACIÓN, POSESIÓN y NOMINACIÓN“ (Hervorhebungen von Iturrioz).

Diese Liste aller für die Untersuchung relevanten linguistischen Disziplinen deckt sich mit einer Vielzahl von Untersuchungen zu diesem Thema (vgl. die Aufsätze in Bosque 1996 sowie Longobardi 1994, Wiese 1997 u.a.). Letztlich sind fast alle Bereiche in Betracht zu ziehen, inbesondere eine mögliche Idiomatisierung der beteiligten Lexeme. So weist Lois (1996: 204f.) darauf hin, daß auch folgende Phänomene zu erklären sind:
(a) No dijo palabra.
(b) *Dijo palabra.
(c) *No dijo frase.
(d) *No distinguió palabra.

Während (4a) korrekt ist, sind alle anderen Beispielsätze ungrammatisch. Lois vermutet, daß sich dies zum einen auf das syntaktische Phänomen der Negation, zum anderen aber - wahrscheinlich in weit größerem Ausmaß - auf lexikalische Restriktionen zurückführen läßt (vgl. auch Longobardi 1994: 612f.). Diese Restriktionen können auch als idiomatisch bezeichnet werden. Es handelt sich um etablierte Redewendungen. Dafür sprechen auch folgende Beispielsätze (Lois 1996: 205):
(a) Tiene perro.
(b) *Tiene mono. 
Das zweite Beispiel ist nur in Opposition zum ersten inkorrekt. Die Redewendung wird für die Entzugserscheinungen eines Drogenabhängigen verwendet und kann folglich nicht mit 'er besitzt einen Affen' übersetzt werden (Oliver 1987: s.v. mono).

Lois beobachtet in Einklang mit Wiese (1997), daß eine mögliche ,interpretación de masa" zur Folge hat, daß ein singuläres Nomen eigenständig eine NP bilden kann. $\mathrm{Da}$ diese Interpretation möglich ist, hängt von semantisch-pragmatischen Faktoren ab. Bei Reumuth / Winkelmann (1993: 36f.) fehlt demgegenüber das einzelne singuläre Nomen in der Liste der möglichen NP-Strukturen. Im folgenden wird das Phänomen eigenständiger, singulärer Nomina als NP systematisch untersucht.

\subsection{Die Syntax}

Spanische Nomina werden durch die Merkmale Numerus und Genus im Lexikon spezifiziert. Sie besitzen die Eigenschaft der dritten Person. Personalpronomina verhalten sich an dieser Stelle differenzierter.

Mögliche Werte des Attributs Numerus sind Singular oder Plural, des Attributs Genus feminin oder maskulin. Nur das unpersönliche Pronomen lo kann neutrum markieren. Ein Nomen kann in der HPSG folgendermaßen gekennzeichnet werden, wobei Nomen für ein beliebiges Nomen und NOMEN für dessen Bedeutung steht:

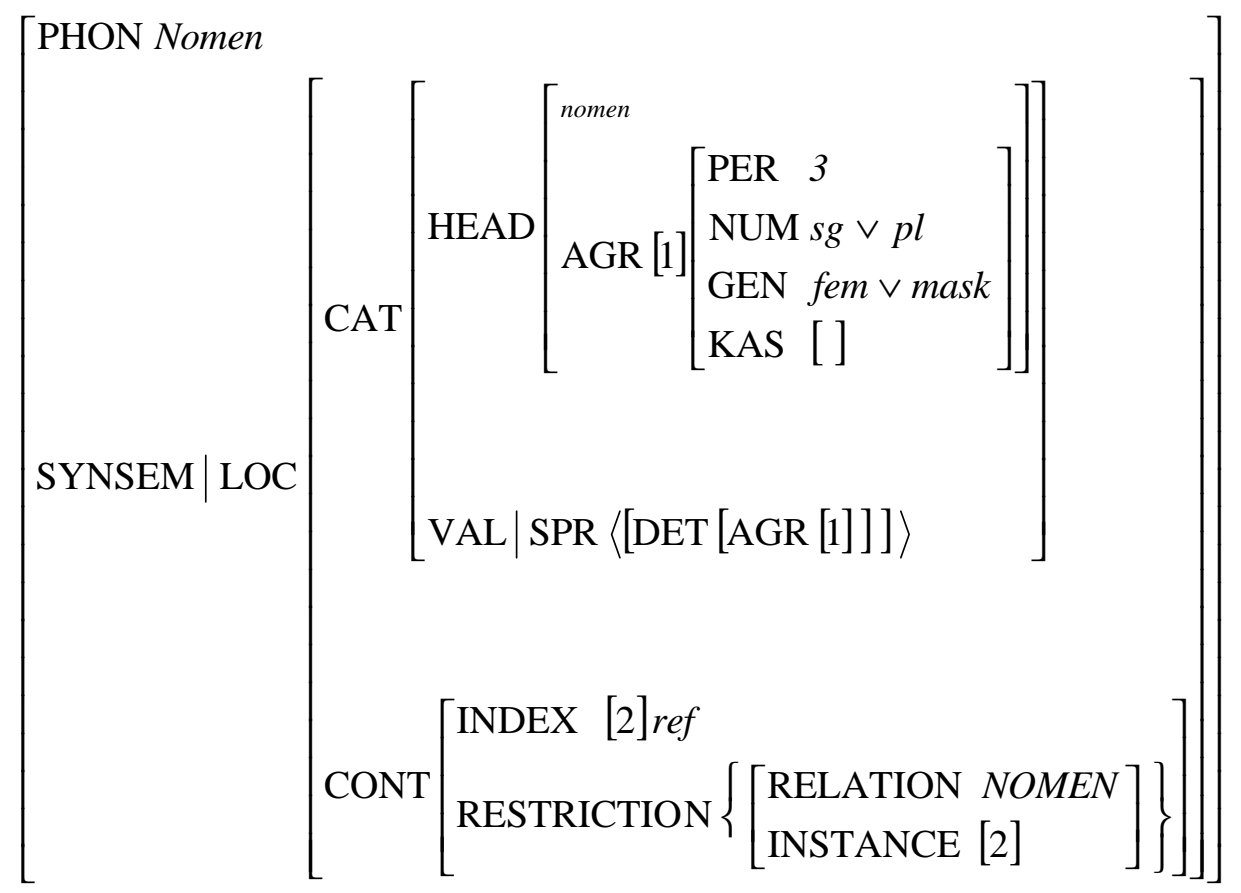


Dies ist eine sehr allgemeine Beschreibung, die nichts darüber aussagt, ob ein beliebiges Nomen auch eigenständig auftreten kann. Eine erste Einschränkung ist, daß das Attribut Genus lexikalisch festgelegt wird, während die meisten Nomina sowohl im Singular wie im Plural auftreten können und somit in der Regel den Numerus nicht festlegen. Dieser kann zuweilen ebenfalls fixiert sein (z.B. las gafas) oder eine Bedeutungsänderung hervorrufen (la gente - las gentes). Im Spanischen ist es außerdem möglich, die Singularform als Kollektivum zu verwenden: ¿Hay mucha trucha en este río? (de Bruyne 1993: 88).

Aber auch beim Genus gibt es Schwankungen: Manche Wörter werden sowohl feminin als auch maskulin gebraucht (análisis, azúcar, calor, vgl. de Bruyne 1993: 71f.), manche variieren dabei ihre Bedeutung (vgl. el mar - la mar bei Seco 1986: 253).

Kasus ist im Spanischen in der Regel nicht markiert. Viele Grammatiken unterscheiden zwischen direktem und indirektem Objekt (sowie Präpositionalobjekt, vgl. Seco 1991: 106ff.) statt Akkusativ-, Dativ- oder Genitivobjekt. Die lateinischen Ausdrücke werden oft vermieden, da sie, den Autoren zufolge, den Sprachgebrauch nicht korrekt wiedergeben. Tatsache ist, daß nur bei den Personalpronomina eine klare Differenzierung des Kasus stattfindet. Somit ist erst durch Paraphrasierung oder Passivbildung erkennbar, um welchen Fall es sich handelt.

Kasus ist somit bei der einzelnen NP nicht erkennbar und nur aus dem Kontext zu erfassen. Dieser Kontext kann folgendermaßen festgehalten werden: Nominalphrasen, die lediglich aus einem Nomen bestehen, dürfen nicht in präverbaler Stellung als Subjekt auftreten (vgl. Longobardi 1994: 616, Casielles 1996: 133ff. sowie Contreras 1996: 143ff. für prädikative Konstruktionen). Somit muß der Kasus indirekt bei der NP realisiert sein. Dies läßt sich auch am Akkusativobjekt beobachten, welches in präverbaler Stellung (betonte Voranstellung) durch ein Personalpronomen verstärkt wird: Producen estos aparatos rapidísimo - Estos aparatos los producen rapidísimo. Durch los ist der Akkusativ grammatisch realisiert (Bsp. aus Matte Bon 1992: I, $302)$.

Da wir bei den HEAD-Merkmalen des Nomens nur bedingt Klassifikationskriterien finden können, ist zu fragen, ob nicht die Subkategorisierungsliste mehr Aufschluß gibt. Dies tut sie insofern, als sie nicht bei allen Nomina Einträge aufweist. Nomina des Spanischen können im Plural, Massennomina auch im Singular ohne Determinierer stehen und so eine leere Subkategorisierungsliste aufweisen. Substan- 
tivierungen benötigen oft einen Determinierer, um die neue Wortart zu kennzeichnen. Eigennamen stehen grundsätzlich ohne Determinierer.

Diese kurzen Ausführungen zeigen bereits, daß auf der syntaktischen Seite keine grundlegende Klassifizierung der Wortart Nomen vorgenommen werden kann. Wir können keine eindeutigen, den Nomina inhärente Merkmale feststellen. Dem gegenüber können Verben nach ihrer Subkategorisierungsliste eingeteilt werden, bei Adjektiven und Präpositionen ist dies in ähnlicher Weise möglich. Die Andeutungen in Richtung auf evtl. Bedeutungsänderungen lassen eine semantische Klassifikationsperspektive bei Nomina vermuten.

\subsection{Die Semantik}

Nomina bezeichnen Individuen und Kollektiva, Abstrakta und Konkreta, Eigenschaften, Vorgänge, Relationen, Maße u.a. Eine solche semantische Klassifikation ist relativ vage und wird je nach grammatischem Ansatz eines Autors entsprechend in eine hierarchische Übersicht gebracht. Ein Nomen hat eine vergleichsweise eindeutige Bedeutung, welche aber trotzdem kontextabhängig variieren kann.

Wir unterscheiden hier zunächst zwischen Eigennamen und Gattungsnamen (Kollektiva), wobei letztere in Abstrakta und Konkreta unterschieden werden (vgl. Alcaraz Varó / Martínez Linares 1997: s.v. nombre). Wiese (1997: 149) beobachtet eine Differenzierung von Nomina in zwei Subklassen: Nomina, die als Massennomina bezeichnet werden, und Nomina, die zählbare Einheiten denotieren, und zumeist als mass nouns und count nouns bezeichnet werden. Die spanischen Termini sind nombre continuo und nombre discontinuo (vgl. Alcaraz Varó / Martínez Linares 1997: s.v. nombre und Martínez 1989: 19ff.). Diese Differenzierung hat syntaktische Auswirkungen. Da Massennomina nicht immer Massen oder Substanzen bezeichnen, sondern eher eine nicht-zählbare Einheit, führt sie die Begriffe „transnumeral“ vs. „numeral“ ein. Dabei wird nach der Numerusrealisierung unterschieden, welcher Klasse ein Nomen angehören soll. Die Definition von Wiese lautet: 
„Ein Nomen gelte als numeral genau dann, wenn es obligatorisch in seiner Pluralform auftritt, sobald es mehr als eine Realisierung des betreffenden Begriffs denotiert. Es sei als transnumeral gekennzeichnet, wenn der Unterschied 'Einheit vs. Vielheit' nicht obligatorisch markiert ist.

Transnumerale Nomina werden als $[+\mathrm{tn}]$, numerale als $[-\mathrm{tn}]$ markiert.“

Somit umfassen transnumerale Nomina die eigentliche Gruppe der Massennomina wie agua, hierro, cemento usw. sowie Abstrakta wie amor oder amistad. Wiese unterscheidet noch transnumerale Stoffnomina wie Wasser und Eisen und nichtsubstanzbezogene Nomina wie Vieh und Obst, die als Sammelbezeichnungen ohne Pluralbildung gelten können, und führt die Unterscheidung [+ mn] für die Stoffnomina und $[-\mathrm{mn}]$ für die Sammelbezeichnungen ein (mn für mass noun). Inwiefern diese Differenzierung für das Spanische gilt, müßte detaillierter untersucht werden, da sich Wieses Beispiele nicht zweifelsfrei übertragen lassen: Die direkte Übersetzung von Obst ist frutos (Plural!), und ob ganado die korrekte Übersetzung für Vieh ist (und nicht auch im Sinne von Herde gebraucht wird), scheint dialektal zu variieren (vgl. das Stichwort ganado bei RAE 1992). Ein von Wiese nicht explizit postulierter, aber inhärenter Charakter des Merkmals [+ $\mathrm{tn}]$ ist logischerweise, daß die mit diesem Merkmal markierten Nomina nie oder nur unter bestimmten Bedingungen im Plural erscheinen.

Wir bekommen auf diese Weise drei Klassen, wobei die zweite als Zwischenkategorie für das Spanische in Zweifel gezogen wurde:

1. [- $\mathrm{tn}],[-\mathrm{mn}]$ für Nomina wie Baum, árbol, libro, cama;

2. [+ $\mathrm{tn}],[-\mathrm{mn}]$ für Nomina wie Vieh, Obst, ganado;

3. [+ $[\mathrm{n}],[+\mathrm{mn}]$ für Nomina wie Wasser, agua, pan, queso, cemento.

Da Nomina grundsätzlich noch nicht dahingehend lexikalisch festgelegt sind, ob sie sich transnumeral oder numeral verhalten, bleibt zu überlegen, ob es sich nicht um ein phrasales Merkmal auf NP-Ebene handelt. Nach Wiese gibt es lediglich eine lexikalische Tendenz zur Transnumeralität oder Numeralität. Tendenziell transnumerale Nomina werden als $\mathrm{N}_{\text {tn }}$, tendenziell numerale als $\mathrm{N}_{\mathrm{n}}$ notiert.

Es gilt nun, die Eigenschaften transnumeraler und numeraler Nomina festzuhalten. Dabei spielen Artikel und Numerusmarker eine wichtige Rolle: Wiese bezeich- 
net sie als num-Einheiten. Da sie eher an einer semantischen Klassifikation interessiert ist, vernachlässigt sie den definiten Artikel mit seiner Referenzeigenschaft (vgl. dazu auch Lavric 2000). Wir können diesen Punkt hier zunächst zurückstellen und sprechen vom Artikel im allgemeinen sowie dem Numerusmarker. Da im Spanischen der Singular die Grundform (coche) darstellt und der Plural gesondert markiert ist $($ coches $=$ coche $+s)$, bedeutet die Einsetzung von num ohne Artikel automatisch die morphologische Pluralmarkierung. Wenn von der Grundform die Rede ist, verweist dies im folgenden auf den Singular als Wert für Numerus. Zur Vereinfachung kann von Artikelgebrauch und Pluralmarkierung statt der abstrakten num-Einheit gesprochen werden.

Als Beispiel für die Notwendigkeit der Pluralmarkierung zur Bildung einer NP mit alleinigem Substantiv betrachten wir folgende Sätze:
(a) Es un árbol. (RA-1)
$\left(\mathrm{N}_{\mathrm{n}}\right.$ mit Artikel)
(b) Un perro mata a un niño. (M-5)
$\left(\mathrm{N}_{\mathrm{n}}\right.$ mit Artikel)
(c) No hay árboles. (M-1)
$\left(\mathrm{N}_{\mathrm{n}}\right.$ mit Pluralmarkierung)
(d) Tengo amigos. (M-1)
$\left(\mathrm{N}_{\mathrm{n}}\right.$ mit Pluralmarkierung)
(e) *Es árbol.
(bloßes $\mathrm{N}_{n}$ )
(f) *Perro mata a un niño.
(bloßes $\mathrm{N}_{\mathrm{n}}$ )
(g) Hice subir agua del pozo. (M-3)
(bloßes $\mathrm{N}_{\mathrm{tn}}$ )
(h) Soldados yugoslavos beben cerveza. (P-4) (bloßes $\mathrm{N}_{\mathrm{tn}}$ )
(i) Los embarques de hierro han posibilitado la (bloßes $\mathrm{N}_{\mathrm{tn}}$ ) vida urbana. (Rch-6)

Während transnumerale Nomina allein eine eigenständige NP bilden können und oft keine Pluralmarkierung besitzen dürfen, ist die Pluralbildung bei numeralen Nomina ohne Artikel zumeist nötig. Welche Einschränkungen unter welchen Bedingungen bei numeralen Nomina im Plural bestehen, kann hier nicht untersucht werden (vgl. dazu aber die Aufsätze zu semantischen Fragestellungen und den plurales escuetos in Bosque 1996). Nach Wiese scheinen für zahlreiche Sprachen noch zusätzliche Angaben erforderlich zu sein. Sie nimmt an, daß das Phänomen der numeralen Nomina mit Pluralmarkierung ein universelles ist (vgl. Wiese 1997: 152ff.).

In Verbindung mit dem Kopulaverb ser können viele numerale Nomina in ihrer Grundform eine NP bilden. Dabei ist kein Indefinitartikel und keine Pluralmarkierung notwendig (vgl. dazu auch ausführlich Contreras 1996). 

(a) Es agua. (RA-5)
(bloßes $\mathrm{N}_{\mathrm{tn}}$ )
(b) Soy profesora de física. (S-3)
(bloßes $\mathrm{N}_{\mathrm{n}}$ )

Aber die Pluralmarkierung und der unbestimmte Artikel können auch bei $\mathrm{N}_{\mathrm{n}}$ stehen:
(a) Somos actores. (M-3)
$\left(\mathrm{N}_{\mathrm{n}}\right.$ mit Pluralmarkierung)
(b) Ella es la fuente. (Rch-6)
$\left(\mathrm{N}_{\mathrm{n}}\right.$ mit Artikel)

Diese Beobachtung wird durch Zähl- und Maßkonstruktionen im Deutschen gestützt. Numerale Nomina tendieren im Deutschen dazu, eine Zählkonstruktion einfach mit dem Zahlwort und Plural zu bilden, eine Maßkonstruktion mit einer Maßeinheit und Plural (vgl. zwei Bäume, drei Personen; zwei Kilo Äpfel, drei Tonnen Bücher). Transnumerale Nomina brauchen im Deutschen eine Mengenangabe, um in Zählkonstruktionen auftreten zu können (sechs Stück Vieh) und verändern in Maßkonstruktionen nicht den Numerus (zwei Kilo Gold). Im Spanischen können transnumerale Nomina nicht in Zählkonstruktionen erscheinen, während dies für numerale Nomina möglich ist:
(a) *tres aguas
(b) *cuatro hierros
(c) cinco días
(d) seis páginas

Maßkonstruktionen werden grundsätzlich über die Präposition de gebildet. Wir haben es nicht mehr mit einer Nominalphrase, sondern mit einer nominalen Konstruktion aus Kopf und Adjunkt zu tun:

(a) selección de paquetes de software (Rch-2)

(b) dos kilos de carne (M-7)

Die bisher betrachteten Beispiele lassen darauf schließen, daß ein frei stehendes Nomen im Singular ohne Determinierer nur dann eine NP bildet, wenn es als transnumeral eingestuft werden kann oder in einer Kopulakonstruktion vorkommt. Die Deskription von Nomina als transnumeral muß aber insofern eingeschränkt werden, als Nomina lediglich eine lexikalische Tendenz zur (Trans)numeralität besitzen. Sie können auf phrasaler Ebene in die konträre Form umgewandelt werden. Wiese (1997: 157) nennt diese Übergänge „Schleifer-, Sortierer- und Packungsnumerus“. 
Die Prozedur des „Sortierers“ bzw. „Packers“ (auch „Universal Sorter / Packer“, Wiese 1997: 159) überführt ein transnumerales Nomen in ein numerales, d.h. von [+ tn] nach [- tn]. Die Prozedur des „Schleifers“ (oder auch des „Fleischwolfs“, sofern man den Ausdruck „Universal Grinder“ wörtlich nimmt) überführt ein numerales Nomen in ein transnumerales, d.h. von $[-\mathrm{tn}]$ nach [+ tn] (vgl. für einen weniger systematischen, aber identischen Ansatz Martínez 1989: 20).

Folgende Sätze sind Beispiele für den Sortierer / Packer (vgl. Wiese 1997: 159):

(13) Sortierer / Packer: $[+\mathrm{tn}] \rightarrow[-\mathrm{tn}]$

(a-Beispiele: Substanz; b-Beispiele: Sorten / Portionen)

(1a) Solo un 5\% son de vino espumoso. (M-2)

(1b) Le gusta cocinar con un buen vino argentino o chileno. (S-15)

(2a) Solo había bebido cerveza. (S-6)

(2b) Es una cerveza bávara. (RA-1)

(3a) Tiene incluso una marca propia de café y otra de té. (M-14)

(3b) Así no llevaría yo nueve cafés. (M-15)

(4a) El dilema de hierro para America del Sur es éste. (RA-5)

(4b) Se podían ver los hierros que formaban las tiendas. (S-13)

Die Sätze in (a) enthalten ein Massennomen, das eine Substanz kennzeichnet. Diese Substanz kann wie in (1b) oder (2b) in Sorten unterteilt werden oder wie in (3b) oder (4b) in Portionen (Wiese unterscheidet Sortierer und Packer nochmals explizit). Beispiel (3b) wird im Alltag oft über eine Maßkonstruktion vermittelt: Así no llevaría yo nueve tazas de café.

Der Übergang des Schleifens, von einem numeralen $\mathrm{zu}$ einem transnumeralen Nomen, kann ein Objekt in eine Substanz umwandeln:

(14) Schleifer: $[-\mathrm{tn}] \rightarrow[+\mathrm{tn}]$

(a-Beispiele: Objekte; b-Beispiele: Substanz)

(1a) Un perro mata a un niño. (M-5)

(1b) Huele a perro. (RA-3)

(2a) Ein Huhn fliegt über das Haus.

(2b) Der Salat enthält Huhn. 
Es ist kein Zufall, daß es sich bei den Beispielen oft um Nahrungsmittel handelt. Hier werden Substanzen in bestimmten Mengen zur Speisezubereitung benötigt. Im Spanischen lassen sich schlechter Beispiele als im Deutschen finden, da mitunter ein neues Lexem für die aus dem lebenden Tier zubereitete Speise verwandt wird: pez 'vertebrado acuático, de respiración branquial' vs. pescado 'pez comestible sacado del agua' (vgl. RAE 1992: s.v. pez und pescado). Wenn dies nicht der Fall ist (z.B. bei pollo), wird unter der eßbaren Speise auch nur der eßbare Teil eines Tieres verstanden.

Für das Spanische kann man nun folgende Phänomene beobachten, die wir noch nicht erfaßt haben (Bsp. aus de Bruyne 1993: 60):
(a) Para ti hay / tenemos cama.
(b) Llevaban pantalón corto.
(c) Portaban traje de gala.
(d) ¿Tienes revólver?

Hier bildet ein numerales Nomen im Singular eine NP. De Bruyne verweist explizit darauf, daß es sich jeweils nur um eine einzige Entität handeln kann, die man nur einmal besitzt oder nur einmal braucht (vgl. auch Martínez 1989: 21). Dabei spielt auch das Verb eine Rolle: haber, tener und ser werden bevorzugt (de Bruyne 1993: 60; möglich sind auch portar, llevar u.a.). Diese Konstruktionen können ebenfalls unter der Übergangsprozedur des Schleifens erfaßt werden, indem wir die neue Kategorie um Klassen erweitern. In (15) werden Klassen der genannten Objekte angesprochen, so z.B. in (15a) irgendeine beliebige Art von 'Bett'. Es ist lediglich klar, daß es sich um ein Bett handelt. Ähnliches kann für 'Hose', 'Anzug' und 'Revolver' gesagt werden. Wir können unsere Beispielsätze für die Operation des Schleifens nunmehr erweitern und benennen die Operation zum besseren Verständnis in „Generalisierer" um:

(16) Generalisierer: $[-\mathrm{tn}] \rightarrow[+\mathrm{tn}]$

(a-Beispiele: Objekte; b-Beispiele: Substanz oder Klasse)

(1a) Un perro mata a un niño. (M-5)

(1b) Huele a perro. (RA-3)

(2a) Preferimos esperar por una cama mullida y caliente. (S-10)

(2b) Por fin hubo cama. (S-10) 
(3a) Tenían ahorros para comprar un revólver. (RA-3)

(3b) La puerta se cerró de golpe y sacó revólver. (Rch-7)

(4a) Todos los pescadores volvieron, menos él. (RA-3)

(4b) Ella me da así la posibilidad de hacer hoy esto, mañana aquello, cazar por la mañana, pescar por la tarde, cuidar el ganado al anochecer, hacer crítica después de cenar según me plazca, sin transformarme nunca en cazador, pescador, pastor o crítico. (RA-2)

Wir hatten bemerkt, daß Nomina dann allein eine NP bilden können, wenn sie transnumeral sind oder in Kopulakonstruktionen auftreten. Dies kann nun erweitert werden um die Klasse der generalisierten Nomina, also der Nomina, die durch die Operation der Generalisierung zu transnumeralen Nomina wurden. Wichtig ist dabei festzuhalten, daß diese Eigenschaften optional sind. Determinierer oder Pluralmarkierung sind trotz einer evtl. Generalisierung möglich:

(17) (a) Somos unos actores. $\left(\mathrm{N}_{\mathrm{n}}\right.$ mit Pluralmarkg. und Artikel, vgl. (10a))

(b) Ella es la fuente. $\left(\mathrm{N}_{\mathrm{n}}\right.$ mit Artikel, vgl. (10b))

(c) Huele a perros. $\left(\mathrm{N}_{\mathrm{n}}\right.$ mit Pluralmarkierung, vgl. (16) (1b))

(d) Para ti hay una cama. $\left(\mathrm{N}_{\mathrm{n}}\right.$ mit Artikel, vgl. (16) (2b))

(e) Solo un 5\% son de un vino espumoso. $\left(\mathrm{N}_{\mathrm{n}}\right.$ mit Artikel, vgl. (13) (1a))

(f) Se podían ver los hierros $\quad\left(\mathrm{N}_{\mathrm{n}}\right.$ mit Pluralmarkg. und Artikel, vgl. que formaban las tiendas. (13) (4b))

Im Anschluß stellt sich allerdings die Frage, ob nun die Eigenschaft der Transnumeralität des Nomens erhalten bleibt. Die Antwort ist ganz klar negativ. In (17e) und (17f) handelt es sich um numerale Nomina, die aus tendenziell eher transnumeralen Nomina abgeleitet wurden. Wir können somit sagen, daß folgende Bedingungen gelten müssen, wenn ein einzelnes Nomen eine NP bilden kann:

\section{(18) Das Nomen}

1. steht in einer Kopulakonstruktion oder

2. ist ein tendenziell transnumerales Nomen oder

3. ist tendenziell numeral und mit Pluralmarkierung versehen oder

4. ist ein tendenziell numerales Nomen, das durch Generalisierung transnumeral wurde. 
Es bleibt zu fragen, ob diese Bedingungen wirklich grundsätzlich für das Spanische gelten. Nach dem bisherigen Erkenntnisstand können wir dies annehmen. In einer detaillierten Untersuchung zur Semantik des Nomens müßte noch zu zeigen sein, inwieweit diese Annahme universell gültig ist und unter welchen Bedingungen Ausnahmen erfolgen können. Zudem bliebe zu untersuchen, ob die Differenzierung [ $\pm \mathrm{tn}]$ und vielleicht noch $[ \pm \mathrm{mn}]$ für eine Klassifikation ausreichend ist.

Nach Iturrioz (1996) sind in einer umfassenden Untersuchung fast alle linguistischen Disziplinen gefordert. Dabei müßten besonders Idiomatisierungen und das verbale Umfeld berücksichtigt werden, um die kontextuellen Bedingungen für eigenständige Nomina zu ergründen. Dies konnte hier nicht geleistet werden. Allerdings lassen sich Ausnahmen von den hier aufgestellten Regeln zumeist als Idiomatisierungen erklären.

\subsection{Integration in eine HPSG-basierte Deskription}

An dieser Stelle soll ein Vorschlag zur Deskription von spanischen Nomina in der HPSG erfolgen. Es wurde zwar mit einigen Einschränkungen von (trans)numeralen Nomina gesprochen, der entsprechende Wert kann aber nur auf der NP-Ebene, der gesättigten phrasalen Ebene zugesprochen werden. Es wird somit noch zu fragen sein, von wo die entsprechende Information für (Trans)numeralität oder expliziter Einzelstellung des Nomens resultiert.

Folgende Bedingungen hatten wir als charakteristisch für das Auftreten von Nominalphrasen festgehalten, die lediglich aus einem Nomen bestehen:

- das finite Bezugsverb der NP ist die Kopula ser,

- das Nomen ist transnumeral (oder durch Generalisierung transnumeral geworden),

- das Nomen ist durch den Numeruswert plural markiert.

In einer formalen Beschreibung können die Bedingungen disjunktiv postuliert werden. Zunächst nehmen wir im semantischen Index einer Nominalphrase das Merkmal Transnumeralität (TN) an: 


$$
\left[\operatorname{SYNSEM}\left[\begin{array}{l}
\text { CAT } \mid \text { HEAD nomen } \\
\text { CONT } \mid \text { INDEX }[\mathrm{TN} \pm]
\end{array}\right]\right.
$$

Folglich kann aus Matrix (19) $\mathrm{NP}_{\mathrm{n}}$ als [SYNSEM | CONT | INDEX [ TN - ] ] definiert werden.

Für das Auftreten der Pluralmarkierung kann folgender Eintrag gemacht werden, der mit dem Merkmal [TN -] einhergeht:

$$
\left[\operatorname{SYNSEM}\left[\begin{array}{l}
\text { CAT } \mid \text { HEAD } \mid \text { AGR [NUM } p l] \\
\text { CONT } \mid \text { INDEX [TN - }]
\end{array}\right]\right]
$$

Als drittes kann die Komplementliste des Verbs ser den Eintrag NP erhalten. Damit sind explizit alle Typen von Nominalphrasen gemeint, auch $\mathrm{NP}_{\mathrm{n}}$.

$$
\left[\operatorname{SYNSEM}|\mathrm{CAT}| \mathrm{VAL}\left[\begin{array}{l}
\mathrm{SUBJ}\langle\rangle \\
\operatorname{COMP}\langle\mathrm{NP}\rangle
\end{array}\right]\right]
$$

Bisher offen blieb an dieser Stelle der Analyse der Aufbau der Nominalphrase. Wie gestalten sich die Ebenen N' und N, und welche Merkmale tragen sie? Die Frage des Kopfes - bisher das Nomen - wird ebenfalls noch zu diskutieren sein, da einige wichtige Untersuchungen von Nominalphrasen den Determinierer als Kopf ansehen (vgl. z.B. Abney 1987, Longobardi 1994, Lois 1996).

\subsection{Das Pronomen}

Der Terminus Pronomen steht ähnlich wie Adjektiv oft als Oberbegriff für eine Reihe von Wortarten. Neben den Personal-, Demonstrativ- und Possessivpronomina werden auch Indefinit- und Relativpronomina dazu gezählt. Zumeist wird dann zwischen adjektivischem und pronominalem Gebrauch unterschieden (vgl. Reumuth / Winkelmann 1993: 61ff.). Es steht außer Frage, daß die syntaktische wie semantische Verwendung dieser sogenannten Pronomina divergiert. 
Auch wenn in manchen Bezeichnungen weiterhin der Begriff Pronomen auftreten wird, soll hier unter dem Oberbegriff Pronomen diejenige Wortart verstanden werden, welche grundsätzlich eigenständig eine Nominalphrase bildet. Dazu gehören nur die Personalpronomina, welche je nach Kasus eine besondere Form bilden: yo, tú, ella vs. mí, ti, ella oder sí. Zudem muß zwischen betonter und unbetonter Form unterschieden werden: betonte Pronomina wie mí, tí oder ella und unbetonte Pronomina wie me, te, le oder la. Für letztere gilt, daß sie klitisch gebraucht werden (vgl. die umfangreiche Literatur zur Deskription von Klitika in der HPSG: z.B. Miller 1992, Monachesi 1995, allgemeiner Van Eynde 1998).

Pronomina können syntaktisch analog zu den Nomina beschrieben werden mit dem Unterschied, daß sie Kasus realisieren und weder Komplemente subkategorisieren noch Adjunkte tolerieren. Pragmatisch erstellen sie eine Referenz auf etwas bereits Erwähntes oder Bekanntes, das im Merkmal CONTEXT abgebildet wird. Eine HPSG-Deskription kann folgendermaßen aussehen (vgl. Pollard / Sag 1994: 52ff.):

$$
\left[\begin{array}{l}
\operatorname{SS} \mid \text { LOC }\left[\begin{array}{l}
\text { CAT } \left.\left[\begin{array}{l}
\text { pron } \\
\text { HEAD }\left[\text { AGR }\left[\begin{array}{ll}
\text { PER } 1 \\
\text { NUM } s g \\
\text { KAS } & N O M
\end{array}\right]\right.
\end{array}\right]\right] \\
\text { CONTENT [] } \\
\text { CONTEXT | BACKGROUND [1] }
\end{array}\right]
\end{array}\right.
$$

Neben den Merkmalen für Kongruenz, zeichnet das Pronomen yo noch eine semantische Relation aus, die nicht näher spezifiziert werden kann. Das Pronomen ist anaphorisch, d.h. es bezieht sich auf eine vom Kontext vorgegebene Einheit. Somit ist die semantische Struktur von yo in (22) völlig offen. Lediglich im pragmatischen Attribut CONTEXT kann eine Referenz auf eine noch näher zu bestimmende Einheit [1] angegeben werden, die sich auf den Sprecher bezieht

Pronomina als Pro-Formen für die Nominalphrase stehen in dieser Arbeit nicht im Vordergrund. Wir werden im Kap. III.3.3. im Zusammenhang mit der Einführung einer Sortenhierarchie für Wortarten nochmals auf sie eingehen. 


\section{Determinierer}

„Artikel [lat. articulus „Gelenk“; engl. determiner. - Auch: Begleiter, Determinans, Determinator, Geschlechtwort]. In der traditionellen, teilweise auch noch in der strukturellen Grammatik angenommene Wortklasse mit zwei Elementen: „bestimmter“ Artikel (der) und „unbestimmter“ Artikel (ein). Diese werden heute zu den Determinantien (bzw. im Fall von ein auch zu den Quantoren) gezählt“ (Bußmann 1990: s.v. Artikel, vgl. auch Alcaraz Varó / Martínez Linares 1997: s.v. determinante).

Diese Definition von Bußmann zeigt bereits, daß es eine Reihe von verschiedenen Ansätzen und Ideen gibt, wie die „Begleiter“ von Nomina einzuordnen sind. Wir werden in dieser Arbeit den Terminus Determinierer ganz allgemein für diese sogenannten Begleiter verwenden. Dabei wird sich sehr rasch herausstellen, daß im Spanischen die Abgrenzung von Determinierern und Adjektiven problematisch ist. Es gilt zu eruieren, welche Klassifikationskriterien für eine eindeutige Differenzierung dieser beiden Wortklassen herangezogen werden können (vgl. auch Kolde 1996: 28ff.).

Auch die Gruppe ${ }^{19}$ der Determinierer selbst erweist sich als überaus heterogen. Sie besteht laut Bußmann im Deutschen aus den Artikeln, Demonstrativpronomina und anderen Pronomina. Gleichfalls zu nennen sind die sogenannten Quantoren, ein nach Bußmann ,,aus der Prädikatenlogik übernommener Terminus zur Bezeichnung von Operatoren, die der Spezifizierung bzw. Quantifizierung von Mengen dienen und alltagssprachlich durch unbestimmte Adjektive / Pronomina (alle, manche, einige u.a.), Numeralia (ein(e), zwei, drei), den definiten Artikel (die Bücher sind kostbar) oder unbestimmte Pluralität (Bücher sind teuer) ausgedrückt werden“ (Bußmann 1990: s.v. Quantor, vgl. auch Alcaraz Varó / Martínez Linares 1997: s.v. determinante und Longobardi 1988 für das Italienische). Die hier beschriebene Gruppe der Quantifizierer wird semantisch zudem sehr vage definiert und erweist sich als überaus heterogen. Sie überschneidet sich außerdem mit den Determinierern und enthält auch traditionell als Adjektive bezeichnete Wörter. Innerhalb der Gruppe der

\footnotetext{
${ }^{19}$ Wir verwenden hier den Begriff Gruppe für eine lose Ansammlung von in irgendeiner Form ähnlich markierten Wörtern. Demgegenüber soll der Begriff Klasse eine Menge an Wörtern bezeichnen, die nach einem zuvor definierten Schema oder Kriterium zusammengestellt worden sind.
} 
Pronomina befindet sich neben den Demonstrativ- und Possessivpronomina mit den Indefinitpronomina (etliche, manche, alle, u.a.) erneut eine Gruppe, die als sehr heterogen anzusehen ist und sich mit Quantoren und Numeralia überschneidet.

Folgende Gruppen werden in allen konsultierten Grammatiken einheitlich erfaßt:

- Artikel: bestimmter (el, la, los, las), unbestimmter (uno/a),

- Demonstrativpronomina: este/a/o(s), ese/a/o(s), aquello/a(s),

- Possessivpronomina: $\quad m i(s), \operatorname{tu}(s), s u(s)$, vuestro/a $(s)$, nuestro/a(s),

- Numeralia: (a) Kardinalia: uno, dos, tres...

(b) Ordinalia: $\quad$ primero/a $(s)$, segundo/a(s), tercero/a $(s) \ldots$

(c) Multiplikativa: doble(s), triple(s) ...

Folgende Gruppen sind aufgrund ihrer Heterogenität problematisch (vgl. Rodríguez Díez 1992a: 232) und werden in den konsultierten Grammatiken uneinheitlich erfaßt:

- Quantoren: Sie umfassen Wörter, die als Adjektive oder Pronomina gesehen werden (muchos, varios), sowie den bestimmten Artikel, Numeralia und Konstruktionen, die in irgendeiner Weise Mengen bezeichnen (z.B. unbestimmte Pluralität: libros, oder bestimmte: dos kilos de manzanas);

- Indefinitpronomina: Die den Quantoren zugeordneten Adjektive oder Pronomina sowie die Numeralia werden oft auch als Indefinitpronomina bezeichnet.

Allegranza (1998: 61f.) erstellt in Anlehnung an Quirk u.a. (1985) für das Italienische sechs Gruppen (vgl. auch die sehr ähnliche Einteilung bei Hernández Alonso 1984: 445 sowie Grevisse 1993: 858f. und Jones 1996: 208-241 für das Französische und Renzi 1988b: 357ff. für das Italienische):

$$
\begin{aligned}
& \text { - Artikel (il, uno, } \left.\mathrm{del}^{20}\right) \\
& \text { - Demonstrativa (questo, quelle, codesto) } \\
& \text { - Possessiva (mio, tuo, suo, nostro, vostro, loro) } \\
& \text { - q-Determinierer (ogni, qualche, entrambi, molti u.a.) } \\
& \text { - Kardinalia (uno, due, tre,...) } \\
& \text { - Ordinalia (primo, secondo, terzo, prossimo, ultimo u.a.) }
\end{aligned}
$$

\footnotetext{
${ }^{20}$ Partitive Konstruktionen mit der Präposition de werden in dieser Arbeit nicht berücksichtigt.
} 
Allegranza faßt die heterogene Gruppe der Quantoren und Indefinitpronomina als q-Determinierer zusammen. Diesen Ansatz können wir fürs erste übernehmen. Die Multiplikativa verhalten sich analog zu den Ordinalia und brauchen von daher nicht explizit berücksichtigt zu werden. Mit prossimo und ultimo zählt Allegranza zwei Wörter zu den Ordinalia, die in der Regel als Adjektive bezeichnet werden.

In vielen spanischen Grammatiken werden Determinierer gar nicht als Wortklasse aufgefaßt, vielmehr stellen sie eine Subklasse der Adjektive dar (vgl. auch Rodríguez Díez 1992a und b sowie Alcaraz Varó / Martínez Linares 1997: s.v. adjetivo; folgende Unterteilung nach Alarcos Llorach 1994: 82):

$$
\begin{aligned}
& \text { I adjetivos determinativos (= Determinierer) } \\
& \text { II adjetivos cualificativos (= Adjektive) }
\end{aligned}
$$

So faßt auch Seco (1991: 89ff.) Artikel und Adjektive als Adjunkte des Nomens in einem Kapitel zusammen. Dabei versteht er unter artículo lediglich den bestimmten und unbestimmten Artikel, alle anderen Determinierer werden als Adjektive bezeichnet. Diese Sichtweise hat ihre Berechtigung, wie sich zeigen wird. Fast alle Determinierer können in einer Weise gebraucht werden, die der Verwendung von Adjektiven ähnelt. Allerdings sind Determinierer zumeist obligatorische Begleiter von Nomina, während Adjektive sich optional einem Nomen anschließen. Von daher bilden Determinierer notwendige und subkategorisierte Elemente der Nominalphrase, während Adjektive als Adjunkte und somit als fakultative Elemente eingeordnet werden. Wenn nun nach Seco alle Determinierer ebenfalls als adjungierend und adjektivisch bezeichnet werden müßten, hätte das nicht unerhebliche Folgen für die strukturelle Deskription der Nominalphrase. Von daher erscheint eine klare Trennung dieser Wortarten notwendig und sinnvoll.

Ein erster Zweifel an der Differenzierung von Seco ergibt sich bereits daraus, daß Seco den Artikel nicht zu den determinierenden Adjektiven zählt. Demonstrativ- und Possessivpronomina beinhalten die Funktion des Artikels und gehen semantisch darüber hinaus, d.h. sie fügen zum definiten einen deiktischen oder possessiven Gehalt hinzu, und sind deshalb den Artikeln so ähnlich, daß sie nicht zugleich mit diesen in einer Nominalphrase erscheinen können. Wenn wir einen idealisierten Vertreter der Wortart Determinierer einführen, stellt sich die Frage, ob diese Pronomina nicht dem Vertreter näher stehen, als es bei den Artikeln der Fall ist. Eine Folge davon wäre, daß Demonstrativ- und Possessivpronomina das Gegengewicht zu den qualifizieren- 
den Adjektiven im Sinne Secos darstellen und nicht der definite Artikel. Sie können zudem betont und substantiviert werden, was bei den klitischen Artikeln nicht möglich ist. Alvarez Martínez (1986: 200) begründet ihre Entscheidung, den Artikel nicht zu den sonstigen Determinierern zu rechnen, mit morphologischen Argumenten. Als unbetonte Form müsse er als nominales Morphem klassifiziert werden. Dieser Argumentation können wir zum einen aufgrund unserer Definition des Wortes - der Artikel ist folglich ein Wort - zum anderen aufgrund seines syntaktischen Verhaltens, z.B. als Substantivierer, nicht folgen.

Im ersten syntaktischen Teil seiner Grammatik differenziert Matte Bon (1992: I, 199-240) zwischen (un)bestimmten Artikeln, Demonstrativ- und Possessivpronomina. Die Indefinitpronomina oder auch Quantoren werden nur im zweiten Teil der Grammatik behandelt, der eher von semantischen Fragestellungen ausgeht. Matte Bon (1992: II, 1-40) ordnet nach den Klassen von Nomina, die ein Determinierer in der Regel beschreibt (vgl. auch die sehr ähnliche Einteilung für das Italienische bei Schwarze 1988: 285ff.):

Individuos (Individuen): fulano, la gente, alguien, cada, lo que, otro ... Cuantidades (Mengen): $\quad$ mucho, bastante, todo, más ... de / que, ... Numerales (Numeralia): dos, segundo, doble ... ${ }^{21}$

Der Versuch, syntaktische Konstruktionen über die Bedeutung zu erfassen, ist in dieser Form relativ ungenau. Sprachliche Zeichen sind oft semantisch unterspezifiziert, und erst durch den situativen Kontext kann Eindeutigkeit oder gegebenenfalls auch Ambiguität entstehen. Es wird somit Weltwissen zum Verständnis notwendig. Wir gewinnen keine zusätzliche Information für eine syntaktische Analyse.

Ziel dieses Kapitels ist eine Klassifikation von Adjektiven und Determinierern. Zunächst erläutern und diskutieren wir den Ansatz von Alvarez Martínez (III.2.1.), um dann Klassifikationskriterien zu erstellen (III.2.2.). Dabei erweisen sich die Bereiche Kookkurrenz (III.2.3.) und Semantik (III.2.4.) als besonders komplex (die Zusammenhänge zwischen beiden in III.2.5.). Da sich die Abgrenzung in einzelne Klassen als zu idealisierend erweist, erstellen wir eine Abstufung, die in einem Dendrogramm graphisch dargestellt werden kann (III.2.6.). Die Ergebnisse werden

\footnotetext{
${ }^{21}$ Aufgrund des häufigen adverbialen Gebrauchs von primero und der Homonymie von uno als Artikel und Zahlwort verwenden wir das Zahlwort zwei (dos, doble, segundo) als Repräsentant der Numeralia.
} 
am Schluß analysiert (III.2.7.). Ein Exkurs wendet das Klassifikationsverfahren auf das Deutsche an, um die Besonderheiten der spanischen Sprache herauszustellen (III.2.8.).

\subsection{Klassifikation nach Alvarez Martínez (1986)}

Alvarez Martínez differenziert die Determinierer nach folgenden Kriterien (für das folgende Alvarez Martínez 1986, bes. 194ff.):

1. morphologisch: Genus / Numerus / Komparation,

2. syntaktisch: Nukleus vs. Satellit

(im Sinne der Dependenzgrammatik),

Substantivierbarkeit,

Substantivierer von Adjektiven,

Adverbialer Gebrauch,

3. distributionell: Voran- vs. Nachstellung,

4. lexikalisch: paradigma limitado und

syntagma,

5. semantisch: valor artículo,

6. kombinatorisch: mögliche Kookkurrenz.

Alvarez Martínez untersucht die morphologischen Merkmale Genus und Numerus: Differenziert der Determinierer zwischen den möglichen Genera oder ist seine Form unveränderlich (z.B. todo/a vs. cada)? Differenziert der Determinierer zwischen Singular und Plural oder ist nur eine Form möglich (z.B. todo(s) vs. cada)? Die Möglichkeit der Komparation und des adverbialen Gebrauchs sind traditionellerweise Eigenschaften von Adjektiven. Beides kommt auch bei den Determinierern vor und muß deshalb berücksichtigt werden.

Im Bereich der Syntax muß die Frage gestellt werden, ob ein Determinierer auch allein eine Nominalphrase bilden kann oder ob er nur als „Begleiter“ operiert. Alvarez Martínez stellt diese Frage im Sinne der Dependenzgrammatik und bezeichnet den Kern einer Phrase als Nukleus - wir können hier vom „Kopf“ sprechen -, den Begleiter als Satellit - wir sprechen von „Spezifizierern“(vgl. Weber 1992: 29ff.). 
Als weitere Möglichkeit erkennt sie den Einsatz von Determinierern als Substantivierern von Adjektiven: Es gibt eine Reihe von Determinierern, die äquivalent zum Artikel Adjektive zu Nomina transponieren können. Dies muß ebenfalls erfaßt werden.

Das distributionelle Kriterium erscheint als ein Merkmal von größter Relevanz. Einige Determinierer können sowohl vor als auch nach dem Nomen stehen, ein typisches Merkmal spanischer Adjektive.

Bei den lexikalischen Merkmalen wird Alvarez Martínez unklar (Nr. 4 in 29). Alle Determinierer werden als ein paradigma limitado eingestuft und gehören so einer geschlossenen Klasse von Wörtern an. Da dies aber für die gesamte Wortart Determinierer angenommen wird, findet keine Differenzierung statt und das Kriterium ist hinfällig. Das zweite Kriterium syntagma wird parallel zur reinen Begleiterfunktion gesehen - mit Ausnahme von mi / tu / su, die in Beziehung zu mío / tuyo / suyo gesetzt werden - und ist damit ebenfalls wenig informativ. Wir werden beide Kriterien nicht übernehmen.

Das semantische Kriterium valor artículo bleibt leider ebenfalls unpräzise. Wenn damit eine artikelähnliche Eigenschaft gemeint ist, wie sieht diese aus? Was ist unter dem semantischen Wert eines Artikels zu verstehen in Abgrenzung z.B. zu den Demonstrativa? Die Demonstrativa werden bei Alvarez Martínez (1986: 194ff.) zwar mit dem Merkmal valor artículo markiert und dies auch zu recht, sofern damit eine artikelähnliche deiktische Funktion gemeint ist. Das Merkmal Transpositor de calificativos (hier Substantivierer von Adjektiven genannt), welches nur auf Determinierer zutreffen soll, die nicht den valor artículo kennen, wird aber für die Demonstrativa ebenfalls als zutreffend markiert. Dies ist unlogisch und läßt die Absicht dieses Merkmals nicht mehr erkennen.

Zuletzt muß Kookkurrenz als Kriterium genannt werden. Determinierer können kombiniert werden und sind dabei nur von einigen semantischen und syntaktischen Restriktionen beschränkt. Alvarez Martínez berücksichtigt leider nur die Kombination mit dem Artikel. Es gibt allerdings noch eine Vielzahl anderer noch zu untersuchender Möglichkeiten.

Die Grenze zwischen Determinierern und Adjektiven definiert Alvarez Martínez (1986: 82) anhand folgender Kriterien: 
(30) 1. Determinierer weisen keine Komparation auf.

2. Sie stehen vor dem Nomen. Adjektive können dagegen sowohl vor als auch nach dem Nomen positioniert sein.

3. Determinierer bilden ein festes Inventar im Lexikon, Adjektive eher ein offenes Inventar.

4. Determinierer sind substantivierbar, während Adjektive dies nur mit bestimmten Determinierern vermögen:

(a) Este es un problema muy serio. (A-3)

(b) Este último serviría para que el boss se diera unas carreritas. (A-1)

(c) Si entregas tu alma a alguien procura que sea de confianza. (M-1)

(d) No es el único. (M-8)

Die vier Kriterien gehören dem morphosyntaktischen und distributionellen Bereich an. Für die Differenzierung von Determinierern und Adjektiven ist das dritte Kriterium (offenes / festes Inventar) nicht relevant, während die anderen eine Reihe von Ausnahmen aufweisen. Es ist von daher zu prüfen, inwieweit die Abgrenzungskriterien in dieser Form sinnvoll sind.

Alvarez Martínez (1986: 200) klassifiziert die spanischen Determinierer letztlich nach folgenden Fragestellungen:

1. Muß der Determinierer immer eine „begleitende“ Funktion ausüben? (Sólo adyacente nominal)

2. Kann er als Adverb operieren? (Se adverbializan)

3. Kann er qualifizierende Adjektive substantivieren? (Transpositores de calificativos)

4. Erlaubt er Komparation? (Admiten gradación)

Es entsteht eine Typologie, die den Grenzbereich zu den Adjektiven definiert und hauptsächlich auf morphosyntaktischen Kriterien aufbaut (nach Alvarez Martínez 1986: 202): 


\begin{tabular}{|c|c|c|c|c|c|c|}
\hline \multicolumn{7}{|c|}{ Adjetivos } \\
\hline \multicolumn{6}{|c|}{ Tipo I } & \multirow[t]{2}{*}{ Tipo II } \\
\hline $\mathrm{I}_{1}$ & $\mathrm{I}_{2}$ & $\mathrm{I}_{3}$ & $\mathrm{I}_{4}$ & $\mathrm{I}_{5}$ & $\mathrm{I}_{6}$ & \\
\hline $\begin{array}{l}\text { cada } \\
\text { sendos } \\
\text { mi, tu, su } \\
\text { mero } \\
\text { cierto }\end{array}$ & $\begin{array}{l}\text { tal } \\
\text { varios } \\
\text { ambos } \\
\text { demás }\end{array}$ & \begin{tabular}{|l} 
bastante \\
demasiado \\
tanto \\
mucho \\
poco \\
más \\
menos
\end{tabular} & $\begin{array}{l}\text { este, ese, aquel } \\
\text { alguno } \\
\text { ninguno } \\
\text { cualquiera } \\
\text { uno } \\
\text { otro } \\
\text { todo }\end{array}$ & $\begin{array}{l}\text { uno, dos.. } \\
\text { primero, } \\
\text { segundo... } \\
\text { doble, triple... }\end{array}$ & $\begin{array}{l}\text { mismo } \\
\text { mío, tuyo... }\end{array}$ & calificativos \\
\hline \multirow[t]{2}{*}{$\begin{array}{l}\text { Sólo } \\
\text { adyacente } \\
\text { nominal }\end{array}$} & & $\begin{array}{l}\text { Se adver- } \\
\text { bializan }\end{array}$ & $\begin{array}{l}\text { Transpositores } \\
\text { de calificativos }\end{array}$ & & \multicolumn{2}{|c|}{ Admiten gradación } \\
\hline & \multicolumn{6}{|c|}{ Adyacente nominal o Núcleus nominal } \\
\hline
\end{tabular}

So erhält Alvarez Martínez zwei Extreme: Determinierer, die immer nur begleiten $\left(\mathrm{I}_{1}\right)$, und solche, die graduierbar und somit den qualifizierenden Adjektiven sehr nahestehen $\left(\mathrm{I}_{6}\right)$. Die Klasse $\mathrm{I}_{2}$ zeichnet sich neben dem Merkmal sólo adyacente nominal von $\mathrm{I}_{1}$ auch durch Substantivierung ohne zusätzlichen Determinierer aus. Die Mitglieder der Klasse $\mathrm{I}_{3}$ können als Adverb auftreten. Die Determinierer von $\mathrm{I}_{4}$ substantivieren qualifizierende Adjektive, $\mathrm{I}_{5}$ besteht aus den Numeralia.

Diverse Punkte an dieser Klassifikation sind problematisch. So können poco und mucho $\left(\mathrm{I}_{3}\right)$ ebenfalls als steigerbar (Kriterium von $\mathrm{I}_{6}$ ) eingestuft werden.

(a) poco $\rightarrow$ muy poco, poquísimo

(b) mucho $\rightarrow$ muchísimo

Die Gruppe $I_{5}$ der Numeralia, die sich nur semantisch begründen läßt, ist in sich ebenfalls heterogen, da sich die Kardinalia ohne weiteren Determinierer substantivieren lassen. Die vor das Nomen gestellten Possessiva $m i$ / $t u$ / su und die nachgestellten mío / tuyo / suyo stehen sich in den Extremgruppen gegenüber, obwohl sie semantisch eindeutig zusammengehören. Zudem weisen die Pluralformen vuestro und nuestro zusätzlich eine morphologische Differenzierung des Genus auf und gehören beiden Gruppen an. Diese Besonderheiten einiger Numeralia werden nicht erfaßt (vgl. die sehr ähnliche, intuitive Einteilung bei Rodríguez Díez 1992a). 
Zahlreiche Ausnahmen lassen sich prinzipiell nur bedingt in ein System einordnen, da sie nur unter bestimmten Umständen gelten (vgl. Moliner 1970 zu den jeweiligen Stichwörtern):

\section{Mögliche Nachstellung}

(a) este, ese, aquel können dem Nomen nachgestellt werden. Sie bezeichnen einen Affekt des Sprechers. Es muß ein Artikel vor dem Nomen stehen.

(b) alguno wird bei Negation nachgestellt.

(c) cualquiera wird u.U. nachgestellt, der unbestimmte Artikel muß dann voranstehen.

(d) todo bewirkt bei Nachstellung eine besondere Betonung der entsprechenden Phrase.

(e) mismo wird nur bei Personalpronomen, Adverbien und Eigennamen nachgestellt.

(f) bastante ist nachgestellt ein Synonym von suficiente ('genügend').

Nachstellung kann Emphase, Affekt oder Bedeutungsänderung nach sich ziehen. Dies sind Eigenschaften, die auch Adjektive besitzen können. In (34b) und (34e) müssen besondere lexikalische Eigenschaften des Determinierers zur Erklärung herangezogen werden.

\section{Besonderheiten in Kombination mit dem definiten Artikel}

(a) mucho / poco stehen mit Artikel nur bei folgendem Relativsatz.

(b) demasiado steht in Ausnahmefällen mit Artikel.

(c) Ordinalia werden ohne Artikel adverbial verwendet.

(d) todo steht grundsätzlich vor dem Artikel.

(e) uno steht mit Artikel nur in Korrelation zu otro: El uno tiene como principal medio de acción la libertad; el otro, la servidumbre. (RA-5)

(f) demás steht in Koordination ohne Artikel: $Y$ es que los muñequitos, naves, maquetas y demás plástico basado en las películas de George Lucas dejaron de ser meros juguetes para convertirse en piezas de coleccionista. (M-9)

(g) Kardinalia stehen fakultativ mit Artikel.

Auch hier müssen einige Merkmale als lexikalische Eigenschaften des entsprechenden Determinierers gelten: (35a) bis (35d). Zwei weitere Besonderheiten können als 
Folge von Koordination betrachtet werden: (35e) und (35f). Die Eigenschaft der Kardinalia, sowohl mit als auch ohne Artikel gebräuchlich zu sein (35g), hat semantische Gründe.

\section{Homonymes Adjektiv zum nachgestellten Determinierer}

(a) varios kann im Sinne des Adjektivs variado verwendet werden.

(b) cierto kann im Sinne des Adjektivs seguro verwendet werden.

Diese zwei Beispiele zeigen, daß es auch im semantischen Bereich Überschneidungen und Unklarheiten gibt, die für eine Klassifikation zu berücksichtigen sind. Ähnlich gelagert ist die Frage, wie Definitheit verstanden und von Indefinitheit und Quantifikation abgegrenzt werden kann, welche Kriterien dabei eine Rolle spielen und welche wiederum nicht. Da sich Determinierer nur schwerlich von Adjektiven abgrenzen lassen, erscheint es zudem sinnvoll, typisch adjektivische Merkmale wie prädikativen Gebrauch und Stellung zu berücksichtigen. Ein letztes schwieriges Phänomen für den Aufbau eines Systems stellt die Besonderheit von mero dar, keine Adjektive innerhalb der gleichen NP zu erlauben.

Wir haben es bei den spanischen Determinierern mit einer begrenzten Menge von Wörtern einer Wortklasse zu tun, die sich nicht nur schwer von anderen Wortklassen abgrenzen lassen, sondern auch neben allgemeinen Eigenschaften ganz spezielle aufweisen (vgl. z.B. (34e) und (35b)). Von daher können an dieser Stelle einige Vorgaben für die weitere Arbeitsweise formuliert werden:

- Es ist nicht möglich, eine Klassifikation zu erstellen, die klar voneinander abgrenzbare Klassen erzeugt. Eher muß davon ausgegangen werden, daß dabei relativ unklare und diffuse Gruppierungen entstehen (wie bei Alvarez Martínez), die auf bestimmten Prioritäten bei der Kriterienauswahl beruhen. Ein solches Ergebnis ist nur bedingt brauchbar.

- Somit sollte ein System angestrebt werden, das eher irrelevante Details vernachlässigt und für die spätere HPSG-Deskription relevante Merkmale hervorhebt. Wir bezeichnen dieses idealisierte System als Supersystem. Infolgedessen sind Auswahl und Gewichtung der entsprechenden Kriterien von größter Bedeutung.

- Da die Eigenarten der spanischen Determinierer nur vordergründig durch eine Einteilung in Klassen erfaßt werden, bietet sich für die Differenzierung der Determinierer eine Skala an. Diese Skala kann auf einer Berechnung der Distanz bzw. der Ähnlichkeit der Determinierer untereinander basieren. Ausgangspunkt 
ist dabei eine Berechnung, welche die positiv und negativ spezifizierten Merkmale eines jeden Determinierers mit den Merkmalen aller anderen Determinierer vergleicht und die Distanz oder Ähnlichkeit mathematisch berechnet (vgl. Altmann / Lehfeldt 1973). Ob dabei die Distanz eines jeden Determinierers zu den anderen Determinierern oder die Ähnlichkeit der Determinierer untereinander berechnet wird, ist zweitrangig. Wir sprechen im folgenden nur noch von der Distanz.

- Daraus folgt wiederum, daß die Auswahl der Kriterien für eine Klassifikation von größter Wichtigkeit ist.

Trotz der bisher erarbeiteten Probleme bei der Klassifikation der Determinierer nehmen wir zunächst die Typologie von Alvarez Martínez als Ausgangsposition. Die Klassifikation müßte durch weitere Merkmale wie prädikativer Gebrauch und Stellung ergänzt werden, die bei ihr zwar Erwähnung, aber keine Verwendung finden. Wir erhalten zur Klassifizierung von Determinierern folgende Liste möglicher Kriterien, die noch im Detail besprochen werden. Alvarez Martínez hat aus der Liste von (37) letztlich nur die Kriterien 2, 3, 5 und 6 für ihre Klassifikation benutzt, wobei wir das Merkmal sólo adyacente nominal ihrer Klasse $\mathrm{I}_{1}$ im Kriterium Substantivierbarkeit behandeln.

1. Genus und Numerus

2. Adverbialisierung

3. Komparation

4. Prädikation

5. Substantivierbarkeit

6. Substantivierung von Adjektiven ${ }^{22}$

7. Quantifikation

8. Vor- vs. Nachstellung

9. Kombinationsmöglichkeiten, Kookkurrenz

10. Semantische Aspekte

${ }^{22}$ Bei der Besprechung der Kriterien werden die beiden Substantivierungskriterien gemeinsam behandelt, so daß die Numerierung der Liste ab 6. nicht mehr mit den Kapitelüberschriften übereinstimmt. 


\subsection{Die Klassifikationskriterien}

Folgende Wörter können im Spanischen als Determinierer bezeichnet werden: alguno, ambos, bastante, cada, cierto, cualquiera, demás, demasiado, doble, dos, el / la, este / ese / aquel, más / menos, mero, mi / tu / su / nuestro / vuestro, mío / tuyo / suyo / nuestro / vuestro, mismo, mucho, ninguno, otro, poco, segundo, sendos, tal, tanto, todo, uno, varios.

Dos, segundo und doble stehen im folgenden für die entsprechende Liste der $\mathrm{Nu}-$ meralia. Este steht stellvertretend für alle Demonstrativa (Singular und Plural), mi bzw. mío für die entsprechenden Possessiva, el für die Gruppe der definiten Artikel (el, la, los und las), más für más und menos.

Für eine exakte Deskription dieser Wörter sollen nun die oben genannten Kriterien untersucht und auf ihre Tauglichkeit hin überprüft werden. Ziel ist es, alle Determinierer genau zu beschreiben und anhand einer Distanzmatrix klassifizieren zu können. Eine Distanzmatrix summiert die unterschiedlichen Merkmale eines Determinierers in Relation zu jedem anderen, so daß eine Tabelle entsteht, in der die jeweilige Distanz zwischen zwei Determinierern durch eine reelle Zahl dargestellt wird. In der Folge wird ein Diagramm konstruiert, das die Determinierer stufenweise zueinander in Beziehung setzt. So wird eine Klassenbildung wie bei Alvarez Martínez verhindert, die den Anschein vermittelt, es gebe relativ homogene, voneinander abgrenzbare Klassen. Voraussetzung für eine solche Matrix ist die Berechnung der Distanz anhand klar definierter binärer Merkmale. Diese Merkmale sollen hier zunächst erstellt werden. Wir beginnen mit Merkmalen, die auch Adjektive beschreiben können, und gehen dann später auf mehr determinierertypische Eigenschaften ein.

\subsubsection{Genus und Numerus}

Spanische Nomina kennen zwei morphologische Merkmale, die in der Regel realisiert werden: das Geschlecht (Genus) als weiblich oder männlich und die Anzahl (Numerus) als Singular (ein Exemplar) oder Plural (zwei oder mehrere Exemplare). Unveränderliche Nomina sind selten, aber vorhanden: z.B. las gafas (nur Plural), el martes - los martes (Singular und Plural identisch), el coma ('das Koma') - la coma 
('das Komma', also unterschiedliches Genus, vgl. die entsprechenden Listen bei Reumuth / Winkelmann 1993: 22).

Diese Unterscheidung findet nicht bei allen Determinierern statt. Hier sind für die fehlende Differenzierung beim Numerus ambos, cada, demás, más, sendos und varios sowie beim Genus bastante, cada, cualquiera, demás, doble, dos, más, mi und tal zu nennen. Bei bastante und doble ist dies morphologisch durch den Auslaut -e begründet, varios wird im Singular nur als qualifizierendes Adjektiv gebraucht (vgl. de Bruyne 1993: 241). Die Kardinalia differenzieren den Numerus ebenso durch unterschiedliche Lexeme uno - dos, tres ... wie die Artikelformen el - la das Genus. Damit bekommen wir zwei Lexikoneinträge für uno: einmal den unbestimmten Artikel uno / una mit den Pluralformen unos / unas und zum zweiten das Numeral uno / una im Singular mit den Pluralformen dos, tres usw.

Während más und menos überhaupt keinen Numerus kennzeichnen, kennt cada nur den Singular und alle übrigen dieser Klasse nur den Plural. Genus wird entweder realisiert oder nicht realisiert, so daß ein Merkmal [Genus \pm ] eingeführt werden kann.

Die Darstellung des Numerus ist komplexer, wenn die Besonderheit, daß más und menos überhaupt keinen Numerus anzeigen, berücksichtigt werden soll. Dies ist insofern gerechtfertigt, als más und menos oft als Determinierer eingeordnet werden, obwohl sie eigentlich eher als Adverbien agieren. Eine korrekte Klassifikation müßte also die besondere Position dieser beiden Wörter herausstreichen. Es ist somit angebracht, sie in die Klassifikation zu integrieren.

Wenn wir nun zwischen Singular- und Pluralrealisierung unterscheiden und die Merkmale [Singular \pm ] und [Plural \pm ] erstellen, dann würden alle Determinierer, die beide Formen realisieren, mit zwei Plus beschrieben, diejenigen, die nur ein Merkmal realisieren, mit einem Plus und einem Minus sowie más und menos mit zwei Minus, z.B.:

$$
\begin{array}{ll}
\text { (a) más / menos: } & \text { [Singular -, Plural -], } \\
\text { (b) ambos, u.a.: } & \text { [Singular -, Plural +], } \\
\text { (c) cada: } & \text { [Singular +, Plural -], } \\
\text { (d) este, poco: } & \text { [Singular +, Plural +]. }
\end{array}
$$

Ein gravierender Nachteil an dieser Darstellung ist, daß sich z.B. cada (nur im Singular) und ambos (nur im Plural) gleich in zwei Merkmalen unterscheiden, obwohl 
eigentlich nur der Wert des einen Kriteriums Numerus unterschiedlich ist. Eine Alternative ist die Unterscheidung in Numerusrealisierung und Numerusdifferenzierung. Es kann ein Merkmal [Numerus realisiert \pm ] eingeführt werden, das zwischen más / menos und allen anderen Determinierern unterscheidet, sowie ein Merkmal [Numerus differenziert \pm ], das zwischen der Gruppe von cada, ambos u.a., die nur ein Merkmal realisieren, und allen anderen Determinierern, die beide Merkmale realisieren, unterscheidet. Damit ergibt sich folgende Dreiteilung:
(a) más / menos: [Num realisiert -, Num differenziert -],
(b) cada, ambos u.a.: [Num realisiert +, Num differenziert -],
(c) este, poco u.a.: [Num realisiert + , Num differenziert +$]$.

Bei dieser Klassifikation wird nicht mehr unterschieden, ob sich fehlende Numerusdifferenzierung durch die Singular- oder die Pluralform äußert. Diese Information wird aber in einer Grammatiktheorie wie der HPSG im Kongruenzmerkmal grundsätzlich angegeben und kann von daher an dieser Stelle vernachlässigt werden. Wichtiger erscheint eine Unterscheidung der Determinierer in bezug auf die Art der Numerusrealisierung und -differenzierung, nicht in bezug auf deren Wert. Cada und ambos verhalten sich insofern ähnlich, als den zu determinierenden Nomina ähnliche Eigenschaften zugesprochen werden: Sie dürfen nur in dem einen, dem Determinierer inhärenten Numerus erscheinen.

Numerusrealisierung findet wie genannt nur bei más / menos nicht statt. Bei ambos, cada, demás, varios und sendos wird der Numerus nicht differenziert.

\subsubsection{Adverbialisierung}

Bei Alvarez Martínez (1986: 194ff.) werden bastante, demasiado, más, mero, mисho, poco, tanto und todo als adverbialisierbar eingestuft, d.h. sie können auch als Adverbien auftreten. Dies sollen einige Beispiele illustrieren (aus Matte Bon 1992: II, 75ff., und de Bruyne 1993: 108, 227):
a. ¿Te ha gustado? Sí, es bastante interesante.
b. ¿Y que te parece? Para mí, es un poco demasiado caro. 
c. Y Esther, toda roja, empezó a chillarme.

d. Este muchacho es más fuerte que su hermano.

e. La última edición de este libro es menos cara que la anterior.

f. ¡No trabajes tanto!

g. iNo articules tan mal!

h. Este hombre trabaja mucho.

i. Una muchacha muy inteligente.

Im Fall eines adverbialen Gebrauchs können tanto, poco und mucho ihre Form verändern. Tanto wird zu tan, mucho zu muy, poco kann in der Form un poco auftreten. Alle drei Formen werden auch in Steigerungskonstruktionen verwendet.

Für mero konnte weder ein entsprechendes Beispiel für die Annahme von Alvarez Martínez noch ein Hinweis in anderen Grammatiken gefunden werden. Tal wird auch als Adverbial klassifiziert (vgl. de Bruyne 1993: 99ff., 243f., 248 u.a.), was aber nur in bestimmten Konstruktionen möglich ist ${ }^{23}$ und von Alvarez Martínez (1986: 178) nicht berücksichtigt wird. Für mismo postuliert de Bruyne (1993: 243) adverbialen Gebrauch und zitiert Beispielsätze wie En la misma Valencia no hay melón mejor que este. In diesem Satz ist die Funktion von misma in syntaktischer Hinsicht als adjektivisch zu werten, auch wenn es der Bedeutung 'sogar' (als Satzadverbial) entspricht.

Wir führen das Merkmal [Adverbialer Gebrauch \pm ] ein und werten es für die bei Alvarez Martínez genannten Determinierer außer mero positiv und für alle anderen negativ. Es muß als eines der Merkmale gesehen werden, die Nähe zu Adjektiven ausdrücken.

\subsubsection{Komparation}

Eng damit zusammenhängend ist das Kriterium der Komparation. Komparierbarkeit ist eine Eigenschaft, die allgemein Adjektiven zugeschrieben wird (vgl. de Bruyne 1993: 108, Bsp. von dort):

\footnotetext{
${ }^{23}$ De Bruyne 1993: 248 verweist auf ¿qué tal ...? wie z.B. in ¿qué tal ese libro? oder tal de + Adjektiv wie in Tal estaba de nervioso que no supo contestar.
} 
(41) Este muchacho es más fuerte que su hermano.

Alle Adjektive sind grundsätzlich steigerungsfähig. Des öfteren ist der Sinn allerdings fragwürdig (was bedeutet más rojo?). Theoretisch ist es möglich, einen Kontext zu konstruieren, in dem diese Konstruktion sinnvoll ist. An dieser Stelle ist es wichtig zu sehen, daß syntaktisch viele Konstruktionen korrekt sind, die auf den ersten Blick semantisch oder pragmatisch nicht sinnvoll anmuten.

Más stellt ein Ausdrucksmittel der Komparation dar. Es ist von daher bedenklich, más und menos als komparierbar einzustufen. Andererseits bedeuten sie stets ein Mehr / Weniger, das vergleichend wirkt: El Ayuntamiento de Madrid necesita 2.000 funcionarios más (M-16). Möglich ist eine Konstruktion wie mucho más, z.B. in Los madrileños hemos pagado mucho más (M-16).

Von den hier als Determinierern eingestuften Wörtern werden in vielen Grammatiken mismo und mío als steigerbar eingeschätzt (vgl. de Bruyne 1993: 187, 243, Bsp. von dort):

(a) La ciudad parece más suya.

(b) ¡Este destino es el mismísimo demonio!

Bei mismo ist dies nur im Superlativ mit dem Suffix -ísimo oder in Vergleichskonstruktionen mit (el) mismo (...) que. Dies berechtigt aber, ein neues Merkmal [Komparation \pm ] einzuführen, das auf mío, mismo und más sowie auf alle qualifizierenden Adjektive zutrifft.

\subsubsection{Prädikation}

Adjektive können in folgenden prädikativen Konstruktionen auftreten (Bsp. aus Matte Bon 1992: I, 185):

(a) ¿Qué te parece? Es precioso.

(b) ¿La conoces? Es simpatiquísima.

Diese Eigenschaft trifft auch auf einige Determinierer zu (Bsp. aus de Bruyne 1993: 223): 
(44) (a) ¿De quién es esta casa? Es nuestra.

(b) Eso es.

(c) Los manifestantes son muchos, son demasiados.

(d) Son dos cualquieras.

Diese Eigenschaft scheint nicht selbständig aufzutreten. Vielmehr hängt ein prädikativer Gebrauch davon ab, ob ein Determinierer allein stehen kann, was wiederum davon abhängig ist, ob er substantiviert werden kann oder ob er nur als Begleiter operiert:

(a) *Es cada

(b) *Son meros

Weder cada noch mero können eigenständig substantiviert werden, d.h. ohne Artikel, und folglich nicht prädikativ gebraucht werden.

Somit stellt sich die Frage, inwieweit ein Merkmal [prädikativ \pm ] distinktive Funktion hat und nicht als Folgeerscheinung anderer Merkmale zu werten ist. Von daher schließen wir die Fragestellung der Substantivierbarkeit hier an und gehen zunächst davon aus, daß ein Merkmal [prädikativ \pm ] nicht notwendig ist.

\subsubsection{Substantivierbarkeit}

Substantivierung wird das Phänomen genannt, daß sich ein Wort einer nichtsubstantivischen Wortart wie ein Substantiv verhält. Dieses Wort wird durch lexikalische Prozesse in ein Nomen überführt und bekommt nomenähnliche Eigenschaften zugesprochen. Determinierer können auf zwei Arten substantiviert werden: entweder analog zu den Adjektiven durch den (definiten) Artikel, durch einen artikelähnlichen Determinierer oder selbständig. Selbständig bedeutet, daß ein Determinierer in sich selbst eine artikelähnliche Eigenschaft trägt, so daß es nicht mehr nötig ist, einen Artikel voranzustellen.

Der Terminus Artikel muß im folgenden eingegrenzt werden. Der Terminus Determinierer - zu Beginn von Kapitel III.2.2. eingeführt - wird als Oberbegriff aufgefaßt. Untergruppen sind Demonstrativa, Possessiva, Indefinita, definiter Artikel usw. 
Mit Artikel wollen wir im folgenden auf typische Eigenschaften des definiten Artikels referieren, wie z.B. die Substantivierung, die auch z.T. auf andere Determinierer zutreffen. „Echte“ Artikel sind demnach Determinierer, die dem definiten Artikel in vielen Eigenschaften sehr nahe kommen, aber nicht unbedingt mit ihm übereinstimmen müssen. Sie verfügen besonders über die Eigenschaft, aus einem Nomen oder der Kombination Nomen + Adjektiv eine vollständige Nominalphrase zu erstellen und damit die Phrase in der X-bar-Theorie auf die N"-Ebene anzuheben. Auf el / la / los / las wird nur mit der Bezeichnung definiter Artikel Bezug genommen. Auch muß der später eingeführte „typische“ Determinierer davon abgegrenzt werden. Er stellt in der Klassifikation einen Vertreter der Wortart Determinierer dar, der zu Vergleichszwecken eingeführt wird und in seinen Eigenschaften einem „echten“ Artikel sehr nahe kommt.

Die mögliche Kombination bei der Substantivierung mit einem Artikel - d.h. dem definiten Artikel oder einem ähnlichen Determinierer - hat Einfluß auf andere Kriterien. Manche Determinierer können nur in Kombination mit einem Artikel substantiviert werden und erhalten dann Charakteristika, die sie allein nicht aufweisen. Ein Beispiel sei hier demás, das überhaupt nur in Kombination mit dem Artikel auftreten kann (Bsp. aus de Bruyne 1993: 235):

(46) (a) Los demás pelos se contagian.

(b) Tus demás pelos se contagian.

(c) *Demás pelos se contagian.

(d) Toda la demás gente lo vio.

(e) *Demás gente lo vio.

(f) Un buen hombre es un hombre bueno para los demás.

Demás ist in substantiviertem Zustand fähig, als prädikatives Element zu fungieren. Diese Tatsache zeigt, daß Prädikation eine Eigenschaft sein könnte, die für substantivierbare Wortarten gilt. Allgemein sind Adjektive substantivierbar und können prädikativ auftreten. Alle Determinierer, die nicht substantiviert werden können, fungieren auch nicht prädikativ. Damit kann das Merkmal [prädikativ \pm ] als redundant bezeichnet werden.

Im folgenden muß die Eigenschaft der Substantivierbarkeit noch differenziert werden. Wir sahen bereits, daß eine Differenzierung in Substantivierung mit oder ohne Artikel möglich ist. So steht z.B. demás nur mit Artikel, este nur ohne. Dazu 
kommt noch die Möglichkeit, sowohl mit Artikel als auch ohne substantiviert werden zu können (z.B. dos), sowie die Eigenschaft, auch selbständig Adjektive substantivieren zu können (transpositor de cualificativos nach Alvarez Martínez 1986: 202). Implizit bedeuten diesen Eigenschaften, daß es eine Gruppe von Determinierern gibt, die sich analog oder ähnlich zum Artikel verhält, und eine andere, die bevorzugt in Kombination mit dem Artikel steht. Die Eigenschaft, nur in Kombination mit dem Artikel aufzutreten, ist wiederum typisch für die Wortart Adjektiv.

Aus diesen Ausführungen ergeben sich folgende Eigenschaften, die auf ihre Distinktivität geprüft werden müssen, d.h. ob sie die Determinierer von den Adjektiven und die Determinierer untereinander differenzieren oder ob das entsprechende Merkmal keine neue Eigenschaft benennt und damit redundant ist:

(a) substantivierbar ohne Artikel

(b) substantivierbar mit Artikel

(c) substantivierbar mit und ohne Artikel

(d) substantivierbar im Neutrum (mit lo)

(e) substantiviert Adjektive

(f) operiert analog zum (definiten) Artikel

(g) steht nur zusammen mit dem Artikel

(h) operiert sowohl mit als auch ohne Artikel

Als erstes ist ein Merkmal [substantivierbar ohne Artikel 士] nötig. Dieses kennzeichnet alle Determinierer, die wie die Demonstrativa eigenständig eine Nominalphrase bilden können (z.B. Eso es). Ob dies für den definiten Artikel zutrifft, hängt davon ab, ob man die Personalpronomina im Nominativ él und ella als substantivierte Form des definiten Artikels versteht. Dieser Vergleich ist problematisch. Es handelt sich bei definitem Artikel und Personalpronomen zwar um zwei historisch miteinander verwandte Wortarten (vgl. Alvar / Pottier 1983: 110ff. und 119ff.), aber trotzdem zwei unterschiedliche Lexeme. Die definiten Artikel sind in den romanischen Sprachen in der Regel klitisch und können im Gegensatz zu den Demonstrativa nie betont werden. Wir gehen somit davon aus, daß der definite Artikel nicht substantivierbar ist und in diesem Fall durch das Personalpronomen ersetzt wird.

Bei genauerer Betrachtung der Verhältnisse zeigt sich, daß wir bei der Substantivierung mit und ohne Artikel eine ähnliche Situation haben wie beim Kriterium Numerus. Einige Determinierer sind nur mit Artikel (demás, mío, mismo), andere wie- 
der nur ohne Artikel substantivierbar (alguno, este, todo, demasiado, mucho), d.h. letztere können pronominal gebraucht werden. Auf eine dritte Gruppe trifft beides (dos, otros, tal), auf eine vierte (cada, el u.a.) beides nicht zu. Dabei nimmt otro noch eine besondere Stellung ein: Im Singular kann es nur mit Artikel substantiviert werden (el otro viene, *otro viene), im Plural ist beides möglich (los otros vienen, otros vienen). Bei vier Möglichkeiten brauchen wir zwei differenzierende Merkmale. Am einfachsten sind diese zwei:

(a) [substantivierbar mit Artikel \pm ]

(b) [substantivierbar ohne Artikel \pm ]

Diese Merkmale teilen die Determinierer in vier Gruppen ein: Determinierer, die

- wie ein Adjektiv (d.h. mit Artikel substantivierbar),

- wie ein Artikel (eigenständig ohne weiteren Artikel substantivierbar),

- nach beiden Verfahren,

- überhaupt nicht

substantiviert werden können.

Die Merkmalszuordnung ergibt sich wie folgt:

(49) (a) demás [substantivierbar mit Artikel +, substantivierbar ohne Artikel -]

(b) alguno [substantivierbar mit Artikel -, substantivierbar ohne Artikel +]

(c) dos [substantivierbar mit Artikel +, substantivierbar ohne Artikel +]

(d) cada [substantivierbar mit Artikel -, substantivierbar ohne Artikel -]

Wie demás verhalten sich noch doble, mismo und segundo. $\mathrm{Zu}$ dos gehört noch tal, $\mathrm{zu}$ alguno noch ambos, bastante, cualquiera, demasiado, mucho, ninguno, poco, todo, tanto, uno und varios. Dabei können poco und uno in bestimmten Kontexten auch mit Artikel stehen: uno in Korrelation zu otro, poco in Verbindung mit anderen Determinierern wie varios, wenn eine Präposition vorangeht oder ein Relativsatz folgt. Solche Besonderheiten werden nicht berücksichtigt. Zweimal Minus bekommen neben cada auch cierto, el, más, mero, mi und sendos. Die Bedeutung des Artikels schränkt Briz Gómez (1989: 125) korrekt ein: „El artículo es índice de sustantividad, pero no sustantivador. En primer lugar cabe destacar las sustantivaciones sin la presencia del artículo (...): negros y blancos se dieron la mano en las elecciones." 
Zwei Merkmale, die erneut eine klare Differenzierung zwischen Adjektiv und Determinierer erlauben, sind [substantiviert Adjektive \pm ] und [substantivierbar im Neutrum \pm ]. Nach obigem Zitat von Briz Gómez mag man die Fähigkeit zur Substantivierung zwar bezweifeln (Adjektive können vor allem im Plural wie Nomina gebraucht werden), doch scheint uns die Substantivierung von Adjektiven eine besondere Fähigkeit derjenigen Determinierer zu sein, die ohne Artikel substantiviert werden können, da sie zumindest den Kontext definieren, in dem dies möglich ist. Eine Sonderstellung nimmt an dieser Stelle der definite Artikel ein.

Das zweite Merkmal ist für alle qualifizierenden Adjektive typisch und kann in diesem Fall auf alle adjektivähnlichen Determinierer erweitert werden:

Substantivierung von Adjektiven

(a) ¿Cómo se le ocurre a este desgraciado espiarme así?. (S-16)

(b) No esperaba tantos invitados. (Seco 1991: 166)

\section{Substantivierung im Neutrum}

(c) Felisberto Hernández y la escritura de 'lo otro'.

(d) Lo mío, mío y lo tuyo de emtrambos. (Bsp. aus de Bruyne 1993: 55)

Wir werten Substantivierung von Adjektiven positiv bei alguno, cualquiera, el, este, ninguno, otro, todo und uno, Substantivierung im Neutrum bei bastante, demás, demasiado, doble, este, más, mío, mismo, mucho, otro, poco, segundo, tal, tanto, todo und uno.

Die letzten drei Merkmale der obigen Liste (47) - operiert analog zum (definiten) Artikel, steht nur zusammen mit dem Artikel, operiert sowohl mit als auch ohne Artikel - erscheinen etwas vage. Gemeint ist eine Verwendung des Determinierers in einer sehr artikeltypischen Umgebung (vgl. Helbig / Buscha 1994: 355), wobei das Adjektiv fakultativ ist:

(a) _ Adj $\mathrm{N}$ wie in Contó para ello con la valiosa ayuda de su hermano Alexander. (Rch-1)

(b) _ N Adj wie in Casi ha desaparecido la / esta violencia callejera. (M-11)

Die mit Unterstrich gekennzeichnete Leerstelle kann immer mit dem definiten Artikel gefüllt werden und vervollständigt die Nominalphrase: [ Stelle kann nur eine begrenzte Zahl von anderen Determinierern stehen. Wie im Zu- 
sammenhang mit der Substantivierung gibt es erneut eine Gruppe, die nur mit dem Artikel zusammen in einer solchen Konstruktion auftreten kann - d.h. sie verhalten sich analog zu den Adjektiven -, und andere, die sowohl mit als auch ohne Artikel auftreten. Wir konstruieren daraus folgende zwei Merkmale:

(a) [steht in Umgebung _ A N \pm ]

(b) [steht in Umgebung Art _ $\mathrm{N} \pm$ ]

Das erste Merkmal kennzeichnet alle Determinierer, die in einer artikeltypischen Umgebung stehen und eine Nominalphrase vervollständigen. Der Artikel und alle ihm ähnlichen Determinierer sind folglich als weiterer Teil der Nominalphrase ausgeschlossen, da der entsprechende Determinierer die Nominalphrase vervollständigt (in der X-bar-Theorie gesprochen: auf die Ebene N" anhebt). Das zweite Merkmal differenziert zwischen den restlichen Determinierern, indem eine adjektivtypische Umgebung definiert wird, d.h. der Determinierer trägt nicht zur Vervollständigung auf N"-Ebene bei. Dies darf nicht mit der möglichen Kookkurrenz von zwei Determinierern verwechselt werden. Es kann eine Zwischenklasse (dos und otro), die beide Merkmale trägt, als Übergangsstufe zwischen Artikel und Adjektiv auch an dieser Position plaziert werden. $\mathrm{Zu}$ dieser Klasse gehört nicht poco, da es nur dann nach dem Artikel stehen kann, wenn die NP Teil einer Präpositionalphrase ist oder einem Relativsatz vorausgeht.

(a) demás [steht in Umgebung _ A N -, steht in Umgebung Art _ $\mathrm{N}+$ ]

(b) mismo [steht in Umgebung _ A N -, steht in Umgebung Art _ $\mathrm{N}+$ ]

(c) dos [steht in Umgebung _ $\left.\mathrm{A} \mathrm{N} \mathrm{+,} \mathrm{steht} \mathrm{in} \mathrm{Umgebung} \mathrm{Art} \_\mathrm{N}+\right]$

(d) otro [steht in Umgebung _ A N +, steht in Umgebung Art _ $\mathrm{N}+$ ]

(e) alguno [steht in Umgebung _ A N +, steht in Umgebung Art _ $\mathrm{N}$-]

(f) este [steht in Umgebung _ A N +, steht in Umgebung Art _ $\mathrm{N}$-]

Demás und mismo können nur in einer Umgebung vorkommen, die durch einen Artikel eingeleitet wird (genauso: doble, mero und segundo). Alguno und este sind dem definiten Artikel in dieser Frage als gleichwertig zur Seite zu stellen. Sie erzeugen somit die entsprechende Umgebung für demás und mismo. Dos und otro gehören in eine Zwischenposition. Sie können sowohl eine artikelähnliche Funktion ausüben als auch in einer Umgebung stehen, die durch den Artikel eingeleitet wurde. 
Als Besonderheit ist hier zunächst mío zu nennen. Da es nur nach der Nominalphrase steht, kann es nicht in den beschriebenen Umgebungen auftauchen. In gleicher Weise, nur aus anderen Gründen, verhält es sich mit todo, welches grundsätzlich vor der Nominalphrase steht und ebenfalls nicht erfaßt wird. Zwar ist eine Abfolge todo - Adjektiv - Nomen möglich, dann füllt todo aber nicht die durch die Leerstelle gekennzeichnete Artikelfunktion aus (Bsp. aus de Bruyne 1993: 225):

(54) Todo buen ciudadano debe ayudar a la justicia.

Todo wird in diesem Fall synonym zu cada verwendet. Da todo einige außergewöhnliche Eigenschaften aufweist, werden wir es zwar weiter berücksichtigen, diese Eigenschaften führen jedoch dazu, daß todo nicht problemlos in die Klassifikation integriert werden kann. Auf dieses Problem wird weiter unten nochmals eingegangen.

Durch diese zwei Determinierer erhalten wir bei den beiden obigen Merkmalen eine vierte Klasse, die sich folgendermaßen darstellt:

(a) todo [steht in Umgebung _ A N -, steht in Umgebung Art _ $\mathrm{N}$-]

(b) mío [steht in Umgebung _ A N -, steht in Umgebung Art _ N -]

Alle in diesem letzten Abschnitt nicht genannten Determinierer verhalten sich in bezug auf das Umgebungsmerkmal wie alguno und este.

\subsubsection{Quantifikation}

Die Gruppe der quantifizierenden Determinierer wurde bereits als problematisch bezeichnet, da sie sich als sehr heterogene Klasse erweist. Allgemein muß die Quantifizierung als Problem gewertet werden. Einige Autoren versuchen, grundsätzlich eine Analyse der Nominalphrase ohne Quantifikation zu erstellen (Gawron / Peters 1990, Kolliakou 1995), andere sehen diese als das herausragende Problem an (vgl. Kiss 1995a, Allegranza 1998).

Es besteht kein Zweifel daran, daß ein Determinierer einen Einfluß auf die ihm folgende Nominalphrase besitzt. Die Frage ist, wie dieser Einfluß aussieht. Wenn wir 
eine Nominalphrase wie el período literario español comprendido entre 98 y $36^{24}$ betrachten, dann ist klar zu erkennen, daß sich der Artikel auf die gesamte folgende Phrase bezieht. Hier ergibt sich kein Quantifikationsproblem, wie es in Kapitel II.2.8. für den Satz Cada estudiante sabe una canción erörtert wurde.

Im Gegensatz dazu stehen die dem Nomen folgenden Adjektive. Hier bildet das erste Adjektiv eine Einheit mit dem Nomen, auf die sich dann das zweite Adjektiv als ganzes bezieht usw. Wir können den Einflußbereich mit eckigen Klammern folgendermaßen darstellen:

[el [[[período literario] español] comprendido entre 98 y 36]]

Das Problem stellen folglich mehrere Adjektive in einer Nominalphrase dar. Ähnliches ergibt sich bei einer Phrase mit mehreren Determinierern. Betrachten wir die aus (56) erstellte Phrase: los demás períodos literarios españoles. Muß hier jeder Determinierer als eine einzelne Einheit verstanden werden, so daß los sich auf die gesamte NP bezieht? Oder sind beide Determinierer als eine Einheit zu verstehen, die sich zusammen auf die NP bezieht? Wir müssen folglich fragen, ob (a) oder (b) die korrekte Klammerdarstellung ist:

(a) [ los [demás [ [ períodos literarios] españoles ] ] ]

(b) [ [los demás ] [ [ períodos literarios] españoles ] ] ]

Demás kann zwar nie allein stehen, los bezieht sich aber auf alles folgende. Wir werden folglich (a) favorisieren. Eine Phrase wie *demás períodos literarios españoles wird durch die syntaktischen Eigenschaften von demás unterbunden. Für echte Kookkurrenz aus zwei Determinierern wie z.B. todo und este stellt sich die Frage ähnlich:

[Todos [estos $[$ cuentos $]]]$ están inéditos. (S-9)

In diesem Beispiel determiniert todos die folgende Nominalphrase, die von estos eingeleitet wird. Damit wird die Menge aller cuentos eingegrenzt auf diejenigen, auf die estos referiert. Todos bezieht sich auf die gesamte Menge von estos cuentos. Der Bezug ergibt sich aus der Stellung.

\footnotetext{
${ }^{24}$ Bsp. aus Ponte Far, José Antonio (1992): Renovación de la novela en el siglo XX: del 98 a la Guerra Civil. Madrid: Anaya, 4.
} 
Diese Beobachtung wirft die grundsätzliche Fragestellung auf, wie Kookkurrenz von Determinierern behandelt werden soll. Zudem hängt sie eng mit der strukturellen Fragestellung zusammen, welche Konstituente als Kopf der Nominalphrase interpretiert wird (D oder N) und wie in der Folge eine HPSG-Matrix einer spanischen Nominalphrase aussehen soll.

Dies zeigt, daß wir es mit einem äußerst komplexen Phänomen zu tun haben, das nicht nur verschiedene Bereiche der Deskription berührt, sondern zudem in der Weise theorieabhängig ist, daß die Struktur der Nominalphrase sich auch aus theoretischen Vorüberlegungen ergibt. Diese Aspekte werden hier zurückgestellt. Wir erstellen kein Merkmal für quantifizierende Eigenschaften von Determinierern.

\subsubsection{Vor- und Nachstellung}

Determinierer stehen normalerweise vor dem Nomen. Wie bereits gezeigt, weisen einige Determinierer Eigenschaften von Adjektiven auf, so daß Nachstellung möglich wird. Es gibt folgende drei Stellungsmöglichkeiten:

Der Determinierer steht

1. immer vor dem Nomen (el, uno u.a.),

2. vor und evtl. nach dem Nomen (este, alguno u.a.),

3. immer nach dem Nomen (nur mío).

Aus diesen Eigenschaften können folgende zwei Merkmale abgeleitet werden:

(a) [vorangestellt \pm ]

(b) [nachgestellt \pm ]

Damit können alle spanischen Determinierer entsprechend differenziert werden. Für die oben genannten ergibt sich folgende Abstufung:
(a) el / la [vorangestellt + , nachgestellt -]
(b) este [vorangestellt + , nachgestellt + ]
(c) mío [vorangestellt -, nachgestellt +]

Diese Einordnung entspricht der Auffassung, daß jeweils Artikel und Demonstrativa sowie Demonstrativa und das nachgestellte Possessivpronomina nur in einem Merkmal differieren sollten. Artikel - und fast alle anderen Determinierer, für die der de- 
finite Artikel hier repräsentativ steht - und das nachgestellte Possessivpronomen differieren in zwei Merkmalen. Damit steht este als Repräsentant der Übergangsklasse in der Mitte. Nicht berücksichtigt wird, daß die Gruppe um este nur unter bestimmten Bedingungen nachgestellt werden kann und nicht, wie man vermuten könnte, frei in ihrer Positionswahl ist. Die Gruppe trotzdem einheitlich zu erfassen, ist insofern gerechtfertigt, als die Bedingungen für Nachstellung erheblich divergieren: este / ese / aquel drücken in Nachstellung einen Affekt des Sprechers aus, mismo folgt nur bei vorangehendem Eigennamen und Personalpronomen, alguno wird nur in negierten Phrasen nachgestellt, segundo und doble können frei positioniert werden. Wir gehen davon aus, daß Voranstellung den Standard bei Determinierern im Spanischen darstellt. So definieren wir die positive Markierung des Merkmals [nachgestellt + ] neben der positiven Markierung [vorangestellt + ] als nur unter bestimmten Bedingungen möglich. Neben den bereits genannten Determinierern können noch cualquiera, más, ninguno, tal und todo in bestimmten Fällen nachgestellt werden.

Bevor die etwas komplexeren Kriterien Kookkurrenz und semantische Aspekte behandelt werden, soll eine Zusammenfassung des bereits Erarbeiteten erfolgen. Dazu werden die Kriterien aus (37) und die daraus resultierenden Merkmale aufgelistet:

1. Genus und Numerus:

2. Adverbialisierung:

3. Komparation:

4. Prädikation:

5. Substantivierbarkeit:
[Genus \pm ]

[Numerus realisiert \pm ]

[Numerus differenziert \pm ]

[Adverbialer Gebrauch \pm ]

[Komparation \pm ]

[substantivierbar mit Artikel \pm ]

[substantivierbar ohne Artikel \pm ]

[substantivierbar im Neutrum \pm ]

[steht in Umgebung _ A $\mathrm{N} \pm$ ]

[steht in Umgebung Art _ $\mathrm{N} \pm$ ]

6. Substantivierer von Adjektiven: [substantiviert Adjektive \pm ]

7. Quantifikation:

8. Voran- vs. Nachstellung: [vorangestellt \pm ]

[nachgestellt \pm ] 
Diese Merkmale können theoretisch ausreichen, um die Syntax der Determinierer zu erfassen, vor allem wenn wir Kookkurrenz als ein syntaktisch-semantisches Phänomen begreifen. Allerdings ist es im Sinne der HPSG, die semantische Seite zu berücksichtigen. Deshalb folgt ein längerer Abschnitt über Kookkurrenz von Determinierern (III.2.3.) sowie über ihre semantischen Eigenschaften (III.2.4.). Inwieweit die semantischen Merkmale für eine Klassifikation nötig sind, kann später leicht festgestellt werden, indem bei der Berechnung der Distanzen diese Merkmale probeweise entfallen. 


\subsection{Kookkurrenz}

Determinierer können in Kombination miteinander auftreten. Neben einem einzelnen Determinierer vor dem Nomen oder Adjektiv sind zwei Determinierer davor nichts Ungewöhnliches. Mitunter stehen sogar drei in einer Phrase (aquellas sus dos obsesiones, Alarcos Lorrach 1994: 14). Diese Beispiele wurden aber von einigen befragten Muttersprachlern als zweifelhaft eingestuft. In diesem Kapitel soll untersucht werden, welche Kombinationen möglich sind und welche Faktoren dabei eine Rolle spielen. Da dies vom semantischen Gehalt eines Determinierers abhängig ist, schließt sich eine Untersuchung der Semantik an.

\subsubsection{Kookkurrenz nach Prado Ibán (1993)}

Nach Prado Ibán (1993: 270) gibt es in bezug auf ihre kombinatorischen Eigenschaften zwei Klassen von Determinierern. Aufgrund der noch zu erläuternden Problematik dieses Ansatzes sprechen wir im folgenden von Gruppen:

A: Demonstrativa, Possessiva, existenzielle Indefinitpronomina (alguno, ninguno, uno, cualquiera), Distributive (sendos, cada) sowie ambos, tal, tanto, cierto und todo,

B: Gradative (mucho, poco, demasiado, bastante), Kardinalia, Ordinalia sowie mismo, varios, otros, más und menos.

Dabei postuliert sie folgende Eigenschaften der Gruppen und belegt dies mit den darauf folgenden Beispielen (alle folgenden Beispiele aus Prado Ibán 1993: 270ff.):

(63) Gruppe A:

- inkompatibel untereinander, aber kombinierbar mit B,

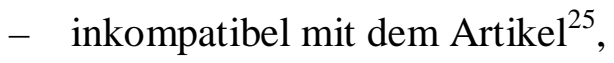

- steht meist vor B.

\footnotetext{
${ }^{25}$ Es sei daran erinnert, daß mit Artikel der definite Artikel gemeint ist.
} 
Gruppe B:

- kompatibel mit A und manchmal untereinander,

- kompatibel mit dem Artikel oder einem artikelähnlichem Determinierer,

- steht meist hinter A.

Die folgenden Beispiele zeigen die Inkompatibilität der Mitglieder aus Gruppe A untereinander, aber die Kompatibilität mit Mitgliedern aus Gruppe B.

(64) (a) estos muchos niños (A vor B)

(b) *este ningún niño $\quad(* \mathrm{~A}$ vor $\mathrm{A})$

(c) *un cualquier amigo $(* \mathrm{~A}$ vor $\mathrm{A})$

(d) *mis algunos amigos (*A vor A)

Hier wird die Kombination von Determinierern der Gruppe B mit Determinierern der Gruppe A und innerhalb der Gruppe B deutlich:
(a) algún otro muchacho (A vor B)
(b) mis pocos enemigos (A vor B)
(c) estas muchas cosas (A vor B)
(d) otros muchos chicos (B vor B)
(e) pocos segundos puestos (B vor B)
(f) varias otras oportunidades (B vor B)

Die Gruppe B steht generell nach Gruppe A:
(a) este mismo niño / *mismo este niño (A vor $\mathrm{B}) /(* \mathrm{~B}$ vor $\mathrm{A})$
(b) ningún otro amigo / *otro ningún amigo (A vor $\mathrm{B}) /(* \mathrm{~B}$ vor $\mathrm{A})$
(c) cada dos manzanas / *dos cada manzanas (A vor B) / (*B vor A)
(d) cada poco tiempo /* poco cada tiempo (A vor B) / (*B vor A)

Prado Ibán nennt aber auch selbst Gegenbeispiele:
(a) ese tal muchacho (beide aus A),
(b) mis tantos amigos (beide aus A).

Es lassen sich problemlos weitere finden: 
(c) la reina es la reina en la medida que es alimentada de una cierta manera. (beide aus A; Rch-4))

(d) Las actitudes políticas de ella son otras tantas causas de desacuerdos. (B vor A; RA-6).

Zwar weist sie selbst auch darauf hin, daß es Ausnahmen in ihrer Klassifikation gibt, dennoch scheint die Einteilung sehr ungenau. Determinierer der Gruppe B können zuweilen vor A stehen. Die Gruppe B ist nicht frei untereinander kombinierbar, wie die Klassifikation von Prado Ibán glauben machen will. Allerdings ist die Tendenz der Untersuchung richtig. Determinierer der Gruppe A stehen eher als 1. Glied einer Kombination, Determinierer der Gruppe B eher als 2. Glied. Wie zu sehen sein wird, besteht aber z.B. zwischen cierto und varios kein großer Unterschied in ihrem Kombinationsmöglichkeiten, obwohl sie bei Prado Ibán unterschiedlichen Gruppen zugeordnet werden.

Aus diesen Überlegungen kann die Schlußfolgerung gezogen werden, daß es Regelmäßigkeiten bei den Kombinationsmöglichkeiten der Determinierer gibt. Um alle Möglichkeiten zu erfassen, wird untersucht, wie alle Determinierer zueinander in Beziehung stehen.

Zwei Problembereiche sind vorweg zu nennen: Zum einen sind nicht-mögliche Kombinationen natürlich auch nicht nachweisbar. Es kann also nur aus dem NichtVorhandensein die Hypothese abgeleitet werden, daß die Kombination nicht zulässig ist. Zum anderen wurden mögliche, aber zweifelhafte Kombinationen von Muttersprachlern sehr unterschiedlich bewertet. Es gab z.B. große Unterschiede zwischen Spaniern und Chilenen. Folglich können wir vermuten, daß neben diesen geographischen wahrscheinlich auch noch soziale und andere Faktoren zu unterschiedlichen Sprecherurteilen beitragen können. Wir können also nur versuchen, uns den wahren Verhältnissen anzunähern, und ein idealisiertes Supersystem erstellen. Es gibt keine eindeutig als falsch oder richtig zu deklarierenden Kombinationen.

\subsubsection{Eine erste umfassendere Klassifikation}

Es wurden alle Determinierer als erstes und zweites Glied erfaßt und Problemfälle in Beispielsätzen erfragt. Dabei ergab sich, daß die Numeralia sowie mismo, mero, 
demás und mío nie an erster Stelle stehen können, und somit werden sie in der Tabelle als erstes Glied nicht erfaßt. Todo, ambos, sendos, cada, ninguno, alguno können nicht an zweiter Stelle stehen und werden ebenfalls dort nicht aufgeführt. Más / menos können nur nachgestellt kombiniert werden und werden nicht berücksichtigt. Kombinationen mit mío und cualquiera in Nachstellung werden nur in der folgenden Tabelle aufgeführt und sonst nicht weiter bearbeitet. Dos, segundo und doble stehen jeweils stellvertretend für die Klasse der Kardinalia, Ordinalia und Multiplikativa. Die Numeralia können untereinander kombiniert werden, wenn ein Artikel voransteht. Diese und andere Kombinationen aus drei Determinierern werden in der folgenden Tabelle nicht berücksichtigt.

\section{Kombinationsmöglichkeiten spanischer Determinierer}

\begin{tabular}{|c|c|c|c|c|c|c|c|c|c|c|c|c|c|c|c|c|c|}
\hline & & & & & & & & & & & & & & & & \multicolumn{2}{|c|}{ nachgest. } \\
\hline $1 . \quad$ & 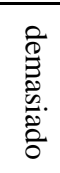 & $\frac{2}{0}$ & 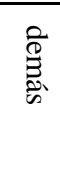 & $\overrightarrow{\tilde{F}}$ & 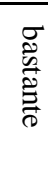 & $\vec{\Xi}$ & 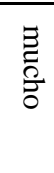 & 今. & $\begin{array}{l}\text { E. } \\
\text { : } \\
\text { z. }\end{array}$ & ठ্ & 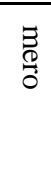 & ' & $\stackrel{\circ}{\circ}$ & 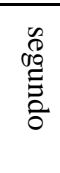 & $\frac{\partial}{\frac{\sigma}{\sigma}}$ & 跑 & 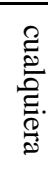 \\
\hline $\mathrm{mi} / \mathrm{tu}$ & $X$ & $\mathrm{X}$ & $X$ & $\mathrm{X}$ & $X$ & & $X$ & $\mathrm{X}$ & $\mathrm{X}$ & $X$ & & $\mathrm{X}$ & $\mathrm{X}$ & $X$ & $X$ & 1 & \\
\hline este & $?$ & & & & $\mathrm{X}$ & $X$ & $X$ & $X$ & $X$ & $X$ & $X$ & $X$ & $X$ & $X$ & $X$ & $X$ & \\
\hline uno & & $X$ & & & & $X$ & & $?$ & $X$ & $X$ & & $X$ & $\mathrm{X}$ & $X$ & $X$ & $X$ & $\mathrm{X}$ \\
\hline el / la & & & $X$ & & & $\mathrm{X}$ & & & $\mathrm{X}$ & $X$ & $\mathrm{X}$ & & $\mathrm{X}$ & $\mathrm{X}$ & $X$ & $X$ & \\
\hline otro & & & & $X$ & & & $X$ & $X$ & & $X$ & & $X$ & 1 & $X$ & $X$ & $X$ & \\
\hline cada & $?$ & & & & $?$ & & $X$ & $X$ & & & & $X$ & $?$ & $X$ & $X$ & $X$ & \\
\hline cualquiera & & & & & & & & & $?$ & & $X$ & $\mathrm{X}$ & $\mathrm{X}$ & $\mathrm{X}$ & $\mathrm{X}$ & $X$ & 1 \\
\hline todo & & & & & & & & & & & $\mathrm{X}$ & $\mathrm{X}$ & $\mathrm{X}$ & $\mathrm{X}$ & $\mathrm{X}$ & $X$ & $\mathrm{X}$ \\
\hline alguno & & & & & & & & & & & $X$ & $X$ & $\mathrm{X}$ & $X$ & $X$ & $X$ & \\
\hline bastante & & & & & 1 & & $?$ & & & & & $\mathrm{X}$ & $\mathrm{X}$ & $X$ & $X$ & $X$ & $\mathrm{X}$ \\
\hline ninguno & & & & & & & & & & & $X$ & & $\mathrm{X}$ & $X$ & $X$ & $X$ & $\mathrm{X}$ \\
\hline demasiado & 1 & & & & & & & & & & & $X$ & $\mathrm{X}$ & $X$ & $X$ & $X$ & $X$ \\
\hline cierto & & 1 & & & & & & & & & & $X$ & $X$ & $X$ & $X$ & $X$ & $X$ \\
\hline tanto & & & & 1 & & & & & & & & $?$ & $\mathrm{X}$ & $X$ & $\mathrm{X}$ & $X$ & $\mathrm{X}$ \\
\hline varios & & & & & & & & 1 & & & & $?$ & $\mathrm{X}$ & $X$ & $X$ & $X$ & $\mathrm{X}$ \\
\hline mucho & & & & & & & I & & & & & & $\mathrm{X}$ & $\mathrm{X}$ & $X$ & $X$ & $\mathrm{X}$ \\
\hline poco & & & & & & & & & & & & 1 & $X$ & $\mathrm{X}$ & $X$ & $?$ & $\mathrm{X}$ \\
\hline tal & & & & & & 1 & & & & & & & $\mathrm{X}$ & $X$ & $\mathrm{X}$ & & \\
\hline ambos & & & & & & & & & & & & & & $X$ & $X$ & $?$ & \\
\hline sendos & & & & & & & & & & & & & & $X$ & $X$ & $?$ & \\
\hline
\end{tabular}

Legende: $\mathrm{X}$ kombinierbar,

? fragwürdige Möglichkeiten,

/ Aufeinandertreffen des gleichen Determinierers in Spalte 1. und 2.

(Leerraum) nicht-kombinierbar

Folgende Kombinationen sind als Besonderheiten zu werten und werden in (68) nicht erfaßt: 
- todo este / mi / uno. Todo steht grundsätzlich der gesamten Nominalphrase voran und ist kein notwendiger Bestandteil zum Aufbau der Phrase. Folglich sind die Merkmale (steht in der Umgebung _ A N und steht in der Umgebung Art _ $\mathrm{N}$ ), welche den Aufbau der NP beschreiben, nicht zutreffend. Damit müssen auch die Kombinationsmöglichkeiten von todo anders gewichtet werden. Die Einschränkungen sind eher semantisch begründet.

- uno als 2. Glied steht nur in der Kombination mit todo und in der Folge cada uno. Bei letzterer Folge darf kein Nomen folgen, so daß uno als substantivierte Form gelten muß. Gleiches gilt für cada tanto. Wir können somit davon ausgehen, daß bei substantivierten Determinierern mehr Kombinationsmöglichkeiten gegeben sind als sonst. Die Folge *unos dos ist nicht erlaubt. Da dos für die Klasse der Kardinalia steht und unos cientos, unos cincuenta usw. erlaubt ist, wird die Kombination als möglich markiert: ya está presente en unos cincuenta centros sanitarios de toda Europa (P-5).

- cada muß in cada dos als Adverbial interpretiert werden (vgl. Allegranza 1998: 93). Dies zeigt der unterschiedliche Numerus der NP im Beispielsatz Venía cada dos meses a Madrid (S-13). Cada kann nur im Singular auftreten, dos meses steht im Plural. Folglich ist die Phrase * cada meses ungrammatisch.

- $m i$ als 2. Glied steht nur in der Kombination mit todo und este: todas mis cosas, este mi coche.

- Alvarez Martínez wertet tanto mismo als mögliche Kombination, bringt aber kein Beispiel. Da wir ebenfalls keines vorweisen können, wird die Kombination als unzulässig angesehen.

- Die Numeralia sind in den folgenden Beispielen untereinander kombinierbar: los dos primeros capítulos, los primeros dos capítulos, el primer doble salto usw. Diese Kombinationen aus drei Determinierern lassen sich folgendermaßen erklären: 1. Es handelt sich um eine Kombination wie z.B. in aquellas sus dos obsesiones, wo jeweils die Kombination erster und zweiter Determinierer (aquellas sus als Ausnahme s.o.) sowie die Kombination zweiter und dritter Determinierer (sus dos s. Tab. 68) erlaubt sind. Dieses konkrete Beispiel wurden aber von vielen Informanten als ungewöhnlich eingestuft. Die Nachstellung eines Elements gilt als stilistisch korrekter: aquellas dos obsesiones suyas. Auch ein Komma nach dem ersten Determinierer wurde als stilistisch besser bewertet: aquellas, sus dos obsesiones. 2. Einer der Determinierer operiert wie ein Adjektiv. Das ist in 
den genannten Beispielen der Kombinationen mit Numeralia der Fall. Wie die weitere Untersuchung zeigen wird, verhalten sich primero und doble syntaktisch wie Adjektive und können auch vor einem Determinierer, hier dos, stehen.

- Es scheint viele fragwürdige Konstruktionen zu geben: Informanten ließen eine Vielzahl an Kombinationen zwar zu, bezeichneten sie aber als nichtssagend, da sie beide Determinierer als semantisch synonym oder sehr ähnlich einstuften, z.B. varios und pocos. Ihre Kombination galt als fragwürdig. Zudem wurden auch Kombinationen, die Prado Ibán (1993: 271) als möglich angab, von Informanten als falsch angesehen (z.B. aquel demasiado sol, u.U. regional bedingt).

Es lassen sich von daher folgende erste Beobachtungen zu den Ergebnissen von Tabelle (68) machen:

1. Die ersten fünf Erstdeterminierer ( $m i$ bis otro) lassen sich mit vielen Zweitdeterminierern verbinden, in der Gruppe der Zweitdeterminierer von poco bis mío sogar mit fast allen. Nicht-mögliche Verbindungen sind wahrscheinlich auf semantische Ähnlichkeit oder Unverträglichkeit zurückzuführen (z.B. *este cualquie$r a)$. Es muß untersucht werden, inwieweit die Kombinationsmöglichkeit auf semantischer Grundlage beruht. Der definite Artikel ist natürlich nicht mit dem indefiniten kombinierbar u.ä.

2. Cualquiera und mío sind gesondert zu behandeln. Cualquiera muß im Lexikon mit zwei Einträgen behandelt werden, mío kann nur nachgestellt werden.

3. Die letzten drei Zweitdeterminierer, otro, segundo und doble, sind so auffallend kombinationsfähig, daß sie als Adjektive eingeordnet werden könnten. Poco und mero sind als eine Art Zwischengruppe zu werten.

4. Die restlichen Zweitdeterminierer lassen sich außer mit mi, este, cada und otro nur schlecht kombinieren. Alle stehen eher als einziger Determinierer alleine vor dem Nomen.

5. Wenn man die Zweitdeterminierer von poco bis mío als eine Gruppe erfaßt (2.2) und den Rest als eine zweite (2.1) sowie bei den Erstdeterminierern ähnlich vorgeht ( $m i$ bis otro als 1.1, der Rest als 1.2), so ergibt sich folgende Tabelle. Da mero eine Zwischenposition einnimmt, wird es zunächst nicht berücksichtigt: 


\begin{tabular}{|c|c|c|c|}
\hline \multicolumn{2}{|c|}{ Gruppe } & 2.1 & 2.2 \\
\hline & Determinierer & $\begin{array}{l}\text { bastante, cierto, demás, } \\
\text { demasiado, dos, mismo, } \\
\text { mucho, tal, tanto, varios }\end{array}$ & $\begin{array}{l}\text { cualquiera, } \\
\text { doble, mío, otro, } \\
\text { segundo, poco }\end{array}$ \\
\hline 1.1 & el / la, este, mi / tu, otro, uno & $\mathrm{X}$ & $\mathrm{X}$ \\
\hline 1.2 & $\begin{array}{l}\text { ambos, alguno, bastante, cada, cier- } \\
\text { to, cualquiera, demasiado, mucho, } \\
\text { ninguno, poco, sendos, tal, tanto, } \\
\text { todo, varios }\end{array}$ & & $\mathrm{X}$ \\
\hline
\end{tabular}

Dieses Ergebnis ist aus verschiedenen Gründen unbefriedigend. Erstens zeigt Tabelle (68) einige Fragezeichen, die in Tabelle (69) nicht berïcksichtigt werden. Zweitens finden sich auch viele unzulässige Kombinationen, für die eine passende Erklärung gefunden werden muß. Auch dies ist oben nicht berücksichtigt. Drittens wird die Struktur der Nominalphrase außer acht gelassen, welche die Besonderheit von todo herausstellt. Diese kann aber nach der Klassifikation von Quirk u.a. (1985), die Allegranza fürs Italienische übernimmt, eindeutiger umrissen werden.

\subsubsection{Klassifikation nach Allegranza (1998) und Quirk u.a. (1985)}

Allegranza (1998) übernimmt von Quirk u.a. (1985: 253) eine Klassifikation der Determinierer, die auf der Wortfolge basiert. Quirk u.a. unterteilen in

\section{(70) I Prädeterminierer \\ II Zentraldeterminierer \\ III Postdeterminierer}

Die drei Termini unterscheiden die Determinierer aufgrund ihrer Stellung im Falle von Kookkurrenz. Prädeterminierer stehen grundsätzlich an erster Position, Postdeterminierer stehen immer an letzter bzw. folgen den Prä- und / oder Zentraldeterminierern. Adjektive stehen nach Zentraldeterminierern. Als Prädeterminierer nennt Quirk fürs Englische all und both, Allegranza findet mit entrambi und tutti entsprechende Beispiele fürs Italienische (vgl. eine ähnliche Diskussion über tutti und 
entrambi bei Renzi 1988b: 357ff.). Im Spanischen haben wir nur todo gefunden, das sich identisch verhält. Es steht vor der gesamten Nominalphrase und kann auch vor einigen Determinierern stehen, die dann als Zentraldeterminierer eingeordnet werden. Allerdings läßt es nicht alle Kombinationen zu, wie Tabelle (68) zeigt. Wir müssen also die Kombinationsmöglichkeiten von todo weiterhin mit untersuchen. Die Kombinationsmöglichkeiten legen aber dar, daß todo sich mit Determinierern verbinden kann, die in keiner anderen Kombination die zweite Stelle einnehmen können. Diese Einschränkungen sind anscheinend semantischer Natur. Es gilt nun zu untersuchen, ob todo den Erfordernissen eines Prädeterminierers nach Quirk genügt.

Im direkten Vergleich mit ambos, dessen italienisches Pendant entrambi ebenfalls prädeterminierende Eigenschaften aufweist, zeigt sich die besondere Stellung von todo:

(71) (a) Todos sus / los cuentos son autobiográficos. (S-8)

(b) * Todos cuentos son autobiográficos.

(c) Una gran confusión se ha apoderado de toda Europa. (A-6)

(d) Ambos segundos puestos son atractivos. (aus Prado Ibán 1993: 276)

(e) Ambos puestos son atractivos.

(f) *Ambos los puestos son atractivos.

(71c) zeigt, daß ein Eigenname auf den Prädeterminierer todo folgen kann. Der Eigenname Madrid erfüllt alle Voraussetzungen für eine gesättigte Nominalphrase. Die Beispiele (71d-f) belegen wiederum, daß ambos (als das spanische Äquivalent zu beide) nicht als Prädeterminierer gewertet werden kann. Die Klasse der Prädeterminierer beinhaltet somit nur ein Element. Dies ist aufgrund der beschriebenen besonderen Eigenschaft von todo aber syntaktisch zu rechtfertigen.

Todo ergänzt eine komplette Nominalphrase. Diese Eigenschaft steht in Kontrast zu allen anderen Determinierern (vgl. auch Alvarez Martínez 1986: 171ff.; Bsp. aus Beckmann 1994: 305ff.):
(a) Venid (todos) aquí.
(b) (Todo) está en orden.
(c) He leído (todos) los libros.
(d) ¿Has visto algún libro interesante?
(e) ¿Has visto algún el libro interesante? 
(f) Cada alumno recibe un libro.

(g) *Cada este alumno recibe un libro.

Todo kann auch vor einer Nominalphrase mit Demonstrativ- oder Possessivpronomen, dem Artikel oder otro stehen:

(73) (a) Todos estos cuentos están inéditos / Estos cuentos están inéditos. (S-9)

(b) Tiene todo mi apoyo. (M-12)

(c) Lo sentíamos por todo el cuerpo / Lo sentíamos por el cuerpo. (S-2)

(d) toda otra pretension (Prado Ibán 1993: 270)

Todo kann auch vor einem einzelnen singulären Nomen auftreten. Es muß dann mit 'jeder/s' übersetzt werden und ist mit cada vergleichbar. Caso, elemento und clase bezeichnen in (74) Sachverhalte, die als pars pro toto stellvertretend für die Gesamtmenge stehen. Im ersten Beispiel kann die Phrase todo caso auch als feste Redewendung interpretiert werden:

(a) En todo caso sólo puede hablarse en términos más estrictos de un cereal. (RA-1)

(b) Se lo separa así de todo elemento extraño que pueda arrastrar. (RA-1)

(c) Se han destapado toda clase de corrupciones. (A-5)

Die Sonderstellung von todo beweist, daß wir die Struktur der Nominalphrase berücksichtigen müssen. Todo übernimmt offensichtlich eine andere Funktion in der Nominalphrase als der folgende Determinierer, der immer auch allein vor dem Nomen stehen kann. Damit haben wir es bei todo mit einer ganz anderen Art von Determinierer zu tun, da todo eine Nominalphrase nicht eigenständig determinieren kann, es sei denn, es wird im Sinne von cada verwendet (vgl. auch Longobardi 1988: 661ff. für ital. ogni vs. tutti). Diese Ergebnisse müssen als strukturelle Eigenart von todo gewertet werden, die sich nur bedingt in eine Klassifikation integrieren lassen. Wir können zwar ein Attribut [Prädeterminierer \pm ] einführen, das nur auf todo positiv zutrifft, müssen aber die Sonderstellung unter den spanischen Determinierern hervorheben. Im folgenden wird todo als Prädeterminierer und im Sinne von todo + Artikel (z.B. todos los coches) und nicht analog zu cada analysiert. 


\subsubsection{Zentral- und Postdeterminierer}

Die Aufteilung in Zentral- und Postdeterminierer erweist sich aufgrund der Menge der verbleibenden Determinierer als schwierig. Allegranza (1998: 61ff.) überträgt englisch each und few als ogni und pochi ins Italienische und deklariert ogni als Zentraldeterminierer und pochi als Postdeterminierer. Auf mehr empirische Beispiele geht er leider nicht ein und bringt auch keine Gesamtübersicht der italienischen Determinierer.

Im Spanischen verhält es sich zumindest mit den bereits genannten Determinierern genauso: cada ist ein Zentraldeterminierer und poco ein Postdeterminierer. Sie werden auch bei Prado Ibán in die ihrer Klassifikation entsprechenden Gruppen A und B eingeordnet (Beispiele aus Prado Ibán 1993: 270ff.).

(a) Cada segundo domingo me visita.

(b) Es aconsejable visitar al dentista cada poco tiempo.

(c) cada libro.

(d) *un/el cada libro.

(e) (los) pocos niños / *pocos los niños.

(f) esos pocos niños.

(g) algún otro muchacho / *otro algún muchacho.

(h) jotros dos cafés, por favor! / ?dos otros cafés.

(i) otros muchos chicos / muchos otros chicos.

Cada kann eine NP determinieren und dabei sowohl allein als auch mit folgendem Postdeterminierer stehen (75a-c). Es ist mit dem Artikel nicht kombinierbar (75d). Poco hingegen kann mit dem Artikel kombiniert werden (75e) und nach Zentraldeterminierern (75f) stehen wie auch allein (75e). Die Unterscheidung Zentral- vs. Postdeterminierer ergibt sich also aus der Stellung bei Kookkurrenz: Wenn ein weiterer Determinierer folgen kann, handelt es sich beim ersten um einen Zentraldeterminierer (vgl. cada in (75b)). Wenn ein Determinierer einem Zentraldeterminierer folgen kann, handelt es sich um einen Postdeterminierer (vgl. poco in (75b)).

Bei einigen Determinierern ist die Frage der Einordnung schwierig. Otro z.B. ist mit sehr vielen Zentraldeterminierern kompatibel und könnte von daher als Postdeterminierer eingeordnet werden (z.B. 75g). Allerdings stellen die Beispiele (75h) und (75i) dies in Frage, denn otro steht vor dos und mucho, die ebenfalls als Postdetermi- 
nierer eingeordnet werden müßten. Wir lassen also entweder Kombinationen zwischen Postdeterminierern zu, wie Prado Ibán sie auch für ihre Klasse B annimmt allerdings mit den schon genannten zahlreichen Gegenbeispielen -, oder wir erlauben Ausnahmen, z.B. auf der Grundlage von semantischer Unverträglichkeit oder anderen nicht diesem Bereich zugehörigen Merkmalen.

Ein weiteres Problem wird von Allegranza beim Italienischen thematisiert: die möglichen Kombinationen mit Adjektiven. Er weist für das Italienische nach, daß ein Zahlwort und ein Possessivpronomen völlig frei miteinander und zudem mit jedem beliebigen Adjektiv kombiniert werden können. Dies ist für das Spanische äußerst fragwürdig, da die Funktion des Artikels vom Possessivpronomen übernommen wird. Zunächst die Beispiele von Allegranza (Allegranza 1998: 74):
(a) $i$ tuoi due famosi libri (Abfolge: $i$ Pos Zahl ${ }^{26}$ Adj libri)
(b) $i$ due tuoi famosi libri (Abfolge: $i$ Zahl Pos Adj libri)
(c) $i$ due famosi tuoi libri (Abfolge: $i$ Zahl Adj Pos libri)
(d) $i$ tuoi famosi due libri (Abfolge: $i$ Pos Adj Zahl libri)
(e) ifamosi tuoi due libri (Abfolge: $i$ Adj Pos Zahl libri)

In der Umgebung $i_{\text {_ }}$ libri sind bis auf die Reihenfolge *Adjektiv < Zahlwort < Possessiv (* i famosi due tuoi libri) alle Kombinationen möglich. Im Spanischen können nur die folgenden als wohlgeformt gelten. Da die Possessiva in vorangestellte $\left(\operatorname{Pos}_{1}\right)$ und nachgestellte $\left(\mathrm{Pos}_{2}\right)$ unterteilt werden, erfolgt eine Differenzierung durch Index:
(a) tus dos famosos libros
(Abfolge: Pos $_{1}$ Zahl Adj libros)
(b) tus famosos dos libros
(Abfolge: Pos $_{1}$ Adj Zahl libros)
(c) los dos primeros capítulo
(Abfolge: Art Zahl Ord libros)
(d) los primeros dos capítulos
(Abfolge: Art Ord Zahl libros)

Die Beispiele in (c) und (d) stehen parallel zu (a) und (b). Die Ordinalia verhalten sich auffällig ähnlich zu den Adjektiven.

Es gibt im Spanischen noch die Möglichkeit der Nachstellung. Folgende Beispiele sind ebenfalls korrekt:

\footnotetext{
${ }^{26}$ Zur Vermeidung von Verwechslungen steht hier „Zahl“ als Abkürzung für „Zahlwort“. „Num“ für „Numeral“ könnte mit Numerus verwechselt werden.
} 

(a) tus dos libros famosos
(Abfolge: Pos $_{1}$ Zahl libros Adj)
(b) los dos famosos libros tuyos (Abfolge: Art Zahl Adj libros $\operatorname{Pos}_{2}$ )
(c) los famosos dos libros tuyos (Abfolge: Art Adj Zahl libros $\mathrm{Pos}_{2}$ )

Das vorangestellte Possessivpronomen $\left(\mathrm{Pos}_{1}\right) \mathrm{mu}$ an erster Stelle der Determiniererfolge stehen. Es übernimmt die Funktion des Artikels. Die Kombinationsmöglichkeiten sind also im Spanischen wesentlich eingeschränkter und lassen sich auf folgende Formel bringen (vgl. Zimmermann 1992: 257 für eine ähnliche Regel des Deutschen; „২" bedeutet ,steht vor"):

\section{Abfolgeregeln für spanische Determinierer}

1. Possessiv 1 / Artikel < Zahlwort < Nomen; und

2. Possessiv $_{1} /$ Artikel $<$ Adjektiv

Die Stellung des Adjektivs ist innerhalb des gegebenen Rahmens frei. Nur Possessivpronomen oder Artikel müssen vorangehen. Dies ist ein erster Hinweis auf das Merkmal valor artículo von Prado Ibán. Eine bessere Umschreibung ist valor identificador (Alvarez Martínez 1986: 55-65). Es muß im Spanischen immer ein Determinierer vorangehen - wenn das Nomen nicht allein stehen kann (s. Kapitel III.1.). Dieses Merkmal wird in unserer Klassifikation durch das Merkmal [steht in Umgebung _ A N ] erfaßt.

Die spanischen Determinierer mit der Annahme von drei Positionsklassen zu erklären, scheint nur eingeschränkt möglich zu sein. Es bleibt die Frage, wie die Kombinationsmöglichkeiten von Postdeterminierern zu verstehen sind. Allegranza (1998) z.B. geht davon aus, daß Numeralia wie dos und u.U. auch quantifizierende Ausdrücke wie mucho anders behandelt werden müssen.

Wenn wir den Zentral- und Postdeterminierern die genannten Eigenschaften und zusätzlich die der Klassen A und B (nach Prado Ibán) zusprechen, ergibt sich folgende Liste:

(80) Zentraldeterminierer:

- stehen vor Postdeterminierern,

- stehen immer vor Adjektiven,

- sind nicht frei untereinander kombinierbar. 
Postdeterminierer:

- stehen nach Zentraldeterminierern,

- stehen evtl. nach Adjektiven,

- sind frei untereinander kombinierbar.

Todo wurde als einziges Element der Klasse Prädeterminierer definiert. Bei der Klassifikation der restlichen Determinierer stellen sich nun zwei Fragen:

- Welche Determinierer gehören in welche Klasse?

- Ist die Aufteilung in zwei Klassen (s. die Einteilung bei Prado Ibán und die jetzt nach Abzug des Prädeterminierers verbleibenden zwei bei Allegranza) korrekt und bildet die empirisch gewonnenen Sachverhalte adäquat $a b$ ?

Wir gehen die Beispiele durch und versuchen mit möglichst eindeutigen Fällen zu beginnen.

Wie Tabelle (68) zeigt, heben sich segundo und doble klar von allen anderen Determinierern ab. Sie können als Postdeterminierer gewertet werden. Cualquiera und mío müssen als nachgestellte Determinierer wahrscheinlich eher adjektisch betrachtet werden, d.h. es sind grundsätzlich alle Kombinationsmöglichkeiten erlaubt, sofern sie einen Sinn ergeben. Damit können sie in dieser Klassifikation außen vor bleiben.

Poco dürfte ebenfalls als Postdeterminierer zu werten sein, da sich die fehlenden Kombinationsmöglichkeiten semantisch begründen lassen. Ob auch mero als Postdeterminierer betrachtet und analog zu poco als semantisch eingeschränkt gewertet werden kann, erscheint anhand der vielen nicht-möglichen Kombinationen von mero fraglich. Otro kann problemlos ebenfalls als Postdeterminierer eingestuft werden. Es läßt aber auch in Erststellung zahlreiche Kombinationen zu. Damit entsteht eine kleine Klasse von Postdeterminierern mit drei eindeutig zugeordneten Mitgliedern: segundo, doble und poco. Bei otro und mero bleiben Zweifel. Cualquiera und mío werden als frei kombinierbare, adjektisch zu wertende Determinierer eingeschätzt, die nur semantisch bedingte Restriktionen kennen.

Bei den Zentraldeterminierern lassen sich schnell zwei Klassen ausmachen: das Possessivpronomen $\mathrm{mi} / \mathrm{tu}$, die Demonstrativpronomina este / ese / aquel, cada und otro sowie in eingeschränktem Maß die Artikel el / la und uno sind mit fast allen anderen Determinierern kombinierbar. Zwar sind auch hier wieder einige Ausnahmen zu beobachten, aber diese Gruppe erlaubt wesentlich mehr Möglichkeiten als 
die übrigen Determinierer, die in einer zweiten Klasse zusammengefaßt werden können.

Wenn wir mit diesen Annahmen eine Tabelle wie (69) erstellen, dann sieht diese wie folgt aus. Inwieweit die Annahme der Kombinierbarkeit oder NichtKombinierbarkeit sich als korrekt erweist, geben die Prozentzahlen in der jeweiligen Zelle an (X bedeutet kombinierbar, - nicht kombinierbar). Das Aufeinandertreffen des gleichen Determinierers wird nicht erfaßt:

\section{Klassifikationsversuch der Kombinationsmöglichkeiten}

\begin{tabular}{|l|l|l|l|l|}
\hline \multicolumn{1}{|c|}{2.} & $\begin{array}{l}\text { Zentraldeterminierer B: } \\
\text { tal, cierto, demasiado, ba- } \\
\text { stante, tanto, demás, mucho, } \\
\text { varios, mismo, dos }\end{array}$ & $\begin{array}{l}\text { Postdeterminierer: } \\
\text { mero, poco, segun- } \\
\text { do, doble }\end{array}$ & $\begin{array}{l}\text { otro } \\
\text { mío }\end{array}$ \\
\hline $\begin{array}{l}\text { Zentraldeterminierer A: } \\
\text { el / la, mi / tu, este, cada, otro, uno }\end{array}$ & $\begin{array}{l}\mathrm{X} \\
(51,6 \%)\end{array}$ & $\begin{array}{l}\mathrm{X} \\
(79,2 \%)\end{array}$ & $\mathrm{X}$ & $\mathrm{X}$ \\
\hline $\begin{array}{l}\text { Zentraldeterminierer B: } \\
\text { cualquiera, alguno, ninguno, todo, } \\
\text { tanto, bastante, demasiado, cierto, } \\
\text { varios, mucho, poco, tal, ambos, } \\
\text { sendos }\end{array}$ & $(100 \%)$ & $\mathrm{X}$ & $(60 \%)$ & $(54,5 \%)$ \\
\hline
\end{tabular}

Tabelle (81) ist in vielerlei Hinsicht eine so extreme Idealisierung, daß Zweifel an der Untersuchungsmethode aufkommen müssen, da wir in Tabelle (68) viele Fragezeichen und unerlaubte Kombinationen an Stellen hatten, die hier als kombinierbar erfaßt werden. Wenn wir als Beispiel die Kombination eines Zentraldeterminierers A mit einem Zentraldeterminierer B betrachten, so finden sich bei theoretisch insgesamt 60 möglichen Kombinationen 25 unerlaubte und vier fragwürdige Kombinationen. Damit trifft die Markierung als kombinierbar nur auf 51,6\% der entsprechenden Fälle tatsächlich zu. 48,3\% sind fragwürdige oder unerlaubte Kombinationen. Bei den anderen Kombinationsmöglichkeiten ergeben sich nur vereinzelt höhere Werte. Der Durchschnitt liegt bei 79,3\%. Damit erweist sich die Klassifikation von Quirk u.a. (1985) in dieser Form als unzureichend für die Kombinationsmöglichkeiten spanischer Determinierer.

Für die Abgrenzung von Determinierern und Adjektiven hatten wir festgehalten, daß es wahrscheinlich kaum möglich ist, halbwegs eindeutige Klassen zu erstellen. Stattdessen wurde eine Abstufung vorgeschlagen, um anhand von Merkmalen die Distanz zu berechnen. Diese Idee könnte auch wegweisend für die Kombinations- 
möglichkeiten der Determinierer sein. Es läßt sich relativ schnell erkennen, daß die Kombinationsfähigkeit als Erst- oder Zweitdeterminierer von einem Determinierer zum nächsten leicht ab- oder zunimmt. Von daher erstellen wir im folgenden eine graduelle Abstufung.

\subsubsection{Abstufende Klassifikation}

Die einfachste Methode, um die in der Tabelle (68) erkennbare Abstufung darzulegen, ist es, die Kombinationsmöglichkeiten als Erst- bzw. Zweitdeterminierer zu summieren und danach jeweils für jede der beiden möglichen Positionen Klassen zu bilden. Damit entstehen zwei Gruppen: die Klassen der Erstdeterminierer (im folgenden die Klassen a, b, c, ...) und die der Zweitdeterminierer (die Klassen 1, 2, $3, \ldots)$, geordnet nach der Anzahl ihrer Kombinationsmöglichkeiten. Da es sich jeweils um die gleichen Wörter handelt, werden neue Klassen in der Weise gebildet, daß der Durchschnitt aus den Klassen der Erst- und der Zweitdeterminierer erstellt wird. Diese Klassen nennen wir entsprechend a1, a2, b2, b3 usw. Im ungünstigsten Fall befinden sich im Endergebnis keine zwei Wörter in der gleichen Klasse, und es entstehen so viele Klassen wie Wörter. Im günstigsten Fall sind beide Klassifikatio-

nen identisch, und es treten keine Veränderungen ein. Diese Abstufung kann wie bei der Abgrenzung Determinierer - Adjektiv nur eine Annäherung darstellen, da immer wieder Ausnahmen zu finden sind.

Wir erhalten folgende zwei Listen, geordnet nach der Anzahl der Kombinationsmöglichkeiten, ergänzt durch die Anzahl der fraglichen Kombinationen. Nachgestellte Determinierer wie mío und cualquiera werden nicht berücksichtigt. Da wir todo schon besonders klassifiziert haben, werden die als Ausnahmen deklarierten Kombinationsmöglichkeiten nicht erfaßt, auch wenn todo hier nochmals aufgeführt wird: 
(82) Liste der Kombinationen in erster und zweiter Position

\begin{tabular}{|c|c|c|c|c|c|c|c|}
\hline \multicolumn{4}{|c|}{ Erste Position } & \multicolumn{4}{|c|}{ Zweite Position } \\
\hline 1. & $m i$ & 13 & \multirow[t]{2}{*}{$\mathrm{a}$} & 1. & segundo & \multirow[t]{2}{*}{20} & \multirow[t]{2}{*}{1} \\
\hline 2. & este & $11(+1)$ & & 2. & doble & & \\
\hline 3. & uno & $8(+1)$ & \multirow[t]{3}{*}{$\mathrm{b}$} & 3. & otro & $16(+1)$ & 2 \\
\hline 4. & $e l / l a$ & 8 & & 4. & poco & $11(+2)$ & 3 \\
\hline 5. & otro & 7 & & 5. & mero & 6 & \multirow[t]{5}{*}{4} \\
\hline 6. & cada & $5(+3)$ & \multirow[t]{13}{*}{$\mathrm{c}$} & 6. & $d o s$ & 5 & \\
\hline 7. & cualquiera & $5(+1)$ & & 7. & mismo & \multirow[t]{3}{*}{$4(+1)$} & \\
\hline 8. & todo & \multirow[t]{2}{*}{5} & & 8. & varios & & \\
\hline 9. & alguno & & & 9. & mucho & & \\
\hline 10. & bastante & $4(+1$ & & 10. & tal & 3 & 5 \\
\hline 11. & ninguno & \multirow[t]{3}{*}{4} & & 11. & bastante & $2(+1)$ & \\
\hline 12. & demasiado & & & 12. & tanto & \multirow[t]{3}{*}{2} & \\
\hline 13. & cierto & & & 13. & cierto & & \\
\hline 14. & tanto & \multirow[t]{2}{*}{$3(+1)$} & & 14. & demás & & \\
\hline 15. & varios & & & 15. & demasiado & $1(+2)$ & \\
\hline 16. & mucho & \multirow[t]{3}{*}{3} & & 16. & $m i$ & \multirow[t]{11}{*}{0} & \multirow[t]{11}{*}{6} \\
\hline 17. & poco & & & 17. & este & & \\
\hline 18. & tal & & & 18. & uno & & \\
\hline 19. & ambos & \multirow[t]{2}{*}{2} & \multirow[t]{2}{*}{$\mathrm{d}$} & 19. & el / la & & \\
\hline 20. & sendos & & & 20. & cada & & \\
\hline 21. & mero & \multirow[t]{6}{*}{0} & \multirow[t]{6}{*}{$\mathrm{e}$} & 21. & cualquiera & & \\
\hline 22. & dos & & & 22. & todo & & \\
\hline 23. & mismo & & & 23. & alguno & & \\
\hline 24. & demás & & & 24. & ninguno & & \\
\hline 25 . & segundo & & & 25. & ambos & & \\
\hline 26. & doble & & & 26. & sendos & & \\
\hline
\end{tabular}


Die umfangreiche Klasse c könnte bei den Erstdeterminierern nochmals aufgeteilt werden. Dies ist aufgrund der nun folgenden Verknüpfung der beiden Klassifikationen nicht nötig, da die Mitglieder dieser Klasse ohnehin getrennt werden. Durch die Durchschnittsbildung entstehen folgende elf Klassen. Die Bezeichnung spiegelt die Verknüpfung wider: c6 ist der Durchschnitt der Klasse c in erster Position mit der Klasse 6 in zweiter Position.

Durchschnitt der Klassen aus (82)

\begin{tabular}{|c|c|c|c|c|c|c|c|c|}
\hline 1. & $m i$ & \multirow[t]{2}{*}{ a6 } & 11. & bastante & \multirow[t]{5}{*}{$\mathrm{c5}$} & 19. & ambos & \multirow[t]{2}{*}{ d6 } \\
\hline 2. & este & & 12. & demasiado & & 20. & sendos & \\
\hline 3. & uno & \multirow[t]{2}{*}{ b6 } & 13. & cierto & & 21. & mero & \multirow[t]{3}{*}{ e4 } \\
\hline 4. & el / la & & 14. & tanto & & 22. & dos & \\
\hline 5. & otro & b2 & 15. & tal & & 23. & mismo & \\
\hline 6. & cada & \multirow[t]{5}{*}{ c6 } & 16. & varios & \multirow[t]{2}{*}{$\mathrm{c} 4$} & 24. & demás & e5 \\
\hline 7. & cualquiera & & 17. & mucho & & 25. & segundo & \multirow[t]{2}{*}{ e1 } \\
\hline 8. & todo & & 18. & poco & c3 & 26. & doble & \\
\hline 9. & alguno & & & & & & & \\
\hline 10. & ninguno & & & & & & & \\
\hline
\end{tabular}

Wir können nun die Kombinationsmöglichkeiten dieser Klassen zueinander in Beziehung setzen und dabei einige Klassen bereits zusammenfassen, die identische oder fast identische Kombinationsmöglichkeiten kennen. Dabei kennzeichnet „,“ das Aufeinandertreffen der gleichen Klasse, „,+“ zeigt an, daß alle Mitglieder der beiden Klassen jeweils miteinander in der betreffenden Reihenfolge kombiniert werden können, „,-“ bedeutet nicht kombinierbar und „o“ meint, daß die Mitglieder der beiden Klassen teilweise miteinander kombiniert werden können. Die Klassen c4 und c5 sowie e4 und e5 wurden aufgrund sehr geringer, unerheblicher Unterschiede zusammengefaßt. 
Kombinationsmöglichkeiten der vereinigten Klassen aus (83)

\begin{tabular}{|c|c|c|c|c|c|c|c|c|c|}
\hline${ }_{1 .}^{2} \quad 2$. & $\mathrm{a} 6$ & b6 & b2 & c6 & $\begin{array}{c}\mathrm{c} 4+ \\
\mathrm{c} 5\end{array}$ & $\mathrm{c} 3$ & d6 & $\begin{array}{c}\mathrm{e} 4+ \\
\mathrm{e} 5\end{array}$ & e1 \\
\hline a6 & 1 & - & + & - & $\mathrm{O}$ & + & - & $\mathrm{O}$ & + \\
\hline b6 & - & 1 & + & - & $\mathrm{O}$ & 0 & - & O & + \\
\hline b2 & - & - & 1 & - & 0 & + & - & $\mathrm{O}$ & + \\
\hline c6 & - & - & $\mathrm{O}$ & I & $\mathrm{O}$ & $\mathrm{O}$ & - & $\mathrm{O}$ & + \\
\hline $\mathrm{c} 4+\mathrm{c} 5$ & - & - & + & - & 1 & 0 & - & - & + \\
\hline c3 & - & - & + & - & - & 1 & - & - & + \\
\hline d6 & - & - & - & - & - & - & 1 & - & + \\
\hline $\mathrm{e} 4+\mathrm{e} 5$ & - & - & - & - & - & - & - & 1 & - \\
\hline e1 & - & - & - & - & - & - & - & - & I \\
\hline
\end{tabular}

Die Klassen können nun charakterisiert werden: a6 und b6 weisen Charakteristika auf, die auf Zentraldeterminierer zutreffen. Sie sind mit Postdeterminierern kombinierbar und stehen nie in Zweitstellung außer nach einem Prädeterminierer. Das gleiche gilt für die Klassen c6 und d6. Allerdings sind ihre Kombinationsmöglichkeiten bereits eingeschränkt, denn c6 läßt sich nicht mit den eine größere Anzahl von Determinierern enthaltenden Klassen $\mathrm{c} 4+\mathrm{c} 5$ verbinden, d6 kann nur mit e1 verbunden werden. Wir können hier eine Abstufung bei den als Zentraldeterminierern bezeichneten Determinierern wahrnehmen und eine Subklassifikation der Zentraldeterminierer in drei Subklassen vorschlagen.

Für die Postdeterminierer gilt ähnliches. Die Determinierer in den Klassen e4, e5 und e1 treten nie in Erststellung auf. Die Kombinationsmöglichkeiten sind allerdings erneut ziemlich unterschiedlich: e1 ist mit allen Determinierern außer e4 und e5 kombinierbar, für e4 und e5 sind die Möglichkeiten erheblich eingeschränkt. Die restlichen drei Klassen b2, c4 + c5 und c3 unterscheiden sich wiederum in ihren Möglichkeiten. Eine Trennung der Postdeterminierer zunächst nach dem Kriterium der möglichen Erststellung scheint angebracht. Eine weitere Einteilung, wie wir sie für die Zentraldeterminierer vorschlugen, ist natürlich auch hier möglich.

Allerdings muß an dieser Stelle überlegt werden, wie genau eine Klassifikation überhaupt sein kann. Wie bereits mehrfach erläutert wurde, handelt es sich in Tabelle (68) nicht um eine klare Darstellung der Kombinationsmöglichkeiten, sondern um eine Annäherung. Einige Kombinationen sind eindeutig möglich und werden häufig verwendet (los dos coches), andere sind möglich, aber ziemlich selten (algún otro ingrediente, S-5). Manche sind fragwürdig und wurden von befragten Muttersprachlern z.T. abgelehnt (aquel demasiado sol, Prado Ibán 1993: 271). Wenn nun Klassen 
von kombinierbaren Determinierern gebildet werden, kann es sich weiterhin nur um eine Annäherung handeln, die mit ziemlicher Sicherheit erlaubte und unerlaubte Kombinationen enthält, die dem Leser fragwürdig erscheinen. Konsequenterweise ist eine genaue Klassifizierung nicht möglich. Wir definieren von daher Klassen, die über bestimmte Merkmale verfügen, wie z.B. daß ein Determinierer nie in Erststellung steht. Damit folgen wir dem Ansatz von Alvarez Martínez, ausgewählte Merkmale zu gewichten. Wir schlagen folgende Einteilung vor:

1. Prädeterminierer: todo (aus c6).

2. Zentraldeterminierer: mi, este, cada, uno, el / la, cualquiera, alguno, ninguno, ambos, sendos (Klasse a6 + b6, c6, d6).

3. Postdeterminierer A: otro, varios, mucho, tanto, bastante, demasiado, cierto, tal, poco (Klasse b2, c4 + c5, c3).

4. Postdeterminierer B: mismo, dos, mero, demás, segundo, doble (Klasse e4 + e5, e1).

Diese Klassifikation basiert auf folgenden Merkmalen:

1. Prädeterminierer: steht vor der gesamten Nominalphrase.

2. Zentraldeterminierer: mit Postdeterminierern kombinierbar, aber nicht untereinander.

3. Postdeterminierer A: untereinander und mit Postdet. B kombinierbar.

4. Postdeterminierer B: nie in Erststellung und folglich auch nicht untereinander kombinierbar.

Die oben genannte Eigenschaft von Postdeterminierern, sowohl vor als auch nach qualifizierenden Adjektiven zu stehen, wenn diese vor dem Nomen positioniert sind, wurde für dos nachgewiesen. Inwieweit dies auf andere Determinierer dieser Klasse zutrifft und nicht von der Eigenschaft von dos abhängig ist, ein Zahlwort zu sein, müßte noch belegt werden.

Es wurde bereits betont, daß es sich nur um eine Annäherung handeln kann. Wir können dies sehr einfach verdeutlichen, indem in die Tabelle (68) die jetzt postulierten Klassen integriert werden. Wir teilen dabei einmal in die Gruppen Prä-, Zentralund Postdeterminierer (A und B) sowie in die oben gewonnenen Klassen nach der Häufigkeit des Vorkommens (e1 bis a6) ein. Todo wird dabei weiterhin als Prädeterminierer gewertet und nicht in die Klasse c6 integriert. 


\begin{tabular}{|c|c|c|c|c|c|c|c|c|c|c|c|c|c|c|c|c|c|}
\hline \multirow{2}{*}{\multicolumn{3}{|c|}{$\begin{array}{l}\text { Klassifikation nach } \\
\text { Zentral- und Postdet. } \\
\text { Klassifikation }\end{array}$}} & \multicolumn{9}{|c|}{ Postdeterminierer A } & \multicolumn{6}{|c|}{ Postdeterminierer B } \\
\hline & & & \multirow[t]{2}{*}{ b2 } & \multirow[t]{2}{*}{ c3 } & \multicolumn{7}{|c|}{$\mathrm{c} 4+\mathrm{c} 5$} & \multicolumn{4}{|c|}{ e4 +e5 } & \multicolumn{2}{|l|}{ e1 } \\
\hline & $\begin{array}{l}\text { nach } \\
\text { Tab. } \\
\text { (83) }\end{array}$ & & & & ミ & $\frac{2}{8}$ & 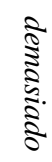 & 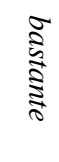 & $\widehat{\Xi}$ & 亏ั & 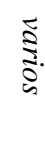 & 离 & 密. & Бे & $\frac{\Im}{5}$ & 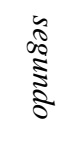 & $\frac{2}{\delta}$ \\
\hline Prä. & (c6) & todo & $\mathrm{X}$ & $\mathrm{X}$ & & & & & & & & & & & $\mathrm{X}$ & $\mathrm{X}$ & $\mathrm{X}$ \\
\hline \multirow{10}{*}{ 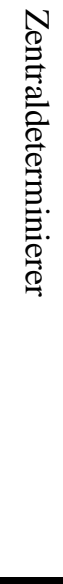 } & \multirow[t]{2}{*}{ a6 } & $m i, t u$ & $\mathrm{X}$ & $\mathrm{X}$ & & $\mathrm{X}$ & $\mathrm{X}$ & $\mathrm{X}$ & $\mathrm{X}$ & $\mathrm{X}$ & $\mathrm{X}$ & $\mathrm{X}$ & $\mathrm{X}$ & $\mathrm{X}$ & & $\mathrm{X}$ & $\mathrm{X}$ \\
\hline & & este & $\mathrm{X}$ & $\mathrm{X}$ & $\mathrm{X}$ & & $?$ & $\mathrm{X}$ & & $\mathrm{X}$ & $\mathrm{X}$ & & $\mathrm{X}$ & $\mathrm{X}$ & $\mathrm{X}$ & $\mathrm{X}$ & $\mathrm{X}$ \\
\hline & \multirow[t]{2}{*}{ b6 } & uno & $\mathrm{X}$ & $\mathrm{X}$ & $\mathrm{X}$ & $\mathrm{X}$ & & & & & $?$ & & $\mathrm{X}$ & $\mathrm{X}$ & & $\mathrm{X}$ & $\mathrm{X}$ \\
\hline & & $e l, l a$ & $\mathrm{X}$ & & $\mathrm{X}$ & & & & & & & $\mathrm{X}$ & $\mathrm{X}$ & $\mathrm{X}$ & $\mathrm{X}$ & $\mathrm{X}$ & $\mathrm{X}$ \\
\hline & \multirow[t]{4}{*}{ c6 } & cada & $?$ & $\mathrm{X}$ & & & $?$ & $?$ & & $\mathrm{X}$ & $\mathrm{X}$ & & & & & $\mathrm{X}$ & $\mathrm{X}$ \\
\hline & & cualquiera & $\mathrm{X}$ & $\mathrm{X}$ & & & & & & & & & $?$ & & $\mathrm{X}$ & $\mathrm{X}$ & $\mathrm{X}$ \\
\hline & & alguno & $\mathrm{X}$ & $\mathrm{X}$ & & & & & & & & & & & $\mathrm{X}$ & $\mathrm{X}$ & $\mathrm{X}$ \\
\hline & & ninguno & $\mathrm{X}$ & & & & & & & & & & & & $\mathrm{X}$ & $\mathrm{X}$ & $\mathrm{X}$ \\
\hline & \multirow[t]{2}{*}{ d6 } & ambos & & & & & & & & & & & & & & $\mathrm{X}$ & $\mathrm{X}$ \\
\hline & & sendos & & & & & & & & & & & & & & $\mathrm{X}$ & $\mathrm{X}$ \\
\hline \multirow{9}{*}{ 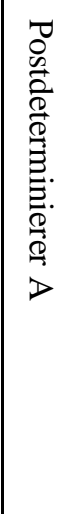 } & b2 & otro & I & $\mathrm{X}$ & & & & & $\mathrm{X}$ & $\mathrm{X}$ & $\mathrm{X}$ & & & $\mathrm{X}$ & & $\mathrm{X}$ & $\mathrm{X}$ \\
\hline & c3 & poco & $\mathrm{X}$ & I & & & & & & & & & & & & $\mathrm{X}$ & $\mathrm{X}$ \\
\hline & \multirow{7}{*}{$\begin{array}{l}\mathrm{c} 4+ \\
\mathrm{c} 5\end{array}$} & varios & $\mathrm{X}$ & $?$ & & & & & & & I & & & & & $\mathrm{X}$ & $\mathrm{X}$ \\
\hline & & mucho & $\mathrm{X}$ & & & & & & & I & & & & & & $\mathrm{X}$ & $\mathrm{X}$ \\
\hline & & tanto & $\mathrm{X}$ & ? & & & & & I & & & & & & & $\mathrm{X}$ & $\mathrm{X}$ \\
\hline & & bastante & $\mathrm{X}$ & $\mathrm{X}$ & & & & I & & $?$ & & & & & & $\mathrm{X}$ & $\mathrm{X}$ \\
\hline & & demasiado & $\mathrm{X}$ & $\mathrm{X}$ & & & I & & & & & & & & & $\mathrm{X}$ & $\mathrm{X}$ \\
\hline & & cierto & $\mathrm{X}$ & & & I & & & & & & & & & & $\mathrm{X}$ & $\mathrm{X}$ \\
\hline & & tal & $\mathrm{X}$ & & I & & & & & & & & & & & $\mathrm{X}$ & $\mathrm{X}$ \\
\hline
\end{tabular}

Die Klassifikation in Prä-, Zentral- und Postdeterminierer erweist sich als starke Idealisierung gegenüber der Aufteilung in die neun Klassen e1 bis a6. Eine Einteilung der Determinierer nach dem Verfahren von Quirk u.a. (1985) bringt letztlich keine zufriedenstellenden Ergebnisse. Wenn die Kombinationsmöglichkeiten nach den hier vorliegenden Informationen erfaßt werden sollen, müssen sie in die neun Klassen eingeteilt werden, die offenbar die Verhältnisse relativ gut erfassen.

Für eine Klassifikation nach Merkmalen, die in die HPSG übertragbar ist, sind diese Klassen zu komplex. Wir können aber auf die Definitionskriterien zurückgreifen, die oben zur Klassenbildung verwendet wurden. Dies waren die folgenden drei: 

(a) steht vor der gesamten Nominalphrase (Prädeterminierer)
(b) mit Postdeterminierern kombinierbar (Zentraldeterminierer)
(c) untereinander kombinierbar
(Postdeterminierer A)

Werden diese Merkmale auf die vier Klassen verteilt, ergibt dies folgende Tabelle.

\section{Merkmale der Kombinationsklassen (vorläufig)}

\begin{tabular}{|l|c|c|c|c|}
\hline & Prädet. & Zentraldet. & Postdet. A & Postdet. B \\
\hline steht vor der NP & + & - & - & - \\
\hline mit Postdet B. kombinierbar & + & + & + & - \\
\hline untereinander kombinierbar & - & - & + & - \\
\hline
\end{tabular}

Es ist anhand der drei Merkmale möglich, die vier Gruppen zu differenzieren. Allerdings hatten wir schon vorher bemerkt, daß der Prädeterminierer todo sich von allen anderen unterscheidet, da er vor der Nominalphrase steht und somit schon ausreichend durch die Merkmale über den Aufbau der Nominalphrase [steht in Umgebung _ A N -] und [steht in Umgebung Art _ $\mathrm{N} \mathrm{-]} \mathrm{abgegrenzt} \mathrm{wird.} \mathrm{Die} \mathrm{anderen} \mathrm{beiden}$ Merkmale können in [Erststellung \pm ] und [Zweitstellung \pm ] umbenannt werden wobei sie sich nur auf die Stellung innerhalb der Nominalphrase beziehen - und erfassen weiterhin die gleiche Information. Zentral- und Prädeterminierer sowie Postdeterminierer A können in Erststellung stehen, alle Postdeterminierer (A und B) können in Zweitstellung stehen.

\section{Merkmale der Kombinationsklassen (endgültig)}

\begin{tabular}{|l|c|c|c|}
\hline & Zentraldet. & Postdet. A & Postdet. B \\
\hline Erststellung & + & + & - \\
\hline Zweitstellung & - & + & + \\
\hline
\end{tabular}

Da diese Einteilung auf Quirk u.a. (1985) basiert, ist sie nach den obigen Ergebnissen noch unzureichend, um alle Kombinationsmöglichkeiten zu erfassen. Wir können sie aber durch eine semantische Betrachtung der Kookkurrenz ergänzen (s. Kap. III.2.5.). Mit Erststellung werden alle Determinierer außer demás, doble, dos, más, mero, mío, mismo und segundo markiert. Zweitstellung ist positiv zu werten für bastante, cierto, demás, demasiado, doble, dos, mero, mismo, mucho, otro, segundo, tal, tanto und varios. 


\subsection{Semantische Aspekte}

In diesem Kapitel erfolgt eine semantische Analyse der Determinierer. Die Ansätze dazu sind in den konsultierten Grammatiken zum Teil sehr unterschiedlich (vgl. z.B. Matte Bon 1992 und de Bruyne 1993). Ziel ist die Bildung von booleschen Merkmalen in der Weise, daß sie der begonnenen Gesamtklassifizierung dienen. Helbig / Buscha (1994: 234f.) stellen drei Möglichkeiten vor, wie deutsche Indefinitpronomina klassifiziert werden können:

„So kann man zwischen Indefinitpronomina, die die Personen und NichtPersonen aus einer Vielzahl aussondern (einige, etwas, irgendeiner / irgendwelche, irgendwer, jemand, mancher, mehrere), und Indefinitpronomina, die diese zu einer Gesamtheit zusammenfassen (positiv: alle(s), jeder, negativ: keiner, niemand, nichts), unterscheiden.

Eine andere Einteilung ergibt sich, wenn man die Pronomina nach dem unterschiedlichen Grad der Unbestimmtheit - von positiv bis negativ - anordnet: alle(s), jeder, mancher, mehrere, einige, man, jemand, etwas, irgendwer, irgendeiner / irgendwelche, keiner, niemand, nichts.

Eine dritte Möglichkeit besteht darin, die Indefinitpronomina danach einzuteilen, ob sie nur Personen (jemand, irgendwer, man, niemand), nur NichtPersonen (etwas, nichts) oder beides (alle(s), einige, irgendeiner / irgendwelche, jeder, keiner, mancher, mehrere) bezeichnen.“

Die ersten beiden Einteilungen sind in spanischen Grammatiken ebenfalls zu finden (z.B. in Matte Bon 1992: II, 1ff.). Die dritte verspricht nicht sonderlich viele neue Ergebnisse, da Helbig / Buscha auch die substantivischen und adverbialen Indefinitpronomina einbeziehen und die dritte Einteilung z.B. in der Klasse der auf NichtPersonen referierenden Pronomina nur substantivisch gebrauchte Indefinitpronomina kennt (vgl. im Deutschen etwas, nichts; im Spanischen algo, nada, für einen ähnlichen Ansatz vgl. Rodríguez Díez 1992a: 248f.). Damit verbleiben nach Helbig / Buscha zwei mögliche Klassifikationsansätze:

1. Einzelnes wird durch das Pronomen aus einer Vielzahl ausgesondert oder mehrere einzelne Dinge / Personen zu einer Gesamtheit zusammengefaßt. Damit kann zwischen Indefinitpronomina, die sich auf Einzelnes oder Individuelles beziehen, und solchen, die sich auf Mengen beziehen, unterschieden werden. 
2. Die Pronomina werden nach dem Grad der Unbestimmtheit angeordnet. Wir erhalten eine abstufende Skala, ähnlich der Ergebnisse im Kapitel über Kookkurrenz.

Nach Allegranza (1998) und Quirk u.a. (1985) können die Artikel, Demonstrativa, Possessiva und Numeralia als eigenständige Klassen betrachtet werden. Lamíquiz (1987: 145-158) nimmt die Numeralia zu der Klasse der Quantifizierer und differenziert in der Folge zwischen exakten und relativen Quantifizierern, während Alcina Franch / Blecua (1975: 585ff.) die Numeralia als eigenständige Klasse führen. Ähnlich uneinheitlich werden tanto und tal gesehen: Während Fernández Ramírez (1985: 137ff.) diese als Demonstrativa einordnet, klassifizieren Alcina Franch / Blecua (1975: 587) tan(to) als cuantitativo intensivo und tal als identificador. Uneinheitlich ist ebenfalls die Sichtweise bei den Artikeln, besonders beim unbestimmten.

Wir werden uns im folgenden zunächst an Lamíquiz und später an Alcina Franch / Blecua orientieren. Die daraus gewonnenen Ergebnisse können dann anhand der Ausführungen von Matte Bon und de Bruyne vervollständigt werden.

\subsubsection{Demonstrativa}

„Los demonstrativos, como los posesivos y el artículo determinado, forman parte de los determinantes de segunda mención: su función es situar un sustantivo que ya ha aparecido explícita o implícitamente en el contexto [...]“ (Matte Bon 1992: I, 223, vgl. auch Asenjo Orive 1990). Lamíquiz (1987: 145) bezeichnet die Determinierer als presentadores und benennt die Subklasse von Demonstrativ- und Possessivpronomina sowie des bestimmten Artikels als actualizadores. Aus seinen Ausführungen geht hervor, daß er dabei die gleichen Funktionen meint wie Matte Bon.

Das Demonstrativpronomen besitzt Hinweischarakter, d.h. deiktische Funktion. Dies grenzt es von allen anderen Determinierern ab. Dabei sind die drei Formen este, ese und aquel insofern zu differenzieren, als sie jeweils unterschiedlich auf die Sprecher-Hörer-Nähe referieren: este bezeichnet Dinge oder Personen, die sich zwar nah beim Sprecher, aber weiter entfernt vom Hörer befinden. Ese bezeichnet Dinge oder Personen, die nah beim Hörer, aber weiter entfernt vom Sprecher sind. Bei aquel befindet sich das Bezeichnete für beide Seiten nicht in Reichweite. Damit hat este 
stärkeren Bezug zum Personalpronomen der ersten Person, ese zum Personalpronomen der zweiten Person und aquel zu dem der dritten Person. Ese wird öfter als die anderen beiden auch in einer dem Nomen nachgestellten Position verwendet und erzeugt dann eine abschätzige Wertung des entsprechenden Sachverhalts (vgl. Asenjo Orive 1990: 49f.).

Inwieweit können nun tanto und tal als Demonstrativa bezeichnet werden, wie dies bei Fernández Ramírez (1987: 137ff.) der Fall ist? Er nennt tanto ein quantifizierendes und tal ein qualifizierendes Demonstrativpronomen. Tanto steht sowohl in komparativen Konstruktionen als auch in gewöhnlichen Nominalphrasen, in denen es mit mucho vergleichbar verwendet wird (vgl. de Bruyne 1993: 239): No podíamos prever que iba a venir tanta gente. Hier verdeutlicht der Gebrauch von tanto, daß in einer Situation nach Meinung des Sprechers ein „mehr als erwartet“ eintrat. Mucha statt tanta hätte objektiv die Situation beschrieben. Wir ordnen von daher tanto mit Alcina Franch / Blecua (1975: 587f.) in die Klasse der Quantifizierer ein.

Anders sieht es aus bei tal. Es wird von Alcina Franch / Blecua (1975: 587f.) als Identifizierer bezeichnet, ein Begriff der neben Aktualisierer auch für den bestimmten Artikel gebraucht wird. Eine Sache oder Person wird für die Gesprächspartner als eindeutig identifiziert oder im Kontext als in diesem Moment relevant aktualisiert. Tal referiert auf Eigenschaften, und zwar identifizierend. Insofern hat es etwas Demonstratives (und Aktualisierendes): No quiero nada con tal individuo (de Bruyne 1993: 246). Es bleibt die Frage, ob diese Verwendung als demonstrativ bezeichnet werden kann.

In diesen Kontext müßte nach Matte Bon (1992: II, 20) dann auch mismo als Determinierer mit demonstrativer Funktion aufgefaßt werden. Er definiert wie folgt: „Para referirse a un elemento que ya ha sido mencionado explícitamente, o que ha aparecido implícitamente en el contexto, subrayando que se trata de ese individuo y que no es necesario o no se quiere mencionar / buscar otro de identidad distinta, se usa el operador mismo/a(s): ¿Quién era? El mismo señor de hace un rato.“ Mismo referiert immer auf etwas bereits Genanntes und wird von Alcina Franch / Blecua (1975: 587f.) mit tal zusammen als Identifizierer genannt. Diese Funktion entspricht nicht der von Demonstrativa. Da unter ,demonstrativ“ allgemein eine deiktische Funktion verstanden wird, könnte man die Klasse erweitern und generell von „verweisend" sprechen. Danach sind die Demonstrativa este, ese und aquel Pronomina, die deiktisch auf etwas in Zeit oder Raum hinweisen, tal weist auf eine gewisse, im 
Kontext bestimmte Beschaffenheit hin und mismo auf etwas bereits Erwähntes zurück. Alcina Franch / Blecua gruppieren auch otro in die Klasse der Demonstrativa, da es auf etwas Erwähntes verweise, das sich in irgendeiner Art von dem im gegebenen Kontext Genannten unterscheidet.

Die Ausführungen zu mismo, tanto, tal und otro zeigen, daß je nach Ansatzpunkt und Intuition des Autors das Etikett demonstrativ verschiedenes bedeutet. Da die Demonstrativa die definite Funktion des Artikels um eine deiktische Komponente ergänzen, sind sie ebenfalls als definit einzuordnen und unterscheiden sich in diesem Punkt stark von allen genannten anderen Pronomen. Wir bezeichnen nur este, ese und aquel als Demonstrativa, die der Oberklasse Aktualisierer zugeordnet sind. Für die Demonstrativa wird das Merkmal [demonstrativ +] eingeführt.

\subsubsection{Possessiva}

Possessivpronomina zeigen eine Relation an, die eine Person oder Sache mit anderen Personen oder Sachen verbindet (vgl. für eine übersichtliche Einführung Rodríguez Díez 1992b oder Repiso Repiso 1989, bzw. auch Carpenter 1992: 290). Dabei kann es sich um Zugehörigkeit, Interesse, Besitz usw. handeln. Wie bereits gesehen, kennt das Spanische zwei Arten von Possessivpronomina: dem Nomen vorangestellte ( $m i$, $t u$, su) und nachgestellte (mío, tuyo, suyo). Nuestro und vuestro gehören beiden Gruppen an. Ihre Bedeutung in bezug auf den besitzanzeigenden Charakter ist identisch. Allerdings lassen sie durch ihre Position unterschiedliche Kombinationsmöglichkeiten zu. Während die vorangestellten die Funktion des bestimmten Artikels beinhalten, ist dies bei den nachgestellten nicht der Fall, und so sind sie mit diesem kombinierbar (Bsp. aus de Bruyne 1993: 186): Pedro se ha marchado a América, donde vive una hermana suya. Stünde im Nebensatz donde vive su hermana, wäre klar, daß Pedro nur eine Schwester hat. So muß der Nebensatz im Deutschen mit 'wo eine seiner Schwestern lebt' wiedergegeben werden. Mio besitzt folglich nicht den definiten Charakter von $m i$, was durch die mögliche Kombination mit uno deutlich wird.

Bei Lamíquiz (1987: 145ff.) werden die Possessiva zu den Aktualisierern gezählt, die sich auf bereits Erwähntes oder den Sprechern Geläufiges beziehen. Alcina Franch / Blecua erstellen für die Possessiva eine neue Klasse. Andere Determinierer 
können nicht zu dieser Klasse gerechnet werden. Da sich das vorangestellte Possessiv $m i$ in seinem syntaktischen Verhalten grundlegend vom nachgestellten mío unterscheidet, erstellen wir zwei Klassen, die beide das Merkmal [possessiv +] tragen, und unterscheiden diese durch tiefergestellten Index:

(91) Possessiv 1 : mi, tu, su, nuestro, vuestro

Possessiv $_{2}$ : mío, tuyo, suyo, nuеstro, vuestro

\subsubsection{Die Artikel}

Wir müssen zwischen dem bestimmten und dem unbestimmten Artikel differenzieren, die Matte Bon als Artikel „de la primera mención y [...] de la segunda mención“ bezeichnet (zu den folgenden Ausführungen und Beispielen vgl. Matte Bon 1992: I, 199ff.). Der Artikel der „Ersterwähnung“ kennzeichnet etwas, das in die Gesprächswelt erst eingeführt wird. Der Artikel der „Zweiterwähnung“ referiert auf in irgendeiner Form im Kontext bereits Bekanntes. Matte Bon verweist zudem auf die generelle und die spezielle Referenz, die beide Artikelformen ausüben können und so keine großen Bedeutungsunterschiede aufweisen. Er zitiert folgende Beispiele:

(a) El cardenalito de Venezuela es un pájaro en peligro de extinción.

(b) En una oficina (...) tiene que haber fotocopiadora.

El cardenalito de Venezuela und una oficina verweisen allgemein auf die gesamte mögliche Referenzmenge, die das entsprechende Nomen umfassen kann, also alle venezolanischen Kardinäle (als Vogelart) und alle möglichen Büros. Demgegenüber bezeichnen diese beiden in den folgenden Sätzen etwas Eindeutiges und dienen einer speziellen Referenz:

(a) ¿Qué tal tus pájaros?

¿No te lo he contado? Se me ha muerto el cardenalito de Venezuela.

(b) ¿Y qué haces?

Trabajo en una oficina. 
Matte Bon zeigt auch, daß der Numerus des Nomens (bzw. seine Numeralität oder Transnumeralität) ebenfalls gewichtige Auswirkungen auf die Verwendungsmöglichkeiten der Artikel hat.

Da die Artikel traditionell in spanischen Grammatiken gesondert vermerkt sind, werden sie bei Alcina Franch / Blecua konsequenterweise nicht mit quantifizierenden Determinierern in Verbindung gebracht. Lamíquiz zählt den bestimmten Artikel zu den Aktualisierern, den unbestimmten zu den Quantifizierern. Die Trennung mag künstlich erscheinen, da vielfach nur schwer ein Bedeutungsunterschied auszumachen ist. Dies mögen folgende zwei Beispielsätze verdeutlichen, die fast identisch erscheinen (aus Vera Luján, erscheint):

(a) El mendigo extendió la mano.

(b) El mendigo extendió una mano.

Ein Vergleich mit den Eigennamen ist hilfreicher: „el uso de un nombre propio de persona se sitúa ya de por sí en el ámbito de la segunda mención“" (Matte Bon 1992: II, 1). Damit kann die Kombination definiter Artikel plus Nomen mit dem Eigennamen in Verbindung gebracht werden, der ebenfalls der Zweiterwähnung dient. Einzige Ausnahme ist nach Matte Bon die Situation, daß eine Person sich oder andere vorstellt und damit einen Eigennamen zum ersten Mal erwähnt. Betrachten wir folgenden Kontext zur genaueren Klärung (Hervorhebung durch Absatz und nichtkursive Schreibweise von mir, AK):

(95) (a) En la década de los 20, soplaron con fuerza los vientos regeneradores del vanguardismo estético en Europa. A este movimiento pertenecieron personalidades españolas de excepción como Pablo Picasso, Salvador Dalí y Luis Buñuel. (...)

Fue Picasso quien, con el estilo cubista, escribió la primera página de la pintura del siglo $\mathrm{XX}$.

Los admiradores de este pintor malagueño pueden apreciar en el Museo de Arte Reina Sofía de Madrid su Guernica, el retrato del horror del bombardeo nazi sobre un refugio vasco durante la Guerra Civil. (P-2)

(b) Fue este pintor quien, con el estilo cubista, escribió ...

(c) Fue el pintor quien, con el estilo cubista, escribió ..

(d) ?Fue un pintor quien, con el estilo cubista, escribió ... 
Betrachten wir den Absatz (95a): Picasso wird neben Dalí und Buñuel durch einen Eigennamen eingeführt. Ob ein Hörer diesen Satz begreift, hängt von seiner Kenntnis der Person Picassos ab. Ersetzen wir im markierten Satz Picasso mit este pintor (=95b), ist der Absatz zweifelsohne weiterhin grammatisch korrekt, da der Folgesatz in (95a) die gleiche Konstruktion wie (95b) aufweist. Es ist nunmehr nicht mehr möglich, zwischen den drei genannten Personen den gemeinten Referenten auszumachen. Syntaktisch kann sich die Phrase este pintor auf Picasso, Dalí oder Buñuel beziehen. Da Buñuel kein Maler war und deshalb als Bezugsperson nicht in Frage kommt, entsteht die Möglichkeit einer falschen Referenz, die den Unkundigen verwirren dürfte. Ersetzen wir den markierten Satz in (95a) durch Satz (95c), bleibt weiterhin die Frage ungeklärt, um welchen Maler es sich handelt. Das Verständnis bleibt erschwert. Bei einer Ersetzung durch (95d) schließlich bekommen wir eine Konstruktion, die zwar syntaktisch korrekt ist, semantisch aber keinen Sinn mehr ergibt. Da bereits einige Maler erwähnt wurden, kann nur unter der Annahme eines Stilbruchs ein neuer Maler eingeführt und so „ersterwähnt“ werden.

Hieraus können wir schlußfolgern, daß eine NP mit definitem Artikel eine dem Eigennamen ähnliche Funktion erfüllt. Wir integrieren somit den definiten Artikel als eigene Subklasse in die Klasse der Aktualisierer neben Demonstrativa und Possessiva. Der indefinite Artikel wird zur bisher nicht betrachteten Klasse der Indefinita gezählt (vgl. auch das Referenzmodell bei Lavric 2000: 28).

Allerdings muß diese Argumentation eingeschränkt werden. Nach Habel (1986: 94ff., vgl. auch Habel / Rollinger 1982: 138ff.) kann für eine definite Kennzeichnung der logische Operator IOTA eingeführt werden:

IOTA $\mathrm{x}: \mathrm{p}(\mathrm{x})$

bezeichnet das eindeutig bestimmte Objekt mit der Eigenschaft $\mathrm{p}$.

Allwood u.a. (1973: 90) definieren: „Es gibt bestimmte Bedingungen dafür, daß der Ausdruck IOTA $x(F(x))$ gebraucht werden kann: es muß ein $x$ geben, das die Eigenschaft $F$ hat, und es darf nicht mehr als ein $x$ geben, das diese Eigenschaft hat". Habel erweitert von daher die Definition der definiten Kennzeichnung, daß der Kontext eine Sache als in diesem Fall einmalig einschränkt. Die definite NP der Universitätspräsident (Habel 1986: 101) ist von daher nur dann als im streng logischen Sinne definit zu betrachten, wenn der Kontext dies ermöglicht, da wir über die Welt wissen, daß es mehr als einen Universitätspräsidenten gibt. Der Kontext kann eine ein- 
deutige Referenz auf nur eine Person erstellen, die dann logisch mit einem Eigennamen vergleichbar ist.

Dies gilt aber nur für singuläre Termini. Im Plural sind Nomina logisch auf Mengen bezogen und somit nicht mehr eindeutig definit. Der Beispielsatz Die Universitätspräsidenten trafen sich zu ihrer jährlichen Sitzung in Bonn (Habel 1986: 101f.) kann evtl. aus dem Kontext als definite Kennzeichnung interpretiert werden. Habel nennt dies versteckte Definitheit (vgl. dazu auch Lavric 2000: 27f.). Dies ist insofern relevant, als es zeigt, daß der definite Artikel nicht eine eindeutig definite Kennzeichnung nach sich zieht. Somit erstellen wir zwar ein Merkmal [definit +] für den spanischen Artikel el / la / los / las. Dies ist aber nach logischen Gesichtspunkten eine intuitive Klassifizierung. Gleiches gilt für die Demonstrativa und Possessiva ${ }_{1}$

Die Klasse der Aktualisierer nach Lamíquiz wird in drei Unterklassen aufgeteilt. Dies ist auch im Hinblick auf eine HPSG-Klassifikation sinnvoll, da alle drei Klassen differenziert betrachtet werden müssen:

(97) 1. Aktualisierer

a. Demonstrativa: este, ese, aquel

b. Possessiva 1 : $m i$ / tu ...

c. def. Artikel: el / la / los / las

2. Possessiva 2 : mío / tuyo ...

3. Indefinitpronomina ... 


\subsubsection{Indefinitpronomina}

Die komplexesten Differenzierungen semantischer Art bei den Indefinitpronomina erstellen Lamíquiz und Alcina Franch / Blecua. Lamíquiz (1987: 145-158) unterscheidet zwischen numerales und extensivos. Dabei charakterisiert er die Numeralia als präzise (mit den Subklassen Kardinalia, Ordinalia, Multiplikativa und Bruchzahlen), während die restlichen Pronomina in Abhängigkeit vom subjektiven Empfinden des Sprechers stehen und in einem objektiven Sinn als unpräzise gelten können.

Alcina Franch / Blecua (1975: 587-686) unterteilen genauer, wobei sie für Numeralia die gleichen Ergebnisse wie Lamíquiz aufweisen. Sie erstellen folgende Klassifikation:

Semantische Klassifikation

\begin{tabular}{|c|c|}
\hline Possessiva & (s.o.) \\
\hline Demonstrativa & (s.o. + otro, harto, asî) \\
\hline \multirow[t]{3}{*}{ Quantifizierer } & $\begin{array}{l}\text { gradativos } \\
\text { (mucho, poco, bastante, demasiado, harto, todo) }\end{array}$ \\
\hline & existenciales $\quad$ (alguno, ninguno) \\
\hline & $($ más, menos, $\tan ($ to $))$ \\
\hline \multirow[t]{2}{*}{ Numeralia } & Zahlwörter \\
\hline & distributivos $\quad($ sendos, cada, ambos) \\
\hline Identifizierer & identificadores $\quad$ (mismo, tal, asî) \\
\hline
\end{tabular}

Possessiva und Demonstrativa wurden bereits behandelt. Otro wird nicht zu den Demonstrativa gerechnet. Harto wird als Adjektiv, así als Adverb betrachtet. Als eindeutige Klasse können die Numeralia mit den Zahlwörtern gelten. Die Subklasse der Distributiva muß genauer betrachtet werden, da sich die Klasse sehr heterogen zeigt und z.T. äußerst selten Verwendung finden. Gleiches gilt für mero. Folglich werden sie in manchen Grammatiken gar nicht erwähnt (z.B. Beckmann 1994).

Die Tabelle nach Alcina Franch / Blecua beinhaltet eine Differenzierung, die bei Matte Bon ähnlich geäußert wird. Matte Bon nennt das diesbezügliche Kapitel „Individuos y Cantidades“ und unterteilt es in zwei entsprechende Unterkapitel, die zwischen Quantifizierern, die sich in irgendeiner Form auf Mengen beziehen, und Identi- 
fizierern, die auf Individuen referieren, differenzieren. Hernández Alonso (1984: 448) nennt die zweite Gruppe singularizantes. Die Zielsetzung von Matte Bon ist allerdings die Deskription von Sprechakten und der dabei verwendeten syntaktischsemantischen Mittel. Er orientiert sich folglich an Situationen und ihrer sprachlichen Realisierung und nicht an einzelnen Lexemen und deren Verwendungsmöglichkeiten.

Die Differenzierung in Indefinitpronomina, die eher auf Individuen referieren, und solche, die sich eher auf Mengen beziehen, kann die Tabelle nach Alcina Franch / Blecua genauer gestalten. Allerdings hatten wir durch die Einführung des IOTA-Operators bereits gesehen, daß dies nur für Singularformen gilt. Von daher handelt es sich bei der genannten Differenzierung erneut um eine intuitive Einteilung. Indefinitpronomina im Plural referieren immer auf Mengen, auch wenn sie tendenziell eher Individuen kennzeichnen.

Gradativos kennzeichnen Mengen und sagen nach dem subjektiven Empfinden des Sprechers etwas über deren Größe aus, ob es sich um viele oder wenige Elemente, ausreichend viele oder zu viele handelt. Existenciales kennzeichnen demgegenüber einzelne Dinge oder Personen als vorhanden. Anzahl und Beschaffenheit bleibt offen. So definiert Matte Bon existenciales wie alguno u.a. folgendermaßen: „para referirse a uno o varios elementos de un grupo que ya se ha mencionado“ (Matte Bon 1992: II, 9). Für ninguno gilt das gleiche in der verneinten Form (vgl. auch die Ausführungen zu ital. nessuno bei Longobardi 1988: 647). Demgegenüber definiert Matte Bon (1992: II, 20) mucho und tanto, die den gradativos und intensivos zugeordnet sind: ,para hablar de manera imprecisa de una gran cantidad de elementos“.

So stellt sich die Frage, ob möglicherweise alle Determinierer danach unterschieden werden können, ob sie sich im Singular tendenziell eher auf Individuen beziehen und das Beschriebene als existent, nichtexistent oder in sonst irgendeiner Weise hervorheben (z.B. bezeichnet cada eine Eigenschaft, die auf jedes Element einer bestimmten Gruppe zutrifft) oder ob sie eher die Anzahl beschreiben (z.B. dos oder тисho). Dabei würden wir eine Skala der Genauigkeit erhalten: Die Numeralia z.B. bezeichnen die genaue Anzahl von Elementen in einer Menge. Mucho und poco geben hinsichtlich einer Anzahl den subjektiven Eindruck des Sprechers wieder, während tanto und demasiado diesen Eindruck bewerten. Wir könnten somit hinsichtlich Anzahl und Subjektivität von einer Abstufung sprechen. 
Es gibt mehrere problematische Fakten bei dieser Einteilung: Erstens handelt es sich grundsätzlich nur um eine Tendenz. Es ist sehr oft möglich, einen Kontext zu finden, mit dem die Hypothese in Frage gestellt werden kann. Matte Bon (1992: II, 9ff.) nennt zudem zahlreiche syntaktische Konstruktionen mit ähnlichem semantischen Gehalt (cantidad de, la mayoría de usw.), die mit diesem lexikalischen Ansatz nicht erfaßt werden. Ein Beispiel für einen solchen Kontext gibt varios: Prinzipiell verhält es sich synonym zu unos mit der Bedeutung 'mehrere, manche', allerdings ergänzt durch 'verschieden': Hay varias versiones de lo sucedido (de Bruyne 1993: 241). Damit manifestiert es zwar eine Menge von mehreren Elementen, diese werden aber auch als verschieden charakterisiert und mit einer individuellen Eigenschaft markiert (wenn auch nur in Abhängigkeit von der Menge).

Zweitens gilt die für definite Artikel notwendige Numerusdifferenzierung auch bei Indefinitpronomina: alguno bezieht sich im Singular auf etwas mit der Anzahl 1, algunos im Plural auf eine vage Anzahl mehrerer Dinge. Damit ist die vorgeschlagene Unterteilung in Mengen- und Individuenmarkierer höchst zweifelhaft.

Drittens gibt es einige problematische Fälle wie tal, mismo, mero u.a. Diese haben auch qualifizierende Eigenschaften integriert, so daß z.B. mero nicht mit qualifizierenden Adjektiven kombiniert werden kann. Tal enthält eine demonstrative Komponente, mismo ist reflexiv. Damit sind die spanischen Determinierer in ihren semantischen Eigenheiten so komplex, daß eine Klassifikation in der vorgeschlagenen Form nicht sinnvoll ist. Allerdings ist es möglich, die Kombinationsmöglichkeiten durch semantische Informationen genauer zu bestimmen. Dies soll im folgenden geschehen.

\subsection{Semantisches und Kookkurrenz}

Bei den Gruppierungen von Lamíquiz und Alcina Franch / Blecua können wir zwar eine Tendenz erkennen, zwischen individuen- und mengenbezogenen Determinierern zu unterscheiden, diese Tendenz läßt sich aber nicht allgemein bestätigen. Statt dessen ist es möglich, die Kombinationsmöglichkeiten anhand der genannten semantischen Tendenz explizit zu differenzieren. Bisher werden nicht alle erlaubten oder unerlaubten Kombinationen durch die erstellten Merkmale erfaßt. Semantische Aspekte spielen bei der Kookkurrenz eine wichtige Rolle, stellen aber nicht das 
Grundgerüst dar. Poco und otro weisen z.B. deutlich mehr Kombinationsmöglichkeiten auf als viele andere Determinierer, ohne daß dies semantisch begründet werden könnte. Wir starten mit der bereits genannten, semantisch nicht verwertbaren Einteilung zwischen individuen- und mengenbezogenen Determinierern.

Zur ersten Gruppe können die existenciales von Alcina Franch / Blecua gezählt werden, sowie die identificadores und distributivos. Zur zweiten Gruppe können wir die gradativos und intensivos rechnen. Die Numeralia bilden eine gesonderte Gruppe, ambos und mío stehen einzeln. Folgende drei Kriterien werden verwendet (nach Matte Bon 1992: II, 1ff., vgl. auch Hernández Alonso 1984: 448 und Alvarez Martínez 1986: 162):

(99) A: Tendenziell werden Individuen aus einer Vielzahl ausgesondert (bei Negation wird kein Element explizit ausgesondert);

B: Tendenziell werden Individuen zu einer Gesamtheit zusammengefaßt;

C: Tendenziell wird eine genauere Angabe gemacht (zumindest im Singular).

Unter $\mathrm{C}$ werden die Numeralia, die Aktualisierer sowie ninguno, cada und todo positiv markiert, wobei wir für die Aktualisierer dies nur in der Singularform annehmen können und Pluralformen wie los / las ausgliedern müssen. Ninguno, cada und todo werden aufgrund der tendenziell genaueren Mengenangabe nicht den semantischen Gruppen der existenciales, distributivos, gradativos (nach Alcina Franch / Blecua) zugeordnet: 
(100) Zuordnung der semantischen Klassen anhand der Merkmale in (99)

\begin{tabular}{|l|l|c|c|c|}
\hline Klasse & Determinierer & $A$ & $B$ & $C$ \\
\hline s1 Aktualisierer + Numeral & ambos & + & + & + \\
\hline $\begin{array}{c}\text { s2 Aktualisierer (Art., Pos } 1, \\
\text { Dem.) + ninguno u. cada }\end{array}$ & $\begin{array}{l}\text { el / la, mi / tu, este, ninguno, } \\
\text { cada }\end{array}$ & + & - & + \\
\hline $\begin{array}{l}\text { s3 existenciales, distribu- } \\
\text { tivos, identificadores }\end{array}$ & $\begin{array}{l}\text { uno, alguno, cualquiera, demás, } \\
\text { otro, sendos, mismo, tal, cierto }\end{array}$ & + & - & - \\
\hline s4 Numeralia + todo & doble, segundo, dos, todo & - & + & + \\
\hline s5 gradativos, intensivos & $\begin{array}{l}\text { bastante, demasiado, mucho, } \\
\text { poco, varios, tanto, más, mero }\end{array}$ & - & + & - \\
\hline s6 Possessiv 2 & mío & - & - & - \\
\hline
\end{tabular}

Um die Kombinationsmöglichkeiten einzubeziehen, müssen folgende Zusätze erfolgen:

- die Numeralia müssen in Kardinalia sowie Ordinalia und Multiplikativa unterteilt werden, da die Kardinalia weniger Möglichkeiten zulassen;

- ambos und sendos sind zwar nicht der gleichen Klasse zugeordnet (wenn auch beide als Distributiva bezeichnet wurden), verhalten sich aber gleich in ihren Kombinationsmöglichkeiten;

- otro und poco werden aufgrund ihres Verhaltens in einer separaten Kombinationsklasse erfaßt;

- es muß zwischen Determinierern, die nur in Erststellung, nur in Zweitstellung oder in beiden Stellungen vorkommen, unterschieden werden;

- mío, más und der Prädeterminierer todo werden aufgrund ihrer Außenseiterrollen ausgesondert.

Damit ergibt sich folgende neue Tabelle, wobei wir die Merkmale Erststellung (1.) und Zweitstellung (2.) ergänzen, die in Kapitel III.2.3. erarbeitet wurden. Bei den auf reinen Kombinationseigenschaften basierenden Klassen (A, B, C aus (100)) werden die Merkmale kombiniert und mit o markiert, wenn sie sich widersprechen. 
(101) Semantische Klassen, erweitert durch Kombinationsklassen und-merkmale

\begin{tabular}{|c|c|c|c|c|c|c|c|}
\hline \multicolumn{2}{|c|}{ Klasse } & \multirow{2}{*}{$\begin{array}{l}\text { Determinierer } \\
\text { ambos, sendos }\end{array}$} & \multirow{2}{*}{$\begin{array}{l}A \\
+\end{array}$} & \multirow{2}{*}{$\begin{array}{c}B \\
\mathrm{o}\end{array}$} & \multirow{2}{*}{$\begin{array}{l}C \\
\mathrm{o}\end{array}$} & \multirow{2}{*}{$\begin{array}{l}1 . \\
+\end{array}$} & \multirow{2}{*}{2.} \\
\hline s1 & Komb.klasse d6 + Distributiv & & & & & & \\
\hline s2 & $\begin{array}{l}\text { Aktualisierer + ninguno u. } \\
\text { cada }\end{array}$ & $\begin{array}{l}\text { el / la, mi / tu, este, } \\
\text { ninguno, cada }\end{array}$ & + & - & + & + & - \\
\hline \multirow[t]{3}{*}{ s3 } & \multirow{3}{*}{$\begin{array}{l}\text { existenciales, } \\
\text { identificadores }\end{array}$} & uno, alguno, cualquiera & + & - & - & + & - \\
\hline & & tal, cierto & + & - & - & + & + \\
\hline & & demás, mismo & + & - & - & - & + \\
\hline & Komb.klassen b2, c3 & otro, poco & $\mathrm{o}$ & $\mathrm{O}$ & - & + & + \\
\hline \multirow[t]{2}{*}{ s4 } & Ordinalia, Multiplikativa & doble, segundo & - & + & + & - & + \\
\hline & Kardinalia & dos & - & + & + & - & + \\
\hline \multirow[t]{2}{*}{ s5 } & \multirow[t]{2}{*}{$\begin{array}{l}\text { gradativos, } \\
\text { intensivos }\end{array}$} & $\begin{array}{l}\text { bastante, demasiado, } \\
\text { mucho, varios, tanto }\end{array}$ & - & + & - & + & + \\
\hline & & mero & - & + & - & - & + \\
\hline & Prädeterminierer & todo & - & + & + & - & - \\
\hline & (kaum kombiniert) & mío, más & & & & & \\
\hline
\end{tabular}

Eine Diskussion der einzelnen Gruppen bei Alarcos Llorach (1994: 123ff.), Fernández Ramírez (1987: 297ff.), de Bruyne (1993: 204-224) und Matte Bon (1992: II, 9, 16 ; 22) ist insofern nicht notwendig, als sie das Intuitive der Einteilung nur bestätigen würde. Zum einen hatten wir bereits gesehen, daß definite Determinierer nur eingeschränkt eine genaue Angabe erbringen. Letztlich ist sie immer vage. Gleiches gilt für die Klasse s3, welche z.B. mit uno und alguno Indefinitpronomina umfaßt, die zwar tendenziell eher ein Individuum aus einer Gesamtheit aussondern, dies allerdings lediglich im Singular explizit tun. Im Plural handelt es sich ebenfalls um eine vage Angabe.

Wir haben im Zuge der Untersuchung der Kombinationsmöglichkeiten von Determinierern immer wieder behauptet, eine mögliche Nicht-Kombination könne auf semantischer Unverträglichkeit beruhen. Nun können wir dieser Hypothese nachgehen. Wir betrachten dazu die Tabelle (87) mit den möglichen Kombinationen und bilden den Durchschnitt mit den neuen, aus der semantischen Analyse hervorgegangenen Klassen und verschieben die Zeilen und Spalten, so daß zusammengehörige Determinierer auch nebeneinander stehen. Das Ergebnis ist Tabelle (102). Steuerer (in den Spalten mit S markiert) verweist auf ein noch zu erläuterndes neues Merk- 
mal. Die Klassen z1-z9 bezeichnen die Determinierer in Zweitstellung, e1-e12 in Erststellung.

Kombinationsklassen spanischer Determinierer (Endfassung)

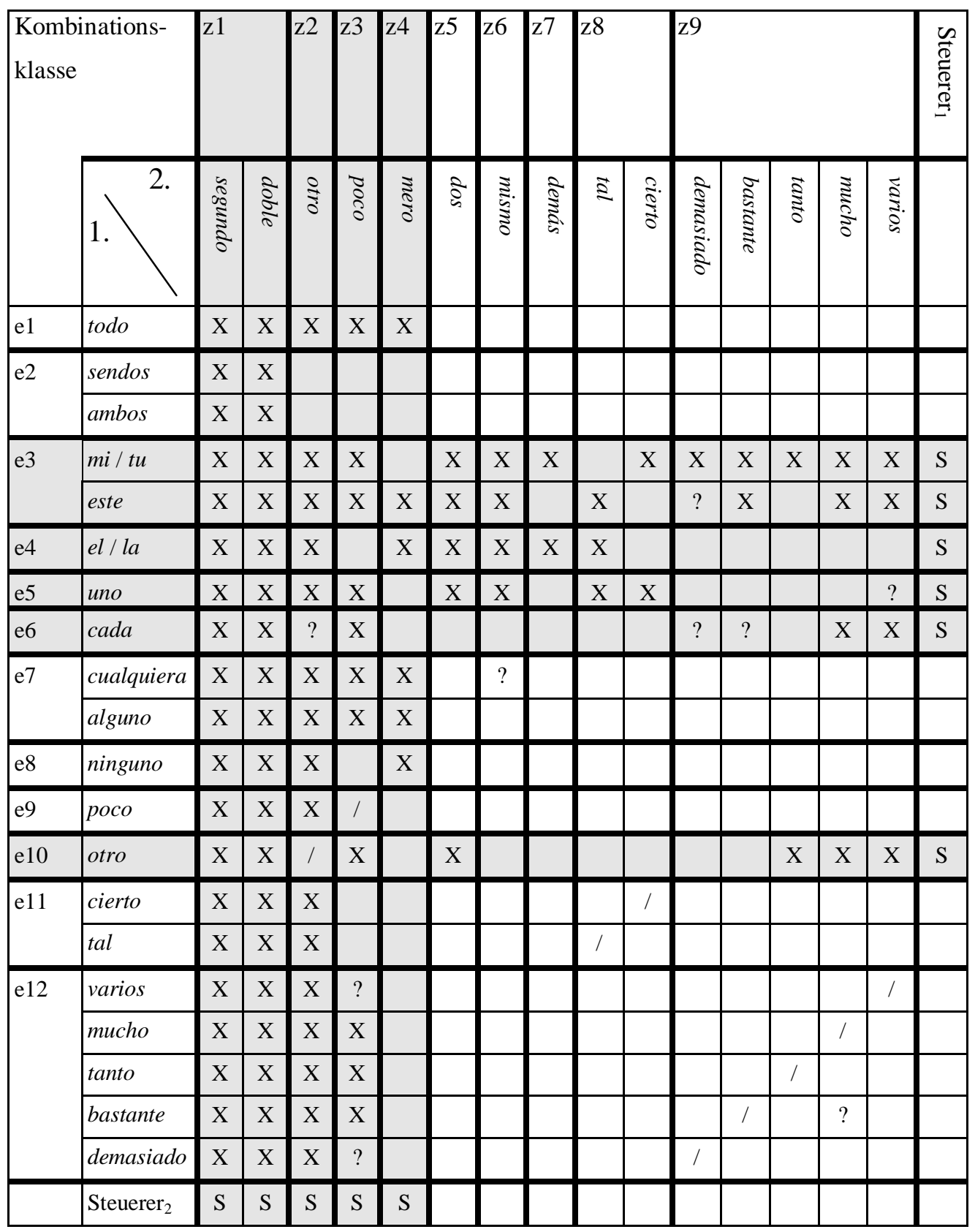

Anhand dieser Ergebnisse lassen sich folgende Beobachtungen machen:

- Es ist nicht unproblematisch, die Kombinationsmöglichkeiten spanischer Determinierer zu beschreiben. Weiterhin bleiben einige Fragezeichen. Es gibt sowohl erlaubte wie unerlaubte Kombinationen, die sich nur schwer in das System integrieren lassen. 
- Determinierer, die sich aufgrund ihres semantischen Gehalts widersprechen und deshalb auch nicht kombiniert werden können, wurden bisher nicht in die Deskription integriert. Semantische Inkompatibilität erklärt einige unerlaubte Kombinationen im obigen System.

So kann der Artikel el / la als gegensätzlich zu cierto, demasiado, bastante und tanto gesehen werden, cada als gegensätzlich zu demás und mismo, poco zu dos, cierto und tal, uno zu demasiado, bastante, tanto und mucho u.a. Dabei handelt es sich nicht um eine binäre Antonyme wie bei schwarz - weiß, schlau-dumm, groß - klein, sondern um eine (teilweise diskutierbare) Gegensätzlichkeit.

Die Kombinationsmöglichkeiten können an bestimmten Wörtern festgemacht werden, die wir hier Kombinationssteuerer (mit S markiert) nennen wollen. Dabei ist die Reihenfolge (Erststellung, Zweitstellung oder beides) nicht grundlegend entscheidend. Als Kombinationssteuerer in Erststellung können wir nennen: mi, este, el, cada, uno sowie otro. In Zweitstellung sind dies segundo, doble, mero, otro und poco. Otro kommt dabei in beiden Positionen vor. Sie sind in (102) in der letzten Spalte bzw. Zeile mit „S“ markiert. Diese Wörter „steuern“ die Kombinationen insofern, als ein Vertreter an einer der beiden Position vorkommen muß. Dieses Wort erhält im Lexikon alle mit ihm kombinierbaren Determinierer individuell zugeteilt. Damit lassen sich alle Kombinationen vorhersagen, da entweder eine Klasse von Determinierern, die mit diesem Steuerer kombinierbar sind, oder aber einzelne Determinierer eingetragen werden, wenn die gesamte Klasse nicht erfaßt wird. Wir können dies an folgenden Beispielen erläutern:

(a) ningún otro amigo

(b) ese tal muchacho

In (103a) ist otro der Kombinationssteuerer, d.h. daß die Kombination von ninguno und otro möglich ist, wird durch ein Merkmal im lexikalischen Eintrag von otro festgelegt, welches ninguno als vorangehenden Determinierer zuläßt. Da otro sowohl in Erst- als auch in Zweitstellung stehen kann, ninguno aber nur in Erststellung vorkommt, erlaubt otro als Kombinationssteuerer ninguno nur als vorangehenden Determinierer. Folglich ist die Kombination *otro ningún muchacho ungrammatisch. In (103b) steuert ese diese Information bei und erlaubt tal als folgenden Determinierer. Die einzige Kombination, in welcher die Reihenfolge der Determinierer unerheblich 
scheint, besteht aus poco und otro. Beide können sowohl vor als auch nach dem anderen stehen.

Die Erklärung des Systems durch die Annahme von Kombinationssteuerern läßt nur wenige Ausnahmen zu: cualquiera mismo und bastante mucho sind als evtl. kombinierbar markiert, werden aber nicht erfaßt. Sie stellen aber fragwürdige Kombinationen dar. Ansonsten werden korrekte Vorhersagen gemacht, wenn wir von lexikalischen Einträgen als Kombinationssteuerern ausgehen, die bei jedem Determinierer seine jeweiligen Kombinationsmöglichkeiten erfassen. Die gebildeten Kombinationsklassen sind in sich relativ homogen. Die Aktualisierer können drei Kombinationen nicht bilden, die nach dem vorgeschlagenen System angenommen werden könnten: *este demás, *mi mero, *este tanto. Andere können als gegensätzlich gelten (z.B. *mi tal, *mi cierto). Los pocos ist nur im Kontext eines folgenden Relativsatzes erlaubt.

Wir können nur bedingt Kombinationsklassen bilden der Art, daß Klasse X mit Y immer und mit $\mathrm{Z}$ nie kombinierbar wäre. Zudem sind die Klassen sehr klein (ein bis höchstens fünf Elemente). Die Kombinationsmöglichkeiten der Kombinationssteuerer sind sehr unterschiedlich. Deshalb ist es nicht sinnvoll, ein System in Anlehnung an Allegranza zu erstellen, das indirekt auf syntaktisch-semantischen Faktoren beruht (mit Merkmalen, die Quantifizierer, Ordinalia und Possessiva als Klasse verstehen). Abschließend bilden wir die Merkmale [Kombinationssteuerer ${ }_{1} \pm$ ] und [Kombinationssteuerer $_{2} \pm$ ], die für alle oben als solche genannten Determinierer positiv gewertet werden. Alle anderen sind negativ markiert. Welche Determinierer einem anderen folgen oder vorangehen können, wird folglich lexikalisch festgelegt. 


\subsection{Gesamtklassifikation}

Wir können im folgenden eine Klassifikation der spanischen Determinierer erstellen. Ziel ist eine Abgrenzung zu den Adjektiven. Zunächst werden alle erstellten Merkmale aufgeführt:

(104) Klassifikationsmerkmale spanischer Determinierer

\begin{tabular}{|c|c|}
\hline \multirow[t]{3}{*}{ Genus und Numerus } & 1. [Genus \pm$]$ \\
\hline & 2. [Numerus realisiert \pm$]$ \\
\hline & 3. [Numerus differenziert \pm ] \\
\hline Adverbialisierung & 4. [Adverbialer Gebrauch \pm ] \\
\hline Komparation & 5. [Komparation \pm$]$ \\
\hline Prädikation & - \\
\hline \multirow[t]{5}{*}{ Substantivierbarkeit } & 6. [substantivierbar mit Artikel \pm ] \\
\hline & 7. [substantivierbar ohne Artikel \pm ] \\
\hline & 8. [substantivierbar im Neutrum \pm ] \\
\hline & 9. [steht in Umgebung _ $\mathrm{A} \mathrm{N} \pm$ ] \\
\hline & 10. [steht in Umgebung Art $\_\mathrm{N} \pm$ ] \\
\hline Substantivierung von Adjektiven & 11. [substantiviert Adjektive \pm ] \\
\hline Quantifikation & - \\
\hline \multirow[t]{2}{*}{ Vor- vs. Nachstellung } & 12. [vorangestellt \pm ] \\
\hline & 13. [nachgestellt \pm ] \\
\hline \multirow[t]{2}{*}{ Kookkurrenz } & 14. [Erststellung \pm$]$ \\
\hline & 15. [Zweitstellung \pm ] \\
\hline \multirow{2}{*}{$\begin{array}{l}\text { Semantische Aspekte } \\
\text { und Kookkurrenz }\end{array}$} & 16. [Kombinationssteuerer $\left.{ }_{1} \pm\right]$ \\
\hline & 17. [Kombinationssteuerer $2 \pm]$ \\
\hline
\end{tabular}

Wir erhalten 17 Merkmale, die sich auf morphologische, syntaktische und semantische Eigenschaften der 28 Determinierer beziehen. Der Bereich Prädikation läßt sich über die Merkmale der Substantivierbarkeit erfassen. Quantifikation ist als ein theorieabhängiges Phänomen zu werten, das für die angestrebte Klassifikation keine weiterführenden Ergebnisse erwarten läßt. Hier kann erst eine HPSG-basierte Analyse weitere Ergebnisse erbringen. 
Die Bedeutung der Merkmale [steht in Umgebung _ A N \pm ] und [steht in Umgeb. Art _ $\mathrm{N} \pm$ ] soll an dieser Stelle kurz wiederholt werden: Sie beziehen sich auf den Aufbau der Nominalphrase und kennzeichnen eine artikeltypische Funktion, nämlich die Anhebung der Phrase auf die N"-Ebene. Das erste Merkmal wird positiv für alle Determinierer gewertet, die analog zum definiten Artikel diese Funktion ausüben. Das zweite Merkmal stellt das Gegenteil dar, z.B. bei demás. Bei diesem Determinierer muß ein Artikel voranstehen, damit der Determinierer erscheinen darf. Demás nimmt somit analog zu einem qualifizierenden Adjektiv keinen Einfluß auf die Ebenen nach der X-bar-Theorie. Die Merkmale [Erststellung \pm ] und [Zweitstellung \pm ] dürfen nicht mit Voran- oder Nachstellung verwechselt werden. Sie bezeichnen die Möglichkeit eines Determinierers, in einer Zweierkombination vor dem Nomen in der ersten oder zweiten Position auftreten zu können.

Die Merkmale können nun den Determinierern zugeordnet werden. Anzahl und Art der Merkmale werfen Fragen auf: Zum einen muß noch geprüft werden, ob diese Merkmale wirklich die Menge der Determinierer erfassen und linguistisch zufriedenstellend klassifizieren. Zum anderen wird noch $\mathrm{zu}$ untersuchen sein, ob einige Merkmale nicht überflüssig sind, z.B. die morphologischen Merkmale Genus und Numerus, welche evtl. geringen Einfluß auf die Klassifikation ausüben, oder ob noch Merkmale fehlen. So wird die in vielen Grammatiken übliche Einteilung in Artikel, Possessiva, Demonstrativa und Quantifizierer bisher nicht explizit erfaßt. Merkmale wie definit, possessiv oder demonstrativ blieben unberücksichtigt. Sie wurden zwar in den bisherigen Ausführungen erwähnt, da sie aber nur auf jeweils einen oder zwei Determinierer zutreffen, erscheinen sie nicht besonders repräsentativ für eine Klassifikation. Dem widerspricht die Auffassung vieler Grammatiker, daß die Artikel, Possessiva und Demonstrativa in Opposition zu allen anderen Determinierern stehen.

Von daher bieten sich zwei Alternativen der Klassifikationen an, die es zu testen gilt: Zum einen mit den Merkmalen [definit \pm ] für den definiten Artikel, [possessiv \pm ] für die Possessiva mi und mío sowie [demonstrativ \pm ] für die Klasse von este, ese und aquel. Zum anderen könnte das Merkmal [Aktualisierer \pm ] als Charakteristikum für die gesamte semantische Klasse der Aktualisierer aufgefaßt werden, so daß nur noch ein Merkmal diese Klasse von den restlichen Determinierern trennen würde. Damit käme die Differenz nicht so stark zum Ausdruck wie beim ersten Vorschlag. Der erste Vorschlag hat zusätzlich den Nachteil, daß auch die drei Subklassen der Klasse Aktualisierer durch jeweils zwei Merkmale voneinander geschieden werden. 
Wir werden alle Klassifikationsmöglichkeiten testen und auf signifikante Differenzen überprüfen.

Ziel der Klassifikation muß eine linguistisch sinnvolle Abgrenzung und Unterteilung der Determinierer untereinander sowie zwischen Determinierern und Adjektiven sein. Dazu sehen wir es als notwendig an, Merkmale zu gewichten (vgl. Altmann / Lehfeldt 1973: 20ff.), da manche Merkmale als schwerwiegender angesehen werden als andere. Das genaue Gewichtungsverfahren wird in Kapitel III.2.6.1. erläutert. Die Gewichtung kann bestimmte Merkmale besonders hervorheben und damit z.B. eher determinierertypische Eigenschaften so herausstellen, daß Determinierer, welche diese besitzen, sich deutlicher von adjektivähnlichen Determinierern hervorheben. Determinierer, die sich keiner dieser Klassen anschließen, bleiben unberührt.

Es folgt eine Tabelle der bisher bearbeiteten Merkmale verteilt auf die Determinierer. Die einzelnen Werte der Determinierer gehen aus den Erläuterungen zum jeweiligen Merkmal hervor. 
(105)

Merkmale der spanischen Determinierer

\begin{tabular}{|c|c|c|c|c|c|c|c|c|c|c|c|c|c|c|c|c|c|}
\hline & 1. & 2. & 3. & 4. & 5. & 6. & 7. & 8. & 9. & 10. & 11. & 12. & 13. & 14. & 15. & 16. & 17. \\
\hline & 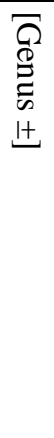 & 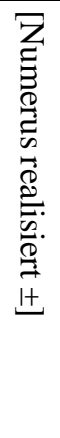 & 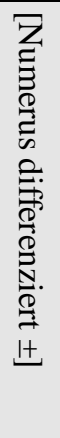 & 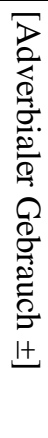 & 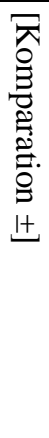 & 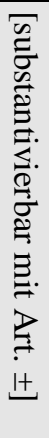 & 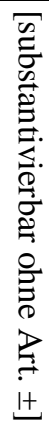 & 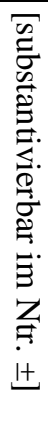 & 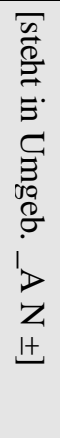 & 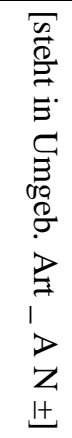 & 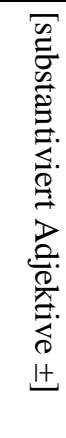 & 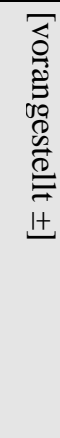 & 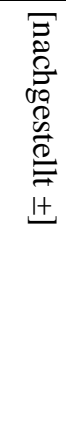 & 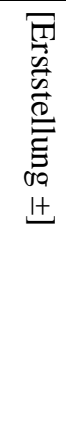 & 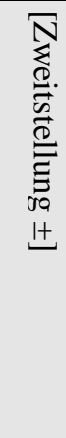 & 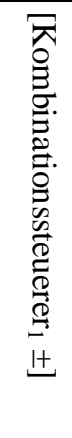 & 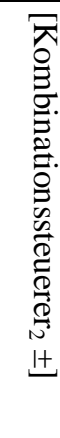 \\
\hline alguno & + & + & + & - & - & - & + & - & + & - & + & + & + & + & - & - & - \\
\hline ambos & + & + & - & - & - & - & + & - & + & - & - & + & - & + & - & - & - \\
\hline bastante & - & + & + & + & - & - & + & + & + & - & - & + & - & + & + & - & - \\
\hline cada & - & + & - & - & - & - & - & - & + & - & - & + & - & + & - & + & - \\
\hline cierto & + & + & + & - & - & - & - & - & + & - & - & + & - & + & + & - & - \\
\hline cualquiera & - & + & + & - & - & - & + & - & + & - & + & + & + & + & - & - & - \\
\hline demás & - & + & - & - & - & + & - & + & - & + & - & + & - & - & + & - & - \\
\hline demasiado & + & + & + & + & - & - & + & + & + & - & - & + & - & + & + & - & - \\
\hline doble & - & + & + & - & - & + & - & + & - & + & - & + & + & - & + & - & + \\
\hline$d o s^{27}$ & - & + & + & - & - & + & + & - & + & + & - & + & - & - & + & - & - \\
\hline$e l / l a$ & + & + & + & - & - & - & - & - & + & - & + & + & - & + & - & + & - \\
\hline este & + & + & + & - & - & - & + & + & + & - & + & + & + & + & - & + & - \\
\hline más & - & - & - & + & + & - & - & + & + & - & - & + & + & - & - & - & - \\
\hline mero & + & + & + & - & - & - & - & - & - & + & - & + & - & - & + & - & + \\
\hline$m i / t u$ & - & + & + & - & - & - & - & - & + & - & - & + & - & + & - & + & - \\
\hline mío & + & + & + & - & + & + & - & + & - & - & - & - & + & - & - & - & - \\
\hline mismo & + & + & + & - & + & + & - & + & - & + & - & + & + & - & + & - & - \\
\hline mисho & + & + & + & + & - & - & + & + & + & - & - & + & - & + & + & - & - \\
\hline ninguno & + & + & + & - & - & - & + & - & + & - & + & + & + & + & - & - & - \\
\hline otro & + & + & + & - & - & + & + & + & + & + & + & + & - & + & + & + & + \\
\hline росо & + & + & + & + & - & - & + & + & + & - & - & + & - & + & + & - & + \\
\hline segundo & + & + & + & - & - & + & - & + & - & + & - & + & + & - & + & - & + \\
\hline sendos & + & + & - & - & - & - & - & - & + & - & - & + & - & + & - & - & - \\
\hline tal & - & + & + & - & - & + & + & + & + & - & - & + & + & + & + & - & - \\
\hline tanto & + & + & + & + & - & - & + & + & + & - & - & + & - & + & + & - & - \\
\hline todo & + & + & + & + & - & - & + & + & - & - & + & + & + & + & - & - & - \\
\hline uno & + & + & + & - & - & - & + & + & + & - & + & + & - & + & - & + & - \\
\hline \multirow[t]{2}{*}{ varios } & + & + & - & - & - & - & + & - & + & - & - & + & - & + & + & - & - \\
\hline & 1. & 2. & 3. & 4. & 5. & 6. & 7. & 8. & 9. & 10. & 11. & 12. & 13. & 14. & 15. & 16. & 17. \\
\hline
\end{tabular}

${ }^{27}$ Dos steht hier stellvertretend für die Kardinalia. Numerusdifferenzierung findet über neue Lexeme statt (dos, tres, cuatro ...) oder morphologisch (ciento - cientos) statt. 


\subsubsection{Klassifikationsmethode}

Die Merkmale der einzelnen Determinierer müssen für eine weitere Klassifikation zueinander in Beziehung gesetzt werden. Das läßt sich am besten mit einer Distanzfunktion realisieren. Jedem Determinierer wurden in Tab. (105) für jedes binäre Merkmal der Wert + bzw. - (entspricht 1 bzw. 0) zugeordnet. Nun werden zwei Determinierer anhand jedes einzelnen Merkmals verglichen, wobei die Differenz errechnet wird.

Altmann / Lehfeldt (1973: 43ff.) schlagen für Berechnungen dieser Art die Euklidische Distanz als Grundlage vor (vgl. auch Sokal / Sneath 1963: 121ff.):

(106) Formel der Euklidischen Distanz:

$\operatorname{Distanz}=\sqrt{\sum\left(\chi_{i} \alpha_{i}\right)^{2}}$, wobei $\chi_{i}$ der mögliche Gewichtungsfaktor und $\alpha_{i}$ der Differenzwert beim Merkmal $i$.

Die Formel besagt, daß bei jedem Merkmal, hier symbolisiert durch die Variable $i$, der Differenzwert $\alpha$ mit dem Gewichtungsfaktor des Merkmals - sofern eingesetzt multipliziert wird. Das Produkt wird anschließend quadriert. Diese Operation findet mit jedem Merkmal statt. Am Schluß werden die gewonnenen Werte aller Merkmale summiert und aus der Summe die Wurzel gezogen. Wenn keine Gewichtung vorgenommen wird, muß lediglich die Summe der unterschiedlichen Werte bei zwei Determinierern gebildet und daraus die Wurzel gezogen werden. Da durch die Operation des Wurzelziehens reelle Zahlen entstehen, kann die Wurzeloperation mit dem Hinweis, daß es sich bei den genannten Werten um die quadrierte Euklidische Distanz handelt, ausgelassen werden.

Wie findet nun nach der Distanzberechnung die Klassifikation statt? Da es das Ziel ist, zwar Klassen von Determinierern zu erstellen, dabei aber eine Abstufung vorzunehmen, wird das Verfahren der Clusterbildung ausgewählt. Nach Bacher (1996: 144) kann dieses Verfahren als hierarchisch agglomerativer Algorithmus bezeichnet werden. Dabei werden Klassen stufenweise gebildet, d.h. erst werden zwei Determinierer zu einer Klasse zusammengefügt, die sich besonders ähnlich sind. In diesem Fall beträgt der kleinstmögliche Abstand zwischen zwei Determinierern 0, wenn sich die Werte bei keinem Merkmalen unterscheiden. In unserer Tabelle ist das 
z.B. bei alguno und ninguno der Fall, sowie bei demasiado und mucho. Wenn wir von einer auf möglicher Ähnlichkeit basierenden Klassifikation ohne Gewichtung ausgehen, ist der größtmögliche Wert dieser Ähnlichkeit die Gesamtanzahl der berücksichtigten Merkmale, in unserem Fall 17. Dieser Wert trifft erneut für die beiden genannten Determinierergruppen zu. Ob diese Klassenbildung berechtigt ist, muß eine spätere Interpretation zeigen.

Nach Bacher (1996:144) findet die Clusterbildung nach folgendem Prinzip statt:

(107) „Schritt 1: Jedes Klassifikationsobjekt bildet zu Beginn ein selbständiges Cluster. Setze daher die Clusterzahl K gleich der Klassifikationsobjektzahl n.

Schritt 2: Suche das Clusterpaar $(\{p\},\{q\})$ mit der größten Ähnlichkeit bzw. der geringsten Unähnlichkeit, verschmelze das Clusterpaar $z u$ einem neuen Cluster $\{\mathrm{p}, \mathrm{q}\}$ und reduziere Clusterzahl $\mathrm{K}$ um 1 $(\mathrm{K}=\mathrm{K}-1)$.

Schritt 3: Prüfe, ob K gleich 1 ist. Ist dies der Fall, beende den Algorithmus, da alle Klassifikationsobjekte einem einzigen Cluster angehören. Bei nein fahre mit Schritt 4 fort.

Schritt 4: Berechne die Ähnlichkeit bzw. Unähnlichkeit des neu gebildeten Clusters $\{\mathrm{p}, \mathrm{q}\}$ zu den verbleibenden Clustern i.

Schritt 5: Gehe zu Schritt 2.“

Wir überführen zunächst zwei Determinierer, z.B. alguno und ninguno, in ein Cluster (Schritt 2). Da an dieser Stelle routinemäßig ein Algorithmus die Abbruchbedingung überprüft und feststellt, daß nach dieser Prozedur die Klassifikation noch nicht beendet ist (Schritt 3), wird nun die Ähnlichkeit bzw. Unähnlichkeit zu allen anderen Clustern berechnet (Schritt 4). Da die Werte von alguno und ninguno identisch sind, müssen auch die Distanzen zu den anderen Determinierern übereinstimmen. Wir können beide zu einem Cluster zusammenstellen und jeweils einen Distanzwert zu jedem anderen Determinierer löschen.

Wenn im Laufe der Klassifikation die Distanz zu den verbleibenden Determinierern differiert - weil Determinierer zu einer Klasse zusammengefaßt wurden, die sich in einem oder zwei, später auch mehreren Merkmalen unterscheiden und somit bereits in einer höheren Distanz als 0 zueinander stehen -, dann gibt es mehrere Mög- 
lichkeiten, mit der Distanz zu den anderen Determinierern in Schritt 4 umzugehen. Für uns sind drei interessant:

1. das Maximumverfahren,

2. das Minimumverfahren,

3. das Mittelwertverfahren.

Wir nehmen als Beispiel die Gruppe um alguno und ninguno, die sich in mehreren Klassifikationsansätzen auf der nächsten Ebene mit cualquiera zu einem neuen Cluster zusammenschließt. Da die Distanzwerte von alguno / ninguno und cualquiera zu allen anderen Determinierern differieren, hat mal alguno eine höhere Distanz (z.B. zu demás), mal cualquiera (z.B. zu demasiado). Das Maximumverfahren wählt die größere Distanz aus und löscht die kleinere. Damit bekäme alguno zu demasiado die gleiche Distanz wie cualquiera, da sie jetzt zu einem Cluster gehören und die kleine Distanz gelöscht wurde. Das Minimumverfahren steht konträr dazu, wählt die kleinere Distanz aus und löscht die größere.

Bacher (1996: 142) zeigt, daß die Maximumvariante sehr hohe Anforderungen an die Homogenität des Clusters stellt. Objekte einer Klasse (auch „Clique“ genannt) sollen jeweils sehr geringe Distanz zueinander aufweisen. Findet sich keine geringe Distanz, wird ein neues Cluster gebildet. Die Minimumvariante stellt dagegen eine sehr schwache Anforderung an die Homogenität. Jedes Objekt im Cluster soll lediglich mindestens einen nächsten Nachbarn im Cluster haben, wodurch die Cluster sehr heterogen werden können. Beide Verfahren sind als extrem zu werten. Das hier favorisierte Mittelwertverfahren gleicht beide genannten Mängel insofern aus, als es eine mittlere Homogenität fordert.

Das Mittelwertverfahren erzeugt aus beiden Distanzen den Mittelwert in Abhängigkeit von der Anzahl der zu einem neuen Cluster zusammengefaßten Cluster. Letzteres besagt, daß bei der Verbindung von z.B. alguno / ninguno und cualquiera die Tatsache berücksichtigt wird, daß die erste Gruppe bereits aus zwei Elementen besteht, während die zweite nur aus einem Element besteht. So wird der Distanzwert zu einem beliebigen Determinierer nun nach der folgenden Formel berechnet (Bacher 1996: 270): 
(108) $\mathrm{u}_{(\mathrm{p}+\mathrm{q}, \mathrm{i})}^{\text {neu }}=\left(\mathrm{n}_{\mathrm{p}} * \mathrm{u}_{\mathrm{p}, \mathrm{i}}+\mathrm{n}_{\mathrm{q}} * \mathrm{u}_{\mathrm{q}, \mathrm{i}}\right) /\left(\mathrm{n}_{\mathrm{p}}+\mathrm{n}_{\mathrm{q}}\right)$

wobei u die Distanz, p und q Cluster, i das Cluster, zu dem die Distanz berechnet wird, und n die Anzahl der Elemente in einem Cluster sind.

p und q symbolisieren die beiden Cluster, die bereits zu einem neuen Cluster zusammengefügt worden sind, i ist der Determinierer oder das Cluster, zu welchem die Distanz berechnet wird, $\mathrm{n}$ beinhaltet die Anzahl der Determinierer im jeweiligen Cluster.

Die Formel besagt, daß die Distanz von alguno / ninguno z.B. zu demás mit der Anzahl der im Cluster bereits vorhandenen Determinierer (hier zwei) multipliziert und daraufhin mit der Distanz von cualquiera zu demás summiert wird. Da das Cluster von cualquiera nur aus einem Determinierer besteht, wird es lediglich mit eins multipliziert. Das Ergebnis wird sodann durch die Gesamtzahl aller in beiden alten Clustern berücksichtigten Determinierern dividiert (hier drei).

Diese Formel gehört nach Bacher zum Verfahren des Weighted-Average-Linkage. Das einfachere Average-Linkage-Verfahren summiert lediglich die Distanzen von Cluster $\mathrm{p}$ und $\mathrm{q}$ und dividiert diese durch zwei:

$$
u_{(p+q), i}=\left(u_{p, i}+u_{q, i}\right) / 2
$$

Dieses Verfahren könnte ebenfalls für die Clusterbildung verwendet werden. Es besteht allerdings die Gefahr einer relativ großen Ungenauigkeit, da wir des öfteren zwei Cluster miteinander verbinden werden, die eine sehr große Differenz in der Anzahl der ihnen jeweils zugehörigen Determinierer aufweisen: Ein Cluster mit fünf Determinierern verbindet sich mit einem Cluster aus lediglich einem Determinierer. Wenn in diesem Fall die Anzahl der enthaltenen Determinierer nicht berücksichtigt wird, kann auf einer späteren Ebene der Klassifikation eine Verzerrung der Distanzberechnung erfolgen, da das Cluster mit nur einem Determinierer höher gewichtet wird als die Determinierer im zweiten Cluster. Dies soll vermieden werden. Da die Berechnung nach dem Weighted-Average-Linkage-Verfahren nur unbedeutend komplexer ist, stellt dieses Verfahren auch kein Problem dar. 


\subsubsection{Anwendung des Klassifikationsverfahrens}

Wir haben 17 boolesche Merkmale erstellt, nach denen die 28 Determinierer klassifiziert werden können. Dabei kann die Distanz zwischen den Determinierern berechnet werden: das Minimum ist 0 für den Fall, daß es keinen Unterschied gibt. Eine zweite Möglichkeit ist, die Ähnlichkeit der Determinierer untereinander zu berechnen, d.h. jedes gemeinsame Merkmal wird summiert: das Maximum beträgt im Falle von 17 Merkmalen natürlich 17. Beide Herangehensweisen führen zu demselben Ergebnis in einer Klassifikation nach dem oben erläuterten Verfahren.

Bedeutender sind folgende Alternativen: Da eine Gewichtung möglich ist, können sowohl determinierer- als auch adjektivtypische Merkmale gewichtet werden. Sinnvoll sind die Faktoren 2 oder 3. Da es sich um morphologische, syntaktische und semantische Merkmale handelt, können jeweils Gruppen von Merkmalen ausgespart oder ergänzt werden. Interessant ist es, nur die syntaktischen Merkmale 4-15 in Tab. (105) zu betrachten. Schon genannt wurde die Möglichkeit, die semantischen Merkmale [definit \pm ], [possessiv \pm ] und [demonstrativ \pm ] einzuführen, um diese traditionell bzw. intuitiv als besondere Klassen empfundenen Determinierer zusätzlich abzugrenzen. Da drei Merkmale zu viel sein könnten, wurde bereits vorgeschlagen, nach dem Ansatz von Lamíquiz (1987) ein Merkmal [Aktualisierer \pm ] für die drei Klassen einzuführen. Zusätzlich ist ein Vergleich zum Maximum- bzw. Minimumverfahren zur Absicherung der Ergebnisse zweckmäßig.

Weiterhin können den Wortarten Determinierer und Adjektiv in unserer Merkmalliste die entsprechenden Werte zugeordnet werden. So ist es direkt möglich, die Determinierer mit den generalisierten Wortartenvertretern $\mathrm{zu}$ vergleichen. Voraussetzung dafür bildet eine geeignete Zuordnung der Merkmale. Wir schlagen folgende Zuordnung vor: 
(110) Merkmale der Wortartenvertreter Adjektiv und Determinierer

\begin{tabular}{|c|c|c|c|c|c|c|c|c|c|c|c|c|c|c|c|c|c|}
\hline & 1. & 2. & & 4. & 5. & 6. & 7. & 8. & 9. & 10. & 11. & 12. & 13. & 14. & 15. & 16. & 17. \\
\hline & 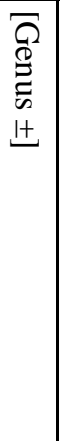 & 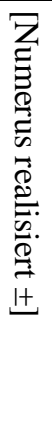 & 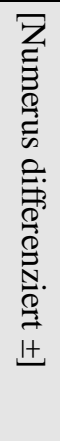 & 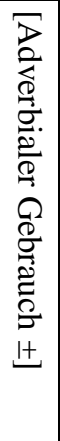 & 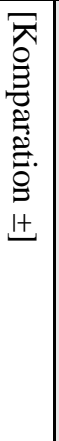 & 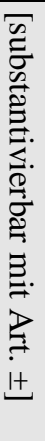 & 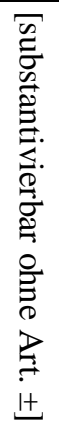 & 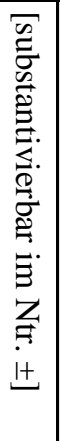 & 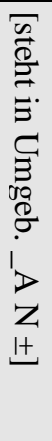 & 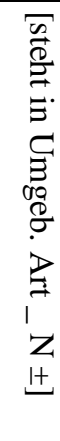 & 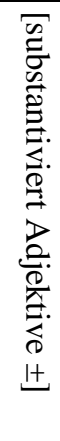 & 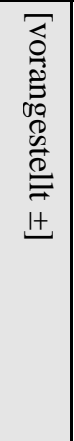 & 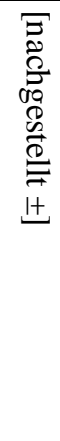 & 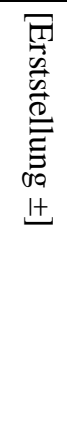 & 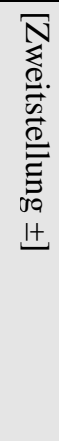 & 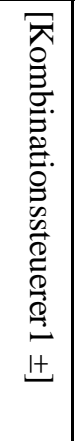 & 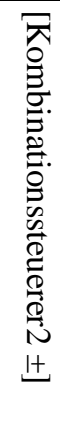 \\
\hline Adjektiv & $\mathrm{O}$ & $\mathrm{O}$ & $\mathrm{O}$ & + & + & + & - & + & - & + & - & + & + & - & - & - & - \\
\hline Determ. & O & O & o & - & - & - & + & - & + & - & + & + & - & + & - & + & - \\
\hline
\end{tabular}

Die Zuordnung der morphologischen Merkmale Genus, Numerus realisiert und $\mathrm{Nu}-$ merus differenziert erweist sich als wenig sinnvoll, da es zumindest bei den Determinierern Ausnahmen gibt. Für das ,typische“ Adjektiv werden sie analog nicht berücksichtigt. Ansonsten verhalten sich beide Wortarten konträr zueinander mit der Ausnahme der Voranstellung, die für Determinierer normal und bei Adjektiven in der Regel möglich ist.

Wie ergibt sich diese Zuordnung? Wir beginnen mit der Wortart Determinierer. In der Regel wird als typischer Determinierer der definite Artikel im Singular gesehen. Damit könnten wir unserem typischen Determinierer dessen Merkmale zuweisen: [steht in Umgeb. _ A N +] (9.), [substantiviert Adjektive +] (11.), [vorangestellt +] (12.), [Erststellung +] (14.), [Kombinationssteuerer ${ }_{1}+$ ] (16.). Alle anderen wären negativ. Allerdings sind die meisten Determinierer ohne einen anderen Determinierer substantivierbar, während der definite Artikel in diesem Fall durch das Personalpronomen ersetzt wird. Wir fügen also hinzu: [substantivierbar ohne Artikel +] (7.). Damit unterscheiden sich uno und el jeweils nur durch ein Merkmal vom typischen Wortartenvertreter, wobei die ersten drei Merkmale nicht gewertet werden, also statt 17 nur 14 zur Verfügung stehen. Im Klassifikationsverfahren werden diese Merkmale nur im Vergleich mit den Wortartenvertretern unberücksichtigt gelassen, da wir eine gesonderte Klassifikation ohne diese Merkmale erstellen. Im Vergleich zwischen einzelnen Determinierern werden sie in die Berechnung einbezogen.

Für die Wortart Adjektiv können wir das Gegenteil annehmen: [Adverbialer Gebrauch +] (4.), [Komparation +] (5.), [substantivierbar mit Artikel +] (6.), [substantivierbar im Neutrum +] (8.), [steht in Umgebung Art _ N +] (10.), [vorangestellt +] (12.), [nachgestellt +] (13.). Das Merkmal Erst- bzw. Zweitstellung muß in diesem 
Kontext unberücksichtigt bleiben, da die Untersuchung nur Kombinationen von Determinierern ermittelt. Zwar wurden Adjektive erfaßt, wobei sich zeigte, daß sie vor Postdeterminierern, aber nach Zentraldeterminierern stehen können. Beide Merkmale sind aber nur zur internen Differenzierung von Determinierern notwendig.

Adjektive können theoretisch ohne Artikel substantiviert werden, wenn sie im Plural stehen (vgl. den Satz negros y blancos se dieron la mano en las elecciones von Briz Gómez 1989: 125). Wir gehen davon aus, daß auf plurale Adjektive als nominale Wortart bei einer Substantivierung die gleichen Regeln angewendet werden können wie auf numerale Nomina. Damit wäre die Substantivierung ohne Artikel der markierte Fall, welche die vorige Anwendung der lexikalischen Regel der Generalisierung benötigt. Zudem trafen einige Überlegungen, besonders bei den definiten Artikeln, nur auf die Singularform zu. Für einen Vergleich mit den Determinierern werten wir deshalb das Merkmal Substantivierung ohne Artikel beim idealisierten Wortartenvertreter Adjektiv negativ.

Welche verschiedenen Berechnungen sind nun möglich, und welche davon erweisen sich als gewinnbringend? Folgende Übersicht soll diese Frage erläutern:

1. Distanz- oder Ähnlichkeitsberechnung: Es wurde bereits gesagt, daß es sich um eine formale Differenzierung handelt. Zwei Determinierer können entweder mit der Distanz 0 oder der Ähnlichkeit 17 bei 17 Merkmalen als identisch in ihren Eigenschaften gewertet werden. Wir entscheiden uns hier für die Distanz. Der Unterschied ist belanglos.

2. Maximum-, Minimum- oder Mittelwertverfahren: Wir favorisieren das Mittelwertverfahren, da es die Schwächen der beiden anderen Verfahren ausgleicht. Das Maximumverfahren wird zum Vergleich herangezogen. Das Minimumverfahren bildet zu große Cluster, so daß es bei der geringen Menge an zu klassifizierenden Objekten kaum noch Aussagekraft besitzt (vgl. Bacher 1996: 140ff.).

3. Berechnung der Distanz mit oder ohne Wurzelziehen: beim Maximum- und Minimumverfahren ist der Unterschied unbedeutend. Beim Mittelwertverfahren kann das Weglassen der Operation des Wurzelziehens durch die neuerliche Berechnung der Distanz nach der Clusterbildung zu leichten Differenzen führen. Da wir das Mittelwertverfahren favorisieren, soll immer die Wurzel gezogen werden. Da dies Teil der verwendeten Formel ist, erhalten wir auch nur so das eigentliche Ergebnis. 
4. Anzahl der Determinierer: Wir werden immer alle 28 spanischen Determinierer berücksichtigen. Zum Vergleich werden die Wortarten Determinierer und Adjektiv als idealisierte Vertreter in die Klassifikation eingebunden. Bei den Klassifikationen mit Gewichtung ergibt sich kein Unterschied zwischen 28 und $28+2$ Determinierern. Wir betrachten der Einfachheit halber bei Gewichtung die Klassifikationen mit den idealisierten Wortartenvertretern.

5. Anzahl der Eigenschaften: Wie oben bereits erwähnt, könnte es interessant sein, die semantischen Merkmale zu erweitern. Diese Vermutung hat sich aber nicht bestätigt, so daß diese Klassifikationsergebnisse nicht erwähnenswert sind. Als aussagekräftiger erweist es sich, morphologische Eigenschaften wie Genus, Numerusrealisierung und -differenzierung sowie Komparation nicht zu berücksichtigen, so daß nur 13 der 17 Merkmale beachtet werden.

6. Gewichtung der Eigenschaften: Neben dem Faktor 1 (keine Gewichtung) sind Klassifikationen auch mit Faktor 2 oder 3 durchführbar. Da Faktor 3 keine nennenswerten Veränderungen ergab, kann diese Möglichkeit entfallen. Bei der Frage, welche Merkmale gewichtet werden sollen, bestehen zwei Möglichkeiten: die determinierer- oder die adjektivtypischen. Die beiden Varianten müßten die jeweils zu der entsprechenden Wortart tendierenden Determinierer hervorheben.

Damit erhalten wir sechs Klassifikationswege:

(111) Klassifikationswege

\begin{tabular}{|l|l|l|l|l|}
\hline Nr. & $\begin{array}{l}\text { Anzahl } \\
\text { der Det. }\end{array}$ & $\begin{array}{l}\text { Anzahl } \\
\text { der Eig. }\end{array}$ & $\begin{array}{l}\text { Gewichtungsfaktor } \\
\text { (gewichtete Wortart) }\end{array}$ & Verfahren \\
\hline 1. & 28 & 17 & $1 \mathrm{x}$ & Mittelwert \\
\hline 2. & $28+2$ & 17 & $1 \mathrm{x}$ & Mittelwert \\
\hline 3. & $28+2$ & 17 & $2 \mathrm{x}(\mathrm{Det})$ & Mittelwert \\
\hline 4. & $28+2$ & 17 & $2 \mathrm{x}(\mathrm{Adj})$ & Mittelwert \\
\hline 5. & 28 & 13 & $1 \mathrm{x}$ & Mittelwert \\
\hline 6. & 28 & 17 & $1 \mathrm{x}$ & Maximum \\
\hline
\end{tabular}

Wir beginnen mit der Klassifikation der 28 Determinierer ohne Gewichtung und erstellen ein Dendrogramm (vgl. Altmann / Lehfeldt 1973: 34ff., bes. 41, und Sokal / Sneath 1963: 262ff). Dendrogramme stellen die Information einer abstufenden Klassifikation in einer Graphik dar, so daß die Klassenbildung und die entsprechenden 
Abstände einfach erfaßt werden können. Cluster werden in Klammern abgebildet, die alle Determinierer erfassen, die an dieser Stelle der Klassifikation (hier Durchlauf genannt) berücksichtigt wurden.

(112) Dendrogramm von 28 Determinierern bei 17 Eigenschaften ohne Gewichtung mit Mittelwertverfahren (nach Tab. 105)

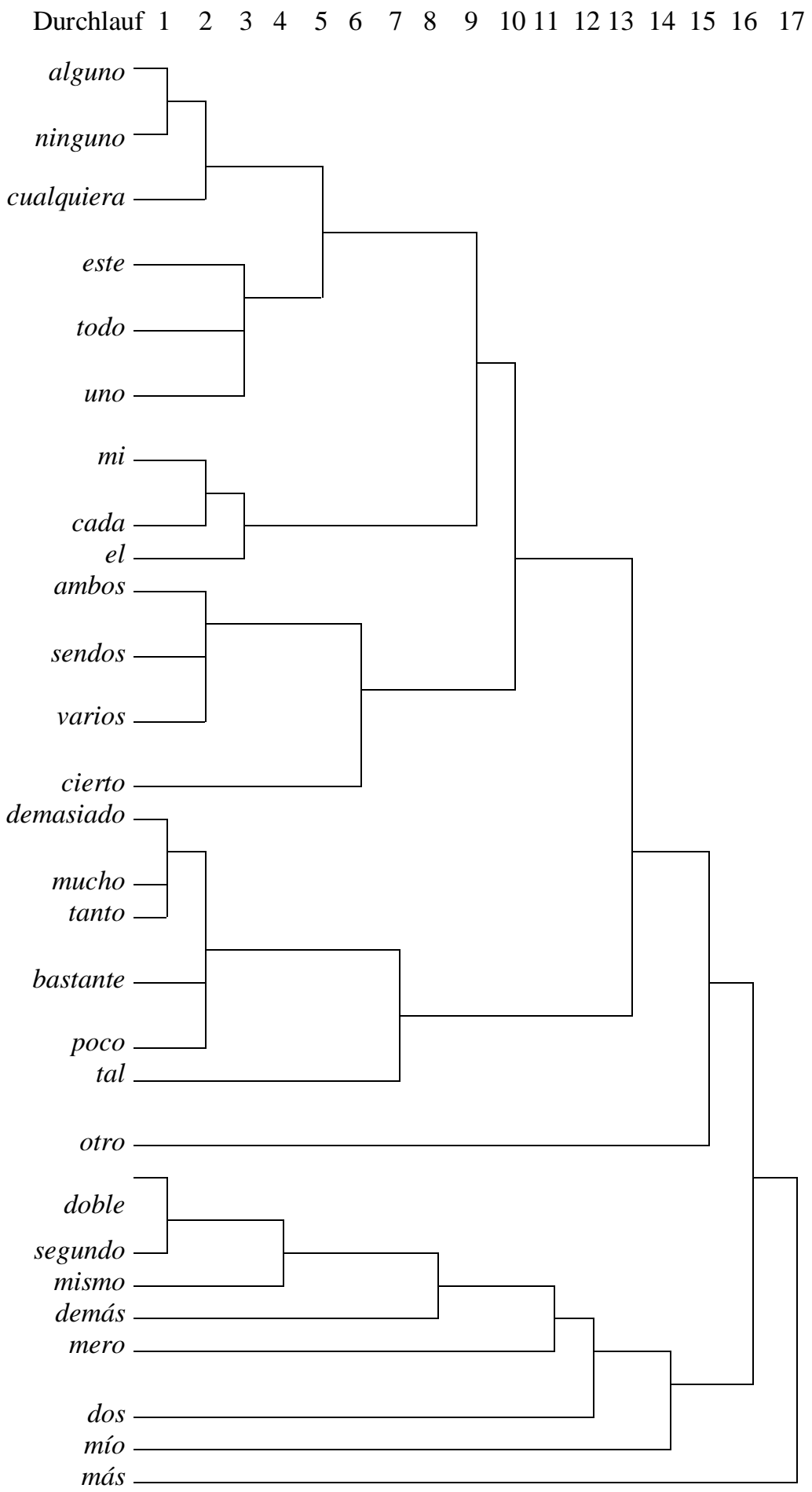


Die Zahlenangaben beziehen sich auf die Durchläufe der Klassifikation. Ein Durchlauf bezeichnet eine Clusterbildung, bei der zwei Cluster zusammengeschlossen werden. Die Distanz, welche der Clusterbildung zugrunde liegt, wird in der folgenden Tabelle genannt (nach der dritten Stelle hinter dem Komma abgebrochen):

\section{Distanzen bei der Clusterbildung}

\begin{tabular}{|r|r|r|r|r|r|}
\hline Durchlauf & Distanz & Durchlauf & Distanz & Durchlauf & Distanz \\
\hline 1 & 0 & 7 & 1,816 & 13 & 2,392 \\
\hline 2 & 1 & 8 & 1,866 & 14 & 2,590 \\
\hline 3 & 1,41 & 9 & 1,912 & 15 & 2,594 \\
\hline 4 & 1,626 & 10 & 1,992 & 16 & 2,914 \\
\hline 5 & 1,634 & 11 & 2,131 & 17 & 2,956 \\
\hline 6 & 1,652 & 12 & 2,161 & & \\
\hline
\end{tabular}

Das Dendrogramm (112) zeigt, daß die spanischen Determinierer in einer Abstufung zu den Adjektiven hin angeordnet werden müssen. Eine Klassenerstellung ist relativ schwierig, wenn wir die in (111) genannten anderen Verfahren und Gewichtungen einbeziehen, da sich nur wenige Determinierer immer an der gleichen Stelle befinden. Allerdings befinden sich bestimmte Determinierer immer in festen Gruppen, da die größte Distanz zwischen ihnen 1 beträgt. Diese Gruppen variieren zwar ihre Position innerhalb der Klassifikationen, wir können sie aber als Fixpunkte definieren, während alle anderen Determinierer sich wie Satelliten um diese herum anordnen. Als Fixpunkte können wir immerhin die folgenden 15 der 28 Determinierer bezeichnen, welche sich spätestens im zweiten Durchlauf bei Distanz 1 zu einem Cluster zusammengeschlossen haben:

(114) 1. alguno, ninguno, cualquiera,

2. mi, cada,

3. ambos, sendos, varios,

4. demasiado, mucho, tanto, bastante, poco,

5. doble, segundo.

Wir können zudem die gemeinsamen Eigenschaften dieser Fixpunkte bestimmen (o bei Uneinheitlichkeit der Eigenschaft): 


\begin{tabular}{|c|c|c|c|c|c|c|c|c|c|c|c|c|c|c|c|c|c|}
\hline & 1. & 2. & 3. & 4. & 5. & 6. & 7. & 8. & 9. & 10. & 11. & 12. & 13. & 14. & 15. & 16. & 17. \\
\hline & $\begin{array}{l}\bar{Q} \\
\stackrel{Q}{0} \\
\stackrel{\vec{Z}}{6} \\
\text { it }\end{array}$ & 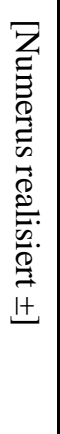 & 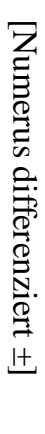 & 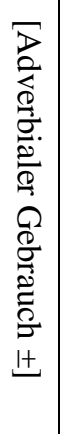 & 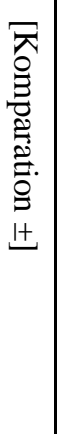 & 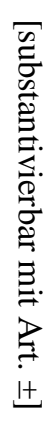 & 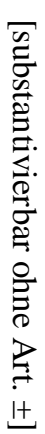 & 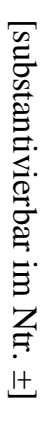 & 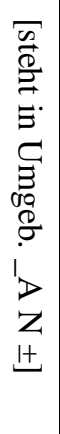 & 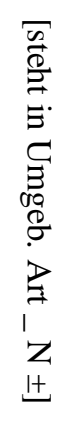 & 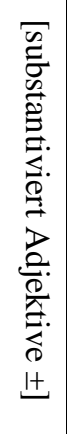 & 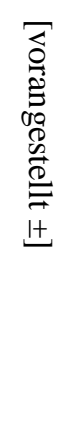 & 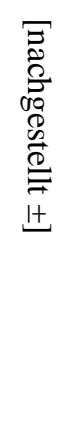 & 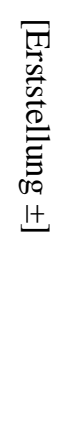 & 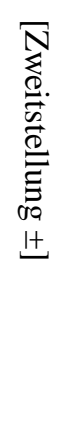 & 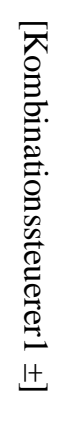 & 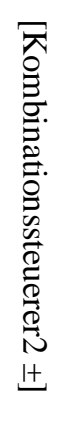 \\
\hline alguno & + & + & + & - & - & - & + & - & + & - & + & + & + & + & - & - & - \\
\hline ninguno & + & + & + & - & - & - & + & - & + & - & + & + & + & + & - & - & - \\
\hline cualquiera & - & + & + & - & - & - & + & - & + & - & + & + & + & + & - & - & - \\
\hline Fixpunkt 1 & o & + & + & - & - & - & + & - & + & - & + & + & + & + & - & - & - \\
\hline$m i / t u$ & - & + & + & - & - & - & - & - & + & - & - & + & - & + & - & + & - \\
\hline cada & - & + & - & - & - & - & - & - & + & - & - & + & - & + & - & + & - \\
\hline Fixpunkt 2 & - & + & o & - & - & - & - & - & + & - & - & + & - & + & - & + & - \\
\hline ambos & + & + & - & - & - & - & + & - & + & - & - & + & - & + & - & - & - \\
\hline sendos & + & + & - & - & - & - & - & - & + & - & - & + & - & + & - & - & - \\
\hline varios & + & + & - & - & - & - & + & - & + & - & - & + & - & + & + & - & - \\
\hline Fixpunkt 3 & + & + & - & - & - & - & o & - & + & - & - & + & - & + & $\mathrm{O}$ & - & - \\
\hline demasiado & + & + & + & + & - & - & + & + & + & - & - & + & - & + & + & - & - \\
\hline mucho & + & + & + & + & - & - & + & + & + & - & - & + & - & + & + & - & - \\
\hline росо & + & + & + & + & - & - & + & + & + & - & - & + & - & + & + & - & + \\
\hline bastante & - & + & + & + & - & - & + & + & + & - & - & + & - & + & + & - & - \\
\hline tanto & + & + & + & + & - & - & + & + & + & - & - & + & - & + & + & - & - \\
\hline Fixpunkt 4 & O & + & + & + & - & - & + & + & + & - & - & + & - & + & + & - & o \\
\hline doble & - & + & + & - & - & + & - & + & - & + & - & + & + & - & + & - & + \\
\hline segundo & + & + & + & - & - & + & - & + & - & + & - & + & + & - & + & - & + \\
\hline Fixpunkt 5 & O & + & + & - & - & + & - & + & - & + & - & + & + & - & + & - & + \\
\hline
\end{tabular}

Die gemeinsamen Eigenschaften dieser Fixpunkte können nun mit den idealisierten Wortartenvertretern verglichen werden. In den Spalten Det und Adj steht die jeweilige Differenz der Wortartenvertreter zu den Klassen, symbolisiert durch die Fixpunkte. Uneinheitliche Eigenschaften (o) werden dabei ignoriert, es zählen nur + / Differenzen: 


\begin{tabular}{|l|c|c|c|c|c|c|c|c|c|c|c|c|c|c|c|c|c|c|c|}
\hline & 1. & 2. & 3. & 4. & 5. & 6. & 7. & 8. & 9. & 10. & 11. & 12. & 13. & 14. & 15. & 16. & 17. & Det & Adj \\
\hline Fixpunkt 1 & o & + & + & - & - & - & + & - & + & - & + & + & + & + & - & - & - & 2 & 9 \\
\hline Det & o & o & o & - & - & - & + & - & + & - & + & + & - & + & - & + & - & X & 11 \\
\hline Fixpunkt 2 & - & + & o & - & - & - & - & - & + & - & - & + & - & + & - & + & - & 2 & 9 \\
\hline Fixpunkt 3 & + & + & - & - & - & - & o & - & + & - & - & + & - & + & o & - & - & 2 & 8 \\
\hline Fixpunkt 4 & o & + & + & + & - & - & + & + & + & - & - & + & - & + & + & - & o & 5 & 8 \\
\hline Fixpunkt 5 & o & + & + & - & - & + & - & + & - & + & - & + & + & - & + & - & + & 11 & 4 \\
\hline Adj & o & o & o & + & + & + & - & + & - & + & - & + & + & - & - & - & - & 11 & X \\
\hline
\end{tabular}

Als erste Ergebnisse können folgende Beobachtungen gemacht werden:

- Der typische Determinierer steht zwischen den Fixpunkten 1 und 2. Fixpunkt 1 zeichnet sich durch determinierertypische Eigenschaften mit möglicher Nachstellung und ohne Kombinationssteuerung aus. Fixpunkt 2 kennt weder Substantivierung ohne Artikel noch von Adjektiven. Der konstruierte, typische Determinierer erhält somit keinen expliziten Vertreter und stellt eindeutig eine Idealisierung dar. Fixpunkt 3 verhält sich gegenüber dem typischen Determinierer wie Fixpunkt 2, steht aber etwas näher zu den Adjektiven.

- Fixpunkt 5 befindet relativ nah am typischen Adjektiv und sehr weit entfernt vom typischen Determinierer.

- Fixpunkt 4 befindet sich am ehesten in einer Zwischenposition, die mit einer Differenz von 5 bzw. 8 relativ weit von beiden Wortarten entfernt ist.

An dieser Stelle sollen die anderen in (111) genannten Klassifizierungsansätze in die Besprechung einfließen. Sie bestätigen die Fixpunkte, die grundsätzlich zusammen stehen. Allerdings ist die Position der Fixpunkte innerhalb der Klassifikation unterschiedlich. So folgt z.B. im Dendrogramm der zweifachen Gewichtung adjektivtypischer Merkmale dem Fixpunkt 1 (alguno u.a.) Fixpunkt 3 (ambos u.a.), welcher damit vor Fixpunkt 2 ( $m i$ u.a.) steht. Dies wird im folgenden vereinheitlicht, da es keine weitere Auswirkungen auf die Abgrenzung der Determinierer von den Adjektiven nach sich zieht. Daß jede Klassifikation andere Positionen der als Satelliten bezeichneten Determinierer zur Folge hat, ist in diesem Zusammenhang wichtiger. Wir erstellen eine vergleichende Tabelle, die um die schattierten Fixpunkte gruppiert ist. Jede Gruppe um einen Fixpunkt kann als Klasse bezeichnet werden. 
(117) Fixpunkte und Satelliten der sechs Klassifikationen

\begin{tabular}{|c|c|c|c|c|c|c|}
\hline Nr. & 1 & 2 & 3 & 4 & 5 & 6 \\
\hline Anzahl Det: & 28 & $28+2$ & $28+2$ & $28+2$ & 28 & 28 \\
\hline $\begin{array}{l}\text { Anzahl Ei- } \\
\text { genschaften: }\end{array}$ & 17 & 17 & 17 & 17 & 13 & 17 \\
\hline Gewichtung: & $1 \mathrm{x}$ & $1 \mathrm{x}$ & 2x (Det) & $2 \mathrm{x}(\mathrm{Adj})$ & $1 \mathrm{x}$ & $1 \mathrm{x}$ \\
\hline Verfahren: & Mittelwert & Mittelwert & Mittelwert & Mittelwert & Mittelwert & Maximum \\
\hline \multirow[t]{6}{*}{ Fixpunkt 1} & alguno & alguno & alguno & alguno & alguno & alguno \\
\hline & ninguno & ninguno & ninguno & ninguno & ninguno & ninguno \\
\hline & cualquiera & cualquiera & cualquiera & cualquiera & cualquiera & cualquiera \\
\hline & este & & todo & este & este & este \\
\hline & todo & & & todo & todo & todo \\
\hline & uno & & & uno & uno & uno \\
\hline \multirow[t]{7}{*}{ Fixpunkt 2} & $m i$ & $m i$ & $m i$ & $m i$ & $m i$ & $m i$ \\
\hline & cada & cada & cada & cada & cada & cada \\
\hline & el & el & el & el & & el \\
\hline & & DET & este & DET & & mero \\
\hline & & este & DET & & & otro \\
\hline & & todo & otro & & & \\
\hline & & uno & uno & & & \\
\hline \multirow[t]{5}{*}{ Fixpunkt 3} & ambos & ambos & ambos & ambos & ambos & ambos \\
\hline & sendos & sendos & varios & varios & sendos & sendos \\
\hline & varios & varios & cierto & cierto & varios & varios \\
\hline & cierto & cierto & sendos & sendos & el & cierto \\
\hline & & & & & cierto & \\
\hline \multirow[t]{7}{*}{ Fixpunkt 4} & demasiado & demasiado & demasiado & demasiado & demasiado & demasiado \\
\hline & mucho & mucho & mucho & mucho & mucho & mucho \\
\hline & tanto & tanto & tanto & tanto & tanto & tanto \\
\hline & bastante & bastante & bastante & bastante & bastante & bastante \\
\hline & росо & росо & росо & росо & poco & росо \\
\hline & tal & tal & tal & & tal & \\
\hline & otro & otro & & & otro & \\
\hline \multirow[t]{11}{*}{ Fixpunkt 5} & doble & doble & doble & doble & doble & doble \\
\hline & segundo & segundo & segundo & segundo & segundo & segundo \\
\hline & mismo & mismo & mismo & demás & demás & mismo \\
\hline & demás & ADJ & ADJ & otro & mismo & mío \\
\hline & mero & demás & demás & $d o s$ & mero & demás \\
\hline & dos & mero & mero & mero & dos & dos \\
\hline & mío & dos & mío & tal & menos & tal \\
\hline & más & mío & dos & mismo & mío & más \\
\hline & & más & más & ADJ & & \\
\hline & & & & mío & & \\
\hline & & & & más & & \\
\hline
\end{tabular}

Die Veränderungen bei der Klassenzugehörigkeit können auf alle sechs Klassifikationen in (111) bezogen werden. Wenn wir aber nur die ersten vier Klassifikationen betrachten, die wir aufgrund des Mittelwertverfahrens und der Berücksichtigung aller Eigenschaften für aussagekräftiger halten, vereinfacht sich die Klassifikation durch 
die Zuordnung einiger weiterer Determinierer als feste Satelliten. Wir beschränken uns im folgenden auf die Klassifikationen ohne Gewichtung mit 28 und $28+2$ Determinierern sowie auf die mit zweifacher Gewichtung von determinierer- bzw. adjektivtypischen Eigenschaften bei $28+2$ Determinierern. Die beiden anderen Verfahren (Maximum bzw. mit nur 13 Eigenschaften) verdeutlichen, daß die Klassifikation im Detail je nach Verfahren variiert, aber trotzdem sehr vergleichbare Resultate zu den favorisierten Verfahren zeigt. Somit können wir die spanischen Determinierer trotz der in (118) verdeutlichten Wechsel als eine noch relativ homogene Klasse charakterisieren (z.B. im Vergleich zu der Sprachenklassifikation bei Altmann / Lehfeldt 1973: 28ff.).

Bei den restlichen Determinierern, die wir als Satelliten bezeichnet hatten, können wir zwei weitere Gruppen ersehen: diejenigen, welche immer in der gleichen Klasse stehen, und diejenigen, welche von Klasse zu Klasse wechseln. Zur ersteren Gruppe gehören el und die Wortart Determinierer als fester Bestandteil der Klasse 2, cierto als Bestandteil der Klasse 3 sowie dos, mismo, demás, más, mero, mío und die Wortart Adjektiv als fester Bestandteil der Klasse 5. Damit treffen sich in Klasse 5 diejenigen Determinierer, die syntaktisch als adjektivisch gesehen werden, da sie nicht in der Umgebung _ A N, sondern nur in der Umgebung Art _ $\mathrm{N}$ auftreten können. Neben 15 Determinierern als Fixpunkten haben wir nun noch 8 Determinierer und die Wortartenvertreter als feste Satelliten kategorisiert.

Als nicht feste Satelliten - und damit am schwierigsten zu kategorisieren - bleiben die folgenden fünf Determinierer:

(118) Wechsel der „Satelliten“:

1. todo, este und uno wechseln zwischen 1 und 2,

2. tal wechselt zwischen 4 und 5,

3. otro wechselt zwischen 2, 4 und 5.

Im Vergleich zur einfachen Klassifikation mit 28 Determinierern erzeugt die Berücksichtigung der Wortartenvertreter (28 + 2 Determinierer) eine Akzentuierung der Ergebnisse. Das idealisierte Adjektiv erzeugt ein Cluster mit mismo mit der Differenz 1 und bewirkt in der entsprechenden Klasse 5 kaum eine Veränderung. Der idealisierte Determinierer tritt in der Regel an den definiten Artikel $e l$, zu dem eine Differenz von 1 besteht. Nur wenn das unterscheidende Merkmal gewichtet wird, steht das Demonstrativ este näher. 
Folgendes Schema zeigt die Abstufungen zur Deskription der einfachen Klassifikation mit 28 Determinierern. Die Eigenschaften der Determinierer werden im Verlauf immer ,adjektivischer“, sowohl innerhalb der Klassen wie auch insgesamt von Klasse 1 zur Klasse 5 hin.

Abstufungen der Determinierer

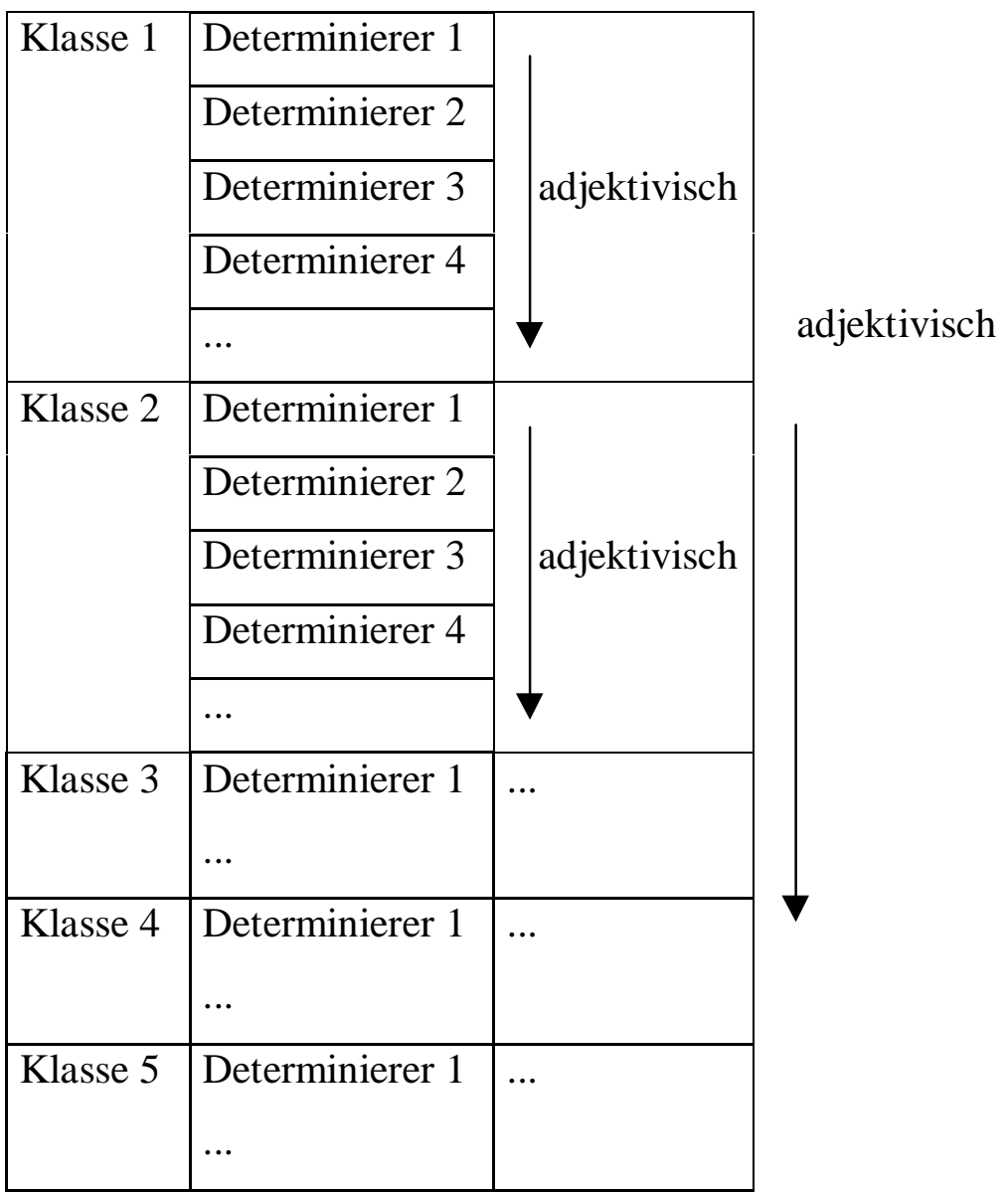

Wir müssen hervorheben, daß der typische Wortartenvertreter Determinierer keinen expliziten Vertreter hat. Die Eigenschaften, die wir für einen typischen Determinierer aufgestellt hatten, waren bei keinem spanischen Determinierer in dieser Zusammenstellung vorhanden. Somit ist es nicht verwunderlich, daß dieser idealisierte Determinierer sich nicht am äußersten Ende der Skala einordnet, sondern sich am Rande der ersten oder zweiten Klasse in der Nähe des definiten, indefiniten oder demonstrativen Determinierers, der als Aktualisierer bezeichneten Klasse, positioniert. Für Klasse 1 gilt, daß diese Determinierer manche zusätzliche Eigenschaft besitzen, die eher als adjektisch gelten muß. In der Regel ist dies die mögliche Nachstellung. 
Was passiert nun bei einer Gewichtung von bestimmten Eigenschaften? Es gibt zwei Modifikationsmöglichkeiten, wenn Eigenschaften besonders hervorgehoben werden:

1. Der Determinierer besitzt diese Eigenschaften. Er wird sich in die Richtung der gewichteten Wortart bewegen: für Determinierereigenschaften in (119) nach oben, für Adjektiveigenschaften nach unten.

2. Der Determinierer hat diese Eigenschaften nicht. Er wird abgegrenzt und dürfte sich in die entgegengesetzte Richtung bewegen.

Wenn wir nun die Klassifikationen mit Gewichtung betrachten, dann sind folgende Bewegungen erkennbar:

Este und uno wandern bei Gewichtung der determinierertypischen Eigenschaften in die Klasse 2, wo sie auch ohne Gewichtung bei Berücksichtigung der Wortartenvertreter stehen. Bei Gewichtung der adjektivtypischen Eigenschaften stehen sie in Klasse 1. Todo verhält sich ähnlich. Tal wandert bei Gewichtung der adjektivtypischen Eigenschaften in die Klasse 5, ansonsten steht es in Klasse 4. Allen Determinierern, die hier als Satelliten bezeichnet wurden, ist gemeinsam, daß sie relativ weit außen in einer Klasse stehen.

Otro wandert bei Gewichtung der determinierertypischen Eigenschaften von Klasse 4 zur Klasse 2, bei der Gewichtung der adjektivtypischen nach Klasse 5. Damit ist otro in unserer Klassifikation eindeutig als eine hybride Form zu sehen. Es kann sowohl adjektivisch wie auch als determinierend in Bezug auf sein syntaktisches Verhalten beschrieben werden. Die Semantik von otro ist stets als determinierend zu bezeichnen. 


\subsection{Analyse der Klassifikationsergebnisse}

Es lassen sich folgende 13 Wörter als „echte“ Determinierer bestimmen:

(120) 1. alguno, ninguno, cualquiera,

2. mi, cada, el,

3. este, uno, todo,

4. ambos, sendos, varios, cierto.

Diese vier Gruppen befinden sich in der Regel in den ersten drei Klassen in (117), und zwar fast immer in dieser Zusammenstellung. Sie besitzen zwar auch Eigenschaften, die als eher adjektivisch eingeordnet wurden, sind sonst aber als determinierende Artikelwörter zu sehen.

Im Gegensatz dazu befindet sich folgende Gruppe:

5. doble, segundo, mismo, demás und mero.

Diese fünf Determinierer sind als relativ stark adjektivisch zu bezeichnen, vor allem im Vergleich zu den unter 1. - 4. genannten. Sie verhalten sich syntaktisch wie Adjektive. Semantisch handelt es sich um Determinierer. Sie haben eher quantifizierende Funktion und keine qualifizierende wie Adjektive.

Ähnlich adjektivisch, aber mit einigen weiteren Eigenschaften, die sie sehr an den Rand der Klasse 5 in (117) stellen, verhält sich folgende Gruppe:

6. dos, mío und más.

In einer Zwischenposition befindet sich folgende Gruppe:

7. demasiado, bastante, mucho, poco, tanto und tal.

Hier handelt es sich um Determinierer, die einige adjektivtypische Eigenschaften, sonst aber kein durchgängig adjektivisches Verhalten aufweisen, wie es bei Gruppe 5 der Fall war. Wir sprechen bewußt von Gruppen, da eine Abgrenzung der Determinierer wie bei Alvarez Martínez (1986) nach den Ergebnissen der Klassifikation abgelehnt werden muß.

Am unmißverständlichsten zeigt sich die Ambiguität der Determinierer zwischen Artikel und Adjektiv bei otro, das wir noch nicht aufgeführt haben. Bei einfacher 
Gewichtung gehört otro zur Zwischenklasse 4 zusammen mit mucho und poco. Werden die determinierertypischen Eigenschaften gewichtet, wandert otro in die Klasse 2 zu este, uno und der Wortart Determinierer. Werden allerdings die adjektivtypischen Eigenschaften gewichtet, wandert otro in die Klasse $5 \mathrm{zu}$ doble, segundo, demás und der Wortart Adjektiv. Damit stellt otro das beste Beispiel für einen spanischen Determinierer mit hybridem Charakter in einer Position zwischen Artikel und Adjektiv dar. Es steht zumeist wie todo am Rand einer Klasse und verdeutlicht die Abstufung nach Schema (119).

Wir wollen die Eigenschaften der Gruppen 1 - 7 in (120) zusammenfassend darstellen. Dabei steht ein „,+“ oder ,,-“, wenn 2/3 der beinhalteten Determinierer diese Eigenschaft aufweisen. Ansonsten stellt „,“ die fehlende Zuordnung dar. Die Merkmale Genus und Numerus werden ignoriert.

Merkmale der Gruppen 1 - 7

\begin{tabular}{|c|c|c|c|c|c|c|c|c|c|c|c|c|c|c|c|}
\hline & & 4. & 5. & 6. & 7. & 8. & 9. & 10. & 11. & 12. & 13. & 14. & 15. & 16. & 17. \\
\hline & & 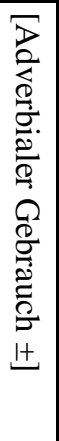 & 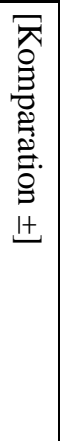 & 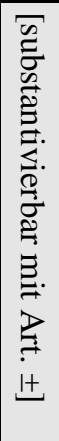 & 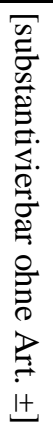 & 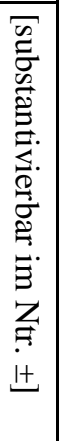 & 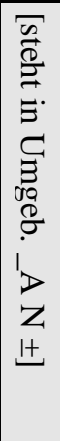 & 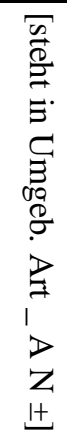 & 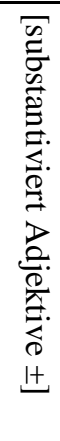 & 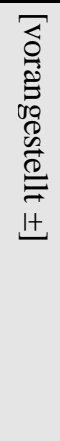 & 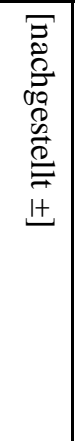 & 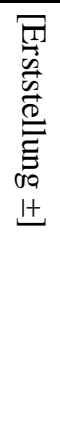 & 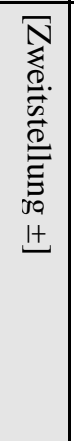 & 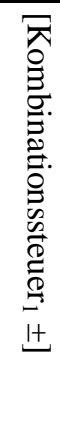 & 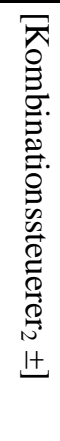 \\
\hline 1. & $\begin{array}{l}\text { alguno, } \text { ningu- } \\
\text { no, cualquiera }\end{array}$ & - & - & - & + & - & + & - & + & + & + & + & - & - & - \\
\hline $\mathrm{D}$ & Determ. & - & - & - & + & - & + & - & + & + & - & + & - & + & - \\
\hline 2. & este, uno, todo & - & - & - & + & + & + & - & + & + & + & + & - & + & - \\
\hline 3. & $\begin{array}{l}\text { mi / tu, cada, el } \\
\text { / la }\end{array}$ & - & - & - & - & - & + & - & - & + & - & + & - & + & - \\
\hline 4. & $\begin{array}{l}\text { ambos, sendos, } \\
\text { cierto, varios }\end{array}$ & - & - & - & $\mathrm{O}$ & - & + & - & - & + & - & + & 0 & - & - \\
\hline 5. & $\begin{array}{l}\text { demasiado, } \\
\text { bastante, } \\
\text { mucho, poco, } \\
\text { tanto, tal }\end{array}$ & + & - & - & + & + & + & - & - & + & - & + & + & - & - \\
\hline 6. & dos, más, mío & - & + & + & - & + & + & - & - & + & + & - & - & - & - \\
\hline A & Adjektiv & + & + & + & - & + & - & + & - & + & + & - & - & - & - \\
\hline 7. & $\begin{array}{l}\text { doble, segundo, } \\
\text { mismo, demás, } \\
\text { mero }\end{array}$ & - & - & + & - & + & - & + & - & + & $\mathrm{O}$ & - & + & - & $\mathrm{O}$ \\
\hline & & & & & & & & & & & & & & & \\
\hline & otro & - & - & + & + & + & + & + & + & + & - & + & + & + & + \\
\hline
\end{tabular}


Die Tabelle zeigt, daß die ersten beiden Gruppen fast identische Merkmale mit dem Wortartenvertreter Determinierer besitzen. Diese Merkmale sind z.T. bei der dritten Gruppe - allerdings mit gleicher quantitativer Differenz zum Wortartenvertreter wie Gruppe zwei - und in noch stärkerem Ausmaß bei der vierten nicht mehr gegeben. Die fünfte Gruppe wird bereits durch weniger Eigenschaften gekennzeichnet, die für Determinierer typisch sind. Die sechste und siebte Gruppe weisen ziemlich eindeutig adjektivische Eigenschaften auf.

Da otro nicht in das Schema paßt und wir es besonders hervorgehoben hatten, soll es hier nochmals mit den Wortartenvertretern verglichen werden:

\section{Eigenschaften von otro}

\begin{tabular}{|l|l|c|c|c|c|c|c|c|c|c|c|c|c|c|c|}
\hline & & 4. & 5. & 6. & 7. & 8. & 9. & 10. & 11. & 12. & 13. & 14. & 15. & 16. & 17. \\
\hline D & Det. & - & - & - & + & - & + & - & + & + & - & + & - & + & - \\
\hline & otro & - & - & + & + & + & + & + & + & + & - & + & + & + & + \\
\hline A & Adj. & + & + & + & - & + & - & + & - & + & + & - & - & - & - \\
\hline
\end{tabular}

Otro zeichnet sich dadurch aus, daß es sehr viele Eigenschaften besitzt und deshalb entsprechend der Gewichtung eingeordnet werden kann. Folglich paßt es nicht zu anderen Determinierern, die weniger Eigenschaften besitzen und bei einer Gewichtung weniger flexibel reagieren. Die Ausführungen bei Schwarze (1988: 33f., 43-52) lassen vermuten, daß sich ital. altro analog zu span. otro verhält. So kann altro z.B. ebenfalls in der Umgebung _ A N wie auch in der Umgebung Art _ $\mathrm{N}$ sowie in Erstund in Zweitstellung auftreten und besitzt damit Eigenschaften, die in dieser Kombination nur bei otro und vermutlich auch nur bei altro vorkommen.

Mit Blick auf die semantischen Eigenschaften gilt es noch zu bemerken, daß die Klassifizierung nicht die semantischen Klassen nach Alcina Franch / Blecua (1975: 585ff.) bestätigt. Nur ansatzweise können die existenciales (alguno, ninguno und cualquiera) sowie die gradativos (demasiado, bastante, mисho, poco) als Klassen betrachtet werden. Zum einen treten mit tal und tanto Determinierer hinzu, die auf keinen Fall in diese semantischen Kategorien gestellt werden dürfen. Zum anderen gilt diese Beobachtung für andere semantische Klassen nicht: Die Aktualisierer, die distributivos und auch die Numeralia lassen sich nicht als Klassen festmachen. Es handelt sich somit bei unserer Klassifikation um eine primär syntaktische Einteilung, zu der die Semantik lediglich einige Aspekte beisteuert. 
Wir können abschließend folgendes festhalten:

- Die spanischen Determinierer lassen sich nur in einer Abstufung zwischen Determinierer und Adjektiv erfassen. Von daher ist die traditionelle Einschätzung der Determinierer von Seiten spanischer Grammatiker als adjectivos determinativos nachvollziehbar und korrekt.

- Der definite Artikel kann problemlos integriert werden. An dieser Stelle sind spanische Grammatiken, die den definiten Artikel oft aus sprachhistorischen oder morphologischen Gründen gesondert behandeln, im syntaktischen Bereich als ungenau zu kritisieren.

- Es handelt sich primär um eine syntaktische Klassifikation. Die Semantik steuert einige für die Kombinationsmöglichkeiten wichtige Aspekte bei.

\subsection{Exkurs: Vergleich mit dem deutschen Determinierersystem}

Für das Spanische konnten wir feststellen, daß es keine klare Abgrenzung zwischen Determinierern und Adjektiven gibt. Wir haben dabei zwar behauptet, dies wäre im Deutschen anders, haben diese Behauptung aber bisher nicht bewiesen. Dies soll hier kurz zu Vergleichszwecken geschehen, wobei sich das Deutsche aufgrund seiner deutlich anderen Nominalsyntax anbietet. Zudem werden wir sehen, daß eine Klassifikation anhand der für das Spanische erarbeiteten Kriterien möglich ist und die gewünschten Ergebnisse bringt. Um einen möglichst brauchbaren Vergleich zu ermöglichen, werden wir die Kriterien auf das Deutsche übertragen. Daß wir damit der deutschen Grammatik nicht voll gerecht werden können, sollte schon aufgrund des deutschen Kasussystems einleuchten. Die folgende Klassifikation deutscher Determinierer und Adjektive ist nur für den Vergleich gedacht.

Nach Helbig / Buscha (1994: 357) können folgende Determinierer berücksichtigt werden: der / die / das, alle, dieser / jener, ein, einige, irgendein / irgendwelche, jeder, kein, mancher, mehrere, mein / dein / sein / euer / ihr, solche. Dazu kommen noch folgende, die Helbig / Buscha (1994: 358) als Indefinitpronomina oder Zahladjektive bezeichnen, die wir aber zum Vergleich mit heranziehen müssen: viele, wenige, beide, sämtliche, zwei / drei ... Wir fügen noch zweiter / dritter und zweifach / dreifach als Gegenstück zu doble, segundo hinzu, die im Deutschen klar adjektivisch sind, sowie andere als Gegenstück zu otro. 
Zuvor sind noch einige Anmerkungen zu machen: Wir führen nicht die Varianten der Determinierer auf, wie sie bei Helbig / Buscha (1994: 356f.) bezeichnet werden, wie derjenige, derselbe, welch ein, manch ein, ein solches, solch ein oder auch jedweder. Diese Varianten verhalten sich meist identisch mit der Ausgangsform. In die Analyse integriert wird das bei Langer / Thümmel (1994: 118) als Demonstrativadjektiv bezeichnete Wort derartige. In Grammatiken des Deutschen variieren die zu den Indefinitpronomina gezählten Determinierer z.T. beträchtlich (vgl. Helbig / Buscha 1994: 356ff. und Engel 1982: 64ff.). Wir erstellen hier eine Zusammenstellung, die in erster Linie dem Vergleich mit dem Spanischen dient und die deutschen Gegebenheiten nicht grundlegend analysiert.

Weiterhin gehen Helbig / Buscha (1994: 356f.) davon aus, daß Kombinationen zwischen Determinierern im Deutschen nicht möglich sind (vgl. dazu auch Engel 1982: 67f.). Dies müssen wir hier aber annehmen, um einen Vergleich mit dem Spanischen erstellen zu können. Mit Langer / Thümmel (1994) liegt außerdem eine Untersuchung der Kombinationsmöglichkeiten deutscher Determinierer vor.

Zuletzt gehen Helbig / Buscha von einem Nullartikel aus, den wir hier ablehnen, und beziehen Relativ- und Interrogativpronomina ein, die in der vorliegenden Arbeit ausgeklammert wurden.

Wir müssen zunächst bei den Kriterien untersuchen, inwieweit sie auf das Deutsche angewandt werden können. Als erstes zeigte sich, daß Genus grundsätzlich bei allen Determinierern differenziert wird. Dies ist im Plural z.T. nicht ersichtlich, zeigt sich aber z.B. bei der Substantivierung. Somit kann dieses Merkmal entfallen. Gleiches gilt für die Numerusrealisierung: Es gibt kein Pendant zum spanischen más. Das deutsche mehr verhält sich in der Regel adverbial. Wir können lediglich feststellen, ob der Determinierer nur in einem Numerus erscheint: So kommt z.B. alle nur im Plural vor, ein nur im Singular (der Plural wird durch einige vertreten). Irgendein und irgendwelche werden als Vertreter des jeweiligen Numerus gemeinsam betrachtet.

Sodann können die Merkmale Voran- und Nachstellung entfallen, da es Nachstellung im Deutschen nicht gibt (eine bekannte Ausnahme: Vater unser) Gleiches gilt für die Kombinationssteuerung: Die Kombinationsmöglichkeiten werden mit den Merkmalen Erst- und Zweitstellung abgedeckt. Es verbleiben somit elf Merkmale.

Die meisten Werte bei den Merkmalen der deutschen Determinierer dürften einsichtig sein. Erklärungsbedürftig sind die Kombinationsmöglichkeiten, wie sie bei 
Langer / Thümmel (1994:116ff.) untersucht wurden. Einige Kombinationsmöglichkeiten, die Langer / Thümmel als solche betrachten, können nicht berücksichtigt werden: einige der, mehrere dieser, manche jener. Hier liegt eine Genitivkonstruktion vor, wie folgendes Beispiel zeigt. So kann die Kombination mehrere dieser in dem Satz mehrere dieser Gattung verhalten sich anders als mehrere Exemplare dieser Gattung verhalten sich anders umgeformt werden, um den Genitiv offensichtlich zu machen. Eine Form der Kombination auf gleicher Ebene wäre die ungrammatische Phrase *mehrere diese Gattung. Wir gehen davon aus, daß hier zwei Phrasen vorliegen: [mehrere [dieser Gattung] NP ] NP. Die Determinierer mehrere und dieser sind auf derselben Einbettungsebene nicht kombinierbar. Ebenfalls ausgeklammert werden Phrasen der Art welch ein, solch ein, all der Ärger u.a. aus den oben genannten Gründen (vgl. Engel 1982: 69).

Eine Kombinationstabelle kann wie folgt aussehen. Der, alle, einige, irgendein, kein, manch und mehrere können nicht in Zweitstellung, solch, beide, sämtliche, derartige, zweiter und zweifach nicht in Erststellung auftreten. Die Kombinationen alle diese, all der, diese eine bzw. diese meine sind im Deutschen ähnlich wie im Spanischen erlaubt. Wir hatten sie für das Spanische als Ausnahmen deklariert und verfahren hier für das Deutsche analog. Damit können folglich auch dieser, ein und mein in Zweitstellung entfallen. Kombinationen, die nur bei Substantivierung des zweiten Determinierers möglich sind, werden nicht berücksichtigt, z.B. alle beide kommen mit, aber *alle beide Freunde kommen mit. Für die Numeralia beide, zwei, zweite und zweifach können wir annehmen, daß sie als Adjektive in Dreierkombinationen analog zum Spanischen viele Kombinationen zulassen, z.B. die anderen beiden Bücher, die beiden anderen Bücher, die zwei anderen Bücher, die anderen zwei Bücher usw. Diese Fälle werden hier ebenfalls nicht berücksichtigt. 


\begin{tabular}{|l|c|c|c|c|c|c|c|c|c|c|c|}
\hline & jeder & $\begin{array}{l}\text { sämt- } \\
\text { lich }\end{array}$ & solche & beide & viele & wenige & $\begin{array}{l}\text { derar- } \\
\text { tige }\end{array}$ & zwei & zweite & $\begin{array}{l}\text { zwei- } \\
\text { fach }\end{array}$ & andere \\
\hline mein & & $\mathrm{x}$ & & $\mathrm{x}$ & $\mathrm{x}$ & $\mathrm{x}$ & $\mathrm{x}$ & $\mathrm{x}$ & $\mathrm{x}$ & $\mathrm{x}$ & $\mathrm{x}$ \\
\hline der & & & & $\mathrm{x}$ & $\mathrm{x}$ & $\mathrm{x}$ & & $\mathrm{x}$ & $\mathrm{x}$ & $\mathrm{x}$ & $\mathrm{x}$ \\
\hline diese & & & & $\mathrm{x}$ & $\mathrm{x}$ & $\mathrm{x}$ & $\mathrm{x}$ & $\mathrm{x}$ & $\mathrm{x}$ & $\mathrm{x}$ & $\mathrm{x}$ \\
\hline einige & & & $\mathrm{x}$ & & & $\mathrm{x}$ & $\mathrm{x}$ & & $\mathrm{x}$ & $\mathrm{x}$ & $\mathrm{x}$ \\
\hline irgendein & & & $\mathrm{x}$ & & & & & $\mathrm{x}$ & $\mathrm{x}$ & $\mathrm{x}$ & $\mathrm{x}$ \\
\hline kein & & & $\mathrm{x}$ & & & & $\mathrm{x}$ & $\mathrm{x}$ & $\mathrm{x}$ & $\mathrm{x}$ & $\mathrm{x}$ \\
\hline mancher & & & & & & & $\mathrm{x}$ & $\mathrm{x}$ & $\mathrm{x}$ & $\mathrm{x}$ & $\mathrm{x}$ \\
\hline andere & & & $\mathrm{x}$ & & & & $\mathrm{x}$ & $\mathrm{x}$ & $\mathrm{x}$ & $\mathrm{x}$ & $/$ \\
\hline ein & $\mathrm{x}$ & & $\mathrm{x}$ & & & & $\mathrm{x}$ & & $\mathrm{x}$ & $\mathrm{x}$ & $\mathrm{x}$ \\
\hline mehrere & & & $\mathrm{x}$ & & & & $\mathrm{x}$ & & $\mathrm{x}$ & $\mathrm{x}$ & $\mathrm{x}$ \\
\hline wenige & & & $\mathrm{x}$ & & & $/$ & $\mathrm{x}$ & & $\mathrm{x}$ & $\mathrm{x}$ & $\mathrm{x}$ \\
\hline viele & & & $\mathrm{x}$ & & $/$ & & $\mathrm{x}$ & & $\mathrm{x}$ & $\mathrm{x}$ & $\mathrm{x}$ \\
\hline jeder & $/$ & & & & & & $\mathrm{x}$ & & $\mathrm{x}$ & $\mathrm{x}$ & $\mathrm{x}$ \\
\hline alle & & & & & & & $\mathrm{x}$ & & $\mathrm{x}$ & $\mathrm{x}$ & $\mathrm{x}$ \\
\hline zwei & & & $\mathrm{x}$ & & & & $\mathrm{x}$ & $/$ & $\mathrm{x}$ & $\mathrm{x}$ & $\mathrm{x}$ \\
\hline
\end{tabular}

Es lassen sich folgende Beobachtungen machen:

- Mit den Numeralia und andere sind sehr viele Kombinationen erlaubt. Wir nehmen an, daß nur für solche, beide, viele, wenige, derartige, andere und die $\mathrm{Nu}-$ meralia das Merkmal [Zweitstellung +] akzeptabel ist. Jeder und sämtliche werden in die Gruppe der Ausnahmen mit Kombinationen wie alle diese und diese meine eingeordnet.

- der, diese und mein sind mit fast den gleichen Determinierern kombinierbar und bilden auch im Deutschen eine Klasse, die als Aktualisierer bezeichnet werden kann.

- solche und derartige sind auffallend häufig kombinierbar.

- Wir hatten für spanische Determinierer die Merkmale Kombinationssteuerer ${ }_{1}$ und Kombinationssteuerer 2 eingeführt. Diese könnten im Deutschen auf alle Determinierer in Zweitstellung übertragen werden. Somit kann das Kriterium Kombinationssteuerer zur Differenzierung entfallen.

Es folgt die Zusammenstellung aller Merkmale. Die Numerierung bleibt so, wie sie aus der Klassifikation für spanische Determinierer bekannt ist: 


\begin{tabular}{|c|c|c|c|c|c|c|c|c|c|c|c|}
\hline & 3. & 4. & 5. & 6. & 7. & 8. & 9. & 10. & 11. & 14. & 15. \\
\hline & 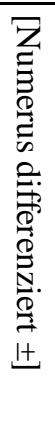 & 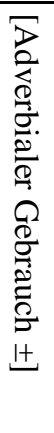 & 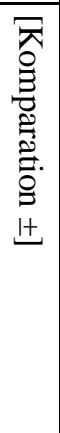 & 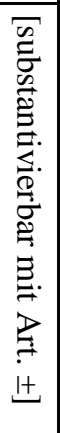 & 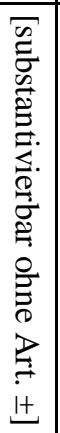 & 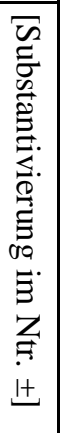 & 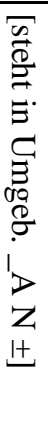 & 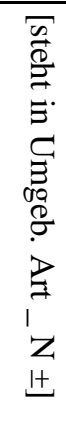 & 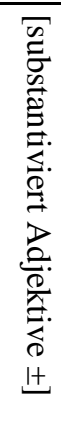 & 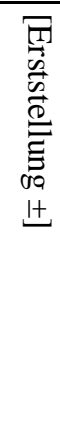 & 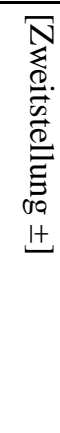 \\
\hline der & + & - & - & - & + & + & + & - & + & + & - \\
\hline alle & - & - & - & - & + & + & + & - & + & + & - \\
\hline dieser & + & - & - & - & + & + & + & - & + & + & - \\
\hline ein & - & - & - & - & + & + & + & - & + & + & - \\
\hline einige & - & - & - & - & + & + & + & - & + & + & - \\
\hline irgendein & + & - & - & - & + & + & + & - & + & + & - \\
\hline jeder & - & - & - & - & + & + & + & - & + & + & - \\
\hline kein & + & - & - & - & + & + & + & - & + & + & - \\
\hline mancher & + & - & - & - & + & + & + & - & + & + & - \\
\hline mehrere & - & - & - & - & + & + & + & - & + & + & - \\
\hline mein & + & - & - & - & + & + & + & - & + & + & - \\
\hline solche & - & - & - & - & + & + & + & - & + & + & + \\
\hline andere & + & - & - & + & + & + & + & + & + & + & + \\
\hline beide $^{28}$ & - & - & - & + & + & + & - & + & + & - & + \\
\hline sämtliche & + & - & - & - & - & - & + & - & + & + & - \\
\hline derartige & + & + & - & - & + & + & + & - & + & - & + \\
\hline viele & + & + & + & + & + & + & + & + & + & + & + \\
\hline wenige & + & + & + & + & + & + & + & + & + & + & + \\
\hline$z w e i^{29}$ & + & - & - & + & + & - & + & + & + & + & + \\
\hline zweiter & + & + & - & + & - & + & - & + & - & - & + \\
\hline zweifacher & + & + & - & + & - & + & - & + & - & - & + \\
\hline
\end{tabular}

Ein erster Blick zeigt, daß bis auf das erste Merkmal der Numerusdifferenzierung alle Determinierer von der bis mein identisch sind. Die idealisierte Wortart Determinierer erhält die gleichen Merkmale wie der. Damit sind bereits elf der neunzehn erfaßten Determinierer in einer Klasse (im folgenden DET) zusammengefaßt. Identisch sind ebenfalls viele und wenige, die interessanterweise alle erstellten Kriterien innehaben, sowie die Numeralia zweiter und zweifacher. Eine übersichtlichere Ta-

\footnotetext{
${ }^{28}$ Für beide müssen unterschiedliche Sprechereinschätzungen angenommen werden: Wir beurteilen eine Phrase wie *beide großen Autos als inakzeptabel. Eine gegenteilige Einschätzung hat das Merkmal [steht in Umgebung _ A N +] zur Folge.

${ }^{29}$ Für zwei gilt das gleiche wie für dos: es steht für die Kardinalia und differenziert durch neue Lexeme $($ drei, vier , ....) oder morphologisch (Million Millionen).
} 
belle sieht wie folgt aus, wobei wir die idealisierte Wortart Adjektiv ergänzen. Für die Merkmale des Wortartenvertreters Adjektiv gelten die gleichen Einschränkungen, wie wir sie bereits für die spanische Klassifikation genannt hatten: Die Merkmale Erst- und Zweitstellung werden nur für die Kombination von Determinierern verwandt. Das Merkmal substantivierbar ohne Artikel trifft bei Adjektiven nur im Plural $\mathrm{zu}$ und setzt eine Generalisierung als lexikalischen Prozeß voraus. Wir weisen nochmals darauf hin, daß es sich um eine Klassifikation zum Vergleich mit den spanischen Determinierern handelt. Sie kann keinen Anspruch auf eine vollständige Erfassung des deutschen Determinierersystems stellen.

Merkmale der deutschen Determinierer (einfach klassifiziert)

\begin{tabular}{|c|c|c|c|c|c|c|c|c|c|c|c|}
\hline & 3. & 4. & 5. & 6. & 7. & 8. & 9. & 10. & 11. & 14. & 15. \\
\hline & 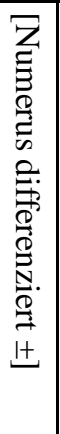 & 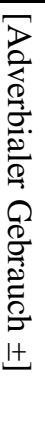 & 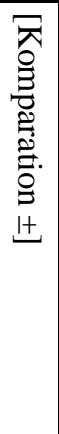 & 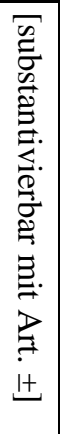 & 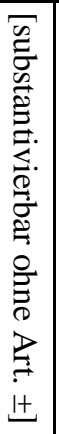 & 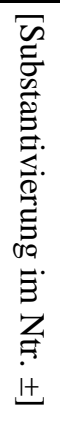 & 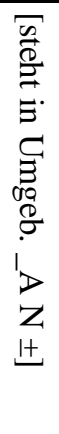 & 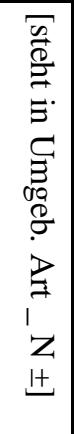 & 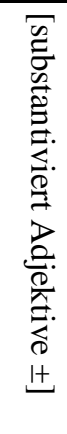 & 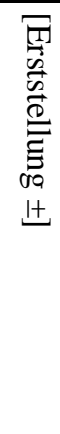 & 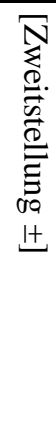 \\
\hline Klasse DET & $\mathrm{O}$ & - & - & - & + & + & + & - & + & + & - \\
\hline solche & - & - & - & - & + & + & + & - & + & + & + \\
\hline sämtliche & + & - & - & - & - & - & + & - & + & + & - \\
\hline derartige & + & + & - & - & + & + & + & - & + & - & + \\
\hline zwei & + & - & - & + & + & - & + & + & + & + & + \\
\hline andere & + & - & - & + & + & + & + & + & + & + & + \\
\hline viele & + & + & + & + & + & + & + & + & + & + & + \\
\hline zweiter & + & + & - & + & - & + & - & + & - & - & + \\
\hline ADJEKTIV & $\mathrm{O}$ & + & + & + & - & + & - & + & - & - & - \\
\hline beide & - & - & - & + & + & + & - & + & + & - & + \\
\hline
\end{tabular}

Wenn wir diese Ergebnisse in ein Dendrogramm überführen, erhalten wir folgendes Ergebnis bei einer einfachen Klassifikation ohne Gewichtung mit elf Merkmalen und der Wortart Adjektiv (mit Gewichtung ergeben sich keine gravierenden Unterschiede, statt der Durchläufe werden direkt die Distanzen genannt): 
(126)

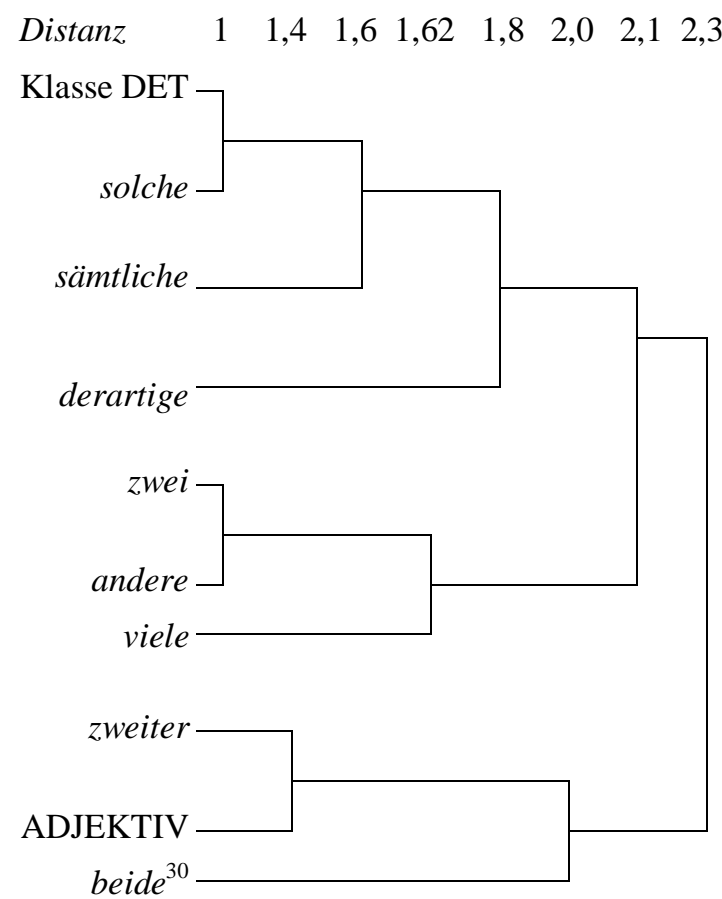

Folgende Beobachtungen lassen sich machen:

- Dadurch, daß eine Klasse bereits aus elf Determinierern besteht, erhalten wir ein sehr viel übersichtlicheres Dendrogramm, als es beim Spanischen der Fall war.

- Die Numeralia zweiter / zweifacher sowie beide können in ihrem syntaktischen Verhalten als Adjektive angesehen werden.

- Auch wenn eine sehr kleine - im Vergleich zum Spanischen bedeutend kleinere Übergangsgruppe mit zwei, andere und viele entsteht, so hat das Deutsche im Vergleich zum Spanischen ein klareres Determinierersystem. Trotzdem bleiben Abgrenzungsprobleme, wie wir sie aus der romanischen Sprache kennen. Solche, sämtliche und derartige stehen ihr syntaktisches Verhalten betreffend nur in der Nähe der Determinierer, auch wenn sie als solche klassifiziert werden müssen.

Es bleibt noch, erneut darauf hinzuweisen, daß wir ein Klassifikationssystem benutzt haben, welches für das Spanische entwickelt wurde. Dieses System wurde hier fast eins zu eins auf das Deutsche angewandt. Inwieweit diese Vorgangsweise dem Deutschen gerecht wird, müßte eine detailliertere Untersuchung zeigen. Es ist sehr wahrscheinlich, daß einige neue Kriterien geschaffen oder bereits erstellte modifiziert

\footnotetext{
${ }^{30}$ Wenn das Merkmal steht in Umgebung _ A N positiv gewertet wird, fällt beide mit der gleichen Distanz wie viele in die Gruppe zu zwei und andere.
} 
werden müßten. Dabei ist besonders die Kasusrealisierung im Deutschen zu beachten, die von unserem Klassifikationssystem nicht erfaßt wird (vgl. Engel 1982: 57ff., der seine Wortartenklassifikation auf Kriterien der Kombinierbarkeit mit Flexemen basiert).

Zusammenfassend kann auch für das Deutsche eine Übergangsklasse erstellt werden, die sich weder den Determinierern noch den Adjektiven eindeutig zuordnen läßt. Diese Klasse ist allerdings wesentlich kleiner und wesentlich einfacher einzugrenzen als im Spanischen. Man kann nicht von einer Klasse der determinierenden Adjektive sprechen, höchstens von einigen wenigen Determinierern, die in eine solche Richtung tendieren. Die deutschen Determinierer stellen das einheitlichere System dar. 


\section{Spanische Determinierer und Nomina in der HPSG}

Anhand ausgewählter Kriterien haben wir im letzten Kapitel eine Klassifikation der spanischen Determinierer erstellt. Diese Klassifikation ergab, daß sich die spanischen Determinierer nicht als eine homogene Wortart erfassen lassen. Vielmehr haben einige von ihnen Eigenschaften, die eine Einordnung als determinierende Adjektive begründen, wie auch in traditionellen spanischen Grammatiken angenommen wird (z.B. doble, segundo, mismo, demás und mero). Demgegenüber verhalten sich andere Determinierer erwartungsgemäß analog zum definiten Artikel, weisen aber trotzdem auch adjektivtypische Eigenschaften auf (z.B. alguno, ninguno, cualquiera). Eine letzte Gruppe verhält sich ähnlich wie der definite Artikel und kann nicht als adjektivähnlich betrachtet werden (cada, mi). Diese Determinierer weisen in ihrem syntaktischen Verhalten eine große Nähe zum definiten Artikel des Deutschen auf. Das Ergebnis ist eine Abstufung, die von Determinierern, die sich syntaktisch wie Adjektive verhalten, über die Zwischenklassen bis zu Determinierern reicht, die sich syntaktisch rein determinierend verhalten.

Es stellt sich nun die Frage, wie dieses Ergebnis in einer deskriptiven Grammatik wie der HPSG erfaßt werden kann. Ein Ergebnis der Klassifikation war, daß wir eher auf syntaktische als auf semantische Merkmale zählen können. Semantische Aspekte sorgen dafür, daß auch die determinierenden Adjektive noch als Determinierer gesehen werden müssen, da sie quantifizierend und nicht qualifizierend verwendet werden. Allerdings waren die semantischen Eigenschaften nur schwer herauszuarbeiten, so daß wir lediglich Anhaltspunkte für die Kombinationsmöglichkeiten erstellen konnten. Von daher wird die Semantik nur einen Teilaspekt der folgenden Betrachtungen ausmachen.

Als erstes wollen wir die Beziehung zwischen Nomina und Determinierern in der Nominalphrase, besonders bezüglich Valenz und Kongruenz, betrachten (Kap. III.3.1.). Als Valenz wird die Eigenschaft eines Lexems bezeichnet, morphologische Kategorien eines abhängigen Elements zu bestimmen (vgl. auch Weber 1992: 17, 34ff., der von „Leerstellen“ spricht). Hier müssen wir zunächst einmal erörtern, ob in der spanischen Nominalphrase eine Abhängigkeit besteht und ob diese vom Nomen ausgeht. Zudem wird die Beziehung zwischen Nomen und Determinierer wie auch zwischen Nomen und Adjektiv durch Kongruenz bestimmt (III.3.2.). Dabei ist nach 
Zwicky (1993) die Frage zu stellen, welches Element die Kongruenz auslöst und welches Element Zielpunkt der Kongruenz ist.

Über diese Fragestellungen sollte es möglich sein, die Wortarten voneinander abzugrenzen und eine Sortenhierarchie zu erstellen (III.3.3.). Diese zeigt die Abstufung zwischen Determinierer und Adjektiv auf und integriert sie in ein Gesamtsystem. Es werden den Determinierern bestimmte Eigenschaften je nach Sorte zugesprochen, die aber unterschiedlich realisiert sein können. Es muß dabei möglich sein, daß ein Determinierer zugleich zwei Sorten angehört, um der Abstufung gerecht zu werden. Innerhalb der Sortenhierarchie sorgen lexikalische Regeln dafür, daß eine Wortart in eine andere überführt werden kann. Diese Regeln unterstehen aber bestimmten Bedingungen.

Zudem müssen Stellungsfragen zur Sprache kommen (III.3.4.). Das Thema der Kombinationsmöglichkeiten spanischer Determinierer sowie die Fragestellung, wie diese erfaßt werden können, werden erörtert. Dabei handelt es sich um eine Annäherung an die Verhältnisse. Je nach Hintergrund der befragten Informanten wurden dieselben Kombinationen erlaubt, ausdrücklich befürwortet oder abgelehnt. Diese Vagheit kann hier nur bedingt berücksichtigt werden.

Die Struktur der Nominalphrase wird in Kap. III.3.5. thematisiert, um über Lexikoneinträge (III.3.6.) und mögliche lexikalische Regeln (III.3.7.) eine Deskription der als engere NP bezeichneten Phrase zu stellen (III.3.8.). Als engere NP gilt die Kombination Nomen und Determinierer, in dieser Arbeit ergänzt durch Analysen von Adjektiven und rudimentär von Präpositionen.

Zuletzt sind die semantischen Aspekte einzubeziehen (III.3.9.). Dazu gehört auch das Thema Quantifikation.

\subsection{Valenz}

Pollard / Sag $(1987$; 1994) gehen davon aus, daß das Nomen einen Determinierer als Komplement benötigt, um eine Nominalphrase zu bilden. Dazu wurde in früheren Versionen das HEAD-Attribut als nominal bezeichnet und in der Subkategorisierungsliste eine Determiniererphrase (DP) verlangt. Sie nehmen grundsätzlich an, daß es sich um eine Determiniererphrase handelt, da im Englischen oder Deutschen dort auch ein Genitivobjekt diese Funktion übernehmen kann: Vaters Auto, father's car. 
Dies ist im Spanischen in dieser Form nicht der Fall, auch wenn mehrere Determinierer vor dem Nomen den Eindruck erwecken können, es handle sich um eine komplexere Phrase (z.B. eine DP). Der entsprechende Eintrag sieht wie folgt aus (Pollard / Sag 1987: 139ff.):

$$
\left[\text { SYNSEM } \mid \text { LOC } \mid \text { CAT }\left[\begin{array}{l}
\text { HEAD nomen } \\
\text { SUBCAT }\langle D P\rangle
\end{array}\right]\right]
$$

Wir hatten nun in Anlehnung an die Vorschläge von Borsley (1987, vgl. Pollard / Sag 1994: 344ff., Kiss 1995a und Müller 1999) die SUBCAT-Liste durch das Merkmal VALENZ (VAL) ersetzt, welches die Attribute SUBJECT (SUBJ), COMPLEMENTS (COMPS) und SPECIFIER (SPR) aufweist, die wiederum durch Listen repräsentiert sind. Für Nomina müssen wir davon ausgehen, daß sie keine Subjekte binden und Komplemente grundsätzlich fakultativ sind und nicht in der Subkategorisierungsliste erscheinen. Es bleibt also das Attribut für Spezifizierer. Zusätzlich wurde das Attribut ARGUMENT-STRUCTURE (ARG-S) eingeführt, das die Subkategorisierungsliste ersetzt, um die Obliquitätshierarchie der Komplemente zu erfassen, wenn das alle drei Valenzmerkmale zusammenbindende Merkmal fehlt. Dieses Merkmal ist für unsere Absichten nicht weiter relevant und wird vernachlässigt. Die allgemeine Struktur spanischer Nomina sieht in der neuen Version wie folgt aus (vgl. Pollard / Sag 1994: 385, 391):

$$
\left[\text { SYNSEM } \mid \text { LOC } \mid \text { CAT }\left[\begin{array}{l}
\text { HEAD nomen } \\
\operatorname{VAL}[\operatorname{SPR}\langle\text { det }\rangle]]
\end{array}\right]\right.
$$

Wir gehen davon aus, daß das Valenzattribut von Nomina nur ein Merkmal, nämlich SPR für Spezifizierer, enthalten kann und daß in diesem eine Liste mit dem einzelnen Wert det erscheint.

Nun bleibt allerdings zu fragen, ob das Nomen überhaupt Kopf der Phrase ${ }^{31}$ ist. Es gibt zahlreiche Ansätze in der HPSG und in verwandten Modellen, die das Nomen als Kopf sehen, aber auch einige andere, die in Anlehnung an Abney (1987) von der Determiniererphrase und dem Determinierer als Kopf sprechen (z.B. Abb 1994,

\footnotetext{
${ }^{31}$ Zur Diskussion des möglichen Kopfes einer NP oder DP siehe: Zwicky 1985 vs. Hudson 1987 und wiederum Zwicky 1993, sodann McGlashan 1993, Cann 1993, Borsley 1993, vgl. auch Börjars 1994 und Bouma 1988 für einen kategorialgrammatischen Ansatz.
} 
Oepen 1994, Winhart 1997). Pollard / Sag (1994: 364ff.) nennen einige Argumente, warum in ihrem System Determinierer als Komplemente und Nomina als Köpfe behandelt werden. Die Argumentation fußt im wesentlichen auf der Möglichkeit von Komparativkonstruktionen durch Determinierer und ist eher semantischer Natur. Somit bezeichnen sie zwar das Nomen als Kopf der Nominalphrase, gehen aber zugleich davon aus, daß Determinierer auch ihre Köpfe selektieren: „While continuing to assume that N's are the heads of NPs and subcategorize for their determiners, we will also assume that determiners reciprocally select their $\mathrm{N}^{\prime}$ sisters" (Pollard / Sag 1994: 50).

Diese Annahme hat nach Oepen (1994) und Winhart (1997) eine zyklische Struktur zur Folge. Das Nomen subkategorisiert den Determinierer, wie in (128) gezeigt wurde. Gleichzeitig verweist der Determinierer durch das Attribut SPECIFIED (SPEC) auf das Nomen und selektiert seinen Kopf, wie in der HPSG für Adjunkte angenommen wird (vgl. Oepen 1994: 58 und Winhart 1997: 375):

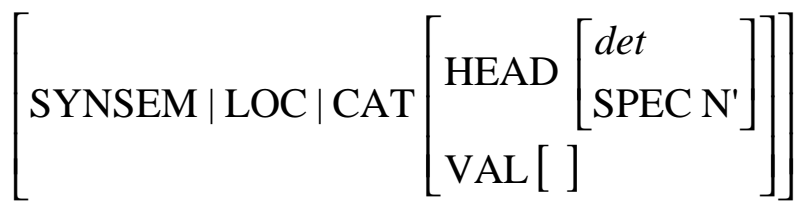

Der Wert des Attributs SPEC besteht hier aus den Werten des Nomens, auf das sich der Determinierer bezieht. In der Regel folgt auf N' ein Index, der auf das Nomen weist.

Oepen (1994: 57) weist zu Recht daraufhin, daß diese Relation - das Nomen subkategorisiert den Determinierer durch seine SUBCAT-Liste, der Determinierer selektiert seinen Kopf über das Attribut SPECIFIED (SPEC) - weder eine typische Subkategorisierung darstellt noch mit dem Kopf-Marker-Schema übereinstimmt. Ein Marker hat keinen Einfluß auf die Subkategorisierungsliste seines Kopfes. Diese Liste bleibt unangetastet und verändert sich nicht. Damit erhalten wir ein System, das zwischen Komplementation und Adjungierung eingeordnet werden muß. Dies sieht aber die HPSG-Theorie von Pollard und Sag in dieser Form nicht vor.

Oepen und Winhart äußern sich zudem unzufrieden mit dem Lösungsansatz für deutsche Nominalphrasen. Pollard / Sag (1994: 371f.) führen das Attribut DECLENSION (DECL) ein, welches das Flexionsparadigma von Adjektiven bestimmt. Adjektive selektieren als Adjunkt ihren Kopf, in diesem Fall ein Nomen, über das Merkmal MOD. Pollard / Sag schlagen vor, daß die Flexionsklasse des Ad- 
jektivs im Nomen verankert ist, das wiederum den Determinierer subkategorisiert. Dies ist aber nicht adäquat, da eindeutig der Determinierer für die Flexion des Adjektivs veranwortlich ist: das kleine Mädchen vs. ein kleines Mädchen. Mädchen verhält sich hier in bezug auf die Flexion des Adjektivs neutral.

Die Überlegungen von Pollard / Sag (1994) sowie auch von Oepen (1994) und Winhart (1997) orientieren sich am Deutschen und zum Teil am Englischen. Wie sieht es nun im Spanischen aus? Wir hatten festgestellt, daß transnumerale Nomina im Spanischen eigenständig eine NP bilden können. Das gleiche gilt für numerale Gattungsnomina, die durch den Prozeß der Generalisierung in transnumerale umgewandelt wurden. Eigennamen können in Ausnahmefällen mit einem Determinierer auftreten. Pronomina sind in der Regel nicht mit Determinierern kombinierbar, auch wenn die folgende Ausnahme im Korpus zu finden ist. Yo übt in (130b) aber nicht die Funktion eines Pronomens aus:

(130) (a) El Juan encontrado fue ilusión o real. (Rch-5)

(b) Ostentación del egocentrismo: el yo a través de la obra literaria. (RA-4)

Somit gilt nur für singuläre zählbare Nomina, daß sie mit dem Determinierer auftreten müssen. Transnumerale Nomina sowie Eigennamen und Pronomina können nur unter gewissen Umständen mit dem Artikel stehen. Wenn wir von den zwei folgenden Klassen ausgehen, dann scheint es immer einen Kontext zu geben, in dem sich eine Klasse wie die andere und damit konträr zu den hier aufgezeigten Eigenschaften verhält:

(131) Klassen von Nomina

a. singuläre zählbare Gattungsnamen

b. plurale zählbare Gattungsnamen, Eigennamen, Pronomina

Angesichts der Tatsache, daß Nomina anscheinend nicht eindeutig klassifizierbar sind, stellt sich die Frage, ob die Annahme einer Determiniererphrase hier nicht Abhilfe schafft. Damit wäre der Determinierer Kopf der Phrase und würde ein Nomen als Komplement oder Spezifizierer (je nach Terminologie) subkategorisieren. Vorteil dieser Regelung wäre auch, daß die lexikalische Regel der Substantivierung von Determinierern entfallen könnte, da diese ohnehin eigenständig eine NP bzw. DP bilden. 
Ein Nachteil dieser Analyse ist allerdings, daß im Fall von alleinstehenden Nomina zumeist ein phonologisch leerer Determinierer angenommen werden muß, da in der Folge kein den Kopf repräsentierendes Lexem mehr zur Verfügung steht. So setzt Abbs DP-Analyse (1994) diesen leeren Kopf voraus. Damit erhält man ein Element, dessen Eigenschaften nicht erkennbar sind und das u.E. der Intuition widerspricht. Netter (1994; 1996) erstellt eine Analyse, die auf seiner Theorie der funktionalen Köpfe basiert. Er variiert den Ansatz von Pollard / Sag aber nicht entscheidend. Der Ansatz von Winhart (1997) ist in seiner Hauptausrichtung auf die Deklination deutscher Adjektive nur bedingt auf das Spanische übertragbar. Alle Ansätze (Abb 1994, Oepen 1994, Pollard / Sag 1994, Netter 1994; 1996 und Winhard 1997) liefern aber wertvolle Ideen, die im folgenden noch in unsere Analyse einfließen werden.

Im Kapitel (III.1.) über ein eigenständiges Nomen als NP hatten wir festgestellt, daß der Spezifizierer nicht voranstehen muß. Damit kann davon ausgegangen werden, daß der Spezifizierer im Spanischen in den meisten Fällen fakultativ ist. Als Bedingungen dafür, daß ein Nomen eigenständig eine NP bildet, hatten wir folgendes genannt:

(132) Bedingungen für die NP-Bildung durch ein einzelnes Nomen:

Das Nomen

1. steht in einer Kopulakonstruktion oder

2. ist ein tendenziell transnumerales Nomen oder

3. ist tendenziell numeral und mit Pluralmarkierung versehen oder

4. ist ein tendenziell numerales Nomen, das durch Generalisierung transnumeral wurde.

Es kommen jetzt folgende Möglichkeiten hinzu:

(132) Es handelt sich um

5. einen Eigennamen oder

6. ein Pronomen.

Gleichzeitig können aber alle Nomina auch mit Spezifizierer gebraucht werden: 
(133) (a) Borrell dice tener „la misma ambición“ que el Felipe González de 1982. (M-10)

(b) El agua empezó a rebotar en su rostro y en sus manos. (S-9)

(c) ¿Si usted piensa en los hombres, les encuentra admirables? (RA-3)

In (133) gibt es Beispiele für Eigennamen (a), transnumerale Nomina (b) und plurale zählbare Nomina (c) mit dem definiten Artikel als Determinierer. Somit müssen wir festhalten, daß ein Determinierer auch dann stehen kann, wenn es syntaktisch nicht unbedingt nötig ist (Beispiele a und c). Es gibt anscheinend nur einen Fall, in dem ein Determinierer stehen muß: Wenn ein singuläres zählbares Gattungsnomen auftritt, das nicht generalisiert wurde. Für diese Nomina muß eine Subkategorisierung eines Determinierers angenommen werden.

Einige bereits erwähnte Autoren kritisieren den Ansatz von Pollard / Sag (1994) als zyklisch. Das Nomen wählt den Determinierer, dieser wählt das Nomen. Es handelt sich weder um eine Beziehung, wie sie in der HPSG bei Adjunkten vorgesehen ist (das Adjunkt wählt den Kopf, ohne daß dieser Restriktionen ausübt), noch wie sie bei Komplementen gedacht ist (der Kopf selektiert das Komplement, ohne daß das Komplement Restriktionen ausübt). In unserem Fall würde nach den bisherigen Ergebnissen ebenfalls das Nomen den Determinierer als Komplement selektieren und der Determinierer als eine Art Adjunkt seinen Kopf wählen. Da jeweils unterschiedliche Informationen übertragen werden, ist die Struktur u.E. nur vordergründig zyklisch.

Demgegenüber unterstützt Zwicky (1993: 294ff.) diese Analyse: Spezifizierer operieren nach seinen Untersuchungen weder wie Komplemente noch wie Adjunkte. Sie stehen vielmehr in einer Art Zwischenposition. Zwicky erstellt zunächst zwei Beziehungsmuster: Kopf vs. Abhängiges Element (head vs. dependent) und Funktor vs. Funktorbezugspunkt (functor vs. functee). Dabei versteht er die Dependenzbeziehung als eher syntaktisch und die Funktorbeziehung als eine eher semantisch geprägte Relation. Adjungierung (hier Modifizierung) und Komplementierung (hier Operator und Argument) werden nun wie folgt eingeordnet (nach Zwicky 1993: 294ff.): 


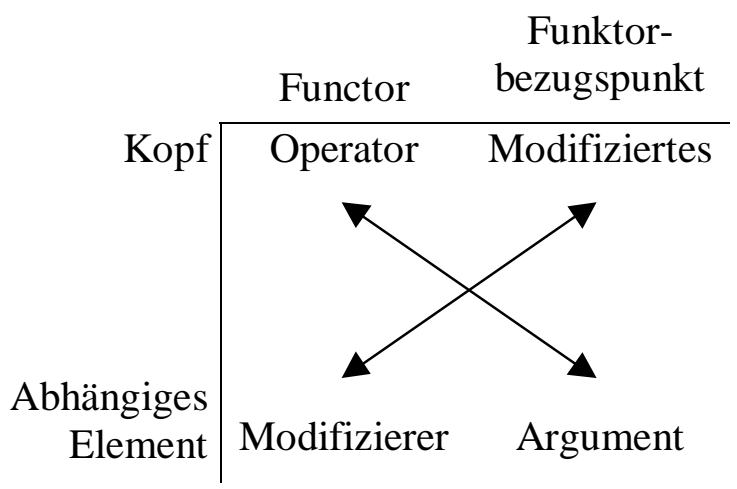

Zwicky weist nun den Beziehungen Merkmale zu und kommt in der Folge zu dem Schluß, daß Spezifiziererbeziehungen so nicht erfaßt werden können. Das folgende Schema ist Ergebnis seiner Betrachtungen, wobei die Spalten a., b. und c. alternative Beschreibungsmuster darstellen:

Funktor-

\begin{tabular}{|c|c|c|c|c|}
\hline a. & b. & c. & Funktor & bezugspunkt \\
\hline Kopf & Kopf: +1 & + Kopf, - Abhängig & Operator & $\overline{\text { Modifiziertes }}$ \\
\hline Begleiter & Kopf: 0 & - Kopf, - Abhängig & Spezifizierer & Spezifiziertes \\
\hline $\begin{array}{l}\text { Abhängiges } \\
\text { Element }\end{array}$ & Kopf: -1 & - Kopf, + Abhängig & Modifizierer & Argument \\
\hline
\end{tabular}

Die von Zwicky eingeführte Bezeichnung Begleiter soll lediglich in einer neutralen Terminologie kennzeichnen, daß es sich weder um einen Kopf noch um ein abhängiges Element handelt. Zwicky baut seine Argumentation auf den Eigenschaften der Oppositionen Kopf vs. Abhängiges Element, Funktor vs. Funktorbezugspunkt und Basis vs. Nicht-Basis auf. Basis soll dabei die Zwischenstellung kennzeichnen, die mit dem Begleiter als einem Element, das weder Kopf noch abhängiger Bestandteil der Phrase ist, dargestellt wurde. Zwicky (1993) gibt keine linguistische Erklärung für den Begriff Basis, so daß wir ihn lediglich als einen Platzhalter für das bezeichnete grammatische Phänomen verwenden. Die Opposition Basis vs. Nicht-Basis unterscheidet dann die drei Dependenzfunktionen in (135) oben rechts (Operator, Modifiziertes und Spezifiziertes) von denen unten links (Spezifizierer, Modifizierer und Argument).

Zwicky weist den einzelnen Relationen Eigenschaften zu, die wir in der folgenden Tabelle auflisten. Wir versuchen, die Eigenschaften auf den spanischen Determinierer zu beziehen (,,+“ kennzeichnet eine mögliche Zuordnung, ,,““ die nicht-mögliche, „o“ eine nicht-entscheidbare Zuordnung). 


\begin{tabular}{l|l|c} 
Funktion & Merkmal & Wert \\
\hline \hline \multirow{4}{*}{ Funktor } & semantischer Funktor & + \\
\cline { 2 - 3 } & Zielpunkt der Kongruenz & $\mathrm{o}$ \\
\cline { 2 - 3 } & Rektionsauslöser & $\mathrm{o}$ \\
\cline { 2 - 3 } & lexikalisch subkategorisiert & + \\
\hline \multirow{3}{*}{ Kopf } & Ort der Morphosyntax & $\mathrm{o}$ \\
\cline { 2 - 3 } & Wortebene & + \\
\cline { 2 - 3 } & die Kategorie bestimmend & $\mathrm{o}$ \\
\hline \multirow{3}{*}{ Basis } & extern repräsentativ & - \\
\cline { 2 - 3 } & gefordert & - \\
\cline { 2 - 3 } & klassifizierend &
\end{tabular}

Die Eigenschaften der Basis entsprechen dem spanischen Determinierer nicht: Er ist kein externer Repräsentant der Nominalphrase, sondern das Nomen. Der Determinierer wird in der Regel weder unbedingt gefordert, noch klassifiziert er die Phrase. Dagegen kann er als semantischer Funktor betrachtet werden und wird zumindest teilweise subkategorisiert. Er befindet sich grundsätzlich auf Wortebene, d.h. es kann keine Nominalphrase anstelle eines Determinierers vor dem Nomen auftreten.

Allerdings zeigen vier nicht entscheidbare Merkmale die relativ schwierige Übertragung von Zwickys Überlegungen. Es läßt sich nicht klären, ob Nomen oder Determinierer für Kongruenz und Rektion verantwortlich sind. Ist das Nomen oder der Determinierer Träger der morphologischen Information? Und welches Element zwingt das andere, in Kongruenz zu ihm zu treten? Verlangt das Nomen den Determinierer oder ist es umgekehrt? Diese Fragen sind mitunter abhängig von der zugrunde gelegten Syntaxtheorie und der untersuchten Sprache. Im Deutschen ist die Flexion der Adjektive abhängig vom Determinierer, so daß Winhart (1997) davon ausgeht, daß der Determinierer der Kopf der Phrase ist und seine Eigenschaften auf das Nomen überträgt. Sie erstellt eine Sortenhierarchie, die Mehrfacheinträge ermöglicht. Zlatic (1997) geht nach ihren Untersuchungen für das Serbische davon aus, daß die Fragestellung des Kopfes der NP sprachabhängig ist. Für Englisch oder Deutsch befürwortet sie eine DP-Analyse. Da diese auf das Serbische nicht übertragbar ist, geht sie für diese Sprache von einer NP-Analyse aus. Diese Schlußfolgerung 
erscheint stimmig (vgl. Zlatic 1997: 25) und wird von Berman / Frank in ihrer Implementierung eines Grammatikfragment jeweils für Französisch und Deutsch in der Lexikalisch-Funktionalen Grammatik gestützt. Im Deutschen wird die Nominalphrase nach Abney (1987) als Determiniererphrase (DP) interpretiert (Berman / Frank 1996: 165ff.), während die Nominalphrase im Französischen als solche erhalten bleibt (Berman / Frank 1996: 54ff., für einen entgegengesetzten Ansatz vgl. Parodi 1994). Gleiches gilt für die Analyse der hebräischen Nominalphrase als NP durch Wintner (1998) und der deutschen als DP durch Kathol (1998).

Allegranza (1998) erweitert den Ansatz der Funktorbeziehung und nimmt an, daß Marker, Adjunkte und Spezifizierer grundsätzlich Funktoren sind. Sie selektieren den Kopf, auf den sie sich beziehen, und sind in der Regel fakultativ. Allegranza nimmt keine Subkategorisierung des Determinierers an, so daß der Determinierer fakultativ ist. Wir gehen hier davon aus, daß nur singuläre zählbare Nomina das Merkmal

\section{(137) $[\mathrm{VAL}[\mathrm{SPR}<\operatorname{det}>]]$}

tragen. Alle anderen erlauben nur fakultative Determinierer. Gleichzeitig verweist der Determinierer auf das Nomen, das er näher spezifiziert, und weist ihm einige Eigenschaften zu. Damit erhalten wir eine Beziehung zwischen Nomen und Determinierer, die erneut als Zirkelstruktur kritisiert werden kann. Dem muß entgegengehalten werden, daß beim Nomen lediglich markiert wird, ob ein Determinierer nötig ist oder nicht und welches Genus dieser tragen muß. Es ist nicht festgelegt, welche Eigenschaften der Determinierer sonst einbringt und wie er realisiert wird. Seine beigesteuerten Eigenschaften überträgt der Determinierer auf das Nomen. Beide Wortarten ergänzen sich und unifizieren die entsprechenden Merkmale. Damit erhalten wir ein Abhängigkeitsverhältnis, das zwischen Subkategorisierung und Adjungierung steht, wie es letztlich auch Zwicky (1993) vorschlägt.

Determinierer bekommen nach Allegranza eine Funktorfunktion zugesprochen. Somit muß ein Schema eingeführt werden, das die Relation zwischen einem Funktor und seinem Kopf reguliert. Dieses Schema ersetzt folglich das von Pollard / Sag (1994: 56, 362ff.) gebräuchliche Head-Adjunct-Schema und das Head-SpecifierSchema, welche die Relation zwischen Kopf und Adjunkt sowie zwischen Kopf und Spezifizierer darstellen (vgl. Kapitel II.2.3.). Ob Allegranzas Funktorschema auch für Komplementierer geeignet ist, wie er behauptet, kann hier nicht untersucht werden. Allegranza (1998: 83) erstellt folgende Matrix für die Funktorfunktion: 


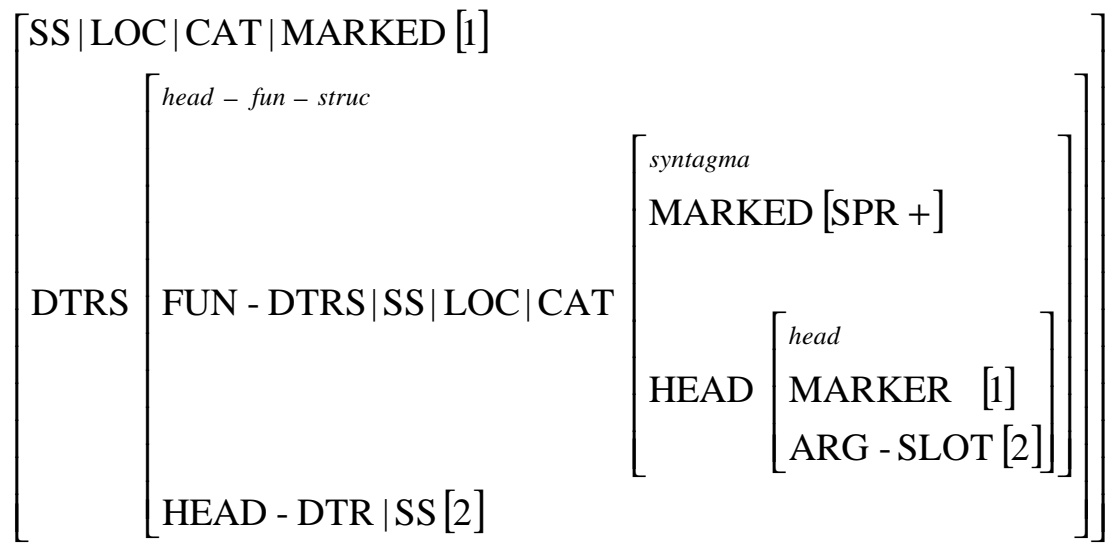

Die gesamte Phrase wird als MARKED gekennzeichnet, wie der Pfad [SS | LOC | CAT | MARKED [1] ] aufzeigt. Innerhalb der Phrase muß es eine Funktortochter und eine Kopftochter geben. Nach Allegranza trägt die Funktortochter das Attribut [MARKED | SPR +]. Die Bezeichnung SPR kann in dieser Arbeit nicht aufrecht erhalten werden, da wir dieses Attribut im Gegensatz zu Allegranza weiterhin in der Valenz annehmen. Wir benennen es daher in STATUS um (vgl. Winhart 1997: 350), welches die Ebene der Phrase innerhalb des X-bar-Schemas kenntlich macht. Der Kopf des Funktors trägt die Merkmale MARKER und ARG-SLOT. MARKER bezeichnet die funktortypischen Eigenschaften, die auf die Phrase übertragen werden, ARG-SLOT bezieht sich auf den Kopf und muß mit den Merkmalen des Kopfes in der Kopftochter unifizierbar sein. ARG-SLOT ersetzt somit das Merkmal MOD bei Adjunkten und SPEC bei Spezifizierern.

Revidiertes Funktorschema, wobei $n \geq m$

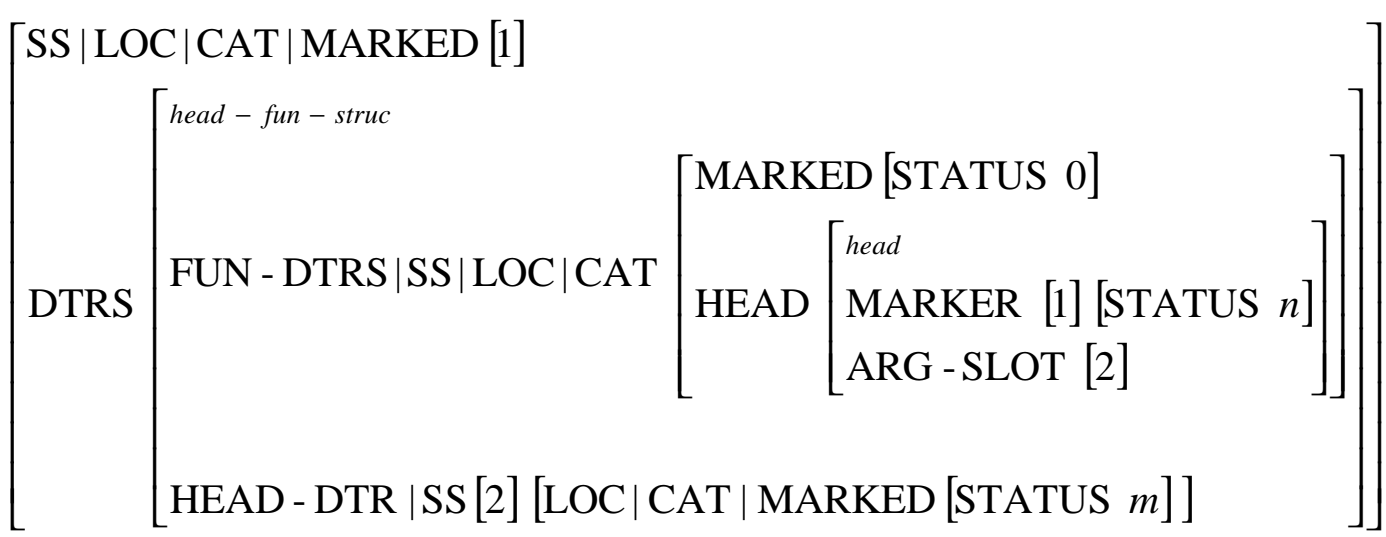


Die Indizierung ist folgendermaßen zu verstehen:

Der Pfad SS | LOC | CAT | MARKED bestimmt die Ebene der Phrase, d.h. ob es sich um N' oder N" handelt. Dies muß mit dem Wert des Determinierers übereinstimmen, der für die Anhebung auf diese Ebene verantwortlich ist. Dies ist der Wert des Pfades DTRS | FUN-DTRS | SYNSEM | LOC | CAT | HEAD | MARKER. Er ist dem Determinierer inhärent und trägt, sofern der Determinierer die Phrase auf die NP-Ebene anhebt, den STATUS-Wert 2, während im Attribut HEAD | ARG-SLOT markiert wird, auf welcher Ebene das Nomen steht (hier mit dem Index [2] ). Die Angabe [MARKER [ STATUS 0 ] ] bei der Funktortochter zeigt, daß sich grundsätzlich um ein lexikalisches Zeichen handelt, da an dieser Stelle im Spanischen keine Phrasen auftreten können. Wir können von daher das Funktorschema leicht modifiziert übernehmen. Eine genauere Erläuterung der Lexikoneinträge erfolgt in Kapitel III.3.6.

\subsection{Kongruenz}

Kongruenz bezeichnet die Übereinstimmung zwischen Satzteilen hinsichtlich morphologischer Merkmale wie Kasus, Numerus oder Genus (vgl. auch Alcaraz Varó / Martínez Linares 1997: s.v. concordancia). Es kann zwischen Kongruenz über die Satzebene hinaus, etwa bei Anaphern, und satzinterner Kongruenz unterschieden werden. Das erste Phänomen kann hier unberücksichtigt bleiben.

Satzinterne Kongruenz findet in drei Bereichen statt: zwischen Subjekt und Verb, zwischen Subjekt und prädikativ gebrauchten Nominalphrasen sowie innerhalb der Nominalphrase zwischen dem Nomen und möglichen Begleitern. Dabei sind die Bereiche hinsichtlich der Merkmale zu unterscheiden, welche die Differenzierungen ausmachen. Bei der Subjekt-Verb-Kongruenz müssen Person und Numerus übereinstimmen. Kasus ist im Spanischen an dieser Stelle in der Regel nicht realisiert. Bei der Subjekt-Prädikativ-Kongruenz und der NP-internen Kongruenz sind es Genus und Numerus, die korrespondieren müssen. Die dritte Person ist bei der NP-internen Kongruenz vorgegeben.

In der Standard-HPSG von Pollard / Sag (1994) wird Kongruenz über das Attribut CONTENT | INDEX definiert und damit über die Semantik. Dies ist z.B. von Kathol (1999) kritisiert worden. Pollard / Sag (1994: 148, 67-73, 95) differenzieren zwar 
zwischen syntaktischer, semantischer und pragmatischer Kongruenz, hauptsächlich findet Kongruenz aber über die Semantik statt. Nach Pollard / Sag (1994: 47ff.) muß der SYNSEM-Wert des Nomens libro wie folgt dargestellt werden:

\section{Lexikoneintrag für libro}

$$
\left.\left[\begin{array}{c}
\text { CAT }\left[\begin{array}{l}
\text { HEAD nomen } \\
\text { SUBCAT }\langle\text { DP }\rangle
\end{array}\right] \\
\text { INDEX [1] }\left[\begin{array}{l}
\text { ref } \\
\text { PER 3 3 } \\
\text { NUM sg } \\
\text { GEN mask }
\end{array}\right] \\
\text { RESTR }\left\{\left[\begin{array}{l}
\text { RELN LIBRO } \\
\text { INST [1] }
\end{array}\right]\right\}
\end{array}\right]\right]
$$

Die drei für die Kongruenz relevanten Merkmale stehen im semantischen Teil der Deskription. Nach unserer Auffassung widerspricht diese Deskription der sprachlichen Intuition. Es handelt sich bei der Kongruenz um ein grammatisches Phänomen, das den syntaktischen Aufbau eines Satzes dem Hörer verdeutlichen soll. Dabei ist die Kongruenz für das Verständnis oft redundant. Folgende Beispiele belegen, daß z.B. zwischen syntaktischem und semantischem Genus unterschieden werden muß (aus Kathol 1999: 218f.):

(141) (a) Die Samper-Gesellschaft ist Bauherr/in der Frauenkirche.

(b) Schau dir dieses Mädchen an, wie gut sie / es Tennis spielt.

(c) Su majestad suprema está contento.

(d) Vous êtes loyal.

Die Beispiele bestehen aus drei Fällen von prädikativer und einem von anaphorischer Kongruenz. Im Beispiel (a) kann auf das feminine Subjekt durch eine feminine oder eine maskuline NP referiert werden. Bei der Kongruenz über feminines Genus besteht eine syntaktische, bei der über maskulines Genus eine semantische Relation. Gleiches gilt für Beispiel (b): Der Sprecher kann syntaktisch auf das Genus neutrum von Mädchen referieren oder semantisch auf das Geschlecht feminin, da es sich um eine weibliche Person handelt. Allerdings wurde dieser Beispielsatz von einigen Be- 
fragten angezweifelt, wahrscheinlich weil die Distanz zwischen Pronomen und Bezugsnomen noch nicht groß genug ist.

Im dritten Beispiel (c) referiert das prädikative Adjektiv auf das semantische Genus der mit majestad bezeichneten Person. Interessanterweise ist die Phrase *su majestad supremo ungrammatisch. In (d) referiert das prädikative Adjektiv auf den semantischen Numerus der mit der Höflichkeitsform vous bezeichneten einzelnen Person. Damit können wir festhalten, daß für den hier besonders relevanten Bereich der NP-internen Kongruenz die Syntax entscheidend ist. Dazu sollte aber auch das Phänomen der Sinnkongruenz in Betracht gezogen werden (vgl. Metzeltin 1997: 33). Sowohl de Bruyne (1993: 565f.) als auch Seco (1986: 112f.) bezeichnen den Gebrauch des Plurals nach Kollektiva als korrekt. Nach Alcina Franch (1992: 163) sind solche Konstruktionen besonders typisch für den madrilenischen Dialekt (erstes Beispiel aus Metzeltin 1997: 33, zweites aus de Bruyne 1993: 565):

\section{(141) (e) Aquel grupo de estudiantes nos invitaron a un café.}

(f) La mitad de los habitantes han emigrado.

Hier kann die gesamte NP aquel grupo de estudiantes als semantisch im Plural angegeben werden. Es findet in der Folge eine semantische Kongruenz mit dem Verb (Plural statt Singular) statt, wobei die syntaktische Kongruenz im Singular weiterhin sowohl möglich als auch korrekt ist. Damit ergeben sich Alternativen für den Sprecher: Er kann unter verschiedenen Optionen auswählen. Pollard / Sag (1994: 62) nennen dies pragmatische Kongruenz: ,pragmatic agreement [...] arises when contextual background assumptions are required to be consistent". Im Fall des Beispiels (e) wird der Numeruswert der Präpositionalphrase, welche ein Adjunkt der SubjektNP darstellt, auf diese übertragen.

Die Aufteilung in semantische, syntaktische und pragmatische Kongruenz bei Pollard / Sag (1994: 61ff.) ist verwirrend. Wir gehen hier zunächst von syntaktischer Kongruenz aus, vor allem innerhalb der NP. Wie bereits gesehen, muß berücksichtigt werden, daß auch andere Faktoren die Kongruenz beeinflussen können. Diese Faktoren müssen aber im einzelnen untersucht werden. Zunächst nehmen wir ein Kongruenzattribut AGREEMENT (AGR) an, daß im Kopfattribut (HEAD) verankert ist: 


$$
\left[\text { SYNSEM } \mid \text { CAT } \mid \text { HEAD } \mid \text { AGR }\left[\begin{array}{l}
\text { PER } \ldots . \\
\text { NUM } \\
\text { GEN } \ldots
\end{array}\right]\right]
$$

Innerhalb der Nominalphrase spielt dabei das Attribut PERSON keine entscheidende Rolle, da alle Gattungsnomina und Eigennamen per definitionem als [PER 3] markiert sind. Nur für Pronomina muß es als variabel angesehen werden.

Um Sinnkongruenz und andere ähnlich gelagerte Fälle erfassen zu können, müssen wir zusätzlich von einer semantischen Komponente ausgehen. Somit kann eine Doppelung der Kongruenzeinträge angenommen werden: syntaktisch und semantisch. Um die Differenzierung offensichtlich zu machen, benennen wir die semantischen Kongruenz-Merkmale in sNUM, sGEN und sPER um.

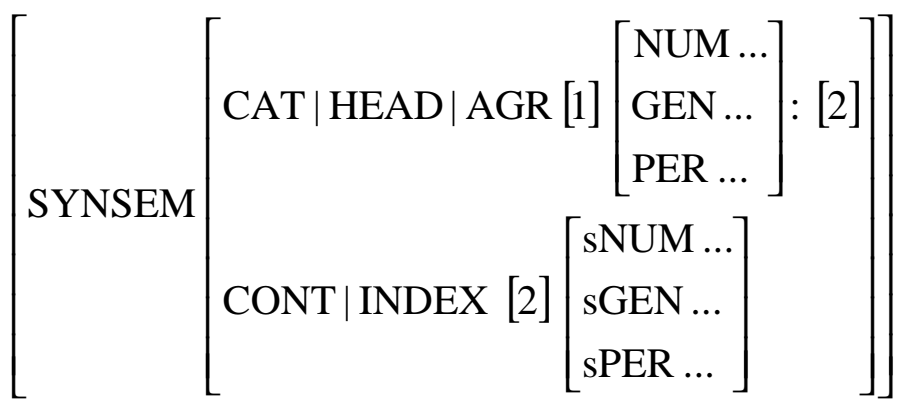

Die Parallelität der grammatischen Information wird durch den Verweis über den Index ( : [2] ) realisiert. Da es sich grammatisch nicht um dieselbe Information handelt, sondern nur um möglicherweise identische Werte ähnlicher Attribute, kann keine Indizierung AGR zum INDEX stattfinden.

Im Normalfall (default-Wert) kann der syntaktische Eintrag mit dem semantischen koindiziert werden, um die Übereinstimmung kenntlich zu machen. In anderen Fällen muß eine Differenzierung erfolgen. Matrizen für die Lexeme majestad und vous sowie die Deskription für die Phrase aquel grupo de estudiantes aus den obigen Beispielsätzen (141c-e) können wie folgt dargestellt werden. Die entsprechenden lexikalischen Einträge müssen diverse Disjunktionen enthalten. In den Beispielsätzen ist bereits ein bestimmter Wert realisiert: 
(144)

(c) PHON majestad $\left.\operatorname{SYNSEM}\left[\begin{array}{l}\text { CAT | HEAD | AGR }\left[\begin{array}{l}\text { NUM } s g \\ \text { GEN fem }\end{array}\right] \\ \text { CONT | INDEX }\left[\begin{array}{l}\text { sNUM sg } \\ \text { sGEN mask }\end{array}\right]\end{array}\right]\right]$

(d) $\mathrm{PHON}$ vous

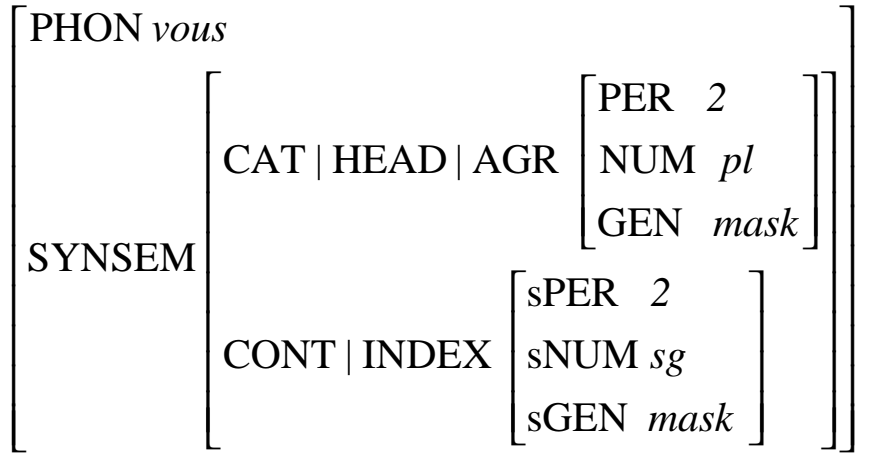

(e) $\Gamma$ PHON aquel grupo de estudiantes $\left.\operatorname{SYNSEM}\left[\begin{array}{l}\text { CAT | HEAD | AGR }\left[\begin{array}{l}\text { NUM } \\ \text { GEN } g \text { mask }\end{array}\right] \\ \text { CONT | INDEX }\left[\begin{array}{l}\text { sNUM } p l \\ \text { sGEN mask }\end{array}\right]\end{array}\right]\right]$

Ein Nomen wie majestad in (c) wird von Corbett (1991: 225) als hybrides Nomen bezeichnet. Im Beispiel (d) wird die lexikalische Unterspezifizierung von vous (Genus: mask oder fem, Numerus: $s g$ oder $p l$ ) durch den Kontext aufgelöst. Bei (e) kann der differenzierte Eintrag für syntaktische oder semantische Kongruenz nur auf der NP-Ebene erfolgen. Die lexikalischen Einträge der einzelnen Lexeme sind nicht davon betroffen.

Somit kann jetzt für die prädikative Kongruenz postuliert werden, daß dort teilweise semantische Kongruenz über das Attribut INDEX stattfindet (vgl. Kathol 1999: 223). Die Frage ist, ob dies in irgendeiner Weise systematisch erfolgt. Wir könnten sagen, daß die Kongruenz über den INDEX abläuft, sofern dort Einträge vorliegen. Wenn für den INDEX keine besonderen Einträge vorliegen und dieser mit der syntaktischen Kongruenz koindiziert, dann findet die Kongruenz über die Syntax statt.

Für die Subjekt-Verb-Kongruenz kann dies nicht angenommen werden. Hier liegen in den obigen Beispielen zwei sich widersprechende Fälle vor. Bei vous êtes loyal besteht zwischen dem Subjekt vous und dem Verb êtes syntaktische Kongru- 
enz, das Verb steht ebenfalls im Plural, während das prädikative Adjektiv loyal sich nach der Semantik richtet. Der Satz un grupo de estudiantes nos invitaron a un café stellt ein Gegenbeispiel dar: Das Subjekt steht syntaktisch im Singular, das Verb im Plural. An dieser Stelle müssen wir vom Einfluß einer pragmatischen Komponente ausgehen. Der Sprecher will evtl. die Gruppe als Menge mehrerer Studenten besonders herausstellen oder erstellt die Kongruenz mit estudiantes als letztem Wort des Subjekts (vgl. auch de Bruyne 1993: 566, también la ultraderecha tienen frases de ...). Auf jeden Fall ist es sinnvoll, hier von pragmatischer Hintergrundinformation auszugehen, wie sie in der HPSG im Attribut CONTEXT dargestellt wird. In diesem Fall nehmen wir an, daß der Phrase auf N"-Ebene das semantische Attribut [sNUM $p l$ ] zugewiesen werden kann.

Hybride Nomina scheinen vom Kongruenzwechsel besonders betroffen zu sein. Da diese sich in der Regel auf menschliche Personen beziehen, kann ein Merkmal HUMAN in Erwägung gezogen werden. Dieses steht für eine semantische Eigenschaft von Lexemen, die im Spanischen noch weitere Veränderungen nach sich zieht, z.B. den obligatorischen Gebrauch der Präposition $a$ vor Nominalphrasen im Akkusativ. Wir können folgende Regel aufstellen: Ist ein Nomen mit dem Merkmal [HUMAN +] versehen, dann muß es im Akkusativ in einer Präpositionalphrase mit $a$ stehen (für einige Ausnahmen vgl. Meisterfeld 1998: 89ff.). Ähnliches gilt anscheinend für die Kongruenz von Nominalphrasen: Ist eine NP mit dem Merkmal [HUMAN +] versehen, dann besteht verstärkt die Möglichkeit, daß seine semantischen Indexwerte von den syntaktischen Agreementwerten abweichen. Wir fügen somit dem INDEX eines Nomens das Merkmal HUMAN hinzu (vgl. auch Hadlich 1975: 38f., 109).

\subsection{Das Lexikon}

\subsubsection{Die Wortart Determinierer}

Die Untersuchung ist nun so weit fortgeschritten, daß wir die lexikalischen Einträge der Wortarten genauer untersuchen können. Dabei war bisher nicht klar, wie die Determinierer einzustufen sind. Alarcos Llorach (1994) u.a. sprechen in ihren Grammatiken von determinierenden Adjektiven, wenn sie auf die Determinierer im allgemei- 
nen zu sprechen kommen. Dabei wird der definite Artikel ausgeklammert. Es hat sich bei unseren Untersuchungen als richtig herausgestellt, daß Determinierer, was die Syntax angeht, teilweise ähnlich, teilweise analog zu Adjektiven operieren. Der Wortartenvertreter Determinierer ordnete sich nicht am Rande der Skala ein, sondern eher in der Mitte. Gleichzeitig zeigte sich, daß die Ausklammerung des definiten Artikels unbegründet ist. Er ist in seinem Verhalten anderen Determinierern durchaus vergleichbar.

Die Merkmale, die tendenziell eher auf Adjektive zutreffen, sind vor allem folgende:

1. [Adverbialer Gebrauch + ],

2. [komparierbar +$]$,

3. [substantivierbar mit Artikel +] und

4. [substantivierbar im Neutrum + ].

Stellung und Kombinierbarkeit differenzieren eher die Determinierer untereinander und sind für eine Abgrenzung zu den Adjektiven weniger geeignet. Allerdings muß erneut herausgestellt werden, daß die Wortart der Determinierer sich nur unzulänglich als homogene Klasse beschreiben läßt. Von daher sind Abgrenzungen grundsätzlich ein Problem. Wir finden nie alle Merkmale bei einem Determinierer realisiert.

Es läßt sich erkennen, daß ein Determinierer für [substantivierbar im Neutrum +] markiert sein muß, damit [Komparation +] und [Adverbialer Gebrauch +] auf ihn zutreffen können. Wir können diese somit miteinander kombinieren: Ist ein Determinierer nicht im Neutrum verwendbar, dann sind die anderen beiden Merkmale nicht vorhanden. Wir nennen dieses Merkmal NEUTRUM (NTR) und erstellen die zwei Merkmale ADVERBIAL (ADV) und KOMPARATION (KOMP) als abhängige Werte des Merkmals NTR.

Für die Determinierer, die tendenziell als den Adjektiven ähnlich eingestuft werden, muß noch das Merkmal [substantivierbar mit Artikel \pm ] berücksichtigt werden. Dieses steht in Opposition zu [substantivierbar ohne Artikel \pm ]. Beide Merkmale beschreiben idealisierte Verhaltensmuster von Determinierern: das Merkmal [substantivierbar mit Artikel +] ist eher typisch für adjektivische, das Merkmal [substantivierbar ohne Artikel +] eher für determinierende Artikelwörter, so daß wir sie als ADJEKTIVTYPISCHE_SUBSTANTIVIERUNG (ADJ_SUBST) und DETERMI- 
NIERERTYPISCHE_SUBSTANTIVIERUNG (DET_SUBST) bezeichnen können. Damit erhalten wir folgendes Bündel an Merkmalen, das adjektivähnliche Determinierer beschreibt:

$$
\left[\begin{array}{l}
\text { ADJ_SUBST } \pm \\
\text { NTR }\left[\begin{array}{ll}
\text { ADV } & \pm \\
\text { KOMP } & \pm
\end{array}\right]
\end{array}\right]
$$

Analog können wir nun für die determinierenden Artikelwörter verfahren. Dabei sind vor allem die Merkmale [substantivierbar ohne Artikel \pm ] und [substantiviert Adjektive \pm ] relevant. Letzteres Merkmal kann in TRANSPOSITION (TRANS) umbenannt werden. Folgende Merkmale beschreiben tendenziell eher determinierende Artikelwörter:

$$
\left[\begin{array}{lr}
\text { DET_SUBST } \pm \\
\text { TRANS } & \pm
\end{array}\right]
$$

Wir haben bisher eine dritte Klasse unberücksichtigt gelassen, die in der Klassifikation deutlicher abgrenzbar war: diejenigen Determinierer, die sich syntaktisch wie Adjektive verhalten. Sie können nicht in der Umgebung [ _ A N $]_{N P}$ auftreten bzw. treten immer in der Umgebung [Art _ $\mathrm{N}]_{\mathrm{NP}}$ auf. Diese Determinierer werden semantisch weiterhin als determinierend eingeordnet. Syntaktisch stehen sie den qualifizierenden Adjektiven näher und stärken die Argumentation spanischer Grammatiker, welche die Determinierer als determinierende Adjektive bezeichnen. Diese Klasse besteht aus demás, doble, mero, mismo und segundo. Die Umgebungsmerkmale können über die Sorten erfaßt werden und müssen nicht explizit als Merkmale auftreten.

Die drei Klassen - determinierende und adjektivische Determinierer sowie adjektivähnliche Determinierer, die auch als determiniererende Adjektive bezeichnet werden können - werden in eine Sortenhierarchie integriert, wie sie in der HPSG gebräuchlich ist. Dafür sprechen mehrere Gründe:

- die schlechte Abgrenzung der Klassen voneinander. In einer Sortenhierarchie kann angenommen werden, daß für einen Determinierer mehrere Einträge möglich sind (vgl. Winhart 1997: 347 und Malouf 1998: 88ff.).

- die Kombination von determinierender Semantik und adjektivischer Syntax. Dies kann in einer Sortenhierarchie durch Einträge kenntlich gemacht werden, die gleichzeitig zu mehreren Supertypen gehören. 
- der Einfluß von Determinierern auf das mögliche Auftreten von Adjektiven. Mero verhindert weitere Adjektive in der NP, andere Determinierer beschränken die Wortstellung. Nur die Numeralia lassen sich relativ frei mit Adjektiven kombinieren.

Wir erstellen einen Typ $D$ für Determinierer, der eine determinierende Semantik beinhaltet und den Supertyp aller spanischen Determinierer darstellt. Ein Subtyp von $D$ ist d-element (in Anlehnung an Winhart 1997: 346f.). D-element kennzeichnet alle Determinierer, die in der Lage sind, die Nominalphrase von der Ebene $N^{\prime}$ auf die Ebene N" anzuheben und somit die Artikelfunktion auszuüben ( [steht in Umgebung _ A N +]). Die Sorte d-element kennt wiederum zwei Subtypen: adjektivische Determinierer (adj_Det) und Determinierer (Det). Adjektivische Determinierer zeichnen sich durch die Merkmale in (146) aus, Determinierer durch die Merkmale in (147). Die verbleibende Sorte determinierende Adjektive (det_Adj) beschreibt die Determinierer, welche sich syntaktisch adjektivisch verhalten (mit dem Merkmal [steht in Umgebung _ A N -] ).

Beide Strukturen (146) und (147) können somit als vom Typ d-element gekennzeichnet werden und gelten damit für die Subsorten adj_Det und Det. Für die Vertreter der Sorte adj_Det gilt, daß sie ebenfalls Merkmale aus (146) tragen können. Allerdings muß es trotzdem möglich sein, daß Determinierer sowohl als adj_Det als auch als Det im Lexikon eingetragen sind. Durch den gemeinsamen Supertyp $d$ element wird folglich die Notation vereinfacht. Nur auf diese Weise kann die Abstufung ersichtlich gemacht werden. Wir erhalten folgende Sortenhierarchie für Determinierer, wobei Adjektive als die benachbarte Wortart mit einbezogen werden müssen und Adj für die Untersorte der qualifizierenden Adjektive steht: 


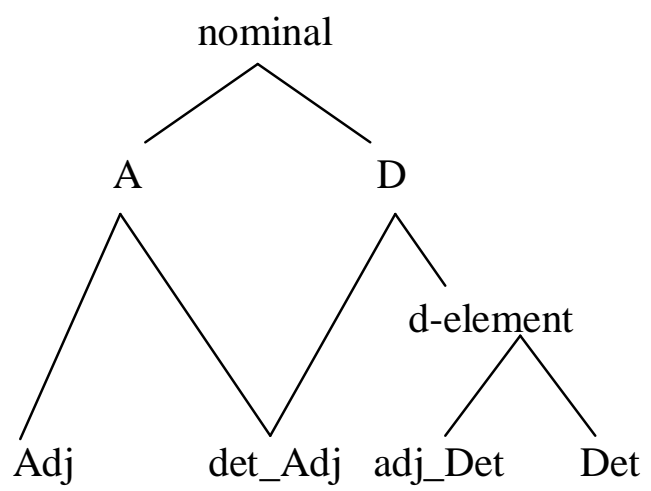

Um den Lexikoneintrag für einzelne spanische Determinierer erstellen zu können, müssen zuvor noch Stellungsfragen bearbeitet werden. Wir gehen zunächst auf die Wortart Nomen ein.

\subsubsection{Die Wortart Nomen}

In Kapitel III.1. hatten wir bereits ausführlicher die Wortart Nomen behandelt. Dabei ging es aber in erster Linie um die Frage, wann Gattungsnomina eigenständig eine NP bilden können. Hier soll die Wortart Nomen als solche bearbeitet und in die Sortenhierarchie unter (148) integriert werden.

Wir hatten bereits Gattungsnomina in singuläre zählbare und plurale Nomina sowie Massennomina aufgeteilt. Dieser Differenzierung lag die Beobachtung zugrunde, daß singuläre zählbare Nomina nur unter bestimmten Bedingungen eigenständig eine NP bilden, während plurale Gattungsnomina sowie Massennomina dies jederzeit können. Dabei sind pragmatische Einschränkungen zu berücksichtigen. Zu der komplexen Wortart Nomen sind zudem noch Eigennamen und Pronomina zu zählen. Diese bilden grundsätzlich eigenständig eine NP. Der Gebrauch eines Determinierers ist zwar möglich, verlangt aber sehr spezielle Bedingungen. Wir gehen deshalb davon aus, daß Nomina im allgemeinen nach ihrem gewöhnlichen Gebrauch in zwei Klassen einzuteilen sind: diejenigen, die grundsätzlich eigenständig eine NP bilden können, und diejenigen, die einen Determinierer fordern. Nach unseren bisherigen Ergebnissen ist die erste Klasse als tendenziell transnumeral, die zweite als tendenziell numeral klassifizierbar. Dabei muß aber bedacht werden, daß diese Tendenz durch einige Prozesse revidiert werden kann und erst auf N"-Ebene zu klären ist, ob es sich um eine Konstruktion handelt, der Transnumeralität zugesprochen werden 
kann. Wir bezeichnen die erste Klasse als $\mathrm{N}_{\mathrm{tn}}$, die zweite als $\mathrm{N}_{\mathrm{n}} \cdot \mathrm{Zu} \mathrm{N}_{\mathrm{tn}}$ sind Eigennamen, Pronomina, plurale zählbare Gattungsnamen und Massennomina zu rechnen, zu $\mathrm{N}_{\mathrm{n}}$ lediglich singuläre zählbare Gattungsnomina. Beide unterscheiden sich durch ihre Valenz (vgl. die Matrix in (128)). In Anlehnung an die in der Literatur verbreitete englische Terminologie (vgl. Winhart 1997: 347) kürzen wir singuläre, zählbare Nomina mit sg-count, plurale Massennomina mit pl-mass ab, en steht für Eigennamen, pron für Pronomen. Wir nehmen folgende Sortenhierarchie an:

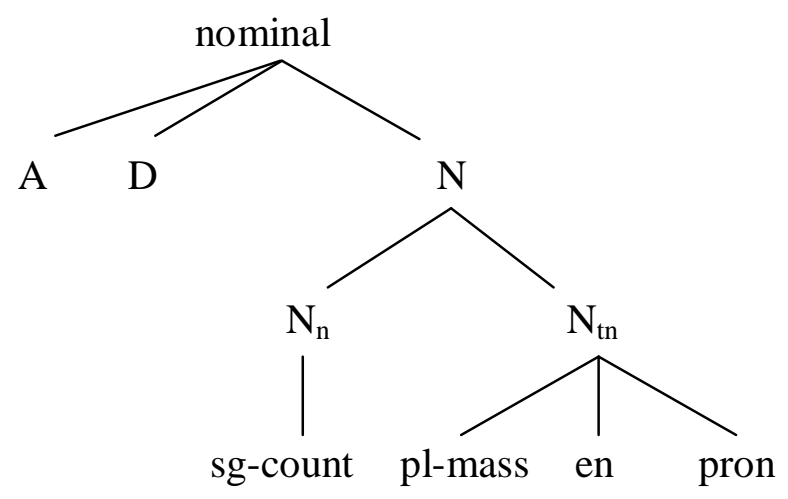

Wie bereits angesprochen gibt es mögliche Verschiebungen in der Sortenhierarchie auf horizontaler Ebene. Ein Eigenname oder ein Pronomen kann wie ein singulärer Gattungsname verwandt werden, ein singuläres Nomen wie ein Massennomen. Determinierer können substantiviert oder auch wie Adverbien gebraucht werden und in der Folge ganz aus der nominalen Sortenhierarchie herausfallen, da Adverbien als verbal eingestuft werden. Bevor diese Übergänge mit lexikalischen Regeln erläutert und die genauen Lexikoneinträge für jede Wortart vorgenommen werden können, müssen wir die Wortstellung als wichtigen Bestandteil der lexiklaischen Einträge in die Diskussion integrieren.

\subsection{Wortstellung}

Grundsätzlich stehen Determinierer vor dem Nomen. Deswegen sah die Klassifikation auch lediglich ein Merkmal Nachstellung für Ausnahmen vor. Allerdings gibt es im Spanischen mit mío einen Determinierer, der nur nachgestellt wird, und die folgenden zehn Determinierer, die mitunter nachgestellt werden können: alguno, cualquiera, doble, este, más, mismo, ninguno, segundo, tal, todo. Sie verlieren in Nach- 
stellung nicht ihre determinierende Semantik, auch wenn diese mit einer pragmatischen Komponente der subjektiven Sprecherempfindung kombiniert werden muß. Somit können sie nicht als Adjektive gelten. Wir gehen davon aus, daß im Spanischen eine Standardlinearisierung vorliegt. Diese besagt:

$$
\begin{aligned}
\text { (150) Determinierer } & <\text { Nomen } \\
\text { Nomen } & <\text { Adjektiv }
\end{aligned}
$$

Somit gilt folgende Reihenfolge, sofern Vertreter der jeweiligen Wortart vorhanden sind:

$$
\text { (151) Determinierer }<\text { Nomen }<\text { Adjektiv }
$$

Wir müssen folglich nur den Ausnahmefall Nachstellung eines Determinierers markieren. Dies kann über das Merkmal NACHSTELLUNG (NACH) geschehen, welches bereits für die Klassifikation der Determinierer eingeführt wurde. [NACH +] bedeutet, daß dieser Determinierer keine artikeltypischen Eigenschaften übernimmt, sondern nach dem Nomen in Adjunktposition steht. Wenn ein Determinierer wie este normalerweise artikelähliche Eigenschaften besitzt, verliert er diese in Nachstellung.

Ein bereits behandeltes Thema ist die Kombination von Determinierern. Dabei hatten wir zwei Klassen von Determinierern erstellt, die Kombinationssteuerer genannt wurden: diejenigen, die sehr viele Kombinationen in Erststellung erlauben, und diejenigen, die sehr viele Kombinationen in Zweitstellung erlauben. Die Kombinationssteuerer bekommen alle Determinierer zugeschrieben, die nach oder vor ihnen stehen können. Somit bekommt ein in Erststellung als Kombinationssteuerer bezeichneter Determinierer im Stellungsattribut das Attribut KOMBINATIONSSTEUERER_1 (KOMB_1) zugeteilt, das als Wert die Menge aller Determinierer enthält, die in Zweitstellung folgen können. Folglich erhält z.B. mi alle entsprechenden Determinierer aus der Liste in (102) zugeteilt. Demgegenüber bekommt ein in Zweitstellung als Kombinationssteuerer bezeichneter Determinierer das Attribut KOMB_2 zugeteilt, das als Wert die Menge aller Determinierer enthält, die vor ihm stehen können, z.B. wären dies bei doble alle Determinierer, die überhaupt in Erststellung auftreten können. Diese Angabe ist im Lexikon enthalten und entfällt, wenn sie nicht realisiert wird. Nicht als Kombinationssteuerer vermerkte Determinierer erhalten keine Einträge. Diese Darstellung der Kombinationsmöglichkeiten bietet folgende Vorteile: 
- Nur elf Determinierer erhalten lexikalische Einträge im Merkmal Kombination.

- Es ist sofort erkennbar, ob und in welcher Kombination ein Determinierer in der Regel auftritt.

- Mit otro haben wir einen Determinierer, der in beiden Kombinationssteuerungsmengen auftritt und somit beide Einträge bekommen muß.

- Wir können genau festhalten, welche Kombinationen erlaubt sind und welche nicht. Die als Kombinationssteuerer bezeichneten Determinierer erlauben oder verbieten viele Kombinationen, so daß diese Information lexikalisch registriert wird. Über das Lexikon ist es folglich möglich, Änderungen so zu erfassen, daß das Merkmal Kombinationssteuerer oder ein bestimmter Determinierer der Wertemenge von Kombinationssteuerer hinzugefügt wird. Dies gilt z.B. für das Pos$\operatorname{sessiv}_{1} m i$, das nach Demonstrativa folgen kann und bisher nicht erfaßt wurde. Dadurch sind die jeweiligen Angaben relativ leicht anzugleichen, z.B. wenn sich herausstellen sollte, daß in einem bestimmten sozialen oder geographischen Umfeld eine sonst nicht erlaubte Kombination gebräuchlich ist (oder umgekehrt). Die Darstellungsart erweist sich als sehr flexibel.

Wir können für diesen Teil folgende Matrizen erstellen, die noch in eine Gesamtdarstellung der Determinierer integriert werden müssen:

$$
\left[\begin{array}{l}
\mathrm{NACH} \pm \\
\mathrm{KOMB} \_1\{\text { menge }\}
\end{array}\right] \vee\left[\begin{array}{l}
\mathrm{NACH} \pm \\
\mathrm{KOMB} \_2\{\text { menge }\}
\end{array}\right]
$$

$M i$ erhält somit folgenden Eintrag:

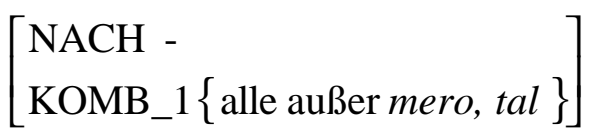

Und folgendes gilt für mero:

$$
\left[\begin{array}{l}
\mathrm{NACH}- \\
\left.\mathrm{KOMB} \_2 \text { este, el, cualquiera, alguno, ninguno, todo }\right\}
\end{array}\right]
$$


Otro erhält folgenden Eintrag:

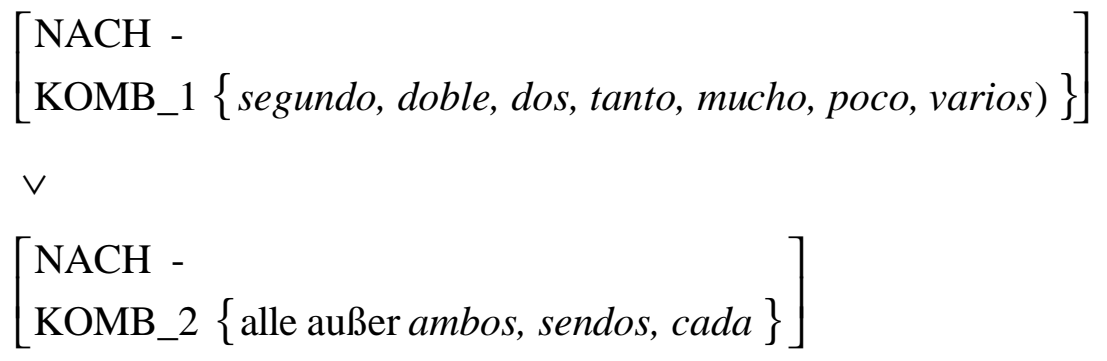

Nachstellung kann bereits im Lexikon als nicht möglich festgehalten werden ( [NACH - ] ). Die Menge der möglichen Einträge bei otro zeigt, daß eine Zusammenfassung in Klassen sinnvoll ist, sofern sich bei den Kombinationen Determinierer identisch verhalten. Diese können wiederum in Ausnahmefällen aufgelöst werden (vgl. die Kombinationsklassen e1 - e12 und z1 - z9 in 102).

Durch diese Einteilung sind die zusätzlichen Merkmale $^{32}$, die Allegranza zur Vermeidung doppelter Einträge bei Nominalphrasen einführt, nicht mehr nötig. Das doppelte, ungrammatische Auftreten eines Determinierers wird durch das Kombinationsattribut verhindert. Dies gilt allerdings nur für Voranstellung. Wenn ein Determinierer nachgestellt wird, kann er nicht gleichzeitig auch vor dem Nomen auftreten. Dies wird durch eine lexikalische Regel verhindert, die bei Nachstellung das Merkmal [MARKER [ STATUS 2] ] nach [MARKER [ STATUS 1] ] korrigiert (vgl. Kap. III.3.7.).

Bevor wir die lexikalischen Einträge erstellen können, fehlt uns noch die interne Struktur der Nominalphrase. Dazu müssen wir den hierarchischen Aufbau der NP erklären.

\footnotetext{
${ }^{32}$ Dies sind die Merkmale POSSPRO für Possessiva, QUAMARK für Quantifizierer und ORDMARK für Ordinalia, vgl. Allegranza 1998: 90f.
} 


\subsection{Ein X-bar-Schema für die spanische NP}

Das X-bar-Schema (Chomsky 1970, Jackendoff 1977) geht von folgender Hierarchie innerhalb der Nominalphrase aus (vgl. auch die Schemata bei Badia 1994: 252ff.):

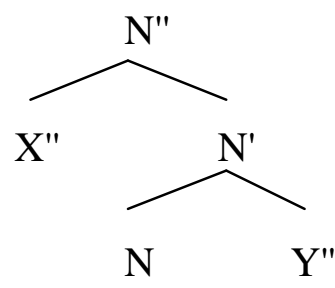

X" steht für einen Spezifizierer, Y" für ein Adjunkt, das auch durch einen nachgestellten Determinierer dargestellt werden kann. Das in unserer Klassifikation verwendete Merkmal [steht in Umgebung _ A N +] weist darauf hin, daß ein entsprechend markierter Determinierer die Nominalphrase von der Ebene N' zur Ebene N" überführt. Allegranza nennt dies eine Funktorbeziehung. In unserem Fall ist diese nur bei singulären Nomina obligatorisch. Das Nomen kann auch eigenständig in die nächste Ebene wechseln und muß dann als transnumeral eingestuft werden, d.h., wir gehen davon aus, daß, auch wenn die Nominalphrase lediglich aus einem Nomen besteht, die Ebenen N", $N^{\prime}$ und $\mathrm{N}$ in (156) realisiert sind. $\mathrm{X}^{\prime \prime}$ und $\mathrm{Y}^{\prime \prime}$ sind dagegen optionale Komponenten.

Allegranza schlägt nun neben dem Kopfmerkmal ein Attribut MARKED | SPR vor, welches den Status der Phrase kennzeichnet. Dieses ist in Opposition zu Pollard / Sag $(1987 ; 1994)$ boolesch und keine Liste. Wir bezeichnen dieses Merkmal nach Winhart (1997: 350) als STATUS. Es ähnelt dem Attribut LEX aus Pollard / Sag (1987), welches zur Abgrenzung von lexikalischer und phrasaler Information eingeführt wurde. Lexikalische Einträge waren als [LEX +], phrasale Einträge als [LEX -] markiert. STATUS übernimmt eine ähnliche Funktion, indem es die X-bar-Ebene bestimmt, auf der sich das Lexem oder die Phrase befindet. Lexeme sind somit durch [STATUS 0], Phrasen durch [STATUS 1 $\vee 2$ ] gekennzeichnet. Damit ist es identisch mit dem Attribut BAR aus der GPSG (Gazdar u.a. 1985: 22ff.).

Über STATUS können Determinierer danach eingeordnet werden, inwieweit sie die Phrase spezifizieren. Determinierer mit dem Merkmal [steht in Umgebung _ A N -] kennzeichnen wie Adjektive nur Phrasen, die mit [STATUS 1] markiert sind, und belassen sie in diesem Status. Diejenigen mit [steht in Umgebung _ A N +] kennzeichnen Phrasen mit [STATUS 1] und überführen sie nach [STATUS 2]. Dos 
und otro, die beide Merkmale tragen, müssen als Determinierer eingestuft werden, welche die Nominalphrase nach [STATUS $1 \vee 2$ ] überführen. Wenn wir nun den Konstituentenbaum bottom-up verfolgen, müssen daher viele Informationen zunächst als unterspezifiziert gelten. Da Nomina mitunter auch eigenständig als phrasal gelten, kann das Merkmal [STATUS 2] erst dann vergeben werden, wenn klar gestellt ist, daß die Phrase vollständig ist.

An dieser Stelle ist zu klären, wie die Struktur einer Nominalphrase mit mehreren (meist zwei) Determinierern aussehen muß. Es gibt zwei Möglichkeiten:

1. Der Determinierer in Zweitstellung hebt die Phrase bereits auf die N"-Ebene an, und der voranstehende Determinierer bewirkt eine Wiederholung dieser Ebene.

2. Der Determinierer in Zweitstellung beläßt die Phrase auf der N'-Ebene, und der voranstehende Determinierer übernimmt die Funktorfunktion der Überführung nach N".

Im Prinzip sind beide Möglichkeiten gleichwertig. Allerdings hatten wir in Kapitel III.1. festgestellt, daß folgende Konstruktionen möglich sind:

(a) tus dos famosos libros (Struktur: $\operatorname{Pos}_{1}$ Zahl Adj libros)

(b) tus famosos dos libros (Struktur: $\operatorname{Pos}_{1}$ Adj Zahl libros)

Für Adjektive muß nach dem X-bar-Schema angenommen werden, daß sie die NP auf N'-Ebene adjungieren. Wenn nun in (157b) folgende Reihenfolge möglich ist

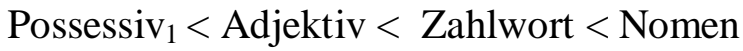

und Adjektive weiterhin auf der X'-Ebene operieren, dann befindet sich sowohl die Phrase Kardinalzahl + Nomen als auch die Phrase Adjektiv + Kardinalzahl + Nomen auf dieser Ebene. Folglich müssen Kardinalia als Determinierer interpretiert werden, welche die Nominalphrase auf der X'-Ebene belassen. Die Reihenfolge in (158) kann demnach folgendermaßen generalisiert werden:

Determinierer $<$ Adjektiv $<$ Determinierer $<$ Nomen

Somit gehen wir davon aus, daß Determinierer, die innerhalb der NP in Zweitstellung auftreten können und mehrere Möglichkeiten in bezug auf das X-bar-Schema zulassen, Spezifizierer sind, die N' auf N' belassen. Allegranza (1998: 99f.) nennt sie N' zu N'-Funktoren. Adjektive, nachgestellte Determinierer sowie adjungierte Präpo- 
sitionalphrasen, die alle als fakultative Adjunkte des Nomens in Frage kommen, stellen somit ebenfalls N' zu N'-Funktoren dar. Determinierer, welche die Nominalphrase grundsätzlich abschließen, überführen diese in die nächst höhere Ebene, welche hier immer die N"-Ebene ist. Somit können sie als N' zu N"-Funktoren bezeichnet werden. Beispielsatz (157b) erhält folglich folgendes Baumdiagramm nach dem X-bar-Schema:

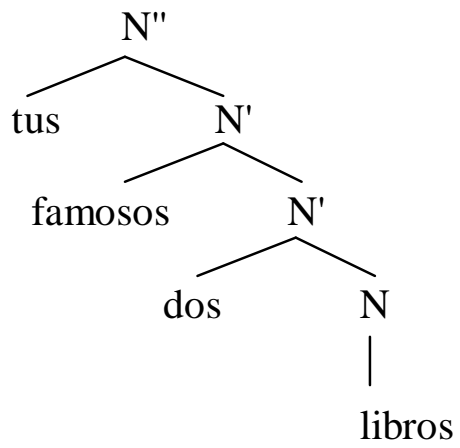

Der Prädeterminierer todo fällt nun insofern aus dem Rahmen, als er als N" zu N"Funktor zu verstehen ist, wobei wir ihn teilweise in die Darstellung der Kombinationsmöglichkeiten integriert haben. Danach entstehen auch bei todo relativ viele Re-

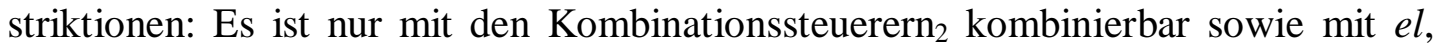
este und mi, d.h. mit den als definit deklarierten Determinierern. Damit kann todo über STATUS und KOMB_1 erfaßt werden. Damit wird todo im Gegensatz zu Ta-

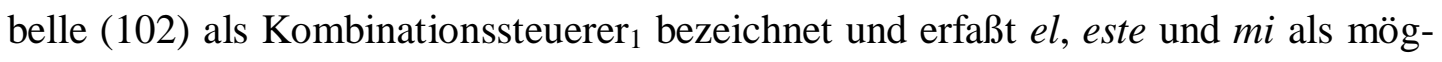
liche Determinierer in Zweitstellung. Gleiches kann für este vor mi angenommen werden. Das MARKER-Attribut wird als Kopfmerkmal aufgefaßt und bestimmt, ob ein Determinierer sein Argument auf eine höhere Ebene anhebt oder auf derselben Ebene beläßt.

\subsection{Lexikoneinträge}

Wir können nun Lexikoneinträge für Determinierer und Nomina erstellen. Adjektive werden aufgrund ihrer Abhängigkeit von der Sprecherintention, welche direkten Einfluß auf die Wortstellung hat, gesondert behandelt.

Anhand der bisherigen Untersuchungen können wir folgenden Vorschlag für singuläre Nomina machen, z.B. für coche: 
(161)

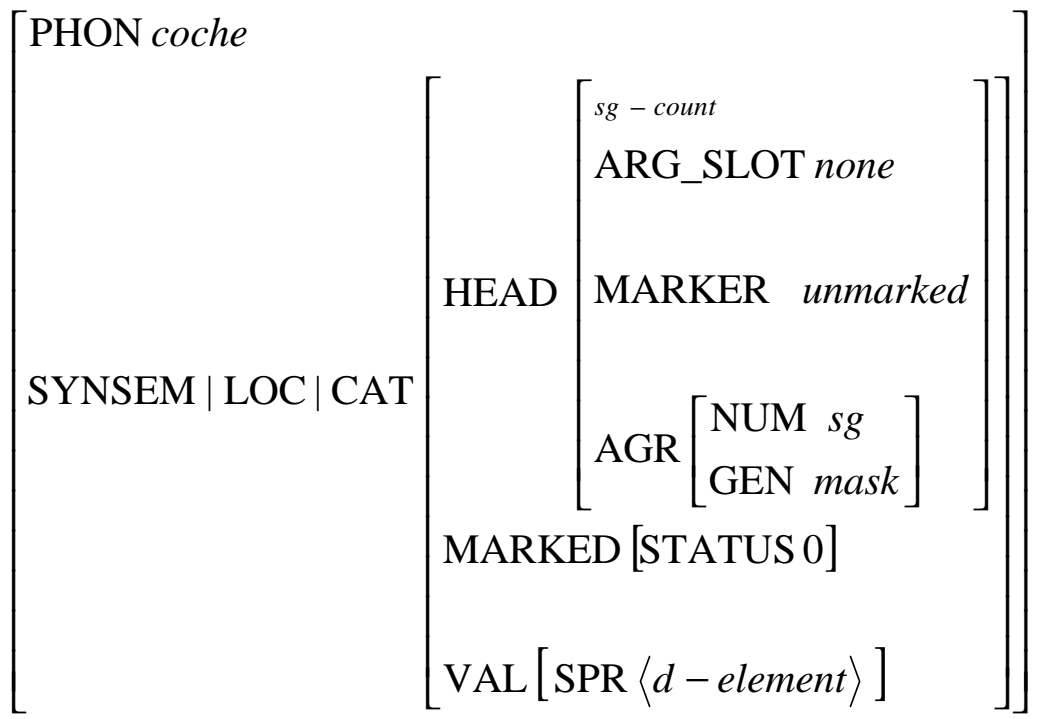

Coche wird als singuläres zählbares Nomen interpretiert. Dies verdeutlicht zum einen die Sortenangabe sg-count oben links im HEAD-Attribut, zum anderen das Valenzmerkmal, das nur bei diesem Nomentyp auftritt (VAL [SPR $<d$-element $>$ ] ). ARG_SLOT und MARKER sind nicht spezifiziert und werden als nicht vorhanden angegeben, da Nomina nicht als Adjunkte oder Spezifizierer für andere Komponenten verwendet werden (vgl. das Funktorschema in (139)). Die Kongruenz wird über AGR angegeben, das Merkmal PERSON steht dabei explizit nur bei Pronomina oder wenn es für die Subjekt-Verb-Kongruenz benötigt wird. Unter MARKED findet eine Differenzierung der Nominalphrase hinsichtlich der X-bar-Ebene statt. Da es sich hier um einen lexikalischen Eintrag handelt, muß der STATUS 0 sein. In der Spezifiziererliste unter Valenz wird die Sorte $d$-element (als Det oder adj_Det) angegeben. Sie kann durch die mögliche Prozedur der Generalisierung auch als fakultativ gesehen werden.

Im Vergleich dazu erhalten plurale Nomina einen fakultativen Eintrag in der Valenzliste. Bei Eigennamen, Massennomina und Pronomina kann die Valenzliste entfallen. Wir können sie wie folgt darstellen, wobei nur spezifizierte Information dargestellt wird: 
(162)

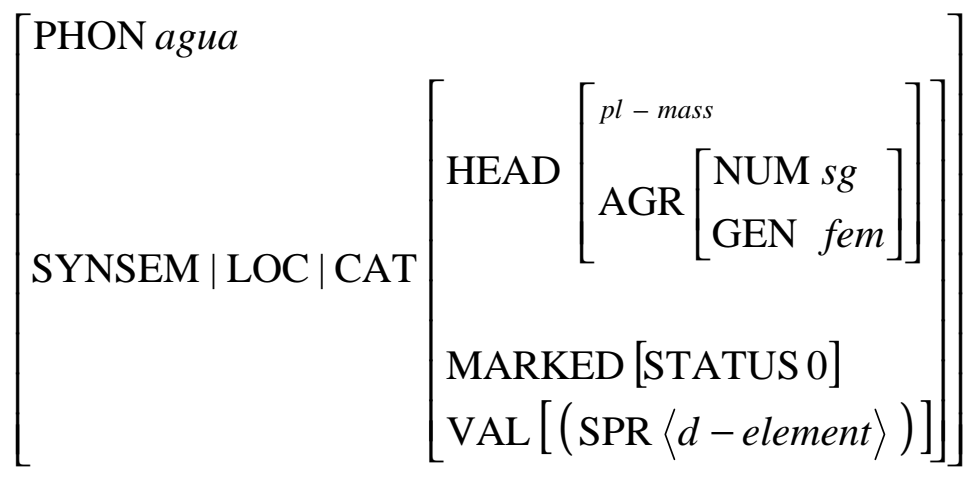

(163)

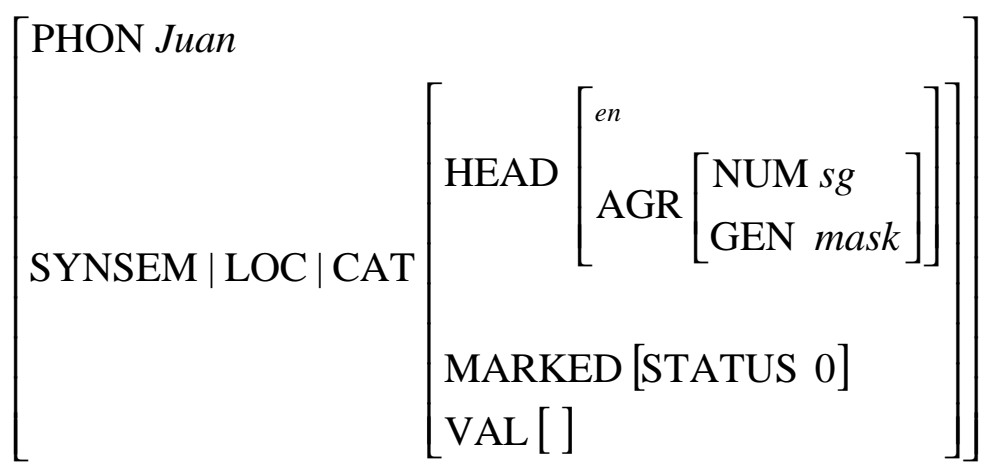

(164)

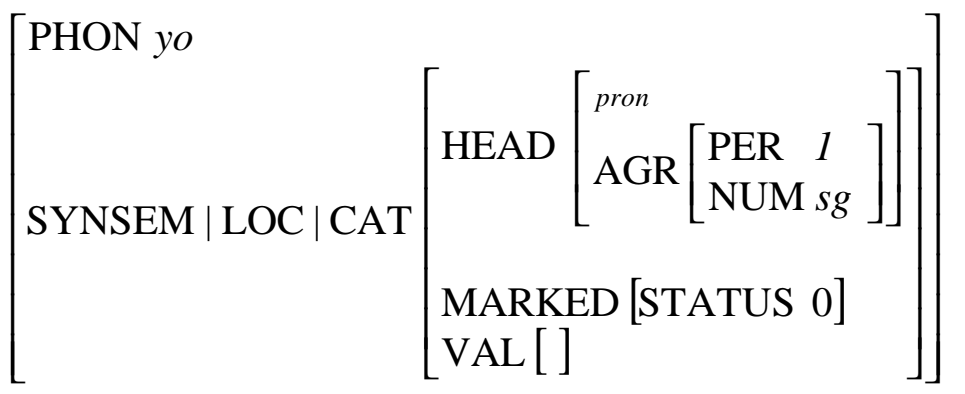

Im Unterschied zu Gattungsnomina können Eigennamen und Pronomina nicht modifiziert werden. Dies ist erst möglich, nachdem diese durch lexikalische Regeln zu Gattungsnomina umgeformt wurden.

Die folgende Matrix enthält einen Vorschlag für den lexikalischen Eintrag für Determinierer, hier am Beispiel von uno. Die Kombinationsmöglichkeiten werden durch die möglichen Klassen in (102) wiedergegeben. STATUS wird im folgenden mit ST abgekürzt. 
(165)

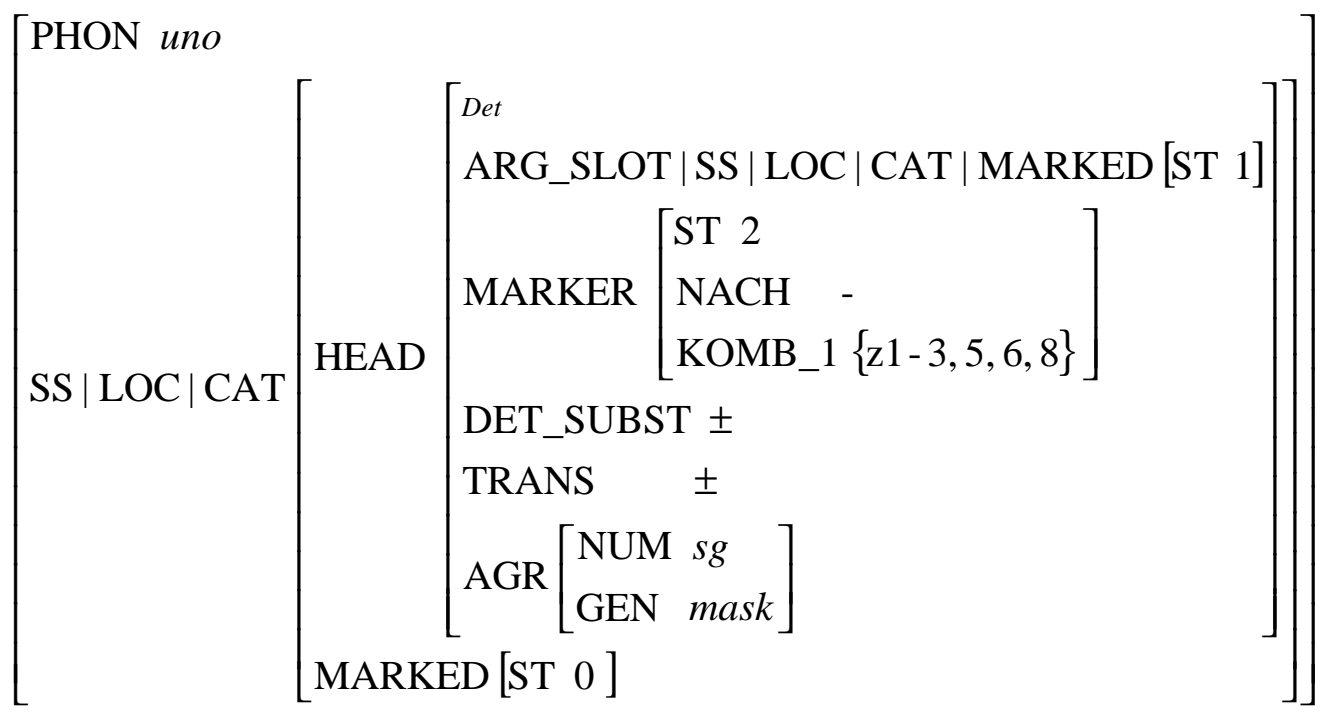

ARG_SLOT bezieht sich auf den Status der zu determinierenden Phrase mit dem Wert [STATUS 1] und bezeichnet damit eine Nominalphrase auf der Ebene N'. MARKER enthält die Attribute für die Stellung von Determinierern. Kongruenz ist im konkreten Fall der Form uno auf Singular und maskulin festgelegt. Welche Attribute in bezug auf Substantivierung auftreten dürfen, wird durch die entsprechende Sorte, hier Det, festgelegt und nur bei Lexemen spezifiziert. Das Merkmal Valenz ist bei Determinierern immer leer und wurde deshalb weggelassen.

Da das STATUS-Attribut dreimal vorkommt, ist eine genauere Differenzierung nötig. Alle drei Angaben sind Werte des Kategorieattributs mit dem Pfad SYNSEM | LOC | CAT:

1. HEAD | ARG_SLOT | SS | LOC | CAT | MARKED | STATUS

bestimmt die Ebene, auf der sich das zu markierende Nomen befindet $\left(1=\mathrm{N}^{\prime}\right.$ oder $\left.2=\mathrm{N}^{\prime \prime}\right)$, in (165): 1 = N'.

2. HEAD | MARKER | STATUS

bestimmt die Ebene, auf der sich die Kombination von Determinierer und Nomen befindet bzw. auf die der Determinierer die Gesamtphrase anhebt $\left(1=\mathrm{N}^{\prime}\right.$ oder $\left.2=\mathrm{N}^{\prime \prime}\right)$, in (165): 2 = N".

3. MARKED | STATUS

bestimmt die Ebene, auf der sich das Lexem (Determinierer oder Nomen) befin$\operatorname{det}\left(0=\mathrm{N}^{\circ} \vee \mathrm{D}^{\circ}, 1=\mathrm{N}^{\prime} \vee \mathrm{D}^{\prime}, 2=\mathrm{N}^{\prime \prime} \vee \mathrm{D}^{\prime \prime}\right)$, in $(165): 0=\mathrm{D}^{\circ}$.

Uno kann also als Determinierer eingeordnet werden, der auf der lexikalischen Ebene als MARKED [ STATUS 0 ] beschrieben wird, sich aber auf ein Nomen mit 
[STATUS 1] $\left(=\mathrm{N}^{\prime}\right)$ bezieht und dieses in [STATUS 2] (= N" oder NP) überführt. Determinierer können sich grundsätzlich nur auf Nomina der Ebene N' beziehen, da Adjunkte an $\mathrm{N}^{\prime}$ herantreten und die Adjungierung vorher erfolgen muß. Adjunkte haben damit eine Wiederholung dieser Ebene zur Folge, die wir als grundsätzlich gegeben annehmen.

Im Vergleich dazu listen wir nun die wichtigsten Merkmale anderer Determinierer auf. Kongruenz-, Kombinations- und Substantivierungseigenschaften können aus Gründen der Übersicht zunächst vernachlässigt werden. So kann die wichtige Angabe über den Statuswechsel besser eingesehen werden. Hier zunächst die Einträge für este und demás:

$$
\left[\begin{array}{l}
\text { SHON este } \mid \text { LOC } \mid \text { CAT }\left[\begin{array}{l}
\text { HEAD }\left[\begin{array}{l}
d-\text { element } \\
\text { ARG_SLOT } \mid \text { SS } \mid \text { LOC } \mid \text { CAT } \mid \text { MARKED [ST 1] } \\
\text { MARKER }[S T 2]
\end{array}\right] \\
\text { MARKED }[\text { ST 0] }
\end{array}\right]
\end{array}\right]
$$

$$
\left[\begin{array}{l}
\text { SS } \mid \text { LOC } \mid \text { CAT }\left[\begin{array}{l}
\text { HEAD }\left[\begin{array}{l}
d e t \_A d j \\
\text { ARG_SLOT } \mid \text { SS } \mid \text { LOC } \mid \text { CAT } \mid \text { MARKED [ST 1 } \\
\text { MARKER }[\text { ST 1 } 1]
\end{array}\right] \\
\text { MARKED [ST } 0]
\end{array}\right]
\end{array}\right]
$$

Das Possessiv $_{2}$ mío muß nach unseren Untersuchungen als Adjunkt interpretiert werden. Es steht grundsätzlich nach dem Nomen. Wir erstellen einen ausführlicheren Lexikoneintrag zum Vergleich mit uno: 
(168)

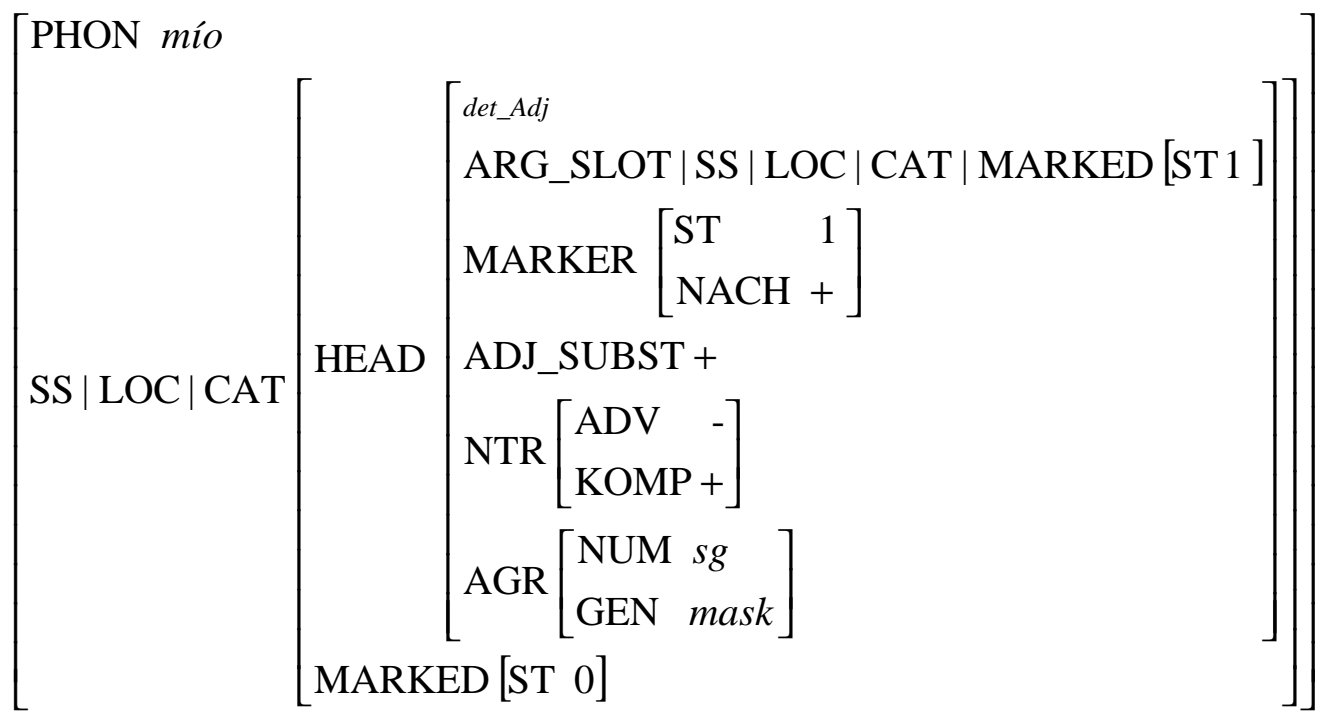

Semantisch ist mío als Determinierer zu interpretieren. Der Hauptunterschied zu este als potentiell nachstellbarer Determinierer besteht im bereits auf lexikalischer Ebene festgelegten Attribut [NACH +].

Der Prädeterminierer todo stellt eine Ausnahme dar, indem er als einziger Determinierer von der N"- zur N"-Ebene überführt:

$$
\left[\begin{array}{l}
\text { SS | LOC } \mid \text { CAT }\left[\begin{array}{l}
\text { HEAD }\left[\begin{array}{l}
d-\text { element } \\
\text { ARG_SLOT } \mid \text { SS } \mid \text { LOC } \mid \text { CAT } \mid \text { MARKED [ST 2 }] \\
\text { MARKER [ST 2 }]
\end{array}\right] \\
\text { MARKED [ST 0 }]
\end{array}\right]
\end{array}\right]
$$

Wir können nun die Determinierer insgesamt über ihre Sortenzugehörigkeit, eine mögliche Nachstellung und die Frage, auf welche Ebene sie die Nominalphrase anheben, definieren (Attribut MARKER | STATUS). Dabei ergibt sich folgendes Bild, mit Ausnahme von dos in der Reihenfolge des Dendrogramms (112) angeordnet. Die in (117) als Fixpunkte herausgestellten Determinierer werden schattiert dargestellt. 
(170) Sorten und Merkmale der Determinierer

\begin{tabular}{|c|c|c|c|c|c|}
\hline Det & Sorte & & & Stellung & Ebene \\
\hline & $\begin{array}{l}d e t_{-} \\
\text {Adj }\end{array}$ & $\begin{array}{l}\text { adj }_{-} \\
\text {Det }\end{array}$ & Det & Nach & MARKER | ST \\
\hline alguno & & & + & + & 2 \\
\hline ninguno & & & + & + & 2 \\
\hline cualquiera & & & + & + & 2 \\
\hline este & & + & + & + & 2 \\
\hline todo & & + & + & + & $2 \Rightarrow>2$ \\
\hline uno & & + & + & & 2 \\
\hline$m i$ & & & + & & 2 \\
\hline cada & & & + & & 2 \\
\hline el & & & + & & 2 \\
\hline ambos & & & + & & 2 \\
\hline sendos & & & + & & 2 \\
\hline varios & & & + & & 2 \\
\hline cierto & & & + & & 2 \\
\hline demasiado & & + & + & & $1 \vee 2$ \\
\hline mucho & & + & + & & $1 \vee 2$ \\
\hline tanto & & + & + & & $1 \vee 2$ \\
\hline bastante & & + & + & & $1 \vee 2$ \\
\hline росо & & + & + & & $1 \vee 2$ \\
\hline tal & & + & + & + & $1 \vee 2$ \\
\hline otro & & + & + & & $1 \vee 2$ \\
\hline dos & & + & + & & $1 \vee 2$ \\
\hline doble & + & & & + & 1 \\
\hline segundo & + & & & + & 1 \\
\hline mismo & + & & & + & 1 \\
\hline demás & + & & & & 1 \\
\hline mero & + & & & & 1 \\
\hline mío & + & & & + & 1 \\
\hline más & & + & & + & 1 \\
\hline
\end{tabular}

Die Klassifikation aus Kap. III.2.7. wird durch dieses Ergebnis bestätigt. Lediglich dos, welches als Numeral in die adjektivische Gruppe 5 eingeordnet wurde, muß aufgrund seiner an dieser Stelle relevanten Eigenschaften zur Gruppe 4 gezählt werden. Die Einteilung ist stimmig, die Merkmale sind übertragbar. Gleichzeitig bleibt 
klar erkennbar, daß die Klassen nicht vollständig voneinander trennbar sind. Die Annahme einer Abstufung erweist sich als richtig. Más erweist sich als besonderer Determinierer, der einzige, der nur mit der Sorte adj_Det gekennzeichnet wird. Die Erwartung, daß sich más besonders abheben muß, wird somit bestätigt.

\subsection{Lexikalische Regeln}

In der HPSG ist das Lexikon eine wichtige Komponente der modularen Grammatik. In ihm sind Informationen festgehalten, die durch grammatische Prozesse verändert oder ergänzt werden. Dabei macht das Lexikon eine Reihe von Vorgaben (defaultWerte). Jeder Sorte von Determinierern entsprechen bestimmte Eigenschaften. Diese sind im Lexikon eingetragen und ermöglichen oder verhindern grammatische Prozesse. Die als grammatische Prozesse bezeichneten Abläufe werden auch durch lexikalische Regeln dargestellt, sofern sie auf der lexikalischen Ebene ablaufen. Sie erscheinen nicht in einem Konstituentenbaum, sondern müssen bereits vor dessen Erstellung Anwendung finden. Mit Hilfe dieser Regeln können default-Werte verändert werden. ${ }^{33}$ Ein bereits zitiertes Beispiel ist die Verwendung von Determinierern als Adverbiale und damit in einer für Determinierer sonst untypischen Weise. Der Determinierer besitzt folglich bestimmte Standardmerkmale, die revidiert werden können.

Ein anderes Beispiel dafür war die Generalisierung von singulären zählbaren Nomina. Diese können im Prozeß der Generalisierung wie Massennomina verwendet werden und erhalten dann Eigenschaften, die mit den Eigenschaften von Massennomina identisch sind. Der Unterschied besteht darin, daß die entsprechende Verwendung nicht als Standard angesehen werden kann. Der Prozeß der Modifikation lexikalischer Eigenschaften wird durch lexikalische Regeln beschrieben (nach Pollard / Sag 1987: 391ff., vgl. auch Flickinger 1987).

Für den Prozeß der Generalisierung ist diese Regel denkbar einfach. Die Sortenhierarchie kennt die Sorte $s g$-count als Untersorte von $\mathrm{N}_{\mathrm{n}}$. Der Sorte war als Eigenschaft zugeschrieben worden, daß die Valenz nicht leer ist, sondern einen Determinierer verlangt. Diese beiden Merkmale müssen von einer lexikalischen Regel revi-

\footnotetext{
${ }^{33}$ Vgl. aber auch Bouma 1997: 25 für die Nachteile von lexikalischen Regeln: „Lexical rules of this type are procedural, require defaults, and may easily lead to spurious ambiguity“. Dementsprechend versucht Warner 2000 englische Auxiliarkonstruktionen ohne lexikalische Regeln darzustellen.
} 
diert werden. Dabei erhält die gleiche phonologische Kette neue Eigenschaften, die aber in sich stimmig sein müssen, d.h. sie sind in dieser Kombination existent, nur nicht mit diesem Lexem.

Zur Illustration wird folgendes Beispiel angegeben (aus de Bruyne 1993: 60):

(171) Para ti hay cama.

Cama kann hier nur verwendet werden, wenn zuvor die lexikalische Regel der Generalisierung angewandt wurde. Diese Regel kann in der HPSG folgendermaßen dargestellt werden:

(172) Lexikalische Regel für Generalisierung am Beispiel cama

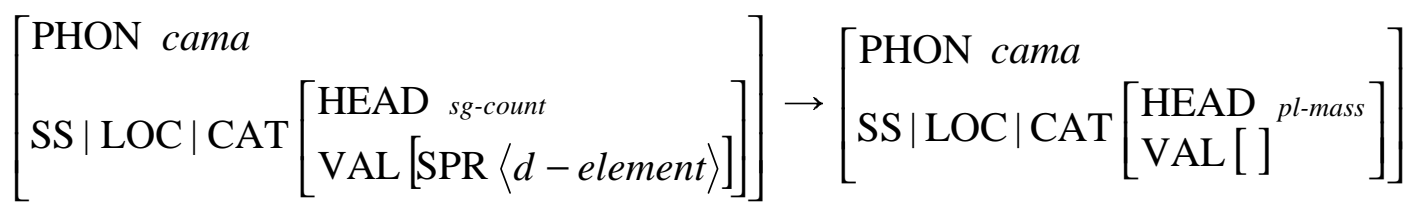

Die phonologische Kette bleibt erhalten. Die Merkmale werden durch andere innerhalb der Sortenhierarchie ersetzt. Dabei bleiben alle nicht erwähnten Merkmale wie z.B. Kongruenz (AGR) in der bisherigen Form erhalten. Cama bleibt Singular und feminin, obwohl seine Sorte in pl-mass modifiziert wurde. Alternativ könnte das Valenzattribut als grundsätzlich fakultativ angegeben werden und so in der lexikalischen Regel entfallen.

In gleicher Weise kann auch der umgekehrte Fall behandelt werden. Eigennamen können wie singuläre zählbare Nomina verwendet werden. Folgendes Beispiele wurden bereits in (130) zitiert:

\section{El Juan encontrado fue ilusión o real.}

Theoretisch ist es auch möglich, daß Eigennamen in den Plural gesetzt und der Sorte pl-mass zugeordnet werden. Dies geschieht zum einen sehr selten, zum anderen würde es aber einen ganz normalen Prozeß darstellen, der problemlos abgeleitet werden kann. Singuläre und plurale Nomina werden grundsätzlich gesondert in der Sortenhierarchie erfaßt, wobei eine lexikalische Regel die eine Form in die andere überführt. Die Annahme zweier Lexikoneinträge würde übermäßig Redundanz erzeugen.

Wir nehmen folgende lexikalischen Regeln für Eigennamen an: 
(174) Lexikalische Regel für Eigennamen

$$
\left[\begin{array}{l}
\mathrm{PHON}[1] \\
\mathrm{SS}|\mathrm{LOC}| \mathrm{CAT}\left[\begin{array}{l}
\mathrm{HEAD} \text { en } \\
\operatorname{VAL}[]
\end{array}\right]
\end{array}\right] \rightarrow\left[\begin{array}{l}
\text { PHON [1] } \\
\mathrm{SS}|\mathrm{LOC}| \mathrm{CAT}\left[\begin{array}{l}
\mathrm{HEAD} \text { sg-count } \\
\operatorname{VAL}[\operatorname{SPR}\langle\text { d-element }\rangle
\end{array}\right]
\end{array}\right]
$$

Bei den Determinierern gibt es vier Merkmale, welche die mögliche Substantivierung steuern. Wir konstruieren lexikalische Regeln, die immer dann anwendbar sind, wenn ein Determinierer dieses Merkmal trägt. Wir beginnen mit den Determinierern der Sorte Det, z.B. alguno:

(175) Lexikalische Regel für die Substantivierung der Sorte Det

$$
\left[\operatorname{HEAD}\left[\begin{array}{l}
\text { Det } \\
\text { DET_SUBST }+
\end{array}\right]\right] \rightarrow\left[\mathrm{HEAD}_{s g}-\text { count } \vee p l-\text { mass }\right]
$$

Bei dieser lexikalischen Regel werden Determinierer der Sorte Det zunächst einmal für die Nomensorte unterspezifiziert. Je nach Numerus muß dann die entsprechende Sorte gewählt werden. Etwas anders sieht die Regel für die Sorte adj_Det aus, z.B. für mío.

(176) Lexikalische Regel für die Substantivierung der Sorte adj_Det

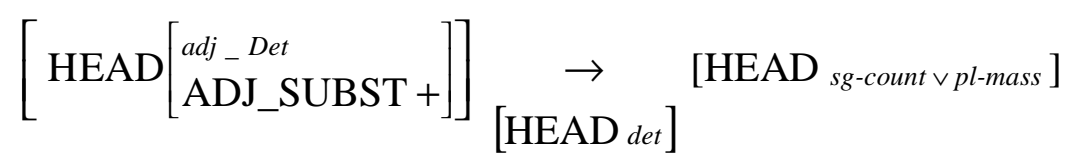

Diese Regel ist kontextabhängig, da sie einen Determinierer erfordert, der als Substantivierer operiert. Analog dazu erscheint die folgende Regel:

(177) Lexikalische Regel für alleinstehende substantivierte Adjektive

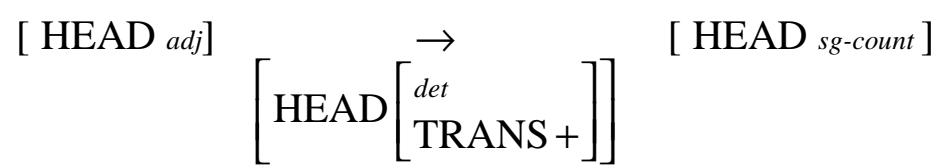

Auch diese lexikalische Regel ist kontextabhängig. Sie besagt, daß die Substantivierung eines Adjektivs im Singular nur in der Umgebung eines transponierenden De- 
terminierers möglich ist. Die Kombination ,transponierender Determinierer + Adjektiv“ kann in eine Kombination von „Determinierer + numerales Nomen im Singular oder Plural“" umgeformt werden (vgl. Varela Ortega 1990:142). Nach Flickinger (1987: 118ff.) können lexikalische Regeln kontextabhängige Ausnahmen aufweisen, deren Deskription und Anwendungsbereich für die entsprechende Regel von höchster Relevanz ist.

Analog kann eine lexikalische Regel für die Nachstellung von Determinierern aufgestellt werden, z.B. bei este:

\section{Lexikalische Regel für die Nachstellung von Determinierern}

$$
\left[\mathrm{HEAD}\left[\begin{array}{l}
\text { MARKER [STATUS 2] } \\
\mathrm{NACH}-
\end{array}\right]\right]\left[\operatorname{HEAD}\left[\begin{array}{l}
\text { MARKER [STATUS 1] } \\
\mathrm{NACH}+
\end{array}\right]\right]
$$

Wenn sich der Determinierer in Nachstellung befindet, kann er zwangsläufig nicht mehr die Phrase auf die Ebene N" (= STATUS 2) anheben.

In gleicher Weise kann auch die Pronominalisierung des definiten Artikels beschrieben werden. Wir waren davon ausgegangen, daß der definite Artikel an den Stellen durch das Personalpronomen ersetzt wird, wo bei anderen Determinierern eine Substantivierung möglich ist. Diese Eigenschaft ist eine Besonderheit des definiten Artikels. Ähnlich kann die semantische Nähe der beiden Possessivpronomina gewertet werden, da eine Phrase wie mi coche durch el mío pronominalisiert werden kann. Wir ordnen dieses Phänomen aber unter anaphorischer Referenz ein, die als gesondertes Phänomen betrachtet wird, wie auch das folgende Beispiel zeigt:

(179) Hay otros ejemplos, como los de países europeos que tienen dietas carentes de vegetales (...). (RA-1)

Der definite Artikel los referiert auf otros ejemplos und ist nicht als Substantivierung zu sehen. Vielmehr wird die Referenz-NP nicht wiederholt. Sie ist aber als semantischer Bezugspunkt zu interpretieren. 


\subsection{Die Struktur der NP}

Bevor wir auf semantische Aspekte zu sprechen kommen, soll der syntaktische Strukturaufbau einer Nominalphrase erläutert werden. Folgender Satz dient als Grundlage:

(180) Los demás cuentos, ordenados por el autor entre estos dos, adquieren también con el tiempo una nueva resonancia. (RA-3)

Bei der folgenden Darstellung der Nominalphrase los demás cuentos werden nach den bisherigen Ergebnissen nicht relevante Attribute vernachlässigt, z.B. Nachstellung und Kombination. Nachstellung wird auf allen Ebenen als [NACH - ], Kombination als [KOMB_1 < demás >] für los beschrieben. Die Kongruenzmerkmale sind immer Plural und maskulin und werden der Übersicht halber ausgelassen. Aus den gleichen Gründen verzichten wir auf das Attribut DTRS. STATUS wird mit ST abgekürzt:

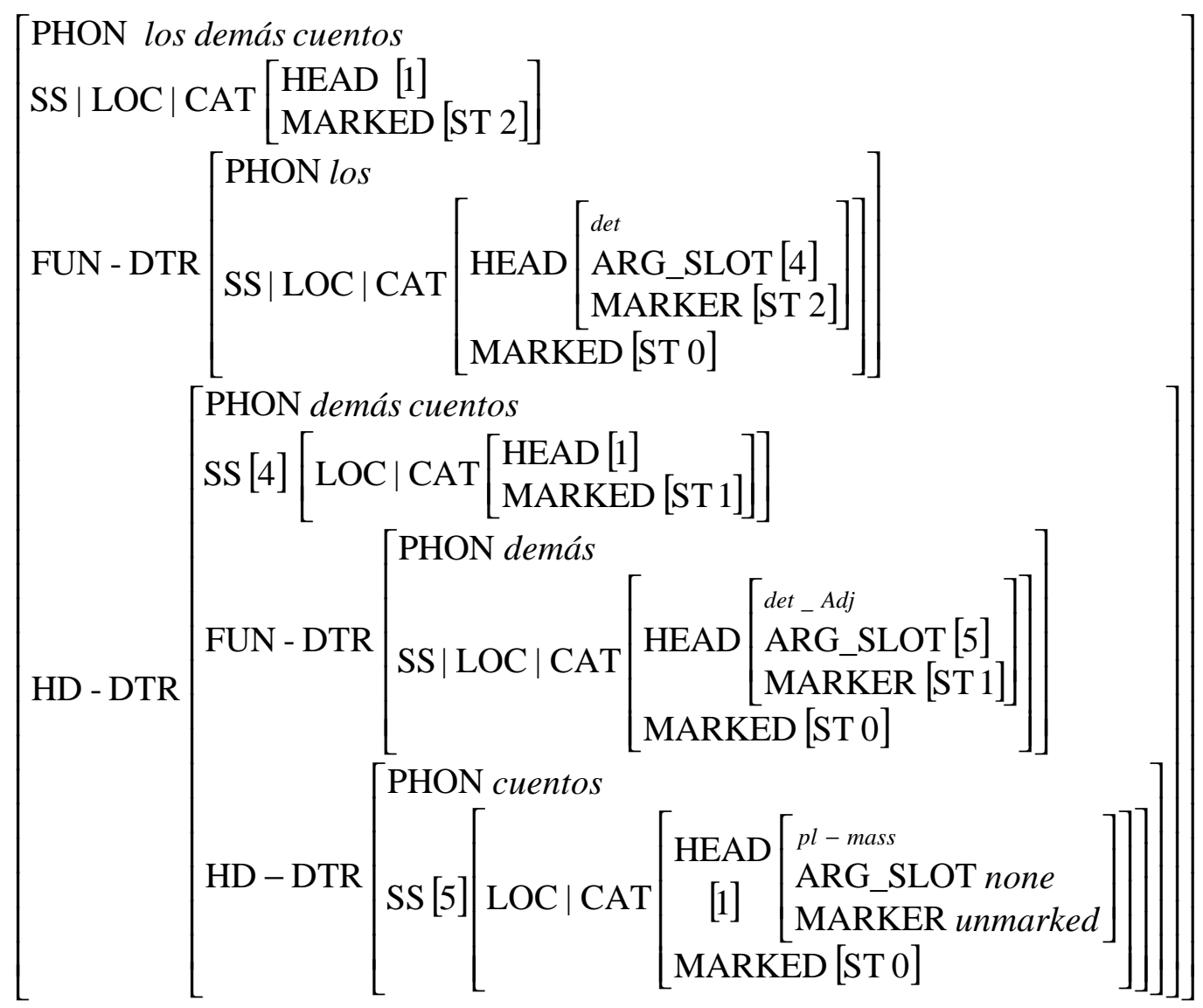


Die Attribute [MARKED [ STATUS 0 ] ] stehen auf lexikalischer Ebene und beschreiben das entsprechende Lexem. Analog kennzeichnen die Attribute MARKED | STATUS die entsprechende X-bar-Ebene bei den Phrasen los demás coches und demás coches mit dem Wert 2 bzw. 1. Zudem verweisen die Determinierer los und demás in ihrem Kopfattribut durch das Attribut ARG_SLOT auf die Kopftochter der bezeichneten Phrase und heben die Phrase auf die im Kopfattribut MARKER | STATUS bezeichnete Ebene an.

\subsection{Die Semantik}

Bisher haben wir nur die syntaktische Seite von Determinierern bearbeitet. In der Folge sollen einige semantische Aspekte erörtert werden, wobei wir eher Probleme aufzeigen als lösen werden. Dies liegt zum einen an der Komplexität der Materie, zum anderen daran, daß viele in der Literatur diskutierte Fragestellungen über den hier gesetzten Rahmen hinausweisen. Wir werden z.B. die Disambiguierung von Quantifizierern innerhalb von Satzstrukturen nicht bearbeiten (vgl. Pollard / Sag 1994: 319ff. und Allegranza 1998: 99ff.). Ziel dieses Abschnittes ist es vielmehr, die Semantik von Determinierern in Anlehnung an Pollard / Sag (1994) zu beschreiben und von der adjektivischen abzugrenzen. Wir gehen davon aus, daß eine Deskription der semantischen Aspekte nach Pollard / Sag (1994) möglich ist. Schwierigkeiten bereitet die prädikatenlogische Einordnung von Determinierern, die nicht einem logischen Operator entsprechen, was bei Pollard / Sag (1987; 1994) nicht thematisiert wird. Wir werden Vorschläge von Habel (1986 und Habel / Rollinger 1982) und Lohnstein (1996) aufgreifen, um die Schwierigkeiten zu verdeutlichen.

In Pollard / Sag (1987: 83ff.) wird in Anlehnung an die Situationssemantik zwischen Individuen und Relationen unterschieden. In einer vereinfachten Deskription werden Eigennamen als auf Individuen referierend bezeichnet, auf denen Relationen operieren. In einer ersten Teildeskription wird so der Eigenname Kim mit [SEM | CONT KIM] dargestellt. In einem zweiten Schritt gehen sie davon aus, daß es eine Entität gibt, auf die eine Relation der Benennung referiert und daß dieser Entität der Name Kim zugeordnet wird. Diese Relation kann als existent oder auch als nicht existent beschrieben werden. Pollard / Sag (1987: 94) erstellen folgende formale Notation für diesen Sachverhalt: 
(182) $\mathrm{x} \mid$ «naming, name: 'Kim', named: $\mathrm{x} ; 1 »$

Diese Formel besagt: es gibt ein x, auf das die Relation naming angewendet wird. Benannt wird x mit dem Namen Kim, und diese Relation ist gültig. In einer Matrix wird dies folgendermaßen dargestellt $(\mathrm{VAR}=$ VARIABLE, RESTR = RESTRICTION, POL = POLARITY):

$$
\left[\begin{array}{l}
\text { RAR [1] } \\
\text { RESTR }\left[\begin{array}{ll}
\text { RELN } & \text { NAMING } \\
\text { NAME } & \text { Kim } \\
\text { NAMED [1] } \\
\text { POL } & 1
\end{array}\right]
\end{array}\right]
$$

In der Folge werden Objekte bzw. das Ein-Objekt-Sein als Relation verstanden, um die Notation zu vereinfachen. Damit erhalten wir z.B. für Katze ein Objekt VAR, für das die Restriktion gilt, daß ihm die Relation Katze zugeschrieben wird. Um nun quantifizierte Objekte erfassen zu können, führen Pollard / Sag die Attribute DETERMINIERER (DET) und INDEX (IND) ein. DET beinhaltet Quantifizierer, INDEX kennzeichnet die entsprechende Bezugsmenge. Innerhalb eines Satzes kann nun die Semantik durch eine verbale Relation erfaßt werden, die sich auf die quantifizierende Nominalphrase bezieht. Das Beispiel every cat howls wird wie folgt aufgefaßt, die Polarität wird nur beim Wert 0 aufgeführt (nach Pollard / Sag 1994: 94ff.):

$$
\left[\begin{array}{c}
\text { QUANT }\left[\begin{array}{l}
\text { DET forall } \\
\text { IND }\left[\begin{array}{l}
\text { VAR [1] } \\
\text { RESTR }\left[\begin{array}{ll}
\text { RELN cat } \\
\text { INST [1] }
\end{array}\right]
\end{array}\right]
\end{array}\right] \\
\text { SCOPE }\left[\begin{array}{lc}
\text { RELN howl } \\
\text { HOWLER [1] }
\end{array}\right]
\end{array}\right]
$$

In Pollard / Sag (1994: 319ff.) wird dies aufgrund satzsemantischer Überlegungen revidiert. Die Autoren ersetzen die Sorte psoa (vgl. Kap. III.3.6.2.) durch eine neue, wenn auch nur in Details differenzierte Sorte quantifier-free-psoa (qfpsoa), die zwischen dem Kern einer Phrase und seiner Quantifikation unterscheidet: 


$$
\left[\begin{array}{ll}
p s o a & \\
\text { QUANTIFIERS } & \text { (list of quantifiers) } \\
\text { NUCLEUS } & (\text { qfpsoa })
\end{array}\right]
$$

NUCLEUS ist identisch mit der oben eingeführten Bezugsmenge. Der Wert ist vom Typ qfpsoa. Da die Angabe der Quantifizierer berechtigterweise als eine Liste angegeben wird, angeordnet nach dem Bezugsbereich (SCOPE), können wir annehmen, daß in Kombination auftretende spanische Determinierer an dieser Stelle die bei Pollard / Sag einfach besetzte Liste nun mehrfach besetzen. Nach ihrem Ansatz können wir die Nominalphrase unos cuentos wie folgt darstellen (Pollard / Sag 1994: 320):

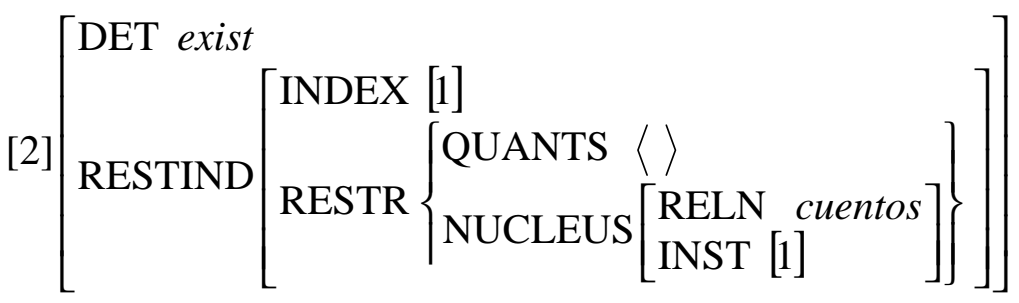

Die Semantik eines Satzes wie yo sé unos cuentos stellt nun lediglich die Erweiterung der Struktur (186) insofern dar, als die mit dem Index [2] bezeichnete Matrix (186) den Wert zum Pfad CONTENT | QUANTS in (187) darstellt:

$$
\text { CONTENT }\left[\begin{array}{l}
\text { QUANTS }\langle[2]\rangle \\
\text { NUCLEUS }\left[\begin{array}{l}
\text { afpsoa } \\
\text { RELATION saber } \\
\text { EL - QUE -SABE [3] }[1, s g] \\
\text { LO-SABIDO }[2]
\end{array}\right]
\end{array}\right]
$$

Nun entstehen zwei Probleme: Zum einen wird die doppelte Quantifizierung bei Pollard / Sag (1987; 1994) nicht explizit berücksichtigt. Zum anderen stellt sich die Frage, wie der definite Artikel oder auch andere Quantifizierer dargestellt werden können. Bisher war eine auf der Prädikatenlogik basierende Darstellung üblich, die hier aber nicht mehr möglich ist, es sei denn, wir greifen auf den IOTA-Operator zurück, was aber nur beim singulären definiten Artikel weiterhilft.

Wir greifen für das zweite Problem Vorschläge von Habel und Lohnstein auf. Habel / Rollinger (1982: 138f., vgl. auch Habel 1986) beschreiben innerhalb einer Text- 
repräsentation in der semantischen Repräsentationssprache MSRL (Modal Semantic Representation Language) Objekte durch Terme. Ein Term kann dabei auch eine Angabe der Quantität mit einer entsprechenden Einheit sein und bildet einen festen Bestandteil der Semantik. Eine quantitative Angabe wie 4,3 Millionen Mark wird dann wie folgt dargestellt. Die Bezeichnung reln1 (reln für Relation) verweist auf die Relation, welche die erwähnte Einheit mark wiedergibt:

(188) OBJEKT: quantity (EINHEIT: reln1 = 4,3 mio), reln1 - EINHEIT: mark.

Ganz ähnlich greifen auch Gawron / Peters (1990: 62) dieses Problem auf, indem sie in der Phrase two critics das Numeral mit

(189) Quantificational Force: TWO

notieren. Das Numeral wird somit als ein fester Bestandteil der Notation, der nicht weiter untergliedert werden kann, sozusagen als Faktum der beschriebenen Welt, repräsentiert. Dieser Ansatz steht in Opposition zu Allegranza (1998: 102ff.), der den semantischen Wert von Numeralia als modifizierend interpretiert. Diese Vorgehensweise ist für Adjektive korrekt, aber nicht für Quantifizierer. Lohnstein (1996: 158ff.) gibt ein Beispiel zur Differenzierung der beiden Phänomene: Die Nominalphrase brauner Hund beinhaltet letztlich die Schnittmenge zwischen Hund und braun:

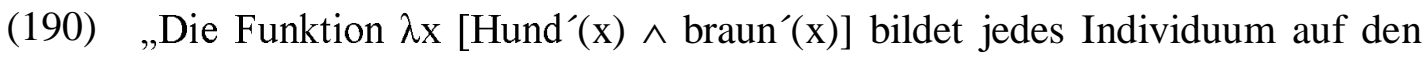
Wert $1 \mathrm{ab}$, das sowohl die Eigenschaft, ein Hund zu sein, als auch die Eigenschaft, braun zu sein, aufweist. Alle Individuen, die nicht beide Bedingungen gemeinsam erfüllen, werden auf 0 abgebildet““.

Demgegenüber beschreibt die Nominalphrase zwei Studenten in zwei Studenten rauchen „die Menge aller Teilmengen (...), deren jeweilige Schnittmengen mit dem Denotat von Student genau zwei Elemente enthält"“. Dazu führt Lohnstein (1996: 158) den Begriff der Kardinalität ein:

(191) Die Kardinalität $|\mathrm{M}|$ einer Menge $\mathrm{M}$ ist die Anzahl der Elemente von M. 
Damit beschreibt das Numeral zwei nicht eine eigene Menge, deren Schnittmenge mit dem nominalen Denotat eine neue Menge ergibt, sondern stellt eine Eigenschaft des nominalen Denotats dar. Dieser Bezug auf die Eigenschaften einer Menge muß als Quantifikation interpretiert werden (z.B. mehr / die meisten / viele / wenige / der Student(en)).

Durch diese Überlegungen bedingt gehen wir davon aus, daß im Attribut DETERMINIERER (DET) nicht mehr nur exist oder forall als analoge Formen der formalen Logik akzeptabel sind, sondern jeder Determinierer der natürlichen Sprache. Dieser schränkt in einer ihm inhärenten Weise den Kern der NP ein. Somit ist für die NP los demás cuentos der Pfad CONTENTS | QUANTS | DET mit dem Wert los oder demás akzeptabel.

Wie gehen wir nun mit dem Problem der doppelten Quantifizierung durch los demás um? Wenn die Quantifizierer in einer Liste erscheinen, müßte es möglich sein, daß bei mehrfachen Einträgen diese Liste lediglich erweitert wird, wobei für Listen angenommen wird, daß diese geordnet sind. Sowohl der erste Eintrag als auch der zweite Eintrag halten das Nomen in ihrer Bezugsmenge.

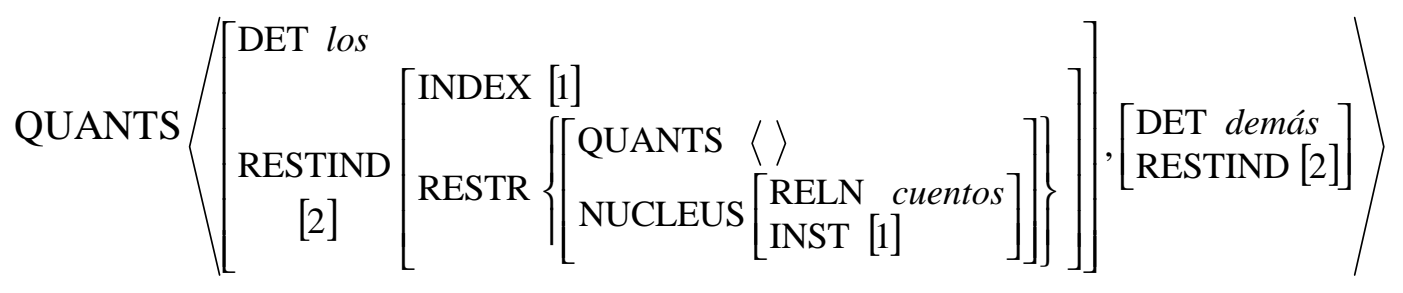

Im restringierten Index (RESTIND) wird das Nomen als der Kern der NP dargestellt. Auf diesen Kern beziehen sich jetzt zwei Determinierer: zunächst der Quantifizierer demás, dann der definite Artikel los. Daß nur diese Reihenfolge möglich ist, wird über die Syntax geklärt. Genauso ist es eine syntaktische Eigenheit von demás, nie allein aufzutreten. Der bedeutungsverwandte Determinierer otros müßte in diesem konkreten Fall analog zu demás dargestellt werden, auch wenn otro grundsätzlich auch andere Konstruktionen zuläßt.

Allegranza führt für die Quantifizierung zwei neue Attribute ein, die sich aber analog zu den bekannten bei Pollard / Sag verhalten. Seine Intention ist es, die Bezugsmenge der Quantifizierer auch innerhalb der NP auf der $\mathrm{N}^{\circ}$ - und $\mathrm{N}^{\prime}$-Ebene zu steuern. Dies sollte zwar schon jetzt nach dem skizzierten Ansatz möglich sein, ist 
aber bei Allegranza deutlicher dargestellt. Zunächst nehmen wir als lexikalischen Eintrag des Nomens cuentos folgendes an:

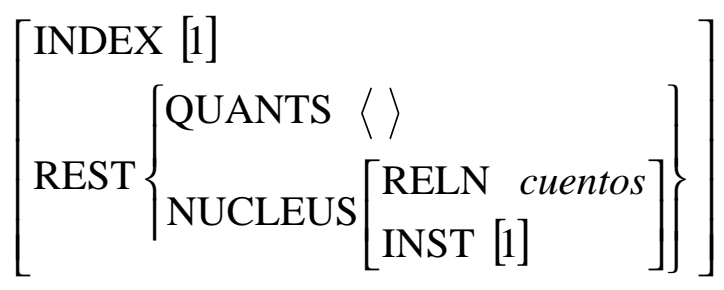

Allegranza stellt hier die berechtigte Frage, inwieweit nicht der bereits semantisch beinhaltete Existenzquantor bei pluralen Gattungsnomina wie cuentos - oder in seinem Beispiel it. libri - berücksichtigt werden muß. Bei singulären Nomina ist er nicht enthalten. Er schlägt von daher vor, daß ein entsprechendes DET-Attribut bereits auf lexikalischer Ebene eingeführt wird und das semantische Prinzip um eine Operation des Zusammenführens der determinierenden Eigenschaften der verschiedenen NP-Ebenen ergänzt wird. So existiert auf $\mathrm{N}^{\circ}$-Ebene ein Existenzquantor, auf N'-Ebene tritt im genannten Beispiel demás hinzu, auf N"-Ebene dann los (nach Allegranza 1998: 104).

Die Semantik von cuentos

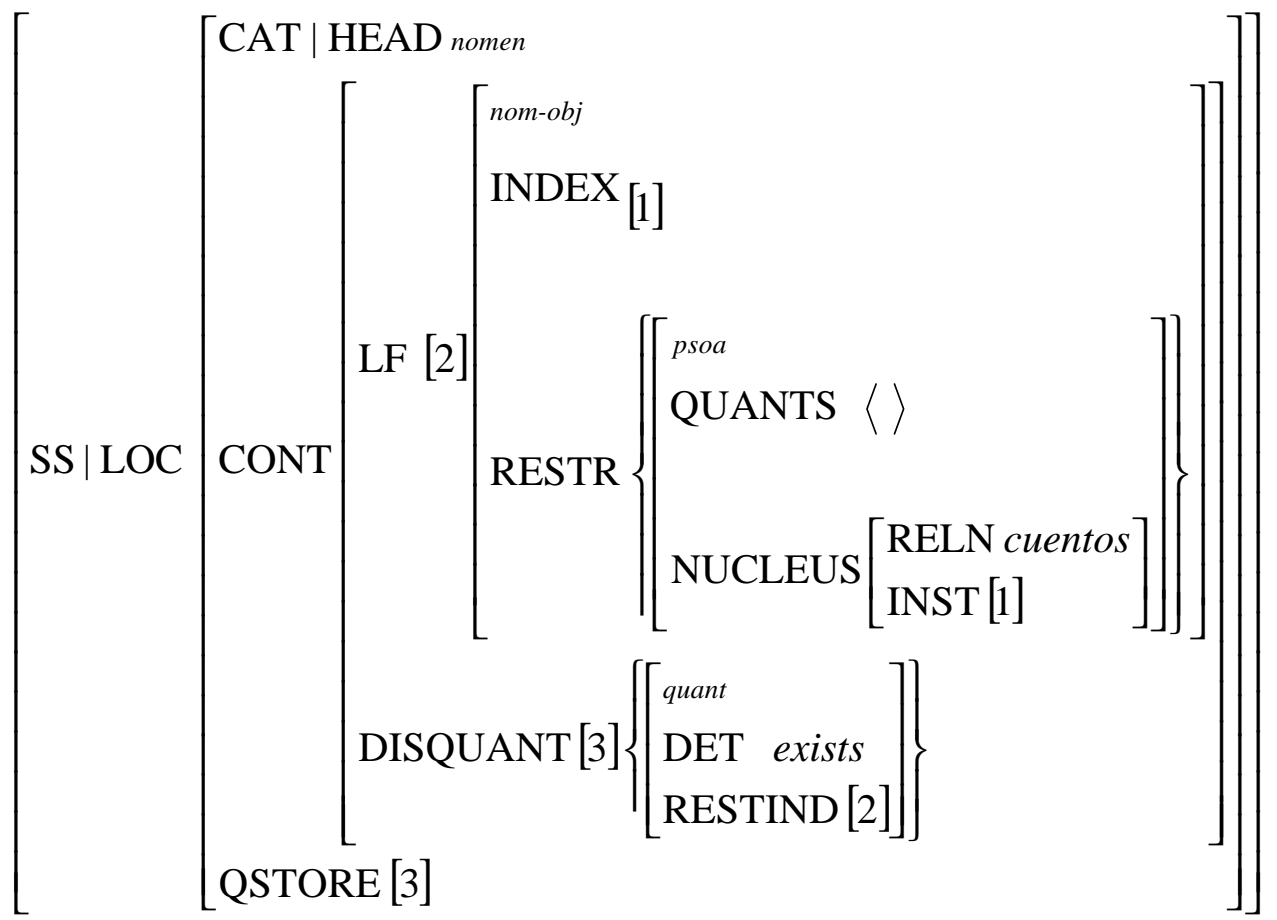

Allegranza beläßt in Anlehnung an die Standard-HPSG von Pollard / Sag (1994) das Attribut QSTORE auf einer Ebene mit SYNSEM. Wir stellen hier QSTORE in An- 
lehnung an Pollard / Yoo (1997: 424) auf die lokale Ebene, da Ambiguität auf Satzebene sonst nicht hinreichend erklärt werden kann. Diese Differenzierung ist für spanische Nominalphrasen insofern wichtig, als es sich grundsätzlich um lokale Merkmale handelt. Das Abbinden von Information über das Merkmal RETRIEVED wird somit grundsätzlich auf lokaler Ebene festgelegt ( SS | LOC ) und dadurch vereinfacht (vgl. Pollard / Sag 1994: 321ff.).

Wie in (194) bereits dargestellt, ersetzt Allegranza im Attribut CONTENT den restringierten Index RESTIND durch LOGICAL-FORM (LF) und die Determiniererangabe DET durch DISTINGUISHED-QUANTIFIER (DISQUANT). Zugleich führt er eine Operation compute-content ein, die einen durch einen Determinierer instantiierten semantischen Wert in die phrasale Deskription integriert (Allegranza 1998: 105).

$$
\text { compute-content }\left([i],\left[\begin{array}{l}
\text { DISQUANT }[h]\{\} \\
\operatorname{LF}\left[\begin{array}{l}
p \text { soa } \\
\text { QUANTS }[k] \\
\text { NUCLEUS }[l]
\end{array}\right]
\end{array}\right]\right)=\left[\begin{array}{l}
\text { DISQUANT }[h] \\
\operatorname{LF}\left[\begin{array}{l}
p \text { soa } \\
\text { QUANTS }[i] \oplus[k] \\
\text { NUCLEUS }[l]
\end{array}\right]
\end{array}\right]
$$

In der Folge entwickelt Allegranza (1998) ein neues semantisches Prinzip. Für weitere Details sei auf seine Ausführungen verwiesen, da die Änderungen gegenüber unserem ersten Ansatz nicht gravierend sind. Kritisch muß der modifizierende Charakter von Numeralia in Allegranzas Ansatz gesehen werden. 


\section{4. $\quad$ Adjektive}

In den vorigen Kapiteln ist es gelungen, die sogenannten determinierenden Adjektive in einer adäquaten Art und Weise zu klassifizieren. Dabei wurden die qualifizierenden Adjektive in die Klassifikation einbezogen, um einen Bezugspunkt zu erhalten. Ein Ergebnis dabei war, daß es zwar eine Klasse von Determinierern gibt, die sich syntaktisch genau wie Adjektive verhalten, wie auch eine Klasse, die sich optional wie Adjektive verhalten kann, daß es sich bei beiden Klassen aber grundsätzlich um Wörter handelt, die von ihrer Semantik her als Determinierer bezeichnet werden müssen.

Im Gegensatz dazu untersuchen wir im folgenden die Klasse der Adjektive, die in spanischen Grammatiken zumeist als qualifizierende Adjektive gesehen werden (vgl. Seco 1991: 89ff.). Sie sind syntaktisch grundsätzlich fakultative Angaben bzw. Adjunkte, die in Opposition zu den Determinierern niemals von irgendeinem Kopf subkategorisiert werden. Die Subkategorisierung von Determinierern hatten wir in der spanischen Nominalphrase bereits stark eingeschränkt und behauptet, daß sie zumeist nicht notwendig ist, da spanische Nomina auch eigenständig eine NP bilden können. Für Adjektive gilt - im folgenden sind mit diesem Terminus nur qualifizierende gemeint -, daß sie keinen obligatorischen Anteil an der NP darstellen und als Adjunkte interpretiert werden.

$\mathrm{Zu}$ diesem Bereich der möglichen Adjunkte zählen drei Konstruktionen: neben Adjektiven auch Präpositionalphrasen und Relativsätze. Präpositionalphrasen werden im folgenden Kapitel kurz angeschnitten (vgl. Verspoor 1996 und Winhart 1997). Der Bereich der Relativsätze soll hier ausgeklammert werden, da er ein neues Untersuchungsfeld erschließt (vgl. Lehmann 1984, Müller 1997 und Sag 1997). Für beide Bereiche können wir allerdings annehmen, daß Ergebnisse für Adjektive teilweise übertragen werden können, da sie ebenfalls immer adjungierend auftreten. Somit ist die Syntax vergleichbar. Der hauptsächliche Unterschied besteht im phrasalen Status von Präpositionalphrasen und Relativsätzen, was besonders in der Art des Anschlusses an die Nominalphrase deutlich wird. Während es sich bei Adjektiven um einzelne Wörter handelt - auch wenn Adjektive Köpfe von sogenannten komplexeren Adjektivphrasen sein können -, müssen die Wortarten der Präpositionen und Relativpronomina als heterogene Klassen verstanden werden. 
Als Präpositionen können z.B. in einer nominalen Konstruktion de, con, en, entre, $\sin$ u.a. auftreten. Ihre semantischen Eigenschaften variieren in beträchtlichem Maße je nach Kontext. Es ist zwar möglich, sie innerhalb eines nominalen Umfeldes zu beschreiben, wie z.B. folgende Strukturbeschreibung für die Nominalphrase im Satz el buen nombre de la Cruz Roja se vea manchado por estas prácticas zeigt:

$$
\left[\left[\text { el }_{\mathrm{D}} \text { buen }_{\mathrm{A}} \text { nombre }_{\mathrm{N}}\right]_{\mathrm{NP}}\left[\left[\operatorname{de}_{\mathrm{P}}\left[\text { la }_{\mathrm{D}} \text { Cruz }_{\mathrm{N}} \text { Roja }_{\mathrm{A}}\right]_{\mathrm{NP}}\right]_{\mathrm{PP}}\right]_{\mathrm{NP}}\right.
$$

Ob diese Deskription allerdings die Untersuchung von Präpositionalphrasen entscheidend weiterführt, muß doch arg bezweifelt werden. Dafür sind die syntaktischsemantischen Eigenschaften von Präpositionen zu komplex.

Gleiches gilt für Relativsätze. Zum einen kann eine Reihe von sehr unterschiedlichen Relativpronomina den Anschluß an die Nominalphrase erstellen, z.B. quien, que, el cual, cuyo u.a., welche in der Regel zusätzlich mit Präpositionen kombinierbar sind, z.B. de / en que, de quien u.a. Zum anderen folgt dem Relativpronomen ein Satz und damit eine verbale Konstruktion, deren Deskription nicht das Ziel dieser Arbeit darstellt. Als drittes sind Relativpronomina in ihrem syntaktischen Verhalten aufgrund der Nähe zu Personal- und Interrogativpronomina sehr komplex (vgl. Lehmann 1984: 399ff.).

Neben Präpositionalphrasen und Relativsätzen als möglichen syntaktischen Bestandteilen von Sätzen werden folgende syntaktische Phänomene ebenfalls nicht behandelt: Komparation, Subordination und Koordination. Wie die Klassifikation ergab, verhalten sich die Komparativadverbien más und menos teilweise den Determinierern sehr ähnlich. Sie standen allerdings im Dendrogramm sehr weit außen in der Klassifikation und müssen gesondert gewertet werden, da sie grundsätzlich eine komparierende Funktion übernehmen. De Bruyne (1993: 273ff.) führt sie von daher unter den Adverbien (Grad-, Quantitäts- und Komparativadverbien) auf (vgl. auch Romero Cambrón 1998: 17ff.).

Als zweites können wir Subordination, die in der Regel durch Relativsätze realisiert wird, ausschließen, sowie als drittes Koordination. Beide Phänomene sind nur bedingt in Grammatiktheorien erforscht (vgl. für einen kategorialgrammatischen Ansatz z.B. zur Koordination Hendriks 1995: 115ff.) und stellen für sich größere Problembereiche dar (vgl. Alcina Franch 1992: 174f.).

Im folgenden werden zunächst spanische Grammatiken in bezug auf qualifizierende Adjektive konsultiert (Kap. III.4.1.). Es schließt sich eine Betrachtung der 
Syntax (III.4.2.) sowie eine Untersuchung der Stellungsfrage nach Braselmann (1993), Dietrich (1993) (III.4.3.) und Pelzing (1981) (III.4.4.) an, welche syntaktische und semantische Fragestellungen berücksichtigt. Ein gesondertes Kapitel ist den bipositionalen Adjektiven gewidmet (III.4.5.). Abschließend erfolgt eine Deskription in der HPSG (III.4.6).

\subsection{Qualifizierende Adjektive in spanischen Grammatiken}

Adjektive sind syntaktisch immer als Adjunkte zu werten. Zudem sind sie von den Determinierern abzugrenzen. In diesem Kapitel soll nun die Stellung der Adjektive, die sehr eng mit ihrer Semantik zusammenhängt, untersucht werden. Das spanische Adjektiv kennt grundsätzlich drei Positionsmöglichkeiten (nach Pelzing 1981: 5):

(197) 1. Einzig Nachstellung (Abfolge: Nomen Adjektiv):

ácido sulfúrico

2. Einzig Voranstellung (Abfolge: Adjektiv Nomen): mera casualidad

3. Bipositionale Adjektive (beide Abfolgen möglich):

actual presidente / presidente actual; común denominador / denominador común

Das zweite Beispiel mera casualidad nennt kein qualifizierendes Adjektiv. Es stellt sich die Frage, ob es qualifizierende Adjektive gibt, die ausschließlich in Voranstellung erscheinen. Dies kann verneint werden. In der Regel ist meist von Adjektiven die Rede, die tendenziell eher voran- als nachstehen. Wörter, die nur vor Nomina stehen, sind Determinierer.

In der letzten Gruppe müssen bisemantische Adjektive hervorgehoben werden. $U n$ empleado triste muß mit 'ein trauriger Angestellter' übersetzt werden, un triste empleado aber mit 'nur ein Angestellter'. Diese Differenzierung kann auf den grundsätzlichen Bedeutungsunterschied zwischen nachgestellten und vorangestellten Adjektiven zurückgeführt werden. Während dem nachgestellten eine objektivbeschreibende Bedeutung nachgesagt wird, sollen vorangestellte eher eine subjektiv- 
affektive Bedeutung beinhalten. Nach Pelzing (1981: 9f.) lassen sich diese „traditionellen Beschreibungsverfahren“ in zwei Standpunkte unterteilen:

1. der logische, der zwischen ,función (...) determinativa, definitoria, restrictiva de la significación del sustantivo“ bei Nachstellung und „función explicativa, pero no definidora“" bei Voranstellung (RAE 1985: 410) unterscheidet, und

2. der psychologische, welcher die subjektive Intention des vorangestellten Adjektivs und den objektiven Charakter bei Nachstellung unterstreicht: „el adjetivo pospuesto viene a sumarse a la representación previa del objeto“ (Gili Gaya, zit. n. Pelzing 1981: 9).

Ein Mangel dieser Ansätze ist die schlechte Abgrenzung der qualifizierenden Adjektive von den determinierenden Adjektiven, so daß als Beispiele für stets vorangestellte Adjektive Determinierer herangezogen werden. Beispielhaft für einen solchen Ansatz sei auf Seco (1991: 169) verwiesen. Seco unterteilt folgendermaßen:

(198) (a) Das Adjektiv steht immer voran: cada, sendos, ambos u.a.

(b) Es kann auch nachgestellt werden: No he visto a hombre ninguno.

(c) Es verändert nachgestellt den Sinn:

el mismo hombre vs. el hombre mismo.

(d) Es steht sowohl voran als auch nach, aber mit einer bestimmten Priorität:

- Demonstrativa und Possessiva stehen in der Regel voran.

- Qualitativa stehen normalerweise nach:

un vestido caro, los países extranjeros u.a.

- Manche erlauben beide Stellungen in der Umgangssprache:

No tengo suficiente valor vs. No tengo valor suficiente.

(e) Hinzu kommen Redewendungen, in denen die Stellung des Adjektivs fest ist: Felices Pascuas.

(198a) bis (198c) nennen Determinierer, die hier nicht als Adjektive klassifiziert werden. (198d) enthält eine sehr allgemeinen Aussage, wie wir sie in (197) bereits getroffen haben. (198e) trägt der Tatsache Rechnung, daß idiomatische Redewendungen nicht in dieser Weise erfaßt werden können. Letztlich versucht Seco die Stellung des Adjektivs über eine Liste der gegebenen Möglichkeiten zu erfassen (für einen ähnlichen Ansatz vgl. de Bruyne 1993: 98-106). 
Damit erweist sich die Klassifikation des vorigen Kapitels III.2. als sehr hilfreich, da sie eine begründete Abgrenzung ermöglicht, die den Untersuchungsgegenstand „Adjektiv“ folgendermaßen einschränkt:

1. Wir bearbeiten nur qualifizierende Adjektive.

2. Diese stehen in der Regel in Nachstellung.

3. Wenn qualifizierende Adjektive in Voranstellung vorkommen, gegebenenfalls verbunden mit einem Bedeutungswechsel, muß dies erklärt werden.

Bei der Untersuchung der Stellungsfrage sind Braselmann (1993) und Dietrich (1993) besonders aufschlußreich. Die genaueste Untersuchung liegt mit Pelzing (1981) vor. Bei Luján (1973 und 1980) steht ein Vergleich der Adjektive mit Relativsätzen in einem transformationsgrammatischen Ansatz im Vordergrund. Ihre Ergebnisse werden eingearbeitet. Zuvor soll die Syntax von attributiven Adjektiven erläutert werden.

\subsection{Syntax von Adjektiven}

Adjektive können nur in attributiver Funktion an Nominalphrasen herantreten und bilden dann Adjunkte. Für Adjunkte wurde in Kap. II.2. ein Kopf-Adjunkt-Schema aufgestellt, welches die Beziehung zwischen dem Kopf und dem Adjunkt regelt. Dabei wählt das Adjunkt seinen Kopf. Der Kopf selbst stellt keine Restriktionen an das Adjunkt. Da wir im letzten Kapitel das Kopf-Funktor-Schema nach Allegranza (1998) eingeführt haben, welches auch für Adjunkte gelten soll, müssen wir das alte Schema aktualisieren. Dabei ändert sich aber lediglich die Terminologie: anstelle des Attributs MOD für Modifizierer steht nun ARG-SLOT für den Bezugspunkt des Adjunkts. Dieser Bezugspunkt stellt grundsätzlich den Kopf der Nominalphrase. Dabei bleibt das STATUS-Attribut der Nominalphrase unverändert. Es handelt sich immer um die X-bar-Ebene $1\left(\mathrm{~N}^{\prime}\right)$. Dies können wir hier als Grundvoraussetzung annehmen und in der Folge dieses Attribut weglassen. So gilt als allgemeine Struktur für Adjektive folgende Matrix (nach Pollard / Sag 1994: 55): 


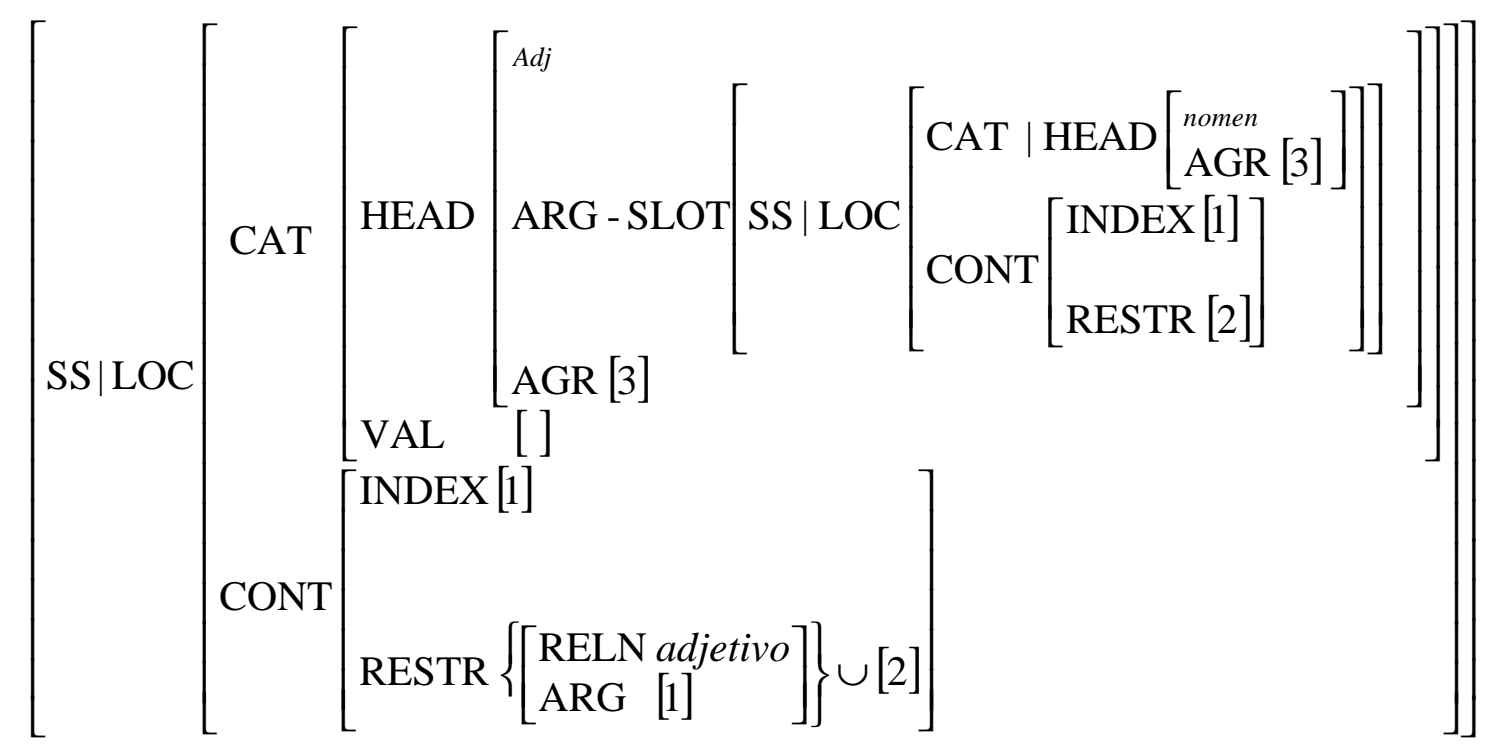

Der Kopf erhält die Sorte $A d j$, die in der Sortenhierarchie für qualifizierende Adjektive stand. Der Bezugspunkt des Adjektivs ist ein Nomen, was der folgende Pfad anzeigt: [ARG-SLOT | SS |LOC | CAT [ HEAD nomen] ]. Das Valenzmerkmal bleibt grundsätzlich leer. Adjektive können zwar selbst eine Adjektivphrase bilden. Dabei treten aber weitere adjungierende Modifizierer an das Adjektiv heran (z.B. Adverbien).

Es gelten außerdem folgende Korrespondenzen: Da Nomen und Adjektiv in Kongruenz zueinander stehen, muß der Wert AGR identisch sein und wird in (199) mit dem Index [3] gekennzeichnet. Gleiches gilt für die Semantik. Die Nominalphrase wird in ihrer Gesamtheit durch das Attribut [ INDEX [1] ] dargestellt, auf welche sich zwei Restriktionen beziehen: die des Nomens [2] und die des Adjektivs [RELN adjetivo]. Diese Information wird im Semantikattribut der Phrase durch die Vereinigung der nominalen [2] mit der adjektivischen Information beschrieben. ${ }^{34}$

Im weiteren Verlauf der Untersuchung müssen wir überprüfen, ob die semantische Komponente in dieser Form korrekt ist oder erweitert werden muß. Zum anderen müssen die Stellung des Adjektivs und die damit zusammenhängenden Fragestellungen erörtert und in das obige Schema eingearbeitet werden. Wir können also bereits jetzt davon ausgehen, daß ein Merkmal STELLUNG, wie es für die Determinierer angenommen wurde, auch für spanische Adjektive nötig ist. Wir beginnen die Besprechung der Sekundärliteratur mit den Ansätzen von Braselmann und Dietrich.

\footnotetext{
${ }^{34}$ Für den sehr ungewöhnlichen Ansatz, Adjektiv + Nomen als eine lexikalische Einheit zu verstehen, vgl. Sadler / Arnold 1994.
} 


\subsection{Die Stellung nach Braselmann (1993) und Dietrich (1993)}

Braselmann nimmt an, daß es eine „Normalposition“ für jedes Adjektiv gibt (Braselmann 1993: 339). Sie erstellt Klassen für Adjektive, die in der Regel nachgestellt, und für solche, die meist vorangestellt werden. Dazwischen steht eine Gruppe, die in beiden Stellungen auftritt und je nach Position ihre Bedeutung ändert. Voranstellung wird z.B. bei Explikativa gebraucht: Der Sprecher möchte Affektivität, Subjektivität oder Betonung ausdrücken. Nachgestellt werden „Determinativa, die restriktiv eingesetzt werden und die eine objektive Qualität bezeichnen“ (Braselmann 1993: 339). Für die markierte Position, d.h. für die nicht normalerweise eingenommene Position, stellt Braselmann Regeln auf, die diese Erscheinungen erklären soll. Soll ein nachgestelltes Adjektiv explikativ / figurativ verwendet werden, überführt es die Regel in die Voranstellung. Äquivalent dazu wird ein normalerweise vorangestelltes bei Modifikation oder Reihung in die Nachstellung überführt. Beide Regeltypen können auch bei Emphase angewandt werden (Braselmann 1993: 349):

\begin{tabular}{|l|l|}
\hline I. & $\begin{array}{l}\text { Basisklassifikation: } \\
\text { Adj in Voranstellung (VS) vs. Adj in Nachstellung (NS) }\end{array}$ \\
\hline II. & $\left.\begin{array}{l}\text { 1. Adj in NS }+\left\{\begin{array}{l}\text { Explikativität } \\
\text { Figurativi tät }\end{array}\right. \\
\text { 2. Adj in VS }+\left\{\begin{array}{l}\text { Modifikation } \\
\text { Reihung }\end{array}\right.\end{array}\right\} \rightarrow$ VS (Adj in VS unberührt) \\
\hline III. & Emphase $\left\{\begin{array}{l}\text { NS } \rightarrow \text { VS } \\
\text { VS } \rightarrow \text { NS }\end{array}\right\}$ (unabhängig vom primären / sekundären Status) \\
\hline
\end{tabular}

Das System von Braselmann erscheint auf den ersten Blick wohl geordnet und übersichtlich. Es stellen sich aber schnell folgende Fragen und Zweifel:

- Was genau bedeutet „Emphase“? Der Terminus wird nicht explizit erläutert (vgl. dazu Dietrich 1993: 90, der Emphase als Intonations- und Stellungswechsel bei gleichzeitig beibehaltener Grundfunktion beschreibt). Braselmann beobachtet allerdings richtig, daß Intonation oder Betonung eines Adjektivs einen Stellungswechsel hervorrufen können. Dabei bleibt aber die Grundfunktion erhalten (vgl. auch Luján 1980: 80). 
- Die Annahme, Reihung durch Koordination oder Komparation geschehe nur in der Nachstellung, ist falsch, wie Pelzing (1981: 141ff.) mit zahlreichen Beispielen belegt:

(201) (a) una profunda, casi religiosa convicción política.

(b) Alto y Bajo Egipto.

(c) sus más o menos acariciadores y sugerentes trinos.

(d) tan delicioso y bello bocado.

(e) la pequeña gran revista.

- Braselmann läßt Adjektive, die je nach Position ihre Bedeutung ändern, unberücksichtigt und postuliert zwei Lexikoneinträge. Dies ist nicht korrekt, da es sich eindeutig um dasselbe Adjektiv handelt und die jeweiligen Bedeutungen sehr ähnlich sind oder aufeinander referieren. Intuitiv wird ein Sprecher stets von der gleichen Wortform ausgehen. Dazu Dietrich (1993: 86): „In Wirklichkeit handelt es sich hier nicht um einen Sonderfall oder eine Ausnahme. Auch hier ist der Unterschied der Lesart aus der Opposition 'restriktiv' - 'nicht-restriktiv' zu erklären. Der 'Bedeutungs'unterschied scheint deshalb größer als in anderen Fällen zu sein, weil sich bei diesen Adjektiven im Zusammenspiel mit dem Substantiv deutlich eine Aufspaltung in unterschiedliche Bezugsbereiche ergibt.“ (Hervorhebungen von Dietrich)

- Aus Dietrichs Kritik läßt sich ein weiterer Punkt formulieren: Braselmann übersieht, daß die Semantik des Nomens (bzw. der gesamten untersuchten Nominalphrase) einen nicht zu unterschätzenden lexikalischen Faktor für die Analyse ergibt. Pelzing (1981: 68) zeigt klar auf, daß „,dem Nomen (...) bei der Gewichtung der Entscheidung, ob AN [= Adjektiv-Nomen-Abfolge] oder NA [= NomenAdjektiv-Abfolge] in Frage kommt, in den Fällen unter Berücksichtigung des zugrunde liegenden Referenzbereichs die Hauptgewichtung beizumessen [ist], in denen das bivalente Adjektiv (semantisch) zu schwach ist, um durch seine Semantik allein Klarheit für die Positionierung zu schaffen“. Bivalent bedeutet bei Pelzing, daß ein Adjektiv sowohl voran- als auch nachgestellt werden kann.

Zusammenfassend läßt sich sagen, daß Braselmann das Phänomen unter mehr oder minder syntaktischen Gesichtspunkten sieht und die Semantik nur tangiert. Dietrich (1993: 78) geht demgegenüber davon aus, daß ,die Stellungsfrage primär und durch- 
gehend eine semantische Frage ist, aus der sich alles andere (also etwa Normfixierungen, Beobachtungen über Durchbrechungen der Norm, Kommunikationsstrategien usw.) ergibt“".

Dietrich berücksichtigt die grammatische Nähe von Adjektiven zu den Determinierern, denn Zahlwörter, Possessivpronomina und indefinite Numeralia operieren als grammatische Determinanten des Nomens und stehen in Voranstellung. Dietrich folgert daraus für qualifizierende Adjektive: „Je geringer die lexikalische Intension des Adjektivs, umso näher steht es den grammatischen Determinanten, umso weniger ist die Oppositionsmöglichkeit der lexikalisch-extensionalen Adjektive sinnvoll“ (Dietrich 1993: 83). Dabei hat jede Sprache ihre eigene Norm, die Ähnlichkeiten und Differenzen zu anderen Sprachen aufweist. Auch die von Dietrich untersuchten romanischen Sprachen unterscheiden sich in ihrem Verhalten, z.B. Französisch und Italienisch. Während das Italienische eine adverbiale Steigerung des Adjektivs in der Nachstellung bevorzugt, ist diese beim Französischen in der Voranstellung sehr wohl möglich:
(a) una bella donna => una donna molto bella (ital.),
(b) une joliefemme => une très joliefemme (frz.).

Für das Spanische zeigt er anhand der Beispiele mala suerte, rara vez, alta frecuencia und alta tensión, daß mit der Voranstellung oft folgende Eigenschaften einhergehen (Dietrich 1993: 83f.):

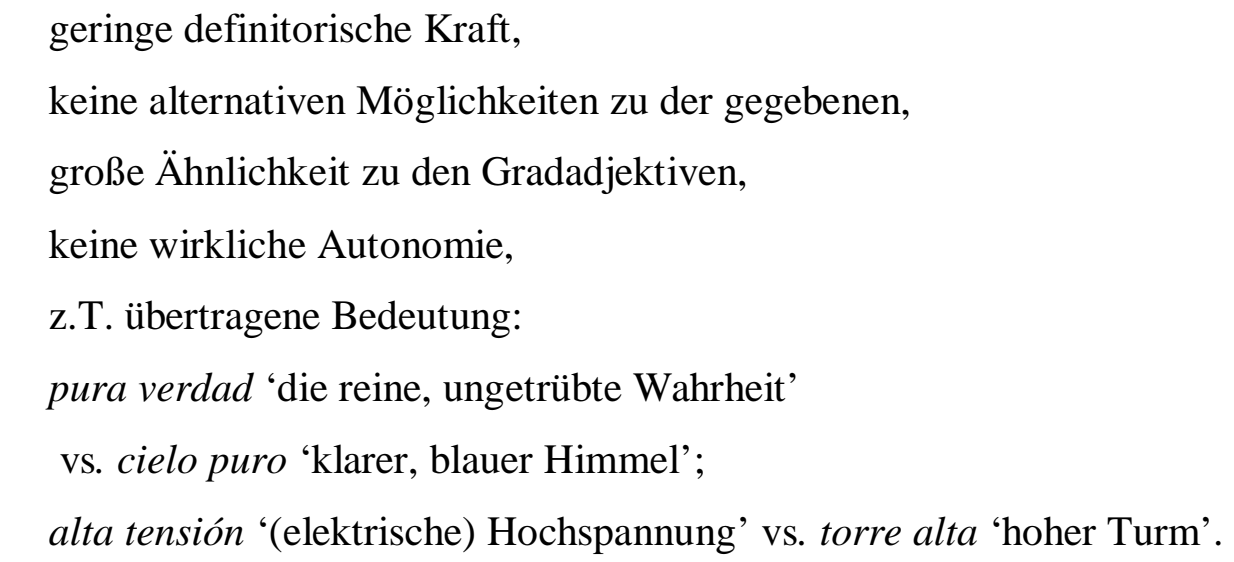

Somit ist die Stellungswahl semantisch begründet. Es gibt wenig handfeste Argumente, die eine rein syntaktische Analyse motivieren könnten. Im Prinzip bleibt die Wahl frei: dulce miel vs. miel dulce. Letztere Verbindung suggeriert, es gebe auch nicht-süßen Honig, was bekanntlich nicht der Wirklichkeit entspricht. Allerdings 
kann es in manchen Sprechsituationen durchaus möglich und legitim sein, süßen Honig von anderem Honig oder anderen Dingen abgrenzen zu wollen und damit einem unkundigen Zuhörer den Sachverhalt zu erklären. Daß eine Sprechsituation ungewöhnlich und selten eintrifft, bedeutet nicht, daß es sie nicht geben kann. Pelzing (1981: 29) stellt bei seinen Untersuchungen per Fragebogen fest, daß z.B. die Phrase artesanía única ('einzigartiges Kunsthandwerk') in der Regel falsch eingeordnet wurde, da die Informanten den beschreibenen Sachverhalt nicht aus eigener Anschauung kannten, und zieht die Schlußfolgerung, „daß fehlende Kenntnis einer Realität (...) zu Fehlleistungen in der Positionsgrammatik führen kann“. Miel z.B. gibt im genannten Beispiel einen Bezugsbereich vor, der 'süß' impliziert und folglich dulce in die Voranstellung drängt. Die Nachstellung impliziert eine mögliche Antonymie, die es zwar nicht gibt, die aber theoretisch denkbar und konstruierbar ist (z.B. eine Lehrsituation in der Grundschule).

Dietrich (1993: 86) zeigt die Antonymie für den Fall der Adjektive, die von Braselmann mit zwei lexikalischen Einträgen bearbeitet werden:

(204) (a) una noticia cierta: die Nachricht ist in einem objektiven Sinn als sicher anzunehmen.

(b) cierta noticia: eine gewisse Nachricht, ähnlich den grammatischen Determinanten tal, vario, diverso, diferente, alguno u.a., welche je nach Kontext in ihrer Bedeutung variieren können.

Dietrich faßt seine Ergebnisse wie folgt zusammen:

(205) „Semantisch extensive Adjektive erscheinen ähnlich wie grammatische Determinanten üblicherweise in der Voranstellung, weil sie nur eine geringere Restriktion gegenüber dem Bedeutungsumfang des determinierten Substantivs ausüben. In der Nachstellung wirken sie dagegen stark restringierend. Semantisch als spezifizierend, stark restringierend empfundene Adjektive können diese Wirkung in der Nachstellung zur Geltung kommen lassen. In der Voranstellung wird ihnen dieser Charakter ausdrücklich genommen.“ (Dietrich 1993: 90, vgl. aber auch die Kritik von Meisterfeld 1998: 100)

Wir nehmen somit folgende Grundsätze für die Stellung der Adjektive an:

1. Es lassen sich keine syntaktischen Restriktionen aufstellen. Die Opposition markiert-unmarkiert (Braselmann 1993) wird abgelehnt. 
2. Die Stellungsfrage ist nicht nur abhängig von der lexikalischen Bedeutung des Adjektivs, sondern auch von der des Nomens und von der Semantik der NP. Nach Pelzing (1981) ist der Referenzbereich des Nomens entscheidend.

3. Die bereits genannte Opposition 'restriktiv'- 'nicht-restriktiv' als grammatische Bedeutung kann folgendermaßen erweitert werden (nach Dietrich 1993: 79, vgl. auch Hölzer 1996: 9):

(206)

\begin{tabular}{|l|l|}
\hline Voranstellung & Nachstellung \\
\hline nicht-restriktiv & restriktiv \\
\hline nicht-distinktiv & distinktiv \\
\hline schildernd & klassifizierend \\
\hline subjektiv-bewertend & objektiv \\
\hline affektiv & determinativ \\
\hline
\end{tabular}

Meisterfeld (1998: 97) zeigt eine ähnliche Opposition für das Italienische (vgl. auch Nespor 1988: 431ff.).

4. Phänomene wie „Reim, Inkonsistenzakzent, Silbenzahl, Textsorte, Stil“ sind zu berücksichtigen (Braselmann 1993: 339). Je nach Kontext und Sprecherintention muß das Kommunikationsumfeld weniger oder stärker zur Erklärung herangezogen werden. Ein poetischer Text wird sich immer von Umgangssprache oder Fernsehwerbung unterscheiden. Diese eher auf der pragmatischen Untersuchungsebene anzusiedelnden Phänomene können hier nicht behandelt werden. Auch Redewendungen müssen aus demselben Grund ausgeschlossen werden.

5. Je größer der Bedeutungsumfang eines Adjektivs, desto eher erscheint es in der Voranstellung, da es kaum Restriktionen gegenüber dem Nomen ausübt. Diese Relation ist vergleichbar mit der Skala der Determinierer, wie sie in Kapitel III.2. erarbeitet wurde. Im Gegensatz zu den Adjektiven mit weitem Bedeutungsumfang stehen wissenschaftliche Termini, die in der Regel sehr spezifisch sind und sehr restriktiv auf den Bedeutungsumfang des Nomens einwirken. Sie treten in Nachstellung auf.

Die genaueste Untersuchung zur Stellungsfrage bei spanischen Adjektiven liegt mit Pelzing (1981) vor. Seine Ergebnisse und Klassifikationen können die hier erarbeiteten Prinzipien ergänzen. Zunächst erläutert er die Valenztheorie von Bull (1954), die zum besseren Verständnis kurz referiert wird. 


\subsection{Die Stellung nach Pelzing (1981)}

Pelzing übernimmt den Ansatz von Bull (1954, referiert nach Pelzing 1981), welcher sich an der Valenztheorie von Tesnière orientiert. Der Begriff der Valenz, im Sinne von Tesnière und nicht $\mathrm{zu}$ verwechseln mit dem hier verwendeten Merkmal VALENZ der HPSG, wird von Bull auf spanische Adjektive übertragen. Nach Tesnière bezieht sich der Valenzbegriff auf Strukturklassen von Verben, denen ein bestimmtes syntaktisches Verhalten inhärent ist. Da die Position von Adjektiven aber nicht rein syntaktisch zu analysieren ist, werden wir den Begriff Valenz durch Position ersetzen.

Bull unterscheidet drei Gruppen: Adjektive,

1. die nur nachgestellt werden,

2. die vor- oder nachgestellt werden können, ohne einen Bedeutungswechsel zu erfahren, und

3. die bei Positionswechsel auch einen Bedeutungswechsel vollziehen.

Die erste Gruppe besteht in der Regel aus eher technisch-wissenschaftlichen Termini, die das modifizierte Nomen in seiner semantischen Referenz näher bestimmen wie z.B. in ácido sulfúrico. Von daher kann angenommen werden, daß der Referenzbereich des Nomens ácido sich mit dem Referenzbereich des Adjektivs sulfúrico überschneidet. Auf diese Schnittmenge wird im gegebenen Kontext referiert. Bull nennt Adjektive, welche sich in dieser Weise logisch erfassen lassen, „holotomic“ oder „whole-splitting“ und erstellt eine monopositionale partitive Klasse, welche auf Nachstellung fixiert ist. Partitiv meint in diesem Kontext die Aufteilung des nominalen Referenzbereiches in einen Teil, auf den die Referenz des Adjektivs zutrifft, und einen Teil, auf den die Referenz nicht zutrifft (Pelzing 1981: 19ff.).

Demgegenüber stehen Adjektive, die sowohl vor als auch nach dem Nomen auftreten können. Dabei geht Pelzing davon aus, daß Adjektive in Voranstellung eine sogenannte integrale Funktion ausüben. Bei der integralen Funktion bilden Adjektive keine Unterklasse des nominalen Referenzbereichs, sondern charakterisieren den gesamten Referenzbereich. In den Beispielen áridos desiertos und gloriosos apóstoles charakterisieren die Adjektive árido und glorioso lediglich einen Sachverhalt, der dem Nomen semantisch schon inhärent ist. Eine Restriktion der Referenz des Nomens durch Nachstellung wäre zudem unsinnig. Die integrale Funktion kann also als eine Integration der Semantik des Nomens durch die Semantik des Adjektivs ver- 
standen werden. Andersherum gibt es natürlich Dinge, auf die das Adjektiv árido Bezug nehmen kann, auf die aber das Nomen desierto nicht referiert. Somit kann der Referenzbereich des Nomens auch als ein Teilbereich des adjektivischen Referenzbereichs angesehen werden.

Wir können beide Phänomene graphisch differenzieren (vgl. Lohnstein 1996: 158). Zunächst stellen wir die Überschneidung der Referenzbereiche des Nomens und eines restriktiv gebrauchten Adjektivs anhand des Beispiels ácido sulfúrico dar:

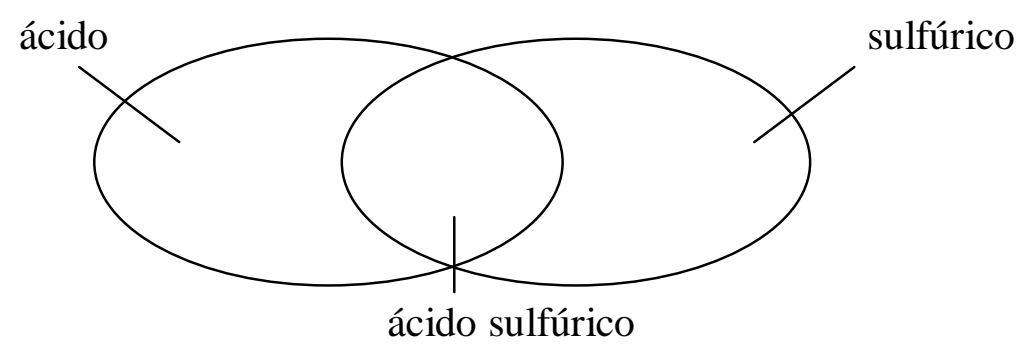

In Opposition dazu steht das Beispiel áridos desiertos. In diesem Fall kann der Referenzbereich des Nomens als Teilbereich des Adjektivs angesehen werden. Im genannten Beispiel kann dies allgemein angenommen werden. In anderen Fällen stellt der Sprecher ein Ereignis in seinem subjektiven Empfinden so dar:

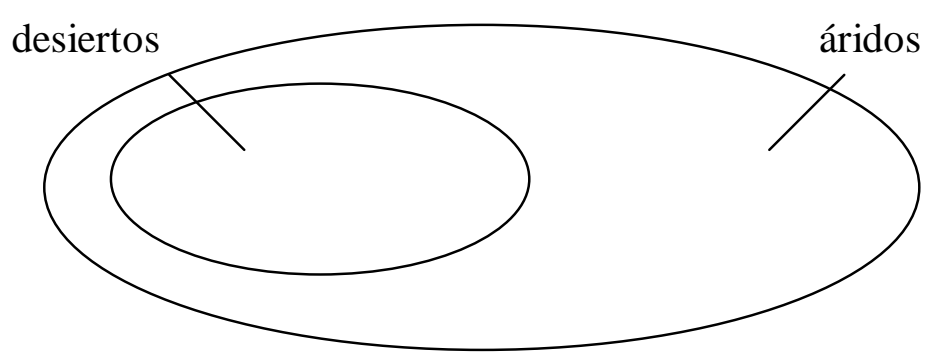

Das hat zur Folge, daß die Semantik des Nomens von höchster Bedeutung ist, da die Kombination von Nomen und Adjektiv ausschlaggebend ist. Daher hat Pelzing (1981: 24) recht, wenn er die partitive oder integrale Funktion von Adjektiven hervorhebt und den Begriff Klasse in diesem Zusammenhang ablehnt:

(209) „Das soll anhand der NPs los campos áridos und los áridos campos gezeigt werden. Dem Adjektiv árido kommt in [Voranstellung] eine integrale und in [Nachstellung] eine partitive Funktion zu. Das Nomen campos erlaubt die Stellung des Adjektivs in [Voranstellung] oder [Nachstellung], da Felder eine Reduktion des Referentiellen in Form einer Adjektivnachstellung zulassen. Denn Felder sind nicht nur trocken, sondern sie können auch andere Eigen- 
schaften haben. Es kann also nicht von einer integralen Klasse von Adjektiven gesprochen werden, da das angesprochene Adjektiv offensichtlich beiden Klassen zuzuordnen wäre, da es in [Voranstellung] oder [Nachstellung] stehen kann.“ (Hervorhebungen v. Pelzing, Einfügungen zur Vermeidung der bei Pelzing gängigen Abkürzungen)

\subsection{Bipositionale Adjektive}

Damit erhalten wir nun folgendes Zwischenergebnis bei der Untersuchung der adjektivischen Stellungsfrage:

- Die Semantik der Phrase ergibt sich aus dem Zusammenspiel der jeweiligen Referenzbereiche von Nomen und Adjektiv.

- Adjektive sind in Nachstellung durch partitive Funktion gekennzeichnet. Der Referenzbereich der Phrase ergibt sich aus der Schnittmenge der Referenzbereiche von Nomen und Adjektiv.

- Adjektive in Voranstellung sind durch integrale Funktion gekennzeichnet. Der Referenzbereich der Phrase entspricht dem Referenzbereich des Nomens. Dabei stellt der nominale Referenzbereich eine Teilmenge des Referenzbereichs des Adjektivs dar.

Die bipositionalen Adjektive erlauben dem Sprecher die Wahl zwischen Nach- und Voranstellung, d.h. beide Optionen sind möglich. Wenn also die integrale Funktion gewählt wird, können wir davon ausgehen, daß der Referenzbereich des Nomens durch den Referenzbereich des Adjektivs abgedeckt wird. Es handelt sich um eine subjektive Option des Sprechers. Alternativ dazu können wir auch festhalten, daß in Nachstellung und folglich in partitiver Funktion das Adjektiv eine Opposition innerhalb des Referenzbereichs des Nomens aufbaut, z.B. die trockenen Felder vs. die nicht-trockenen Felder. In integraler Funktion bei Voranstellung wird diese Opposition vermieden. Das Adjektiv beschreibt lediglich den Referenzbereich des Nomens genauer.

Bipositionale Adjektive besitzen eine geringe semantische Dichte und eine hohe Okkurrenz, wie Pelzing am Beispiel von alto und bajo erläutert. Dietrich weist darauf hin, daß diese Adjektive mit geringer semantischer Dichte gerade aufgrund dieser 
Eigenschaft eine geringe Restriktion auf den Referenzbereich des Nomens ausüben. Werden diese Adjektive nachgestellt, bauen sie eine Opposition zu ihrem Antonym auf, die in der Voranstellung ausdrücklich fehlt. Bei nachgestellten Adjektiven ist diese Opposition vorhanden. Dies kann am Beispiel áridos desiertos nochmals verdeutlicht werden. Árido ist ein tendenziell eher in Nachstellung auftretender Fachbegriff, der eine Opposition zu anderen Adjektiven wie z.B. fértil, fecundo errichtet. Da das Nomen desierto diese Opposition kaum unterstützt, präzisiert das Adjektiv in Voranstellung den Bedeutungsumfang des Nomens.

Adjektive, die in Voranstellung eine etwas andere Bedeutung annehmen und als bisemantisch bezeichnet werden können, bilden nach Pelzing (1981: 112) eine sehr kleine Klasse von 18 Wörtern, die durch bestimmte Kriterien gewonnen wurde: antiguo, bueno, cierto, clásico, extraño, falso, gran(de), mal(o), medio, nuevo, pobre, propio, rico, simple, triste, único, verdadero und viejo. Pelzing weist daraufhin, daß die Abgrenzung zu den rein bipositionalen Adjektiven häufig willkürlich sei, da „die Bisemantizität (die über die Bipositionalität zum Tragen kommt) durch die semantische Breite der BBA (= bipositionale, bisemantische Adjektive) und die NomenCharakteristika 'gestört' werden kann“. Zudem zeigen einige Adjektive aufgrund ihres weiten Bedeutungsumfangs ein „komplexes kombinatorisches Potential“, welches mehr Kombinationsmöglichkeiten mit Nomina zuläßt, als dies gewöhnlich der Fall ist.

Somit ist eine Deskription des semantischen Bedeutungsumfangs von Adjektiven nur in Relation zum Nomen möglich. Der Sprecher bestimmt dabei allerdings, inwieweit er beide Referenzbereiche in Opposition setzt. Pelzing faßt seine Ergebnisse wie folgt zusammen:

(210) „Die für die Adjektivpositionsgrammatik beschreibungsrelevanten Kriterien sind in drei Bereiche zu unterteilen:

(i) Den Adjektivbereich

(ii) Den NP-Bereich

(iii) Den Mischbereich“

Die unter (i) erfaßten Adjektive sind ausreichend determiniert, um ihre Position selbst zu bestimmen. Pelzing faßt an dieser Stelle determinierende und qualifizierende Adjektive zusammen. Da wir die erste Klasse bereits erfaßt haben, kann in dieser Untersuchung eine Adjektivklasse erstellt werden, die sich aufgrund ihrer Semantik 
grundsätzlich durch Nachstellung ausweist. Die bei Pelzing unter (i) genannten voranstehenden Wortformen sind als Determinierer zu werten.

Unter (ii) erfaßt Pelzing diejenigen Adjektive, welche bipositional erscheinen können und deshalb in ihrer Positionszuweisung eine integrale oder partitive Funktion übernehmen. Dies ist nur über die referentielle Semantik des Nomens bzw. der Nominalphrase entscheidbar. Pelzing weist daraufhin, daß einige Adjektive wie concreto, femenino oder primitivo, die scheinbar nur in Nachstellung auftreten, bei entsprechenden Nomina auch in Voranstellung auftreten können.

Unter (iii) faßt Pelzing die bisemantischen, bipositionalen Adjektive zusammen. Er postuliert für diese Adjektive ein Zusammenspiel von adjektivischer und nominaler Semantik, wie z.B. in el empleado triste vs. el triste empleado.

Abschließend können wir folgendes festhalten:

(211) 1. Viele qualifizierende Adjektive sind technisch-wissenschaftlicher Natur und treten nur in Nachstellung auf (210i)).

2. Wenn ein Adjektiv alternativ vor- oder nachgestellt werden kann, gelten folgende Restriktionen: Es übt eine integrale oder partitive Funktion aus, die in Abstimmung mit dem Nomen ermittelt werden kann. Dabei können Termini wie subjektiver vs. objektiver Gebrauch zur Beschreibung verwendet werden (210ii).

3. Wenn das Adjektiv seine Bedeutung durch Vor- oder Nachstellung verändert, ist dies ebenfalls in Abhängigkeit vom Nomen zu sehen. Inwieweit wirklich eine fundamental andere Bedeutung entsteht, muß nach Dietrich (1993) kritisch hinterfragt werden (210iii).

Die Stellungsfrage bei Adjektiven muß letztlich als ein kompliziertes Phänomen beschrieben werden (vgl. die Zusammenfassung von Meisterfeld 1998: 100f.). Da die Semantik sowohl des Adjektivs als auch des Nomens eine entscheidende Rolle spielt, kann der Sprecher je nach Intention und Kontext relativ frei die Stellung variieren. Es stellt sich die Frage, inwieweit die Abgrenzung zwischen (ii) und (iii) überhaupt sinnvoll ist, da es sich um die Problematik der adjektivischen Semantik im Verhältnis zur nominalen handelt. Je öfter ein Adjektiv gebraucht und je weiter sein Bedeutungsumfang angenommen wird, desto freier sind die Möglichkeiten einer integralen oder partitiven Funktion anzusehen. Pelzings Untersuchung verdeutlicht diese Verwendungsmöglichkeiten, ohne daß sich daraus ein adjektivisches Merkmal ableiten 
ließe, das integrale oder partitive Funktion fordert. Es handelt sich wie bei singulären zählbaren Nomina um eine Eigenschaft, die erst auf NP-Ebene deutlich markiert werden kann. Auf untererer Ebene können lediglich die vorhandenen Möglichkeiten dargestellt werden.

Ernst (1986) versucht anhand der Klasse der Relationsadjektive eine in diesem Zusammenhang interessante Deskription der nominalen Semantik. Als Relationsadjektive bezeichnet er vom Nomen abgeleitete Adjektive, die nicht prädikativ verwendet, nicht durch Adverbien modifiziert oder gesteigert und nicht auf gleicher Hierarchieebene koordiniert werden können (Ernst 1986: 318f., vgl. auch Bosque 1993: 118ff. und Alcaraz Varó / Martínez Linares 1997: s.v. adjetivo relacional). Im Verlauf seiner Untersuchung versucht er festzuhalten, in welchen Fällen eine Kombination von Nomen und Relationsadjektiv unzulässig ist und auf welchen Kriterien die Unzulässigkeit beruht. So z.B. „Nomina, deren Semantik bereits das Element 'Bezug zu ...' enthält, können eben diesen Bezug nicht durch ein RA [= Relationsadjektiv] explizieren“. Ernst kommt zu dem Schluß, daß angesichts der „Vielfalt denkbarer semantischer Beziehungen“ eine „systematische und exhaustive Beschreibung (...) kaum möglich [scheint], allenfalls - wie oben versucht - eine Ausgrenzung dessen, was nicht möglich ist“" (Ernst 1986: 336, vgl. Bosque / Picallo 1996 für eine semantische und syntaktische Analyse der Relationsadjektive in der GB-Theorie).

In Anbetracht der Differenziertheit der Ergebnisse erscheinen eine semantische Komponentenanalyse oder ähnliche Verfahren wenig sinnvoll. Das bereits in einem anderen Kontext in Erwägung gezogene Merkmal [HUMAN \pm ] erbringt keine neuen Erkenntnisse (vgl. Pelzing 1981: 115ff.).

\subsection{Adjektive in der HPSG}

Für eine weiterführende Beschreibung spanischer Adjektive in der HPSG stellen sich zwei Fragen:

1. Wie kann die Stellungsproblematik erfaßt werden?

2. Wie kann der Bedeutungsunterschied zwischen Voran- und Nachstellung erfaßt werden? 
Für die erste Frage können wir das Attribut NACHSTELLUNG (NACH) aus der Analyse der Determinierer übernehmen. Wir bekommen folgenden Zusatz für das Kopfattribut eines Adjektivs, hier exemplifiziert am Beispiel sulfúrico aus der Phrase ácido sulfúrico:

$$
\left[\begin{array}{l}
\text { PHON sulfúrico } \\
\text { SS } \mid \text { LOC } \mid \text { CAT } \mid \operatorname{HEAD}\left[\begin{array}{l}
\text { adj } \\
\mathrm{NACH}+
\end{array}\right]
\end{array}\right]
$$

Dabei muß der Wert + für die Nachstellung im Spanischen als der Normalfall gelten. Wir erstellen aber keinen default-Wert, da die Opposition markiert vs. unmarkiert für adjektivische Stellungsfragen wie bei Braselmann (1993) abgelehnt wird.

Die zweite Frage ist weitaus schwieriger zu beantworten. Wir hatten mit Dietrich (1993) festgestellt, daß Voranstellung oft mit nicht-restriktivem Einsatz des Adjektivs verbunden ist. Eine andere Bezeichnung in spanischen Grammatiken gebraucht den Begriff subjektiv. Damit ist zumeist ein Sachverhalt gemeint, der sich nach Einschätzung des Sprechers in einer bestimmten, nicht konventionell gegebenen Weise verhält. Diese Einschätzung wird von einem vermeintlich objektiven Status der Deskription explizit abgegrenzt.

In der HPSG ist es möglich, solche Phänomene über das Attribut CONTEXT abzubilden. Dafür bleiben wir zunächst bei dem genannten Beispiel áridos desiertos. Die Semantik dieser Phrase kann wie folgt abgebildet werden (nach Pollard / Sag 1994: 36):

$$
\left[\operatorname{CONT}[3]\left[\begin{array}{l}
\operatorname{INDEX}[1] \\
\left.\operatorname{RESTR}\left\{\left[\begin{array}{l}
\text { RELN desiertos } \\
\text { INST [1] }
\end{array}\right],\left[\begin{array}{l}
\text { RELN áridos } \\
\text { ARG [1] }
\end{array}\right]\right\}\right]
\end{array}\right]\right.
$$

Das besondere an der Relation zwischen dem Nomen desiertos und dem Adjektiv áridos ist die vom Sprecher gesetzte Stellung. Wir können diese Beziehung im Attribut CONTEXT als sprecherabhängig bezeichnen und eine entsprechende Relation als bewertend einführen. Das semantische Attribut der Phrase áridos desiertos, mit dem Index [3] in (213) kenntlich gemacht, wird dadurch zusätzlich von der Sprechsituation gekennzeichnet. In welcher Weise und aus welchen Gründen die Nominalphrase bewertet wird, bleibt unberücksichtigt (vgl. Pollard / Sag 1994: 27 und 332ff.). 
(214)

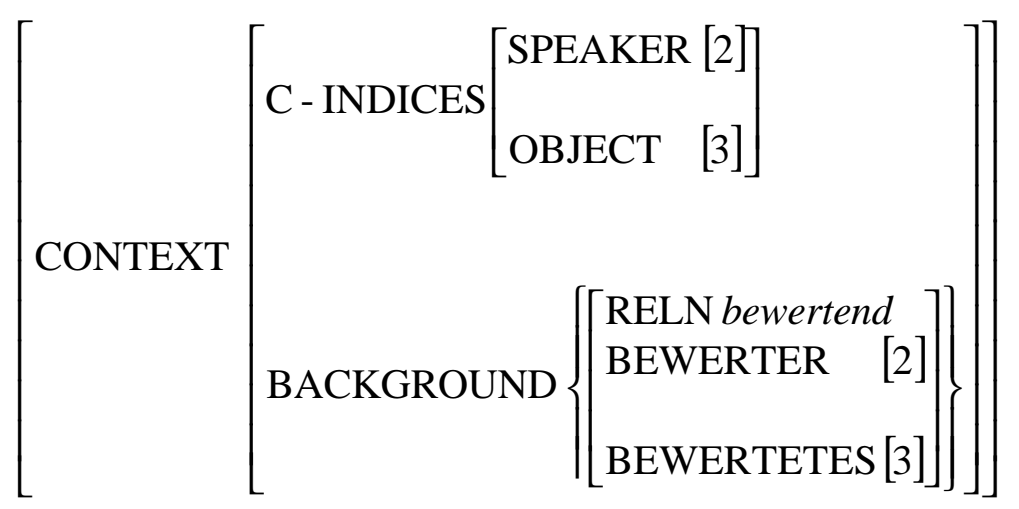

Der Sprecher [2] bewertet das Objekt [3], welches auf die semantische Deskription der Nominalphrase verweist. Dies wird durch das adjektivische Kopfmerkmal [NACH -] ausgelöst, das die Voranstellung beschreibt. Damit können wir eine mögliche nicht-restriktive Interpretation eines vorangestellen Adjektivs in der HPSG erfassen.

Ähnlich versuchen Engdahl / Vallduví (1996), pragmatische Information in die HPSG zu integrieren. Der Terminus information packaging beschreibt ein Phänomen, das auch mit der Dichotomie Thema / Rhema wiedergegeben werden kann, und bestimmt das „Informationszentrum“ eines Satzes. Engdahl / Vallduví (1996: 40) verwenden dazu Merkmale, die zwischen der eigentlichen Information (ground) und dem Schwerpunkt der Informationsdarstellung (focus) differenzieren. Im Englischen werden dafür zumeist prosodische Merkmale verwandt, im Katalanischen dagegen bildet die Wortstellung diese Information ab (Engdahl / Vallduví 1996: 49).

Dieser Ansatz ist nicht direkt auf das hier untersuchte Gebiet übertragbar, stellt aber einen sehr ähnlichen Ansatz dar. Die HPSG stellt somit ein reichhaltiges Inventar an Deskriptionsmethoden zur Verfügung, um auch pragmatische Information zu erfassen (vgl. auch Wilcock 1999 zur Konstruktion kontextueller Information aus dem Lexikon). 


\section{Präpositionen}

Die Untersuchung von spanischen Nominalphrasen soll ein Kapitel über Präpositionen am Beispiel von $a$ und $d e$ abschließen. Sie bilden im Rahmen der HPSG ein bisher relativ wenig beachtetes Phänomen (vgl. Pollard / Sag 1987: 65, 156; 1994 : 255f., 347, Verspoor 1996, Winhart 1997, Badia 1998). Als einzige im romanischen Bereich liegt mit Badia (1998) eine Analyse katalanischer Präpositionen vor, die auch kurz auf das Spanische eingeht und hier als Basis verwendet wird.

Präpositionalphrasen müssen im Spanischen als relativ komplexe Erscheinung angesehen werden. Sie können außer der Subjektfunktion alle Funktionen im Satz übernehmen und dabei sowohl Komplemente als auch Adjunkte sein. Dabei beziehen sich Präpositionen stets auf einen Kopf und subkategorisieren ihrerseits eine Nominalphrase (oder eine Infinitivphrase, einen Nebensatz usw.). Die semantische Ambiguität, die sie dabei an den Tag legen, stellt Winhart (1997: 378ff.) anhand der folgenden deutschen Beispielsätze dar:

(215) (a) Otto kommt in temp,lokal $_{\text {dieser Woche }}$ temp.

(b) Otto kommt diese Woche $\mathrm{temp}_{\text {. }}$

(c) Otto kommt in temp,lokal $_{\text {diese }}$ Stadt $_{\text {lokal }}$.

Diese Beispielsätze belegen die Abhängigkeit deutscher Präpositionen von der semantischen Information des subkategorisierten Nomens, wie wir sie auch für Adjektive beobachten konnten. Badia kommt in einem ähnlichen Kontext zu dem Schluß, daß es zwei Typen von Präpositionen gibt: semantisch leere und semantisch vollwertige. Im folgenden ersten Beispiel kann die Präposition de als semantisch leer bezeichnet werden, da sie vollständig vom Verb depender abhängt und keinen eigenen Beitrag zur Semantik des Satzes beisteuert. Im darauf folgenden Beispielsatz (b) ist dies nicht der Fall.

(216) (a) El porvenir de Ocalan, en caso de una presumible condena a muerte, depende de la generosidad del Parlamento. (M-6)

(b) La carretera que viene de San Fernando. (P-3) 
Im Beispielsatz (216a) kann die Präposition nicht variiert werden:

(217) (a) El porvenir de Ocalan (...) depende de la generosidad del Parlamento.

(b) El porvenir de Ocalan (...) depende *en / *por / *a / *con la generosidad del Parlamento.

Für semantisch vollwertige Präpositionen zeigen Berschin / Fernández-Sevilla / Felixberger (1987: 252) die möglichen Alternativen bei Bewegungsbezeichnungen, z.B. zur Präposition de in (216b) (vgl. auch die katalanischen Beispiele bei Badia 1998: 110):

$$
\text { Vengo }\left\{\begin{array}{l}
\text { a } \\
\text { hasta } \\
\text { para } \\
\text { contra } \\
\text { por } \\
\text { hacia } \\
\text { de } \\
\text { desde }
\end{array}\right.
$$

Folgende Beispiele zeigen, daß wir es im Kontext von Nominalphrasen mit der semantisch vollwertigen Variante der Präposition de zu tun haben. Die Präposition ist bedeutungstragend:

(219) (a) 77 días de bombardeo y aislamiento internacional la han dejado a solas con su enloquecido presidente. (A-5)

(b) Ramiro Villapadierna (...) y la Alianza Atlántica firmaron ayer el acuerdo militar para la retirada yugoslava de Kosovo. (A-4)

(c) Así archiva una inversión de 1.200 millones de pesetas y deja al edificio en un estado de ruina y sin perspectivas de solución. (S-12)

Die Präposition de agiert im gegebenen Kontext [N [pP de NP]] und kann im Deutschen mit einer Genitivphrase oder der Präposition von wiedergegeben werden. Die Präposition baut eine Relation zwischen der subkategorisierten Nominalphrase und dem nominalen Bezugspunkt auf: Allerdings zeigt de Bruyne (1993: 305ff.), daß sich bei diesen syntaktischen Gegebenheiten die semantische Klassifikation von de als äußert komplex erweist. Die Präpositionalphrase kann u.a. folgende Bedeutungen anzeigen: 
(220) (a) ein Besitzverhältnis: la casa de mi padre,

(b) den Stoff, aus dem etwas besteht: la estatua de mármol,

(c) eine Eigenschaft (de bedeutet 'mit'): un hombre de dinero,

(d) die Art und Weise eines Vorgangs: almuerza de pie,

(e) temporale Eigenschaften: Viajaremos de noche y descansaremos de día,

(f) emphatische Eigenschaften: las pobrecitas de las mujeres.

In (220) handelt es sich lediglich um eine Auswahl aus der Liste bei de Bruyne. Je nach Präposition variieren zudem Anzahl und Beschaffenheit der Kategorien.

Wir können also von zwei Typen der Präposition de ausgehen: dem semantisch vollwertigen Typ, der im Kontext von Nominalphrasen auftritt, und dem semantisch leeren Typ, der in verbalen Kontexten auftreten kann, wobei beide Varianten hinsichtlich ihres Auftretens empirisch genauer belegt werden müßten. Beide verhalten sich syntaktisch unterschiedlich: die semantisch leere Variante ist vom Kontext, in der Regel von einem Verb, in der Weise abhängig, daß auch die Präposition ausgetauscht werden muß, wenn das Verb ersetzt wird. Mitunter handelt es sich um traditionell als Verbalperiphrasen bezeichnete Konstruktionen. Bei Maßkonstruktionen bekommen wir eine Zwischenkategorie: Die Präposition ist nicht von einem Verb abhängig. Allerdings ist in einer Phrase wie tres kilos de oro die Präposition de weder austauschbar, noch ist ihr eine eigenständige semantische Bedeutung zuzuschreiben. De erstellt die Relation zwischen tres kilos und oro. Ob dies bereits einen eigenen semantischen Beitrag darstellt, müßte in einem geeigneten Rahmen untersucht werden.

In der HPSG kann der semantische Unterschied sehr einfach dargestellt werden: Semantisch leere Präpositionen erhalten ein entsprechend leeres semantisches Attribut, semantisch vollwertige Präpositionen können im CONTENT-Attribut allgemein mit dem jeweils entsprechenden Eintrag kenntlich gemacht werden. Da de wie viele andere Präpositionen in beiden Formen auftreten kann, müssen im Lexikon beide Möglichkeiten als Disjunktion vermerkt werden.

Badia (1998: 120ff.) stellt die Frage, ob semantisch leere Präpositionen analog zu Hilfs- und Modalverben trotzdem als syntaktische Köpfe interpretiert werden sollten. Badia zeigt daß in Konstruktionen mit Verben wie deber, haber, estar der semantische Beitrag dieser Verben gering ist, während sie syntaktisch eindeutig als Köpfe operieren (z.B. als Träger der Kongruenzmerkmale), und plädiert dafür, semantische und syntaktische Information im Fall von Präpositionen parallel zu setzen und se- 
mantisch leere Präpositionen folglich nicht als syntaktische Köpfe zu sehen. Dann wäre das Nomen innerhalb der Präpositionalphrase der Kopf der Phrase. Dafür sprechen auch folgende parallele Konstruktionen:

(221) (a) Apenas vio llegar a Juan. (S-7)

(b) *Apenas vio llegar Juan.

(c) *Apenas vio llegar al tigre.

(d) Apenas vio llegar el tigre.

Die Nominalphrase in (221) stellt das Akkusativkomplement des Vollverbs ver dar. Wenn die NP das Merkmal [HUMAN +] trägt, wird sie in eine Präpositionalphrase mit $a$ umgeformt. Dieser Prozeß kann als eine syntaktische Modifikation aufgrund semantischer Information betrachtet werden. Nach Badia ist an dieser Stelle eindeutig das Nomen der semantische Kopf der Phrase, und dies spricht folglich dafür, das Nomen auch als syntaktischen Kopf zu sehen. Meisterfeld unterstützt diese Analyse insofern, als er die Dichotomie attributive vs. referenzielle Lesart aufstellt. Die Präposition $a$ steht dabei nur bei identifiziertem Persönlichen (Meisterfeld 1998: 87). In Busco a un médico „hat der Sprecher eine Vorstellung von der Identität des Arztes, setzt aber eine entsprechende Kenntnis bei dem Hörer nicht voraus“ (Meisterfeld 1998: 89), in busco un médico ist dies nicht der Fall. Die Opposition beweist, daß in beiden Fällen das Nomen den Kopf der Phrase darstellt.

Für semantisch leere Präpositionen kann nun angenommen werden, daß sie syntaktisch von einem Verb wie z.B. depender in (216a) oder einem Nomen wie z.B. dem Eigenname Juan in (221a) subkategorisiert werden. Für Nomen muß dann eine lexikalische Regel eingeführt werden, welche besagt, daß ein Nomen mit der Merkmalkombination aus dem syntaktischen Merkmal [KASUS AKK], dem semantischen [HUMAN +] und dem kontextuellen Merkmal [IDENTITÄT +], welches vom Sprecher mit einer bestimmten Identität charakterisierte Personen oder personifizierte Dinge markiert, die Präposition $a$ subkategorisiert. Dies bleibt ein rein syntaktischer Vorgang: 

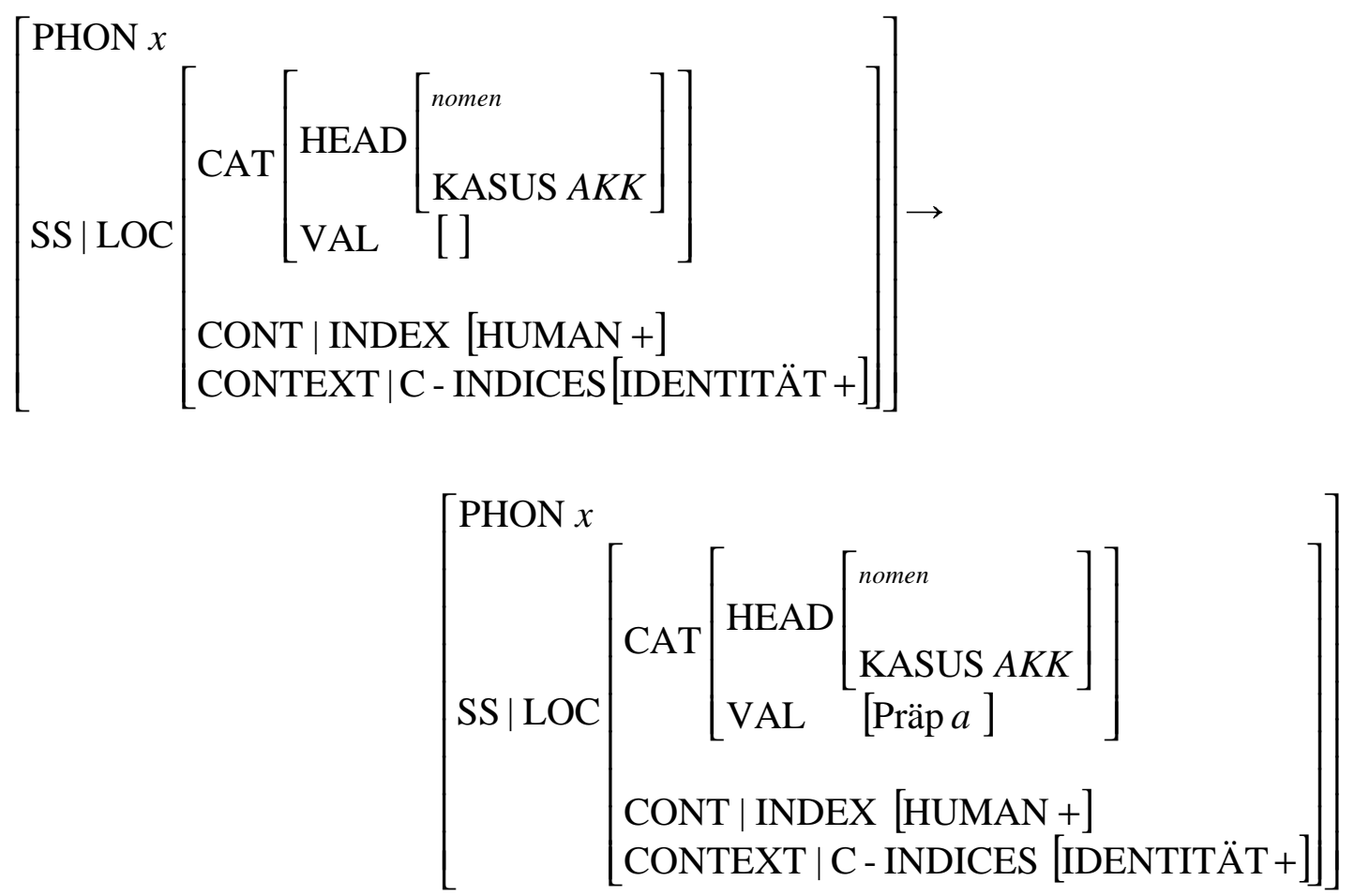

Der lexikalische Eintrag der semantisch leeren Präposition besteht folglich nur noch aus dem Eintrag [HEAD präp]. Für fast alle Präpositionen des Spanischen muß analog zum von Badia untersuchten Katalanischen ein disjunkter lexikalischer Eintrag vorgesehen werden. Einige Präpositionen wie entre, según, sin u.a. können nur semantisch vollwertig verwendet werden (vgl. Badia 1998: 113).

Damit können wir auch Präpositionalphrasen innerhalb nominaler Strukturen erfassen. Für eine weitergehende Deskription mit Bezug auf relativischen Anschluß sei auf Badia (1998: 144ff.) verwiesen. 


\section{Zusammenfassung}

Traditionelle spanische Grammatiken (z.B. Seco 1991, RAE 1985) verstehen das Nomen als Zentrum der Nominalphrase. Weitere nominale Wortarten werden als Adjektive klassifiziert, welche in determinierende und qualifizierende Adjektive unterteilt werden. Als gesonderte Wortart wird der definite Artikel gesehen und nicht zu den Adjektiven gezählt. Ziel dieser Arbeit war es, diese Klassifikation zu überprüfen, evtl. zu modifizieren und sie in eine kopfgesteuerte Phrasenstrukturgrammatik (HPSG) zu integrieren. Dafür mußten zunächst die sprachlichen Gegebenheiten ermittelt werden.

Nach unseren Ergebnissen werden Nomina in folgende Klassen unterteilt: zählbare Gattungsnomina (auch als numerale Gattungsnomina bezeichnet) und nicht zählbare Gattungsnomina (Massennomina oder transnumerale Nomina), Eigennamen und Pronomina. Dabei müssen die zählbaren Gattungsnomina in singuläre und plurale unterschieden werden, da Nomina im Plural eigenständig eine Nominalphrase bilden können. Da letzteres auch für Eigennamen und Pronomina gilt, subkategorisieren nur die singulären zählbaren Nomina einen Determinierer. Bei den pluralen Nomina ist der Determinierer fakultativ.

Mit Wiese (1997) konnten wir feststellen, daß es im Spanischen analog zu anderen Sprachen die Möglichkeit gibt, daß auch singuläre zählbare Nomina eigenständig eine Nominalphrase bilden können. Dazu gibt es bestimmte Vorbedingungen: Das Nomen referiert auf ein einzelnes Objekt und bezeichnet dabei die gesamte Klasse aller entsprechenden Objekte. Wenn diese beiden Bedingungen gegeben sind, kann das singuläre zählbare Nomen eigenständig eine NP bilden, wie z.B. in Para ti hay cama. Der Prozeß der Überführung eines singulären zählbaren Nomens in den $\mathrm{Zu}$ stand, daß es nicht mehr einen Determinierer subkategorisiert und allein stehen darf, nannten wir Generalisierung. Durch die in der HPSG gebräuchlichen lexikalischen Regeln ist es möglich, die Sorte $s g$-count für singuläre zählbare Nomina in pl-mass zu modifizieren, wobei sich die morphologische Komponente des Nomens nicht ändert. Es verbleibt im Singular und geht als Subjekt eine entsprechende Kongruenz mit dem Verb ein.

Dieser Prozeß ist rein lexikalischer Natur und benötigt von daher die besonders ausgereiften lexikalischen Mechanismen des deklarativen Grammatikmodells der 
HPSG, in der lexikalische Informationen über Prinzipien und Schemata zusammengebunden werden, welche den strukturellen Aufbau der Nominalphrase beschreiben. Da es sich bei der Generalisierung auch um einen semantischen Prozeß handelt, kommt der Deskription die Berücksichtigung semantischer Information neben der Syntax in der HPSG besonders zugute. Ohne diese enge Verknüpfung von Syntax und Semantik ist im Spanischen eine zufriedenstellende Deskription zumindest erschwert, da einige Phänomene, wie die singulären zählbaren Nomina, die Abgrenzung Determinierer - Adjektiv, Infinitivkonstruktionen (u.a. Kiss 1995a) oder auch die Fragestellung, unter welchen Bedingungen ein einzelnes Wort als Subjekt vor dem Verb stehen kann (u.a. Casielles 1996), rein syntaktisch nicht zu erklären sind. Der Ansatz von Engdahl / Vallduví (1996) zeigt, daß auch der Bereich der Pragmatik integrierbar ist.

Für die traditionell als determinierende Adjektive bezeichneten Determinierer, erweitert um den definiten Artikel, zeigte eine Analyse der Klassifikation von Alvarez Martínez (1986), daß eine relativ große Zahl an Faktoren die Deskription erschwert. Es lassen sich keine abgrenzbaren Klassen erzeugen, die eine zufriedenstellende Analyse ermöglichen. Von daher wurden die Kriterien von Alvarez Martínez überprüft, modifiziert und ergänzt. Zwei Bereiche benötigten besondere Aufmerksamkeit: Kookkurrenz und semantische Aspekte. Da Determinierer in einer Nominalphrase auch mehrfach auftreten können und dies mit syntaktischen Eigenschaften korreliert, wurde nach Prado Ibán (1993) eine Klassifikation erstellt, die sich aber als ebenso problematisch erwies wie die Gesamtklassifikation von Alvarez Martínez. In beiden Fällen stellt u.E. eine abstufende Klassifikation - was den Verzicht auf abgrenzbare Klassen beinhaltet - den besseren Weg zu einer zufriedenstellenden Deskription dar. Diese Abstufung ist z.B. in der Dialektrometrie (Goebl, erscheint) oder in der Sprachtypologie (Altmann / Lehfeldt 1973) gebräuchlich. Graphisches Ergebnis ist ein Dendrogramm, das übersichtlich die Distanzen der einzelnen Determinierer zueinander zeigt.

Neben morphologischen (Genus und Numerus) und syntaktischen Merkmalen (Adverbialisierung, Komparation, Substantivierung) wurden in die Klassifikation die Thematiken Wortstellung (Voran- vs. Nachstellung) und Semantik integriert. Für die Semantik ergab sich kein explizites Merkmal, da die Ansätze von Alcina Franch / Blecua (1975) oder Lamíquiz (1987) sich als zu ungenau erwiesen. Der Bereich Kookkurrenz konnte aber über den semantischen Ansatz verfeinert und durch die 
Einführung des Merkmals Kombinationssteuerer neben Erst- und Zweitstellung gelöst werden.

Bei der Wortstellung mußte berücksichtigt werden, daß z.B. auch die Demonstrativpronomina als nicht adjektivische Determinierer nachgestellt werden können und dann eine leichte Bedeutungsmodifizierung aufweisen. Dies wurde über die Merkmale Voranstellung und Nachstellung erfaßt. Voranstellung als Standardwert wurde integriert, um das grundsätzlich nachgestellte Possessiv mío zu erfassen.

Damit erhalten wir 17 Merkmale zur Differenzierung spanischer Determinierer.

Da jeder Determinierer folglich über die 17 Merkmale erfaßt wird, können die Determinierer jeweils miteinander in Beziehung gesetzt werden. Diese Relation wird über die euklidische Distanz berechnet. Die Clustererstellung erfolgt hauptsächlich durch das Mittelwertverfahren. Als Ergebnis erhalten wir mehrere leicht divergierende Klassifikationen, die einige Determinierer als Fixpunkte und andere als deren Satelliten bestimmen. Während die als Fixpunkte bezeichneten Determinierer sich grundsätzlich bei jeder Berechnungsmethode an der gleichen Stelle der Klassifikation befinden, ergeben sich bei den Satelliten zwei Klassen: diejenigen, die sich immer demselben Fixpunkt anschließen, und diejenigen, die ihren Fixpunkt variieren. Als Endergebnis können wir folgendes festhalten: Die Wörter alguno, ninguno, cualquiera, mi, cada, el, este, uno, todo, ambos, sendos, varios und cierto sind als Determinierer zu bezeichnen. Der Artikel el kann dabei nach unseren Ergebnissen problemlos integriert werden. Doble, segundo, mismo, demás und mero verhalten sich syntaktisch wie Adjektive, auch wenn sie semantisch als Determinierer einzustufen sind. Als eine Zwischenklasse, die eher den Adjektiven nahe steht, erhalten wir dos, mío und más. Eine wiederum mehr determinierende Eigenschaften aufweisende Zwischenklasse bilden demasiado, bastante, mucho, poco, tanto und tal. Otro erwies sich als bester Repräsentant des hybriden Charakters spanischer Determinierer zwischen Adjektiv und Artikel. Eine Klassifizierung der Determinierer in abgrenzbare Klassen ist folglich nur dann halbwegs möglich, wenn die Zwischenklassen ausgeblendet werden.

Das Klassifikationsverfahren für spanische Determinierer wurde anschließend zu Vergleichszwecken auf das deutsche Determinierersystem übertragen, wobei die für das Spanische erarbeiteten Kriterien nur geringfügig modifiziert wurden. Dabei stellte sich das deutsche System als das homogenere heraus. Die Hälfte aller berücksichtigten deutschen Determinierer stimmen in ihren Eigenschaften überein. Eine 
Übergangsklasse von Determinierern zu Adjektiven ist zwar konstruierbar, aber wesentlich kleiner als im Spanischen.

Die Ergebnisse bei der Beschreibung von Nomina und Determinierern wurden in Kap. III.3. in die HPSG integriert. Für den Bereich der Valenz zeigte sich, daß Determinierer nach Zwicky (1993) als eine Kategorie zwischen Komplementation und Adjungierung interpretiert werden können. Wenn singuläre zählbare Gattungsnomina einen Determinierer subkategorisieren und dieser wiederum über das Merkmal ARGSLOT auf das Nomen referiert, kann das zwar als Zyklus gedeutet werden, entspricht aber zum einen den sprachlichen Gegebenheiten, zum anderen werden jeweils andere Merkmale der Phrase zugeordnet, so daß von gegenseitiger Ergänzung die Rede sein muß. Aufgrund der Ergebnisse bei den Determinierern wird das Nomen als Kopf der spanischen Nominalphrase interpretiert.

Auch der Bereich der Kongruenz wurde modifiziert. Statt der primär semantischen Darstellung von Pollard / Sag (1994) wurde eine Darstellung nach Kathol (1999) eingeführt, die primär syntaktisch operiert, semantische Kongruenz aber berücksichtigt.

Den größten Raum der Deskription spanischer Determinierer nimmt die lexikalische Komponente ein. Die über die Merkmale erstellte Klassifikation wurde in eine Sortenhierarchie integriert, so daß einem Determinierer aufgrund seiner Sorte bereits bestimmte Eigenschaften zugesprochen werden. Wir differenzieren die Sorten det_Adj für determinierende Adjektive, welche Wörter beschreibt, die semantisch als Determinierer, syntaktisch aber als Adjektive eingestuft werden, $a d j \_D e t$ für adjektivische Determinierer, welche Wörter beschreibt, die adjektivische Eigenschaften wie z.B. Nachstellung aufweisen, und Det für Determinierer, die dem idealtypischen Vertreter eines Determinierers nahe stehen. Dabei müssen wir annehmen, daß ein Determinierer zugleich als adj_Det und Det im Lexikon markiert sein kann, was mit der Sorte d-element festgehalten wird. Adj steht für qualifizierende Adjektive.

Für Nomina können wir zwischen der Klasse der singulären zählbaren Gattungsnomina (sg-count) als numeralen Nomina $\left(\mathrm{N}_{\mathrm{n}}\right)$ und der Klassen der pluralen zählbaren Gattungsnomina (pl-mass), der Eigennamen (en) und der Pronomina (pron) als transnumeralen Nomina $\left(\mathrm{N}_{\mathrm{tn}}\right)$ differenzieren. Die nominale Sortenhierarchie des Spanischen ergibt sich damit wie folgt: 


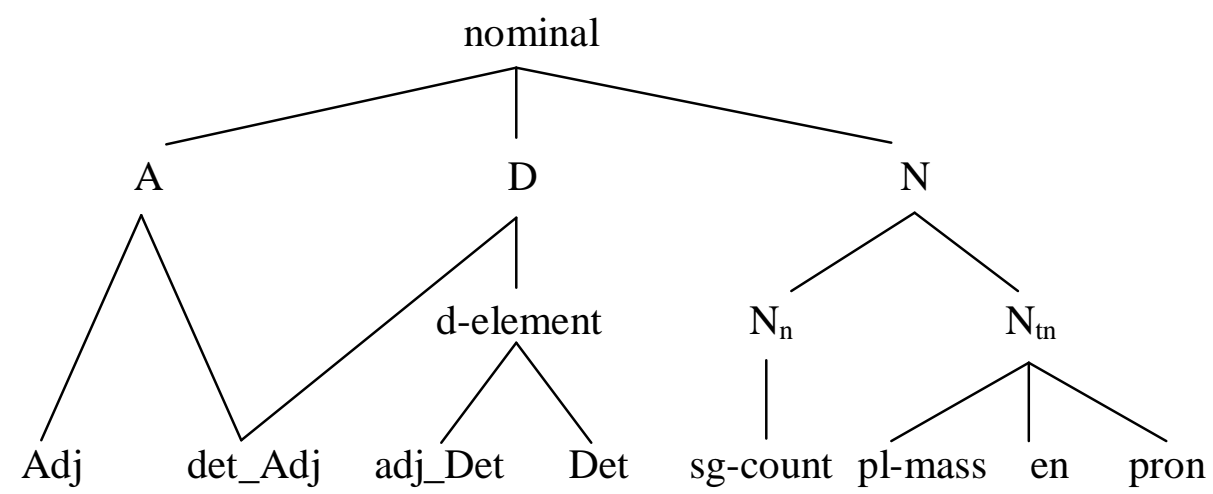

Um einen Lexikoneintrag zu erstellen, ist zudem die Wortstellung relevant. Qualifizierende Adjektive des Spanischen können in der Regel auch vor dem Nomen auftreten. Die Nachstellung ist der Regelfall. Determinierer als determinierende Adjektive können folglich ebenfalls ihre Stellung variieren und nachgestellt werden. Hier stellt aber Voranstellung den Regelfall dar. Für Nachstellung wurde ein Merkmal NACH im Attribut HEAD des Determinierers eingeführt, das entweder bereits festgelegt ist, weil der Determinierer nicht nachgestellt bzw. im Fall von mío nicht vorangestellt werden kann, oder im Lexikon noch nicht festgelegt ist, so daß beide Möglichkeiten in Betracht kommen. Kookkurrenz wird dabei über die Kombinationssteuerer erfaßt. Diese bilden in unserer Klassifikation zunächst eine Gruppe von elf Determinierern, die in einer Verbindung auftreten müssen. Die Kombination zweier Determinierer ist nicht möglich, wenn keiner der beiden als Kombinationssteuerer deklariert ist. Der Kombinationssteuerer steht entweder in Erststellung und bekommt im Merkmal KOMB_1 alle Determinierer zugeteilt, die folgen dürfen, oder er steht in Zweitstellung und bekommt entsprechend die voranstehenden Determinierer im Merkmal KOMB_2 zugeteilt. Die Registrierung dieser Information im Lexikon hat den Vorteil einer größeren Flexibilität. Gerade für die Kookkurrenz konnte festgestellt werden, daß sie je nach Herkunft des Befragten unterschiedlich bewertet wurde.

Damit kann für jeden Determinierer ein entsprechender Eintrag im Lexikon erfolgen. Lexikalische Regeln ermöglichen nun zum einen die Substantivierung von Determinierern nach ihrem Wert beim Merkmal determinierertypische bzw. adjektivtypische Substantivierung, zum anderen die Wiederholung der Ebene 1 (N') bei Nachstellung. Im letzten Fall hebt der Determinierer die Phrase nicht auf die Ebene einer 
vollständigen Nominalphrase an, sondern verhält sich als Adjunkt, auch wenn er mit der Sorte Det vermerkt wurde.

Für Nomina gelten ähnliche lexikalische Regeln: Bei Generalisierung überführt die lexikalische Regel ein singuläres zählbares Nomen der Sorte sg-count in die Sorte pl-mass. Eigennamen und Pronomina können analog in singuläre Gattungsnomina überführt werden, wobei die Sorte und das Valenzattribut modifiziert werden.

Quantifikation innerhalb der Nominalphrase wurde nach Allegranza (1998) in das HPSG-Fragment eingebunden. Sein Ansatz muß hinsichtlich der Deskription von Numeralia als modifizierende Adjunkte kritisiert werden.

Die sprachlichen Gegebenheiten des Spanischen lassen es angebracht erscheinen, neben Determinierern auch qualifizierende Adjektive zu berücksichtigen. Deren Syntax als Adjunkte stellt in der HPSG kein Problem dar. Demgegenüber ist die Stellung problematisch. Zwar treten viele Adjektive eher technisch-wissenschaftlicher Natur nur in Nachstellung auf, doch kann eine Vielzahl auch vor dem Nomen stehen. Für diese Gruppe konnten folgende Restriktionen erarbeitet werden: Das Adjektiv übt eine integrale oder partitive Funktion aus, die in Abstimmung mit dem Nomen ermittelt wird. Dabei können Termini, die in traditionellen Deskriptionen gebräuchlich sind, wie subjektiver vs. objektiver Gebrauch, verwendet werden. Es muß offen gelassen werden, inwieweit eine Differenzierung der Bedeutung tatsächlich vorliegt. Viele Adjektive modifizieren ihre Bedeutung bei Stellungsänderung nur geringfügig und vor allem grundsätzlich in Abhängigkeit vom Nomen.

In der HPSG erhalten Adjektive analog zu Determinierern ein Merkmal NACH. Ist dieses negativ spezifiziert, kann eine pragmatische Relation bewertend angenommen werden, die das Adjektiv in eine Abhängigkeit von der Sprecherintention stellt.

Zuletzt wurden auch Präpositionalphrasen behandelt, welche als phrasale Adjunkte an die Nominalphrase herantreten. Nach Badia (1998) kann zwischen semantisch vollwertigen und semantisch leeren Präpositionen differenziert werden, wobei nur erstere semantische Merkmale erhalten. Dabei zeigt sich, daß der Bedeutungsumfang von Präpositionalphrasen sehr weit ist. Semantisch leere Präpositionen scheinen zudem nicht den Kopf der Phrase zu stellen.

Damit können wir abschließend folgende Ergebnisse festhalten:

1. Das Nomen bildet im Spanischen den Kopf der Nominalphrase. Alle anderen Wortarten stehen in der Regel in einem Abhängigkeitsverhältnis. 
2. Die Wortarten Adjektiv und Determinierer lassen sich im Spanischen nicht eindeutig voneinander trennen. Es ist zwar möglich, durch eine Sortenhierarchie alle Abstufungen zu differenzieren, Mehrfacheinträge sind aber zur adäquaten Deskription notwendig.

3. Der spanische Artikel el / la ist syntaktisch und semantisch als Determinierer zu werten. Eine Sonderstellung kann nur morphologisch oder etymologisch begründet werden.

4. Nomina des Spanischen können fast immer auch eigenständig eine Nominalphrase bilden. Spanische Determinierer müssen als Begleiter zwischen Komplementation und Adjungierung eingeordnet werden, während Adjektive eindeutig Adjunkte sind.

5. Nach Zwicky (1993) können Determinierer in der HPSG als Spezifizierer zwischen Komplementen und Adjunkten interpretiert werden. Dafür wurden die HPSG-Schemata durch die Einführung des Funktorschemas nach Allegranza (1998) modifiziert. Wir können den Aufbau der Nominalphrase durch das X-barSchema zufriedenstellend erfassen.

6. Das Grammatikmodell der HPSG demonstrierte besonders bei der Integration von syntaktisch-semantischer Information seine Stärken. Trotz der nicht unproblematischen Nähe der Determinierer zu den qualifizierenden Adjektiven zeigte sich, daß das spanische Determinierersystem zwar komplex, aber trotzdem in einem konkreten Grammatikmodell handhabbar ist. Dabei kommen HPSG-typische Beschreibungsstrategien wie die Sortenhierarchie und die Einführung des Funktorschemas der Untersuchung zugute. Auch der Bereich Kongruenz konnte durch leichte Änderungen zufriedenstellend gelöst werden. 


\section{Bibliographie}

Abb, Bernd (1994): Die Generierung deutscher Nominalstrukturen - eine funktionale Analyse in der HPSG-Basisgrammatik eines kognitiv orientierten Sprachproduktionssystems. Hamburger Arbeitspapiere zur Sprachproduktion V, Universität Hamburg.

Abb, Bernd / Maienborn, Claudia (1994): Adjuncts in HPSG. In: Trost, Harald (Hrsg.): KONVENS '94. Verarbeitung natürlicher Sprache. Berlin: Springer, $13-22$.

Abeillé, Anne (1993): Les nouvelles syntaxes. Grammaires d'unification et analyse du français. Paris: Colin.

Abney, Steven (1987): The English Noun Phrase in its Sentential Aspect. Diss., Cambridge, Mass.: Microproduction Laboratory.

Aissen, Judith / Perlmutter, David M. (1983): Clause Reduction in Spanish. In: Perlmutter, David M. (Hrsg.): Studies in Relational Grammar. Chicago: Chicago University Press, 360-403.

Alarcos Llorach, Emilio (1994): Gramática de la lengua española. Madrid: Espasa Calpe.

Alcaraz Varó, Enrique / Martínez Linares, María Antonia (1997): Diccionario de Lingüística moderna. Barcelona: Ariel.

Alcina Franch, Juan (1992): Spanisch: Syntax. Español: Sintaxis. In: Holtus / Metzeltin / Schmitt, 160-178.

Alcina Franch, Juan / Blecua, José Manuel (1975): Gramática Española. Barcelona: Ariel.

Allegranza, Valerio (1998): Determiners as Functors: NP Structure in Italian. In: Balari / Dini, 55-107.

Allwood, Jens / Andersson, Lars-Gunnar / Dahl, Östen (1973): Logik für Linguisten. Tübingen: Niemeyer.

Altmann, Gabriel / Lehfeldt, Werner (1973): Allgemeine Sprachtypologie. Prinzipien und Meßverfahren. München: Fink.

Alvar, Manuel / Pottier, Bernard (1983): Morfología histórica del español. Madrid: Gredos. 
Alvarez Martínez, Maria Angeles (1986): El artículo como entidad funcional en el español de hoy. Madrid: Gredos.

Asenjo Orive, María R. (1990): Los demostrativos. Salamanca: Colegio de España.

Bach, Emmon (1988): Categorial Grammar as Theories of Language. In: Oehrle, Richard T. / Bach, Emmon (Hrsg.): Categorial Grammars and Natural Language Structures. Dordrecht: Reidel, 7-34.

Bacher, Johann (1996): Clusteranalyse. Anwendungsorientierte Einführung. München / Wien: Oldenbourg.

Badia, Toni (1994): Aspectes del sintagma nominal en català des de la perspectiva de la traducció automàtica. Barcelona: Publicacions de l'Abadia de Montserrat.

Badia, Toni (1998): Prepositions in Catalan. In: Balari / Dini, 109-149.

Balari, Sergio / Dini, Luca (Hrsg.) (1998): Romance in HPSG. Stanford: CSLI.

Barwise, John / Perry, Jon (1987): Situationen und Einstellungen. Grundlagen der Situationssemantik. Berlin / New York: de Gruyter.

Beckmann, Hans-Georg (1994): Neue spanische Grammatik. Göppingen: Das neue Fachbuch.

Berman, Judith / Frank, Anette (1996): Deutsche und französische Syntax im Formalismus der LFG. Niemeyer: Tübingen.

Berschin, Helmut / Fernández-Sevilla, Julio / Felixberger, Josef (1987): Die spanische Sprache. Verbreitung. Geschichte. Struktur. München: Hueber.

Bierwisch, Manfred (1988): On the Grammar of Local Prepositions. In: Bierwisch, Manfred / Motsch, Wolfgang / Zimmermann, Inge (Hrsg.): Syntax, Semantik und Lexikon. Rudolf Ruzicka zum 65. Geburtstag. Berlin: Akademie-Verlag, 1- 64.

Bird, Steven / Klein, Ewan (1993): Enriching HPSG Phonology. Centre for Cognitive Science, University of Edinburgh.

Börjars, Kersti E. (1994): Feature Distribution in Swedish Noun Phrases. Diss., University of Manchester.

Borsley, Robert D. (1987): Subjects and complements in HPSG. Stanford: CSLI.

Borsley, Robert D. (1993): Heads in Head-driven Phrase Structure Grammar. In: Corbett / Fraser / McGlashan, 186-203.

Borsley, Robert D. (1996): Modern Phrase Structure Grammar. Cambridge, Mass. I Oxford: Blackwell. 
Borsley, Robert D. (1997): Syntax-Theorie. Ein zusammengefaßter Zugang. Tübingen: Niemeyer.

Borsley, Robert D. (Hrsg.) (2000): The Nature and Function of Syntactic Categories. San Diego u.a.: Academic Press.

Bosque, Ignacio (1993): Las categorías gramaticales, relaciones y diferencias. Madrid: Síntesis.

Bosque, Ignacio (Hrsg.) (1996): El sustantivo sin determinación. La ausencia de determinante en la lengua española. Madrid: Visor Libros.

Bosque, Ignacio / Picallo, Carme (1996): Postnominal adjectives in Spanish DPs. Journal of Linguistics 32, 349-385.

Bouma, Gosse (1988): Modifiers and Specifiers in Categorial Unification Grammar. Linguistics 26, 21-46.

Bouma, Gosse (1997): Valence Alternation without Lexical Rules. In: Landsbergen, Jan, u.a. (Hrsg.): Computational Linguistics in the Netherlands 1996. Papers from the Seventh CLIN Meeting. Eindhoven: Eric, 25-40.

Bouma, Gosse / Kruijff, Geert-Jan M. / Oehrle, Richard T. (Hrsg.) (1998): Proceedings of the Joint Conference on Formal Grammar, Head-Driven Phrase Structure Grammar and Categorial Grammar. Saarbrücken.

Braselmann, Petra M. E. (1993): Zur Stellung des attributiven Adjektivs im gegenwärtigen Spanisch. Archiv für das Studium der neueren Sprachen und Literaturen $230,335-351$.

Bresnan, Joan (1982): Control and Complementation. In: Bresnan, Joan (Hrsg.): The Mental Representation of Grammatical Relations. Cambrigde, Mass.: MIT, 282-390.

Briscoe, Ted / Copestake, Ann / Paiva, Valeria de (Hrsg.) (1993): Inheritance, Defaults and the Lexicon. Cambridge: Cambridge University Press.

Briz Gómez, Antonio (1989): Sustantivación y lexicalización en español (la incidencia del artículo). Valencia: Servei de Publicacions Universitat de València.

Bruyne, Jacques de (1993): Spanische Grammatik. Tübingen: Niemeyer.

Bußmann, Hadumod (1990): Lexikon der Sprachwissenschaft. Stuttgart: Kröner.

Bull, William E. (1954): Spanish Adjective Position: The Theory of Valence Classes. Hispania 37, 32-38.

Cann, Ronnie (1993): Patterns of headedness. In: Corbett / Fraser / McGlashan, 44-72. 
Carpenter, Bob (1991): The Generative Power of Categorial Grammars and HPSGs with Lexical Rules. Computational Linguistics 17 / 3, 301-314.

Carpenter, Bob (1992): The Logic of Typed Feature Structures with Applications to Unification-based Grammars, Logic Programming and Constraint Resolution. New York: Cambridge University Press.

Casielles, Eugenia (1996): On the Misbehavior of bare Nouns in Spanish. In: Parodi, Claudia, u.a. (Hrsg.): Aspects of Romance Linguistics. Selected Papers from the Linguistic Symposium on Romance Language XXIV, March 10-13 1994. Washington D.C.: Georgetown University Press, 133-148.

Chomsky, Noam (1957): Syntactic Structures. The Hague: Mouton.

Chomsky, Noam (1965): Aspects of the theory of syntax. Cambrigde, Mass: MIT.

Chomsky, Noam (1970): Remarks on Nominalization. In: Jacobs, Roderick A. / Rosenbaum, Peter S. (Hrsg.): Readings in English transformational grammar. Waltenham, Mass.: Ginn, 184-221.

Chomsky, Noam (1981): Lectures of Government and Binding. Dordrecht: Foris.

Chomsky, Noam / Lasnik, Howard (1995): The Minimalist Program. Cambridge, Mass. / London: MIT.

Contreras, Helen (1996): Sobre la distribución de los sintagmas nominales no predicativos sin determinante. In: Bosque, 141-168.

Cooper, Richard (1983): Quantification and syntactic theory. Dordrecht: Reidel.

Cooper, Richard P. (1990): Classification-based Phrase Structure Grammar: An Extended Revised Version of HPSG. Diss., University of Edinburgh.

Copestake, Ann (1993): Defaults in Lexical Representation. In: Briscoe / Copestake / Paiva, 223-245.

Copestake, Ann / Sanfilippo, Antonio / Briscoe, Ted / Paiva, Valeria de (1993): The ACQUILEX LKB. An Introduction. In: Briscoe / Copestake / Paiva, 148-163.

Corbett, Greville G. (1991): Gender. Cambridge: Cambridge University Press.

Corbett, Greville G. / Fraser, Norman M. / McGlashan, Scott (Hrsg.) (1993): Heads in grammatical theory. Cambridge: Cambridge University Press.

Crystal, David (1995): Die Cambridge Enzyklopädie der Sprache. Frankfurt am Main / New York: Campus.

Dietrich, Wolf (1993): Lateinische und romanische Adjektivstellung. In: Schmitt, Christian (Hrsg.): Grammatikographie der romanischen Sprachen. Akten der 
gleichnamigen Sektion des Bamberger Romanistentages (23.-29.9.1991). Bonn: Romanistischer Verlag, 78-96.

Egli, Urs / Egli-Gerber, Renate (1991): Sprachsysteme - logische und historische Grundlagen der erweiterten Phrasenstrukturgrammatik. Arbeitspapier Nr. 28 der Fachgruppe Sprachwissenschaft, Universität Konstanz.

Engdahl, Elisabet / Vallduví, Enric (1996): Information packaging and grammar architecture: A constraint-based approach. In: Engdahl, Elisabeth (Hrsg.): Integrating information structure into constraint-based and categorial approaches. Amsterdam: ILLC, 39-79.

Engel, Ulrich (1982): Syntax der deutschen Gegenwartssprache. Berlin: Schmidt.

Ernst, Gerhard (1986): Morphologie und Syntax der Relationsadjektive (RA) im Rumänischen: Spezifisches und Gemeinromanisches. In: Holtus, Günter / Radtke, Edgar (Hrsg.): Rumänistik in der Diskussion. Tübingen: Narr, 317338.

Fernández Rámirez, Salvador (1985): Gramática del español. 5 Bde. Madrid: Arco Libros.

Figge, Udo L. (erscheint): Computerlinguistik. Linguistique computationelle. In: Holtus / Metzeltin / Schmitt.

Flickinger, Daniel P. (1987): Lexical Rules in the Hierarchical Lexicon. Diss., Stanford University.

Frank, Anette (1991): Argumentstruktur, grammatische Relationen und lexikalische Regeln. Ein LFG-Fragment zu Partizipialkongruenz, Auxiliarselektion und Clitic-Climbing im Französischen. In: Rolshoven, Jürgen / Seelbach, Dieter (Hrsg.): Romanistische Computerlinguistik: Theorien und Implementationen. Tübingen: Niemeyer, 19-76.

Gawron, Jean Mark / Peters, Stanley (1990): Anaphora and Quantification in Situation Semantics. Stanford: CSLI.

Gazdar, Gerald / Klein, Ewan / Pullum, Geoffrey K. / Sag, Ivan A. (1985): Generalized Phrase Structure Grammar. Cambrigde, Mass.: MIT.

Goebl, Hans (erscheint): Dialektometrie. Dialectométrie. In: Holtus / Metzeltin / Schmitt.

Grevisse, Maurice ( $\left({ }^{13} 1993\right)$ : Le bon usage. Grammaire française avec des remarques sur la langue française d'aujourd'hui, refondue par André Goosse. Paris / Gembloux: Duculot. 
Grewendorf, Günther / Hamm, Fritz / Sternefeld, Wolfgang (1994): Sprachliches Wissen. Eine Einführung in moderne Theorien der grammatischen Beschreibung. Frankfurt am Main: Suhrkamp.

Habel, Christopher (1986): Prinzipien der Referenzialität: Untersuchungen zur propositionalen Repräsentation von Wissen. Berlin u.a.: Springer.

Habel, Christopher / Rollinger, Claus-Rainer (1982): EZH-Struktur als Textrepräsentation - Ein Beispiel. In: Bátori, István / Krause, Jürgen / Lutz, HansDieter (Hrsg.): Linguistische Datenverarbeitung. Versuch einer Standortbestimmung im Umfeld von Informationslinguistik und Künstlicher Intelligenz. Tübingen: Niemeyer, 137-153.

Hadlich, Roger L. (1975): Gramática Transformativa del Español. Madrid: Gredos. Haegeman, Liliane (1991): Introduction to Government and Binding Theory. Cambridge, Mass. / Oxford: Blackwell.

Helbig, Gerhard / Buscha, Joachim (1994): Deutsche Grammatik. Ein Handbuch für den Ausländerunterricht. Leipzig u.a.: Langenscheidt.

Hendriks, Petra (1995): Comparatives and Categorial Grammar. Diss., Groningen.

Hernández Alonso, César (1984): Gramática funcional del español. Madrid: Gredos.

Hinrichs, Erhard / Meurers, Detmar / Richter, Frank / Sailer, Manfred / Winhart, Heike (Hrsg.) (1997): Ein HPSG-Fragment des Deutschen, Teil 1: Theorie. Universität Tübingen.

Hölzer, Peter (1996): Das Relationsadjektiv in der spanischen und deutschen Gegenwartssprache. Wilhelmsdorf: Egert.

Holtus, Günter / Metzeltin, Michael / Schmitt, Christian (Hrsg.) (1992): Lexikon der Romanistischen Linguistik (LRL). Bd. VI, I: Aragonesisch / Navarresisch, Spanisch, Asturianisch / Leonesisch. Aragonés / Navarro, Español, Asturiano y Leonés. Tübingen: Niemeyer.

Holtus, Günter / Metzeltin, Michael / Schmitt, Christian (Hrsg.) (erscheint): Lexikon der Romanistischen Linguistik (LRL). Bd. I: Geschichte des Faches Romanistik / Methodologie. Histoire de la philologie romane / Méthodologie. Tübingen: Niemeyer.

Hudson, Richard A. (1987): Zwicky on heads. Journal of Linguistics 23, 109-132.

Hudson, Richard (2000): Grammar without Functional Categories. In: Borsley, 7-35. Iturrioz, José L. (1996): Los artículos y la operación de determinación. In: Bosque, 339-389. 
Jackendoff, Ray (1977): X-bar-Syntax. A study of phrase structure. Cambridge, Mass.: MIT.

Jones, Michael Allen (1996): Foundations of French syntax. Cambridge: Cambridge University Press.

Kathol, Andreas (1995): Linearization-based German Syntax. Diss., Ohio-StateUniversity.

Kathol, Andreas (1998): Determiners as Nominal Heads. In: Bouma / Kruijff / Oehrle, 155-163.

Kathol, Andreas (1999): Agreement and the Syntax-Morphology Interface in HPSG. In: Levine, Robert / Green, Georgia (Hrsg.): Studies in Contemporary Phrase Structure Grammar. Cambridge / New York: Cambridge University Press, 209-260.

Kaufmann, Ingrid (1995): Konzeptuelle Grundlagen semantischer Dekompositionsstrukturen. Die Kombinatorik lokaler Verben und prädikativer Komplemente. Tübingen: Niemeyer.

King, Paul (1989): A Logical Formalism for Head-Driven Phrase Structure Grammar. Diss., University of Manchester.

Kiss, Tibor (1991): Modals and the scope of negation. On the treatment of optionality in HPSG. Some properties of negation in German. Stuttgart: Institut für wissensbasierte Systeme.

Kiss, Tibor (1995a): Infinitive Komplementation: neue Studien zum deutschen Verbum Infinitum. Tübingen: Niemeyer.

Kiss, Tibor (1995b): Merkmale und Repräsentationen. Opladen / Wiesbaden: Westdeutscher Verlag.

Klenk, Ursula (1980): Formale Sprachen: mit Anwendungen auf die Beschreibung natürlicher Sprachen. Tübingen: Niemeyer.

Klenk, Ursula (1995): Formale Sprachen. In: Jacobs, Joachim, u.a. (Hrsg.): Syntax. Ein internationales Handbuch zeitgenössischer Forschung. Berlin / New York: de Gruyter, 1576-1605.

Klenk, Ursula (1996): Die Beschreibung von Sätzen ohne Subjektsphrase in der kopfgesteuerten Phrasenstrukturgrammatik. In: Weber, Nico (Hrsg.): Semantik, Lexikographie und Computeranwendungen. Tübingen: Niemeyer, 237244. 
Klenk, Ursula (1997): Zur Hypothese einer Hierarchie der Verbkomplemente im Spanischen. In: Gather, Andreas / Werner, Heinz (Hrsg.): Semiotische Prozesse und natürliche Sprache: Festschrift für Udo L. Figge zum 60. Geburtstag. Stuttgart: Steiner, 314-321.

Klenk, Ursula (1998): Zur Kategorisierung spanischer Infinitivkonstruktionen mit el in einer kopfgesteuerten Phrasenstrukturgrammatik. In: Briesemeister, Dietrich / Schönberger, Axel (Hrsg.): Ex nobili philologorum officio: Festschrift für Heinrich Bihler zu seinem 80. Geburtstag. Berlin: Domus Editoria Europaea, 905-917.

Kolde, Gottfried (1996): Nominaldetermination. Eine systematische und kommentierte Bibliographie unter besonderer Berücksichtigung des Deutschen, Englischen und Französischen. Tübingen: Niemeyer.

Kolliakou, Dimitra (1995): Definites and Possessives in Modern Greek: An HPSG Syntax for Noun Phrases. Diss., University of Edinburgh.

Krieger, Hans-Ulrich / Nerbonne, John (1993): Feature-Based Inheritance Networks for Computational Lexicons. In: Briscoe / Copestake / Paiva, 90-136.

Kunze, Jürgen (1991): Kasusrelationen und semantische Emphase. Berlin: Akademie-Verlag.

Lamíquiz, Vidal (1987): Lengua Española. Método y estructuras lingüísticas. Barcelona: Ariel.

Langer, Hagen / Thümmel, Wolf (1994): Syntaxen mit multiplen Hierarchien. In: Klenk, Ursula (Hrsg.): Computatio Linguae II. Stuttgart: Steiner, 102-126.

Lavric, Eva (2000): Ein Modell der Referenz determinierter Nominalphrasen. ZrP $116,20-55$.

Lehmann, Christian (1984): Der Relativsatz: Typologie seiner Strukturen. Theorie seiner Funktionen. Kompendium seiner Grammatik. Tübingen: Narr.

Lohnstein, Horst (1996): Formale Semantik und natürliche Sprache: Einführendes Lehrbuch. Opladen: Westdeutscher Verlag.

Lois, Ximena (1996): Los grupos nominales sin determinante y el paralelismo entre la oración y la frase nominal. In: Bosque, 201-240.

Longobardi, Giuseppe (1988): La Quantificazione. In: Renzi (1988a), 647-696.

Longobardi, Giuseppe (1994): Reference and Proper Names: A Theory of $\mathrm{N}$ Movement in Syntax and Logical Form. Linguistic Inquiry 25 / 4, 609-665. 
Luján, Marta (1973): Pre- and Postnominal Adjectives in Spanish. Kritikon Litterarum 2, 398-408.

Luján, Marta (1980): Sintaxis y semántica del adjetivo. Madrid: Cátedra.

Mallén, Enrique (1995): The role of agreement in Spanish, Icelandic, and German. Linguistische Berichte 157, 216-236.

Malouf, Robert P. (1998): Mixed Categories in the Hierarchical Lexicon. Diss., Stanford University.

Marcos-Marín, Francisco A. (1994): Informática y Humanidades. Madrid: Gredos.

Martínez, José A. (1989): El Pronombre. Bd. 2: Numerales, Indefinidos y Relativos. Madrid: Arco.

Matte Bon, Francisco (1992): Gramática comunicativa del Español. 2 Bde. Madrid: Difusión.

McGlashan, Scott (1993): Heads and lexical semantics. In: Corbett / Fraser / McGlashan, 204-230.

Meisterfeld, Reinhard (1998): Numerus und Nominalaspekt. Eine Studie zur romanischen Apprehension. Tübingen: Niemeyer.

Metzeltin, Michael (1997): Sprachstrukturen und Denkstrukturen. Unter besonderer Berücksichtigung des romanischen Satzbaus. Wien: 3 Eidechsen.

Metzeltin, Michael / Winkelmann, Otto (1992): Die Sprachen der Iberoromanischen Halbinsel und ihre Verbreitung. Las lenguas de la Península Ibérica y su difusión. In: Holtus / Metzeltin / Schmitt, 1-36.

Miller, Philip H. (1992): Clitics and Constituents in Phrase Structure Grammar. New York: Garland.

Moliner, María (1970): Diccionario de Uso del Español. Madrid: Gredos.

Monachesi, Paola (1995): A grammar of Italian clitics. Diss., Tilburg University.

Müller, Stefan (1997): An HPSG-Analysis for Free Relative Clauses in German. Saarbrücken: DFKI.

Müller, Stefan (1999): Deutsche Syntax deklarativ. Head-Driven Phrase Structure Grammar für das Deutsche. Tübingen: Niemeyer.

Naumann, Sven / Langer, Hagen (1994): Parsing. Stuttgart: Teubner.

Nerbonne, John / Netter, Klaus / Pollard, Carl (Hrsg.) (1994): German in HeadDriven Phrase Structure Grammar. Stanford: CSLI.

Nespor, Maria (1988): Il sintagmi aggettivale. In: Renzi (1988a), 425-441. 
Netter, Klaus (1994): Towards a Theory of Functional Heads. In: Nerbonne / Netter / Pollard, 297-340.

Netter, Klaus (1996): Functional Categories in an HPSG for German. Saarbrücken: DFKI.

Oepen, Stephan (1994): German Nominal Syntax in HPSG - On Syntactic Categories and Syntagmatic Relations. DFKI-Document D-94-15. Saarbrücken: DFKI.

Oliver, Juan Manuel (1987): Diccionario de Argot. Madrid: Sena

Parodi, Claudia (1994): On case and agreement in Spanish and English dps. In: Mazzola, Michael L. (Hrsg.): Issues and Theory in Romance Linguistics. Selected Papers from the Linguistic Symposium on Romance Languages XXIII. April, 1-4 1993. Washington D.C.: Georgetown University Press, 403-416.

Pelzing, Ernst (1981): Das attributive Adjektiv im Spanischen. Stellenwert und Stellung. Frankfurt am Main / Bern: Lang.

Plann, Susan (1981): The Two el + infinitive Constructions in Spanish. Linguistic Analyses 7 / 3, 203-220.

Pollard, Carl (1988): Categorial Grammar and Phrase Structure Grammar: An Excursion on the Syntax-Semantics Frontier. In: Oehrle, Richard T. / Bach, Emmon / Wheeler, David (Hrsg.): Categorial Grammars and Natural Language Structures. Dordrecht: Reidel, 391-416.

Pollard, Carl (1989): The syntax-semantics interface in a unification-based phrase structure grammar. In: Busemann, Stefan / Hauenschild, Claudia (Hrsg.): Views of the syntax / semantics interface: Proceedings of the workshop 'GPSG and Semantics'. KIT-Report 74. Fachbereich Informatik, Technische Universität Berlin.

Pollard, Carl / Sag, Ivan (1987): Information-based Syntax and Semantics. Bd. 1: Fundamentals. Stanford: CSLI.

Pollard, Carl / Sag, Ivan (1994): Head-driven Phrase Structure Grammar. Chicago / London: CSLI.

Pollard, Carl / Yoo, Eun Jung (1998): A unified theory of scope for quantifiers and wh-phrases. Journal of Linguistics 34, 415-445.

Prado Ibán, Elena (1993): Los determinantes nominales en español: combinatoria y distribución. In: Hilty, Gerold (Hrsg.): Actes du XX Congrès International de 
Linguistique et Philologie Romanes. Tome III, Section IV. Tübingen: Francke, 265-278.

Quirk, Randolph / Greenbaum, Sidney / Leech, Geoffrey / Svartvik, Jan (1985): A Comprehensive Grammar of the English Language. London: Longman.

RAE $(1985)=$ Real Academia Española (1985): Esbozo de una nueva gramática de la lengua española. Madrid: Espasa Calpe.

RAE $(1992)=$ Real Academia Española $\left({ }^{21} 1992\right)$ : Diccionario de la lengua española. Madrid: Espasa Calpe.

Reape, Mike (1994): Domain Union and Word Order Variation in German. In: Nerbonne / Netter / Pollard, 151-197.

Reape, Mike (1996): Getting things in order. In: Bunt, Harry / van Horck, Arthur (Hrsg.): Discontinous Constituency. Berlin / New York: de Gruyter, 209-253.

Renz, Ingrid (1993): Adverbiale im Deutschen: Ein Vorschlag zu ihrer Klassifikation und unifikationsbasierten Repräsentation. Tübingen: Niemeyer.

Renzi, Lorenzo (Hrsg.) (1988a): Grande grammatica italiana di consultazione. Bd. 1: La frase. I sintagmi nominale e preposizionale. Bologna: il Mulino.

Renzi, Lorenzo (1988b): L’articolo. In: Renzi (1988a), 357-423.

Repiso Repiso, Sigifredo (1990): Los posesivos. Salamanca: Colegio de España .

Reumuth, Wolfgang / Winkelmann, Otto (1993): Praktische Grammatik der spanischen Sprache. Wilhelmsfeld: Egert.

Richter, Frank (1997): Die Satzstruktur des Deutschen und die Behandlung langer Abhängigkeiten in einer Linearisierungsgrammatik. Formale Grundlagen und Implementierung in einem HPSG-Fragment. In: Hinrichs u.a., 13-188.

Richter, Frank / Sailer, Manfred (1995): Remarks on Linearization: Reflections on the Treatment of LP-Rules in HPSG in a Typed Feature Logic. Magisterarbeit, Universität Tübingen.

Riehemann, Susanne (1993): Word Formation in Lexical Type Hierarchies: A Case Study of bar-Adjectives in German. SfS-Report-02-93. Universität Tübingen: Seminar für Sprachwissenschaft.

Rodriguez Diez, Bonifacio (1992a): Los cuantificadores en español. In: GrammaTemas 1. Departamento de filología hispánica, Areas de filología española (Lengua) y Lingüística General, Universidad de León, 231-255. 
Rodriguez Diez, Bonifacio (1992b): Los posesivos en español. In: Gramma-Temas 1. Departamento de filología hispánica, Areas de filología española (Lengua) y Lingüística General, Universidad de León, 215-230.

Romero Cambrón, Ángeles (1998): Historia sintáctica de las construcciones comparativas de desigualdad. Cuenca: Universidad de Castilla-La Mancha.

Sadler, Louisa / Arnold, Doug J. (1994): Prenominal adjectives and the phrasal / lexicon distinction. Journal of Linguistics 30, 187-226.

Sag, Ivan A. (1997): English Relative Clause Constructions. Journal of Linguistics $33,431-484$.

Sag, Ivan A. / Godard, Danièle (1994): Extraction of De-Phrases from the French NP. In: Proceedings of the North East Linguistic Society 24. Department of Linguistics, University of Massachusetts: GLSA, 519-541.

Sag, Ivan / Pollard, Carl (1989): Subcategorization and Head-driven Phrase Structure. In: Baltin, Mark R. / Koch, Anthony S. (Hrsg.): Alternative conceptions of phrase structure. Chicago: University of Chicago Press, 139-181.

Sag, Ivan A. / Wasow, Thomas (1999): Syntactic Theory. A Formal Introduction. Stanford: CSLI.

Schwarz, Monika / Chur, Jeanette (1996): Semantik. Ein Arbeitsbuch. Tübingen: Narr.

Schwarze, Christoph (1988): Grammatik der italienischen Sprache. Tübingen: Niemeyer.

Seco, Manuel (1986): Diccionario de dudas y dificuldades de la lengua española. Madrid: Espasa Calpe.

Seco, Manuel (1991): Gramática esencial del español. Introducción al estudio de la lengua. Madrid: Espasa Calpe.

Sells, Peter (1985): Lectures on contemporary syntactic theories. An introduction to government-binding theory, generalized phrase structure grammar and lexical-functional grammar. Stanford: CSLI.

Shieber, Stuart (1986): An Introduction to Unification-based Approaches to Grammar. Stanford: CSLI.

Shieber, Stuart / Robinson, Jane / Tyron, Mary / Uszkureit, Hans (1983): The Formalism and Representation of PATR-II. In: Research on Interactive Acquisition and Use of Knowledge. Menlo Park, Calif.: Artificial Intelligence Center, SRI International. 
Sokal, Robert R. / Sneath, Peter H. A. (1963): Principles of numerical taxonomy. San Francisco: Freeman.

Steedman, Mark (1993): Categorial Grammar. In: Jacobs, Joachim, u.a. (Hrsg.): Syntax - Ein internationales Handbuch zeitgenössischer Forschung. Berlin: de Gruyter, 395-413.

Stein, Achim (1998): Einführung in die französische Sprachwissenschaft. Stuttgart / Weimar: Metzler.

Stolzenberg, Frieder (1992): UBS - A Unification-Based Language for the Implementation of HPSG. Fachberichte Informatik 2. Universität Koblenz-Landau.

Van Eynde, Frank (1998): Minor and Expletive Pronouns. In: Bouma / Kruijff / Oehrle, 136-143.

Varela Ortega, Soledad (1990): Fundamentos de Morfología. Madrid: Síntesis.

Vera Lújan, Agustín (erscheint): Morphologie. I. Flexionslehre. Morflogía. I. Flexión. In: Holtus / Metzeltin / Schmitt.

Verspoor, Cornelia M. (1996): A Perspective on PPs. In: Grover, Claire / Vallduví, Enric (Hrsg.): Edinburgh Working Papers in Cognitive Science. Bd. 12: Studies in HPSG. Centre for Cognitive Science, University of Edinburgh, 229271.

Warner, Anthony R. (1993): English Auxiliaries. Structure and History. Cambridge: Cambridge University Press.

Warner Anthony (2000): English Auxiliaries without Lexical Rules. In: Borsley, 167220.

Weber, Heinz J. (1992): Dependenzgrammatik. Ein Arbeitsbuch. Tübingen: Narr.

Wiese, Heike (1997): Zahl und Numerale. Eine Untersuchung zur Korrelation konzeptueller und sprachlicher Strukturen. Berlin: Akademie-Verlag.

Wilcock, Graham (1999): Lexicalization of Context. In: Webelhuth, Gert / Koenig, Jean-Pierre / Kathol, Andreas (Hrsg.): Lexical and Constructional Aspects of Linguistic Explanation. Stanford: CSLI, 373-387.

Winhart, Heike (1997): Die Nominalphrase in einem HPSG-Fragment des Deutschen. In: Hinrichs u.a., 319-384.

Wintner, Shuly (1998): Noun Phrases as NPs - the case of Hebrew. In: Bouma / Kruijff / Oehrle, 144-154.

Yoon, James Hye-suk / Bonet-Farran, Neus (1991): The ambivalent Nature of Spanish Infinitives. In: Wanner, Dieter / Kibbee, Douglas A. (Hrsg.): New Analy- 
ses in Romance Linguistics. Selected Papers from the XVIII. Linguistic Symposium on Romance Languages in Urbana Champain, April 7-9 1988. Amsterdam, Phil.: Benjamins, 353-370.

Zimmermann, Ilse (1992): Der Skopus von Modifikatoren. In: Zimmermann, Ilse / Strigin, Anatoli (Hrsg.): Fügungspotenzen. Berlin: Akademie-Verlag, 251279.

Zlatic, Larissa (1997): The Structure of the Serbian Noun Phrase. Diss., University of Texas.

Zwicky, Arnold M. (1985): Heads. Journal of Linguistics 21, 1-29.

Zwicky, Arnold M. (1993): Heads, bases and functors. In: Corbett / Fraser / McGlashan, 292-315. 


\section{Das Korpus}

\section{Die Quellen}

Das verwendete Korpus besteht aus folgenden sechs Teilen:

Jeweils eine digitale Ausgabe von drei der größten spanischen Zeitungen. Die Inhalte sind mit der Printversion identisch. Layout und Werbung sind unterschiedlich. In Klammern folgt das Ausgabedatum sowie das Referenzkürzel:

1. $A b c$ (vom 10.6.1999, Kürzel A);

2. El Mundo (vom 9.6.1999, M);

3. El País (vom 8.6.1999, P).

Zudem werden die folgenden zwei Korpora des Departamento de Lógica, Lingüística, Lenguas Modernas y Filosofía de la Ciencia, Facultad de Filosofía y Letras, Universidad Autónoma de Madrid, verwendet. Beide Korpora umfassen verschiedene Textformen (ausführlich Marcos-Marín 1994: 143ff. und 148ff.).

4. Corpus argentino (Kürzel RA, im Internet unter http://www.lllf.uam.es/ $\sim$ fmarcos/informes/corpus/coarginl.html): Corpus lingüístico de referencia de la lengua española en Argentina, Dirigido por: Francisco A. Marcos-Marín (Universidad Autónoma de Madrid), Secretaria Ejecutiva: Verónica Zumárraga, Colaboración entre la Sociedad Estatal del Quinto Centenario y la Agencia Española de Cooperación Internacional, Instituto de Cooperación con Iberoamérica.

Dimensión del corpus y procentajes:

\begin{tabular}{|l|r|r|}
\hline Tipos Textuales & $\begin{array}{l}\text { Cantidad } \\
\text { de palabras }\end{array}$ & Porcentajes \\
\hline Humanísticos & 433.295 & $21.66 \%$ \\
\hline Escolares & 184.019 & $9.20 \%$ \\
\hline Literarios & 181.822 & $9.09 \%$ \\
\hline Científicos & 322.317 & $16.11 \%$ \\
\hline Jurídicos & 126.561 & $6.30 \%$ \\
\hline Técnicos & 135.831 & $6.79 \%$ \\
\hline Periodísticos & 560.000 & $28.00 \%$ \\
\hline Comerciales & 65.124 & $3.25 \%$ \\
\hline
\end{tabular}


5. Corpus chileno (Kürzel Rch, im Internet unter http://www.lllf.uam.es/ fmarcos/ informes/corpus/cochile.html): Corpus lingüístico de referencia de la lengua española en Chile, Dirigido por: Francisco A. Marcos-Marín (Universidad Autónoma de Madrid), Secretaria Ejecutiva: Ernesto Evans Espiñera (Gabinete de la Presidencia de la República), Colaboración entre la Sociedad Estatal del Quinto Centenario y la Agencia Española de Cooperación Internacional, Instituto de Cooperación con Iberoamérica.

Dimensión del corpus y procentajes:

\begin{tabular}{|l|r|r|}
\hline Tipos Textuales & \multicolumn{1}{|c|}{$\begin{array}{l}\text { Cantidad de } \\
\text { palabras }\end{array}$} & Porcentaje \\
\hline Científicos & 300.000 & $15 \%$ \\
\hline Comerciales & 200.000 & $10 \%$ \\
\hline Escolares & 300.000 & $15 \%$ \\
\hline Humanísticos & 100.000 & $5 \%$ \\
\hline Histórico-jurídicos & 200.000 & $10 \%$ \\
\hline Literarios & 200.000 & $10 \%$ \\
\hline Periodísticos & 300.000 & $15 \%$ \\
\hline Publicitarios & 100.000 & $5 \%$ \\
\hline Técnicos & 300.000 & $15 \%$ \\
\hline
\end{tabular}

6. Das Proyecto Sherezade (Kurzgeschichten im Internet seit 1996 unter http://www.princeton.edu/ enriquef/informe.html, Kürzel S): Es handelt sich um ein Projekt von ehemaligen Mitgliedern der Princeton Universität, New Jersey, das Autoren aus dem spanischsprachigen Raum die Möglichkeit geben möchte, ihre Kurzgeschichten zu veröffentlichen. Jeden Monat können zwei bis drei neue cuentos auf dem Server der Universität Princeton abgerufen werden. Diese wurden zuvor von einer Jury ausgewählt, editiert und illustriert. Die Texte stammen daher von allen fünf Kontinenten und sind mit literarischem Anspruch verfaßt. Berücksichtigt wurden die Kurzgeschichten von Februar 1996 bis Juni 1999.

Das Projekt stellt sich auf den Seiten folgendermaßen selbst vor:

„Proyecto Sherezade surgió en 1996 como un espacio virtual para promover el intercambio de narraciones entre hispanohablantes mediante la publicación de cuentos inéditos y las opiniones de sus lectores. 
En estos tres años hemos publicado electrónicamente más de 100 historias de autores de 25 países, quienes han alcanzado así una difusión internacional impensable con los medios tradicionales de edición.

Si desea participar en este proyecto envíenos su cuento. Los cuentos seleccionados aparecerán con el nombre y la dirección de correo electrónico de su autor, quien continúa siendo el dueño exclusivo del cuento que sólo cede temporalmente para su publicación en Proyecto Sherezade.

También puede participar enviando comentarios a los cuentos.

Proyecto Sherezade es posible gracias al desinteresado esfuerzo de cinco personas del medio académico y cultural que realizan la selección, edición e ilustración de los cuentos, así como a los cibernarradores y lectores que nos envían sus cuentos y comentarios desde los cinco continentes." 


\section{Zuordnung der Beispiele}

Die Beispielsätze entstammen entweder aus der angegebenen Sekundärliteratur oder dem erstellen Korpus. Für den zweiten Fall kann an dieser Stelle das Kürzel hinter dem Beispiel dem jeweiligen Text zugeordnet werden, aus dem das Beispiel stammt. Für die Zeitungsausgaben erfolgt in der rechten Spalte die Zuordnung zu der Rubrik oder dem Titel, bei den Korpora und dem Proyecto Sherezade zum Titel des Textes. Bei den Angaben zu den Korpora steht in Klammern der Texttyp.

Abc:

$\begin{array}{ll}\text { A-1 } & \text { Cultura } \\ \text { A-2 } & \text { Economía } \\ \text { A-3 } & \text { Entrevista a Felipe González } \\ \text { A-4 } & \text { Internacional } \\ \text { A-5 } & \text { Opinión } \\ \text { A-6 } & \text { Sociedad }\end{array}$

El Mundo:

M-1 Busco

M-2 Comer

M-3 Cultura

M-4 Economía

M-5 Encuesta

M-6 Europa

M-7 Index

M-8 Internet

M-9 La amenaza está en la Red

M-10 Nacional

M-11 Opinión

M-12 Resumen de noticias

M-13 Sociedad

M-14 Munich

M-15 Encuentros digitales

M-16 Madrid

El País:

P-1 Comer

P-2 Cultura

P-3 Elecciones

P-4 Internacional

P-5 Salud 
Corpus argentino:

RA-1 Alimentos y nutrición. Bromatología aplicada a la salud (técnico)

RA-2 Cultura y contracultura (humanístico)

RA-3 El lado de la sombra (literario)

RA-4 Historia. Tercer curso. La edad contemporánea. La Argentina de 1831 a 1982 (escolar)

RA-5 Los pensadores de la libertad (humanístico)

RA-6 Manucho. Una vida de Mujica Lainez (humanístico)

RA-7 Parálisis cerebral. Clínica y cirugía del aparato locomotor (científico)

Corpus chileno:

Rch-1 Astronomía Contemporánea (científico)

Rch-2 Chile en el siglo XX (histórico)

Rch-3 Chile, o una loca geografía (humanístico)

Rch-4 El árbol del conocimiento (científico)

Rch-5 Emociones y lenguage en educación y política (científico)

Rch-6 Geografía de Chile (escolar)

Rch-7 Papelucho (literario)

Rch-8 Ven y sígueme (escolar)

Proyecto Sherezade:

S-1 Agustín Varilla

S-2 Cambio de definición

S-3 Círculo, la maldición de Everett

S-4 Didier

S-5 Fiesta en la montaña

S-6 La espera

S-7 La reorganización

S-8 Las muertes normales

S-9 Lluvia

S-10 Podrás decir que conociste un motero

S-11 Por qué llamarte Arlena

S-12 Portada

S-13 Raso azul

S-14 Soledad

S-15 Yo, un internetadicto

S-16 Marie, antes y después del amor 


\section{Namenindex}

Abb, Bernd 1, 60, 185, 188

Abeillé, Anne 3, 19

Abney, Steven 1, 85, 185, 192

Aissen, Judith 18

Alarcos Llorach, Emilio 4, 89, 114, 148, 199

Alcaraz Varó, Enrique 6, 77, 87, $89,194,245$

Alcina Franch, Juan 136 - 146, 174, 196, 230, 254

Allegranza, Valerio 88f., 109, 118, 120 - 126, 136, 151, 192f., 207 209, 222, 225 - 228, 233, 258f.

Allwood, Jens 10, 53, 64, 142

Altmann, Gabriel 97, 154, 156, 163, 169,254

Alvar, Manuel 105

Alvarez Martínez, Maria Angeles 1, 4, 90 - 101, 105, 118, 121, 125, $132,146,172,254$

Andersson, Lars-Gunnar 10, 53, 64 Arnold, Doug J. 234

Asenjo Orive, María R. 136f.

Bach, Emmon 18

Bacher, Johann 156, 157, 158, 159, 162

Badia, Toni 19, 64, 208, 248 - 252, 258

Balari, Sergio 3

Barwise, John 52

Beckmann, Hans-Georg 121, 143
Berman, Judith 19f., 192

Berschin, Helmut 72, 249

Bierwisch, Manfred 63

Bird, Steven 38

Blecua, José Manuel 136 - 146, 174,254

Börjars, Kersti E. 185

Borsley, Robert D. 7, 19f., 48 - 50, 185

Bosque, Ignacio 73f., 79, 245

Bouma, Gosse 185, 217

Braselmann, Petra M. E. 231 - 239, 246

Bresnan, Joan 18, 20

Briz Gómez, Antonio 106, 107, 162

Bruyne, Jacques de 4, 76, 82, 99 109, 135 - 138, 145, 148, 196, 199, 218, 230, 232, 249, 250

Bull, William E. 239, 240

Buscha, Joachim 72f., 107, 135, $175 f$.

Bußmann, Hadumod 53, 87

Cann, Ronnie 185

Carpenter, Bob 24, 30, 138

Casielles, Eugenia 76, 254

Chomsky, Noam 7 - 13, 18f., 41, 208

Chur, Jeanette 53

Contreras, Helen 76, 79

Cooper, Richard 52

Cooper, Richard P. 19f., 64f. 
Copestake, Ann 19, 22

Corbett, Greville G. 198

Crystal, David 7, 9, 12

Dahl, Östen 10, 53, 64

Dietrich, Wolf 231 - 239, 242 - 246

Dini, Luca 3

Egli, Urs 12, 19

Egli-Gerber, Renate 12, 19

Engdahl, Elisabet 3, 247, 254

Engel, Ulrich 1, 176f., 181

Ernst, Gerhard 245

Felixberger, Josef 72, 249

Fernández-Sevilla, Julio 72, 249

Figge, Udo L. 19

Flickinger, Daniel P. 31, 48, 60, 217,220

Frank, Anette 19f., 48, 192

Gawron, Jean Mark 109, 225

Gazdar, Gerald 18, 208

Godard, Danièle 3

Goebl, Hans 254

Grevisse, Maurice 1, 10f., 46, 88

Grewendorf, Günther 7, 9, 12

Habel, Christopher 142, 222 - 224

Hadlich, Roger L. 33, 199

Haegeman, Liliane 11, 18

Hamm, Fritz 7, 9, 12

Helbig, Gerhard 72, 73, 107, 135, 175,176

Hendriks, Petra 230
Hernández Alonso, César 88, 107, 144,146

Hölzer, Peter 239

Hudson, Richard A. 73, 185

Iturrioz, José L. 73, 84

Jackendoff, Ray 12, 208

Jones, Michael Allen 18, 88

Kathol, Andreas 10, 46, 58f., 192 198,256

Kaufmann, Ingrid 63

King, Paul 24

Kiss, Tibor 20, 25 - 30, 34, 38f., 49, $58,65-69,109,185,254$

Klein, Ewan 38

Klenk, Ursula 10, 13, 36, 47f., 73

Kolde, Gottfried 87

Kolliakou, Dimitra 109

Krieger, Hans-Ulrich 48

Kunze, Jürgen 53

Lamíquiz, Vidal 136, 138, 140 $145,160,254$

Langer, Hagen 15, 25, 34, 36, 176

Lasnik, Howard 9, 19

Lavric, Eva 79, 141, 142

Lehfeldt, Werner 97, 154, 156, 163, 169,254

Lehmann, Christian 229, 230

Lohnstein, Horst 222 - 225, 241

Lois, Ximena 74f., 85 
Longobardi, Giuseppe 72, 74, 76, $85,87,122,144$

Luján, Marta 73, 140, 233, 235

Maienborn, Claudia 60

Mallén, Enrique 47

Malouf, Robert P. 73, 201

Marcos-Marín, Francisco A. 4

Martínez Linares, María Antonia 1, 4, 6, 73, 77, 81f., 87 - 101, 105, $118,121,125,132,146,172$, $194,245,254$

Martínez, José A. 6, 77, 87, 89, 194,245

Matte Bon, Francisco 4, 76, 90, 100, 102, 135, 136 - 140, 143, $144-148$

McGlashan, Scott 185

Meisterfeld, Reinhard 199, 238, $239,244,251$

Metzeltin, Michael 8, 196

Miller, Philip H. 86

Moliner, María 95

Monachesi, Paola 3, 86

Müller, Stefan 23, 41, 46, 52f., 56, $58,185,229$

Naumann, Sven 15, 25

Nerbonne, John 3, 48

Nespor, Maria 239

Netter, Klaus 3, 188

Oepen, Stephan 1, 186 - 188

Oliver, Juan Manuel 75
Parodi, Claudia 192

Pelzing, Ernst 231 - 245

Perlmutter, David M. 18

Perry, Jon 52

Peters, Stanley 109, 225

Picallo, Carme 245

Plann, Susan 73

Pollard, Carl 3, 18, 20 - 23, 27f., 30, 32, 33f., 36 - 38, 41, 43, 45, 48 - 53, 55 - 69, 86, 184 - 189, 192, 194, 196, 208, 217, 222 224, 226f., 233, 246, 248, 256

Pottier, Bernard 105

Prado Ibán, Elena 4, 114 - 116, 119, $121-126,131,254$

Quirk, Randolph 1, 88, 120, 127, $133-136$

Reape, Mike 46

Renz, Ingrid 61

Renzi, Lorenzo 88, 121

Repiso Repiso, Sigifredo 138

Reumuth, Wolfgang 75, 85, 99

Richter, Frank 46

Riehemann, Susanne 10, 48

Rollinger, Claus-Rainer 142, 222, 224

Romero Cambrón, Ángeles 230 
Sadler, Louisa 234

Sag, Ivan A. 3, 18, 20 - 23, 27f., 30, 32 - 34, 37f., 40f., 43, 45, 48 - 53, 55 - 69, 86, 184 - 189, 192, 194, 196, 208, 217, 222 - 224, 226f., 229, 233, 246, 248, 256

Sailer, Manfred 46

Schwarz, Monika 53

Schwarze, Christoph 90, 174

Seco, Manuel 2, 8, 11, 63, 72f., 76, 89, 107, 196, 229, 232, 253

Sells, Peter 19

Shieber, Stuart 18, 21, 27

Sneath, Peter H. A. 156,163

Sokal, Robert R. 156, 163

Steedman, Mark 18

Stein, Achim 6, 8f.

Sternefeld, Wolfgang 7, 9, 12

Stolzenberg, Frieder 19

Thümmel, Wolf 34, 36, 176

Vallduví, Enric 3, 247, 254
Van Eynde, Frank 86

Varela Ortega, Soledad 220

Vera Lújan, Agustín 140

Verspoor, Cornelia M. 63, 229, 248

Warner, Anthony R. 49, 217

Wasow, Thomas 19, 30

Weber, Heinz J. 10, 92, 183

Weber, Nico 10, 92, 183

Wiese, Heike 74 - 81, 253

Wilcock, Graham 247

Winhart, Heike 1, 186 - 188, 191, 193, 201f., 204, 208, 229, 248

Winkelmann, Otto 8, 75, 85, 99

Wintner, Shuly 192

Yoo, Eun Jung 68f., 227

Yoon, James Hye-suk 73

Zimmermann, Inge 125

Zlatic, Larissa 191

Zwicky, Arnold M. 2, 184f., 189, 190, 192, 256, 259 


\section{Sachindex}

Abfolge

Abfolgeregel 45f., 125

Adjektiv-Nomen-Abfolge 236

Nomen-Adjektiv-Abfolge 236

Normalposition 235

Ableitung 13

Adjektiv 1 - 5, 12f., 15, 31 - 33, 35, 46, 55, 59 - 62, 64, 69f., 72f., 77, 85, 87 - 99, 101 - 112, 114, 118 120, 124 - 128, 132, 143, 145, 152 155,160 - 163, 165 - 177, 179 184, 186, 188, 191, 196, 199 - 203, 205, 208 - 210, 216, 219, 229 - 248, $253-259$

adjektivtypisch 108, 160, 163, 167, 169,171 - 173, 183, 257

bipositionale-bisemantische

Adjektive (BBA) 231, 242 - 244 bivalent 236

adjektivähnlich 3, 107, 154, 183, 201

Adjektivphrase 15f., 33, 229, 234

Adjektivpositionsgrammatik 243

Adjungierung 2 - 4, 186, 189, 192, $214,256,259$

Adjunkt 12, 46, 56 - 58, 60 - 64, 69, 71, 80, 86, 89, 186, 189, 192f., 196, 205, 208 - 211, 214, 229, 231, 233f., 248, $258 f$.

Adjunkttochter 35, 62

Adverb 93 - 95, 99f., 143, 204, 230, 234,245
Adverbial 16, 61, 91, 95, 101, 112, 152, 155, 161, 166, 173, 176, 179f., 200, 217

Adverbialisierung 97, 100, 112, 152, 254

Agreement, s. Kongruenz

Akkusativ 39, 73, 76, 86, 199

Akkusativobjekt 36, 39f., 45, 48, 50, 76,251

Akkusativphrase 36

Aktualisierer 28, 136 - 138, 140 142, 146 - 148, 151, 153, 160, 170, 174,178

Akzent 10, 46, 70, 169

Algorithmus $156 f$.

alguno 64, 94f., 98, 105 - 109, 111f., 114, 116f., 120, 127, 129 - 133, 143 - 149, 155, 157 - 159, 164 - 167, 168, 172 - 174, 183, 204, 216, 219, 238,255

Allquantor 64

Ambiguität $29,64,66,69,90,172$, 248

ambos 94, 98 - 100, 106, 114, 116f., 120f., 127, 129f., 132f., 143, 146 149, 155, 164 - 168, 172f., 216, 232,255

anaphorisch 47, 55f., 86, 194f., 220 Anhebungskonstruktion 69, 153, 194 Antonymie 150, 238, 243 append-Opperation 28, 52 arbiträr 8 
Argument 47, 48, 90, 142, 186, 189, $190,201,210$

Artikel 1, 2, 8, 16, 29, 35, 65, 69, 78 $80,83,87$ - 90, 92, 95 - 99, 103 115, 117, 119, 122 - 126, 136 - 142, 145, 150, 152f., 161f., 167, 169, 172, 175, 180, 183, 187, 189, 200f., $220,253-255,259$

artikeltypisch 107f., 153, 205

artikelähnlich 92, 103, 108, 115

Artikelfunktion 109, 202

atomar 21f., $25 \mathrm{f}$.

Attribut, s. auch Merkmal 21 - 24, 28f., 32f., 35, 38, 41, 43, 48 - 51, 54, 56, 60 - 68, 70, 75f., 86, 122, 185f., 193f., 197 - 199, 205, 208, 213, 215, 233f., 246, 250, 257

attributiv 61, 63, 233, 251

Attribut-Werte-Paar 3, 17, 20 - 22, $37 \mathrm{f}$.

Auslaut 99

Automat 15

Auxiliarverben 16, 33, 43, 52

Average-Linkage-Verfahren 159

Basis 19, 190f., 235, 248

bastante 90, 94f., 98 - 100, 106f., 114, 117, 120, 127, 129f., 132 134, 143, 147 - 151, 155, 164 - 166, 168,172 - 174, 216, 255

Baumdiagramm 3, 15, 23, 39f., 43, 66f., 80, 210

Bedeutung 238f., 243f., 255, 258
Berechnung 96, 98, 113, 156f., 159, $161 f$.

Berechnungsmethode 255

Betonung 95, 235

Bezugsbereich 64 - 66, 84, 196, 236, 238

Bezugspunkt 220, 229, 233f., 249

binär 43, 98, 150, 156

boolesch 33, 135, 160, 208

bottom-up 209

cada 64, 69, 90f., 94, 98 - 100, 103, 106, 109, 114 - 120, 122f., 126f., 129f., 132f., 143f., 146 - 150, 155, 164 - 166, 168, 172f., 183, 216, 232,255

cierto 94, 96, 98, 106, 114, 116f., $120,127,129,130,132$ - 134, 147 151, 155, 164, 168f., 172f., 216, 243,255

Clique 158

Cluster 2, 157 - 159, 162, 164f., 169

Clusteranalyse 2, 156f., 159, 162, 165,255

cualquiera 64, 94f., 98f., 106f., 112, 114, 117, 119f., 127 - 130, 132f., 147 - 149, 151, 155, 158f., 164 166, 168, 172 - 174, 183, 204, 216, 255

Dativ 29, 36, 76, 86

Dativobjekt 45, 63

default-Wert 55, 197, 217, 246 
definit 1f., 69, 79, 87, 89, 95, 98, 103

- 105, 107f., 111, 114, 119, 138, 140 - 142, 145, 148, 153, 160 162, 169f., 175, 183, 189, 200, 210, 220, 253f.

Definitheit 96, 142

deiktisch 89, 92, $136-138$

deklarativ 19, 53, 253

Deklination 188

demás 11, 94f., 98 - 100, 104 - 108, 110, 116f., 120, 127, 129f., 132 134, 147 - 151, 153, 155, 158f., 164, 168f., 172f., 183, 201, 214, 216,255

demasiado 94f., 98, 100, 105 - 107, 114, 117, 119f., 127, 129 - 134, 143, 145, 147 - 150, 155, 157f., 164 - 166, 168, 172 - 174, 216, 255

Demonstrativadjektiv 176

Demonstrativpronomen 1, 85, 87 - 90, $92,98,103,105,111,114,122$, 126, 136 - 138, 141 - 143, 145, 153, 169f., 206, 232, 255

Dendrogramm 91, 163 - 165, 167, 180f., 215, 230, 254

Denotat $77 \mathrm{f}$.

Dependenzgrammatik 10,91

Determinierer 1 - 5, 13, 16, 21, 32, 34, 36, 45f., 64, 69f., 72f., 76, 80, 83, 85, 87, 89 - 194, 199 - 220, 229 - 232, 234, 237, 239, 244, 246, 253 $-259$

determinierertypisch 98, 154, 167, $171,173,257$ deutsche 175 - 182

Determiniererphrase 1, 184 - 188, 191

Determinierersystem 175, 180f., 255, 259

diachron 8

Diagramm 98

Dialekt 8, 78, 196, 254

Dichotomie 247, 251

Disambiguierung 12

Disjunktion 23, 28f., 36, 61, 84, 197, 250,252

Distanz 1, 96, 98, 113, 127, 156 160, 162, 165, 180f., 196, 254f.

Distanzwert 157f.

distinktiv 12, 61, 103, 105, 239

Distribution 2, 12, 91 - 93

Distributiva 114, 143, 147f.

doble 88, 90, 94, 98f., 106 - 108, 112, 117 - 120, 126f., 129f., 132 - 134, 147 - 150, 155, 164 - 166, 168, 172f., 175, 183, 201, 204f., 216 dos 80, 88, 90, 94, 98f., 103f., 106, 108, 114, 115, 117f., 120, 123 134, 144, 147 - 150, 155, 164, 168f., 172f., 179, 209f., 215f., 255

Durchschnitt 127f., 130, 148

Eigenname 56, 72, 77, 95, 112, 121, 140 - 142, 187 - 189, 197, 203f., 211f., 218, 219, 251, 253, 256, 258

el 1f., 6, 10 - 13, 15, 34, 37f., 40, 52f., 60, 63, 72f., 76, 83, 88, 93, 95, 98f., 102, 104, 106f., 109 - 111, 117f., 120 - 123, 126f., 129f., 132f., 136 - 
142,147 - 150, 155, 161, 164, 168f., 172f., 187, 189, 210, 216, 220, 230, 232, 244, 249, 251, 255, 259

Emphase 95, 235, 250

empirisch 11, 123, 126, 250

Englisch 1, 3, 12, 22, 32, 48, 58, 61, $69,87,120,123,184,187,247$

Ersetzungsregel 12f., 16, 18, 71

Erstdeterminierer 119, 128, 130

Ersterwähnung 139, 141

Erststellung 126, 131f., 134, 147, 149, 150, 152f., 155, 161, 166, 173, 177, 179f., 205, 257

este 2, 6, 76, 88, 94f., 98 - 101, 104f., 107 - 112, 115, 117 - 119, 120f., 126, 127, 129 - 133, 136 - 142, 147 - 155, 164, 168f., 172f., 204f., 210, 214 - 216, 220, 255

Euklidische Distanz 156

existenciales 143 - 148, 174

Existenzquantor 64 - 68

Expletiva 55f.

Explikativa 235

fakultativ 16, 35 - 37, 47, 60f., 89, 95, 107, 185, 188, 192, 210f., 218, 229, 253

feminin 16f., 59, 75f., 195, 198, 218

finit $32,39,47-50,84$

Fixpunkt 165 - 169, 215, 255

Flexem 182
Flexion 47, 186, 191

Formalismus 4, 7, 10, 19, 21, 42, 46, $52,54,55$

Französisch 1, 3, 46, 59, 88, 192, 237

Funktor 189 - 194, $208 f$.

Generalisierer 18, 82 - 84, 160, 162, 180, 187 - 189, 209, 211, 217f., 253f., 258

Generalized phrase structure grammar (GPSG) 18f., 208

generativ $12,18 \mathrm{f}$.

Genitiv 177

Genitivobjekt 76, 184

Genitivphrase 249

Genus 16f., 26, 58, 61, 75f., 91, 94, 97 - 99, 112, 152f., 155, 161, 163, 166, 173, 176, 192, 194 - 196, 198, 254

Gerundialkonstruktion 36

Geschlecht 87, 98, 195

Gewichtung 96, 154, 156, 157, 160, 163 - 169, 171, 173f., 180, 236

Gewichtungsfaktor 156, 163

Gewichtungsverfahren 154

Government-Bindung-Theorie (GB) $18,20,245$

Gradative 114, 237

Grammatik 1f., 4, 11 - 19, 52, 72, 76, 87 - 90, 101f., 135, 140, 143, 153, 175f., 183, 192, 199, 201, 217, 229 - 231, 246, 253

Grammatikformalismen 17 Grammatikfragment 20, 192 
Grammatikmodell 3, 11, 18f., 253, 259

Grammatikregel 18, 41

Grammatiksystem 10, 19

Grammatiktheorie 3, 18f., 38, 100, 230

Gültigkeitsbereich 64f.

Head-driven Phrase Structure Grammar (HPSG) 3 - 7, 12, 18 71, 75, 84, 86, 96, 100f., 113, 133, 142, 152, 183, 185f., 189, 194, 199, 201, 217f., 231, 240, 245 - 250, 253 - 256, 258f.

Hebräisch 192

Höflichkeitsform 196

holotomic 240

Homogenität 158

Homonym 96

hybrid 171, 173, 198, 255

identificador 125, 136, 143, 146 - 148

Identifizierer 137, 143f.

Identität 27, 251

idiomatisch $10,74,84$

Immediate-Dominance-Principle 41

Immediate-Dominance-Schemata 41

indefinit 2, 85, 119, 141, 170, 237

Indefinitheit 96

Indefinitpronomen 1, 79, 88 - 90, 103, $114,135,141$ - 145, 148, $175 f$.

Individuum 52f., 77, 90, 144 - 146, 148

Infinitiv 32f., 36, 47 - 49
Infinitivkonstruktionen 3, 44, 50, 63, 254

Infinitivphrase 36,248

Interferenzen 46

Interrogativpronomina 176, 230

Intonation 46, 235

Intuition 7, 94, 138, 142, 144, 160, 188,195

IOTA-Operator 142, 144

Italienisch 3, 87f., 90, 120 - 124, 144, 174, 237, 239

Kardinalia 88, 94 - 96, 99, 114, 117f., 143, 147f., 155, 179, 209

Kasus 29, 58, 73, 76, 86, 175, 181, 194

Katalanisch 19, 64, 248, 249

Kategorialgrammatik 18f., 185, 230

Klitika 3, 86, 90, 105

Kombinationssteuerer 150f., 167, 176, 178, 205f., 255, 257

Kommunikation 6, 237, 239

Komparation 91, 93, 97, 101f., 112, 152, 155, 161, 163, 166, 173, 179f., $186,200,230,236,254$

Komplement 2 - 4, 32, 34 - 36, 39, 42f., 45, 50, 57, 60, 62, 71, 86, 184 - 187, 189, 192, 248, 256, 259

Komplementiererphrase 49

Komplementliste 85

Komplementphrase 47

Komplementtochter 35, 41f.

Komponentenanalyse 245

Kongruenz 27, 199 
Kongruenz (Agreement) 4f., 11, 16f., 26, 58f., 61, 86, 183, 191, 194 199, 211, 213f., 218, 234, 253, 256, 259

Kongruenzmerkmal 55, 58f., 100, 196f., 250

Konjunktiv 36

Konstituente 6, 13, 33 - 36, 39f., 47, 67,110

Konstituentenabfolge 44 - 46

Konstituentenstruktur 12, 20, 41, 209, 217

Kontext 19, 29, 32, 64, 70f., 73, 76, 86, 90, 102, 106f., 137, 139f., 142, $145,151,162,187,198,230,238$ 240, 244f., 248 - 250

kontextabhängig 77, 219

kontextfrei 4, 12 - 14, 18, 31, 36f., 41 $-43,71$

Kookkurrenz 4, 5, 90 - 92, 97, 108, $110,112,114$ - 125, 134, 136, 145f., 152, 157, 177, 254, 257

Koordination 3, 95, 96, 230, 236

Kopf 24, 31 - 49, 58, 60, 62, 80, 85, 92, 110, 185 - 193, 229, 233f., 248 $251,256,258$

Kopfmerkmal 31, 36, 58, 62, 196, 208, 210, $246 f$.

Kopfmerkmalsprinzip 33f., 71

Kopftochter 31 - 35, 41 - 43, 51, 62, 193

Kopulakonstruktion 80, 83f., 188

Kopulaverb 61, 79

Korpus 4, 5, 53, 187
Latein 76,87

Lexem 12, 18, 30, 43, 46, 48, 55, 58, $65,70,74,82,99,105,144,155$, 179, 183, 188, 197 - 199, 208, 213, 218

lexikalisch $3,5,12,15,18,21,23,26$, 30 - 33, 37, 40, 42f., 45 - 51, 71, 74, 76, 78, 80, 91f., 95, 103, 145, 150f., 162, 180, 184, 187, 191, 194, 197 199, 204 - 208, 211 - 220, 234, 236 - 239, 251, 252f., 256, 258

Lexikalisch-Funktionale Grammatik (LFG) 18 - 20

c-structure 20

$f$-structure 20

Lexikon 3, 7f., 18f., 31, 47f., 75, 93, 119, 150, 199, 202, 205 - 207, 217, 247, 250, 256f.

Lexikoneintrag 99, 184, 194f., 203f., $210,214,218,236,257$

liaison 10

Linearitätsregel 46

Liste 28, 31, 34, 41f., 51, 64, 66, 74f., 97 - 99, 107, 125, 128f., 185f., 205, $208,232,250$

Logik 10, 64

lokal 22f., 32, 63, 248

Marker 31f., 60, 62, 186, 192

Markertochter 35

más 73, 90, 94, 98 - 102, 106f., 112, 114, 122, 134, 143, 147f., 155, 164, 168f., 172f., 176, 204, 216f., 230, 236,255 
maskulin 16, 75f., 195, 198, 213

Maßkonstruktion 80f., 250

Maximumverfahren 158, 160, 162f., $168 f$.

Mengenangabe 3, 7f., 10, 13, 26, 28, 38, 42, 55f., 58, 66, 80, 82, 87f., 90, 96, 110, 123, 135, 142 - 146, 153, 162, 199, 205, 207

Merkmal, s. auch Attribut 16 - 40, 43, 46f., 53 - 70, 75, 77f., 84 - 86, 91 112, 118, 124f., 127, 132, 133 135, 138f., 142, 146f., 149 - 157, 160 - 163, 166f., 169, 173f., 176, 178 - 180, 183, 185f., 190 - 195, 199 - 219, 234, 240, 244 - 247, 251, $254-258$

ADJ_SUBST 200

ADJEKTIVTYPISCHE_SUBSTA NTIVIERUNG 200

ADV 200

AGENS 53

AGR 26, 58, 196f., 211, 218, 234

AGR-S 50

AKK 29, 35f., 39, 48, 251

ARG-S 185

ARG-SLOT 193f., 233 - 256

AUX 33, 43

background 196

bewertend 246, 258

Binding 18f., 41

CAT 22, 24, 48, 52, 193f., 213, 234

CATEGORY 22, 33, 54

C-INDEX 70

COMP-DTRS 39 - 41
COMPS 50f., 185

CONT 22, 24, 54f., 65 - 67, 85, 194,250

CONTEXT 23f., 70, 86, 199, 246

CONTEXT-INDEX 70

DECL 186

DET_SUBST 201

DTRS 20, 35, 39 - 43, 51f., 58, 194

fin $32,39,42,48,82$

focus 247

F-Töchter 51

FUN-DTRS 194

F-Wert 51

GOAL 53

ground 247

head 186

HEAD 22, 24, 32 - 34, 36, 39, 45 49, 58, 61, 76, 184, 194, 196, $211,213,219,234,252,257$

HEAD-DTR 39 - 43, 52

HUMAN 199, 245, 251

Index 17, 28, 40, 57, 70

INDEX 54, 65

inf 32,48

Instance $31,53-56$

INV 43

KOMB_1 205, 210, 257

KOMB_2 205, 257

KOMP 200

KONJ 35

LEX 23, 33, 41 - 45, 48, 208

lex_zeichen $23,26,30$

LOC 22f., 39, 48, 52, 55, 69, 193f., 213,234 
(Merkmal)

MARKED 193f., 208, 211, 213

MARKER 210

MOD 60 - 62, 186, 193, 233

MORPH 48

NFORM 32

NOM 15 - 17, 29, 35, 39 - 42, 47, 48

NON-LOCAL $22 \mathrm{f}$.

NORMAL 32, 35

NOUNFORM 32

Ntr 155, 161, 166, 173, 179f.

NUCLEUS $65 \mathrm{f}$.

NUM 21

per $22,28,47 f ., 197,238$

per_num 47f.

PFORM 32

PHON 22, 38

phrasales_zeichen 23, 26

PRD 33, 61f.

psoa $53-56,65 f$.

qfpsoa 66

QSTORE 65 - 67, 70

QUANTIFIERS 65

QUANTIFIERSTORE 65

QUANTS 66

ref-obj 55

RESTIND 65

RESTR 55, 65

RESTRICTED-INDEX 65

RETRIEVED 66f.

RETRIEVED-QUANTIFIERS 66

SEM 22

sGEN 59, 197
SN 73

sNUM 59, 197, 199

SOURCE 53

SPEAKER 70

SPEC 60, 185f., 193

SPER 59, 197

SPR 46, 50f., 185, 192f., 208, 211

SS 39, 193f., 213, 234

ST 212, 216

STATUS 194, 213, 233

STORED $67 \mathrm{f}$.

SUBCAT 24, 28, 35 - 43, 48f.

SUBJ 48 - 51, 185

SYN 22

SYNSEM 22f., 39, 48, 51, 55, 61f., 69, 85, 194f., 213

VAL 50, 52, 185, 192, 211

VFORM 32, 48

mero 94, 96, 98, 100f., 103, 106, 108, 116 - 119, 126 - 134, 143 - 151, 155, 164, 168 - 173, 183, 201, 206, 216,255

mi 53, 69f., 88, 92, 94, 98f., 106, 117 - 122, 126 - 133, 138f., 142, 147 155, 164 - 168, 172f., 183, 205f., $210,216,220,249,250,255$

Minimumverfahren 158, 160, 162 mío 70, 92, 94, 98, 102, 105 - 111, 116 - 120, 126 - 128, 134, 138f., 142,146 - 148, 153, 155, 164, 168 173, 204, 214, 215f., 219, 220, 255, 257 
mismo 94f., 98, 101f., 105 - 108, 112, 114 - 120, 127 - 130, 132 - 134, 137f., 143, 145, 147 - 151, 155, 164, 168f., 172f., 183, 201, 204, 216, 232, 255

Mittelwertverfahren 158, 162, 164, 168,255

Modalverb 250

Modifizierer 62, 189f., 233f.

Modus 47

monopositional 240

Morphem 10f., 13, 90

Morphologie 1, 9f., 31, 47f., 79, 90f., 94, 98f., 152f., 155, 160f., 163, 175, 179, 183, 191, 194, 253f., 259

Morphophonologie 46

Morphosyntax 9, 93, 191

mucho 64, 90, 94f., 98, 100 - 102, 105 - 107, 114, 117, 120, 123, 125, 127, 129 - 134, 137, 143 - 151, 155, 157, 164 - 168, 172 - 174, 216, 255

Multiplikativa 88f., 117, 143, 147f.

Nachstellung 91, 95, 97, 111f., 117f., 124, 152, 153, 167, 170, 176, 204f., $207,215,220,231$ - 246, 254 - 258

Negation 29, 74, 95, 146

Neutrum 59, 75, 195

ninguno 94, 98, 106f., 112, 114, 116f., 120, 127, 129 - 133, 143, 144,146 - 150, 155, 157 - 159, 164 - 166, 168, 172 - 174, 183, 204, $216,232,255$
Nomen 1f., 4f., 8, 10, 12f., 16, 21, 29, 31 - 36, 45f., 48, 50, 53, 55f., 59 65, 69 - 95, 98, 100, 103f., 107, 109 - 111, 114, 118f., 122, 125, 132, 135, 137 - 140, 142, 153, 162, 183 195, 198f., 203 - 214, 217f., 220, $229,231,234-259$

Abstrakta 77f.

Appellativa 72

Gattungsname 77, 187, 204

Gattungsnomen 72, 187, 189, 197, 203, 212, 253, 256, 258

Kollektivum 76f., 196

Konkreta 52, 77

Massennomen 76 - 78, 81, 203f., $211,217,253$

Sorte 211, 219

nomenähnlich 103

nominal $12,50,63 f ., 69,73,80,90$, 162, 204, 230, 234, 240, 242, 244f., 249, 252f., 256

Nominalphrase 1 - 4, 11, 13 - 21, 33 50, 54, 60, 63f., 67, 69, 71 - 91, 96, 104 - 111, 117 - 123, 132, 134, 137, 141f., 153, 177, 183 - 188, 191, 194, 195, 196, 197 - 215, 229 - 239, $243-259$

Nominativ 39, 86, 105

Nominativobjekt 47

nuestro 69, 88, 94, 98, 139

Nukleus $91 f$.

Nullartikel 176

num-Einheit 79 
numeral 77 - 84, 143, 162, 187f., 203, $220,253,256$

Numeralia 1, 87 - 90, 94, 98, 116, 118, 125, 136, 143 - 147, 174, 177 $181,202,237,258$

Numerus 16f., 21, 26, 47, 58, 61, 73, 75 - 80, 84, 91, 97 - 100, 105, 112, $118,124,140,152$ - 155, 161, 166, 173, 176, 179f., 194, 196, 198, 219, 254

Numerusdifferenzierung 100,145 , 155,179

Numerusmarker 78

Numerusrealisierung 77, 100, 163, 176

Oberflächenstruktur 12

Objekt 18, 30, 34, 36, 49, 51, 55, 62, 70, 76, 81f., 142, 158, 162, 247, 253 objektiv 46, 61, 137, 143, 231, 232, 235, 238f-, 244, 246, 258

Objektpronomen 86

Obliquitätshierarchie $34,36,45,50$, 185

Operator 87, 142, 189f-

Opposition 19, 59, 61, 75, 153, 190, $200,208,229,236-251$

Optionalität 2, 34, 83, 89, 208, 229

Ordinalia 88f., 95, 114, 117, 124, 143, 147f., 151, 207

otro 2, 90, 94f., 98, 106 - 108, 115 138, 143 - 150, 155, 164, 168 - 175, $206-209,216$
Packer 81

Packungsnumerus 81

Paraphrasierung 76

Parser 19

Parsing 18

participle-Form 32f.

partitiv $240-244,258$

Passiv 32f., 60, 76

Person 16f., 26, 47f., 58f., 67, 73, 75, 137 - 142, $194-196$

Personalpronomen $1,4,32,56,70$, 75f., 86, 95, 105, 112, 137, 161, 220

Pfad 22, 25 - 28, 48, 52, 55, 69, 193f., 213,234

Phonem 9, 12

Phonetik 9, 12, 46

Phonologie 9, 12, 20, 22, 23, 38, 46, 188,218

phrasal 21, 23, 26, 33, 41f., 51, 78, 80, 84, 208f., 229, 258

Phrasenstrukturgrammatik 3f., 12 20, 31, 36f., 71, 253

Phrasenstrukturregeln 20,39, 41

Phrasentyp 36, 38

Plural 10f., 17, 78 - 85, 94, 99f., 146, 162, 187 - 189, 196, 203, 211, 218, 253,256

Pluralrealisierung 99

росо 94f., 98 - 101, 106 - 108, 114 $120,123,126$ - 133, 143 - 151, 155, 164 - 168, 172 - 174, 216, 255

Polarität 53 - 55

Positionsgrammatik 238 
Possessivpronomen 1, 69f., 85 - 90, 94, 98, 103, 111, 114, 122 - 126, 136, 138, 141, 143, 151, 153, 207, $220,232,237,255$

Postdeterminierer 120, 123 - 127, 131 - 134, 162

Prädeterminierer 120 - 122, 126, 131 - 134, 147f., 210, 215

Prädikat 11, 58, 97, 102, 104, 112, 152

Prädikatenlogik 53f., 69, 87

prädikativ 33, 59, 61f., 76, 96f., 102 104, 194 - 199, 245

Pragmatik 3, 6, 9, 24, 46, 70f., 86, 195f., 199, 203, 205, 239, 247, 254, 258

Präposition 5, 13, 31 - 33, 36, 48 - 50, 63f., 72, 77, 80, 88, 106, 184, 199, 229, 230, 248 - 252, 258

Präpositionalobjekt 73,76

Präpositionalphrase 4, 12, 33, 35, 46, 48, 63f., 71, 108, 196, 199, 210, 229f., 248 - 252, 258

Pro-Formen 86

Pronomen 4, 32, 55f., 70 - 75, 85 - 89, 105, 135 - 138, 143, 187f., 196f., 203f., 211f., 220, 253, 256, 258

prosodisch 247

q-Determinierer $88 \mathrm{f}$.

Qualitativa 232

Quantifikation 58, 65, 96f., 109 - 112, $152,184,258$
Quantifizierer 64 - 70, 87, 109, 136f., 140, 143f., 151, 153, 207

Quantor 64-68, 87 - 90

Redundanz 17, 30, 104f., 195, 218

Referentialität 56

referentiell 55f., 244

Referenzbereich 139, 236, 239 - 243

Regelsystem 8, 19, 45

Regeltyp 235

Rektion 18f., 41, 48, 191

Rektionsauslöser 191

Rekursivität 13, 28

Relationsadjektiv 245

relativisch 252

Relativpronomen 85, 229f.

Rhema 247

Sachverhalt 9, 52 - 55, 122, 126, 137, 238, 240, 246

Satellit 91f., 165 - 171, 255

Schema 18, 36, 41 - 43, 51, 62, 71, 87, 170, 173, 174, 190, 192, 208, 233f., 254

Funktorschema 192 - 194, 211, 259

Kopf-Adjunkt-Schema (headadjunct-schema) 192, 233

Kopf-Funktor-Schema 233

Kopf-Marker-Schema 186

Kopf-Spezifizierer-Schema (headspecifier-schema) 192

Schnittmenge 240, 242

Schruktur

Kopf-Adjunkt-Struktur 62 
segundo 11, 88, 90, 94, 98, 106 - 108, 112, 117 - 134, 147 - 150, 155, 164 - 168, 172 - 175, 183, 201, 204, 216,255

Semantik 1, 3f., 7, 9 - 12, 20, 22, 24, $31,38,52,54,57$ - 66, 71, 77, 79, 84 - 96, 102, 112 - 114, 118 - 121, 124, 126, 134f., 141 - 153, 160, 163, 171, 174f., 183 - 186, 189, 191, 194 - 202, 205, 220, 229 - 259

sendos 94, 98 - 100, 106, 114 - 117, 120, 127 - 133, 143, 147 - 149, 155, 164 - 168, 172f., 216, 232, 255

Singular 11, 49, 76, 144, 146, 162 singulär $75,122,142,187,189,192$, 203f., 208, 211, 217f., 245, 253 258

Sinnkongruenz $196 f$.

Situationssemantik $52 \mathrm{f}$.

Skopus 67, 69

Sorte 3, 21 - 23, 25, 30 - 42, 55, 65f., 81, 184, 201f., 211 - 219, 234, 253, 256,258

adj_Det 202f., 211, 217, 219, 256f.

d-element 202f., 211, $256 f$.

Det 13, 31, 163, 166 - 168, 174, 202f., 211, 213, 216, 219, 256 258

det_Adj 202f., 256f.

pl-mass 204, 218f., 253 - 258

sg-count 204, 211, 217, 219, 253 258
Sortenhierarchie $3,23,25,30,86$, 184, 191, 201 - 204, 217f., 234, 256 $-259$

Sortenidentität 25

Sortensymbol 22

Sortierer 81

Sorter 81

Speicher 65

Spezifizierer 46, 50, 71, 92, 185 193, 208 - 211, 259

Spezifiziererliste 211

Spezifizierung 87

Sprachfamilie 9

Sprachsystem 7f.

Sprachtypologie 254

Sprechakt 144

Sprecher 6 - 8, 61, 66, 70, 86, 95, 112, 116, 136 - 138, 143f., 195f., 199, 205, 210, 235f., 239 - 247, 251,258

sprecherabhängig 246

Sprechsituation 238, 246

Startsymbol 13 - 15

Status 2, 73, 208, 213, 229, 235, 246

Statuswechsel 214

steigerbar 94, 102

Steigerung 101, 237

Steuerer 148, 150

Stoffnomen 78

Struktur

head-adjunct-structure 41, 58, 62

headed-structure 40, 51

head-nexus-structure 41

head-specifier-structure 41 
(Struktur)

head-structure 41

Kopf-Komplement-Struktur (headcomplement-structure) $40-43$, 51

Kopf-Komplement-Strukture (headcomplement-structure 40f., 58

Kopf-Komplement-Strukture (headcomplement-structure) 41

Kopf-Subjekt-Struktur (headsubject-structure) 41, 51

Strukturalismus $8 \mathrm{f}$.

Strukturbaum 20, 35, 65

subject-drop-languages 47

Subjekt 11, 16, 18, 34 - 37, 40, 42, 47

- 52, 58f., 69, 71, 73, 76, 185, 194 198, 248, 253f.

subjektiv 46, 61, 143f., 205, 232, 241

- 246,258

subjektiv-affektiv 232

Subjektivität 145, 235

Subjektkongruenz 11, 17

subjektlos 3, 50

Subjektphrase 39

Subjekt-Prädikativ-Kongruenz 194

Subjektpronomen 34, 86

Subjektsatz 35

Subjekt-Verb-Kongruenz $\quad 27, \quad 194$, 198,211

subjuntivo 35

Subkategorisierung, s. auch Valenz 28, 32 - 39, 50f., 71, 186, 189, 192,229
Subkategorisierungsliste 24, $34-44$, 47, 49, 50, 52, 57, 60f., 71, 76f., $184-186$

Subordination 230

Substantiv, s. Nomen

substantivierbar 11, 73, 90f., 93, 97, 103 - 106, 112, 118, 152, 155, 161f., 166, 173, 179f., 200f., 204, 219

Substantivierer 90 - 92, 112, 219

Substantivierung 77, 94, 97, 103 107, 152, 162, 167, 176 - 180, 187, 213f., 219f., 254, 257

Subsumtion $24-28$

Subsumtionsrelation 25 - 27

Suffix 102

Superlativ 102

Symbol 13 - 15, 18, 26f., 31, 36f. nicht-terminale 13f., 18, 31, 36f.

terminale 13f., 31,37

synchron 8

synonym 21f., 73, 109, 119, 145

tal 94, 98 - 101, 106f., 112 - 117, 120, 127, 129 - 139, 143, 145 - 151, 155,

164, 168 - 174, 204, 216, 238, 255

tanto 94, 98 - 101, 106f., 114, 117 120, 127 - 138, 144 - 151, 155, 164

- 168, 172 - 174, 216, 255

Teilmenge 242

Teilmengenrelation 25

Tempus 47

Tiefenstruktur 12, 19 
todo 73, 90f., 94f., 98, 100, 105 - 114, 117f., 120 - 122, 127 - 134, 143, 146 - 149, 155, 164, 168 - 173, 204, $210,215 f ., 255$

token-identity 27

Transformation 12, 19

Transformationsgrammatik 18f., 233

Translation 18

transnumeral 77 - 84, 187f., 140, 203, 208,256

Typ, s. Sorte

Typ-Identität 27

Unifikation 19, 21, 24 - 30, 58

Unifikationsgrammatik 3, 18f., 26

Unifikationsregel 28

Universalgrammatik 9f.

uno 37, 69, 88, 90, 94f., 98f., 106f.,

111, 114, 117f., 120, 126f., 129 -

133, 138, 144, 147 - 150, 155, 161,

164,168 - 173, 212 - 216, 255

Valenz, s. auch Subkategorisierung 24, 183 - 185, 193, 204, 211, 213, 217f., 240, 256, 258

Valenzliste 211

Valenzmerkmal 51, 185, 211, 234

Valenzprinzip 51, 71

Valenztheorie 239f.

varios 64, 73, 88, 94 - 100, 106, 114 120, 127 - 134, 144 - 149, 155, 164

- 168, 172f., 216, 255
Verbalperiphrase 250

Verbalphrase $12,33,36,38,42,44$, 49f.

Vokabular 13

Vollverb 23, 25, 251

Weighted-Average-Linkage-Verfahren 159

Weltwissen 90

whole-splitting 240

wohlgeformt 7 - 10, 16, 24, 124

Wortart 1 - 4, 11, 32, 36f., 72, 77, 85f., 89, 92, 103 - 105, 160 - 163, 167, 169, 171, 173, 179 - 184, 192, 199 - 205, 229, 253, 258f.

Wortartenvertreter 160 - 163, 166, 169 - 171, 174, $179 f$.

Wortebene 191

Wortklasse 72f., 87, 89, 96

Wurzel 156, 162

X-bar-Ebene 208, 211, 233

X-bar-Schema 193, 208f., 259

$\mathrm{X}$-bar-Theorie $12,15,33,104,108$, 153

Zahladjektive 175

Zahlwort 80, 90, 124f., 132, 143, 209, 237

Zeichen 8, 18 - 24, 27, 30 - 37, 41 $46,51,90,194$

Zeichenkette 9 
Zentraldeterminierer $120,123-127, \quad$ zyklisch $28,186,189$ $131-134,162$

Zyklus 256

Zirkelstruktur 192

Zweitdeterminierer 119, 128

Zweiterwähnung 139f. 